

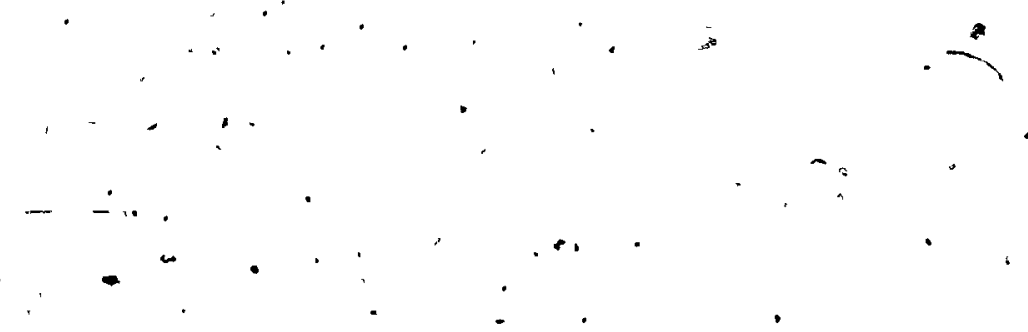

School Mathematics Study Group

Mathematics for the Elementary School, Grade 5
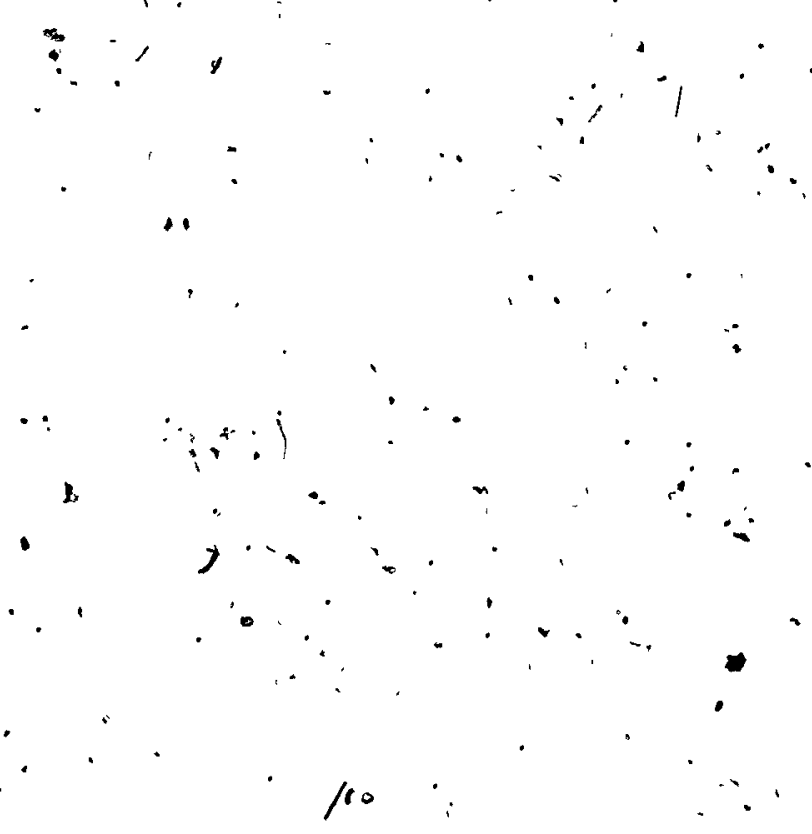

l. Unịit 32 


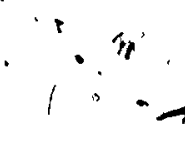

Mathenatics for the Elementary School; Grades

Teacher's Comimentary, Part II

\section{REVISED EDITION}

Prepared under the supervision of the

- Panel on Elementary School Mathematics

of the School Mathematics Study Group:

Leslie Beatty " Chula Vista City School District,

E. Glenadine Gibb $\quad$ Chula Vista, California
Iowa State Teachers Collegc,

Cedar $\bullet$ Falls, lowa

W. T. Guy

University of Texas

S. B. Jackson - . University of Maryland

Irene Sauble, . Detroit Public Schools

M. H. Stone $\cdot$ University of Chicago

J.F. Weaver

Boston University -

R. L. Wilder

Unversity of Michigan -

Now Haven and Londot, Yale University Press 



\section{CONTENTS}

Chapter".

6. ADDITION AND SUBTRACTION OF RATIONAI NUMBERS

- Purpose of the Un1t. ... . . . . . 455

Mathamat1cal Backốround. . . : . 456

Mateplals. . . . . .....".486

Teaching the Unit. : ".". . . .493

Meantng of Rat1onal! Numbers. : * $493^{\circ}, 495$

$257^{\circ}$

Rational Numbers on the Number

Iine.......". . . 501,50

263

P1cturing Rational Numbers on the Number Line.

$508,510 \cdot 269$

Picturing Rational Numbers-wIth

$$
\text { Regions. }
$$

$512,513, \therefore 271$

Rational Numbers with Sets"..

$$
\text { of objects }
$$

518,519

The Simplest Fraction Name for a

- Rational Number.

523,524

27,6

Finding the Simplest Fraction

$$
\text { Name }
$$

.526

282

Common Denominator ......." * 533

Common Muftiple.

535.

290

Least Common Multiple.

538

293

Least Commón Denominator.

544,545 .

Scales on Number Iines:

Addition of Rational Numbers on .

the Number Line.

555,558

'Subtraction of Rationai Numbers." $562,564^{\circ}$

P1cturing. Addition. and. Subtraction

with Regions

$568 ; 569$

299:-

304

Scples for Picturing Addition. ' 572;573

Computing sums and Unknown Addẹnds 577,578

308

312

Diagrams for Probiems:

582,583

316

Properties of Addition of

Ratioral Numbers

$\therefore 587,592^{\circ} 331$

319

323

327

Whole Numbers and Rational

- Numbers. . $\because .$. .

Fractions and Mixed Forms..... . . . .

$\therefore 587,592 \cdot 331$

$597^{\circ}, 335$
$.599,337$


Renaming. Fractions in Mixed Form $603^{\prime}$ Computing with Mixed Forms . . 607,608

Est Imating Sums of Rational Numbets.......

Th1nk1rig About Dec1mals

612,613 345 Addition of Rational Numbers Using Decimals . . . . . Subtraction of Rational Numbers Using Decimals . . . .... Rev̧1ew $\therefore$.

Practice Exercises . . . . . $\because$ Review 618,619

$626,627 \cdot 361$

$636,637 \quad 370$ $646,647: 379$

657.389

Review

660 392

7. MEASUREMENT OF ANGLES: . . . $\therefore 675$

Purpose of Unit.

Mathemat1cal Background. $\therefore \dot{\xi}$

Teaching the Unit.

675.

676

685 .

- Unit Segments and Unit Angles.

Use of Unit Angle in 'Measuring Angles

A Scale for-Measuring Angles.

687,688

407

Drawling ą Angl

Practice in

in Measuing Angiles.

A Standand Unit of Measuring Angles.......$\cdot$

Estimating the Measure of an

Summary.

698,699

417

$-702,703$

420

sum of the Measures of Angles.

713,714

429

431

436

8. AREA ........ $\therefore \ldots$..

Purpose of. Un1t. . . . . . . 743

Mathemat1cal Background. . ... 744

Teaching the Un1t. ....., 760

What is Area?........ 761,763

455

Región and Area. . . . . . . 767

Comparing Areas. 
Chapter

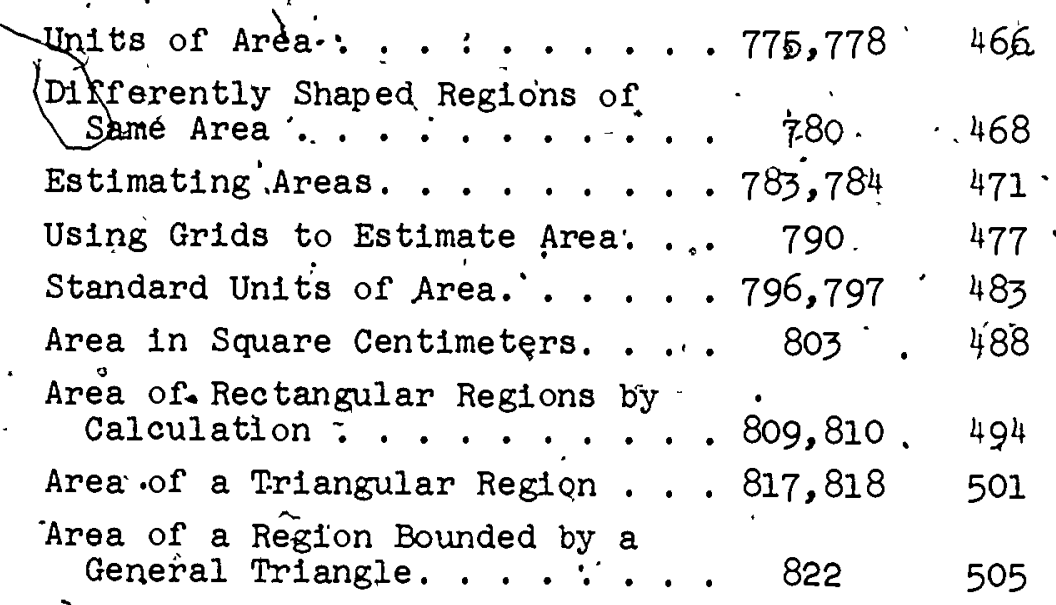

9. : RATIO . . . . . . . . . 827

Purpose of Unit . . . . . . . 827

Mathematical Background:. . . . 828

Tedoring the Unit . . . .. . 836

Introduction to Ratio . . . $837,841 \quad 509$

Different Names for the Same

Rat1o.......'. . 849,852' 517

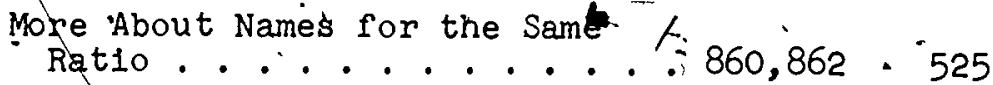

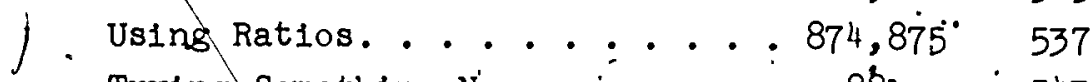

Trying Something New. . . . 881 . 543

Ratios and Rational Numbers... 883

10. REVIEW. . . . . . . . . . 887

Purpose of unit . . . . . . . 887

Teaching Procedures . . .. . . . 888

Concept of Sets......... . 889,549

' Numeration......... $890^{\circ} 550$

Properties and Techniques of
Subtraction, I. . . . . . $892 \quad 552$

Properties of Multiplication and . $896 \quad .556$

Sets of Points. . . . . . $900 \quad 560$

Properties and Techniques of
Addition and Subtraction, II. . 901 
- Chapter

- Tedhniques of Multiplicatian

$$
\text { and Division ...........9.93 }
$$

'Ręcognition' of .Common Geometric

-Figures. . . . . . . . . . 905

Linear Measurement . . . . . . 908

Extendang Systems of Numeration.' 912

572

Factors and Primes . . . . . . 913

573

- Extending Multiplication and Division

Copgruence of Common Gèometric

F1gures............ 919

579

Addition and Subtraction of

Rational Numbers... . . 922

582

Measurement of Angles. . . . . . 925

Area.

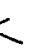

$*$ 


\section{Chapter 6}

ADQITION. AND SUBTRACTION OF RATIONÁL NUMBERS

- PURPOSE OF THE UNIT

The purposes of the unit àre these:

'1. To increase understandifg of the meaning of rational numbers and their use as measures of regions, segments, and . subsets of a set.

2. Tó develop understanding of fraction and decimal notation for rational numbers: to develop fac1lity in renaming rątional numbers,by, fraftion and debimal numerals: to make use of complete factorizations of counting numbers for this purpose.

3. To develop, understanding of the operations of addition and subtraction of rational numbers and of their properties.

4. Tó compute sums and addends using rraction and decimal numerals: to solve problems requiring the use of the operations of addition and subtraction.

5.1 To encourage pupils to discover relations and procedures for themselves, 


\section{Introduction}

In the study of mathematics in the elementary school, a child learns to use several sets of numbers. The first of these is the set of counting numbers,. $1,2,3,4, \ldots$. The second. is the set of whole numbers, $0,1,2,3,4, \ldots$. The child also may have learned certain properties of whole numbers.

During the primary and middle grades the idea of "number" is enlarged, so that by the end of the sixth grade the child regognfzes each of the following as a name for a number:

$$
\frac{4}{3}, \frac{1}{2}, 3.6,2 \frac{1}{2}, 8, i 0, \frac{5}{5}, \frac{6}{2}, .01:
$$

"In traelitional language, we might say that when the ch1ld completed. Fhe first six years of school mathematics he knows about "the whole numbers, fractions, declmals, and mixed numbers." This language is primarily numeral language. It obscures the . fact that a single number can have names of many kinds. "Fract1ons, decimals, and mixed numbers" are kinds of number names rather than different kinds of numbers. Whether we make a plece of ribbon $1 \frac{1}{2}$ in. long, or 1.5 in! long, or $\frac{3}{2}$ in. long makes 'no" difference--our ribbon is the same whatever our cholce of numerar. That is, $1 \frac{1}{2}, 1.5, \frac{3}{2}$ are all names for the same. number. This number is a member of a set of numbers sometimes called the non-negative numbers or the rational numbers of ar1thmet1c. For. our purposes here, we shall call them the ratonal numbers, realizing that they are only a subset of the set or all rational numbers: It also should be realized that within the set of rational numbers is a sjet which corresponds to the set of whole numbers. For example, $0,3,7$ are all rational numbers that are also whole numbers. $\frac{3}{4}, 7$, and .2 . are rational numbers that are not whole numbers. 


\section{First Ideas About Rational Numbers}

Children develop early ideas about rational numbers by working with regions--rectangular regions, circular regions, triangulari regions, e'tc. In Figures A, B, and $C$, rectangular regions have been used. For any "type of region we must first identify the unit region... In Figures $A, B,^{\circ}$ and $C$, the unit region is, a square regtion.

In Figures $A$ and $B$, we see that:

(1) The unit region has been separated anto a number of congruent regions.

(2) Some of the region's have been shaded.

(a) Using regions. Let us see how children use regtons to develop. tneir first ideas of rational numbers. The child learns in simple cases to associate a number like $\frac{1}{2}$ or $\frac{2}{3}$ with a shaded portion of the figure. (Rational numbers can al so be associated with the unshaded portions.).

Using two or more congruent regions (F1g..C.), he can separate each 1nto the same number of congruent parts and shade some of the parts. Again, he can associate a number with the resulting shaded region.

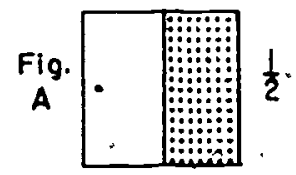

The "unit squäre is separated into 2 , congrueint regions. I 1s. shaded.

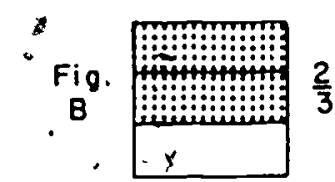

The unit square
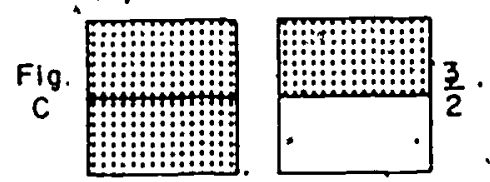

is separated into

3 congment.regions. 2 are shaded.
Each unit square is separated into is congruent regions. 3 are shaded. We have $\frac{3}{2}$ of la unit square.

- At this polnt, the ohild is only at the beginning of $\mathrm{hls}$. concept of rational numbers. However, let us note what we are doing when we introduce, for exampie $\frac{2}{3}$. We separate the (unit) region into 3 congruent parts: "Then we shade 2 of these $\rightarrow$ 
parts. Simflariy, in $\frac{3}{2}$, we separate each (unit) region into' 2 congtruent regions, and shade 3 parts. In using regions to represent a number like $\frac{3}{2}$, we must emphasize the fact that we are thinking of $\frac{3}{2}$ of a unit region, as in Fig. C.

(b) Using the number line. The steps used with regions can be carried out on the number, line. It is easy "to see that this". is a very practical ming to do. If we have a ruler marked only In Inches, we cannot, make certain types of useful measurements. We need to have points between the unit intervals, and we would like to have numbers assoclated with these points.

The way we locate new polnts on the muler parallels the procedure we followed w1th regions. We mark off each unit segment into congment parts. We count off these parts. Thus, in order to locate the point corresponding to $\frac{2}{3}$, we must mark off the unit segrient in 3 "congruent parts. We then count of 2 of them. (Fig. D) If we have separated each unit interval in 2 congruent parts and counted off 3 . of them, we have located the point which we would associate with $\frac{3}{2}$. (F1g. E)

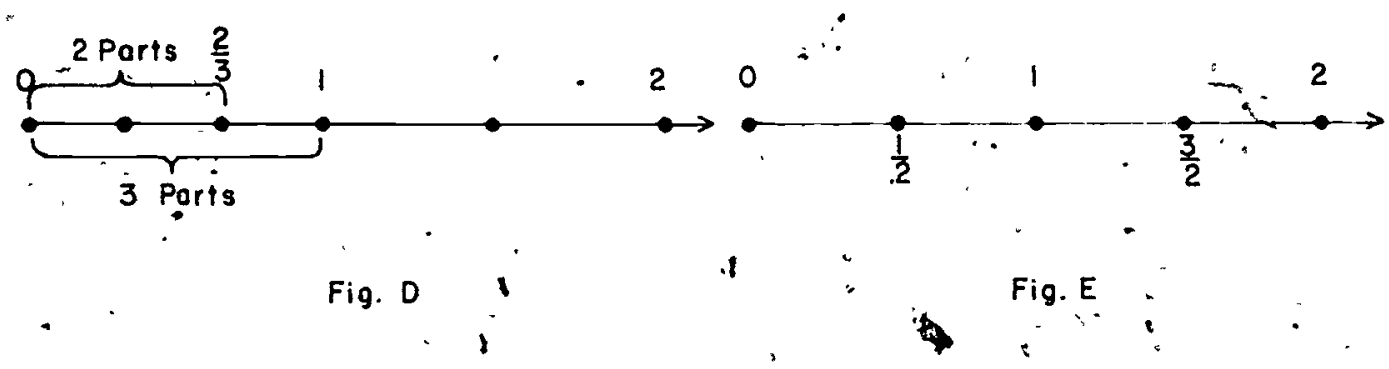

Once, we have this construction in mind, we see that all such numbers as $\frac{3}{4}, \frac{5}{8}, \frac{2}{4}, \frac{1}{3}, \frac{4}{3}, \frac{11}{8}$ can be associated w1th - particuiar points on the number line." To locate $\frac{11}{8}$, for example, we mark the unit segments into 8 congruent segments. (F1;. F)

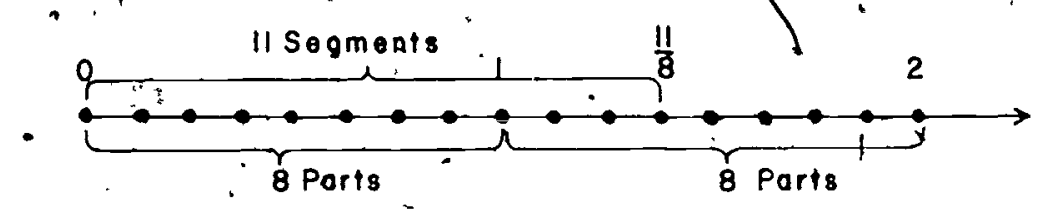

3

F18. F 
(c) Numerals for pairs of numbers. 'Suppose thàt we consider a palr of counting numbers such as 11 and 8 where il 1s the'first, number and 8 is the second number. We can make a symbol, writing theiname of the first number of the pair above the line and that of the second below. Thus for the pair of " numbers; 11 and 8 , our symbol would be $\frac{11}{8}$. If we had thought of 8 as the first number of the pair and $I \bar{I}$ as the second, we would have sald the pair $\beta^{\prime}$ and 11 , and the symbol wouzd have been: $\frac{8}{11}$. For the numbers 3 and 4 , the symbol would be $\frac{3}{4}$. For the numberk 4 and 3 , tne symbol would be $\frac{4}{3}$.

With the symbol fiescribed in the preseding parasraph, we can'assoclate a point on the number line. The second number tells Into how many congment segments to separate each unit segment: The flrst number telis how many segments to count off.

We also can assoclate each of our symbols with a shaded. region as in Fig. $A, B$, and $i C$ : The second number tells us inio how many congruent parts we must separate each unit region. The first number tells us how many of tinese parts shade.

- For young children, 'reglons are easier to see and' to work with than segments. However, the number line has one strong advantage. : For example, we assoclate a number as $\frac{3}{4}$, with exactly one point on the number line. The number line also gives an unamblguous picture for numbers like, $\frac{3}{2}$ and $\frac{7}{2}$. A region corresponding to $\frac{3}{4}$ is less precisely defined in that regions w1th the same measure need not be 1dentical or even congment;

In F18. G, we can see that each shaded region is $\frac{3}{4}$ of a unit square. Recönizing that both shaded regions have $\frac{3}{4}$ sq. units is indeed one part of the area concept.
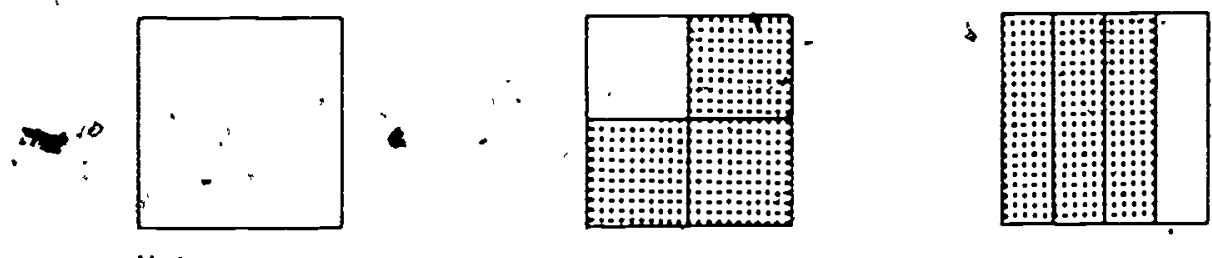

Unst square

Figure $G$ 
When we match numbers with points on the number line, we work with segments that begin at 0 . For tnis reason, though the number line is less intuitive at early stages, it is riell to use It as soon as possible.

\section{Meaning of Ratronal inmber.}

The dtagrams Fig. $\mathrm{H}$, (a), (b), (c), show a number line on which we have located polnts corrsponding to $\frac{1}{2}, \frac{a}{2}, \frac{3}{2}$, etc. and a number line on which we have located potrts zarresponding

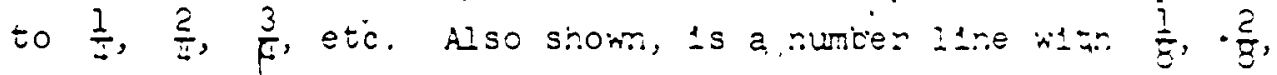
etc. As we look at these lines, re see trat $t:$ seems rer: natural to inink of $\frac{0}{2}$ as ve1-a assoctated We are reaily, so to spear, counttng of: 0 segherts. Similarly, $1 t$ seems ratural to locate $\frac{0}{b}$ and $\frac{0}{g}$ ts irdisated.
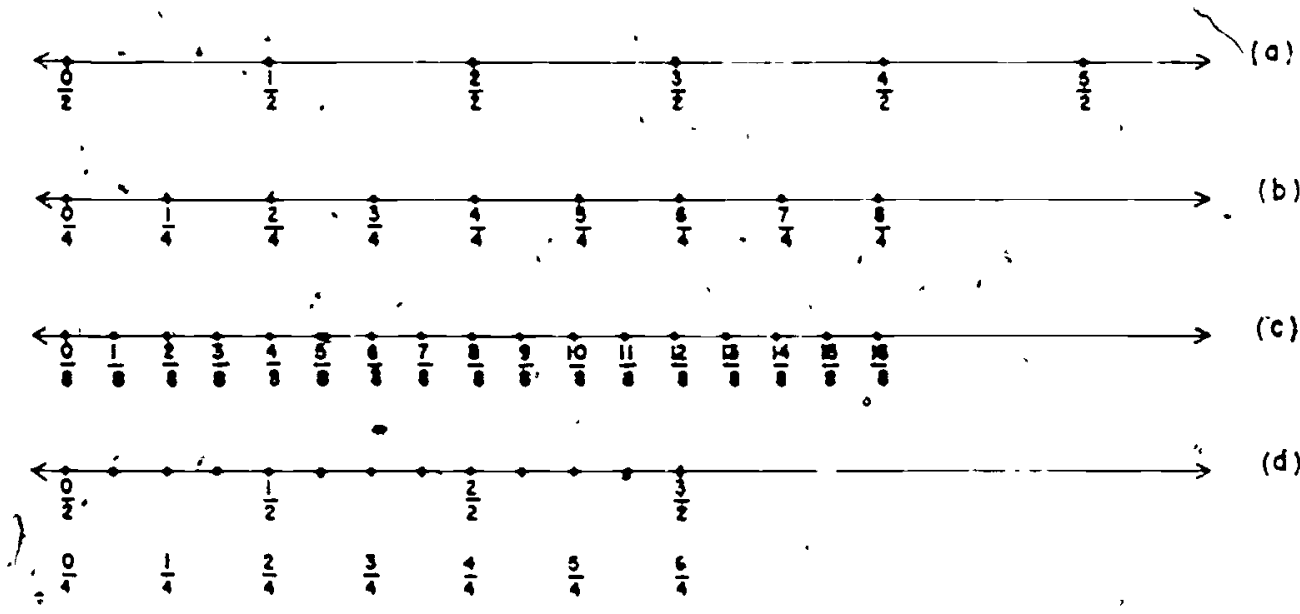

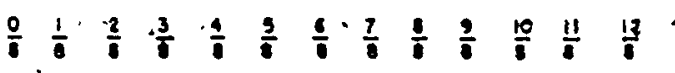

\section{ت2Ture $\because$}

Now lét us put our dtagrams (a), (b), (c) together. "In other words, let us carry out on a single line (d) the process for locating all the points.

When we do'this, we see that $\frac{1}{2}, \frac{2}{4}$, and $\frac{4}{8}$ are all assoclated with the same.point. In the same way, $\frac{3}{4}$ and $\frac{6}{8}$ are associated with the same point. 
Now we are ready to explain more precisely what we mean by fraçtion and by, rational number. Let us agree to call the symbols we-have been using riractions. A fraction, then, is a $\therefore$ symbol associated, with a pair of numbers. The first number of the pair is calied the numerator and the second nimber is called the denominator. So far, we have used only those fractions in wilch the numerator of the number pair is a whole number $(0,1,2, \ldots)$, and the denominator is a counting number . $(1,2,3, \ldots)$.

Each fraction can be used to locate a point on the number Ine. To each point located by a fraction there corresponds a rational number. Thus, a rraction names the rational number. For exarale, if we are told the ifraction $\frac{3}{10}$, we can locate a point that corresponds to 1 t on the number line. $\frac{3}{10}$ is the name of the rational number associated with this point. This point, however; can also be located by means of other, fractions, such as $\frac{6}{20}$ and $\frac{9}{30}$. Thus, $\frac{6}{20}$ and $\frac{9}{30}$ also are names for the rational number named by $\frac{3}{10}$ since they are assoclated w1th the same point. Rational numbers, then, are named by fractions of the type we have been discussing. To each point on the nuppor Ine that can be located by a fraction, there corresponds a nonnegat1ve rational number.

A very unusual child might wonder whether every point in - the number line can be zocated by a fraction of the kind we have described. We must answer "No". There are numbers-m being one of them and $\sqrt{2}$ being another--that have no fraction names of the sort we have described. Introducing such irrational numberis deferred until the seventh and eighth grades.

\section{The hinole Numbers As Rát1onal Numbers}

our pattem for matching rractions with points on the number IIne can be used with these rractions: $\frac{0}{1}, \frac{1}{1}, \frac{2}{1}, \frac{3}{1}$, etc..

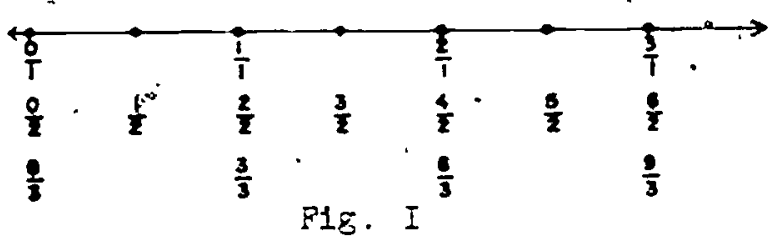


On the number. IIne we see (F1g. I) that we matched ' $\frac{1}{1}, \frac{2}{2}$, $\frac{3}{3}$ with the same point. We note that this point is also matched with the counting number, 1. Thus, to the same point corresponds

(1) the counting number 1

(2) the rational number named by $\frac{1}{1}$.

It seems that 1 would be a convenience to use the symbol 1 as still another name for the rational number named by $\frac{1}{1}, \frac{2}{2}$, etc. This would allow us to write $i=\frac{2}{2}$, for example. In the same way, we would think of 5 as another name for the number named by $=\frac{5}{1}, \frac{10}{2}$, etc. .

We need at this-point to be a little careful in of thinking. There is nothing 1llogical about using any symbol we like as a numeral. A problem does arise, however, when a single symbol has two meanings, because then we are in obvious danger that inconsistencles may result. For example, when we think of 2 , 3 , and 6 as couniting numbers we are accustomed to writins $2 \times 3=6$. We will eventually aefine the product of two rational numbers, and we would be in serious trouble if the product of the rational numbers.named by 2 and 3 were anything but the - rational number named by 6 .

totwever, using $0,1,2,3$, etc., as names for rational numbers never leads us into any inconsistency. For all the. purposes of ari'thmetio--that is, for finding sums, products, etc., and for comparing sizes, we get names for whole numbers or names for rational numberś. In more sophisticated mathenatical terms, we can say that the set of rational numbers contains a : subset--those named by $\frac{0}{1}, \frac{1}{1}, \frac{2}{1}$, etc.--1somorph1c, to the set of whole numbers, that is $\frac{0}{l}, \frac{1}{l}$, etc. behave just like whole ñümbers, 0,1 , etc.

It would be overamb1t1ous to attempt to formulate the 1dea of 1somorphism precisely in our teaching. It is sufficient for - o our purposes to regard $0,1,2$, etc., as names for rational. numbers. It is approprlate to note, however, in connection with operations on rationals, that where the operations are applied to numbers like $\frac{1}{1}$, $\frac{2}{1}$ they lead to results already know from experience with whole numbers. 
Identifying Fractions That. Name The Same Rational Number'

When 'we write $\cdot \frac{1}{2}=\frac{3}{6}$, we are saying " $\frac{1}{2}$ and $\frac{3}{6}$ are. names" for the same number."

(a) Using physical models. The truth of the sentence $\frac{1}{2}=\frac{3}{6}$. an be discovered by concrete experiende. In F1g. J, for example, we have first separated our unit region into two congruent regions. We have then separated each of these parts further into. 3 congruent regions as shown' in the second drawing. The second unit square is thus separated 1nto $2 \times 3$, or 6 parts. Shading 1 part in the first.drawing is equivalent to shading $i \times 3$, or 3 . parts in the. second. We thus irecognize that $\frac{1}{2} x \frac{1 \times 3}{2 \times 3}$.

Shading $\frac{1}{2}$ and $\frac{3}{6}$, of a region.

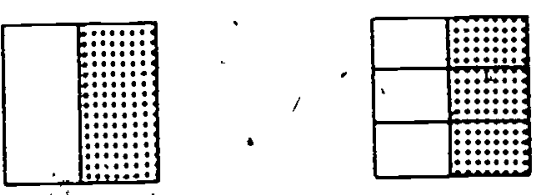

Fig. $J$

Again, our analysis of regions follows a pattern that can be applied on the number 11ne. Let us consider $\frac{1}{2}$ and $\frac{4}{8}$.

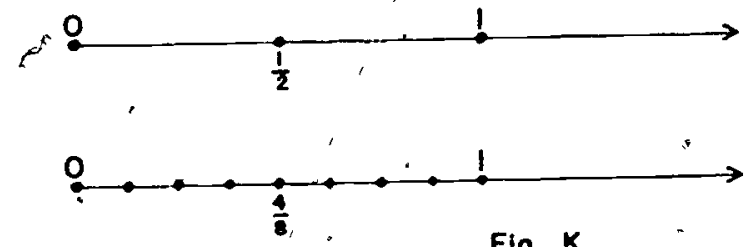

Fig. $K$

In locating $\frac{i}{2}$ on the number line, (Fig. $K$ ) we separate the unit interval into 2 congruent segments. In locating $\frac{4}{8}$, we separate it into 8 congryent segments. We can do this by first separating into 2 parts and then separating each of these 2 segments into 4 ' segments. Th1s process yields $(2 \times 4)$ congruent segments, Taking 1 of 2 congment parts thus leads to the same point as taking $4^{\prime}$ of $8^{\circ}$ congruent parts: .

$$
\frac{1}{2}=\frac{1 \times 4}{2 \times 4}
$$


In other words, when we multiply the numerator and denom1nator of $\frac{1}{2}$ by the same counting number, we can visualize the result using the number 1ine. We have subdivided our $\frac{1}{2}$ intervals Into a number of congruent parts.

After many such experiences, chilidren should be able to make a picture to explain this type of relationship. For example, region and number line pictures for $\frac{3}{4}=\frac{3 \times 2}{4 \times \frac{2}{2}}$ are shown in/ F18. L.
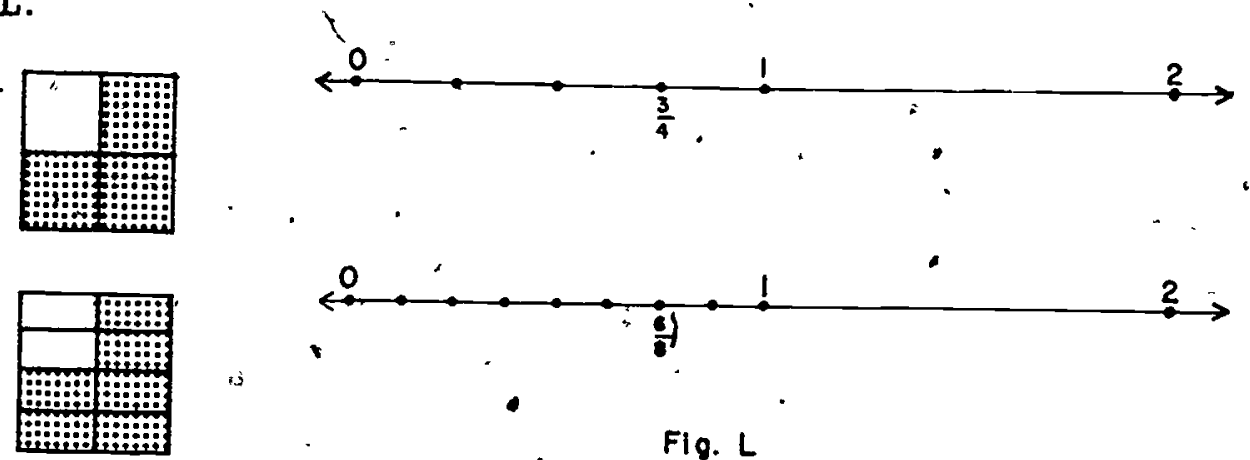

Fig. L

Each $\frac{1}{4}$ ' ${ }^{2}$ part (region or Intervai) is subdivided into 2 ". congment parts; hence $\frac{3}{4}=\frac{3 \times 2}{4 \times 2}$.

(b) Using numerators and denominatiors. In a discussion about two fractions naming the same number, it may appear startling to emphasize multiplying numerator and denominator. by the same counting number. We usually thisk about finding the simplest fraction name if we can. We think, then, $\frac{4}{8}=\frac{1}{2}$. But, of course " $=$ " means "names the same number." Speing $\frac{1}{2}=\frac{4}{8}$, we can think, $\frac{4}{8}=\frac{1}{2}$, and this w111 be particulorly easy if the "names the same number" 1 dea has been emphasized adequatelý.

Another familiar idea also is contained in what has been sa1d." We often think about îjiding numerator and denominator. by the same counting number. For example, we think:.

$$
\frac{6}{8}=\frac{6 \div 2}{8 \div 2}=\frac{3}{4}
$$

This is easy to translate into a multiplicative statement, since multipligation and division are inverse operations: $6 \div 2=3$ means $3 \times 2=6$. 
(c). Using ractoring. The sidea that multiplying the numerator and denominator of a fraction by a counting number gives a new, fraction that names the same number as the original rraction is an idea very well suițed to the discussion in the unit on factoring." "To find a simpler name for $\frac{12}{15}$, we write:

$$
\frac{12}{15}=\frac{2 \times 2 \times 3}{5 \times 3}=\frac{2 \times 2}{5}=\frac{4}{5}
$$

Suppose we are thinking about two fractions. How will we decide. whether or pot they name the same number? There, are two possibilities.

Rule (l). It may be that for such fractions as $\frac{1}{2}$ and $\frac{2}{1 /}$, one fraction is obtained by multiplying the numerator and deriominator of the other by a counting number. In other words, it may be that we can picture the fractions as. was just done. "Since $\frac{2}{4}=\frac{2 \times 1}{2} \frac{1}{2}, \frac{2}{4}$ and $\frac{1}{2}$ belong to the same set--thus name the same number.

Rule (2). It may be that, we cannot use Rule l directly. Fior example, $\frac{2}{4}$ and $\frac{3}{6}$ cannot be tompared directly by Rule $I$. However, we can use. Rule 1 to see that $\frac{2}{4}=\frac{1}{2}$ and $\frac{3}{6}=\frac{1}{2}$, and In this.way, we see that $\frac{2}{4}$ and $\frac{3}{6}$ name the same number.

Notice that in comparing $\frac{2}{4}$ and $\frac{3}{6}$, we might have used Rule $l$ and 2 in a different way. We might have recognized that:

$$
\frac{2}{4}=\frac{2 \times 3}{4 \times 3}=\frac{6}{12} \quad . \quad \text { and } \quad \frac{3}{6}=\frac{3 \times 2}{6 \times 2}=\frac{6}{12} .
$$

or we might have said:

$$
\frac{2}{4}=\frac{2 \times 6}{4 \times 6}=\frac{12}{24} \quad \text { and } \quad \frac{3}{6}=\frac{3 \times 4}{6 \times 4}=\frac{12}{24} .
$$

In the latter example, we have renarded $\frac{2}{4}$ and $\frac{3}{6}$, using fractions with denominator $4 \times 6$. Of course, we recognize that. $4 \times 6 .=6 \times 4 \cdot \cdot$ (Commutąt1ve Property)

$\therefore$ In our example, we sé that $2^{4}$ ' 13 a common denominator. for $\frac{2}{4}$ and $\frac{3}{6}$, though it is not the least common denominator. Nevertheless, one common dendminator for two fractions is always the product of the two denominators. 
(d.) A special test. Let us now consider a special tes't for two fractions that name the same rational number. In our last example we used $6 \times 4$ as the common denominator for $\frac{2}{4}$ and $\frac{3}{6}$. Thus we had

$$
\frac{2}{4}=\frac{2 \times 6}{4 \times 6} \quad \text {. and } \quad \text { ए } \frac{3}{6}=\frac{3 \times 4}{6 \times 4} .
$$

We could say: It is true that $\frac{2}{4}=\frac{3}{6}$, because the two resulting numerators--2 $\times 6$ and $3 \times 4$--are equal, and the denominators. are equal.

In other fwords, to test whether $\frac{2}{4}=\frac{3}{6}$, it is onfy necessary--once you, have understood the reasoning--to test whether" $2 \times 6=3 \times 4$. 'A this last number sentence is true'! In the-same way, we can test.whether $\frac{9}{15}=\frac{24}{40}$ "by testing whether $9 \times 40=8 \times 15$. They do! When we do this, we are. thinking:

$$
\frac{9}{15}=\frac{9 \times 40}{15 \times 40} " \text { and" } \quad \frac{24}{40}=\frac{24, \times 15}{40 \times 1.5}
$$

This is an example of what is sometimes called "cross product mile." It is very usèful in solving proportions. (Sometimes it is stated: The product of the means equals the product of the extremes.).

The rule states: To test whether two fractions $\frac{a}{b}$ and $\cdot \frac{c}{d}$ name the same number, we need only test whether $a \times d=l b \times c$. That is,

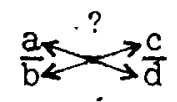

This rule. is important for later applicatios in mathematics such as similar trlangles. In advanced texts on algebra, it is sometimes used as $\bar{a}$ way of defining rational numbers. That is, an advanced text might. say: "A tional number is a set of symbols like $\left\{\frac{1}{2}, \frac{2}{4}, \frac{3}{6}, \frac{4}{8}, . \therefore.\right\}$. Two symbols, $\frac{a}{b}$ and $\frac{c}{d}$, be long to the same set if $a \times d=b \times c$."

What we have done amounts to the same tring, but is developed more intuitively. For teaching purpozes, the "multiply numerator and denominator by the same counting numper." Idea. conveyed by Rule l' can "be visualized more easily "than can the "crass product", rule. 
It would certainly not be our intention to insist that chlldren learn'Rules $l$ and 2 formally. However, these rules. sumarize an experience that is äpropriate for children. "We can form a chain of fractions that name the same number,

$$
\frac{1}{2}=\frac{2}{4}=\frac{4}{8}=\frac{8}{16}=
$$

Each rraction is formed by multiplying the numerator and - denominator of the preceding one by.?. We can visualize this as subdividing repeatediy a segment or a region. (Rule $l_{y}$ ). "s We can form a second chain beginning with $\frac{1}{2}=\frac{3}{6}=\frac{9}{18}$ We can then understand that it is possible to pick out any numeral from one chain and equate it, with any numeral from the other,.. which is Just what Rule 2 says.

\section{Meaning of Rational Number - Summary}

Let. us summarize how far we have progressed in dur development of the rational numbers.

(1) We regard a symbiol like one of the following as naming a rational number:

$$
\text { - } \frac{3}{8}, \frac{0}{5}, \frac{7}{6}, 6, \frac{4}{3}, \frac{6}{4}, 1, \frac{5}{5} .
$$

(2) We know how. ton assoclate each suck symbol with:a point on the, number line.

(3) We know that the same rational number may have many names that are rractions. Thus, $\frac{6}{4}$ and $\frac{3}{2}$ are riaction names for the same number.

(4) We know that when we hąve a rational number named by a.fraction, we can multiply the numerator and denominator of the fraction by the same counting number to obtain a new fraetion name for the same rational number.

(5) We know that in comparing two rational numbers it is useful to use fraction names that have the same..denomfnations. We know, too, that for any two rational numbers, we can always find fraction names of this sort.

Thus far we have not stressed what is oftẹn called, in traditional language, "reducing fractions." to "reduce" $\frac{6}{8}$, 
- for example, is simply to name. It with the name using the smallest possible numbers for the numerator and the denominator. Since 2 is a factor. both of 6 . and 8 , we see that

$$
\frac{6}{8}=\frac{3 \times 2}{4 \times 2}=\frac{3}{4}
$$

We have applied our general idea that "multiplying numerator and denominator by the same counting number" gives a new name for. the same number. We can call $\frac{3}{4}$ the slmplest name for the rational number it' names.

We would say that we have found, in $\frac{3}{4}$, the simplest name For the rational number named by $\frac{6}{6}$. This is more precise than saying we have "reduced" 6 , since we have not made the rational number named by. $\frac{6}{8}$ any smaller. We have used another pair of - numbers to rename $1 \mathrm{t}$.

(6) We know, also, that 2 and $\frac{2}{1}$ name the same number. We thius regard the set. of whole numbers as a subset of the set - of rational numbers. Any number in this subset has a fraction name with denominator 1., $\left(\frac{0}{1}, \frac{1}{1}, \cdot \frac{2}{1}\right.$, etc. belong to this subset.) 2 is a name for a rational number which is a whole number. 2 is not a fraction name for this number, but the number has fraction names $\frac{2}{1}, \frac{4}{2}$, etc.

At this point, it seems reasonable to use "number" for rational numbers where the meaning is clear. We may ask for. the number of inches of measure of a stick, or the number of hours in a school day.

(7) We can agree th speak of the number $\frac{2}{3}$, to avold the . wordiness of "number named by $\frac{2}{3}$." Thus, we might say that the number $\frac{2}{3}$ is greater than the number $\frac{1}{2}$ (as we can verify easily on the number line) This, would be preferable to saying that "the fraction $\frac{2}{3}$ is sreater than the fraction $\frac{1}{2}$," because we do not mear that one name is greater than another.

- (8). We should not say that 3 is the denominator of the . number $\frac{2}{3}$, because the sam̃e number kas other names (I1ke $\frac{4}{6}$ ) with different denominators. $3^{*}$ is rather the denominator of the rraction $\frac{2}{3}$. 
(9) We have seen that the idea of rational number 'is relevant both to regions and line segments. We, will see ston how $y$ t relates to certain problems involving sets.

Now we might introduce some decimals. The numeral, .1, for example, is another name for $\frac{1}{10}$. However, we can expiain. a numeral like I.7- more eas1ly when we have developed the Idea, of adding rational numbers.

\section{Operations on Rational Numbers}

Now let us consider the operations of arithmetic for rational numbers. For each, our treatment will be 'based' on three considerations:

(1) The 1dea of rational number grows out of 1deas ábout regions and the number line. Similariy; each operation on rational numbers can be "vjsualized" in ferms of rejgions or the number line. . Indeed, this is how peofle originally. formed the ideas-of sum, product, etc. of rational numbers. - Each operation was introduced to fit a useful physical situation and not as a way of supplying problems for arithmetie textbooks.

(2) We reoall that some rational numbers are whole numbers. So, iwe want our rules of operation to be consistent with what we al ready kñow about whole numbers.

(3) We must romember that the same rational number has many names. We will want to be sure that the result of an operation on twe. numbers does not depend on the special names. we choose for them. For example, we want the sum of $\frac{1}{2}$ and $\frac{1}{3}$, to be the same number as the sum of $\frac{2}{4}$ and $\frac{2}{6}$.

These three ideas will guide us in defining the operations - of addition, subtraction, multiplication, and division of ratjonai numbers.

\section{Addition and Subtraction}

As. an illustration of addition, we might think of a road by which stand a house, a school and a store. as shown in Fig. M. - If it is $\frac{1}{2}$ mile from the house to the schoól, and. $\frac{1}{4}$ mile 
from the school to the store, then, we can

see that the distance from the house to

the store is: $\frac{3}{4}$ mile.

From such examples we can see the

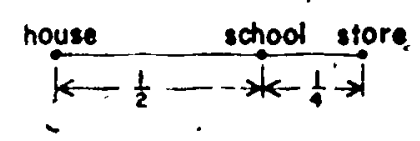

utility of defining addition of rational numbers by using the number line: To find the sum of $\frac{2}{5}$, and $\frac{4}{5}$ we would proceed as in Figure $N$.

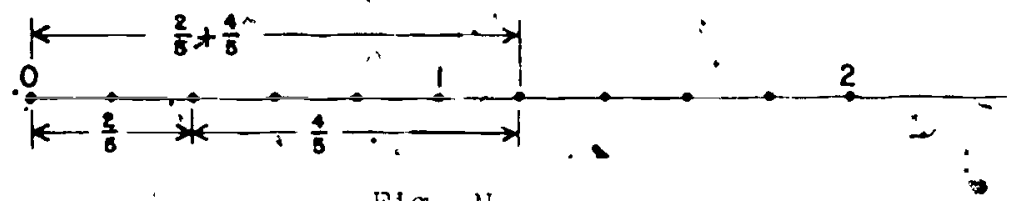

FIE. IS

Using a ruler, we can locate the point on the number line corresponding to the 'sum Qf any' two rational numbers. For example, with appropriate rulers, a ch1ld can locate the point. that corresponds to the sum of $\frac{4}{5}$ and $\frac{3}{8}$. But a child would also like to know that the point for the sum located with a. ruler is one for which he can find a fraction name--a fraction that names a rational number. Of course, one name for tine sum. of $\frac{4}{5}$ and $\frac{3}{8}$ is $\frac{4}{5}+\frac{3}{8}$, but what is the single iraction that names this number? Also, he is interested in knowing whether or not the set of rational numbers is closed under addition,. since, he knows that this is true for the whole numbers.

Using the number line, it is evident that the sum of $\frac{2}{4}$ and $\frac{3}{4}$ is $\frac{5}{4}$. This suggests a way to fald the sum of two rational numbers that are named by fractions with the same denominator. For such fractions, we simply add the niumerators: Thus, $\frac{3}{8}+\frac{4}{8}=\frac{3+4}{8}=\frac{7}{8}$. This definition matches the idea of -joining two line segments.

. But we are not finished! For suppose that we want to add. $\frac{2}{3}$ and $\frac{1}{4}$. We know that there are many other names for the num-; , ber named by $\frac{2}{3}$. Some are: 
Ilkew1se, there are many names for the number named by $\frac{1}{4}$. They Include:

$$
\frac{2}{8},: \frac{3}{12}, \frac{4}{16}
$$

In order to find the sum of those two rational numbers: we simply look for a pair of names with the same denominator-that is, 'with a common denominator. Having found them, we apply gur simple process of. adding numerators.

Thus we can wrote a fraction name for the sum of two Trational numbers if we can write fraction names with the same denominators, for the numbers. This we can always do, for to find the common denominator of two fractions, we need oniy to . find the product of their denominators.

This provides a good argument as to why.

$$
\frac{2}{3}+\frac{1}{4}=\frac{11}{12}
$$

It is clear that $\frac{8}{12}+\frac{3}{12}=\frac{11}{12}$. If the idea that the same number has many names makes any sense at all, it must be true that

$$
\frac{2}{3}+\frac{1}{4}=\frac{8}{12}+\frac{3}{12}
$$

Suppose that in our example we had used a different common denominator, as 24 . Would we get a different result? We see that we would not ror:

$$
\begin{aligned}
& \frac{2}{3}=\frac{2 \times 8}{3 \times 8}=\frac{16}{24} \\
& \frac{1}{4}=\frac{1 \times 6}{4 \times 6}=\frac{6}{24} \\
& \frac{2}{3}+\frac{7}{4}=\frac{22}{24} \\
& \text { and } \frac{22}{24}=\frac{11}{12} .
\end{aligned}
$$

ciftie needs be said here abput subtraction. Using"the number line we can visualize $\frac{3}{4} \cdot-\frac{2}{3}$ as in Figure 0 .

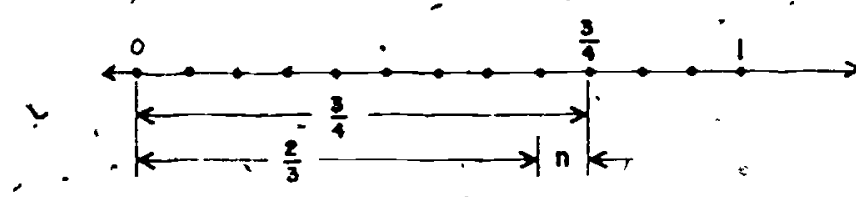

$$
\begin{gathered}
\text { F1E. } 0 \\
471 \\
\therefore 2 i
\end{gathered}
$$


Thus we can define $\frac{3}{4}-\frac{2}{3}$ as the number $n$ such that . $\frac{2}{3}+n=\frac{3}{4}$. Again, skillfully chosen names 'lead at once to the. solution:

$$
\begin{aligned}
\frac{8}{12}+n & =\frac{9}{12} \\
n & =\frac{1}{12}
\end{aligned}
$$

\section{Properties of Addition for Rational Numbers}

our rule for adding rational numbers has some by-products worth noting.

We can see, for one thing, that addition of rational numbers is commutative. Our number line diagram illustrates this. In FIg. ? we see the diagram for $\frac{1}{5}+\frac{2}{5}$ and for $\frac{2}{5}+\frac{1}{5}$.

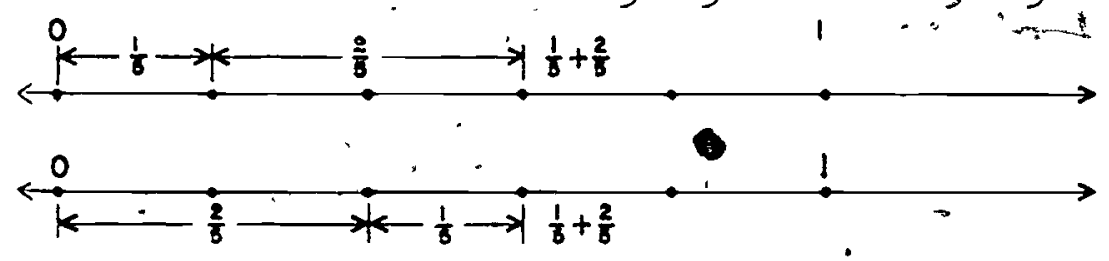

\section{Fig. P}

The commutative property also can be explained in another way.

$$
\frac{1}{5}+\frac{2}{5}=\frac{1+2}{5} \text { and } \frac{2}{5}+\frac{1}{5}=\frac{2+1}{5}
$$

We know" that $1+2=2+1$, so we see that $\cdot \frac{1}{5}+\frac{2}{5}=\frac{2}{5}+\frac{1}{5}$ " In general, to add rational numbers named by fractions with the same - denominator we simply add numerators. Adding numerators involves adding whole numbers. We know that addition of whole numbers is commutative. This leads us to conclude that addition of rational numbers is also commutätive.

We can use this type of discussion or the number line diagram to see that addition of rational numbers is also associative.

- Here is another. Interesting property of addition of rational numbers. We recall that $\frac{0}{1}, \frac{0}{2}, \frac{0}{3}$, etc. are all names for 0 . Thus $0+\frac{3}{4}=\frac{0}{4}+\frac{3}{4}=\frac{0+3}{4}=\frac{3}{4}$. In general we see that the sum of $\underline{0}$ and any rational number is the number. 
Simllarly we recall, for example, that $\frac{2}{1}$ and $\frac{3}{1}$ are. fraction names for 2 and 3 respectively. "Thus

$$
2+3=\frac{2}{1} \div \frac{3}{1}=\frac{2+3}{1}=\frac{5}{1}=5
$$

as we would expect (and hope).

Addition of rational numbers is not difficult to understand, once the- 1dea that the same rational number has many different fraction names has been well established. The technique of computing sums of rational numbers written with fraction names is In essence a matter of finding common denominators. This is essentlally the problem of the least common multiple and thus is a problem about whole numbers.

Addition of Rational Numbers Using Other Numerals

ofter 1t is convenient to use numerals other than fractions to find the sum of two rational numbers. Those commonly used are mixed forms and decimals.

The first kind of numeral can be easily understood once addition has been explained. We can see with line segments that

$$
2+\frac{11}{3}
$$

is a rational number, and 1 th is also easy to see that $\frac{7}{3}$ is another, name for this same number. Similariy, $\frac{15}{1}=3 \frac{3^{3}}{4}$. Indeed, these. 1deas can be introduced before any formal mechanism for adding two rational numbers named by fractions has been developed, because the ldea that $2+\frac{1}{3}^{\circ}=\frac{7}{3}$ goes back to the number line 1dea of sum. To adopt the convention of uriting $2 \frac{1}{3}$ as an abbreviation for $2+\frac{1}{3}$ is then easy, and we may use a numeral like $2 \frac{1}{3}$ as a name for a rational number.. It is these. we call a numeral, in mixed form.

The use of decimais is still another convention for namins. rational numbers. For example, 3.2 names a rational number; other names for this number are

$$
\begin{aligned}
& 3+\frac{c}{10}, \quad 3 \frac{2}{10}, \quad 3 \frac{1}{5}, \quad \frac{16}{5}, \quad \frac{32}{10} ; \quad \frac{320}{100} \\
& 473 .
\end{aligned}
$$

$$
23
$$


of these, $\frac{16}{5}, ' \frac{32}{10}$ ' and $\frac{320}{100}$ are fraction names wille $3 \frac{2}{10}$. and $3 \frac{1}{5}$ are mixed rorms.

The methods for computins witr: decimals are direct outccmes. of their meaning. For example, to compute $3.4-1.7$, we may proceed as folkows:

$$
\begin{aligned}
3 .+ & =3+\frac{1}{10} \\
1.7 & =\frac{1-\frac{7}{10}}{-\frac{11}{10}}=5-\frac{1}{10} .
\end{aligned}
$$

Hence 3.- 1.- = 5.1 .

We wart the child to derelop a more efrictent shcrt-cut procedure for sinding such a sum. Forever, the uncerstanding" of the procedure car. be carrled back, as snown, to the knowledge he al ready has about adding numbers itith names in fraction or mixed form.

In a sinilar way, the procedures for subtracting, multi-. plying and dividing numbers naned by decimals can be underistood In terms of the same operations applied to numbers named by iractions.

\section{Mitiplication}

$3 y$ the tipe the child is ready to find the product of two rational numbers such as $\frac{2}{3}$ and $\frac{3}{4}$, ne has already had a number - of experiences in understanding and computing products of whole numbers.'

Hic thas seen $3 \times 2$ in terms of $a$ rectangular array. ' He can recognize the arrangement in Pig. \& as showing 3 groups of abjects witi 2 objects in each group.

Also, he has.seen $3 \times 2$

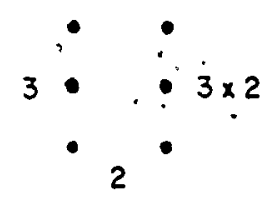

in terms of line segments.

(F1g. R) that is, as a union of 3 two-unit segments.

Furthermore, he has interpreted

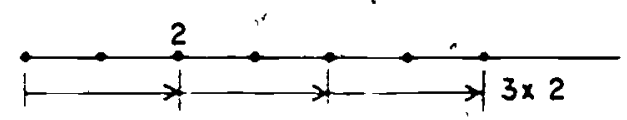

Fig: R

47

$.2=$ 
- Plgure $R$ in terms of travel along̈ a line... For example, if he rows $a$ boat 2 miles an hour across a lake, then in 3 hours he rows 6 miles.

Finaly, $3 \times 2$ can be related. to areas as in Pigure $S$. Thus, a child has seen that the operation of muit1-

- plicatión can be applied to meny physical models. He has related several physical situations to a single number operation.

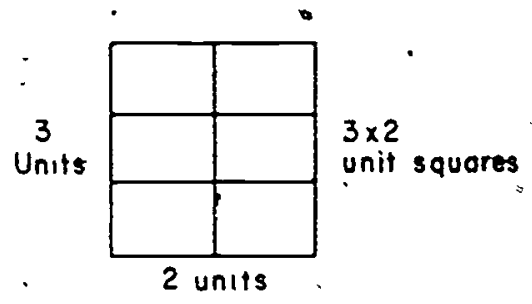

\section{The "Rectangular Reglun" Modez}

- Fig. S

May we remind gou that the 1dea of muitiplying $\frac{2}{3}$ and $\frac{1}{5}$ wes not inverted for the purpose of writing arlthmetic books. Instead, peaple found some applications in which the numbers $\frac{2}{3}$ and $\frac{1}{5}$ appeared and also $\frac{2}{15}$ appeared. Por instance., in P18. I we see a unit square separated

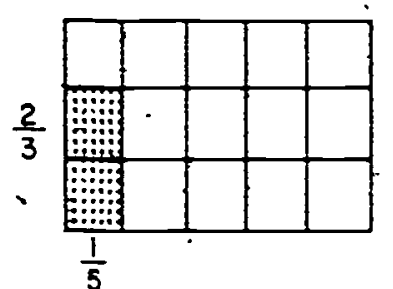

Fig. $T$ Into 15 congruent rectangles: The measure of the shaded region is $\frac{2}{15}$. square units. On the other hand, we have already used the operation of multiplication to compute areas of rectangles having dimensions that are hole numbers. Hence it is natural to say: Let us call $\frac{2}{15}$ the product of $\frac{2}{3}$ and $\frac{1}{5}$ and write $\frac{2}{3} \times \frac{1}{5}=\frac{2}{15}$.

Iogicaily, $\frac{2}{3} \times \frac{\frac{1}{5}}{15}$ a meaningless symbol until we define. 1t. It could mean anything we choose. Our choice of $\frac{2}{15}$ for a meaning seems, however, a useful one, and indeed it is.

Yet, chlldren need many more examples before they can see the general mule that in multiplying rational numbers named by fractions we multiply the numerators and multiply the denominators:

* We should recognize that although the romal introduction of $\frac{2}{3} \times \frac{1}{5}$ is deferred unt1l the sixth grade the development is anticipated by many earlier experiences. Among them are: the 
identification of a fraction with a region and the various steps in finding the measure of a region.

The "Number Line" Model

The product of two whole numbers also can be visualized on the number line. A few natural generalizations to products of rational numbers can be made from these kinds of experiences.

- Por example, if we can think of $3 \times 2$ as illustrated by Figfe $U,(a)$, then it is natural to identify $3 \times \frac{1}{4}$ with the situation pictured in Figure $U$, (b).

(a)

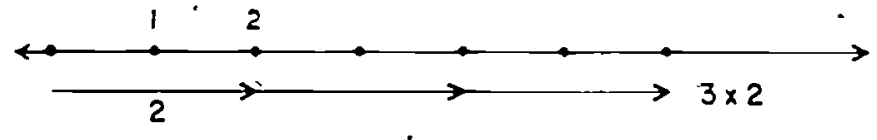

(b)

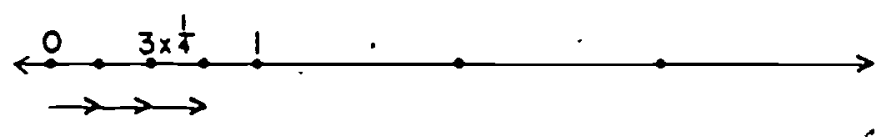

Fiq. U

In the same way we can identify, for example, $3 \times \frac{2}{7}$ with

$$
\frac{2}{7}+\frac{2}{7}+\frac{2}{7}=\frac{6}{7}
$$

Again, if a man walks. 4 miles each hour, then $(2 \times 4)$ miles is the distance he walks in 2 hours. (Fig. V) once more it is also natural to relate $\frac{1}{2} \times 4$ with a distance he travels--this time with his distance in $\frac{1}{2}$ hour.'

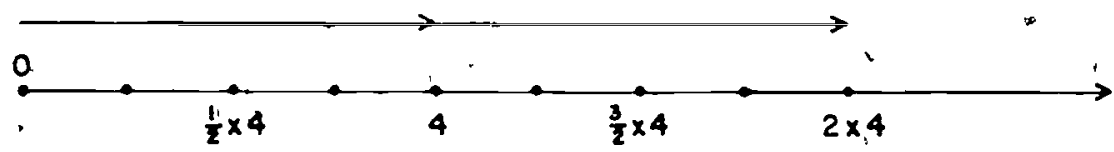

Fig. $V$

Suppose, now, that a turtle travels $\frac{1}{5}$ mile in an hour. In 2 hours, it travels $2 \times \frac{1}{5}$, or $\frac{2}{5}$ miles. We identify the product $\frac{2}{3} \times \frac{1}{5}$ with the'distance it travels in $\frac{2}{3}$ of an hour. 
Fig. W diagrars the turtle's travels.

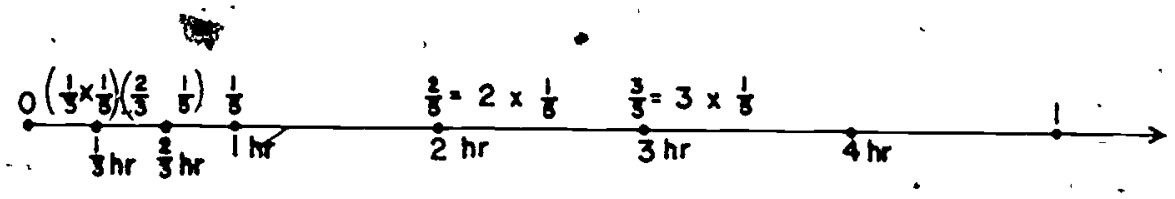

\section{Fig. W}

We locate $\frac{2}{3}$ of $\frac{1}{5}$ on the number line by locating $\frac{1}{5}$, cutting the $\frac{1}{5}$ segment into 3 congruent segments, and counting off two of them, as in Figure $x$.

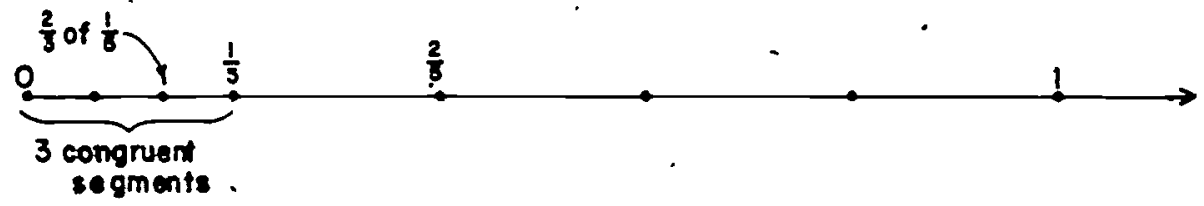

Fig. $X$

More, specifically, we first cut the unit segment into 5 congruent segments. Then éach of these is cut into 3 congruent segments. We thus have $3 \times 5$ segments. We counted $2 \times 1$. of them. We see that $\frac{2}{3}$ of $\frac{1}{5}$ is associated with the point $\frac{2 \times 1}{3 \times 5}$

We had two numbers: $\frac{2}{3}$ and $\frac{1}{5}$. When we talk about $\frac{2}{3}$ of $\frac{1}{5}$ we are explaining a situation in which we have a pair of numbers $\left(\frac{2}{3}\right.$ and $\left.\frac{1}{5}\right)$ associated with a third $\left(\frac{2}{15}\right)$. We have,

* In short, an operation; it is natural to see whether it $t_{m}$ is an operation we know. We fild that it is.

* We alrepdy had agreed, using the rectangle model, that

$$
\frac{2}{3} \times \frac{1}{5}=\frac{2 \times 1}{3 \times 5}=\frac{2}{15} .
$$

'Hence, we now see that we can identify $\frac{2}{3}$ of $\frac{1}{5}$ with $\frac{2}{3} \times \frac{1}{5}$.

Moreover, we notice that if we.use the ldea of travel on the number line ( $\mu_{1 g}, W$ ) it is again natural to ide, ify $\frac{2}{3} \times \frac{1}{5}$ with $\frac{2}{3}$ of $\frac{1}{5}$. 
The "Sets of objects" Madel

We speak, in everyday usage, of " $\frac{2}{3}$ of a dozen of eggs. We visualize this as the result of separating a finite set of 12 - opjects into 3 subsets, each with the same numbor of objects and fhen uniting 2 of the subsets. The relation between this concept and that involving a 12-Inch segment can be seen from Figure $Y$.

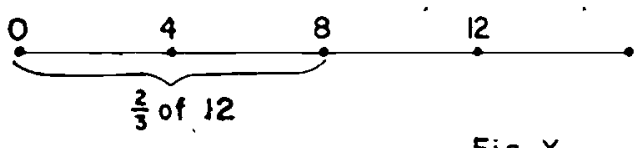

Fig. $Y$

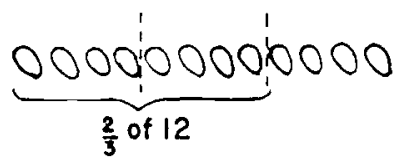

We sometimes use such examples with very young children to emphasize the 1dea of $\frac{2}{3}$. pat this is a little misleading; for we should note that $\frac{2}{3}$ of 12 is again a situation involving two numbers, $\frac{2}{3}$ and 12. Again we can verify that $\frac{2}{3}$ of 12 is computed by finding $\frac{2}{3} \times 12$.

\section{Summary}

From the standpoint of defining the operation of multiplication for rational numbers, it would be entirely sufficient to use one interpretation. However, because products of rational numbers are used in many types of problem situations the child ought to recognize that the definition does fit the needs of " each:

$\frac{2}{3} \times \frac{1}{4}$ can be visualized as:

(1) the area in square inches of a rectangle with length $\frac{2}{3}$ in. and width $\frac{13}{4}$ in.

(2) the length of a line segment formed by taking $\frac{2}{3}$ of a $\frac{1}{4}$. Inch segment.

out of the number line model come many problem stituations. For example, if a car travels $\frac{1}{4}$ mile per minute, it travels $\frac{2}{3} \times \frac{1}{4}$ miles in $\frac{2}{3}$ minute.

Moréover, $\frac{2}{3} \times 12$ can be interpreted in the ways noted and also can be related tinite sets. We use $\frac{2}{3} \times 12$ where we, want to find the number of eggs in $\frac{2}{3}$ of a dozen. 
Mathematics is powerful because á single mathematical idea (like $\frac{2}{3} \times \frac{1}{4}$ ) often has many applicat1. 19. Children can rully understand a product like $\frac{2}{3} \times \frac{1}{4}$ only when they have, had experlences with several applications.

We should observe, that our delinition of the product of two rational numbers is consistent with what we already know about - whole numbers. We know that $2 \times 3$, for example, is another. name for 6. All is well, for the product of $\cdot \frac{2}{1}$ and $\frac{3}{1}$, as computed by our derinition, is $\frac{6}{1}$, and $\frac{6}{1}$ names the same number

4 as 6. We note, too, that our definition leads us to $3 \times \frac{1}{4}=\frac{3}{1} \times \frac{1}{4}=\frac{3}{4}$, as was anticipated earlier.

We should notice, too, that al though our method for finding the product is expressed in terms of specific names for the factors, the product is not changed if we change the names. For example, $\frac{1}{2} \times \frac{3}{4}=\frac{3}{8}$. Renaming $\frac{1}{2}$ and $\frac{3}{4}$ we have

$$
\frac{3}{6} \times \frac{6}{8}=\frac{18}{48}
$$

$\frac{18}{48}$ is another name for $\frac{3}{8}$.

Properties of Multiplication of Rational Numbers

Again our definition has convenient by-products. . For, we observe that the associatif and commutative properties hold multiplication of rational numbers. They hold here as a direct result of the same properties for multiplication of whole numbers.

The following example shows how we may explain the commutative property of multiplication.

our rule for multiplication tells us that $\frac{2}{3} \times \frac{1}{4}=\frac{2 \times 1}{3 \times 4}$. oir mule tells us also that $\frac{1}{4} \times \frac{2}{3}=\frac{1 \times 2}{4 \times 3}$. We know, however, that $1 \times 2=2 \times 1$ and $3 \times 4=4 \times 3$. These racts are Instances of the commutative property of multiplication of whole numbers. Hence we see: $\frac{2}{3} \times \frac{1}{4}=\frac{1}{4} \times \frac{2}{3}$.

We also can visualize this property using rectangular regions as in Figure $\mathrm{z}$.

Figure Z

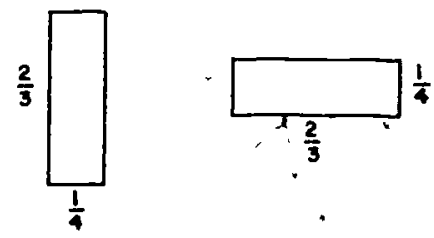

479 
The associative property of multiplication holds, in essence, because it holds for whole numbers. From the associative property, we can compute $\frac{1}{2}(4 \times 5)$ as either $\frac{1}{2} \times(4 \times 5)$. or $\left(\frac{1}{2} \times 4\right) \times 5--a$ fact which is sometimes helprul in using the. formula for the area of a triangle.

We observe, too, an interesting multiplication property of o. our mule for multiplying tworrational numbers named by fractions leads directly to the conclusion that the product of 0 and any rational number is $0:^{\prime}$.

$$
0 \times \frac{2}{3}=\frac{0}{1} \times \frac{2}{3}=\frac{0 \times 2}{1 \times 3}=\frac{0}{3}=0 .
$$

of interest, too, is the multiplication property of 1 . It is easy to see that the product of $I_{4}$ and any number is the number:

$$
1 \times \frac{2}{3}=\frac{1}{1} \times \frac{2}{3}=\frac{1 \times 2}{1 \times 3}=\frac{2}{3} .
$$

Again this is a direct result of the same property of whole numbers. Now 1 , of course, has many names. One of them, for. example, is $\frac{3}{3}$. When we multiply $\frac{4}{5}$ by 1 we can write

$$
1 \times \frac{4}{5}=\frac{3}{3} \times \frac{4}{5}=\frac{3 \times 4}{3 \times 5}=\frac{12}{15}=\frac{4}{5} \text {. }
$$

*This result shows that multiplying the numerator and denominator of a fraction by the same counting number is equivalent to multiplying 1 : by the number named by the fraction.

Finally, the distributive property for rational numbers is an outcome of our definition. The distributive, property tells us that, for, example, $\frac{2}{3}\left(\frac{1}{5}+\frac{2}{5}\right)=\left(\frac{2}{3} \times \frac{1}{5}\right)+\left(\frac{2}{3} \times \frac{2}{5}\right)$. our area picture helps us to understand this easily (FIg. AA) The smaller rectangles have areas $\frac{2}{3} \times \frac{1}{5}$ and $\frac{2}{3} \times \frac{2}{5}$. The area of their union is $\frac{2}{3}\left(\frac{1}{5}+\frac{2}{5}\right)$, or $\frac{2}{3} \times \frac{3}{5}$.

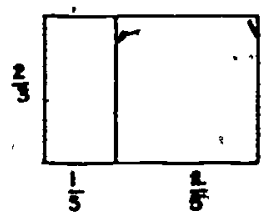

Fig. ÁA

The distributive property is useful in computing a product l1ke. $5 \times 3 \frac{1}{2}$. We can say:

$$
5 \times 3 \frac{1}{2}=(5 \times 3)+\left(5 \times \frac{1}{2}\right)=15+\frac{5}{2}=17 \frac{1}{2} .
$$


1. We can also recognize that we have essentially-applied the distributive property in writing

$$
\frac{3}{4}=3 \times \frac{1}{4}=(1+1+1) \times \frac{1}{4}=\frac{1}{4}+\frac{1}{4}+\frac{1}{4} .
$$

Before leaving the topic of multiplication of rational. numbers", we ought to notice that the product of certain pairs of Atational numbers is 1 . For example, the product of $\frac{2}{3}$ and 1s 1. The number $\frac{2}{3}$ is called the reciprock of $: \frac{3}{2}$, and $\frac{3}{2}$. is the reciprocal of $-\frac{2}{3}$. The reciprocal of a number is the number. 1t must be multiplied by to give $l$ as a product. Every rational number except 0 , has exactly one reciprocal. When the number is named by a fraction, we can easily find the reciprocal by "turning the fraction upside down". Thus the reciprocal of $\frac{3}{8}$ is $-\frac{8}{3}$;

In particular, the reciprocal of the whole number 2 is $\frac{1}{2}$, which can be verifled easily since $2 \times \frac{1}{2}=1$.

0 has no reciprocal. For we know that the product of 0 and every rational number is 0 . Hence there is no number we can murtiply by 0 to give $l$.

\section{Division}

In the rational number system, as in the counting numbers, we "want to use division to answer questions of "what must" we " multiply?". In a division situation we are given one factor and a product. Thus $\frac{5}{3} \div \frac{3}{4}$ is the number such that: $\left(\frac{5}{3}, \frac{3}{4}\right) \times \frac{3}{4}=\frac{5}{3}$, In order to compute $\frac{5}{3}-\frac{3}{4}$ we must solve:

$$
\frac{3}{4} \times \frac{m}{n}=\frac{5}{3}
$$

where $m$, and $n$ are counting numbers.

To acquire an understanding of the division prócess, chilm dren need many concrete experiences in 1ts use. These experi- ences parallel those with multíplication, since division problems can be interpreted as problems in finding an appropriate multi- " * plier. Thus typical problem situations include: lto finding the width of a rectangle when the length and. area are known;

481 
2) finding what fractional part one set is of another;

3) finding the number of segments of given length that can be made by cutting a given segment.

\section{The Idea of Reciprocal and Division:}

'We already have seen that the product of any number and1 ts recipiocal is 1 . For example,

$$
\because \quad \frac{2}{5} \times \frac{5}{2}=\frac{2 \times 5}{5 \times 2}=1 \text {. }
$$

We akso know that $1 \div \frac{2}{5}=n$ means $\frac{2}{5} \times n=1$. Thus, the reciprocal of $\frac{2}{5}$ (which is $\frac{5}{2}$ ) is the number by which one can multiply $\frac{2}{5}$ to obtain the product 1 :

Now suppose we want to find the number $m$ such that

$$
\text { . } \frac{2}{5} \times \mathrm{m}=3 \text {. }
$$

Since $1 \times 3=3$, we can write: $\frac{2}{5} \times \mathrm{m}=(1 \times 3)$. But since $\frac{2}{5} \times \frac{5}{2}=1$, we aiso can write: $\frac{2}{5} \times \mathrm{m}=\left(\frac{2}{5} \times \frac{5}{2}\right) \times 3$. Using the associative property, we, can write again: $\frac{2}{5} \times m=\frac{2}{5} \times\left(\frac{5}{2} \times 3.\right)$ We now see that $m=\frac{5}{2} \times 3$.

We can use the same reasoning to compute $\frac{5}{3} \div \frac{3}{4}$. We must solve:

$$
\frac{3}{4} \times m=\frac{5}{3} \text {. }
$$

We know that $\cdot \frac{3}{4} \times \frac{4}{3}=1$ and that the product or a number and one is the number itself. So,

$$
\therefore \quad\left(\frac{3}{4} \times \frac{4}{3}\right) \times \frac{5}{3}=\frac{5}{3}
$$

and

$$
\frac{3}{4} \times\left(\frac{4}{3} \times \frac{5}{3}\right)=\frac{5}{3} .
$$

Therefore, $\mathrm{m}=\frac{4}{3} \times \frac{5}{3}=\frac{4 \times 5}{3 \times 3}=\frac{20}{9}$.

We note: To divide $\frac{5}{3}$ by $\frac{3}{4}$, we multiply $\frac{5}{3}$ by the reciprocal of $\frac{3}{4}$.

We have seen a way" 1 in which we can derive the general rule: To divide by a non-zero rational number, multiply by 'its reciprocal. 
We see that for whole numbers our rule gives the results we would expect. For example,

$$
6 \div 2=\frac{6}{1} \div \frac{2}{1}=\frac{6}{1} \times \frac{1}{2}=\frac{6}{2}=3: \text {. }
$$

In particular, the reciprocal of $l$ is $l$, since $1 \times 1=1$. Thus when we divide a rational number by $l$ we multiply it by $l$ and obtain the orlginal number, "as we would expect.

our wule for divesion ldentifies dividing by a number with multiplytng by its reciprocal. Thus when we wish to find $\frac{1}{3}$ of 18 we may use either multiplication by $\frac{1}{3}$ or division by 3 .

We now see that: $\frac{3}{4}=3 \times \frac{1}{4}=3 \div 4$. One Important interpretation of $\frac{3}{4}$ as the result of dividing 3 by 4 can be visualized using line segments (F1g. BB)

$\frac{3}{4}$ can be seen, too as the answer to the question "How many $4^{\prime} \mathrm{s}$ in 3 ?" More prec1sely, $\frac{3}{4}$ is the number by which we must multiply 4 to get 3.

Some texts for later grades define rational numbers by using the idea of division. That $1 \mathrm{~s} ; \frac{3}{4} \cdot 1 \mathrm{~s}$ defined from the outset as the number. $x$ satisfying $4 x=3$.

The set of counting numbers is not closed under division-that 1s, with only counting numbers at our disposal, we can not solve an equation ilke $4 \times n=3$. But having introduced the set 7 rational numbers, we can always solve an equation of this type. We can divide any' rational number by any number different from 0 . Henfe the set, made up of all the rational numbers except 0 , 1s closed under division. We can interpret the extension of our idea of number from the counting numbers to the rational numbers as a successful effort to obtain a system of numbers that is closed under division.,

It is an interesting paradox that now, having defined. division by a rational number as multiplication by the reciprocar of the number, we could really get along without division entirely, since to divide by a number we can always multiply by 
Its reciprocal. Later', we introduce the negative numbers to make a system closed under subtraction. Once we have done so, subtratting formber can be replaced by adding the opposite

\section{Fractions-- A Symbol for a Pair of Rational Numbers}

Thus far, we hatye restricted our use of fraction to that of being a symbol naming a pair of whole numbers. Let us now give. meaning to symbols like $\frac{\frac{3}{2}}{\frac{6}{1}}, \frac{1.5}{.5}$, etc. In which the pairs of numbers are rational numbers instead of 'whole numbers.

We call that we already know $\frac{3}{4}$ and $3 \div 4$. are two names for the same number. That is, for $4 \times x=3, n=\frac{1}{4} \times \frac{3}{1}=\frac{3}{4} \cdots$ This suggests that we might say that the symbol $\frac{\frac{3}{2}}{6}$ will mean $\frac{3}{2} \div 6$, and the symbol $\frac{1.5}{.5}$ will mean $1.5 \div 5$.

When we say, let $\frac{3}{2} \div 6=\frac{\frac{3}{2}}{6}$, we are defining the meanings of those symbols which hitherto hąve had. no meaning for us: There is nothing 11 logical with defining a new symbol, in any way we like. However, simply assigning to $\frac{3}{2} \div 6$. the symbor $\frac{\frac{3}{2}}{6}$ does not permit us to treat this new symbol immediately as if it were a fraction of the kind with which we are familiar. For. example, although we know that $\frac{3}{4}=\frac{2 \times 3}{2 \times 4}$. we cannot be certain that $\frac{\frac{3}{2}}{6}=\frac{2 \times \frac{3}{2}}{2 \times \frac{6}{6}}$. Too, just because we know $\frac{1}{3}+\frac{1}{3}=\frac{2}{3}$, we cannot conclude (without argument) that $\frac{1}{1}+\frac{1}{1}=\frac{2}{1}$.

In practice, children in the elementary school are unilikeiy to add or to multiply many numbers named by these new fractions. Yet, examples as $\frac{1.5}{.5}$ will be familiar when using decimal names. in division of rational numbers. In later years, they can find. solutions to such examples as $12 \frac{1}{2} \%$ of 120 by solving

$$
\frac{12.5}{100}=\frac{n}{120}
$$


Thus, it seems necessary to "know" if it is possible to fruityply the numerator and denominator of $\frac{1.5}{.5}$ by 10 to obtain apother name for the same number.

Again, let us make some observations about division. Does.' . 'multiplying dividend and divisor by the same number change the result?. We observe

$$
\begin{aligned}
& 6 \div 2=3 \\
& (6 \times 2) \div(2 \times 2)=3 \text { or } 12 \div 2=3 \\
& \left(6 \times \frac{1}{2}\right)-\left(2 \times \frac{1}{2}\right)=3 \text { or } \frac{6}{2} \div \frac{2}{2}=3 \div 1=3 .
\end{aligned}
$$

We also need to be sure that when we multiply the numerator and denominator of a fraction, as, $\frac{\frac{5}{3}}{\frac{3}{4}}$, by the same number, we obtäin a new fraction equal to the original one.

Does $\frac{5}{3} \div \frac{3}{4} \leq \frac{\frac{5}{3}}{\frac{3}{4}}$ ?

We know that $\frac{5}{3} \div \frac{3}{4}=\frac{3}{4} \times n=\frac{5}{3}$ and $\dot{n}=\frac{4}{3} \times \frac{5}{3}=\frac{20}{9}$.

Let us now multiply both numerator and denominator. of the fraction $\cdot \frac{\frac{5}{3}}{\frac{3}{4}}$ by the same number $\frac{4}{3}$.

$$
\frac{\frac{5}{3} \times \frac{4}{3}}{\frac{3}{4} \times \frac{4}{3}}=\frac{\frac{5 \times 4}{3} \times 3}{\frac{3}{4 \times 4}}
$$

But $\frac{3 \times 4}{4 \times 3}=\frac{12}{12}=1$ and $\frac{5 \times 4}{3 \times 3}=\frac{20}{3}$

Is $\frac{\frac{20}{9}}{1}$. the same as $\frac{20}{9}$ ?

If 1 t is, then $\frac{20}{9} \div 1=\frac{20}{9}$.

We do know that this is true since the product of 1 and a rational number is that same rational number.

Thus $\frac{5}{3} \div \frac{3}{4}=\frac{20}{3}$ and $\frac{\frac{5}{3}}{\frac{3}{4}}=\frac{20}{9}$; so, $\frac{5}{3} \div \frac{3}{4}=\frac{\frac{5}{3}}{\frac{3}{4}}$. 
It is important that extensive use be made of materials ith . " developing understanding of the rational numbers and of the operations of addition and subtraction of rational numbers.

- Some materials which have been found useful are suggested on the next few pages. These may be supplemented by other avallable materials.

Teachers w1ll find coples of these cards made on foot square cardboard useful throughout the. chapter. Colored acetate may' be. used to indicate shaded areas.
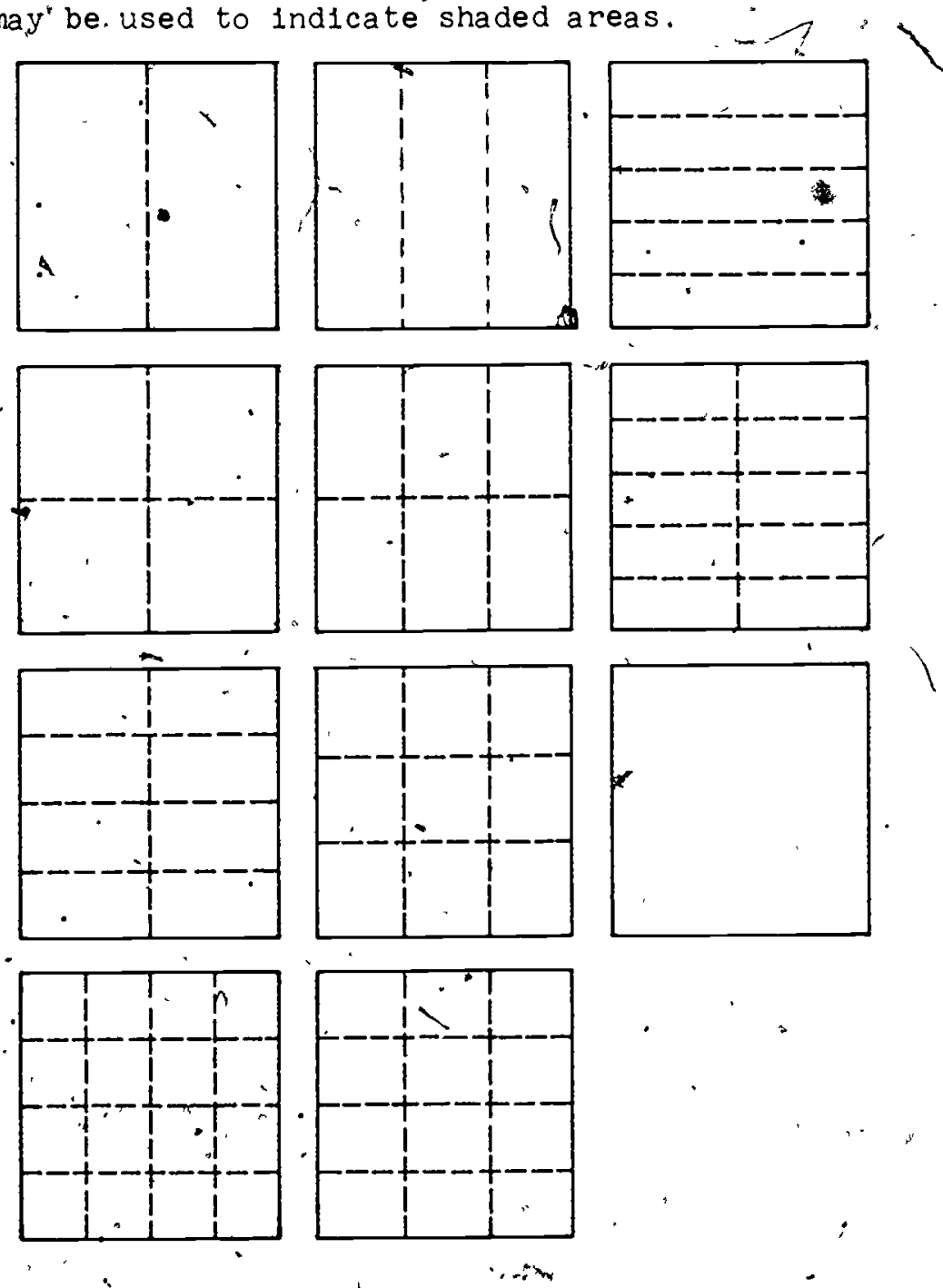
2

Models of circular regions can be copied on cardboard or undecorated paper plates.
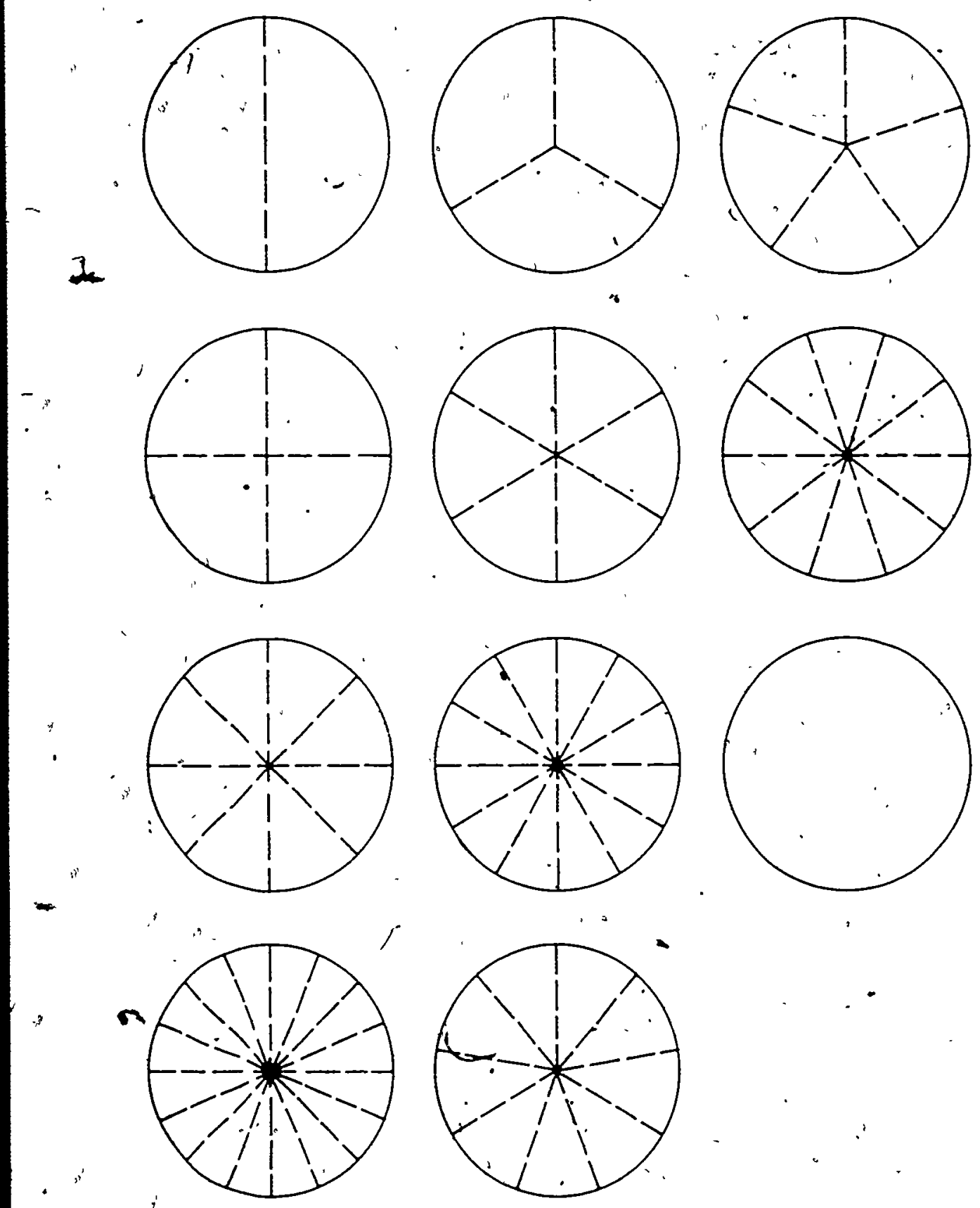

$487^{\circ}$

ERIC

42 


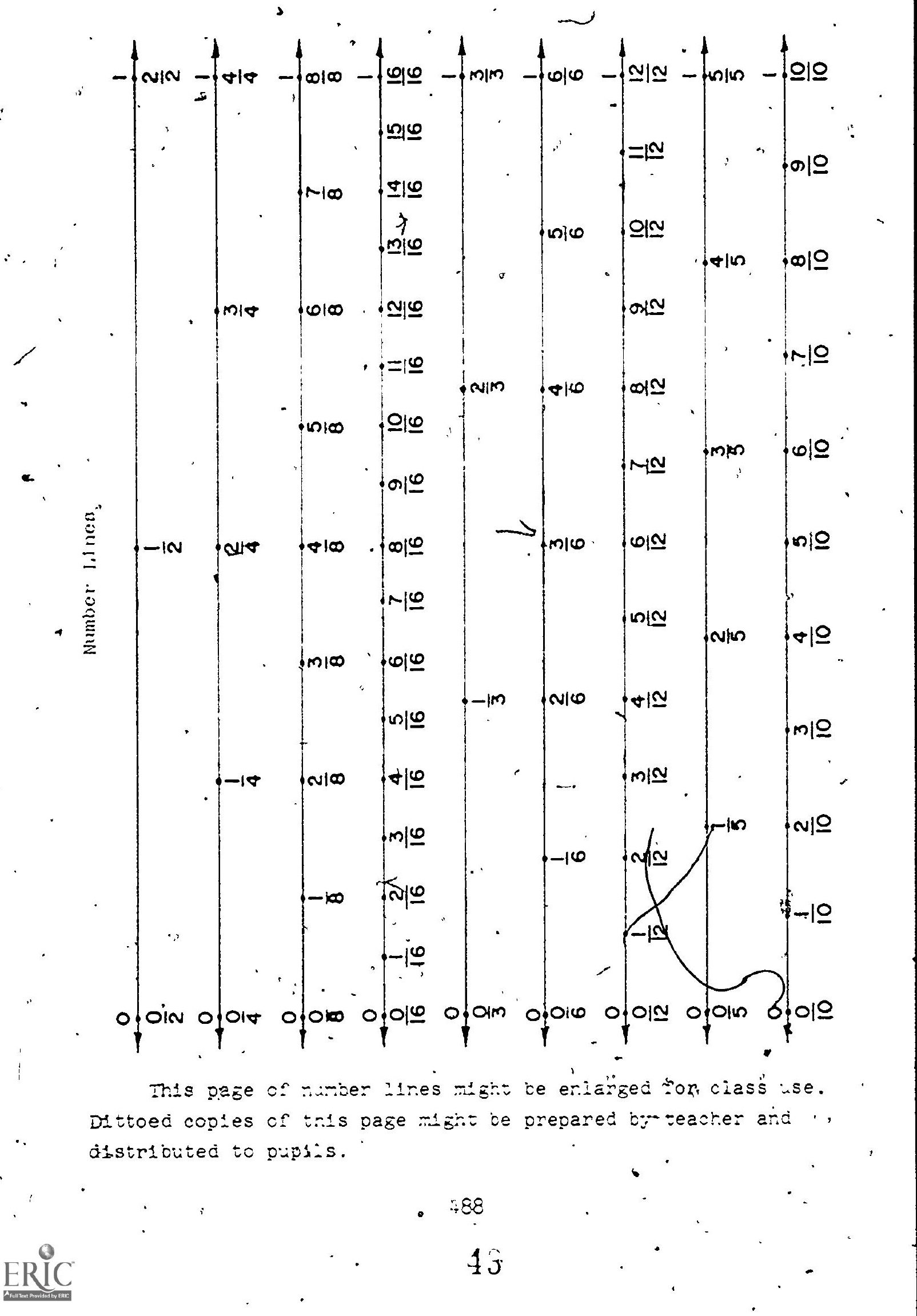





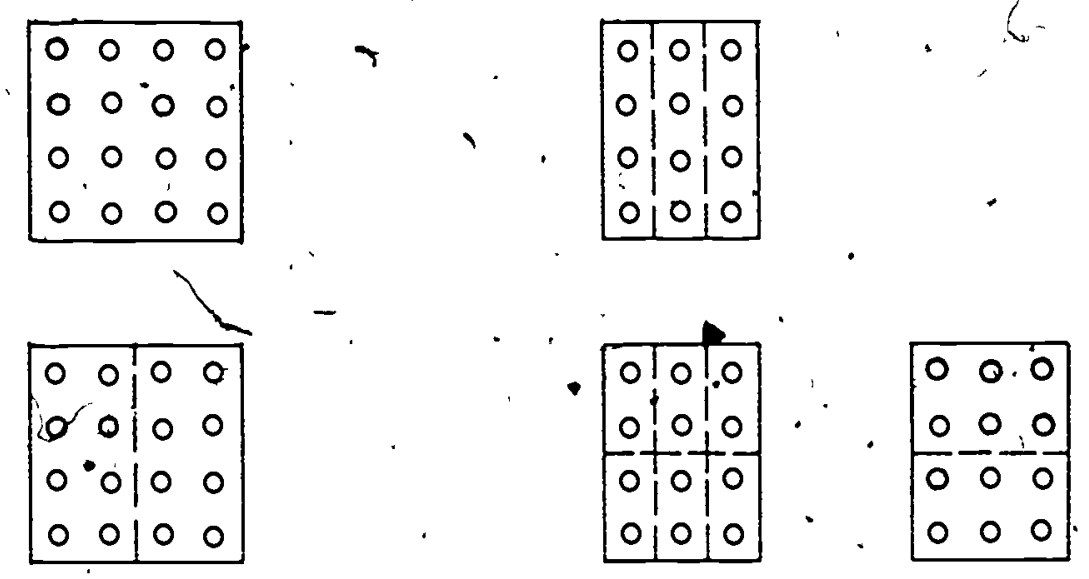

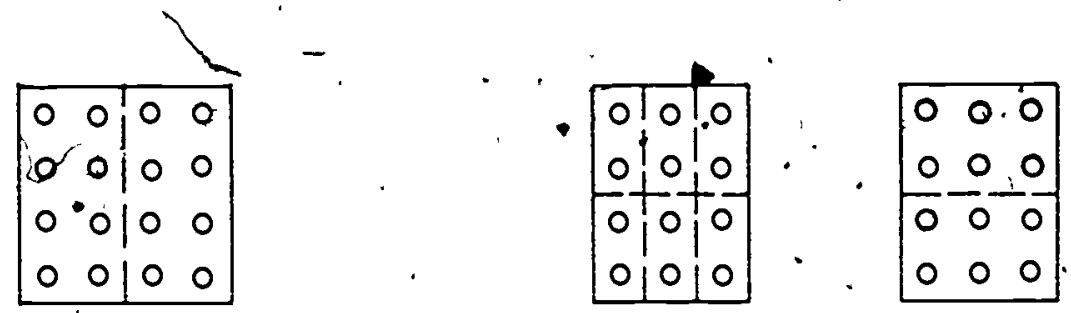

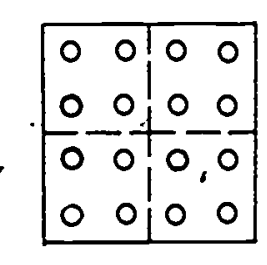
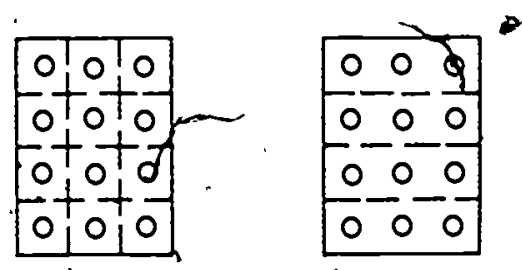

SET B
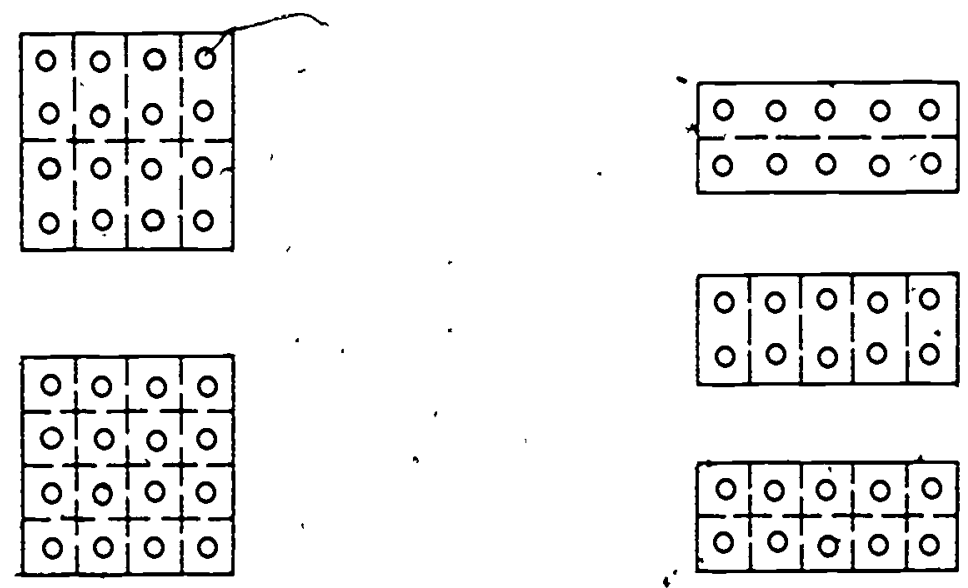

SET A
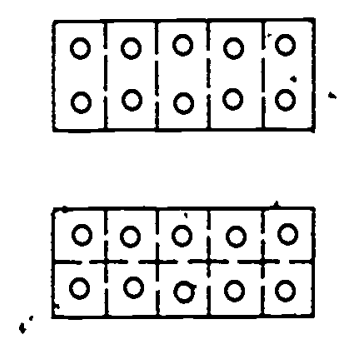

SET C

These arrays may be used to develop the concept of $\frac{1}{2}, \frac{1}{3}, \frac{1}{4}$; etc. of a set of objects. Colored acetate may be used with them to indicate $\frac{2}{3}, \frac{3}{4}, \frac{5}{6}$, etc. of a set of objects. 
Pocket Chart and Cards for. Decimals

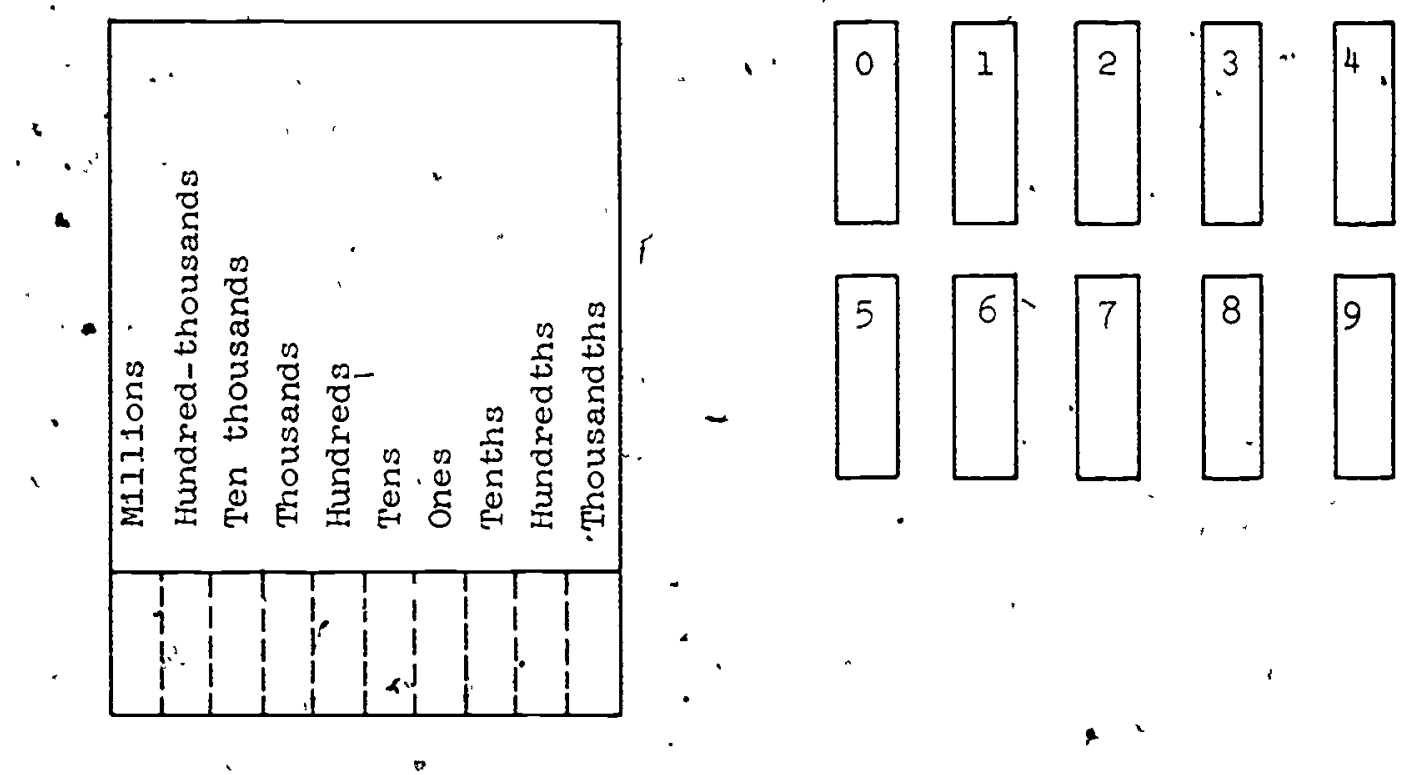

Above is a model of a pocket chart and cards to accompany it for practice as needed in place value work itth decimals. Several duplicates of each card should be - made. 
A sheet of number lines similar to this ?can be dittoed for children to use throughout unit.
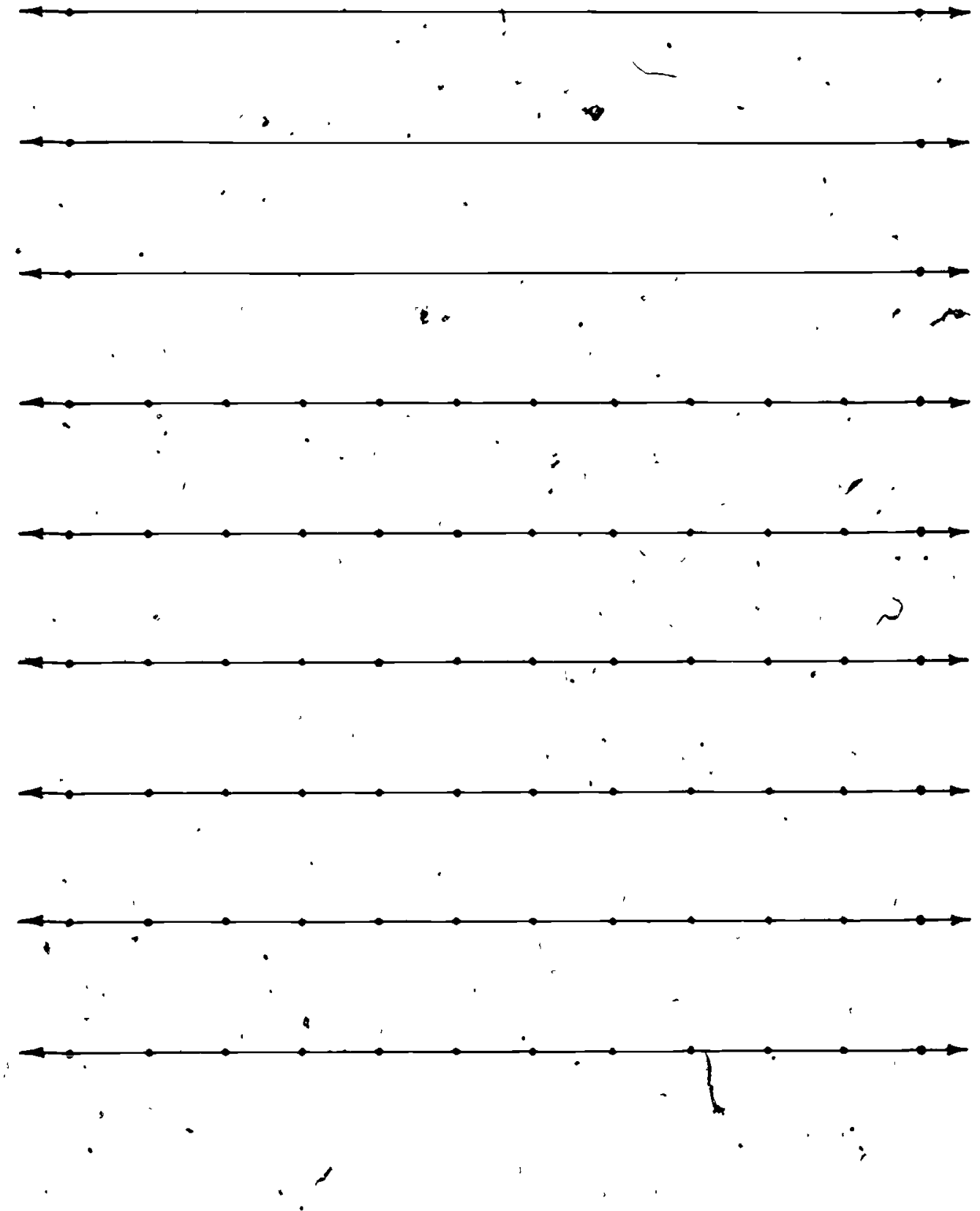

$\therefore$

4921

$4 \%$ 
TEACHING THE UNIT

\section{MEANING 'OF RATIONAL NUMBERS}

Objective: To review

a. the meaning of rational numbers.

b. correct use of the terms symbol, fraction.

c. meaning of numerator and denominator.

d. the use of a rational number to describe

- the measure of a region, a segment, or . a subset of a set. of objects.

Materials: Flannel board with models of circular and square region's separated into congruept parts. Number Iines.

Arrays or sets of objects.

Vocabulary: Rational number; rraction, numerator, denominator, whole number, separate, measure, congruent, circular, unit region, region, line segment) unit segment, represent, union, set.

Suggësted Teaching Procedure

Have children read together and.discuss "Meaning of Rational Numbers" in their books. Before having children do Exercise Set 1, teacher. shows work with concrete materials such as a flannelboard and models of congruent regions, number lines and sets of objects or arrays.

Models of congment regions and sets shown under "Materials" can be used throughout this development." Colored acetate is very useful to indicate shaded regions.

Begin by shading $\frac{1}{2}, \frac{1}{3}, \frac{1}{4}$, etc. of model regions and arrays. Ask questions like the following: 
(a) Into how many congruent regions' is the unit region separated?

(b) How many regions are shaded?

(c) What rational number best describes 1 ts measure? Record this measure.

(d) What does the denominator teil you?

(e) What does the numerator tell you? regions.

Ask similar questions about the unshaded.

Continue with models shading $\frac{2}{3}, \frac{3}{4}, \frac{4}{5}$, etc. and asking similar questions.

It is important to emphasize that:

(a) measure is a comparison with the unit. numbers have meaning only when the unit region is specified.
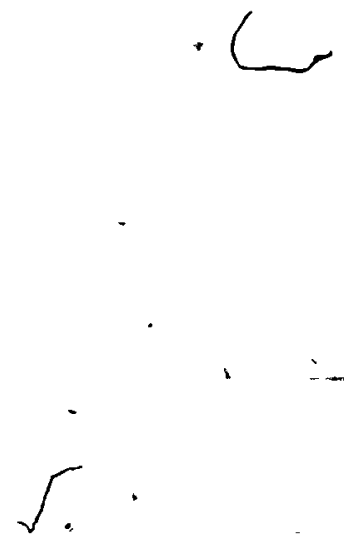
1 ADDITION AND SUBTRACTION OF RATIONAL NUMBERS

\section{MEANING' OF RATIOAAL NUMBERS}

Because of his way of life, early man needed only whole numbers. We. can think of reasons why he came to need other numbers as time went by. For example, he might have wanted to trade more than 2 but less than 3 . hides for a weapon. He might have wished to tell someone that there was"some food but, not. enough for a meal. He could not have handled these situations with whole numbers alone.

Today you would have great difficulty in making yourself understood if you could use only whole numbers. Suppose you knew 'only whole numbers. Could you describe any of these with a whole number?

(a) A trip that took iess than one day

(b) The amount of candy you get when yout share a candy - bar when trong.

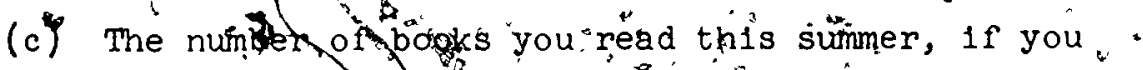
read more then do and iess than

) You would have even more difficulty "In'mathematios." If

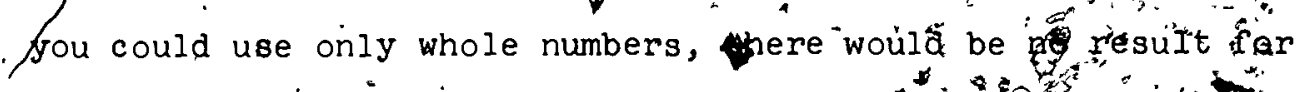
such operationsas $2 \div 5$ or $8 \div 3$. Another sh of number helps you find answers to such operations. The thef numbers. 1s called. the set of rational numbers.

Rational numbers are often used to degcribe the measure of a region, segment, or set of objects.' ' 


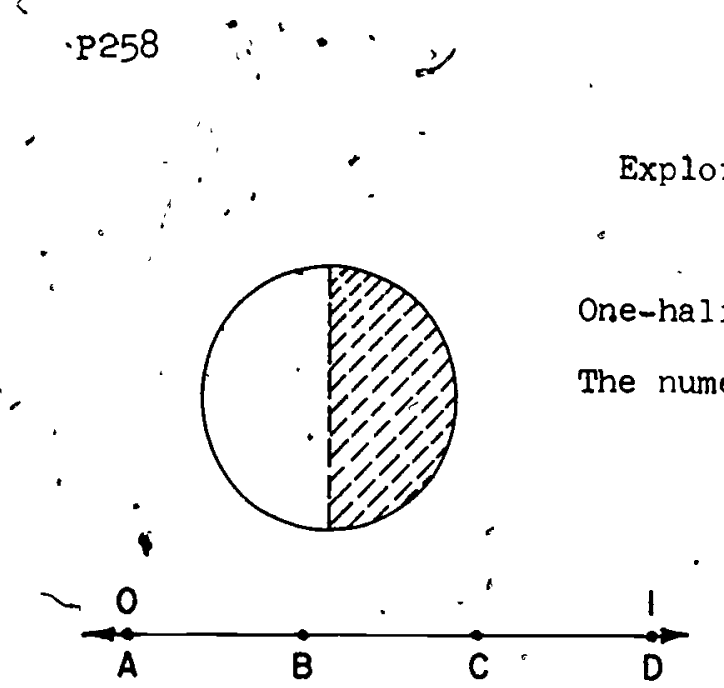

\section{Points $B$ and $C$ separate $\overline{A D}$ into 3 congruent segments:}

If the measure of $\overline{\mathrm{AD}}$ is 1 , the meàsure of $\overline{\mathrm{AC}}$ is two-thirds. - The numeral for two-thirds is $\frac{2}{3}$.

Set $A=\{$ Tom, Jane, Bill, Ann, Sáliy
- Three-ffiths is the number that best'describes what part : of set A the three girls are. The numeral for three-fifths is: $\frac{3}{5}$. One kind of symbol used to name a rational number is called

a fraction. A fraction is written with two names for whole .numbers separated qy a bar. $\frac{2}{3} ; \frac{3}{5}$ and $\frac{1}{2}$ are rractions.

$$
\text { numeral }
$$

The number named below the bar is called the denominator of the fraction and shows into how many parts of equal measure the unit region, unit segment, or set is separated. The number. named above the bar, or numerator, counts the number of these parts that are being uspa.

1. Look at the -circular region above.

a. Into how many congruent regions is it separated? ( 2 )

- $b *$ WiIl this number be represented by a numeral written

$:$. above or below the bar of a fraction? (Lelow ).

c. What is this numeral calied? (denominator)

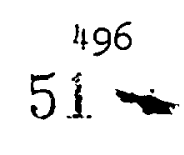


2. a. How many congruent parts of the region are shaded? (1)

b." Where wili you write the numeral that shows this? ( Gave)

$\vec{c}$ What is 1.t called?(mumeratos)

$3_{3}$ Look at.the picture of $\overline{\mathrm{AC}}$. The measure of $\overline{\mathrm{AC}}$ iș.

$\rightarrow$ written $\frac{2}{3}$.

a. 'What does the denominator tell you?( (Anto 3 separated)

b. What does the numerator tell you? $A \overline{A C}$ represerits the zheor of 2 isentw)

4. $\frac{3}{5}$ of the members of set $A$ are girls. What is the relation of the 3 and 5 of the fraction to' Set $A$ ? ( 5 reprecento) summary . - . member.un set. 3 cepresents

1. A rational number is sometimes used to describe the measure of a region, line segment, or set of objeats.

2. Fractions are one of the symbols used to name rational numbers.

3. Fractions are written with 2 names for, whole numbers separated by a bar. (The denominator can not be 0. ).

$$
\frac{4}{5} \text {, numerator }
$$

4. The denominator is the number which tells the number of congruent parts into which the unit segment, unit region, or set has been sepárated.

5. The numerator is the number which counts the number". of these congruent parts that are being. used.

6: The set of rational numbers includes numpers renamed by numerals itke these: $1 \frac{3}{8}, 5,-\frac{2}{2}, \frac{4}{3}, 0,7.2$ and $\frac{1}{2}$. 


\section{Exercisé Set 1 .}

1 .

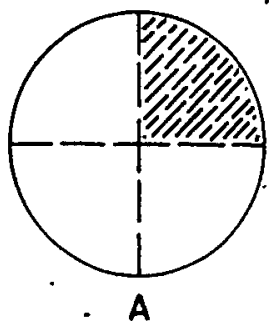

The circulair region $A$ has been separated pnto ( 4 ). congruent regions. (1) region is shaded. The' shaded region is $\left(\frac{1}{4}\right)$ of the circular region.

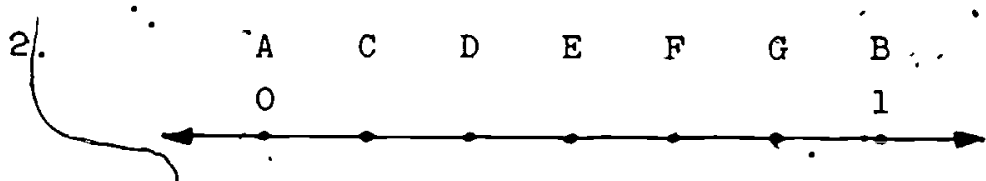

The measure of $\overline{\mathrm{AB}}$ is 1 . Points $\mathrm{C}, \mathrm{D}, \mathrm{E}, \mathrm{F}$, and $G$ separate $\overline{\mathrm{AB}}$ into (6) congruent segments. $\overline{\mathrm{AG}}$ is athe, union of $(5)$ of these congruent segments. The measure of $\overline{A G}$ is $\left(\frac{5}{6}\right)$.

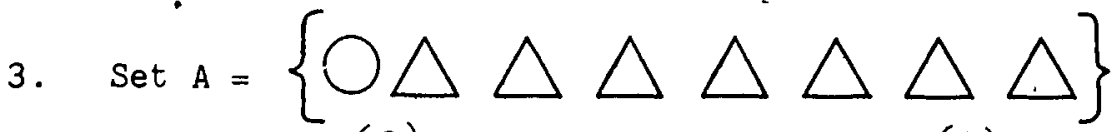

There are (8) members in Set A. (7) members are triangtes. What rational number describes what part of the members of set $A$ are.triangles? ${ }^{2}\left(\frac{\dot{\gamma}}{\varepsilon}\right)$

4.

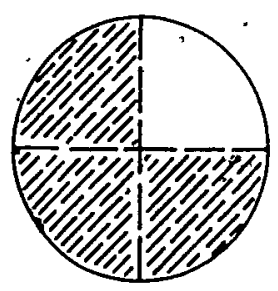

A

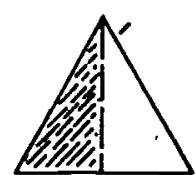

B

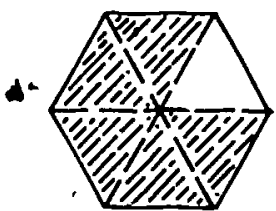

C

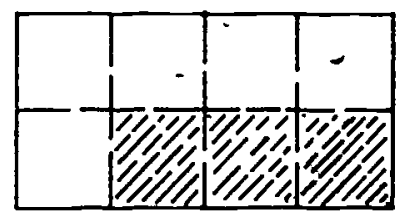

D

Each unit region above is separated into a number of smaller congmuent regions. What rational number best describes the measure of the shaded a rea of each? The unshaded area?

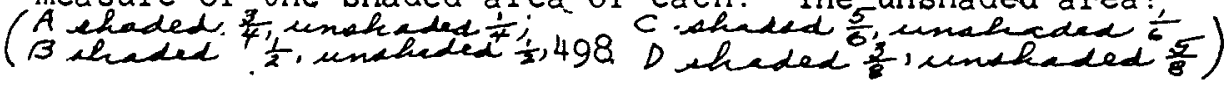


P261

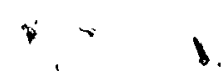

5. Which figures below are not separated into thirds? Explain

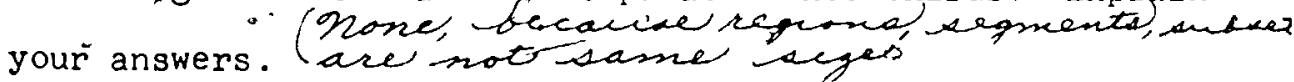
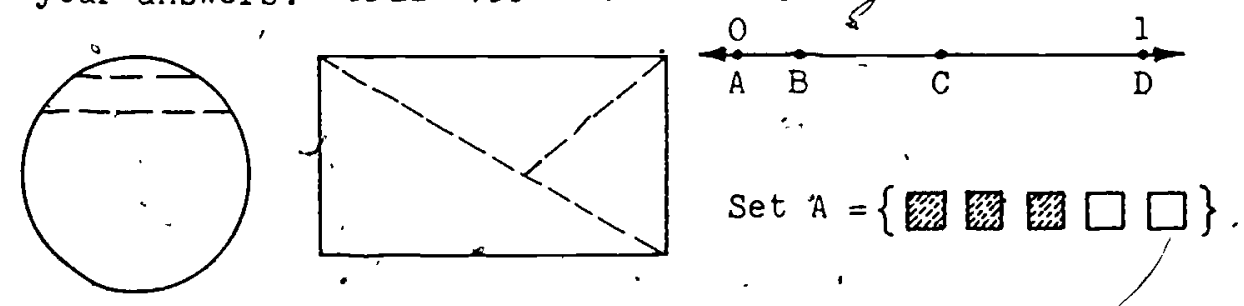

6. Trace the figures below. Then shade a part described by the fraction written below each of the figures.

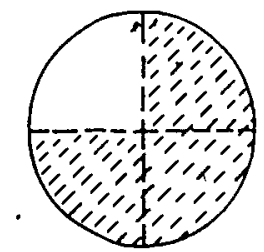

a. $\frac{3}{4}$

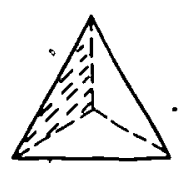

b. $\frac{1}{3}$

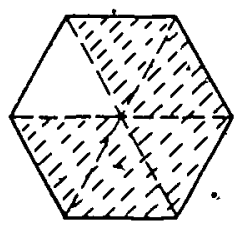

c. $\frac{5}{6}$

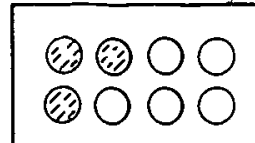

d. $\frac{3}{8}$

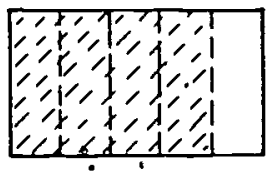

e. $\frac{4}{5}$

(Shade a "3parte, \& part, c 5 parta), \& 3 partal e. 4 pactw)

7. Draw simple figures and shade parts to show:
a. $\frac{1}{6}$
b.. $\frac{5}{8}$
c. $\frac{2}{5}$
d. $\frac{7}{10}$

8. Complete:

Fractions mas be used to nahne(rationall) numbers.

- Fractions are written with numerals separated by a bar.

- The numeral (below) the bar names the dencmetand tells Into how many parts of the same. size the unit is - separated.

The numeral (abre) the bar names the(mumesatay) and counts the number of parts of the same size being used. 
P262

9. Match each rational number named in Column $A$ with its symbol in Column $B$.

Column A

Column B

$\sqrt{7}$

a. two-rifths (iv)

8. $\frac{7}{9}$

b. seven -ninths (qu)

, $\frac{7}{4}$

c. rour-sevenths (ll $)$

1. $\frac{2}{5}$

d. 'five-hakves (h), d. $\frac{9}{7}$

e. nine-sevenths $(y)$ k. $\frac{5}{2}$

i. seven-fourths $(h) \quad i \cdot-\frac{4}{7}$

10. Set $A=$ \{set or. rational numbers\}.

Write names for five members of set A. (Aneweresced)

'11. Complete:"

$$
\begin{aligned}
\text { Set } A & =\{\bigcirc \bigcirc \bigcirc \bigcirc\} \\
\text { Set } B & =\{\bigcirc \bigcirc \bigcirc\} . . \\
\text { Set } C & =\{\bigcirc \bigcirc\} \\
\therefore \text { set } D & =\{\bigcirc\}
\end{aligned}
$$

If the measure of set $D$ is 1 the measure of sept $A$ is"

(4). The measure of Set $B$ is (3). The measure. of

set $C$ is $(2)^{\circ}$.

12. Use sets $A, B, C$, and $D$ in exercise 11 to complete the following: -

$\because$ If the measure of 'Set A is $1, "$ the measure of set $B$. $\therefore \quad$ is $\left(\frac{3}{4}\right)$. The measure of set $C^{-13}\left(\frac{2}{4}\right)$. The measure of set $D$ is $\left(\frac{1}{4}\right)$. 
RATIONAL N̦UMBERS ON THE NUMBER LINE

objectives: To review

- a. the use of 'rational-numbers as measures of segments on the number line.

b. the idea that some rational, numbers are

' whole numbers.

c. order of rational numbers on the number. line.

d. compartiston of rational numbers.

Materlais: Number lines, mulèr, yardstıçk

Vocabulary: Label", point untt sejment, ș̣plest name, $\because \quad$ - order, compare, geñeralization

\section{"Suggested Teaching Procedure}

Have children work through Exploration (first two pages). Then working together. teacher and "pup1 i should wild number lines scaled in fourths and efghtis, halves, thirds, and sixths, etc.

Have chlldren Iinish Expioration together. Before having children do the Exercise" set 2, the teacher may:

(a) have children count by rational numbers using number lines, mier, or yardstick;

(b) examine the order of rational numbers on the number line and their relation to i whole numbers;

(c) compare rational numbers: All' this can be done on number Iines on boardi
enlarged on paper, or dittoed for children.
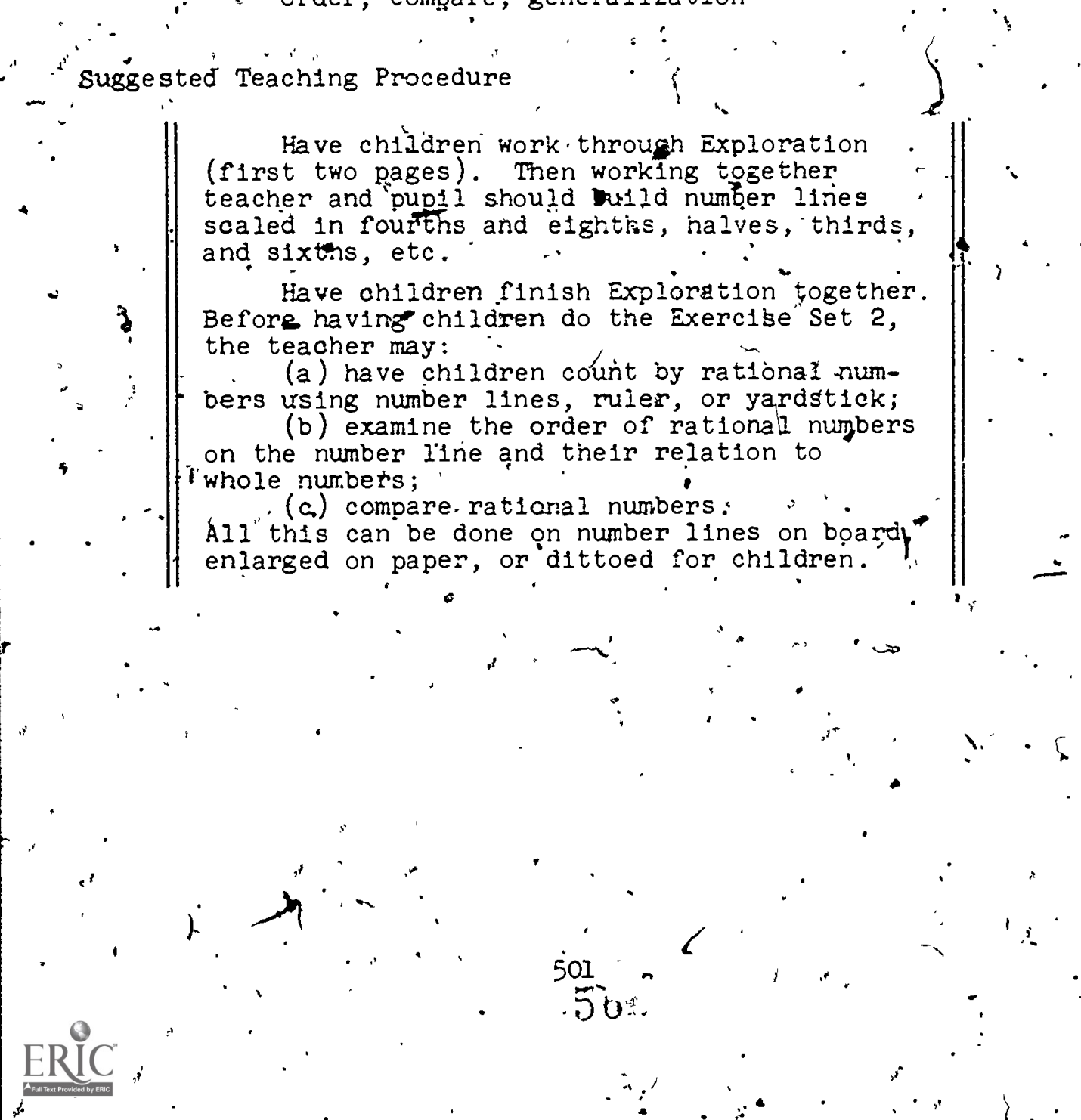
RATTONAL NUMBERS ON THE NUREER IINE
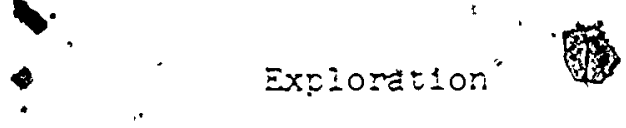

1. Draw a nuricer ine or jour paper. Croose polnt and label، I: 0 . Label otrer poirss equaily spaced in order, $1,2,3$, and $\rightarrow$ Your number ire shouid look like inis.

0

12 3 4

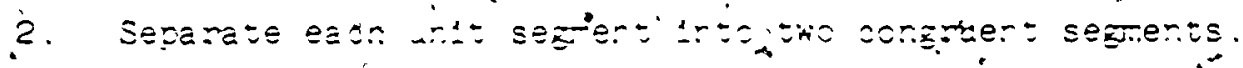

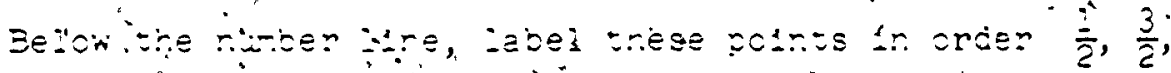
$\frac{2}{2}$, . $a r \dot{z} \frac{7}{2}$.

Does youn ruiber Itre zook line the ore belónt?

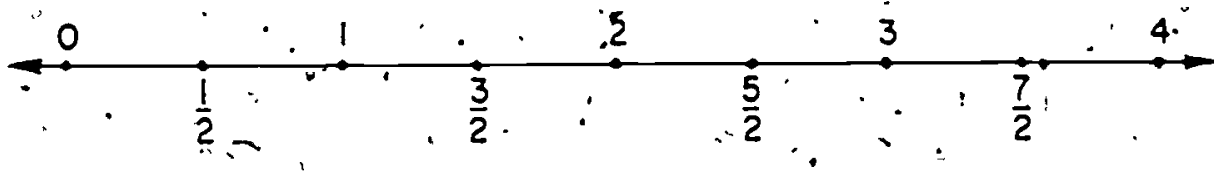

3. Whor potris car now be iabeied $\frac{0}{2}, \frac{2}{2}, \frac{\pi}{2},: \frac{6}{2}$ and $\frac{8}{2}$ ?

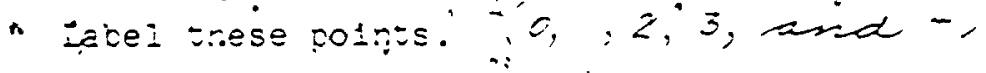

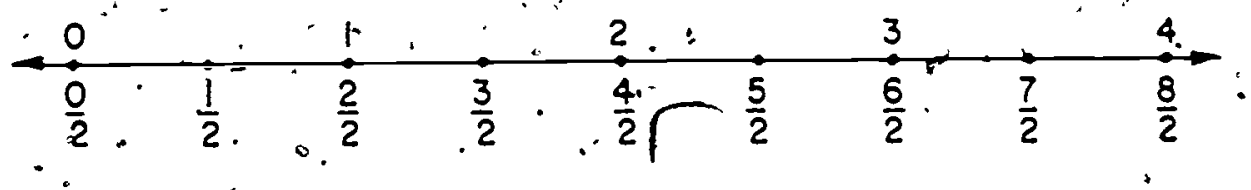

"Wour number istre row shoris a set of segmerts, each" $\frac{1}{2}$ the rength of the orifinal unit segment. The endpolnos are labeled inth"sractions.

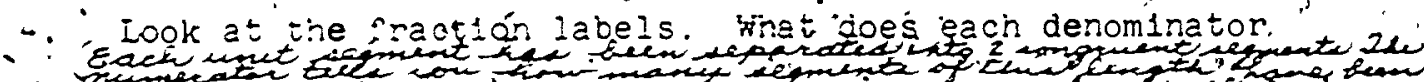
tell yound inat does each nuferator tell you? abo, treginang The points you labeled $\frac{0}{2}, \frac{2}{2}, \frac{4}{2}, \frac{6}{2}$, and $\frac{8}{2}$, were $\therefore$ already labeled with whole numbers 
p264

ij. What other names fid costis labeied $\frac{0}{2}, \frac{2}{2}, \frac{i}{2}, \frac{5}{2}$, and $\frac{8}{2}$ are shown on the rumber itreel 1 Car. a posnt on the number Ine have more inar, one rate . ifee,

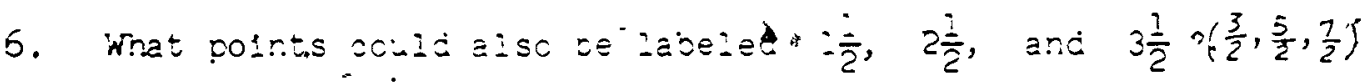

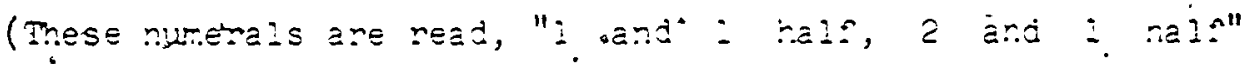
etc.)

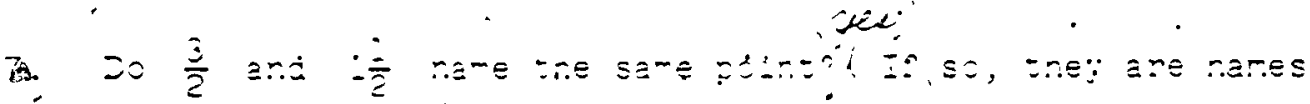
son zre same rimizen. fei

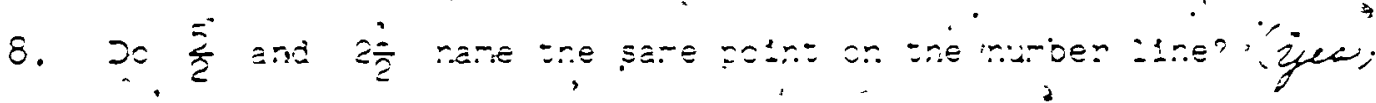
Surimanis:

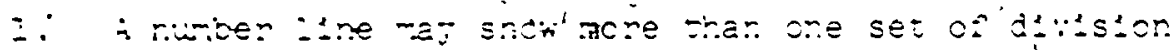
coinis.

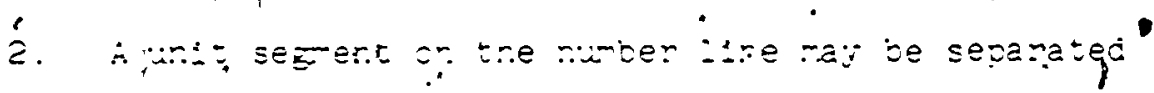

into ary rimger of corizment sezments.

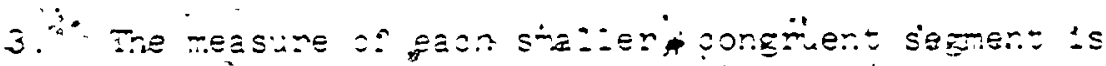
a ra:tcra:- rimicer.

- Sore potris on tre rumber itre can be named by numerels zor whole -umbers ard also by nactiors.

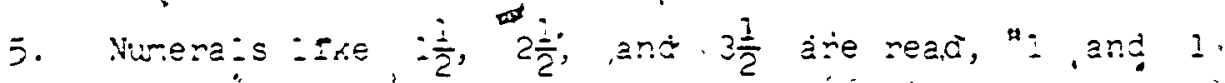
nals," "2 and 2 hals," and. "3 and $i$ nais"

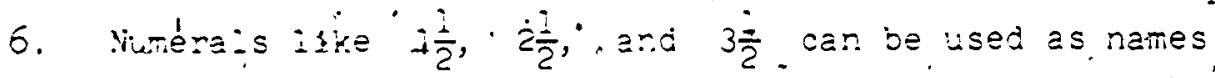
for certaln posnts or tre rumbet Ine.

$5 \mathrm{~s}$ 
P265

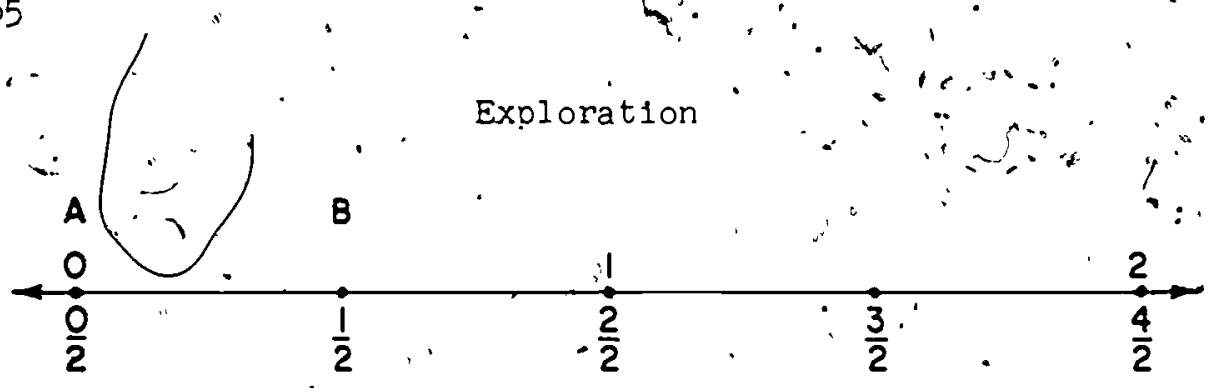
c.
D

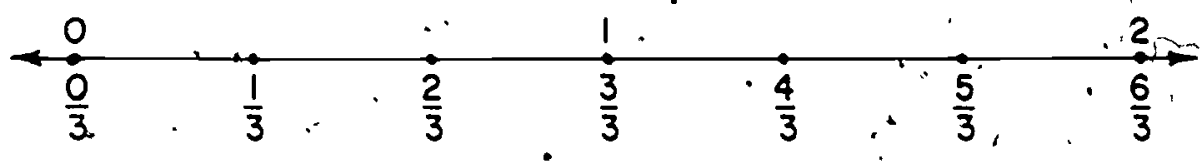

1. Use the number lines above to answer the following.

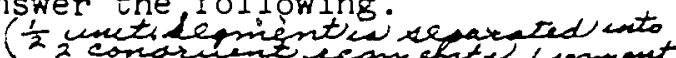
a) what is the measure of $\overline{A B}$ ? why? b. What is the measure of $\overline{C D}$ ?

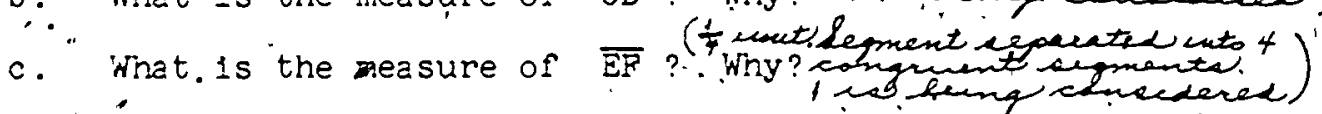

c. Which of the three rational numbers is, the greatest? $\left(\frac{1}{2}\right)$ fe. 'Nhich of the three rational numbers is the least? $\left(\frac{1}{4}\right)$. *.. f. A trrange the measures of $\overline{A B}, \overline{C D}$ and $\overline{E F}$ in ordep - from grieatest to least.' $\left(\frac{1}{2}, \frac{1}{3}, \frac{1}{4}\right)$

8. What generalization can you make about the order of numbers named by iractions whose numergtors are .1 ?

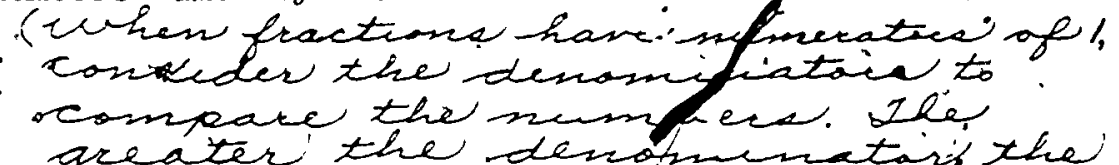
greater the denowberstais the

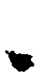


?..

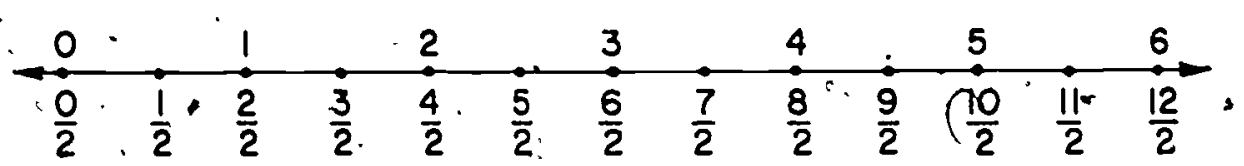

Use the number line above to answer the following questions:

a. Which rational number is greater, $\frac{3}{2}$ or $\frac{5}{2}$ ? $\left(\frac{5}{2}\right)$

b. How can you tell which rational number is greater?

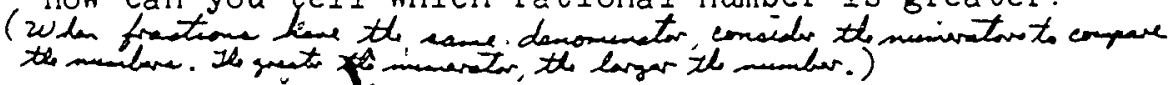

3. Which of the rational numbers in each pair is greater?

a. $\frac{2}{2}$ or $\frac{2}{2}\left(\frac{5}{2}\right)$

$\frac{6}{2}$ or $\frac{8}{2} \quad\left(\frac{8}{2}\right)$

c. $\frac{7}{2}$ or $\frac{3}{2} \cdot\left(\frac{7}{2}\right)^{2} \cdot 1+1$

4. Wnat are other names for $\frac{0^{(0)}}{2}, \frac{4}{2}$, and $\frac{6}{2}$ ? What are 2 . numerals that name:

a. 1 one and 1 half?. $\left(1 \frac{1}{2}\right)\left(\frac{3}{2}\right)$.

$\therefore$ b. 3 ones and 1 half? $\left(3 \frac{1}{2}\right)\left(\frac{7}{2}\right)$

c. 5 . ones and ' 1 hax? ' $\left(5 \frac{1}{2}\right)\left(\frac{11}{2}\right)$

Summary :

1. To compare rational numbers named by fractions whose numerators are 1 , look at the demominators. The greater the number represented by the denomiriator the smaller the rationai number.

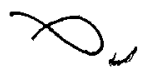

2. On the number I1ne; any fraction to the right of. another names the greater rational number. Any fraction to the left of another represents the smaller rational number. 
3. The order of rational numbers on the number line is the same as the order of whole numbers. As you move to the right along the number line, the rational numbers become greater. As you move to the left, they become smaller.

\section{Exercise Set $?$}

1. Label the points $A-K$ ' shown on the number lines 'below with fractions for rational numbers.
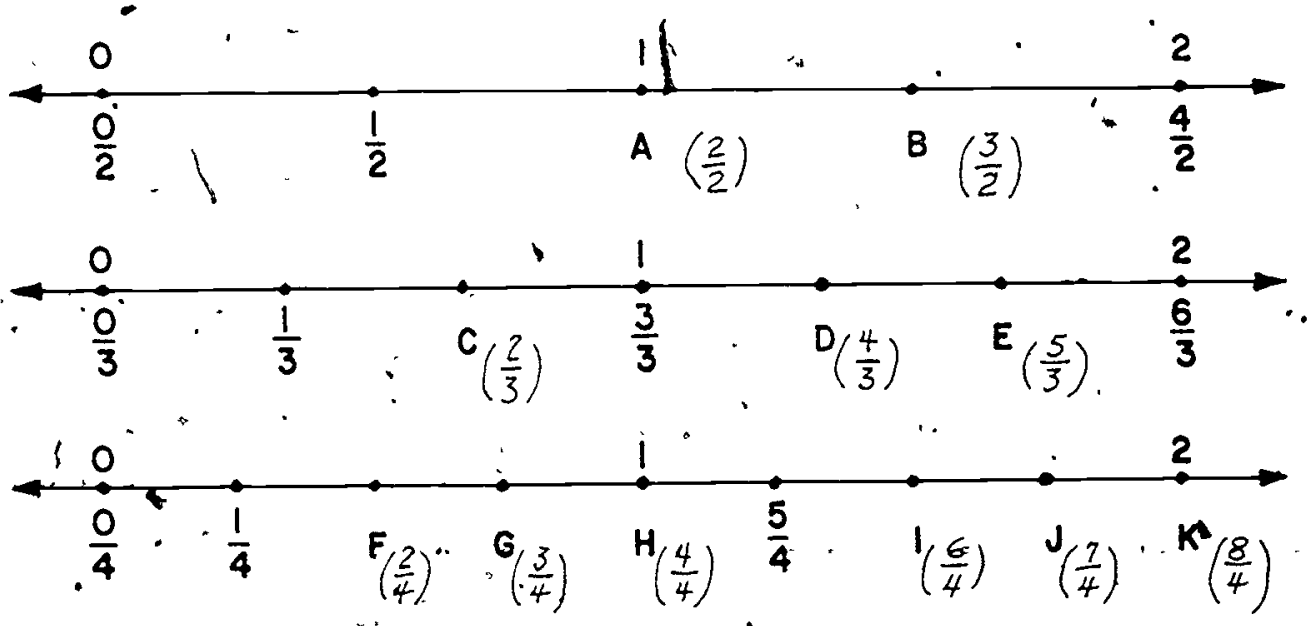

2. domplete each máthematical sentence below. Use $\langle$,$\rangle ,$ and $=$. The number lines in exercise 1 will help you.
a. $\frac{2}{3} \quad(<) \frac{3}{4}$
e. $\frac{7}{4}(<) \frac{6}{3}$
b. $\frac{4}{4}:(=) \frac{3}{3}$
f. $\frac{2}{3}(>) \frac{2}{4}$
c. $\frac{6}{4}(>) \frac{4}{3}$
g. $\frac{1}{4}(<) \frac{1}{3}$
d. $\frac{4}{2}(=) \frac{6}{3}$
h. $\frac{6}{4}(=) \frac{3}{2}$ 
3. Which is greater? How can you tell?
a.. 4 or 1
- e: $\frac{1}{2}$ or $\frac{2}{2}$
b.. $\frac{7}{2}$ or $\frac{5}{2}$.
$\left(\frac{7}{2}\right)$. f. $1 \frac{1}{2}$ or $\frac{4}{3}$
c. $\frac{4}{2}$ or 1
's. $\quad 1 \frac{1}{3}$ or 2
d. 0 or 2
(2)
h. $\frac{5}{4}$ or 1

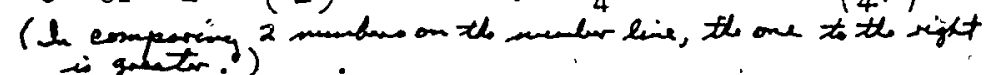

4. Arrange in order from least to greatest:

$$
\frac{7}{4}, .0,1 \frac{1}{2} \text {, and } \frac{4}{3} \quad \cdot\left(0, \frac{4}{3}, 1 \frac{1}{2}, \frac{7}{4}\right)
$$

5. Write the whole numbers that are between $\frac{1}{2}$ and $\frac{7}{2} \cdot(1,2,3)$

6. $1 \frac{1}{2}, \frac{3}{2}$, and 1 one and 1 half are all names for the same point on the number line. Write 2 other names for:
a. 1 one and 1 fourth

b. $\frac{4}{3}$

(one aind

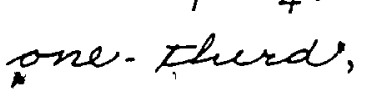

$\left.1 \frac{1}{3}\right)$

7. Write the rational numbers in each set in order of size from least to.greatest.

- Set $A=\left\{\frac{1}{4}, \frac{1}{2}, \frac{1}{3}\right\}\left(\frac{1}{4}, \frac{1}{3}, \frac{1}{2}\right) \cdot \operatorname{set} C=\left\{\frac{1}{6}, \frac{1}{3}, \frac{1}{12}\right\}\left(\frac{1}{12} \times \frac{1}{6}, \frac{1}{3}\right)$ Set. $B=\left\{\frac{3}{4}, \frac{3}{5}, \cdot \frac{3}{8}\right\}\left(\frac{3}{8}, \frac{3}{5}, \frac{3}{4}\right)$ Set $D=\left\{\frac{5}{10}, \frac{5}{2}, \frac{5}{4}\right\}\left(\frac{5}{10}, \frac{5}{4}, \frac{5}{2}\right)$

8. Count by fourths. from 0 to 3. Write your answers in a set. If you need help the number line will help you. The set has been started for you: $A=\left(\frac{0}{4}, \frac{1}{4},\left(\frac{2}{4}, \frac{3}{4}, \frac{4}{4}, \frac{5}{4}, \frac{6}{4}, \frac{1}{4}, \frac{8}{4}\right.\right.$, 


\section{PICTURING RATIONAL NUMBERS ON THE NUMBER LINE}

Objective: To help pupils ase rattonal numbers'to name different points on the number iline.

Vocabulary: Lay off

Materials: Number line on board

Suggested Teaching Procedure

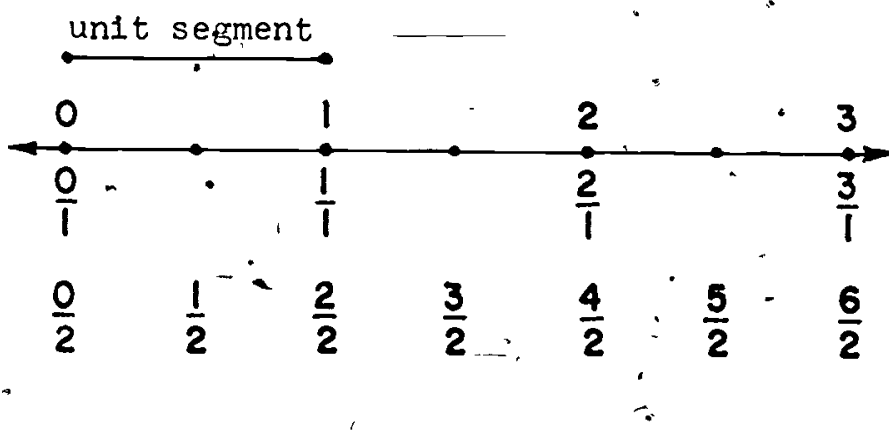

Let us think about the number line pictured above. Compared to the unit segmen't, what number tells the measure - of the segment with end points 'o and. $\frac{1}{2}$ ? What do the 1 and 2 in $\frac{1}{2}$ tell us? (The 2 tells us we are to think of the unit segment"as separated into two congruent segments. The' 1 tells us that if we start at $O$ and lay off one of these congruent segments toward the right, the other endpoint is at ' $\frac{1}{2}$.) Compared to the unit segment, what number telis the measure of the segment with endpoints, 0 and $\frac{3}{2} ?\left(\frac{3}{2}\right)$ What do the 3 and -2 in $\frac{3}{2}$ tell us? (The 2 tells us we. are to think of the unit segment as separated into two congruent segments. The 3 tells us that if we start at 0 and lay of three of these congruent segments end to end toward the right, the other endpoint is at $\frac{3}{2}$.)
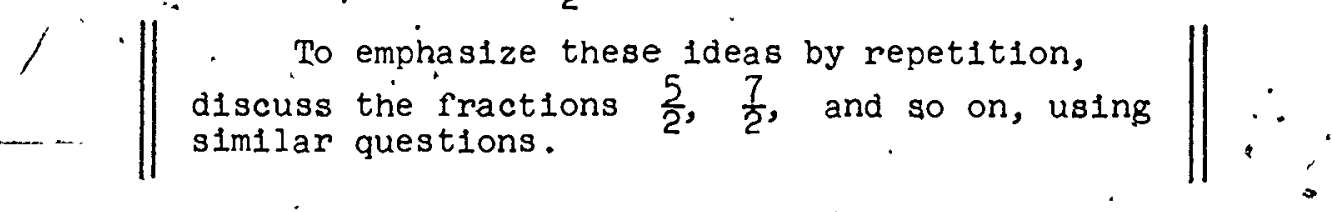
What do the 1 and 3 in the fraction $\frac{3}{1}$.teli us? (The $\mathcal{I}^{\circ}$ tells us that we are to think of the segment of measure 1. The 3 tells that if we start at 0 and lay off this segment 3 times, end-to-end to the right, the endpoint of the last segment $w 111$ be on the point $\frac{3}{1}$.)

Next, separating the unit segment into 4 (instéad of $\dot{1}$ or 2) congment segments, we locate additional points as before.. We label the (old and new) points $\frac{0}{4}, \frac{1}{4}, \frac{2}{4}, \frac{3}{4}$, and so on, as shown below.

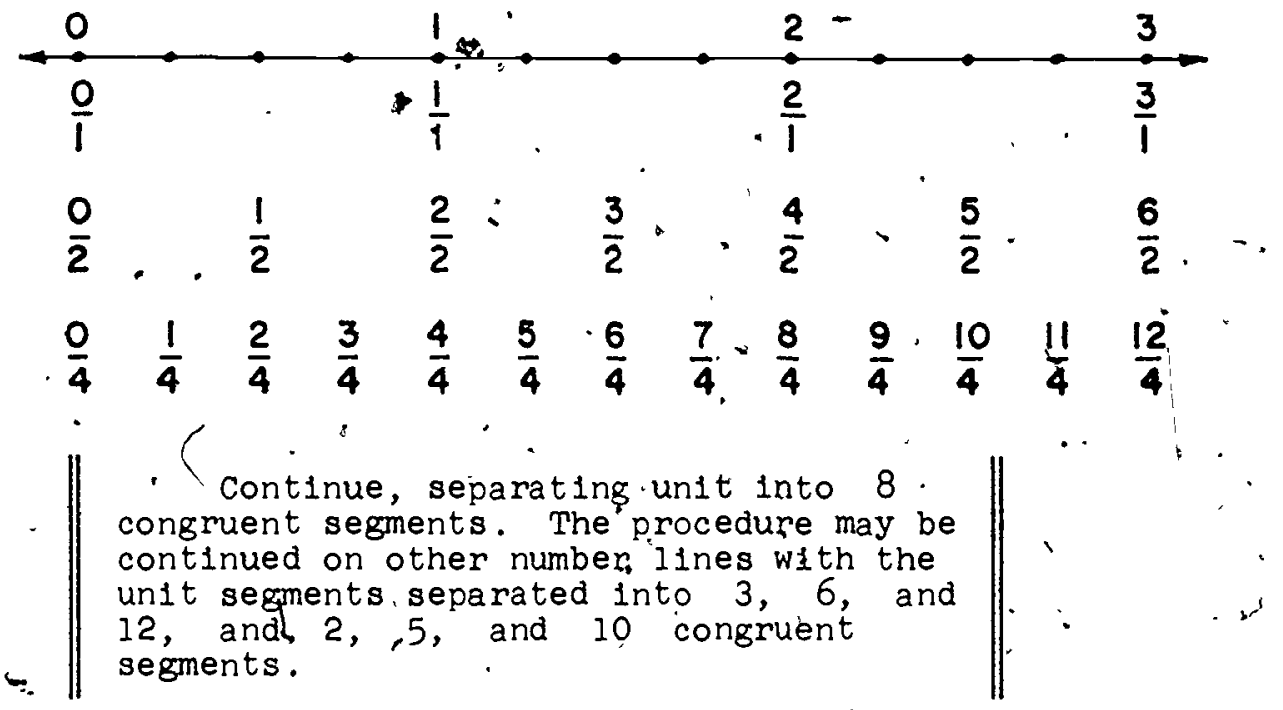


PICTURING RATIONAL NUMBERS ON THE NUMBER LINE

\section{Exercise $\underline{\text { Set }} \underline{3}$}
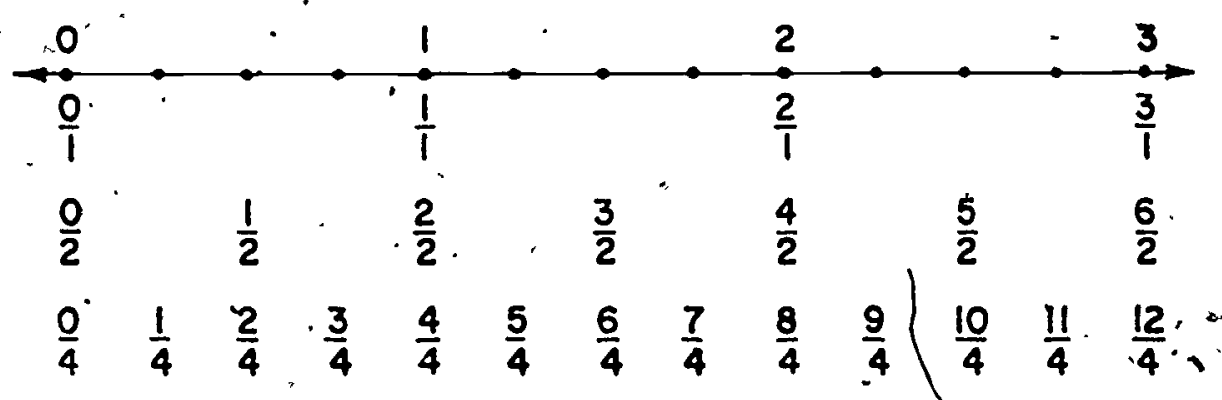

1. "In picturing" $\frac{5 x}{4}$ on the number line, into how many congrüent". segments, do you separate a segment of ength 1? Starting at 0 , hów many times do you lay off to the right a segment of this length to arrive at the point $\frac{5}{4}$ ? (5)

2. In picturing $\frac{3}{4}$ on the number line, into how many segments of the same length do you separate a segment of length 1?(4) Starting at $\dot{0}$, how many times do you lay off to the right a segment of this length to arrive at the point $\frac{3}{4}$ ?. (3).

3. In picturing 3 on the number line, into how many congruent segments do you separate a segment of length 1 ? (1) Starting at 0 , how mány times do you lay off to the right a segment of this length to arrive at the point 3 ?' (3)

4. In picturing $\frac{11}{4}$ on the number line, Into how many congruent segments do you separate a segment of length $1 ?^{(4)}$ Starting at zero, how many times do you lay off to the right a segment of this length to arrive at the point $\frac{11}{4}$ ? (II) 
P270 ,

5. In picturing $\frac{6}{2}$ on the number-ine, into how many congruent segments do "you sẹparate a ségment of length 1(2) Starting at 0 , how many times do you lay off to the right a segment of this length to arrive at the point $\frac{6}{2} ?(6)$

6. How many names are shown on the number line, in exercise 1 for
a. $0\left(0, \frac{0}{1}, \frac{0}{2}, \frac{0}{4}\right)$
b. $\frac{1}{2}\left(\frac{1}{2}, \frac{2}{4}\right)$
c. $3\left(3 ; \frac{3}{1}, \frac{6}{2}, \frac{12}{4}\right)$
d. $2 \frac{1}{2}\left(\frac{5}{2}, \frac{10}{4}\right)$

7. Label with fractions points $A,(B$, and $\dot{C}$ on the number lines below.

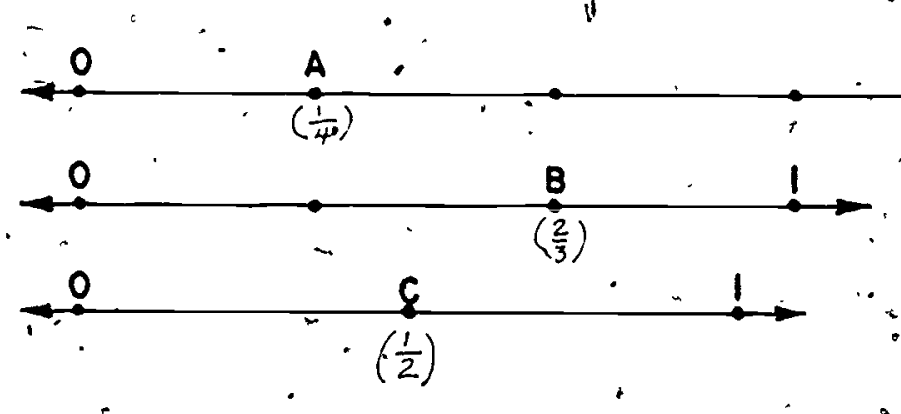

\section{BRAINTWISTER}

Label with rational numbers the points ${ }^{\vee} A, B$, and $C$ of the number ines below.

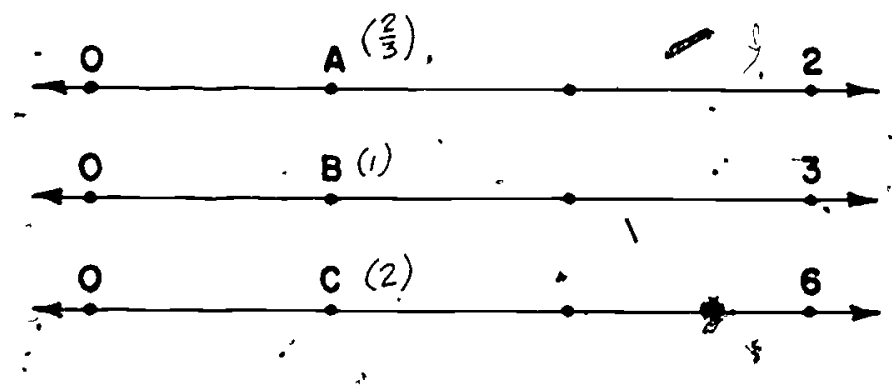


PICTURING RATIONAI NUMBERS WITH REGIONS

\section{I}

Exploration

1. Figure A represents a unit region. It is separated into 3 smaller congruent regions and 2 of these. re'glons are shaded.

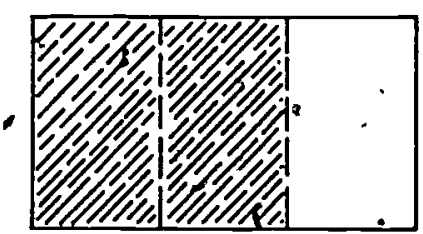

A

a. What is the mêsure, of the shad ded region? $\left(\frac{2}{3}\right)$

b. How are the 2 and the 3 fn the fraction $\frac{2}{3}$

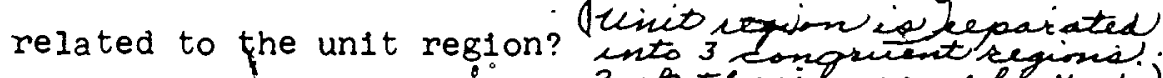
$.2 \mathrm{~g}^{3}$ thesi ace shediodi)

2. Figure $B$ represents the same unit region. II is separated into 6 smaller congrient regions and 4 of these regions - Śre shaded.

* What is the measure of the shaded region? $\left(\frac{4}{6}\right)$

b. How ate the 4 and 6 in the fraction $\frac{4}{6}$ related to

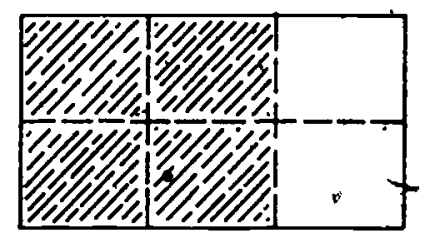

B

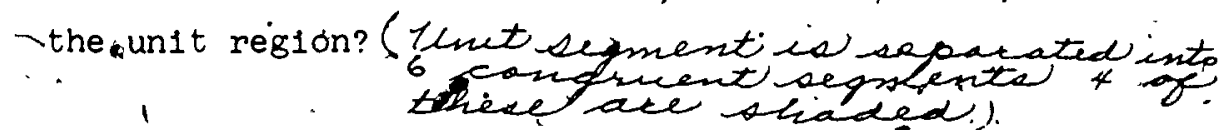

3. - Trace a rectangle congruent to rigure A. Draw broken lines

- to separate the regson into 9 congruent regions"and shade 6: of these regions as shown in figure $C$.

a. What is the measure of the shaded region? $\left(\frac{6}{9}\right)$

b. How are the 6 and the 9 of the iraction $\frac{6}{9}$ related

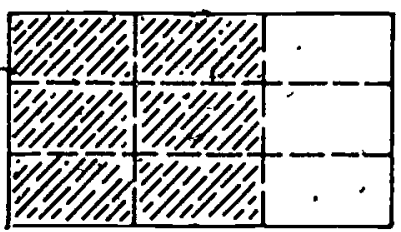
to the unit region? 9 congruent segmentu

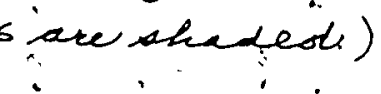<smiles>[AsH]=[AsH]</smiles> 
4. a. Is the unit region the same size in figures $A, B$, "and $C$ ?' '(Yyen')

b. - Are the shaded regions of $A, \cdot B$, and $C$ congruent? (yes)

c. Are the rational numbers $\frac{2}{3}, \frac{4}{6}$ and $\frac{6}{9}$ the measures of these congruent shaded gions?

d. Are $\frac{2}{3}, \frac{4}{6}$, and: $\frac{6}{9}$ names for the same rational' *number?'(zesy

5. Draw 3 congruent rectangular regions: Label: them $A, B$, and $\mathrm{C}$.

a. - Sebarate. A into 3 simalzer congruent regionś.

Shade i region $/$ What is the measure of the shaded region? $\left(\frac{i}{3}\right)$

b. Separate B into 6 smaller congruent regions and shade 2 of them. What is the measure of the straded region? $\left(\frac{2}{6}\right)$

c. Sejparate $c$ into '9 congruent regions. Shàde. 3 of these regions. "Are $\frac{1}{3}, \frac{2}{6}$, and $\frac{3}{9}$ names for the 'same rational number? 1 "Why? (They name the'

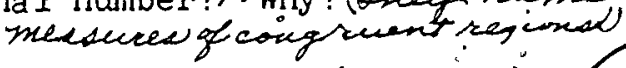

6. The measure of the c1rcular region at the right 1s 1 . The measure of the shaded part is $\frac{4}{8}$. What is the relation of the 4 and 8 of the fraction to the unit pegion? (2he unet sequin es separated anto

7. Trace the circular region above. Separate it into 2 )

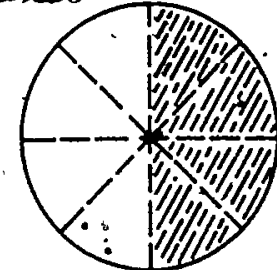
congruent regions. Shade 1 part.

a. What is the measure of the shaded region? $\left(\frac{1}{2}\right)$

b. Are $\frac{4}{8}$ and $\frac{1}{2}$, names for the same number since they name the measures of congruent regtons? (ijes)' 
8. Draw a circular region congment to the one in exercise 6. II Separate. \pm 'Into 4 congment regions and shade 2 , of "

- the,parts. Is $\frac{2}{4}$ another name for $\frac{1}{2} \cdot 3$ and $\frac{4}{8}$ (yes) Why"

A rational number may have many different names

$$
\begin{aligned}
& \cdot i=\frac{2}{2}=\frac{4}{4}=\frac{8}{8} \\
& \frac{1}{2}=\frac{2}{4}=\frac{4}{8} . \\
& \frac{2}{3}=\frac{5}{5}=\frac{5}{3} .
\end{aligned}
$$

\section{Exercise Set}

1. Answer triese questions for each stgure belo\%.

a. Into how many congruent regions is the undt region separated?

b. How many congrueri regions are shaded?

c. What fraction name best jescribes the measure of the shaded region?
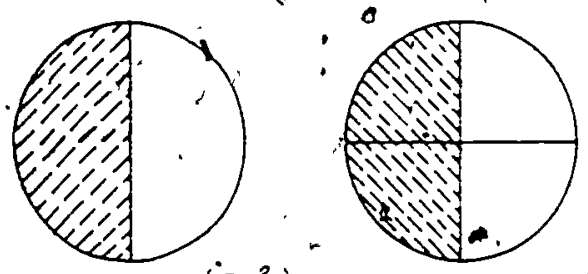

Pigure $1\left(\begin{array}{ll}a & 2 \\ b & 1 \\ c & \frac{1}{2} \\ \frac{b}{2}\end{array}\right)$ Bigure $2\left(\begin{array}{ll}a & 4 \\ b & 2 \\ c & \frac{2}{4}\end{array}\right)$
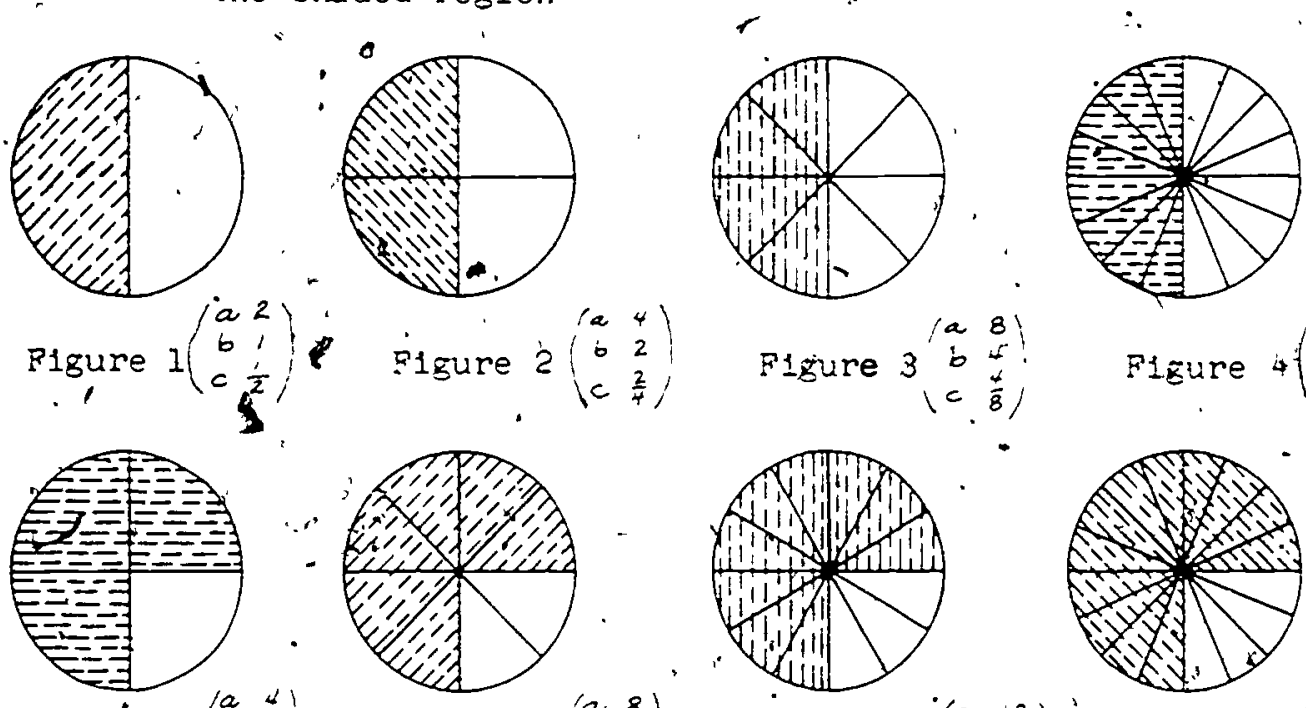

P1gure $5\left(\begin{array}{ll}a & 4 \\ b & 3 \\ c & \frac{3}{4}\end{array}\right) \quad$ Figure $6\left(\begin{array}{ll}a & 8 \\ b & 6 \\ c & \frac{6}{8}\end{array}\right)$.

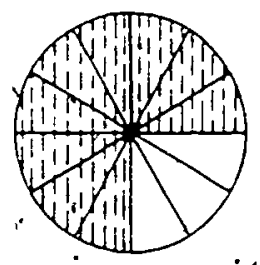

Figure $4\left(\begin{array}{ll}a & 16 \\ b & 8 \\ c & 8 \\ 16\end{array}\right)$.

$\left(\begin{array}{ll}a & 8 \\ b & 5 \\ c & \frac{1}{8}\end{array}\right)$
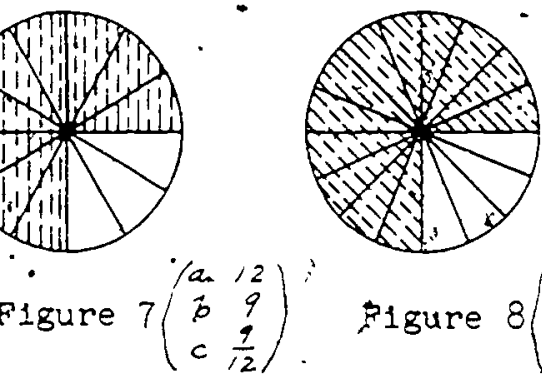

Pigure $8\left(\begin{array}{ll}a & 16 \\ b & 12 \\ c & \frac{12}{16}\end{array}\right)$ 
Pें́

2. Do; the fractions in exercise c "for figures I through" 4 name the same rational number? What rational number do they name? $\left(\frac{1}{2}\right)$

3. Do the fractions in exercise $c$ for figures 5 through 8 - name the-same rational number? What rational number do they - name? $\left(\frac{3}{4}\right)$

4. Write three other rames son $\frac{1}{2} .\left(\frac{2}{4}, \frac{3}{6}, \frac{7}{8}, \frac{5}{10}, \frac{6}{12}, \frac{7}{14}\right.$, eto)

5. Write inree other names ron $\frac{3}{6} \cdot\left(\frac{6}{8}, \frac{9}{2}, \frac{12}{16}, \frac{15}{20}, \frac{18}{2+}, 2\right.$ ted),

6. Wirtte three names for $\frac{1}{4}$. ( $\frac{2}{8}, \frac{1}{16}, \frac{3}{12}, \frac{5}{20}, \frac{\dot{\epsilon}}{24}$, eto $)$

A.

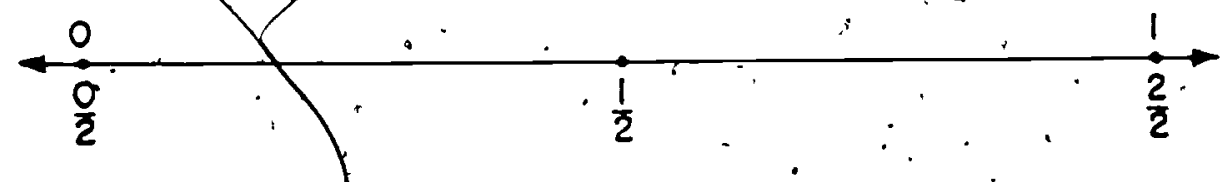

.3
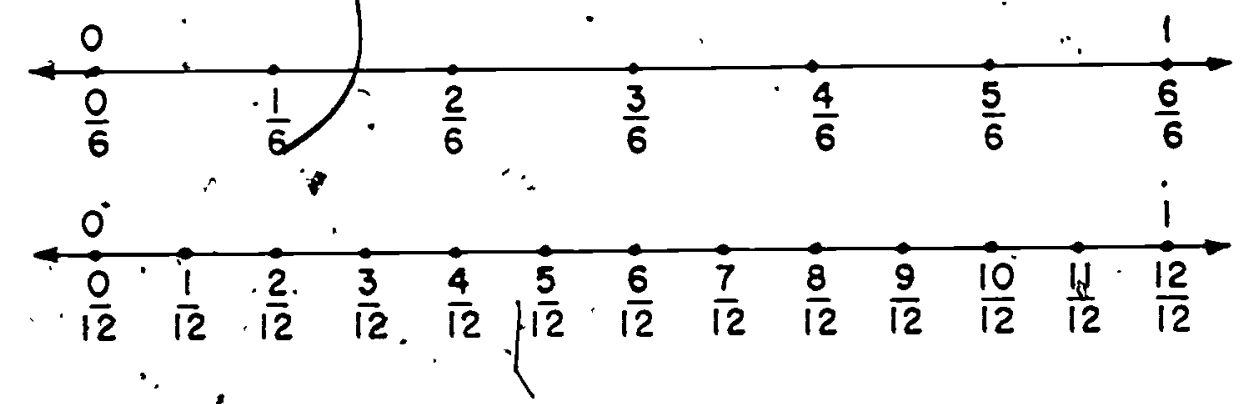

- 7. Look at the number IInes A; B, and C above. Tnree congmient segments, each, having the measure of 1 , have been, separated into smaller congmen't gegments. Answer the following questions about each number line.

a. Into how many congruent, segments has the unit segment been separated?: $\left(\begin{array}{cc}a & 1 \\ b & 6 \\ c & 12\end{array}\right)$

b. : What fraction best names the'measure of each smaller congruent segment? ( $a \frac{i}{2} ; b \frac{1}{6}, c \frac{1}{12}$ ) 
P275

8. Make true statements by writing a different fraction in each space.
a. $\frac{1}{2}=\left(\frac{2}{x}\right)=\underline{\left(\frac{3}{6}\right)}=\frac{\left(\frac{4}{8}\right)}{1}$
d. $\left.\frac{1}{6}=\frac{\left(\frac{2}{8}\right)}{8}=\frac{\left(\frac{3}{12}\right)}{=}=\frac{(4)}{16}\right), \ldots$
b.' $\frac{1}{3}=\left(\frac{2}{6}\right)=\left(\frac{3}{9}\right)$
c. $\frac{2}{3}=\frac{\left(\frac{4}{6}\right)}{2}=\left(\frac{6}{9}\right)$
e. $\frac{3}{1}=\left(\frac{6}{8}\right)=\left(\frac{9}{12}\right)=\left(\frac{12}{16}\right)$
$\therefore I=\left(\frac{2}{2}\right)=\left(\frac{3}{3}\right)=\frac{(4)}{4}$ 


\section{RATIONAL NUMBERS WITH SETS OF OBJECTS}

Objectives: To develop the idea that a rational number shows the relation of a subset to a le set.

To use sets of objects to help children discover ‘. different names for rational numbers.

Materials: Sets of objects, arrays

Vocabuiary: 'Set, subset

Suggested Teaching Procedure

Work througn Exploration together. Follow up with pictures of arrays or sets as shown in. section "Materials", or better yet, concrete gojects. Use sets of 12 objects, and have the pupils show fourths -of the set, thinds of the set, sixths of the set, etc. Also, have. them tell what rational number tells what. part a certain subset is of the whole set: Sets, or pictures of sêts, with 16 objects (or some other number) may be used in a similar manner.
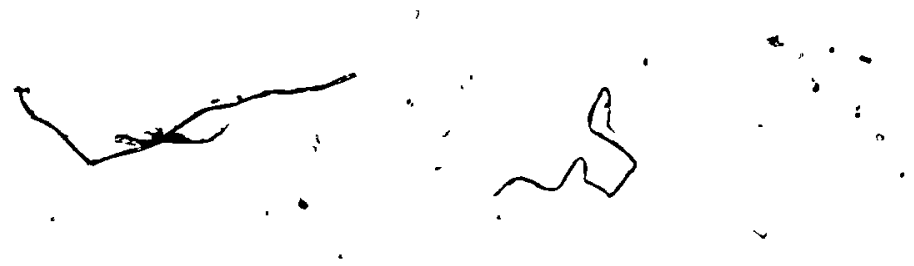

$\cdot 1$ 


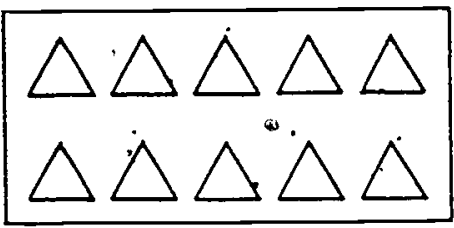

A

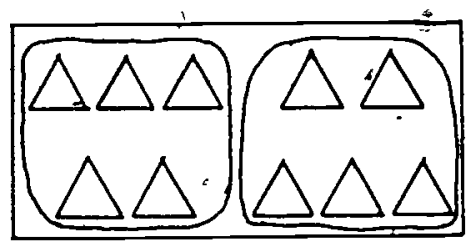

B

1. Figure A shows a picture of a set of 10 objects: What rational number best describes what part of the set each object 1s? $\left(\frac{1}{10}\right)$

- 2. Pigure B "shows the same set separated into subsets each naving the same number of objects.

a. Into how'many, subsets has the set shown if $A$ been.". separated in B? (2)
b. How many" objects are in each" subset?
(5)
c. What part of the set is in each subset? ( $\frac{1}{2} \cdot$ for $\frac{5}{10}$ )

d. Do $\frac{1}{2}$ and $\frac{5}{10}$ name the same rational number? (yed)

3. Trace figure A.' Separate.the objects into 5. subsets,

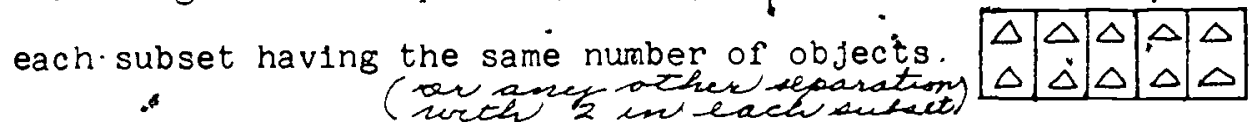
Replace $n$ by a number which makes each sentence true.
a. $\frac{1}{5}=\frac{n}{10}$
c. $\cdot \frac{3}{5}=\frac{i n}{10}$
b: $\frac{2}{5}=-\frac{n}{10}$
d. $\frac{4}{5}=\frac{n}{10}$ 


\section{Exerc1se Set 5}

. $j^{\circ}$

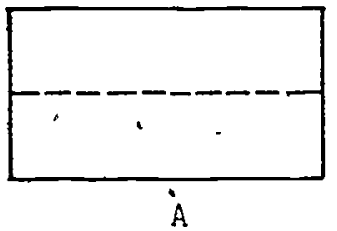

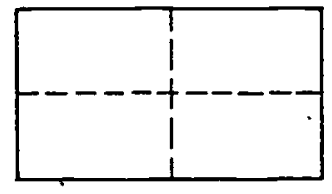

B

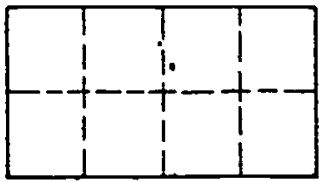

C

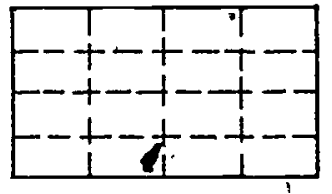

D

$A, B, C$, and $D$ are congruent rectangular regions. The measure of reach is 1 .. Use them to he fo fou answer the following exercises.

1. There are $4^{\circ}$ quarts in a.galion. 3 quarts is the same amount as

a. (3) fourths of gation.

b. (6) eighths of a galion.

c: (1) sixteenths of, a galion.

2. There are 16 ounces in a pound. 8 'ounces is the same amount as

a. I (loge of a pound.

b. L(foerthis) of a pound.

c. 4(eightelior a pound.

d. 8 (sixtienths) of a pound.

3. How many-quarts are there in $\frac{1}{2}$ gallon? (2).

4. How many ounces are there in $\frac{1}{4} \cdot$ pound?

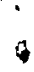


$\therefore \quad \mathrm{P} 278$
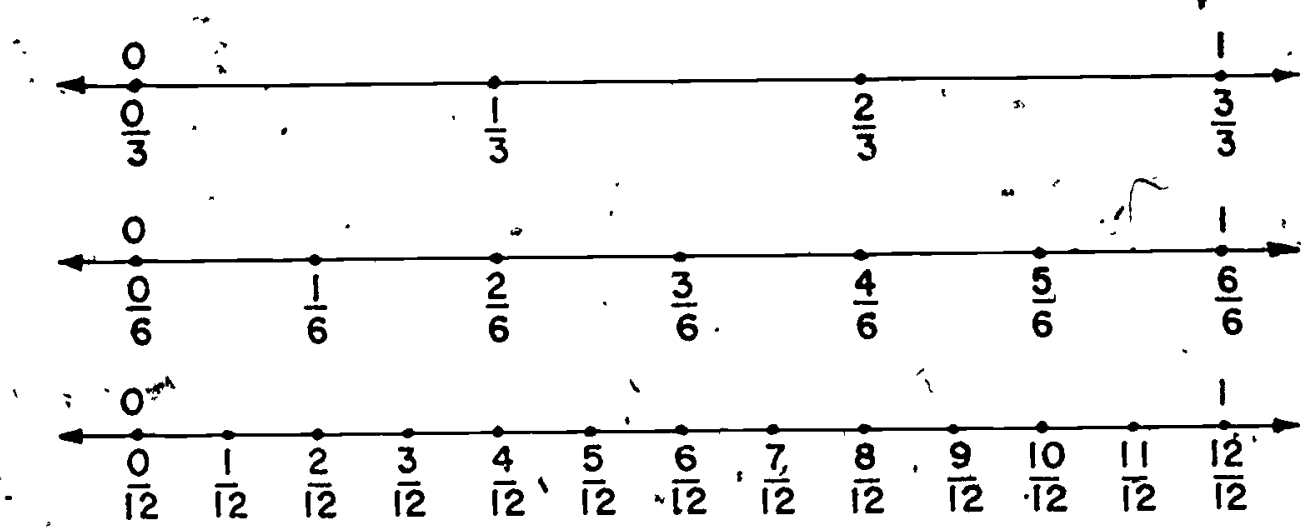

Use the number lines above to answer the following exercises.

5. 8. inches may be written

a. (5) twelfths of a foot.

b. (4) sixths of a foot.

- cr e (2) thirds of a foot.

6. Ten months covid be written as if 10 (twelfore) of a year.

b. 5 (sixths) of a year.

- 7. What part of a dozen cookies are

a. 6 cookies? $\left(\frac{1}{2}\right.$ an $\left.\frac{6}{12}\right)$

- b. 4 cookies? $\left(\frac{4}{12}\right.$ or $\left.\frac{1}{3}\right)$

c. 8 "cookies? $\left(\frac{8}{12}\right.$ as $\left.\frac{2}{3}\right)$

-d. 10 cookies? ( $\frac{10}{12}$ or $\left.\frac{5}{6}\right)$

8. 'What part of a yard is

ia. I' foot? $\left(\frac{1}{3}\right)$

b. 2 feet? $\left(\frac{2}{3}\right)$

䓡

9. What part of a year is

a. 4 months? $\left(\frac{4}{12}\right.$ ias $\left.\frac{1}{3}\right)$.

b. 6 months? $\left(\frac{6}{12}\right.$ ar $\left.\frac{1}{2}\right)$ : 
P279

10. How many inches are there in $\frac{1}{3}$ of a foot? (4)

11. How many eggs are there in " $\frac{1}{\sigma}$ of a dozen? (2)

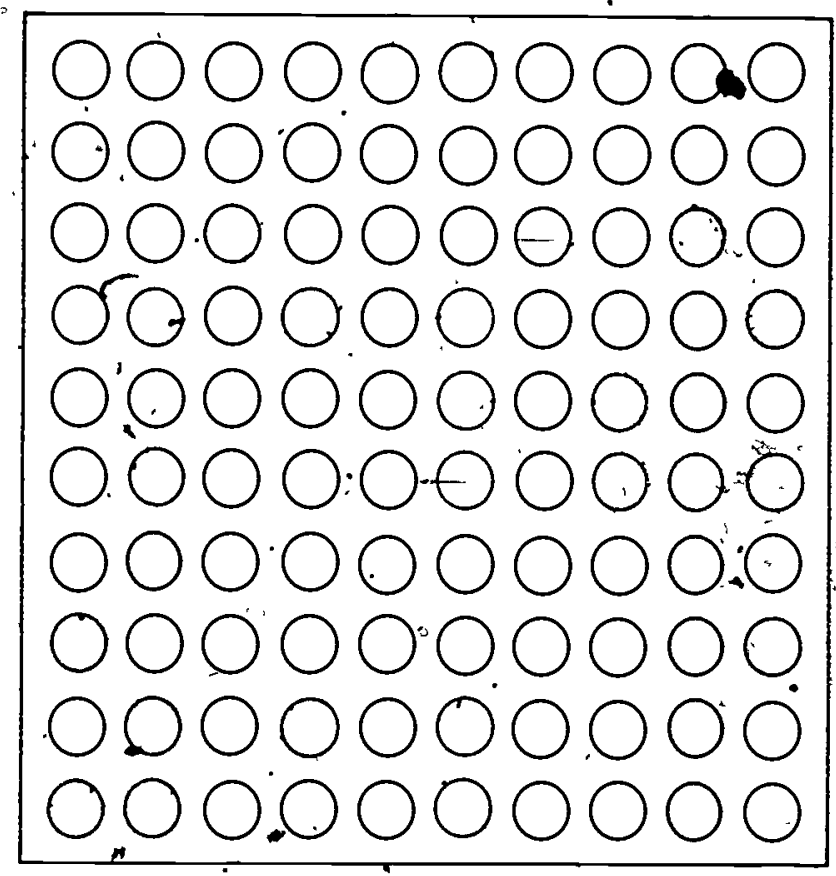

12.. "Above Is a plcture of". a set of 100 pennies. Use it to answer the foliowing questions. Write the fraction with the smallest denominätor for fach rational number used in your ansiswers.

What part of one dollan is

- a. 50 penntes?

b. $19 . \therefore$ pennies? : $\left(\frac{1}{10}\right)$

$\therefore \quad 25$ penniés? $\left(\frac{1}{4}\right)$

d. 5 pennieg! $\left(\frac{1}{20}\right)$

e. $20^{\circ}$ bennies? $\left(\frac{1}{5}\right)$ -

$\because$

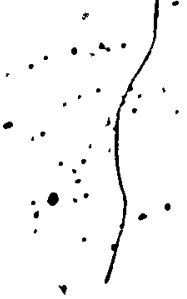


THE SIMPLEST FRACTION NAME FOR A RATIONAL NUMBER

Objectives: To review

(a) many names for one rational number.

(b) meaning of numerator and denominator. To generalize

(a)" any' rational number may be renamed by multiplying numerator and denominator of a fraction name by the same counting. number greater than 1.

(b) although there are more names for a rational number than can be counted, the simplest name for a rational number is that fraction in which numerator and denominator have no common factors except 1.

(c) to find the simplest name, remove greatest common factor of numerator, and denominator.

Vocabulary: Stmplest name, Prime number, common factor; greatest common factor, digit, odd, prime factor, complete factorization, composite

Suggested Tedching. Procedure

and Prief review of Chapter 2, Grade $5^{\text {"Factors }}$ needed.

Have class read and discuss Exploration on "Simplest Name for a Rational Number": It is hoped. children will disc'over intultively how to find many names for any rational number. You w1ll have to judge whether or not your puplis are ready to express the generalization: to find another name for a rational number, multiply the numerator and denominator by the same counting number. When this has been done, proceed with rest of Exploration and Exercise Sets 6 and 7 . 
P280

THE SIMPLEST FRACTION NAME FOR A RATIONAL NUMBER

Exploration

We have found that many fractions name one rational number. For example, we know that

$$
\frac{1}{2}=\frac{2}{4}=\frac{3}{6}=\frac{4}{8} .
$$

1. Find three more fractions that belong on this 11 st. $\left(\frac{5}{10}, \frac{6}{12}, \frac{7}{14}\right.$, eta $)$

. 2. Do these fractions belong on the list? (Yeas).

$$
\frac{50}{100}, \frac{100}{200}, \frac{111}{222}
$$

3. Find $n, m$, and $p$ so that each fraction names $\frac{1}{2}$.

$$
\frac{1}{2}=\frac{n(7)}{14}, \quad \frac{1}{2}=\frac{8}{m}, \quad \frac{1}{2}=\frac{p}{250}(125)
$$

- We have also found several names for $\frac{2}{3}$.

$$
\% \frac{2}{3}=\frac{4}{6}=\frac{6}{9}=\frac{8}{12} \text {. }
$$

4. Do these -fractions belong on this list of names of $\frac{2}{3}$ ? (yes).

$$
\frac{20}{30}, \frac{2 \times 4}{3 \times 4}, \frac{14}{21}, \frac{50}{75}, \frac{2 \times 876}{3 \times 876}, \frac{2 \times 2 \times 5 \times 7}{2 \times 3 \times 5 \times 7}
$$

5. Suppose that $m$ and $n$ are counting numbers.. Give three other names for $\frac{m}{n}$.

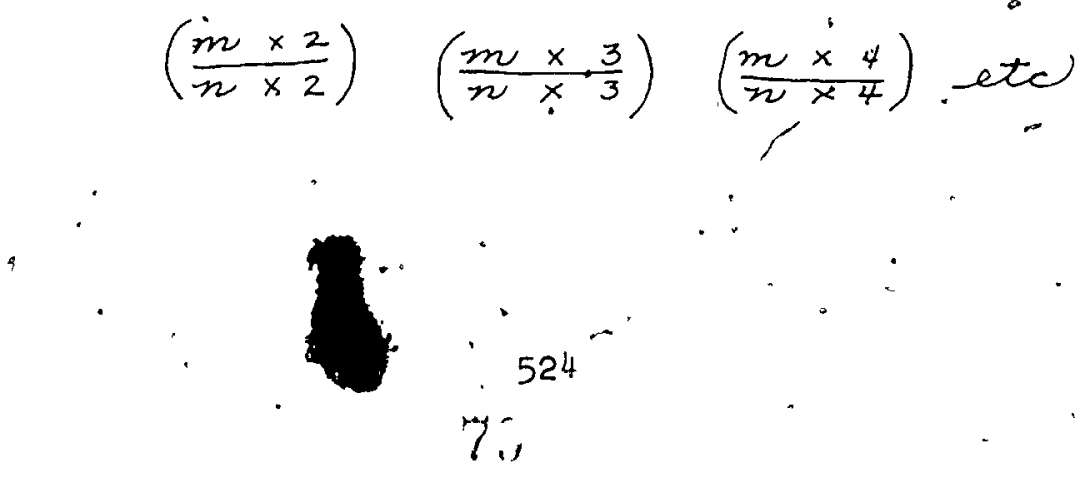


P281

Now try to imagine the set of all fractions which name one rational number:

0

$$
\begin{aligned}
& \left\{\frac{1}{2}, \frac{2}{4}, \frac{3}{6}, \frac{4}{8}, \frac{5}{10}, .\right\} \\
& \left\{\frac{2}{3}, \frac{4}{6}, \frac{6}{9}, \frac{8}{12}, .\right\} \\
& , \quad\left\{\frac{3}{4}, \frac{6}{8}, \frac{9}{12}, \frac{12}{16} . .\right\}
\end{aligned}
$$

Each such set contains a fraction smaller than the rest. We will call this fraction the simplest. fraction name for the rational number. It is a name we often use.

Any other. fraction. in each set can be found from the simplest fraction" name: Do you know the rule for finding the other fractions?

"( Any rational number me y be resumed by miltaging senator

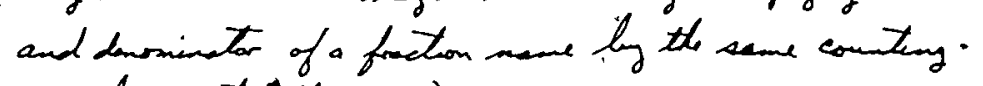
men ter grass 1.)." 
FINDING THE SIMPLEST FRACTION NAME

Exploration

How can you tell whether a' fraction '1s the simplest name

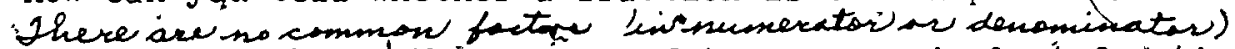
for a rational number? Which ones of these are simplest fraction names? $\left(\frac{5}{11}, \frac{9}{14}, \frac{3 \times 5 \times 5 \times 7}{2 \times 2 \times 11}\right)$

$\frac{3}{12}, \frac{8}{10}, \frac{5}{11}, \frac{9}{14}, \frac{6}{2}, \frac{3 \times 5 \times 5 \times 7}{2 \times 2 \times 11}, \frac{2 \times 5 \times 7 \times 11}{3 \times 5 \times 13}$

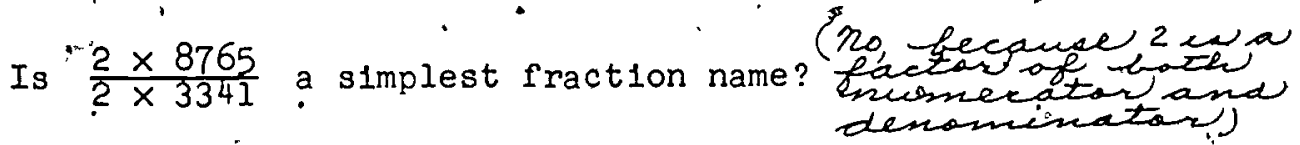

1 Perhaps you remember. that 13 is a prime number. Is

13

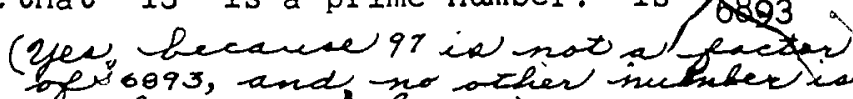

a simplest fraction, name? of 6093 , and no ot

Is $\frac{n}{13}$ always a simplest fraction name if $n<13$ ? (Yei)

These examples should suggest two things to you: .

First, a simplest fraction name is one in which the numerator: and denominator hạve no odmmon factors except 1

Second, you can find the simplest fraction name from any fraction in the set by finding the greatest common factor of 1ts numerator and denominator.

Here are several examples showing how you can find simplest rraction names.

1) Find the simplest fraction name for $\frac{30}{45}\left(\frac{2}{3}\right)$ First factor numerator and denominatór completely.

$$
\frac{30}{45}=\frac{2 \times 3 \times 5}{3 \times 3 \times 5}
$$

Next remove the common prime factors shown ( 3 . ind 5)."

$$
\frac{30}{45}=\frac{2 \times 3 \times 5}{3 \times 3 \times 5}=\frac{2}{3} \ldots
$$

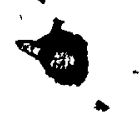

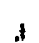

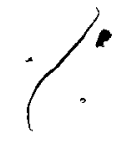

526

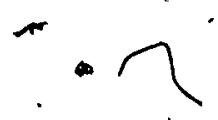

81 . 
2) Find the simplest iraction name for $\frac{96}{375} \cdot\left(\frac{32}{25}\right)$. Hére is a different methọd. First test 2 as a factor of both numerator and denominator. You find that 2 is not a factor of 375 because, the units digit is odd. This means that 2 cannot be a common factor of 96 and 375 .

Now test 3 as a ractor of numerator and denominator. You find:

$$
-\frac{96}{375}=\frac{3 \times 32}{3 \times 125} .
$$

Now remove the common factor.

$$
\frac{96}{375}=\frac{3 \dot{x} 32}{3 \times 125}=\frac{32}{125} \text {. }
$$

Next test 3 again as a factor of 32 and "la 'Since 3 is not a factor of 32 is not a common factor. Continue and try' 5 as a common fractor. Notice, however, that 2 is the only prime factor of 32 and that 5 is the only prime factor of 125. If you see this, it will save you time because you know right away that 32 and .125 have greatest. common factor 1.. This means that $\frac{32}{125}$ is the. simplest fraction hame for $\frac{96}{375}$.

3) Now try the method used in example 1 'to find the, simplest fraction name for $\frac{90}{84}$. $\left(\frac{15}{14}\right)$

4) Next try the method used in example 2 to find the simplest fraction name for $\frac{108}{100}$. ( $\left(\frac{27}{25}\right)$ 
If you know many multiplication facts you cañ often shorten the work in finding simplest fraction names. For example, in, finding the simplest fraction name for ${ }_{88}^{56}$ you might remember that $8 \times 7 \doteq 56$ and $8 \times 11=88$. Then you car write

$$
\frac{56}{88}=\frac{8 \times 7}{8 \times 11}=\frac{7}{11}
$$

5) How can you use the fact: $12 \times 12=144$ in findin: $\checkmark$ the simplest fraction name for $\frac{60}{144} ?\left(\frac{5 \times 12}{12 \times 12}=\frac{5}{12}\right)$ of cour you can always use one of the methods shown - in the examples. .

\section{Exercise Set 6}

1. Write three other fractions, naming each of the following numbers.

a. $\frac{1}{3}\left(\frac{2}{6}, \frac{3}{9}, \frac{4}{12}\right.$, eth $)$ d. $\frac{3}{4},\left(\frac{6}{8}, \frac{9}{12}, \frac{12}{16}\right.$, eto $) 8 . \quad \frac{1}{6}\left(\frac{2}{12}, \frac{3}{18}, \frac{4}{24}\right.$, etw $)$ b. $\quad \frac{2}{5}\left(\frac{4}{10}, \frac{8}{20}, \frac{16}{40}\right.$, eto $)$ e. $\frac{2}{3} \cdot\left(\frac{4}{6}, \frac{6}{9}, \frac{8}{12}\right.$, ete $) h ., \frac{3}{2}\left(\frac{6}{4}, \frac{9}{6}, \frac{12}{8}\right.$, eted $)$ c. $\quad \frac{5}{4}\left(\frac{10}{8}, \frac{15}{12}, \frac{20}{16}\right.$, eto $)$ f. $\frac{7}{8}\left(\frac{14}{16}, \frac{21}{24}, \frac{28}{32}\right.$, ete $) 1 . \frac{3}{5}\left(\frac{6}{10}, \frac{9}{15}, \frac{12}{20}\right.$, etc $)$

2. Copy the fractions which are simplest fraction names.

a. $\frac{6}{12}, \frac{7}{12}, \frac{8}{12}, \frac{9}{12}, \frac{10}{12}, \frac{11}{12},\left(\frac{1}{12}, \frac{11}{12}\right)$

b. $\frac{2}{3}, \frac{11}{4}, \frac{5}{3}, \frac{3}{4}, \frac{4}{6}, \frac{5}{8} \quad\left(\frac{2}{3}, \frac{11}{4}, \frac{5}{3}, \frac{3}{4}, \frac{5}{8}\right)$

c. $\frac{7}{8}, \frac{8}{8}, \frac{6}{8}, \frac{2}{8}, \frac{1}{8}, \frac{3}{8} \quad\left(\frac{7}{8}, \frac{1}{8}, \frac{3}{8}\right)$ 
3. Complete by supplying the missing numerator or denominator,
a. $\frac{3}{6}=\frac{(12)}{24}$.
d. $\frac{9}{18}=\frac{54}{(108)}$
b. $\frac{3}{15}=\frac{12}{(60)}$
e. $\quad \frac{6}{24}=\frac{7 \times 6}{\left(? x^{24}\right)}$
c. $\frac{7}{12}=\frac{(5 \times 7)}{5 \times 12}$
f. $\cdot \frac{\dot{5}}{25}=\frac{\dot{(30})}{150}$

4. Use complete factorization to find simplest fraction names for:

a. $\quad \frac{72}{81}\left(\frac{2 \times 2 \times 2 \times 3 \times 3}{3 \times 3 \times 3 \times 3}=\frac{8}{9}\right)$ b. $\quad \frac{84}{105}\left(\frac{3 \times 2 \times 2 \times 7}{3 \times 5 \times 7}=\frac{4}{5}\right)^{\text {c }} . \quad \frac{98}{196}\left(\frac{2 \times 7 \times 7 \times 1}{2 \times 2 \times 7 \times 7}=\frac{1}{2}\right)$.

5. Find the simplest fraction names for the following; You should be able to do this using miltiplication 'facts only.
a. $\frac{6}{9}$
$\left(\frac{2}{3}\right)$
h. $\frac{21}{24},\left(\frac{7}{8}\right)$
o. $\frac{10}{25}$.
$\left(\frac{2}{5}\right)$
b. $\frac{10}{15}$
1. $\frac{4}{10}\left(\frac{2}{5}\right)$
p. $\frac{15}{20}\left(\frac{3}{4}\right)$
c: $\frac{4}{8}$
J: $\frac{4}{16}:\left(\frac{1}{4}\right)$
q. $\frac{12}{15}$
$\left(\frac{4}{5}\right)$
d. $\frac{9}{12}$
k. $\frac{6}{12}\left(\frac{1}{2}\right)$
r. $\quad \frac{77}{88}$
$\left(\frac{7}{8}\right)$
e. $\frac{12}{8}$
$\left(\frac{3}{2}\right)$
$2 . \frac{8}{16}\left(\frac{1}{2}\right)$
s. $\frac{16}{12}$
$\left(\frac{4}{3}\right)$
×. $\frac{5}{15}$
m. $\quad \frac{8}{12}\left(\frac{2}{3}\right)$
t. $\frac{20}{24}$
$\left(\frac{5}{6}\right)$
8. $\frac{72}{16}\left(\frac{3}{4}\right)$
n. $\frac{7}{21}\left(\frac{1}{3}\right)$
u. $\frac{25}{20}$

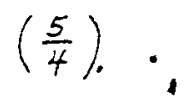

6. Find the simplest fraction name for each number.' Then use $\langle\dot{,}\rangle$, or $=$ in each blank to make a tmue statement.. .
a. $\frac{9}{15}(<) \frac{20}{25}$
a. $\frac{12}{22}(>) \frac{10}{33}$
b: $\frac{8}{10}(>) \frac{9}{15}$
e. $\frac{3}{27} \cdot(<) \frac{8}{18}$
c. $\frac{12}{16} \frac{(=)}{4} \frac{36}{48}$.
f. $\frac{8}{14} \leftrightharpoons \frac{20}{35}$ 


\section{Exercise Set ?}

1. Make true statements by fllling in the blanks.
a. $\frac{2}{3}=\frac{(18)}{27}$
. $\left.\quad \frac{9}{5}\right)=\frac{63}{35}$
b. $\frac{5}{4}=\frac{35}{28}$
f. $\quad \frac{1}{2}=\frac{(1 \times n)}{2 \times n}$
c. $\frac{4}{7}=\frac{4 \times 16}{(4 \times 7)}$
3. $\frac{20}{40}=\frac{27}{(5+)}$
d. $\quad: 5 ;=\frac{-5}{72}$

$$
\therefore \quad \frac{25}{(75)}=\frac{16}{48}
$$

2. 9. The neasure of $\frac{2}{3}$ of a foo in inches is $|8|$

b. The measure of $\frac{1}{2}$ yard. In Inches is. (18).

c. The measure of,$\frac{3}{4}$, hours in minutes 1 s (45).

'i. Twenty minutes is $\frac{(1)}{3}$ of an hour.

e. (4). weeks is $\frac{1}{13}$ of a year.

3. Write "prife" if the number is prime. Name at least one prime factor if the number is composite.

Example: 73 prime.

Nelther' 2, nor 3 , nor 5 is a ractor. (Do you remember: how to tell?) By division we rind that ${ }^{7}$ "is not a factor. 'Th1s is enough to show that 73 , is a prime." (Why?)
a. $58 \quad(2$ - 29$)$.
e. $705(3,5$, or 47)

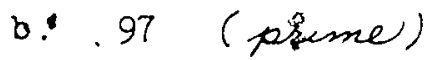
f. $91 .(7$ or 13$)$.
c.. 52 '( 3 /or. 17)
8. $5280 \quad(2,3,5, \text { or } 11)^{\circ}$
d. 365 . ' ( 5 as 73 )
h. 143 (11 of /3) 
4. Use complete ractorization.to find the simplest fraction nàme.
a. $\quad \frac{45}{180}\left(\frac{3 \times 3 \times 5}{2 \times 2 \times 3 \times 3 \times 5}=\frac{1}{4}\right): \frac{105}{143}$
$\left(\frac{3 \times-5 \times 7}{11 \times 13}=\frac{105}{143}\right)$
b. $\frac{126}{60}\left(\frac{2 \times 3 \times 3 \times 7}{2 \times 2 \times 3 \times 5}=\frac{21}{10}\right)$ e. $\quad \frac{90}{300}\left(\frac{1}{2} \times 2 \times 3 \times 3 \times 5 \times 5=\frac{3}{10}\right)$

.. c. $\frac{97}{365}\left(\frac{1 \times 97}{5 \times 73}=\frac{97}{365}\right)$;. $\frac{10 \times 9 \times 11}{5 \times 21 \times 11}\left(\frac{2 \times 5 \times 3 \times 3 \times 11}{2 \times 3 \times 3 \times 7 \times 11}=\frac{5}{7}\right)$

5. By finding the simplest fraction name freach of these numbers, tell which is greater.

a. $\frac{24}{42}$ or $\frac{40}{56} \frac{40}{56}$ b. $\frac{16}{-4}$ or $\frac{45}{99}\left(\frac{45}{99}, \quad\right.$ c. $\frac{81}{117}$ or $\frac{72}{106}$ (scme).

6. In Jefferson school there were. 325 pupils in all and 175 girls. In Washington school there were 312 pupils in ail

- and lil girls in wnich school do.giris form. the zarger

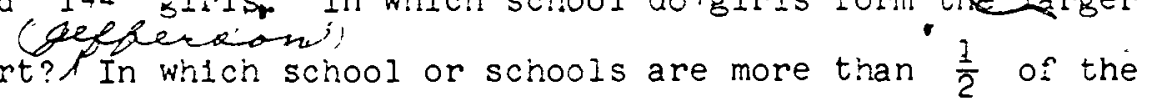
pupils giris?

7. Find the simplest fraction name for:
a. the heasure in feet of 16 inches. ( $\left(\frac{4}{3}\right)$.
$b$. the measure in days of 33 hours. (- $\left.\frac{11}{8 x}\right)$
c.' the measure in m1les of ' 440 yards. $\left(\frac{1}{4}\right)$
d. . the measure in pounds of 20 ounces.. $\left(\frac{5}{4}\right)$
$e$; the measure in nours of 45 , mirute $\left(\frac{3}{4}\right)$

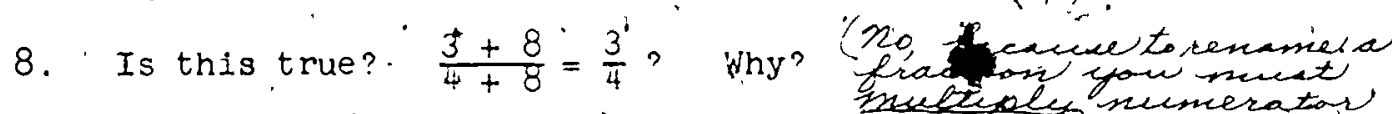
and tiame number

BRAINTWISTER

9. Is $\frac{3843}{10,000}$ a simplest fraction name? (yed) do not need to make any long computation to find the answer. (Becacize the' oney factace of denominator are 2 and 5 .

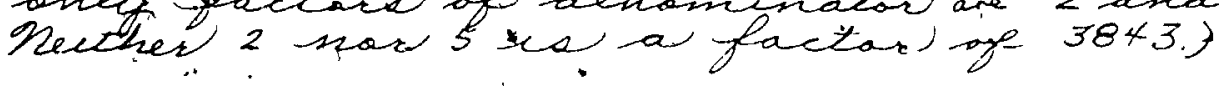




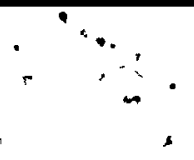

COMMON DENOMINATOR

COMMON MULTIPLE.

LEAST COMMON MULTIPLE

objéctives: To show a need" for renaming rational numbers by fractions with the same denominator.

1. To show that the set of possible common denominators for two fractions is the same as the set of common multiples of their denominators.

To develop a method for finding the least common multiple of two numbers by using their complete. iactorizations.

Vocabulary: Common derominator, multiple, common multiple,. . set of multiples, intersection, $(\cap)$, compare

Suggested Teaching Procedure

The pup1ls kng that any rational number has many fraction names. In the previous lesson. they were.concerned. With finding simplest fraction names. However, the simplest fraction name is parequently not the name most useful for answering gertain questions, e..g., deciding. which of two fractions names the larger number. It is therefore useful to develop a method for finding common denominators for any two fractions. In the next three sections, the problem of rinding common denominators is related to the problem of finding common multiples for the denominatops of
the iractions. .

In the sections, "Common Denominator", "Common Multiple", and "Least Commo Mu"itiple", use is made of the idea's and techniques developed in the unft, "Factors and Primes." Some review of this unit may be needed.

Teachor and pup 113 should work through each. Explpration together. Opportunity should be given for use of the new vocabulary. The dlagram pro-
posed in the section "Least chmon Multiple" for. recording the prime factorizations of the two numbers. for which the least common multiple is to abe found will be particularly helprul to the pup1 is. 
COMMON DENOMINATOR

Sometimes the simplest fraction name is not the name you need to solve a problem. Suppose that you want to know'which is larger, $\frac{2}{3}$ of a mile or $\frac{7}{10}$ of a mile. Would you prefer to have 3 equal stacks, of pennies or to. separate the same set of pènies intorno équa? stacks and take 7 ?

In e1ther case you want to know:

$$
\text { Which is greater, } \frac{2}{3} \text { or } \frac{7}{10} ?^{\prime}\left(\frac{7}{10}\right)
$$

Both names are simplest, but we cannot answer the ques $f 1$ ion with them. Here is another example that may help you to find the answer.

$$
\text { Which is greater, } \frac{7}{2} \text { or } \frac{7}{10} \text { ? }
$$

You know that $\frac{1}{2}=\frac{5}{10}$. You know that $\frac{7}{10}>\frac{5}{10} \cdot$ so you can say $\frac{1}{2}<\frac{7}{10}$

Which is' greater, $\frac{2^{\prime}}{3}$ or $\frac{5}{9} ?\left(\frac{2}{3}\right)$

Which is greater $\frac{2}{6}$ or $\frac{9}{24} ?\left(\frac{.9}{24}\right)$

Which is greater. $\frac{17}{8}$ or ?? $\left(\frac{17}{8}\right)$

The trick is to find for each number names with the same denomirlatior. Thirik again;about $\frac{2}{3}$ and $\frac{7}{10}$ : What other deñominators. do fractions naming $\frac{7}{10}$ have? What other denominators do fractions naming $\frac{2}{3}$ have? . What is the smallëst number. Wh1th is frn both lists of denominators? II

The answers to frese question's help you to see that:

$$
\frac{2}{3}=\frac{20}{30} \text { and } \cdot \frac{7 i}{10} \cdot \frac{21}{30}
$$

fou know then that $\frac{r_{7}}{10}>\frac{2}{3}$ 
P2́89

so

You could answer your question abqut $\frac{2}{3}$ and $\frac{7}{10}$ as soon as you knew that 30 was a denominator, both for $\frac{2}{3}\left(\frac{20}{30}\right)$ and $\frac{7}{10}\left(\frac{21}{30}\right)$

The set of denominators for $\frac{2}{3}$ is $\{\dot{3}, 6,9,12, . .\}=$.

The set of denominators for $\frac{7}{10}$ is $\{10,20,30, . .\}=.\mathrm{L}$

- Set $K$ ts called the set of multiples of. 3 .

Set $I$ is callec the set of miltiples of 10 .

The rumbers common to both sets are called the common multiples of 3 and 10 .

The numbers both sets have in common are a so the numbers

- you can use as denominators for both $\frac{2}{3}$ and $\frac{7}{10}$. They are called common denominators for $\frac{2}{3}$ and $\frac{7}{10}$. Before you study frations any further, you should find out more about common muitiples. .
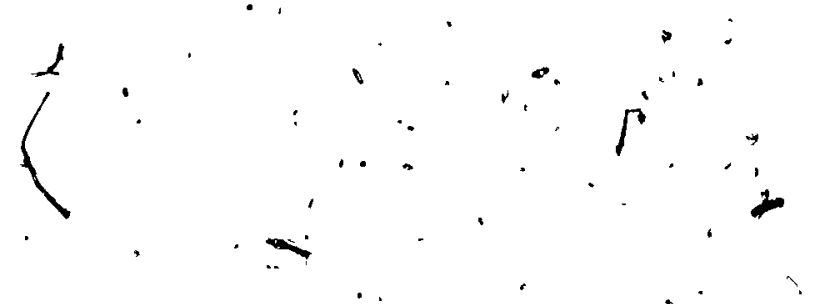
P290

COMMON MULTIPLE

- We use the word "multiple" as another way to talk about.

ractors. Instead of saying

4 is a ractor of 12 .

we may say

12 is a multiple of 4.

Th1s 1dea is not strange. 'Instead of saying;

$$
3 \text { is less than. } 5
$$

we might say

$\therefore$

$$
5 \text { is greater than } 3 \text {. }
$$

\section{Instead of saying}

J

John is younger "than Bruce

we might say

- Bruce is older than John:

What is the other way of saying these?

I am tajiler than you.

- Today is warmer than yesterday.

The Aelation between factor and multiple is another - example of the same 1dea. Put these statements into the language of multiples:

. 1.

7: is a ractor of 21 (2ied immetiple fix)

3 - is not a factor of. 31 (3! wrot amulate of 3 )

12. Is a ractor of 12. (12 wasmetple of:2) 
Put these into the language of factors:

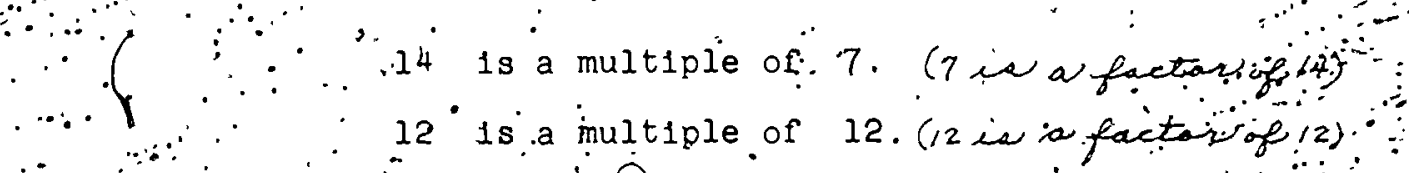
18 is.a multiple of both 9 and 2 (quatiz Because, 18 is a muitiple of both 9 and 2 . 18 is.icalled a common multiple of 9 and 2. Because $12=3 \mid \dot{x} 4$ and $\dot{12}=2 \times 6$, 12 1s. common multiple of 4 and $6 ;$ Is/I2 a common multiple of: 3 and 4 ? of 2 and 3 ? of 4 and i2 $2(y$,

$\because \quad \therefore$ good. way to think äbout common multiples is to use. the language of sets

Let $R$ be the ket of all multiples of $\Rightarrow$ and zet is. be the set of all multiples of:

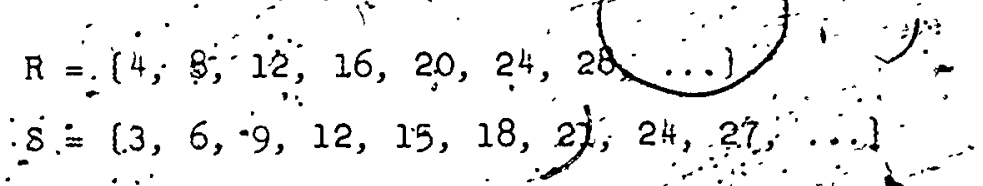

The set 'of.common multiples of 3 "and 4 "is.

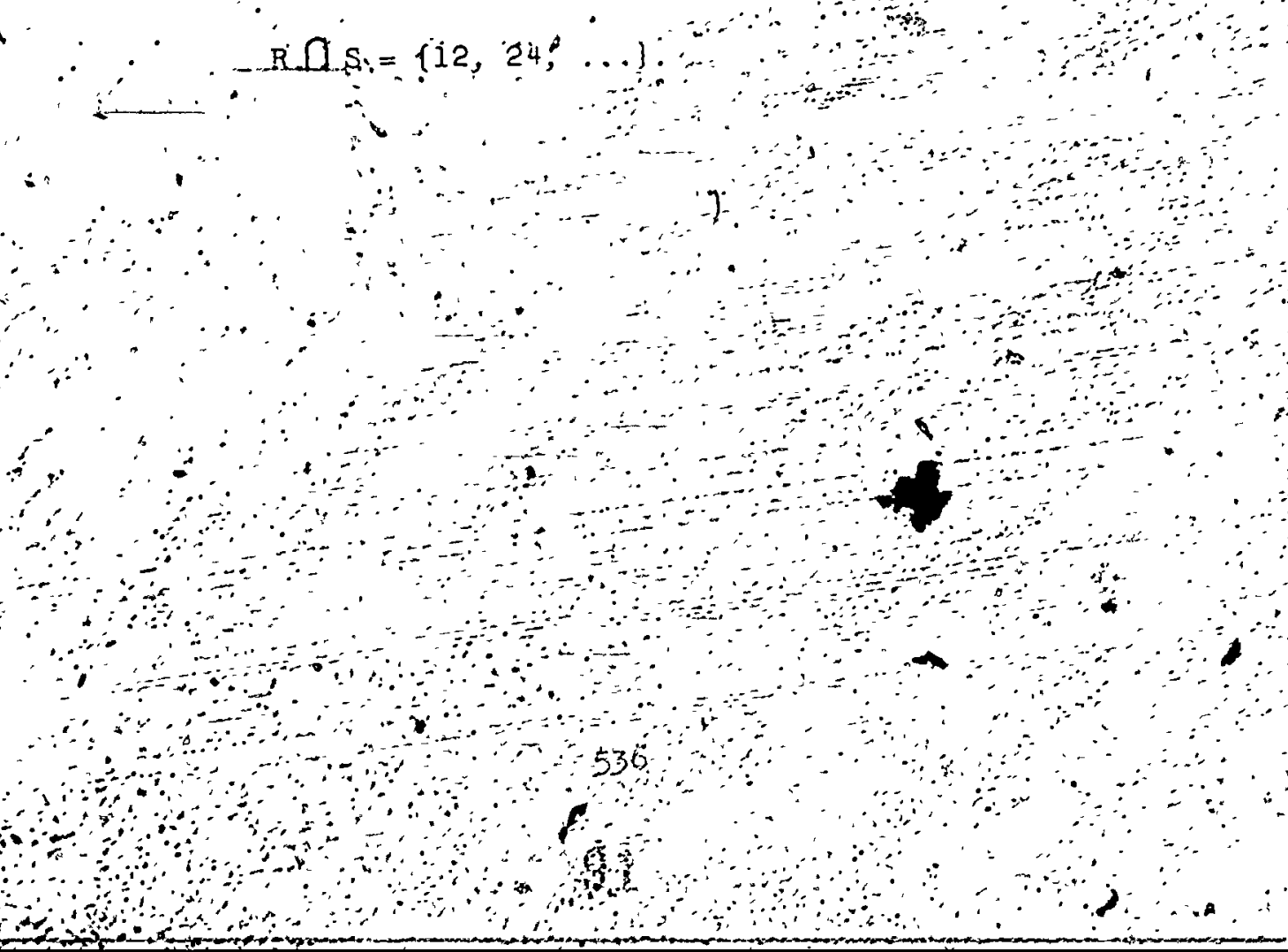




\section{1}

\section{Exercise Set 8}

1. Below are pairs of numbers: Show the set of multiples of each number. Then show the set of common multiples.

Example.: 3,5 a

$A=$ set of multiples of $3=\left\{3,6,9,12,15,18^{\circ} \ldots\right\}$

$\mathrm{B}=$ set. of multiples of $5=[5,10,15,20 \ldots\}$

$A \dot{\Pi}_{B}=$ set of common multiples of 3 and $5=\{15,30 \ldots\}$

a.. $4,6(4,8,12,16,20,24),(6,12,18,24, \ldots)(i 2,24,48, \ldots)$

b. $6,8\left(6,72,18,24^{\circ}, \ldots\right)(8,16,24,32, \ldots)(24,48,72, \ldots)$

c. $15,10(15,30,145, \ldots)(10,20,30, \ldots)(30,60,90, \ldots)$

d. $9,6(9,18,27, \ldots)(6 ;-12,18, \ldots)(18,36,54, \ldots)$

e: $10,20(10,20,30,40, \ldots)\left(20,40,60^{\circ}, \ldots\right)(40,80,120, \ldots)$

2. In the examole in exercise 1 , is 45 a'common multiple of (epes)

3 and 5 If 60 ? If $n$ is a counting number, is n $\times 15$ alwàs a common muitiple of 3 and 5 ? (qup)

3. The product of two numbers is always a multiple common to both numbers." Is it ever the smallest of al common multiplés? both numbers. Is 1 ever (Amaklestmulipifof Is $1 t$ always the smallest? 1 . Give examples. 2 and 3 mat $2 \times 3$ of $4 \times 6$ in mat amalect,

4. I am thinking of two numbers. They have 18 as a common mult 1 ple:

a. Is 36 a common multipie of the two numbers? (2yed)

b. If $n$ is a counting number, is $18 \times$ no always a common multiple of the two numbers? (quis)

c. Couid 9 be a common multtple of the two numbers? (Yjew) Give an example if there is one. ( 3,9 ) , 


\section{LEAST COMMON MULTIPLE}

\section{There are two things which seem to be true about the set} of all the common multiples af any two numbers;

1) Every multiple of the smallest common multiple'is also a common multiple.

- 2) No other numbers are common multiples. For example, tre set of common multiples of 2 and $\beta$ begins

$$
\{6,12,18,24, \cdots\}
$$

It seems to consist of only the multiples of 6.6 is the smal'est common multiple of 2 and 3 .

Because 1) and 2) are always true, we only have to know the smallest common, multiple, then we car find all common. multiples. The smallest common multiple is usualiy called. the least commion. multiple.

(1)
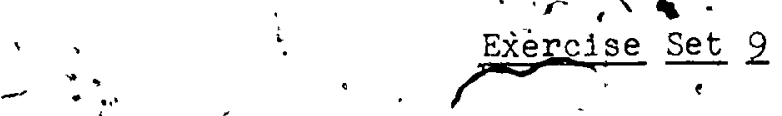

1. The least common multiple of two numbers is 10 . What gre the other common multiples? $(20,30,40, \ldots)$

2. Find two different pairs of numbers with least common multiple 18. (2 astid. 9 ind 9 ; $^{\circ}$ and 18 ; eto)

3. Express th1s 1dea in factatifiguage: $\because$

The least comion multiple 3 , and 4 is 12 .

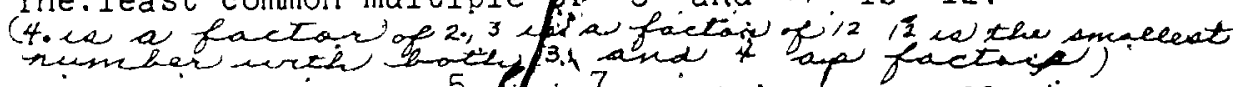

4. If you want to compare $\frac{5}{6}\left(\frac{7}{9}\right.$ what is the smallest denominator you could use? (8)

$5 \times 3$

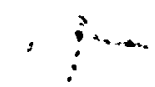


$\mathrm{P} 294$

$\dot{5}$. Find several members the set of multiples for each number below. Underline the laàst common mutiple for each pair.

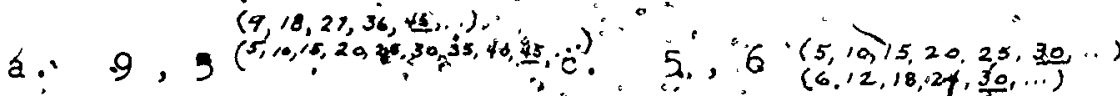

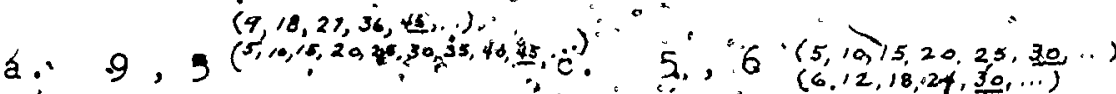

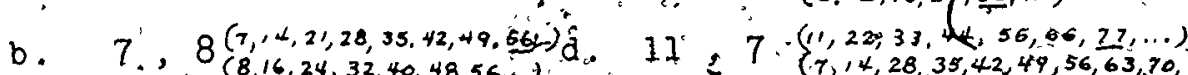
6. Find the lesst common mufthole of each pair. Then show
three of the set of all common multiples .

a. $12,13(156,312,468), c \cdot$ i1, 12 (8), $1168,252, \ldots)$

b. $5,8^{\prime}(40,80,120, \ldots)$ d. $.17,5,(85,170$, $255, \ldots)$.

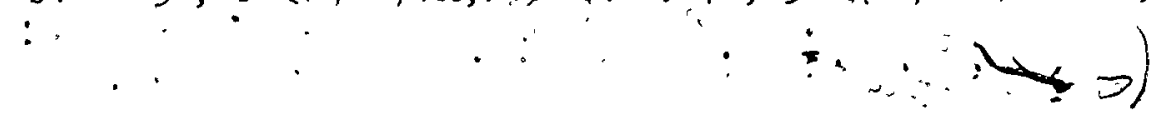

\section{$\because \cdots \quad$ Exploration}

Unt1l now we have found the feast common multiple (l.c.m.) of two numbers by listing multipres of each numger. But this may be a long process even if the numbers are small. For examie, to and l.c.m. For 8 afid 9 iwe find:

$\rightarrow$ Set of multiples of $8=\left\{\left(8,16, q^{4}, 32,40,48,56,64,72 \ldots\right\}\right.$

- Set of multiples of $9=\{9,18,\{27,36,45,54,63,72 \ldots\}$

It $1_{1}$ would be even harder to test "our bellef that the setiof." common multiples of 8 and 9 is

$$
\{72,144,216,28 \dot{8}, \ldots\}
$$

There is a much easier way to do bdth.

A: First we factor the numbers complet
\[ 8=2 \times 2 \times 2 . \]

$$
\begin{aligned}
\therefore & 8=2 \times 2 \times 2 . \\
\therefore \quad 9 & =3 \times 3 .
\end{aligned}
$$


Şûppose that in 1 any common multiple of 8 and 9. Think about the expression for $\mathrm{n}$ as a product of primes. Since $n$ is ä muitiple of $8,2 \times 2 \times 2$-must be a plece of this expression.

\section{$\therefore:=2 \times 2 \times 2 \times$ (any other prime factorst.}

Since $\because \because 1$ a multiple of $9,3 \times 3$ must also he a piece of sing this expression.

$$
\therefore n=3 \times 3 \times \text { (any other prime factors). }
$$

We know then that

If $n=2 \times 2 \times 2 \times 3 \times 3 \times$ (any other prime factors).
is the least common multiple then

$$
n=2 \times 2 \times 2 \times 3 \times 3=8 \times 9=72 \text {. }
$$

$\because$. Ary other common multiple can be expréssed as

$$
8 \times 9 \dot{\times} \text { (other factors). }
$$
This shows that eyery other common multiple of 8 and 99 is

B. Here is another example: Find the l.c.m. of 60 and 270 .

$$
\begin{aligned}
60 & =2 \times 2 \times 3 \times 5 . \\
270 & =2 \times 3 \times 3 \times 3 \times 5 .
\end{aligned}
$$

The /.c.m. must have at least two $2^{\prime} \mathrm{s}$, three $3^{\prime} \mathrm{s}$, and one 5 in its ractorization. So

$$
\text { l.c.m. of } 60^{\circ} \text { and } \begin{aligned}
270 & =2 \times 2 \times 3 \times 3 \times 3 \times 5 \\
& =540
\end{aligned}
$$

We can think of the r.c.m. In this' way

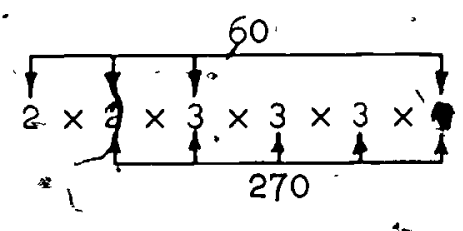

$$
5^{40}
$$

9 
P296

We have put in all the prithee factors we need to get a multiple'. of 270 and a multiple of " 60 . We get the l.c.m. if we". include no mores:

c... Here is one more example. Find the "l.c.m. of 84 and 90.

$$
\begin{aligned}
& 84=? \times 2 \times 3 \times 7 \\
& 90=2 \times 3 \times 3 \times 5^{\circ}
\end{aligned}
$$

Perhaps it will help to think of the problem in j this way: What factors do I have to Include beside

$$
\frac{2 \times 3 \times 3 \times 5}{90}
$$

so that the expression will name 'a multiple of 84 ?

First we mark those numeral's in the complete factorization of 84 that are already written in expressing $90^{\circ}$.

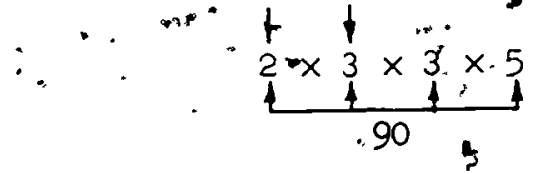

Then we add the remaining piece of the complete factorization of 84.

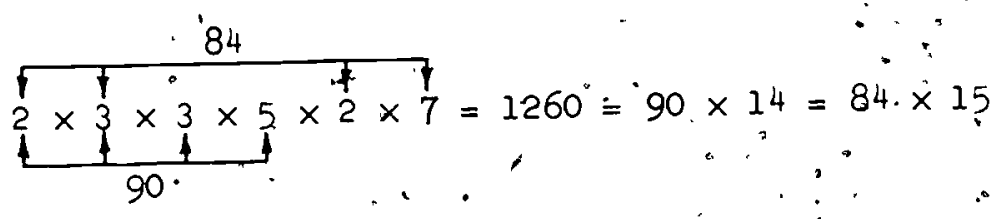

If we show the factors order we get.
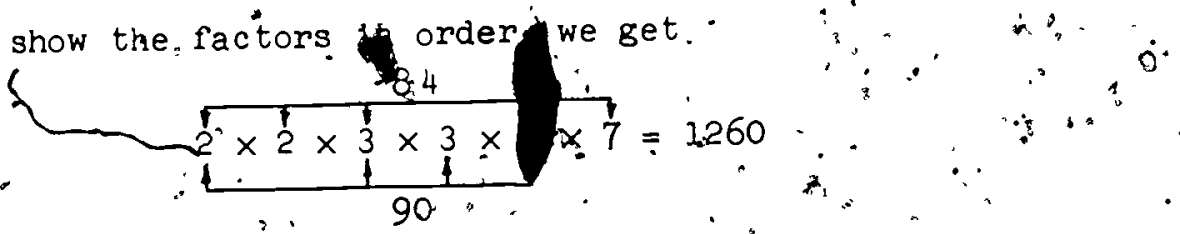

Imagine doing' this problem the long' way!"

Use what we. found in this example to compare

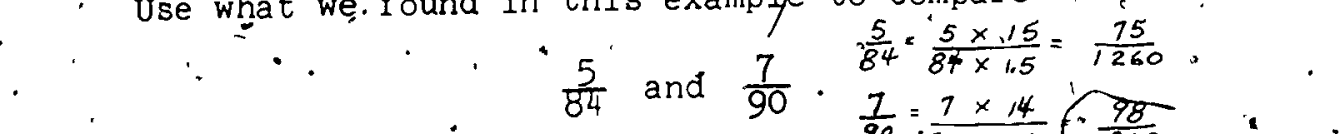

$+$

$\circ$

541

ERIC 
1: Find the least. common. multiple of each pair of numbers.

Then show the set of all common multiples.

Example: 14 and 35

$$
\begin{aligned}
14 & =2 \times 7 \\
35 & =5 \times 7 \\
1 . \mathrm{c} \cdot \mathrm{m} . & =\frac{2}{14} \times \frac{35}{7} \times 5=.70
\end{aligned}
$$

Set of common multiplés $=\{70,140,210 \ldots\}$.

a. 10 and 21 (210, $420,630, \ldots)$

b. 24 and $9 .(72,144,2 \%, \%)$

c. 20 and 36 ( $360,360,540, \ldots)$.

d: $\because 30-$ and ió $(240,480,720, \ldots)$

1. 'Give an example of each:" (Ancuera' whel vary)

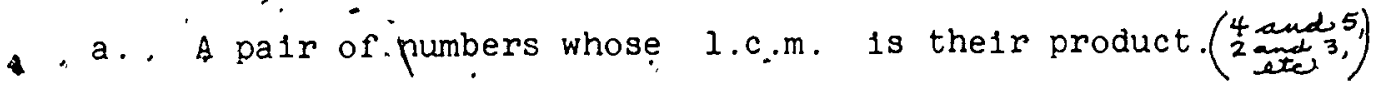

b. A pair of numbers whose l.c.m. 1s one of the numbers (2andi

c: A pair. of numbers for which neither a nor $b$ is timue.

3." A trafific light.at one corner changes every 30 seconds.

- The traffic light at the next corner changes every 3,6 seconds:" At a certain time they both change together." How long will It be until they change together again? ( 180 inutea)

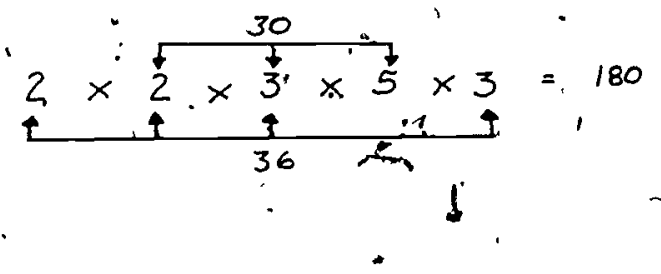


4. A "Discoverer" satelilte goes directly oventhe, north and south poles each time it circles thé earth. It makes one circle in/96 minutes. It is directiy over the north pole, at'noon. When will it next be over the north pole exactiy. on the hour? (houife).

$480=$

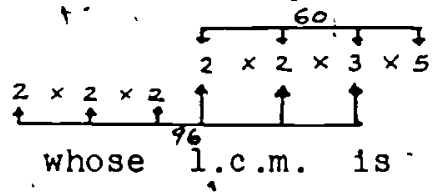

5. a. Find two numbers greater than 1 whose ${ }^{96}$.c.m. is 96. (Hint: use the complete factorization of 96.) (3and 32 and ocher

b. The number 283 is not the l.c.m. of any pair of. numbers except 1 and 283 . What does this show about the factors of 283 ? (2hey are juet l and 283.)

c. Here is what we drew to help us find the l.c.m. of 84 , and 90 :.

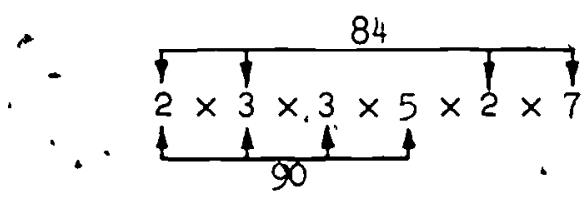

Form the product expression which uses the numerals with two arrows "pointing to them. Can you find a meaning for such an expression? 
'LEAST' COMMON ON DENOMINATOR

Objective: To rename fractions with least common denominators.

Vocabulary: Common denominator, least common denominator

Suggested Teaching Procedure

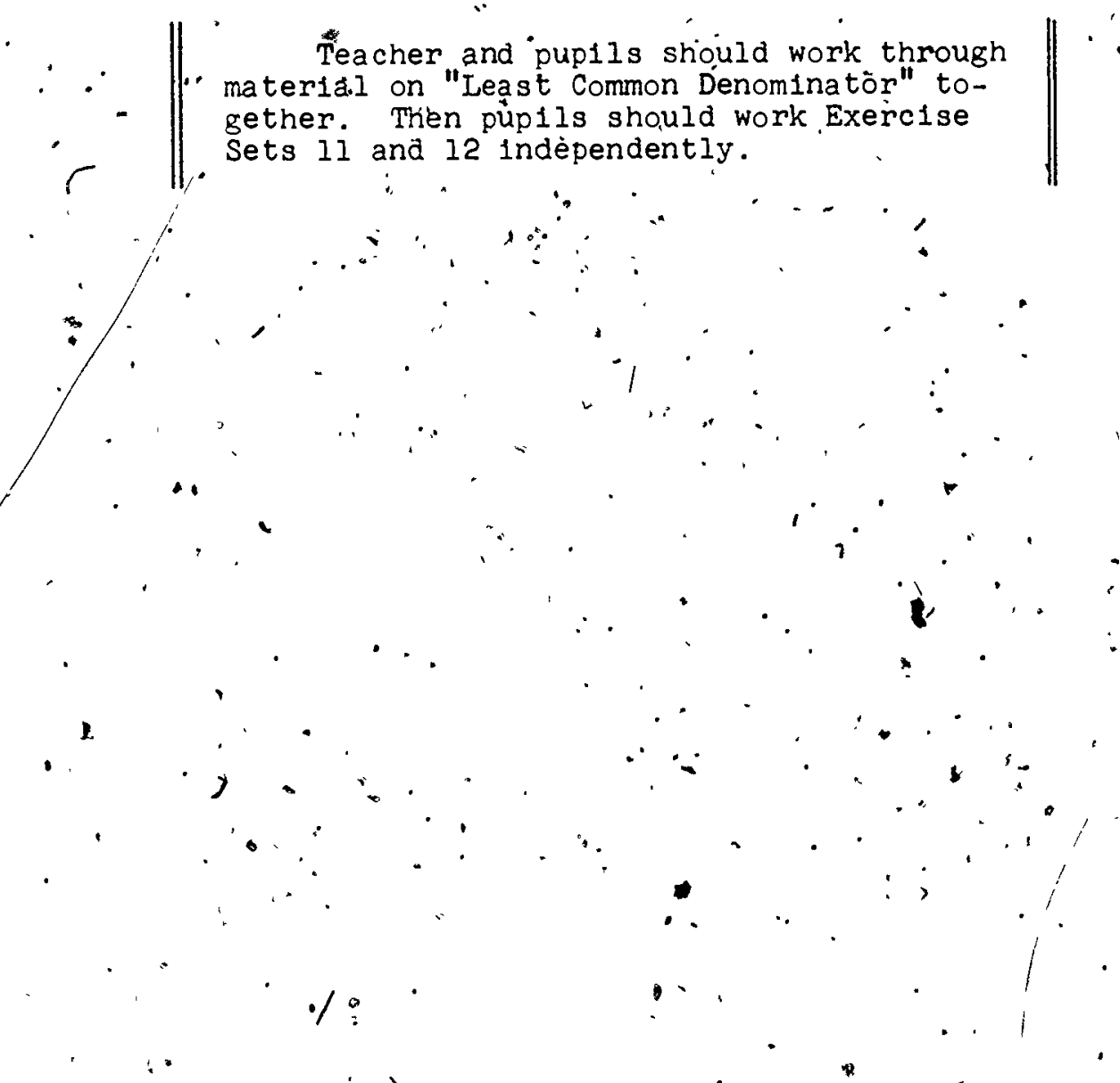

ERIC. 
P299

\section{LEATST COMMON DENOMINATOR}

"Do you' remember why we wan ted to find common multiples? Let us use what have learned to think again' about how to compare $\frac{2}{3}$ and $\frac{7}{10}$.

- We need to fing a name for" $\frac{2}{3}$ and a name for $\frac{7}{10}$. With the same denominator. We say that we want to find a common denominätor for $\frac{2}{3}$ and $\frac{7 \cdot}{10}$. We know that the common denominators for $\frac{2}{3}$ and $\frac{7}{10}$ are the common multiples of 3 and 10 .

The" least common. multiple of 3 and 10 is the least common denominator of $\frac{2}{3}$ and $\frac{7}{10}$.

We can find the least common denominator for $\frac{2}{3}$ and $\frac{7}{10}$ in this way:

$10=2 \times 5$

3 is prime

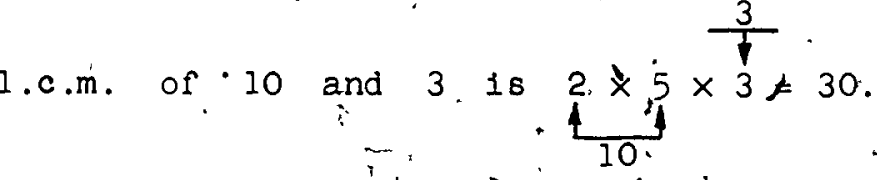

To rename the fractions with the least common denominator we must also find $n^{\prime}$ in $n \times 3=30$. Our diagram shows that

$$
\begin{aligned}
& 3 \times 10=30 . \\
& 10^{\circ} \times 3=30 .
\end{aligned}
$$

Now we know

$$
\begin{aligned}
& \frac{2}{3}=\frac{2 \times 10}{3 \times 10}=\frac{-20}{30} \text { and } \\
& \frac{7}{10}=\frac{7 \times 3}{10 \times 3}=\frac{21}{30} .
\end{aligned}
$$

Notice that $60,20,120 \therefore$ are also common deniominators. 
P300

We didn't really need our method with the small numbers 3. and 10. Here is an example with greater numbers. Which is greater. $\frac{37}{84}$ or $\cdot \frac{13}{30}$ ?

$\therefore \quad$ First, we find the least common denominator.

$$
\begin{aligned}
& 84=2 \times 2 \times 3 \times 7 \\
& 30=2 \times 3 \times 5 \\
& 1.8 . m .=\frac{2 \times 2}{4 \times 3 \times 7 \times 5}, \\
& 30
\end{aligned}
$$

Now we want to express 84 and 30 as factors of the .1.c.m.

By looking at the arrows, we see that

$$
\text { 1.c.m. }=84 \times 5=30 \times 14 \text {. }
$$

Now" we can write:

$$
\begin{aligned}
& \frac{37}{84}=\frac{37 \times 5}{84 \times 5}=\frac{185}{420} \\
& \frac{13}{30}=\frac{13 \times 14}{30 \times 14}=\frac{182}{420}
\end{aligned}
$$

We find, then, that

$$
\frac{37}{84}>\frac{13}{30}:
$$

$\therefore$

.)

0 


\section{Exercise Set 11.}

1. Rename each pair of numbers so the fractions have the léast commón denominator. Hint: . Rename in simplest form before. finding a least common denominator.

a. $\frac{1}{2}$ and $\frac{2}{4}\left(\frac{1}{2} \frac{2}{\left.\frac{2}{4} \text { and } \frac{1}{2}, \frac{2}{4}\right)}\right)$ d. $\frac{8}{5}$. and $\frac{8^{*}}{10}\left(\frac{8}{5}\right.$, arid $\left.\frac{4}{5}\right)$. b. $\frac{3}{5}$ and $\frac{3}{4}\left(\frac{12}{20}\right.$ and $\left.\frac{15}{20}\right)$ e: $\frac{4}{6}$ and $-\frac{2}{3}\left(\frac{2}{3}\right.$ and $\left.\frac{2}{3}\right)$

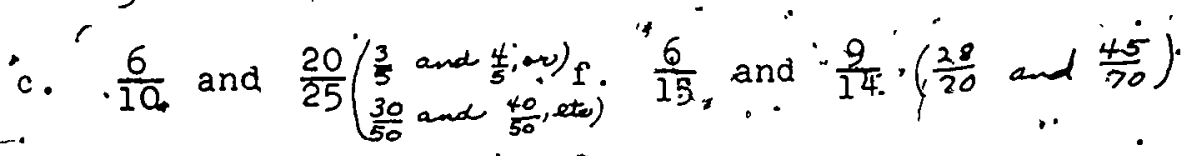

2. For each of the following pairs of fractions, find two other fractions which name the same two numbers and which have the least common dénominator.

a. $\frac{2}{3}$ ' and $\frac{3}{4}\left(\frac{8}{12}\right.$ and $\left.\frac{9}{12}\right)$ e. $\frac{4}{5}$ and $\frac{7}{10}\left(\frac{8}{10}\right.$ and $\left.\frac{7}{10}\right)$

b... $\frac{1}{4}$ and $\frac{2}{5}\left(\frac{5}{20}\right.$ and $\left.\frac{8}{20}\right)$ f. $\frac{7}{8}$ and $\frac{2}{3}$ ( $\frac{21}{24}$ and $\left.\frac{16}{24}\right)$ c., $\frac{5}{8}$ and $\frac{3}{5}\left(\frac{25}{40}\right.$ and $\left.\frac{24}{40}\right) .8 .-\frac{2}{5}$ and $\frac{1}{6}$ ( $\frac{12}{30}$ and $\left.\frac{5}{30}\right)$. d. . . $\frac{3}{8}$ and $\frac{5}{6}\left(\frac{9}{24}\right.$ and $\left.\frac{20}{24}\right)$ h.' $\frac{1}{2}$ and $\frac{2}{3}\left(\frac{3}{6}\right.$ arid $\left.\frac{4}{6}\right)$

$\hat{3}$. 'Wh1ch fraction names the greatest rationai number?
a. $\frac{2}{3}$ or $\frac{\dot{3}}{4}$
$\left(\frac{3}{4}\right)$
e. $\frac{5}{7}$ or $\frac{2}{3}$
$\left(\frac{5}{7}\right)$
b. $\frac{3}{9}$ or $\frac{3}{5}$
f. $\frac{5}{8}$ or ' $\frac{13}{16}$
$\left(\frac{13}{16}\right)$
$\therefore$.c. $\frac{3}{4}$ or $\frac{4}{5} \cdot\left(-\frac{4}{5}\right)$
g. $\frac{3}{5}$ or $: \frac{2}{3}$
d. $\frac{4}{5}$ or $\left(\frac{5}{6}\right.$
$\left(\frac{5}{6}\right)$
h. $\therefore$ : or
$\frac{2}{3} \cdot\left(\frac{2}{3}\right)$

4. Arrange in order from least to greatest.

$$
\frac{3}{4}: \frac{4}{5}, \text { and } \frac{7}{10} \quad\left(\frac{7}{10},: \frac{3}{4}, \frac{4}{3}\right)
$$


$\mathrm{P} 302$

Exerc1se Set 12

1. Find which number is greater:
a. $\frac{7}{18}$ or $\frac{61}{140}\left(\frac{61}{140}\right)$
-b. $\frac{16}{27}$ or $\frac{13}{24} \cdot\left(\frac{16}{27}\right)$

2. a. Which is longer, $\frac{1}{3}$ of a year or 121 days?

b. Wh1ch is lomger, $2000 \mathrm{ft}$ or .3 of a mile? (Hint: First fiñ simplest fraction names.).

3. Which is greater?"
a. $\frac{19}{28}$ or $\frac{140}{210} \cdot\left(\frac{19}{28}\right)$
ib. $\frac{45^{\circ}}{72}$ or' $\frac{275}{400}\left(\frac{275}{400}\right)$

4. List these in order of 's'ze from least to greatest.
a. $\frac{19}{16}, \frac{55}{48}, \frac{43}{36}$
$\left(\frac{55}{48} ; \frac{19}{16}, \frac{43}{36}\right)$
b. $3, \frac{30}{9} ; \frac{23}{8}$
$\left(\frac{23}{8}, 3, \frac{30}{9}\right)$

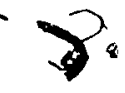

5. Roy is making a hammer toy for h1s little sister. He has wooden peigs - $\frac{19}{32}$ in. In diameter. "To make holes in the boart he has two drills: One makes a hole $\frac{5}{8}$ in; in - diameter. The other makes a hole $\frac{11}{16}$ in. In diameter. Will the peg's itt through both sizes of holes?! Which drill should he use? ( $\frac{5}{8}$ inch driel) - .

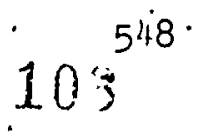


P303:

6. 'On a number' line, if you wanted to show both fourths and sixths you would mark the line in twelfths $\therefore$ How would you mark the line to show both of these? $\because$ 'Tenths and sixths?. (thirtucthe)

b. Sixteenths and thirds? (forty-ightha)

c. Twelfths and ninths? (tyherty-ecitha)

7. . Suppose that there will be either. 4 people or 6 people. - at your party, counting yourself. Suppose also', that you - want to c ut ar cake Before the party and want to divide the whole cake fairly among the people at the party. How
.
.

ERIC 
- SCALES ON NUMBER LINES

i. Objective: ro develop ideas needed for picturing addition and subtraction of rational numbers on the number liné.

Materials: Number lines scaled in ones, fourths, eighths, tenths, twelfths.

Vocabulary: Ségment,'méasure of a segment, congruent segments, scale, scaled in halves, scaled in thirds, etc:

Suggested Teaching Procedure.

In preparation for the following seven sections, the teacher should be thoroughly familiar with the development of addition and subtraction of whole numbers as presented. in "Techniques of Addition and Subtraction", Grade 4, Chapters 3 and 6 .

Work through the Exploration with the class.

Draw a number line scaled in fourths on the board:

\begin{tabular}{ccccccccccc}
$A$ & $B$ & $C$ & $D$ & $E$ & $F$ & $G$ & $H$ & 1 & $J$ & $K$ \\
$O$ & & & & 1 & & $:$ & & 2 & & \\
\hline$\frac{0}{4}$ & $\frac{1}{4}$ & $\frac{2}{4}$ & $\frac{3}{4}$ & $\frac{4}{4}$ & $\frac{5}{4}$ & $\frac{6}{4}$ & $\frac{7}{4}$ & $\frac{8}{4}$ & $\frac{9}{4}$ & $\frac{10}{4}$
\end{tabular}

Whatí's the measure of these segments: $\overline{\mathrm{AB}}\left(\frac{1}{4}\right) ; \overline{\mathrm{AD}} .\left(\frac{3}{4}\right)$; $\overline{\mathrm{AH}} \sigma_{-}\left(\frac{7}{4}\right)$; and so on.

One endpoint of a segment that"has measure $l$ is at $\frac{1}{4}$. What is the other endpoint? ( $\left.\frac{5}{4}\right)_{4}$

$\|$ and $\frac{3^{A s k}}{4}, \mathrm{simpliar}$ questions about points $\frac{2}{4}$.

One endpoint of a segment that hàs measure 1 is at $\frac{6}{4}$.

What is the other endpoint?'. (There are two answers: $\frac{2}{4}$ and $\frac{10}{4}$.)

Ask similar questions about other points.

Draw number lines scaled In sixths, elghths, tenths, and so on, as needed to help pupils. understand the meaning of. "scale" and review the meaning of "the" measure of a segment." 
$\mathrm{P} 304 \cdot$

SCALES ON NUTMBER LINE

\section{Exploration}

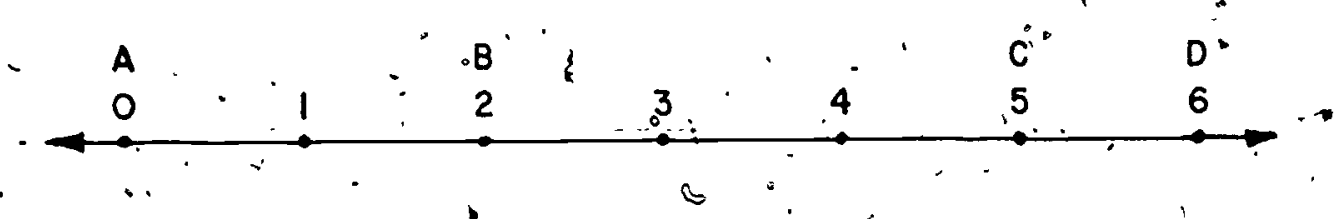

On this number line, the marked points are equally

späced. Each point is labeled with a whole number. Some points are also named with letters.

The segment with endpoints at $a$ and $I$ is the unit

\section{segment.}

1. Look at $\overline{\mathrm{AB}}$. The number at point $A$. is 0 . The number at $B$ is t. $\overline{A B}$ is the union of how many segments, ieach congruent to the unit segment? :

2. What is the measure of $\overline{\mathrm{AC}}$ ?

3. What is the measure of $\overline{\mathrm{AD}} ? \cdot(6)$

4. What is an easy way to tell the measure of any segment if one endpoint is at 0 : (2he number at the onther ).

5. Look at " $\overline{\mathrm{BC}}$. B is the point labeled (2). C 1s the point labeled (5). $\overline{B C}$ is the umion" of "(3) segments, each". congmuent to the unit segment. What is the measure of ${ }^{\prime} \overline{\mathrm{BC}}$ ?

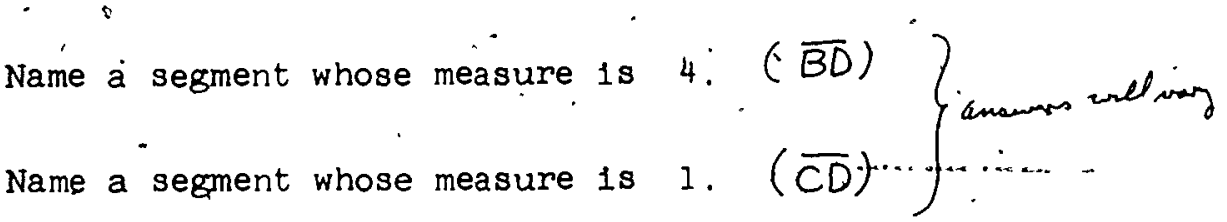

7. Name a segment whose measure is 1 ( ( $\overline{C D}) \cdots . .$. 
E $\quad F$ F $\quad \cdot \quad$ J $\quad$ G

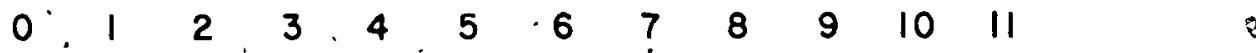

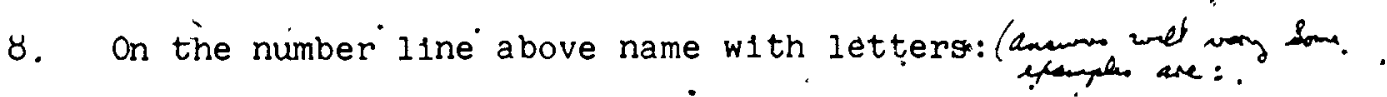

a. Three segmęnts, mea sure of each is $3 .(\overline{E F}, \overline{F J}, \overline{G H})$

$\vec{b}$. Two segments, measure of each is $5 . \quad(\overline{J H}, \overline{F G})$

cc. One segment whose measure is 6 .

d... Two segments, measure of each is $8 . \quad(\overline{F H}, \overline{E G})$

As you know, we may separate a number line into congruent, segments smalfer than unit. segments. Hère are two examplesm
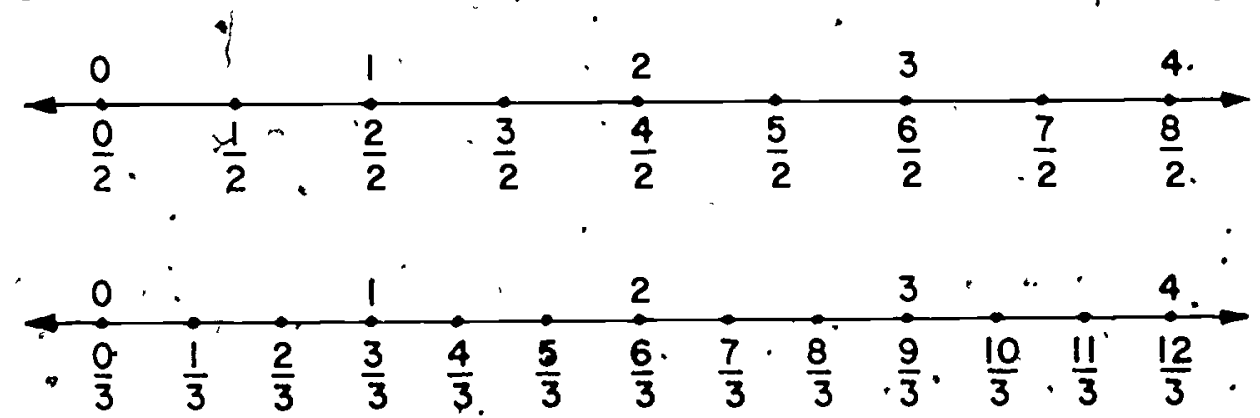

The first line above uses a scale of halves. It is scaled in halves. The second line above uses a scale of thirds. It

is staled in thirds.

Generally we will show, the scale we are using by the denominator of the rraction we use in marking the sçale. If we are marking a scalé in sixths, we wili label a point $\frac{3}{6}$ rather than $\frac{6^{*}}{1 F^{*}}$, or $\frac{2^{\circ}}{4}$, or $\frac{1}{2}$. If we are marking a scale in fifths we will use the label $\frac{2}{5}$ rather than $\frac{4}{10}$, or $\frac{6}{15}$, or $\frac{8}{20}$. 


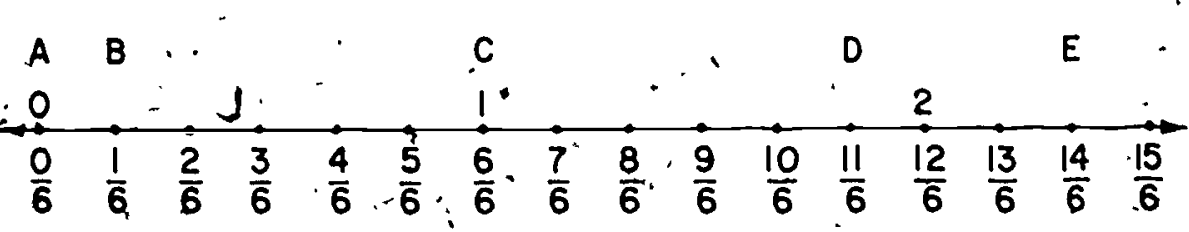

9. a. This number line 13 scaled in (sinth.)

Find the measures of these segments.

b. $\overline{\mathrm{AB}}\left(\frac{1}{6}\right)$

f. $\overline{\mathrm{BC}}^{\prime}\left(\frac{5}{5}\right)$

c. $\overline{\mathrm{AC}}\left(\frac{6}{6}\right)$

8. $\overline{\mathrm{BD}}\left(\frac{10}{6}\right)$

1. $\overline{\mathrm{CD}}$

$\left(\frac{5}{6}\right)$

d. $\overline{A D}\left(\frac{11}{6}\right)$

h. $\overline{\mathrm{BE}}\left(\frac{13}{6}\right)$

j. $\overline{C E}$

e. $\overline{A E}\left(\frac{14}{6}\right)$

k. $\overline{D E}\left(\frac{3}{6}\right)$.

\section{Exerc1se Set 13}

1. a. Look at this number line. Into' how many congruent segments is the unit 'segment. separated? (9)
A.
B
C D
$0^{\circ}$

b. What is the scale? (sintha) ,

$\therefore$ c. Write the set of "ractions you would use to label the points from, $0, \cdot$ to $\cdot 2$. ( $\left(\frac{0}{9} ; \frac{1}{9}, \frac{2}{9}, \cdots \frac{18}{9}\right)$

d. What number of your scale matches point $A$ ? $\left(\frac{0}{9}\right) B\left(\frac{4}{9}\right) C x\left(\frac{13}{9}\right)$ D? $\left(\frac{18}{9}\right)$

e. What is the measure of $\overline{\mathrm{AB}}\left(\frac{4}{9}\right) \overline{\mathrm{AC}} ?^{\left(\frac{13}{9}\right)} \overline{\mathrm{AD}} ?^{\left(\frac{18}{9}\right)} \overline{\mathrm{BC}} ?^{\left(\frac{9}{9}\right)} \overline{\mathrm{BD}} ?^{\left(\frac{14}{9}\right)} \overline{\mathrm{CD}} ?^{\left(\frac{5}{9}\right)}$ 
2. On the number 1 ine below are pictured $\overline{\mathrm{AB}}, \overline{\mathrm{CD}}, \overline{\mathrm{EP}}$ and $\overline{\mathrm{GH}}$.

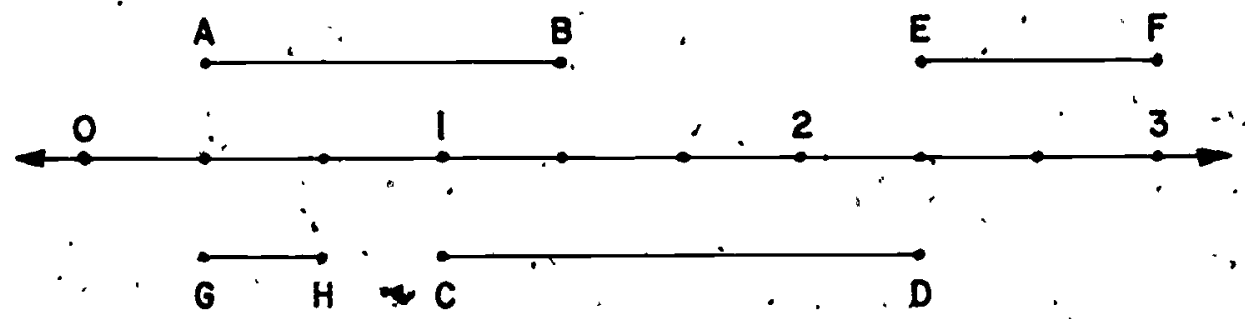

a." The points shown with dot's could be labeled in a - scale of (thurds) : : $\quad \therefore$ A. $\frac{1}{3}$ E. $\frac{7}{3}$

b: What numbers match the points named with letters? $c \cdot \frac{\frac{2}{3}}{3} \dot{G} \cdot \frac{1}{3}$

c. What is the measure of each segment?

3. Do the three segments described in exercise a have the

D. $\frac{7}{3} \quad$ H. $\frac{2}{3}$ same measure? Answer the same question for exerc1ses b and. $\mathrm{c}$.

a. Endpoints $\frac{6}{3}$. and $\frac{9}{3}, \frac{4}{2}$ and $\frac{6}{2}, \frac{2}{8}$ and $\frac{10}{8}$ (yea). b. Endpoints $\frac{5}{4}$ and $\frac{11}{4}, \frac{3}{2}$ and $\frac{6}{2}, \frac{1}{3}$ and $\frac{5}{3}$ ( $(20)$

c. Endpoints $\frac{4}{12}$ and $\frac{10}{12}, \frac{0}{2}$ and $\frac{1}{2}, \frac{3}{8}$, and $\frac{7}{8}($-yes)

4. Rename each of these numbers with a fraction that could be used to label a polnt of a sdale of twenty-fourths. Example: $\frac{1}{2}=\frac{12 i}{24}$.

a. $\frac{1}{3}\left(\frac{8}{24}\right)$ b. $\frac{2}{4}\left(\frac{12}{24}\right)$ c. $\frac{2}{6}\left(\frac{8}{24}\right)$ d. $\frac{11}{12}\left(\frac{22}{24}\right)$ e. $\frac{5}{8}\left(\frac{15}{24}\right)$

5. Which of these numbers cannot be renamed by a fraction which could be used to mark a scale in elighteenths? $(b, c, e, g)$
ia: $\frac{1}{6}$
d. $\frac{2}{9}$
f. $\frac{2}{3}$
b. $\frac{5}{12}$
e. $\frac{3}{4}$
g. $\frac{1}{10}$.
c. . $\frac{7}{16}$ 
ADDITION OF RATIONAL NUMBERS ON THE NUMBER LINE

Obfectives: To develop the meaning of addition of rational numbers by considering the measures of segments on the number line, and the measure of their union." when the segments are placed "end-to-end". To develop a procequre for computing the sum of two rational numbers whose measures are in the $\therefore$ same scale-that is; when the measures are named by fractions which have the same denominator.

To show that addition of rational numbers named by $\therefore$ "fractions with the pame denominator reduces to addition of whole numbers.

Vocabulary: Sum, addend, addition

Materials: - Number lines, dittoed number lines for children.

Suggested Teaching Procedure (See "Materials")

$\|$ Write this problem on the board:

The towns of Justin, Karver and Lind are on a straight road. Karver is between Justin, and Lind. It $1 \mathrm{~s} \frac{2}{10} \mathrm{ml}$. from Justin to Karver and $\frac{7}{10} \mathrm{~m} 1$. from Karver to Lind. How far is it from Justin to Lind?

Do you know the answer to this problem? Can you think of any way to plcture 1t? (W1th a number line) How do we scale the number line? (In tenths) Why? (The measures of the sections of the road are expressed in tenths.)

Here 1 sid number line/aiagram. Imagine its as a picture of the situation.

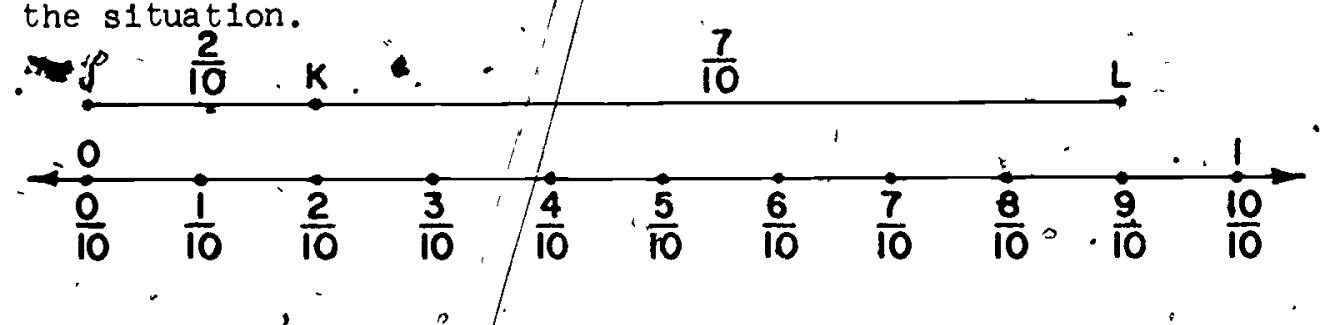


What does $\frac{}{\sqrt{k}}$ represent? (The section of the road between Justin and Karver.) What does $\overline{K L}$ represent? (The section of the road,between Karver and Lind.) What does. $\overline{\mathrm{JL}}$ represent? (The section of the road between Justin and Lind How would you ordinarily express the distance from Justin to Lind? ( $\left.\frac{9}{10}\right)$

Here are, some number line diagrams. They, show how to put coples of segments together "end-to-end", so the1r union is a , segment. For each diagram tell the measures of both segments and the measure of their union.

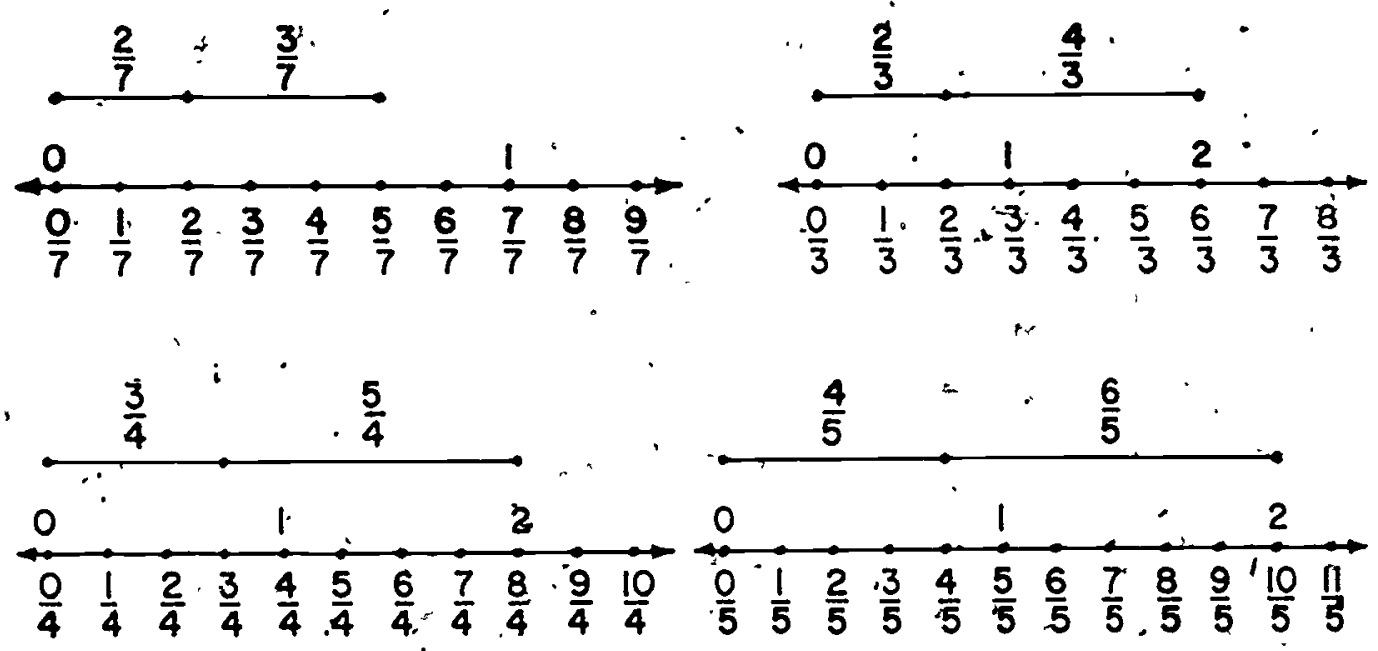

The method we have used for building a new line segment out of two others is way we used earlier of picturing addition of whole numbers. Because the. same method also pictures an operation pn rational numbers, we simply agree to call this operation addition. In this language we can express a relation between measures of length by using the usual symbol $(+)$ to indicate addition. In our examples the relations we have found are:

$$
\begin{array}{rlrl}
\therefore \quad & \frac{2}{7}+\frac{3}{7}=\frac{5}{7} & \frac{3}{4}+\frac{5}{4}=\frac{8}{4} \\
\quad \therefore \frac{2}{3}+\frac{4}{3}=\frac{6}{3} & \frac{4}{5}+\frac{6}{5}=\frac{10}{5}
\end{array}
$$

How is the number ine scaled to show: $\frac{2}{7}+\frac{3}{7}$ ? (in sevenths); $\frac{3}{4}+\frac{5}{4}$ ? (In fourths); $\frac{2}{3}+\frac{4}{3}$ ? (In thirds); $: \frac{4}{5}+\frac{6}{5}$ ? (In fifths): 
$\therefore$ We really don't need diagrams to compute sums like $\frac{3}{4}+\frac{2}{4}$. $\frac{5}{8}+\frac{4}{8}$, or $\frac{7}{3}+\frac{6}{3}$. For $\frac{3}{4}+\frac{2}{4}$ we can count by fourths from $\frac{3}{4}$ : We think $3^{-}$fourths, 4 fourths, 5 fourths. For $\frac{5}{8}+\frac{4}{8}$, how. do we count? (We count by eighths irom $\frac{5}{8}$. We think, "5 eighths 6 eighths, 7 eighths, 8 eighths, 9 eighths.")

This counting really amounts to addition of two whole numbers. We think

$$
\frac{3}{4}+\frac{2}{4}=\frac{3+2}{4}, \quad \frac{5}{8}+\frac{4}{8} \frac{5+4}{8}
$$

Is there any way to get the results for these additions without using the number line or counting? (Yes. The numerator of the result seems to be the sum of the numerators of the addends. The denominator of the result seems to be the common denominator of the addends.)

Are these mathematical sentences true? Use number IInes to decide.
a. $\frac{1}{2}+\frac{1}{2}=\frac{1+1}{2}$
c. $\quad \frac{1}{3}+\frac{1}{3}=\frac{1+1}{3}$
b. $\frac{3}{8}+\frac{2}{8}=\frac{3+2}{8}$
d. $\frac{1}{6}+\frac{3}{6}=\frac{1+3}{6}$ $\frac{1+1}{3}$ ? $\left(\frac{2}{3}\right)$ for $\frac{3+2}{8}$ ? ( $\left.\frac{5}{8}\right)$ for $\frac{1+3}{6} \Rightarrow$ ? $\left(\frac{4}{6}\right)$.

II In computing, the following form 1llustratrating $\frac{3}{4}+\frac{4}{4}$ should be used: $\frac{3}{4}+\frac{4}{4}=\frac{3+4}{4}=\frac{7}{4}$. - In this and succeeding sections, answers to exercises, are often rational numbers. It $1 \mathrm{~s}$, suggested that any appropriate name for that, number be accepted unless there are directions specifying a particular kind of name. Thus $\frac{4}{3}$, $\frac{8}{6}$, and later ' $1 \frac{1}{3}$ would all be accepted. Or course, you may wish to point out advantages of particular kinds of names in particular circumstances, and state that, in the absence of any special considerations, the fraction with the smallest possible denominator is the customarily preferred name. 
-ADDITION OF RATIONAL NUMBERS ON THE NUMBER LINE

\section{Expioration}

Builing a new line segment out Qf two others is a way of . picturing addition of whole numbers. Because the same method also pictures an operation on rational numbers, we agree to call this operation addition. We express a relation between measures " of length" using the usuál symbol "+" to indicate addition.

The addition, $\frac{3}{2}+\frac{4}{2}$ may be shown on a number iline scaled in häl ves.

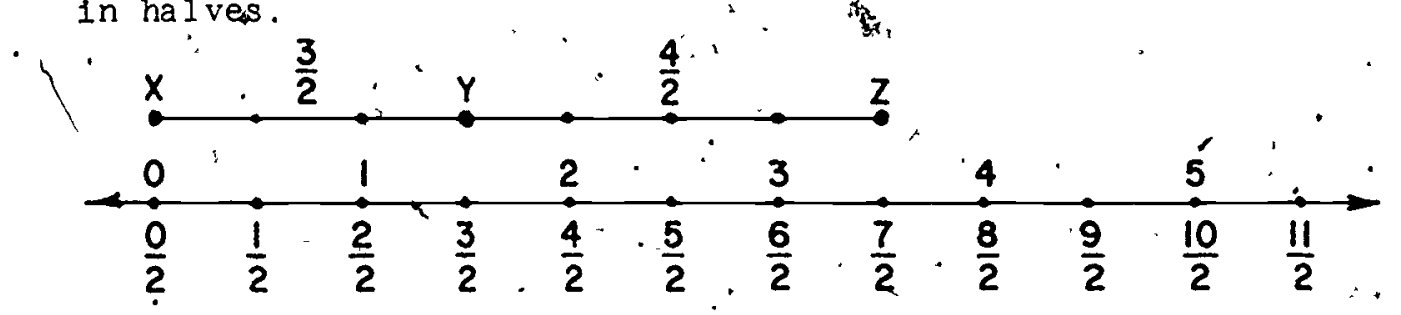

$\overline{X Y}$ is the uniton of three congruent segments, eacb/with 'measure $\frac{1}{2}$, so the measure of $\overline{X Y}$ is $\frac{3}{2}$.

$\overline{\mathrm{YZ}}$ is the union of four congruent segments, each with measure $\frac{1}{2}$, so the measure of $\overline{Y Z}$ is $\frac{4}{2}$..

$\overline{X Z}$ is the union of $(3+4)$, or 7 congruent segiments, , each with, measure $\frac{1}{2}$. The measure of $\overline{X 2}$ is $\frac{7}{2}$.

We write

$$
\frac{3}{2}+\frac{4}{2}=\frac{3+4}{2}=\frac{7}{2}
$$

We can think of $\frac{7}{2}$ as another name for $\frac{3}{2}+\frac{4}{2}$. 
The addition $\frac{6}{5}+\frac{3}{5}$ may be shown on a number line scaled "In fifths.

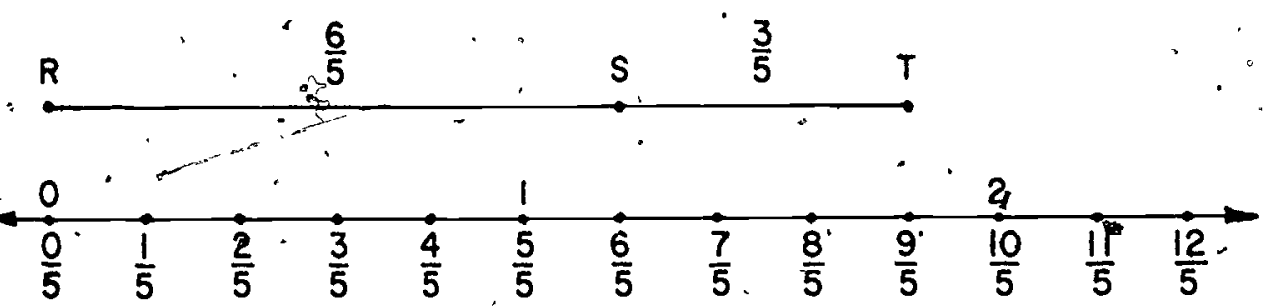

1. $\overline{\mathrm{RS}}$ is the union of (6) congruent segments, each with measure $\left(\frac{1}{5}\right)$ : The measure of $\overline{\mathrm{RS}}$ is $\left(\frac{6}{5}\right)$.

2. - $\overline{S T}$ is the union of (3) congruent segments, each with measure $\left(\frac{1}{5}\right):$ The measure of $\overline{\text { ST }}$ is $\left(\frac{3}{5}\right)$.

3. $\overleftarrow{R T}$ is the union of (9) congment segments, each with measure $\frac{\left(\frac{1}{5}\right)}{}$. The measure of $\overline{R T}$ is $\frac{(6+3)}{5}$ or $\frac{\left(\frac{9}{5}\right)}{\text { i }}$; Ve frite

$$
\frac{6}{5}+\frac{3}{5}=\frac{6+3}{5}=\frac{9}{5}
$$

We can think of $\frac{9}{5}$ as another name for $\frac{6}{5}+\frac{3}{5}$. 
$\therefore$ P3ío

\section{Exerc1se Set $14^{\circ}$}

1. Use number line, diagrams to show each of these sums.

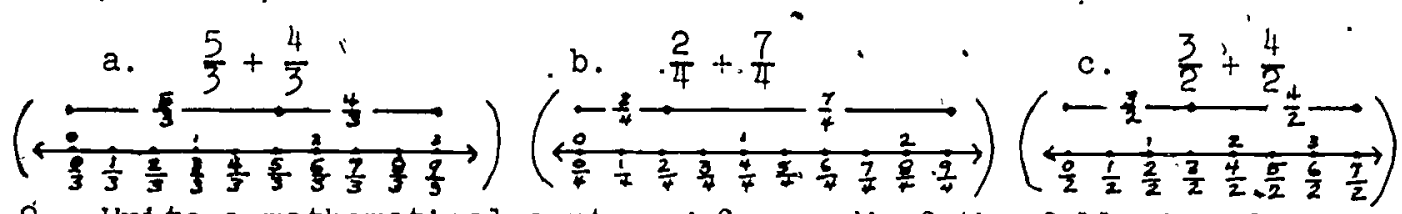

2. Write a mathematical sentence for each of the following diagrams:

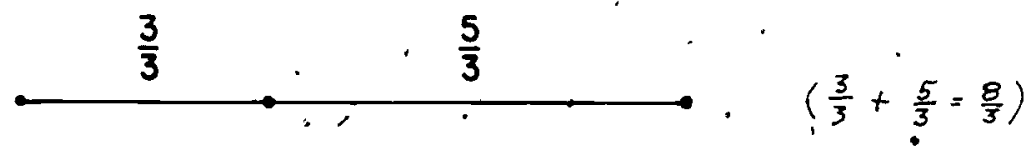

a.
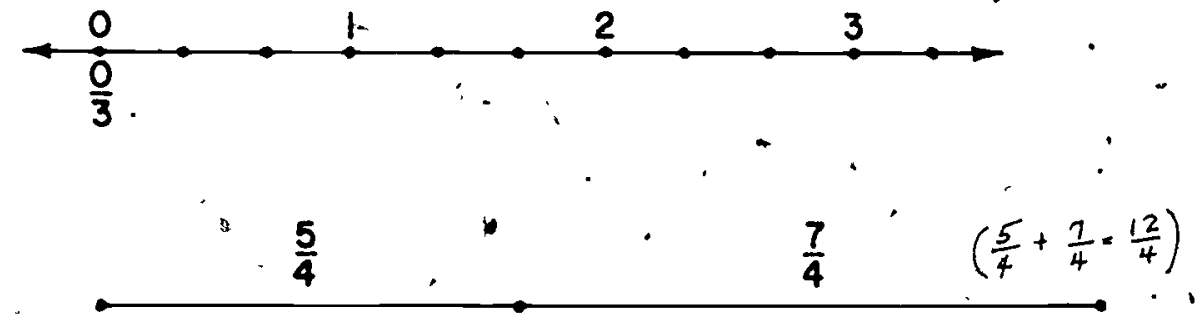

b.
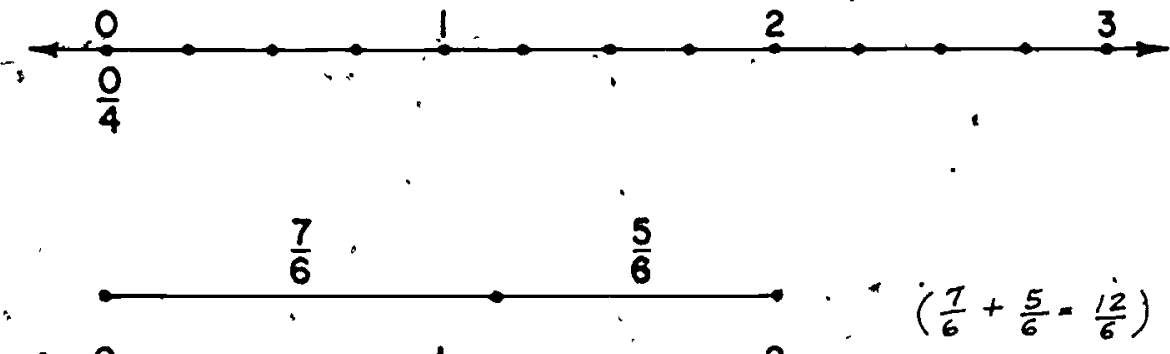

c.

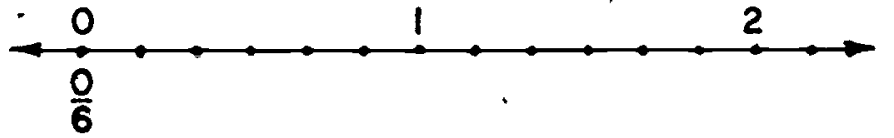

3. Copy and find a rraction name for. each of these sums.

Exercise 2 is done for you.
a. $\frac{3}{4}+\frac{5}{4}=\frac{3+5}{4}=\frac{8}{4}$
b. $\frac{5}{6}+\frac{9}{6}\left(\frac{14}{6}\right)$
d. $\frac{7}{8}+\frac{9}{8}$
$\left(\frac{16}{8}\right)$
c. $\frac{4}{5}+\frac{7}{5}\left(\frac{11}{5}\right)$
$-20-\frac{5}{12}+\frac{9}{12}\left(\frac{14}{12}\right)$ 
P311

.

4. Find a "fraction name for $n$," if:

a $\quad n_{i}=\frac{8}{3}+\frac{7}{3} \cdot\left(\frac{15}{3}\right)$

$\therefore$ b. $\quad \mathrm{n}=\frac{5}{8}+\frac{7}{8} \cdot\left(\frac{12}{8}\right)$

c. $n=\frac{3}{\pi}+\frac{7}{4}$

d. $n=\frac{5}{6}+\frac{3}{6}$

5. Copy each of the foliowing and represent the sum in simplest form. Exercise a 1s done for you

a. $\frac{1}{4}+\frac{1}{4}=\frac{1+2}{4}=\frac{2}{4}=\frac{1}{2}$

b. : $: \frac{2}{8} \div \frac{4}{8}$

c. $\frac{1}{6}+\frac{3}{6}$

d. $\frac{7}{10}+\frac{1}{10}$

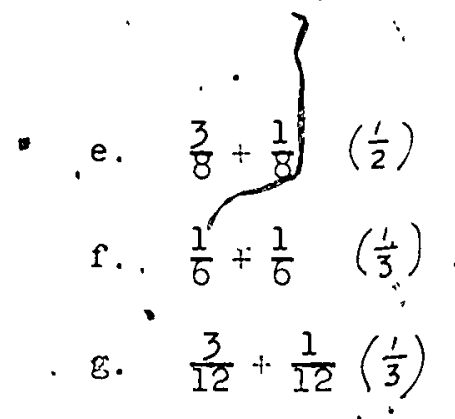

6. "BRAINTWISTERS

a. $\frac{5}{6}$ is the result. of adding tiro rational numbers. The fraction name for ean addend has a denominator 6 . What are two pussible addends? $\left(\frac{1}{6}+\frac{4}{6}\right.$ oi $\left.\frac{2}{6}+\frac{3}{6}\right)$

b. $\frac{5}{6}$ is the result of adding two rational numbers. Each fraction name has a denominator 12. What are two possible addends? $\left(\frac{6}{12}+\frac{4}{12}\right.$ as $\frac{9}{12}+\frac{1}{12}$, eto $)$

c. $\frac{5}{5}$ is the result of adding two rational numbers. One fraction has a denominator of 4 and the other fraction has a denominator 12. What are two possible ' addends? ( $\frac{3}{4}+\frac{1}{12} ; \frac{2}{4}+\frac{4}{1.2}$, eto.)

d. $\frac{7}{12}$. is the result of adding two rational numbers. One fraction has a denominator of. 3 and the other has. a denominator of 4 . What are two possible addends? 
SUBTRACTION OF RATIONAL NUMBERS.

Objectives: To, show that subtraction of rational numbers, I1ke

L. subtraction of whole numbers, is'a process of finding one addend when the other addend and sum are lnown.' Tc picture subtraction of rational numbers isth. segments on the number line.

To show that subtraction of rational numbers named by, fractions with the same denominator requces to subtraction of whole numbers.

Vocabulary: 'sum, addend, unknowm addend.

Materials: - Number lines, ditto'd number lines for children. Suggested Teaching Procedure.

. If your pup1is are not accustomed to thinking
of subtraction of whole numbers as finding an:
addend, you may wish to proceed as follows:
- In the mathematjcal sentence $\frac{2}{3}+\frac{1}{3}=n$, what are the addends? $\left(\frac{2}{3} \cdot \frac{a n d}{6} \frac{1}{3}\right)^{\text {What }}$ is the sum? $(n)$ In the mathemat1cal sentence $\dot{p}=\frac{6}{4}-\frac{2}{4}$, what $1 s^{\circ}$ the sum? $\left(\frac{6}{4}\right)$ What are the addends? $\checkmark \mathrm{p}$ and $\left.\frac{2}{4}\right): i_{0}$.

It imay be necessary to write màthematical. sentences tiltin whole numbers to help pup1ls learn this vocabulary. Draw a number line, as shown, on the board.

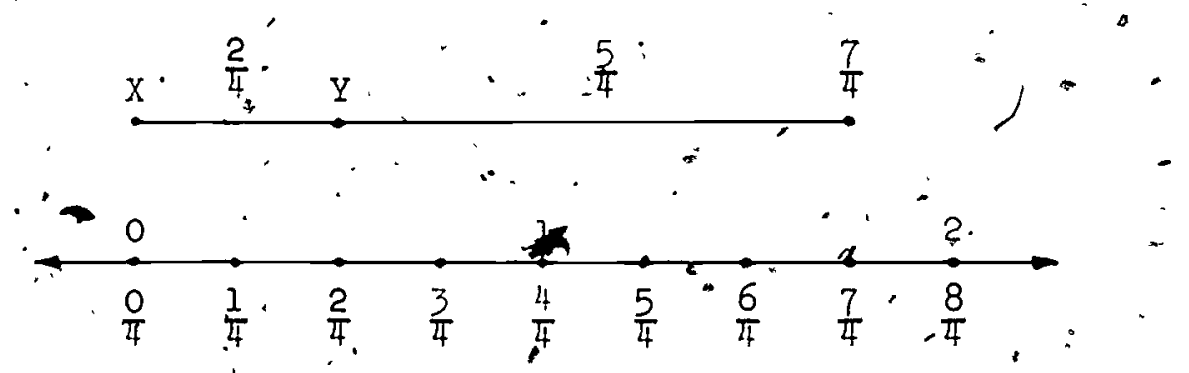

Does the number line show $\frac{2}{4}+\frac{5}{4}=\frac{7}{4} ?$ " (Yes) What scale 1s used in the diagram? (Fourths) what do ve call $\frac{2}{4}$ and $\frac{5}{4}$ ?
(-Addends) What do we call 7 ? (Sum) (Addends) What do we call $\frac{7}{4}$ ? (sum) 
Does the number ine show, $\frac{7}{4}-\frac{5}{4}=\frac{2}{4}$ ? (Yes) In $\frac{7}{4}-\frac{5}{4}=\frac{2}{4}$, what ane the addends? ( $\left(\frac{5}{4}-\right.$ and $\left.\frac{2}{4}\right)$ Fhat is the sum? $\left(\frac{7}{4}\right)^{2}$. Does the sentence $\frac{2}{4}+\frac{5}{4} \div \frac{7}{4}$ express the $\frac{2}{4} ; \frac{5}{4}$, and $\frac{7}{4}$ as does $\frac{7}{4}-\frac{5}{4}=\frac{2}{4}$ ? (Yes)

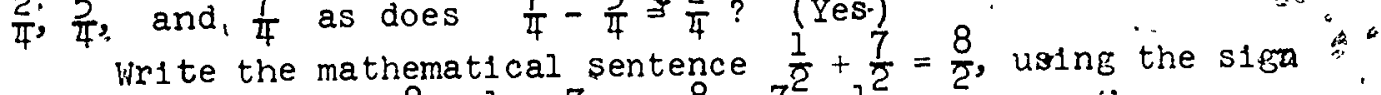
for subtraction. $\quad\left(\frac{8}{2}-\frac{1}{2}=\frac{7}{2}\right.$ or $\left.\frac{8}{2}-\frac{7^{2}}{2}=\frac{1}{2}\right)$

- II Use other examples as needed by your cliass - "II

In $\cdot \frac{5}{3}+\frac{4}{3}=n, n$ is the sum. $D$ the sentences $n=\frac{5}{3}-\frac{4}{3} \cdot s$ and $n-\frac{3}{3}=\frac{5}{3}$. state the same relationship? (Yes).

Look at the number ine. What scale 1s used? (Fourths) What nathematical sentence is shoin? $\left(n+\frac{2}{4}=\frac{8}{4}\right.$, or $\left.\frac{8}{4} ; \frac{2}{4}=n\right)$.

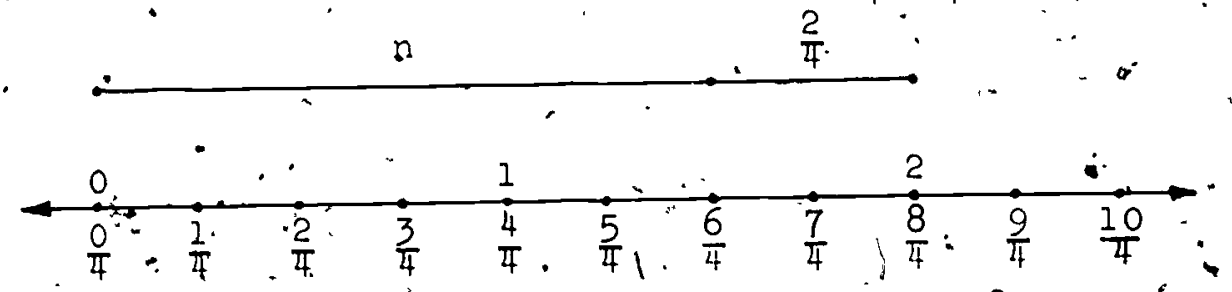

What is one addend? What is the other addend? ( $\frac{2}{4}$ and $\left.\dot{n}\right)$ What is the sum? $\left(\frac{8}{4}\right)$. Look at the pumber line. What numper is is? $\left(\frac{6}{4}\right)$ How could we rind a name for $n$ without using the number I1ne?

$$
\left(n=\frac{8}{4}-\frac{2}{4} ; \quad \text { therefore, } n=\frac{8-2}{4}=\frac{6}{4}\right)
$$

Emphasize the relationship between addion
and subtraction and the fact that there is simi-
larity in the form for obtaining results.
Furnish more examples like the one above.
and ask similar questiops.
There are no new techniques needed for com-
puting addends that are rational numbers. If
the sum and one addend are named by fractions in
the same scale, the subtraction is reduced to
subtraction of whole numbers.
In computing, the following form,
lliustrated for $\frac{10}{8}-\frac{4}{8}$. should be used.

$$
\frac{10}{8}-\frac{4}{8}=\frac{10-4}{8}=\frac{6}{8}
$$




\section{SUBTRACTION OF RATIONAL NỤMERS}

Addition and subtraction are operations on two numbers.

'The result of each operation is a single number.

The result of adding $\frac{2}{3}$ and $\frac{5}{3}$ is $\frac{7}{3}$. We have added. We cali $\frac{2}{3}$ and $\frac{5}{3}$ addends. We call $\frac{7}{3}$ the sum.

- Addition of rational

numbers may be expressed

with fraction numerals as

shown on the right." In

addition, two addends are.

known. We wish to find

the sum:

$$
\begin{aligned}
& \frac{2}{3}+\frac{5}{3}=\frac{7}{3} \quad \frac{2}{3} \text {. addend } \\
& +\frac{5}{3} \text { addend } \\
& \frac{7}{3} \text { sum }
\end{aligned}
$$

When we think about $\frac{7^{\circ}}{3}$ and $\frac{5}{3}$ and get a result of $\frac{2}{3}$, we have subtracted. We call, $\frac{5}{3}$ and $\frac{2}{3}$ addends. We call $\frac{7}{3}$ the sum.

Subtraction of rational

numbers may be expressed with fraction numerais"as shown at the .right. In subtraction, the sum and one addend are known. We wish to find the other addend.

The mathematical sentences

$$
\frac{2}{3}+\frac{5}{3}=\frac{7}{3}, \frac{7}{3}-\frac{5}{3}=\frac{2}{3} \cdot \text { and } \frac{7}{3}-\frac{2}{3}=\frac{5}{3}
$$

express the same relationship among $\frac{2}{3}, \frac{5}{3}$, and $\frac{7}{3}$.

$$
\begin{gathered}
\frac{7}{3}-\frac{5}{3}=\frac{2}{3} \cdot \frac{7}{3} \text { sum }= \\
\frac{-\frac{5}{3}}{\pi} \text { addend } \\
\frac{2}{3} \text { addend }
\end{gathered}
$$


Look at the diagram below. Use the measures of $\overline{\mathrm{AB}}, \overline{\mathrm{BC}}$ and $\overline{\overline{A C}}$ to write three-mathematical sentences which express the same relatiohship.
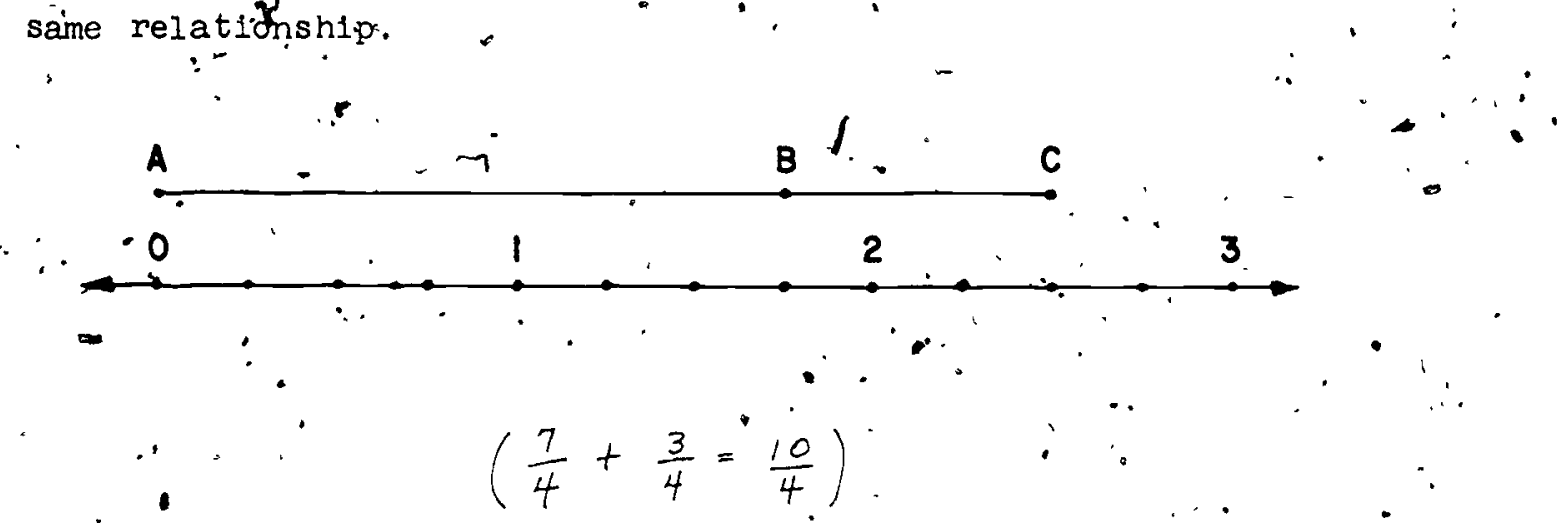

$$
\left(\frac{10}{4}-\frac{7}{4}=\frac{3}{4}\right)
$$$$
\left(\frac{10}{4}-\frac{3}{4}=\frac{7}{4}\right)
$$
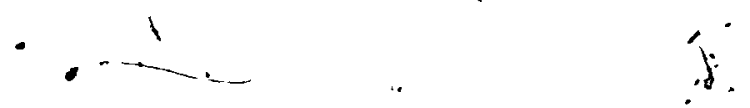
P314

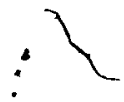

\section{$>$}

\section{Exerc1se Set 15}

1. Use, number Iine'diagrams to picture these relationships.

What number does $n$ represent?

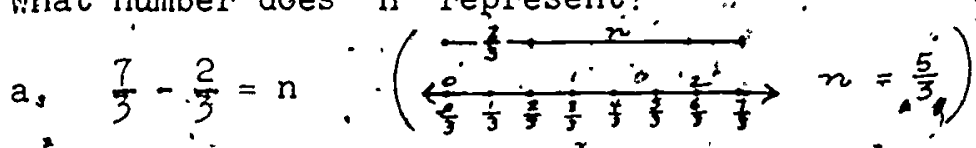

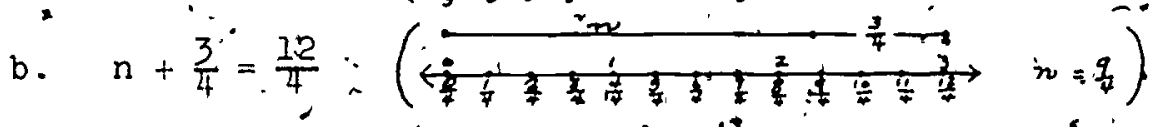

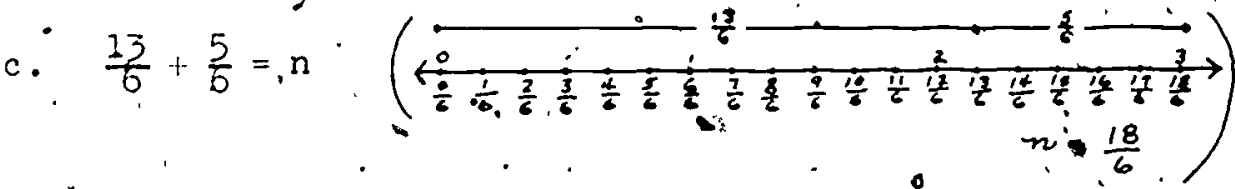

2. What mathematical sentences are pictured in the diagrams below?

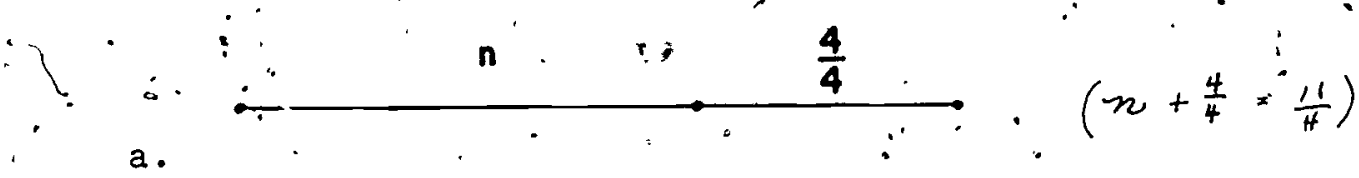
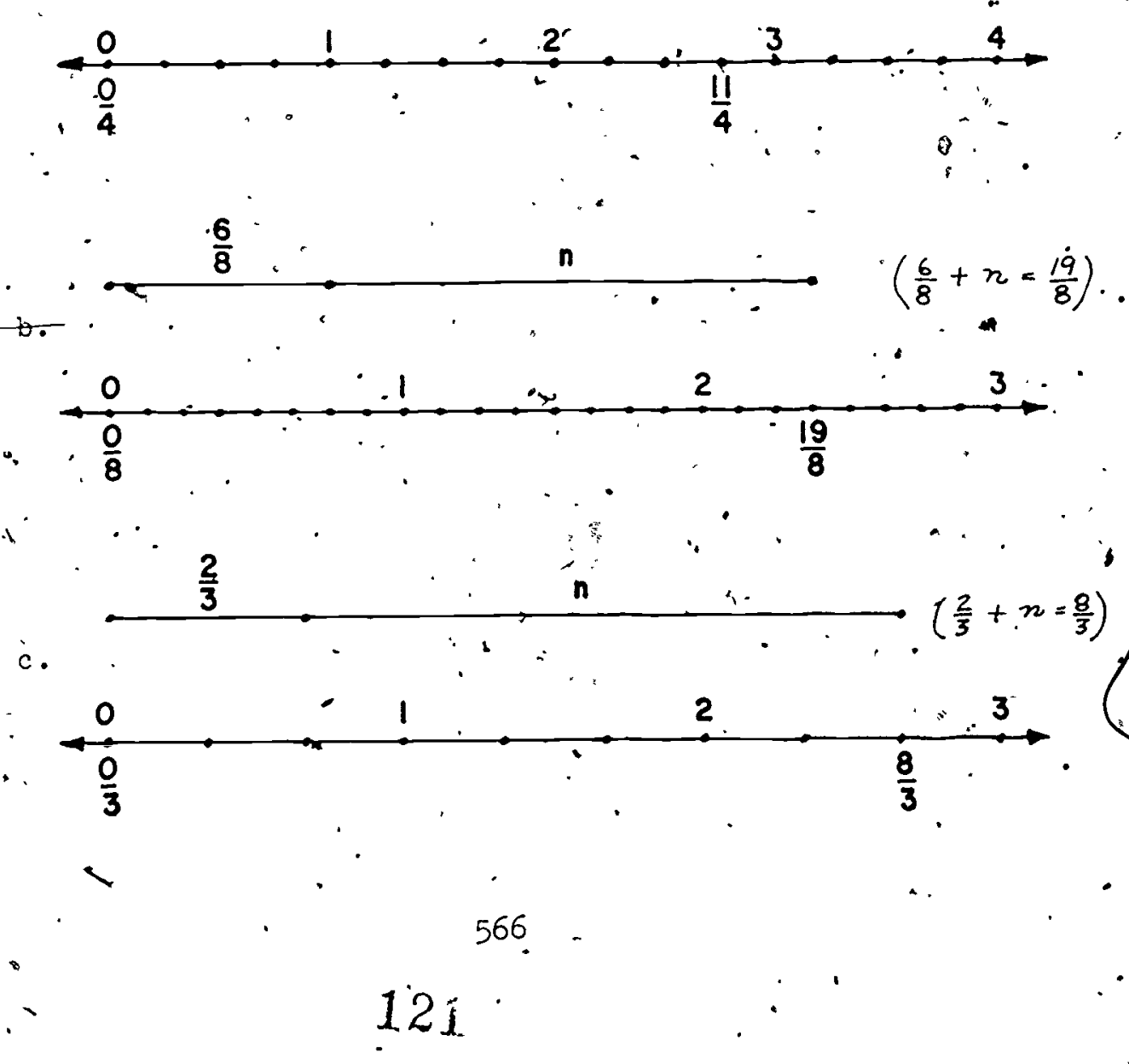
P315

3.' "Copy each sentence and find" a fraction name for " $n$.

Exercise a is done for you.

a. $\quad n=\frac{5}{3}-\frac{1}{3}=\frac{5 \cdots r}{3}=\frac{4}{3}$.

b. $\frac{7}{5}-\frac{3}{5}=n$

$\left(\frac{4}{5}\right) \cdots$

e. $\quad n=\frac{15}{16}-\frac{8}{16}\left(\frac{7}{16}\right)$

$\therefore$ ?
c. $\quad \frac{17}{8}-\frac{9}{8}=n$
f. $\frac{8}{4}-\frac{5}{4}=n$
$\left(\frac{3}{4}\right)$

d. $n=\frac{14}{12}-\frac{9}{12}\left(\frac{5}{12}\right)$

c. $\frac{11}{5}-\frac{7}{5}=n$

4. Copy each sentence and find the other addend. Name each answer in simplest fraction form.
a. $\quad \frac{5}{6}-\frac{\hat{2}}{6}=\bar{n}$
$\left(\frac{1}{2}\right)$
d. $\frac{8}{10}-\frac{4}{10}=\mathrm{n} \cdot\left(\frac{2}{5}\right)$.
b. $\quad \frac{7}{8}-\frac{5}{8}=n$
e. $\frac{9}{16}-\frac{5}{16}=n$
d. $\therefore \frac{7}{4}-\frac{5}{4}=n$
f. $\frac{7}{6}-\frac{5}{6}=n$ 
-PICTURING ADDITION AND SUBTRACTION WITH REGIONS

objective: To show that addition and subtraction of rational numbers can be :plctured with regions, as well as segments: ' $\cdots$

Vocabulary : Un1t region.

Materials: Models of rectangular regions and circular regions

Suggested Teaching Procedure

Work through the Exploration with the class. Use additional models às needed. Emphasize that a relationship between two addends and a sum may be stated by different mathematical sentences. 
P316

PICTURING ADDLTION AND SUBTRACTION WITH REGIONS

Exploration

-You hatve seen how number lines can be used to plcture addition gnd subtraction of rational numbers. Regions can be used also.

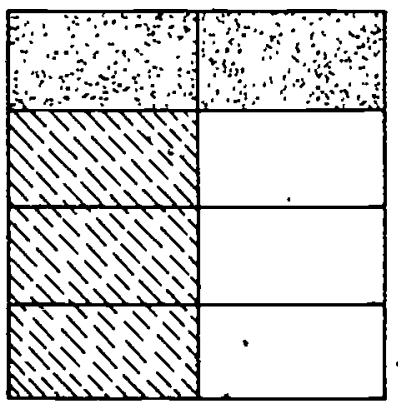

A

1. Figure A represents a unit region. Each of the small regions is $\left(\frac{1}{8}\right)$ of the unit region.

2. The dotted region is $\left(\frac{2}{8}\right)$ of the unit region.

3. The shaded region is $\left(\frac{3}{8}\right)$ of the unit region.

4. The unshaded region is $\frac{\left(\frac{3}{8}\right)}{.}$ of the unlt region.

5. Which regions picture the mathematical sentence "

$$
\frac{3}{8}+\frac{2}{8}=\frac{5}{8} ?
$$

(Ihe shaded and dotced reguonsor ) 
6. Write two other mathematical sentences wh1ch express the same relationship among $\frac{3}{8}, \frac{2}{8}$, and $\frac{5}{8} \cdot\left(\begin{array}{c}\frac{5}{8}-\frac{2}{8}=\frac{3}{8} \\ \frac{5}{8}-\frac{3}{8}=\frac{2}{8}\end{array}\right)$

7. Write a mathematical sentence pictured by the dotted and unshaded regions. $\left(\frac{2}{8}+\frac{3}{8}=\frac{5}{8}\right.$, seto $)$

8. Write three mathematical sentences suggested by the unit region and the dotted regions. $\left(\begin{array}{l}\frac{8}{8}=\frac{2}{8}+n, \frac{8}{8}-\frac{2}{8}=-n, \\ \frac{8}{8}-n=\frac{2}{8}\end{array}\right)$

9. The unit region. and the unshaded region suggest that $\frac{3}{8}+\frac{\left(\frac{5}{8}\right)}{8}=\frac{8}{8}$. Write two other mathematical sentences for this relationship. $\left(\frac{8}{8}-\frac{3}{8}=\frac{5}{8}, \frac{8}{8}-\frac{5}{8}=\frac{3}{8}\right)$

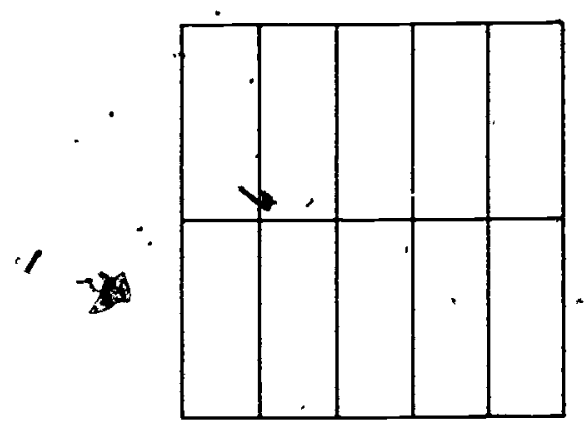

B

8

10. Trace figure $B$ shown above. Shade some parts and write. three mathematical sentences for your picture.

(aniweics ivile varyi) 
P318

\section{Exercise Set 16}

Write a mathematical sentence for each problem. . Use a number

- line if you need help in writing the mathematical sentence or finding the answer. Use simplest names for rational numbers used in your answers.

1. Susan needs $\frac{2}{3}$ yard ribbon to wrap one present and yard of ribbon for another. How much does she need?

$$
\left(\frac{2}{3}+\frac{2}{3}=\infty \text { She needed } \frac{\#}{3} \text { yacds ribbois }\right)
$$

2. Below is a map of a lake. Three friends live at the points marked $X, Y$, and $Z$. $X$ and $Y$ are $\frac{3}{10}$ miles apart. $\dot{Y}$ and. $z$ are $\frac{2}{10}$ miles apart. How long, a boat trip is it from $X$ to $Z$ by way of $Y$ ? $\left(\frac{3}{10}+\frac{2}{10}=n\right.$. The boat trip is $\frac{1}{2}$ mile long.).

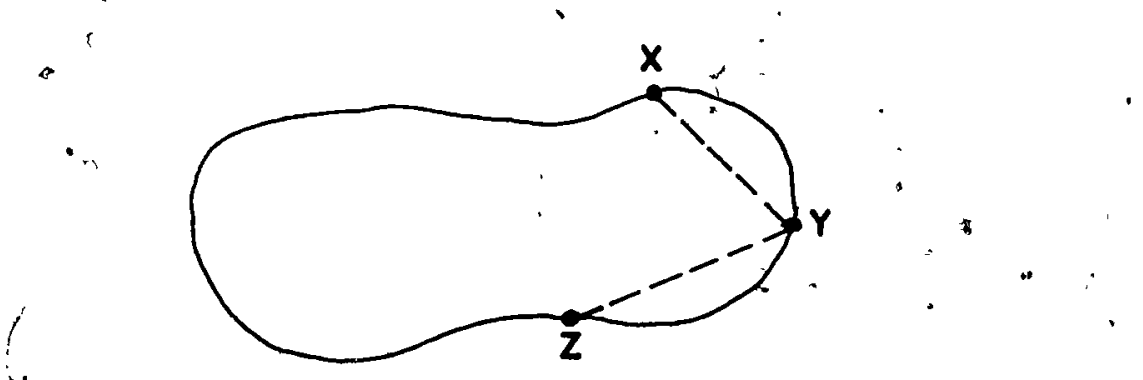

3. "There were 12 chapters in Mary's book. One day she read 2 chapters. The following day she read 1 chapter. What

* rational number best describes the part of the book she read on the two days? ( $\frac{2}{12}+\frac{1}{12}=$ n. Lhe tead $\frac{1}{4}$.of.) the borte oni the two 
Objectives: To show that to picture addition of rational numbers named by fractions with defferent denominators, it is necessary to find a sultable scale. To show that a suitable scale is one in which the number of congruent'segments in each unit segment : is a common multiple of the denominators of the original fraction names.

- To review renaming two rational numbers by fractions using the lowest common denominator.

Materials: Number lines, dittoed number lines for children* $i$ sujgested Teaching Procedure

Addition of rational numbers has been related to finding a measure for the union of two segments placed "end-to-end" on the number line. In that case, the numbers were named by fractions with the same denominator, so the selection of a suitable scale presented no problem. The discussion in this section deals with the question of finding a suitable scale for picturing the sum of two numbers named by fractions with different denominators. It thus provides a geometric picture of the process of finding a common denominator and of renaming the numbers to be added.

It would be well to build up the first diagram in the text in stages, using the chalk board. Show first the scale for halves, then introduce the scale for thirds on the same line, and ask the pupils to think of a scale which will provide fraction labels for the points of both scales. -

Work through the rest of the section with the pupils. 
P319

SCALES FOR PICTURING ADDITION

To picture $\frac{1}{2}+\frac{1}{3}$ on a number line, we need a suitable scale. A scale of sixths can show segments measuring $\frac{1}{2}$ and segments measuring $\frac{1}{3}$.

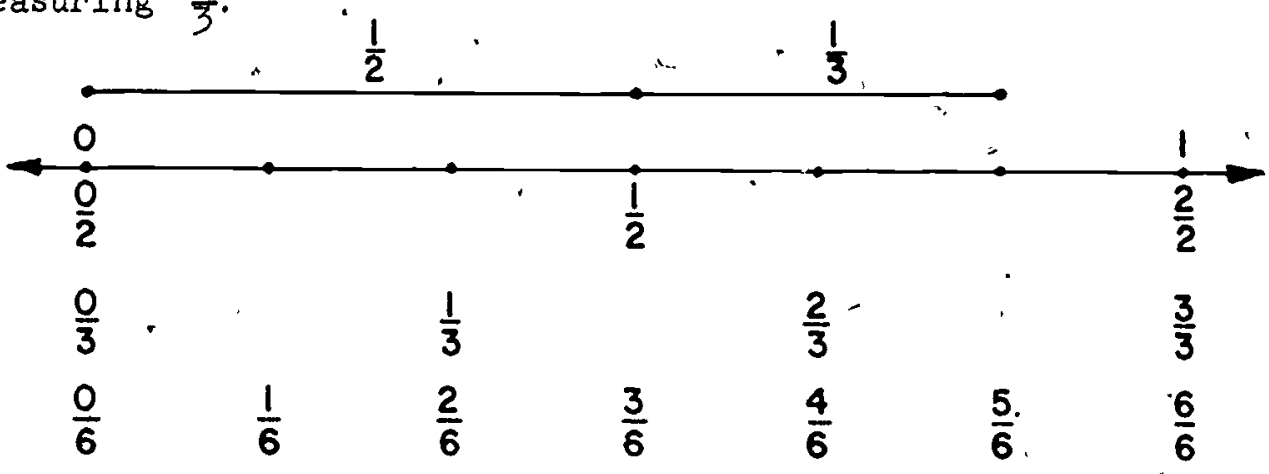

This alagram. suggests that $\frac{1}{2}+\frac{1}{3}$ can be written as

$$
\frac{1}{2}+\frac{1}{3}=\frac{3}{6}+\frac{2}{6} \text {. Now } \quad \frac{3}{6}+\frac{2}{6}=\frac{3+2}{6}=\frac{5}{6} \text {. }
$$

- To show $\frac{3}{4}-\frac{1}{3}$ on a number line we may use a scale of twelf ths.

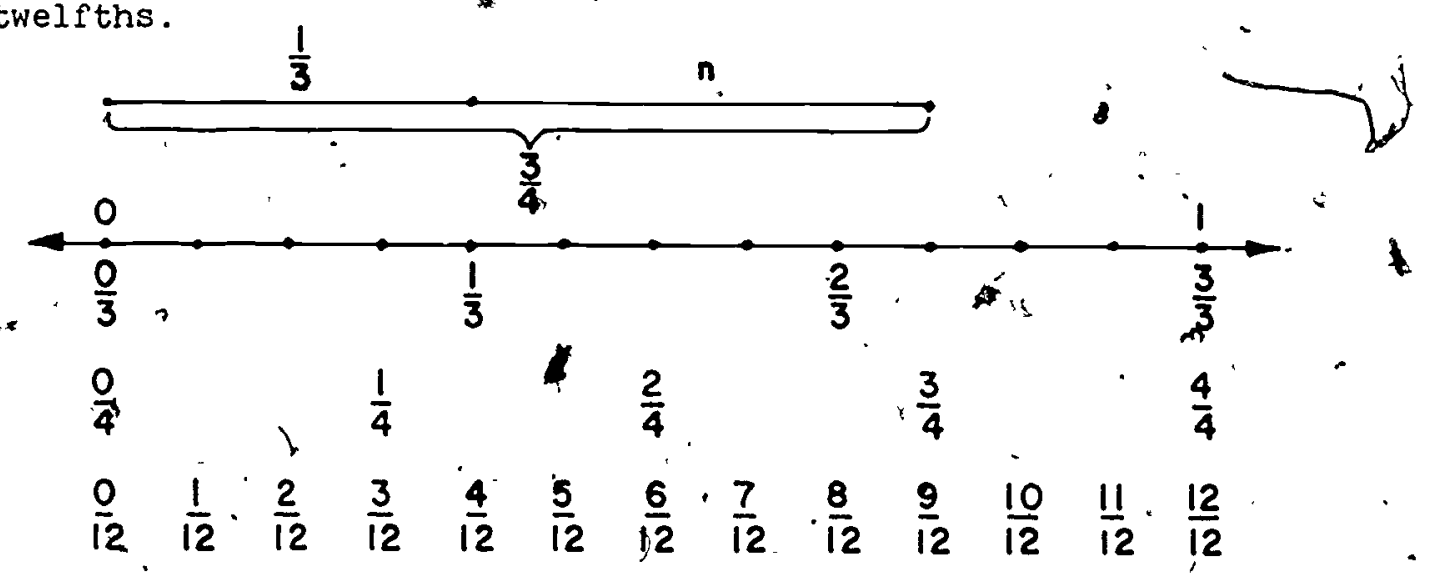

We think, $\mathrm{n}=\frac{3}{4}-\frac{1}{3}$; so $\mathrm{n}+\frac{1}{3}=\frac{3}{4}$. The number that $\mathrm{n}=\frac{5}{12}$. This suggests that $\frac{3}{4}-\frac{1}{3}$ can be written as

$$
\text { - } \frac{3}{4}-\frac{1}{3}=\frac{9}{12}-\frac{4}{12} \text {. iNow, } \frac{9}{12}=\frac{4}{12}=\frac{9-4}{12}=\frac{5}{12} \text {. }
$$


Can we always find a suitable scale to picture the sum of rational numbers? consider $\frac{3}{8}+\frac{1}{6}$.

To find a sultable,scale we must find a scale such that gach $\frac{l}{8}$ segment and each $\frac{1}{6}$ ségment is separated into $a$ whole number of smaller segments. For eighths, we can separate each $\frac{1}{8}$ segment into

2 congmuent segments. Then there $w 111$ be $2 \times 8$ parts in each unit segment.

3 congment segments. Then there will be $3 \times 8$ parts in each unit segment.

4 congmuent segments. Then there w1ll be $4 \times 8$ parits in each unit segment.

Subdividing each $\frac{1}{8}$. segment in these ways suggests scales in which the denominators of the fractions are the set of multiples of 8. $\{8,16,24,3 \dot{2}, 40,48 \ldots\}$ Subdividing sixths suggests the set of multiples of $6 .,[6,12,18,24,30,36, \ldots\}$ A . scale which can be used to picture addition of eighths and sixths will be one in which the denominator of the fraction is a common miltiple of 8 and 6 . The easiest scaly to use is the one in Which the i.c.m.- of 8 and 6 is used, that 1s', the least. common denominator for $\frac{3}{8}$ and $\frac{5}{6}$.

You know how to find the $1.2 . d$. of $\frac{3}{8}$ and $\frac{5}{6}$. It is the 'l.c.m. of 8 and 6 .

$$
\begin{array}{ll}
8=2 \times 2 \times 28 & 6=2 \times 3 \\
\text { l.c.d. }=2 \times 2 \times \frac{2}{2} \times \frac{3}{6} & =2 \times 2 \times 2 \times 3=24
\end{array}
$$

To subdivide the eighths and sixths segments, note that

$$
\begin{aligned}
& 24=8 \times 3 . \\
& 24=6 \times 4 .
\end{aligned}
$$

i. 
P321

So each segment of measure one-eighth is subdivided into 3 congruent segments, and each segment of measure one-sṭth Io subdivided into 4 congruent segments.
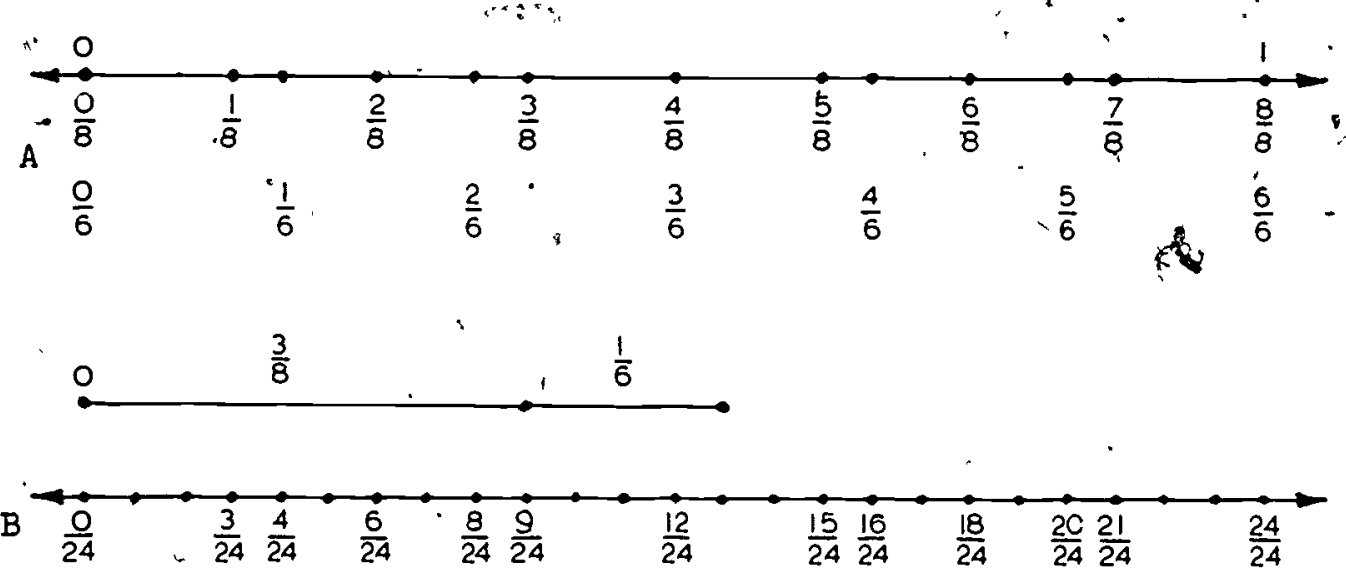

on number line $A$ the eighths and sixths scales are labeled. On $B$, points on the number line are marked for a scale of twenty-fourths. The points corresponding to eighths and sixths are labeled in twenty-fourths.

We write:

$$
\frac{3}{8}+\frac{1}{6}=\frac{9}{24}+\frac{4}{24}=\frac{9+4}{24}=\frac{13}{24} \text {. }
$$

575 
1. Find the scale with the smallest number of divisions you could use to picture these sums.

a. $\frac{3}{8}+\frac{7}{10}$ (fortucthe c. $\frac{3}{14}+\frac{5}{21}$ (forty-secondis).

.b. $\frac{5}{9}+\frac{11}{15}:\left(\right.$ forty-fifthes). d. $\frac{7}{16}+\frac{5}{12}$ (foity-eighths)

?. Use number line diagrams to picture:

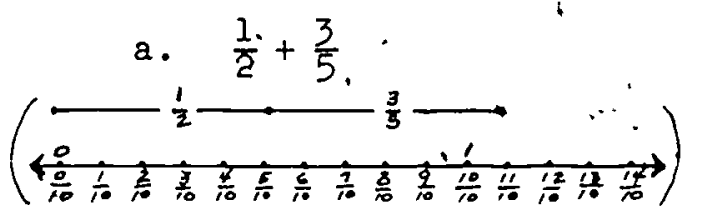

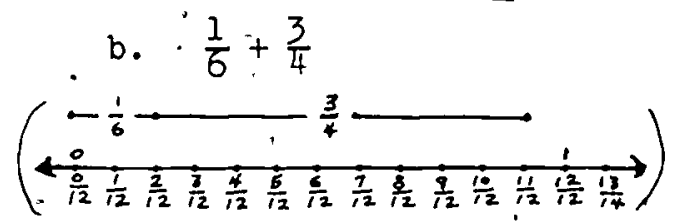

3. For each pair of fractions, write:

$\therefore$ (1) the complete ractorization of each denominator.

(2) the complete factorization of the least commoh denominator.

(3) fraction names using the l.c.d.
a.- $\frac{3}{8}, \frac{7}{20}$
c. $\frac{2}{3}, \frac{4}{15}$
b. $\frac{1}{4} \frac{3}{14}$
d. $\frac{5}{12}, \frac{7}{18}$

(a. $\frac{\underbrace{5}_{20} \times \frac{8}{2} \times 2 \times 5}{20}=40$

$\left.\frac{3}{8}=\frac{15}{40}, \frac{7}{20}=\frac{14}{40}\right)$

(b. $\frac{\frac{4}{2} \times \frac{2}{2} \times 7}{.14}=28$

$\left.\frac{i}{4}=\frac{7}{28} ; \frac{3}{14}=\frac{6}{28}\right)$

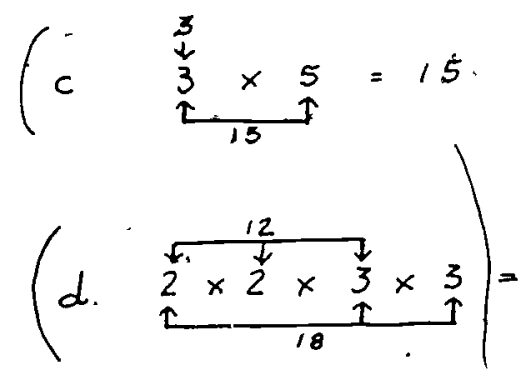

$\left.\frac{2}{3}=\frac{10}{15} ; \frac{4}{15}-\frac{4}{15}\right)$ 
Ob.jectives: To develop computational procedures for adding and subtracting rational numbers named by fráctions with different denominators.

To show that such procedures reduce

(a)

renaming the numbers by fractions olth a common denominator and (b) adding or subtracting whole

- numbers.

Suggested Teaching Procedures Work through the Exploration with the class,
and work out $y$ th them the form in which written work is to be arranged.

Exercise set 18 contains a list of exercises which pupils should now be able to do without using paper and pencil. Similar lists should be: used frequently. Pupils really enfoy making up. such lists for use by the class.
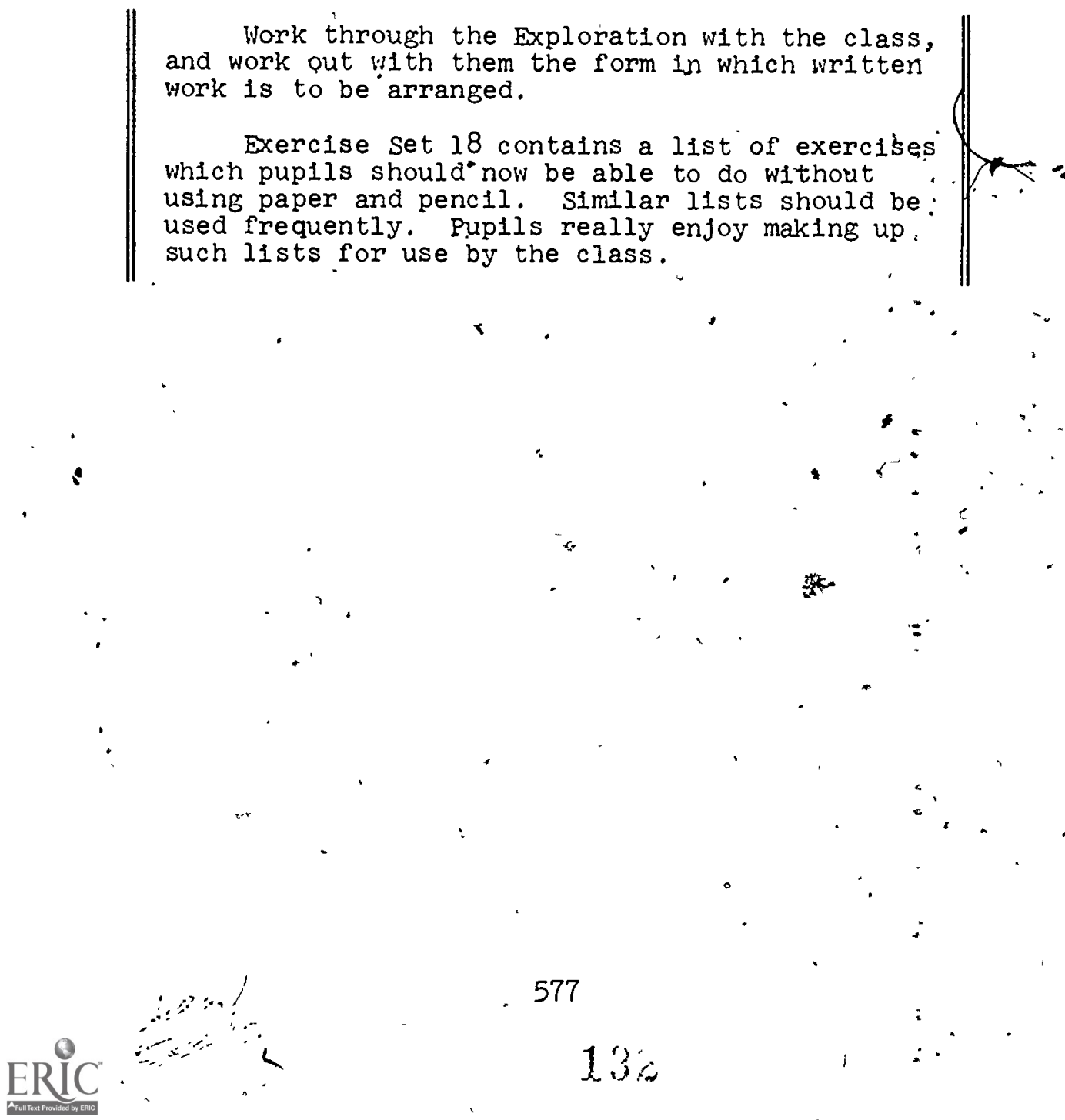
COMPUTING SUMS AND UNKNOWN ADDEND́S.

\section{Exploration}

You have seen that suitable scales can be found for picturing. addition of rational numbers on the number line.

-Rational numbers also can be added without usingidiagrams.

Consider the sum

$$
\frac{1}{2}+\frac{3}{4}
$$

You know how to add rational numbers when they are named by fractions with the same denominator.

1. What is the 1.c.d. for $\frac{1}{2}$ and $\frac{3}{4}$ ?

2. Using the l.c.d., what are, the fraction names for $\frac{1}{2}$ and $\frac{3}{4}$ ?

3. What is the sum of $\frac{1}{2}$ and $\frac{-3}{4}$ ?

You can arrange your work like this:

$$
\begin{aligned}
& n=\frac{1}{2}+\frac{3}{4} \\
& =\frac{2}{4}+\frac{3}{4}=\frac{2+3}{.4}=\frac{5}{4} \\
& \frac{1}{2}+\frac{3}{4}=\frac{5}{4} \quad .1 . \quad . \quad \frac{1}{2}=\frac{2}{4}
\end{aligned}
$$

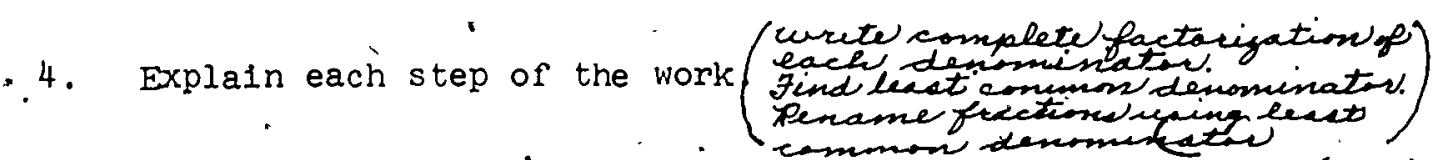

5. Can you rind a comion denominator for any two fractions?(Zew)

6. Can you add any two rational numbers if you have fraction names for them? (yed) 


\section{Exercise Set 18}

1. Find the sum of each pair of numbers: Write the simplest frastion name for the sum.
a. $\frac{1}{2}, \frac{3}{8}\left(\frac{7}{8}\right)$
a. $\frac{2}{3}, \frac{1}{6}$
$\left(\frac{5}{6}\right)$
8. $\frac{3}{4}, \frac{5}{8}$
$\left(\frac{11}{8}\right)$
b. $\frac{1}{2}, \frac{5}{6}\left(\frac{4}{3}\right)$
e. $\frac{2}{3}, \frac{5}{9}$
h. $\frac{3}{4}, \frac{17}{12}\left(\frac{13}{6}\right)$
c. 달 $\frac{7}{12}\left(\frac{13}{12}\right)$
f... $\frac{2}{3}, \ldots \frac{11}{12}\left(\frac{19}{12}\right)$
1. $\frac{3}{4} ; \frac{27}{20}\left(\frac{21}{10}\right)$

2. Find the simplest fraction name for each sum.
a. $\frac{3}{2}+\frac{1}{3}\left(\frac{11}{6}\right)$
d. $\frac{8}{5}+\frac{7}{4}\left(\frac{67}{20}\right)$
g. $: \frac{5}{6}+\frac{3}{4}\left(\frac{19}{12}\right)$
b. $\frac{3}{2}+\frac{4}{5}\left(\frac{23}{10}\right)$
e. $\frac{8}{8}+\frac{2}{3}$
h. $\frac{5}{6}+\frac{3}{5}\left(\frac{43}{30}\right)$
c. $\frac{3}{2}+\frac{6}{7}\left(\frac{35}{14}\right)$
ค. $\frac{8}{5}+\frac{2}{3}$
1. $\frac{5}{6}+\frac{1}{7}\left(\frac{41}{42}\right)$

3. Use number lines to picture these $e_{5}$ mathematical sentences.

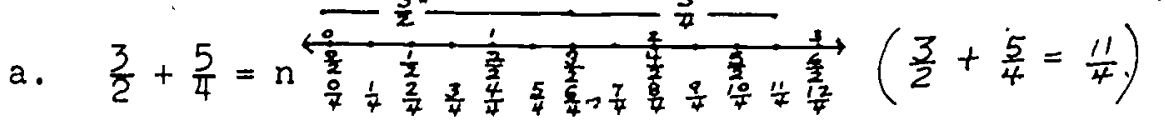

b. $\frac{4}{3}+\frac{\dot{5}}{6}=\left(\frac{13}{6}\right)$

c. $\frac{2}{3}+n=\frac{3}{2}$

a. $\quad \begin{array}{r}\frac{11}{8}-\frac{3}{4}=n \\ \left(\begin{array}{rl}\frac{3}{4} & =\frac{11}{8} \\ n & =\frac{5}{8}\end{array}\right)\end{array}$

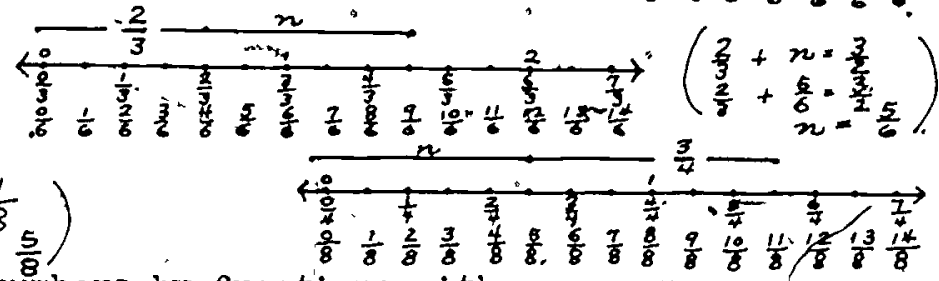

Rename each pair of numbers by fractions with a common

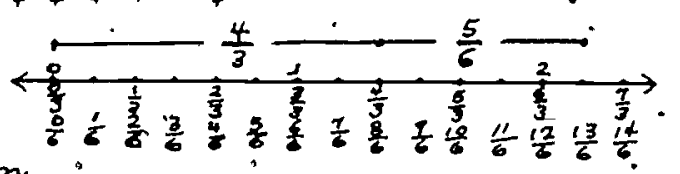
denominator. Then fitdd a fraction name for the number $n$. 4. a. $\frac{5}{6}-\frac{2}{3}=n \cdot\left(\frac{1}{6}\right)$ d. $\quad \frac{5}{6}-\frac{1}{3}=n\left(\frac{1}{2}\right) \quad \frac{3}{4}-\frac{1}{6}=n\left(\frac{7}{12}\right)$

$$
\begin{aligned}
& \text { b. } \frac{2}{3}-\frac{5}{8}=n\left(\frac{1}{24}\right) \text { e. } \frac{4}{5}-\frac{1}{3}=n\left(\frac{7}{15}\right) \text { h. } \frac{4}{5}-\frac{1}{2}=n\left(\frac{3}{10}\right) \\
& \text { c. } \frac{11}{12} \\
& \therefore \quad \text { ค. } \frac{\frac{7}{8}}{-\frac{9}{16} \cdot\left(\frac{5}{16}\right)} \\
& -\frac{5}{6} \\
& \mathrm{n}\left(\frac{1}{12}\right) \\
& \text { 1. } \frac{3}{4} \\
& \downarrow \\
& \frac{-\frac{1}{8}}{n\left(\frac{5}{8}\right)}
\end{aligned}
$$


P325

Find the sum for each pair of numbers and write its simplest fraction name.

5. a. $\frac{1}{3}, \frac{3}{4}\left(\frac{13}{12}\right)$

d. $\frac{3}{8}, \frac{1}{4}\left(\frac{5}{8}\right)$

8. $\frac{2}{3}, \frac{5}{6}\left(\frac{3 \cdot}{2}\right)$

b. $\frac{3}{4}, \frac{1}{2}\left(\frac{5}{4}\right)$

e. $\frac{2}{3}, \frac{1}{4}\left(\frac{11}{12}\right)$

h. $\frac{4}{5}, \frac{1}{2}\left(\frac{13}{10}\right)$

c. $\frac{2}{5}$

f. $\frac{3}{4}$

1. $\frac{1}{3}$

$\frac{\frac{1}{3}}{\left(\frac{11}{15}\right)}$

$\frac{\frac{1}{6}}{\left(\frac{11}{12}\right)} \cdots \frac{\frac{1}{8}}{\left(\frac{11}{24}\right)}$

6. Find $\dot{a}$ fraction name for " $n$ so that each mathematical sentence wi Fl be true.

a. $\frac{2}{3}+n=\frac{3}{4}$

$\left(\frac{1}{12}\right)$

d. $\frac{3}{8}+n=\frac{2}{3} \cdot\left(\frac{7}{24}\right)$

b. $\frac{3}{4}+n=\frac{7}{8} \quad\left(\frac{1}{8}\right)$

e. $\frac{1}{2}+n=\frac{2}{3} \quad\left(\frac{1}{6}\right)$

c. $\frac{2}{5}+n=\frac{3}{4}\left(\frac{7}{20}\right)$

if. $\frac{1}{5}+n=\frac{1}{2}\left(\frac{3}{10}\right)$

7. BRAINTWISTER: Find a fraction which names a number (anam may a. greater than $\frac{3}{10}$ and less than $\frac{3}{8}:\left(\frac{13}{40}, \frac{14}{40}\right)$

b. greater than $\frac{2}{6}$ and less than $\frac{3}{6} \cdot\left(\frac{5}{12}, \frac{7}{18}, \frac{8}{18}\right)$

c. greater than $\frac{3}{6}$ and less than $\frac{4}{6}$. (Find two answers) $\left(\frac{7}{12}, \frac{10}{18}, \frac{11}{18}\right)$

580

$13 i$ 
Certain rational numbers should now be so familiar that you can think of many names for them. You should be able to add and subtract such numbers without writing out your work.

Wi thout doing any writing, try to find what number $\mathrm{n}$ must be.
1. $\frac{1}{4}+\frac{1}{8}=n$
$\therefore 6$.
$n+\frac{5}{8}=\frac{3}{4} \cdot\left(\frac{1}{8}\right)$
2. $\frac{1}{2}+\frac{1}{5}=n \cdot\left(\frac{7}{10}\right)$
$-7 \cdot n+\frac{3}{10}=\frac{4}{5} \cdot\left(\frac{1}{2}\right)$
3. $\frac{3}{4}+\frac{1}{2}=n \quad\left(\frac{5}{4}\right)$.
8. $\quad \frac{5}{6}+\mathrm{n}=\frac{13}{12} \quad\left(\frac{1}{4}\right)$
4: $\frac{1}{6}+\frac{5}{12}=\mathrm{n}\left(\frac{7}{12}\right)$
9. $\mathrm{n}=\frac{7}{2}-\frac{1}{3}\left(\frac{4}{9}\right)$
5. $\frac{7}{8}+\frac{1}{4}=n \cdot\left(\frac{9}{8}\right)$
10. $\frac{10}{10}=n-\frac{7}{10}\left(\frac{17}{10}\right)$

The numbers in the exercises below have fraction names which are probably. less familiar. Show all your work for these exercises.

11. $\frac{5}{6}+\frac{8}{15}=\mathrm{n} \quad\left(\frac{41}{30}\right)$

12. $\frac{5}{14}+\frac{3}{4}=\mathrm{n} \quad\left(\frac{31}{28}\right)$

13. $\frac{13}{12}+\frac{3}{8}=n \quad\left(\frac{35}{24}\right)$

14. $\frac{7}{10}+\frac{11}{6}=\mathrm{n} \quad\left(\frac{38}{15}\right)$

15. $\frac{1}{6}+\frac{9}{14}=n \quad\left(\frac{17}{21}\right)$
16. $\frac{3}{10}+n=\frac{5}{4} \quad\left(\frac{19}{20}\right)$

17. $n+\frac{4}{9}=\frac{11}{12} \quad\left(\frac{17}{36} j\right)$

i8. $\frac{21}{10}=n-\frac{5}{8}\left(-\frac{109}{40}\right)$.

19. $n+\frac{8}{15}=\frac{13}{9}\left(\frac{41}{45}\right)$

20. $\frac{15}{7}=n+\frac{5}{4} \quad\left(\frac{25}{28}\right)$.

Find the number $n$ represents. " In exercise 21 , recall that $\left(\frac{1}{2}+\frac{1}{3}\right)+\frac{1}{4}$ means "Find the sum of $\frac{1}{2}$, and $\frac{1}{3}$, and then add the sum to " $\frac{1}{4}$ ".

2r. . $\left(\frac{1}{2}+\frac{1}{3}\right)+\frac{1}{4}=n \quad\left(\frac{13}{12}\right)$

2. $\quad\left(\frac{3}{4}+\frac{7}{8} z+\frac{2}{3}=\mathrm{n} \quad\left(\frac{55}{24}\right)\right.$

23. $\frac{5}{6}+\left(\frac{3}{2}+\frac{7}{4}\right) \leq n\left(\frac{49}{12}\right)$
24. $n+\left(\frac{7}{12}+\frac{1}{4}\right)=\frac{5}{6}$

25. $\left(\frac{7}{10}+\frac{2}{5}\right)=n-\frac{1}{2}\left(\frac{8}{5}\right)$

26: $\left(\frac{1}{4}+\frac{8}{9}\right)+n=\frac{5}{3}\left(\frac{19}{36}\right)$ 


\section{DIAGRAMS FOR PROBLEMS}

Objectives: - To suggest the use of the number line or a region

to picture number relations in a problem. '

To show that a number line diagram can be used to

- Dicture number relations in a problem even though the problem refers to objects which do not, of themselves, suggest segments.:

Materials: Dittoed copies of number lines and regions.

Suggested Teaching Procedure:

. ? ? : ?

A Work through the exploration in the pupil text with the class. Have them make up ad-' ditional problems solved by addition or subtraction with rational numbers and draw diagrams to picture the number relations ínvolved.

Many children find it very difficult to sosve "word" problems. For such children, thinking about the problem with sufficient care to draw a diagram which pictures the conditions can be very helprul. It is therefore sound.procedure occasionally to require that they make diagrams for problems.

Some children enjoy drawing, and others find it a hard task. For this reason, it is a good 1dea to provide dittoed copies of number lines and circular and rectangular regions which they may use and adapt to their purposes. Models for some of these are included in the sectiol on miterials.

\section{$\wedge$}

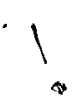


DIAGRAMS FOR PROBLEMS

We have pictured addition and subtraction of rational numbers on the number line, and also with regions. When you wish topsolve a problem using rational numbers, it is sometimes. helpful to picture the relationskips on a number line, or 1na picture of a region. Look at this problem.

Paul found several unusual rocks while he was on vacation. He gave $\frac{3}{8}$ of the rocks to his brother, and gave $\frac{1}{2}$ of them to a friend. What part of the total number of rocks did he give away?

This is not a problem about things which suggest segments, but numbers are used, and numbers may, be repsesented. on the numbexistie.

Suppose the unit segment represents the entire set of rocks Paul found.

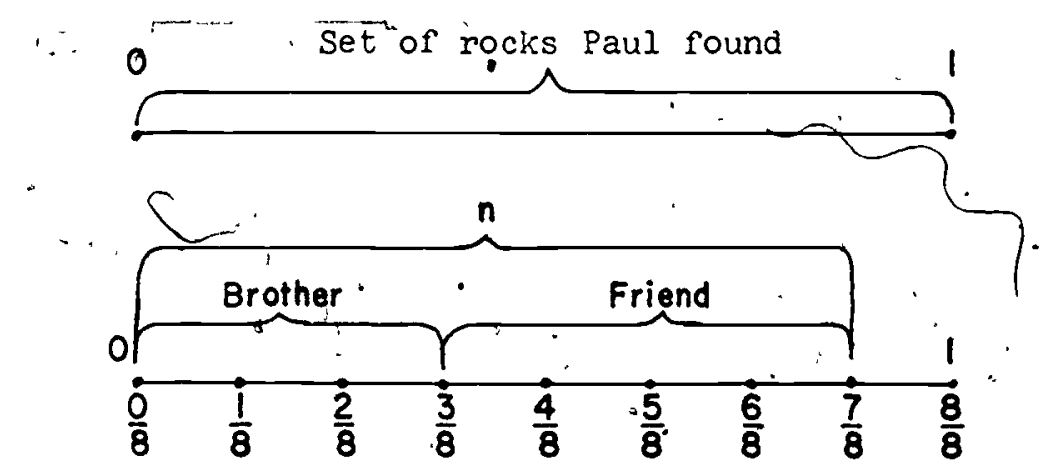

He gave $\frac{3}{8}$ to his brother. He gave $\frac{1}{2}$ (or $\frac{4}{8}$ ) to a friend.

What part did he give away? Represent it by $n$. The diagram suggests: $\quad \mathrm{n}=\frac{3}{8}+\frac{1}{2}$.

$$
=\frac{3}{8}+\frac{4}{8}=\frac{3+4}{8}=\frac{7}{8}
$$

Paul gàve away $\frac{7}{8}$ of the 'rocks.

\section{3.}


Now look at this problem.

Mrs. White cut a ple into 6 pieces. After Bob ate 1 plece, for lunch, $\frac{5}{6}$ was left. Mrs. White served $\frac{1}{2}$ of the whole pie to B1ll. What part of the ple was left then?

Choose a unit segmenty to represent the whole pie, cut into sixths.
$B \circ b\left(\frac{l}{6}\right)$
$\left(\frac{5}{6}\right)$ Left after lunch

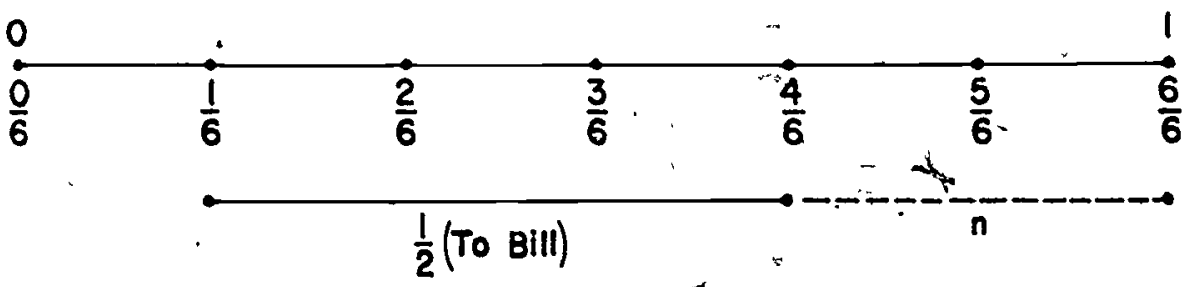

Bob ate $\frac{1}{6}$. B1ll ate $\frac{1}{2}$ or $\frac{3}{6}$. What part of the ple was left?

$$
\begin{aligned}
& \frac{1}{2}+n=\frac{5}{6}, \text { or } \\
& \frac{3}{6}+n=\frac{5}{6} \\
& n=\frac{5}{6}-\frac{3}{6}=\frac{5-3}{6}=\frac{2}{6} \\
& \frac{2}{6} \text { of the ple was left. }
\end{aligned}
$$

You might wish to represènt the whole pie as a unit region separated into sixths.

Bob's piece $\frac{1}{6}$
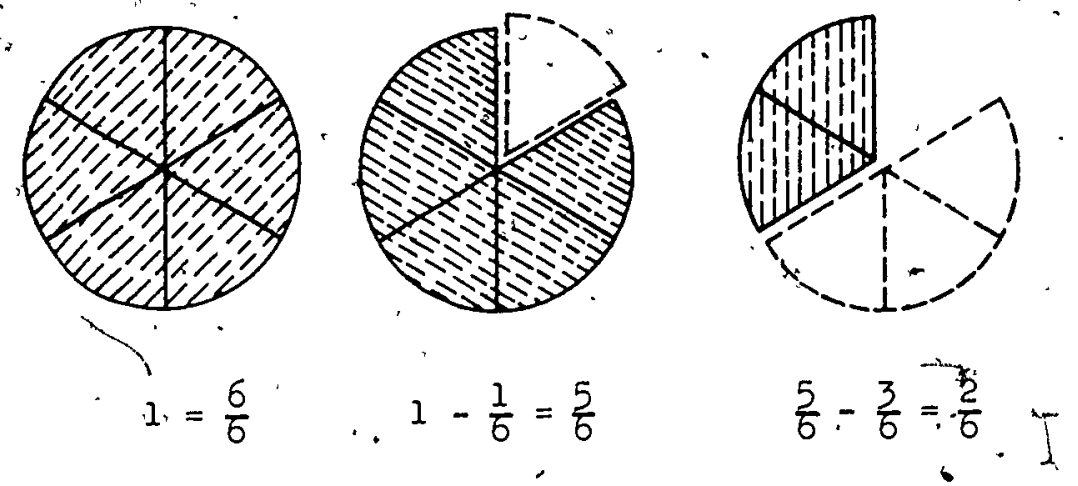

584

$13 j$ 


\section{Exerc1se Set 20}

For each problem, make a dlagram showing the number relationships. Then write a mathematical sentence, and solve 1t." Write your " answer to the problem in a sentence.

I. Susan bought $\frac{7}{8} 1 \mathrm{~b}$. of fudge and $\frac{2}{8} 1 \mathrm{~b}$. of chocolate drops. How much candy did she buy in all?

$\left(\frac{7}{8}+\frac{2}{8}=n ; n=\frac{9}{8}\right.$. Susan bought $\frac{9}{8}$ lb in all)

2. Tom and Jerry went to a Little League game. It took Tom $\frac{1}{4}$ hour to get to the game, aind 1 t took Jerry $\frac{3}{4}$ hour. How much longer did 1 t take Jerry to get to the game than 'Tom? $\left(\frac{3}{4}-\frac{1}{4}=n ; n=\frac{2}{4}\right.$. lt toote Gerry $\frac{2}{4}$ houra

3. David caught a fish welghing $\frac{15}{16} 1 \mathrm{~b}$. John's fish welghed $\frac{7}{16} 1 \mathrm{~b}$. How much more did David's fish welgh?

, $\frac{15}{16}-\frac{7}{16}=n ; n=\frac{8}{16}$. Diasid's firh ivighed $\frac{8}{16}$ lb.)

4. Mrs. Ray had one whole coffee cake. She served $\frac{3}{8}$ of $1 t$ to her neighbor. How much coffee cake did Mrs. Ray have (1) $-\frac{3}{8}=n ; n=\frac{5}{8}$. She had $\frac{5}{8}$ cakei heft $)$

5. Ann was mixing some punch for her friends. She mixed $\frac{2}{3}$ cup orange juice and $\frac{2}{3}$ cup gingerale. How much punch did she have?
$\left(\frac{2}{3}+\frac{2}{3}=n ; n=\frac{4}{3}\right.$.

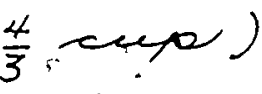


6. Mis. King mixed some liquid plant food for her house plants.

$\because$ The directions said to use $\frac{3}{4}$ tablespoon for each gallon of water. She used 2 gallons of water. How much liquid plant food did wrs. King use? $\left(\frac{3}{4}+\frac{3}{4}=n ; n=\frac{6}{4}\right.$. She used $\frac{6}{4}$ tablispoone plant food)

7. Jack spent $\frac{3}{4}$ hour on Tuesday mowing the lawn:' on Wednesday he sperit $\frac{1}{2}$ hour pulling weeds. How much time did Jack spend doing his work? $\left(\frac{3}{4}+\frac{1}{2}=n ; n=\frac{5}{4}\right.$.

\section{Ke epeent $\frac{5}{4}$. houres)}

8. Larry's mother gave him $\frac{1}{3}$ apple pie for his lungh? She gave his brother, Jim, $\frac{1}{6}$ of the pame pie. How - much of the pie did the two boys. eat? $\left(\frac{1}{3}+\frac{1}{6}=n ; n=\frac{1}{2}\right.$. They ate $\frac{1}{2}$ pie.)

9. Janet bought $\frac{3}{4}$ yd. of material. She used $\frac{2}{3}$ yd. for place mats. How much material was left? $\left(\frac{3}{4}-\frac{2}{3}=\pi ; n=\frac{1}{12}\right.$. There wias $\frac{1}{12}$ yard material lift!)

10. Mrs. Simith used $\frac{7}{8}$ cup brown sugar and $\frac{1}{2}$ cup white sugar in a candy recipe. How much sugar did she use?

1. $\left(\frac{7}{8}+\frac{1}{2}=n ; i=\frac{11}{8}\right.$. Lhe ceed $\frac{11}{8}$ cuipi eugar.)

11. Alice stopped, at the store on the way from her home to the park. It was ' $\frac{3}{5}$ mile to the store. The park was $\frac{9}{10}$ mile from Alice's home. How much farther did she walk to get to the park? ( $\frac{9}{10}-\frac{3}{5}=w ; n=\frac{3}{10}$ :

She wialed $\frac{3}{10}$ mile farther).

12. Jane spent $\frac{2}{3}$ hour doing her homework. Betty spent $\frac{1}{2}$ hour on homework. How much longer did it take Jane to finish?. $\left(\frac{2}{3}-\frac{1}{2}=n ; n=\frac{1}{6}\right.$. It toote her $\frac{1}{6}$-her. Longer.) 


\section{PROPERTIES OF ADDITION OF RATTIONAL NUMBERS}

Objective: To inquire whether the familiar properties of addition of whole numbers are true for addition of rational numbers.

Vocabulary: Assoclative Property for Addition, Commutative Property for Addition, Addition Property of Zero

Suggested Teaching Procedure

"To verify that the sum of two rational numbers. is always a rational number, you. may wish to proceed as folliows:

- We w11l try to find out if properties for the set of whole numbers under addition also hold for the set of rational numbers under addition.

Is the sum of two whole numbers always a whole number? (Yes) Give us some examples. $(7+8=15,11+14=25$, $127+382=509$ )

Is the sum of two rational numbers always a rational number? (If the answer is "yes", ask for some examples.)

$$
\text { Use a number of examples such as } \frac{1}{8}+\frac{7}{8} \text {, }
$$
$\frac{5}{12}+\frac{2}{12},-\frac{3}{4}+\frac{1}{3}, \frac{11}{2}+\frac{8}{3}, \cdot \frac{5}{2}+\frac{2}{4}$. Lead pup1ls to see that in adding two. rational numbers, such as $\frac{3}{4}$ and $\frac{1}{6}$, a common denominator for the fraction names can be found. Then the addition is reduced to one of adding whole numbers. The result of adding will still be a rational number.

Th1s' discussion is concepned with what is technically called the closure property .- We can say that the set of rational numbers is closed under the operation of addition. This language w1ll probably not be lised with ch1ldren. They should understand it in the terms that the sum of any two rational numbers is a rational number.

Is 1t true that the sum of any two rational numbers is a' ways one rational number? (Yes) 
Does this property hold for s'ubtraction? Let's look at some examples and see if we can answer that question.

Are the results of these subtractios rational numbers?.

$$
\frac{7}{4}-\frac{2}{4}, \frac{11}{16}-\frac{5}{16},-\frac{2}{3}-\frac{11}{2}, \frac{2}{2}=\frac{3}{2}, \frac{1}{4}-\frac{3}{4}
$$

(The results of the first three are rational numbers. We do not yet have numbers for results of the last two. We know no rational number which added to $\frac{3}{2}$ has a result of $\frac{2}{2} \cdot 1$ ).

Then w11i you agree that you cannot always subtract two rational. numbers? (Yes)

To verify that the Adaition Property of
Zero is true for rational numbers, you may
wish to proceed in this way:

- Think of two addends that are whole numbers. The sum of these two addends is one of the addends. What are some examples? $(6+0=6$, or $0+15=15$.) Is 1t true that if 0 is, added to any whole number, the result is that whole number? (Yes). Does this property hold for rational numbers? (Y's) Give me some examples.

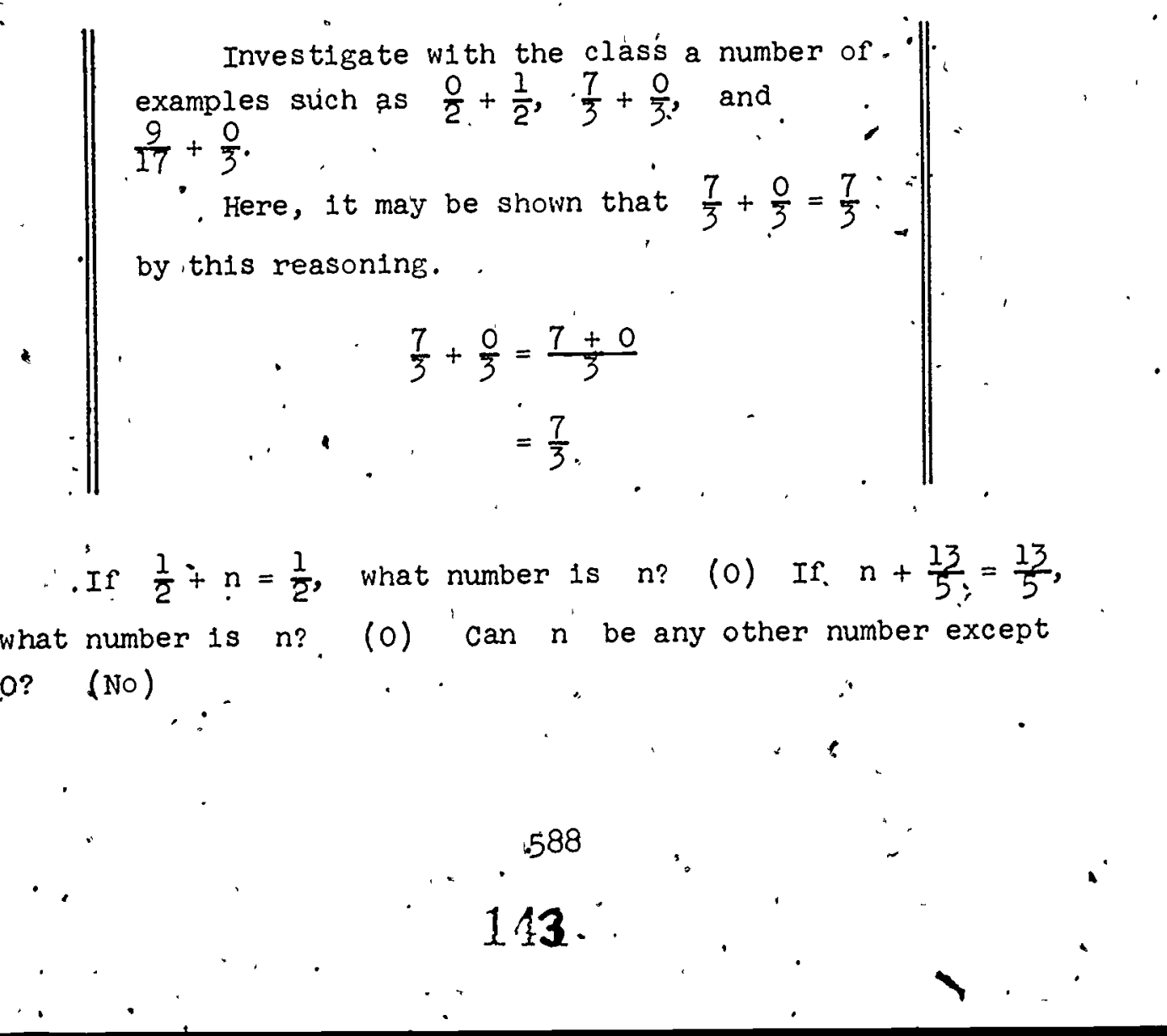


The Commutative Property for Addition may be discussed as follows:

Is 1t trure that $7+\dot{9}=9+7$ ? Is 1t true that $26+41=41+26$ ? (Yes). Try to state the mule for us. (Changing the order of àdding two whole numbers does not change the sum.)

This commutative property for addition of whole numbers is developed in grade 4 . The word "commutative" is used with pupils to name this property.

This is called the commutative property for addition of whole numbers. Whidh of these mathemat1cal sentences are "true because of the commutative property" for addition?

(a) $128+0=0+128$

(b) $256+891=891+256$

(c) $n+621=621+n$ (1f $n$ is any whole number)

(d) $a+b=b+a$ (1f $\underline{a}$ ane $\underline{b}$ are any whole numbersi)

(All are true by the commutative property for addition.).

Does this property hold for the addition of two rational numbers? (Yes). Are you sure?

Have pup1ls show that sums $\frac{5}{8}+\frac{2}{8}$ and
$\frac{2}{8}+\frac{5}{8}$ are the same. Continue with more
examples unt1l pup1ls understand, general1ze,
and freely.use the commutative property:

Does the commutative property hold for the subtraction of two rational numbers? If you don't think so, how many examples do we need to rind? "(One) Give us an example. ( $\frac{3}{2}-\frac{1}{2}$ is not equal to $\frac{1}{2}-\frac{3}{2}$ ) show this on the number line. 


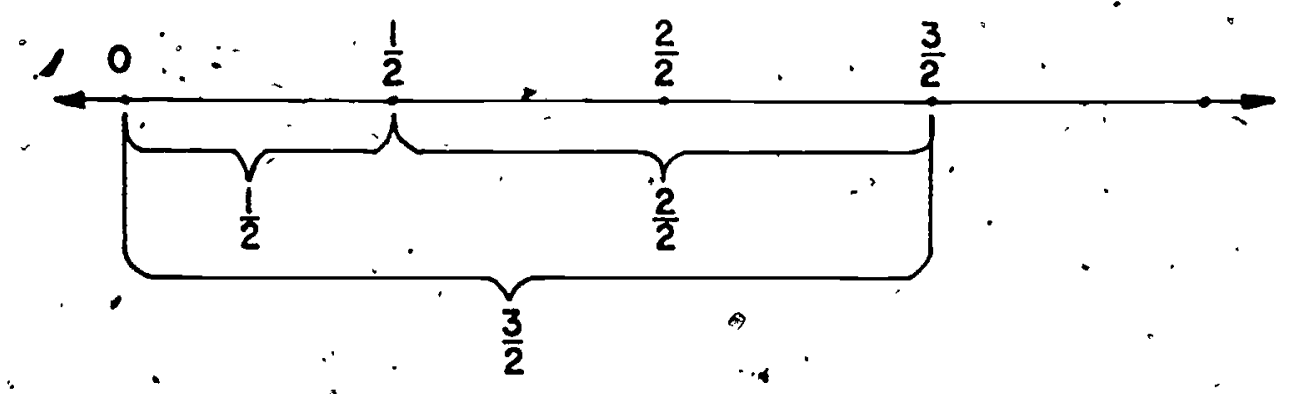

This shows $\frac{1}{2}+\frac{2}{2}=-\frac{3}{2} ;$ therefore, $\frac{3}{2}-\frac{1}{2}=\frac{2}{2}$.

(We cannot show $\frac{1}{2}-\frac{3}{2}$ on the number line. To find $\frac{1}{2}-\frac{3}{2}$, think $\frac{1}{2}-\frac{3}{2}=n .^{2} \frac{1}{2}$ is the sum and $\frac{3}{2}$ one addend. The addend is greater than the sum. We cannot name rational number $n$ such that $n+\frac{3}{2}=\frac{1}{2}$.)

$\|$ To verify the Associative Property:

Addition is an operation on two numbers to obtain a. result

- of one and only one number. How do we add three rational numbers? To answer this question, we might think of how you added three whole numbers. How could you add 6,7 , and 8 , in that order?. (We could think $6+7=13 ; .13+8=21 \%$ ). Tell us another way. (To 6 , add the sum of 7 and 8 . $\left.6+15=2 \frac{1}{N_{2}}\right)^{\circ}$ Is the sum the same in both cases? (Yes). What does $(6+7)+8$ mean? (Add $6+7$ first. Add 8 to that sum.) What does $6+(7+8)$ mean? (Add $7+8$ first. Add that sum to 6.)

Write a statement of this associative property for addition on the board: If three whole numbers are to be added, the third. added to the sium of the first two is equal to the sum of the last two added to the first.

This is a long statement. - Usë a number of illustrations. Arrange your work on the. . board like .this.

$$
\begin{aligned}
20+11 & =8+23 \\
31 & =31
\end{aligned}
$$

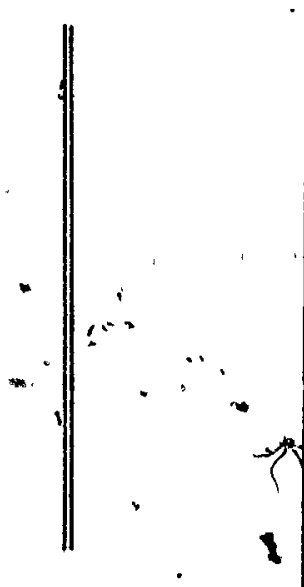


Do you think, the associative property of addition holds for rational numbers? (Yes) Are you sure? Let us try some examples. Show us $\frac{3}{2}+\frac{4}{2}+\frac{7}{2}$ on the number line.
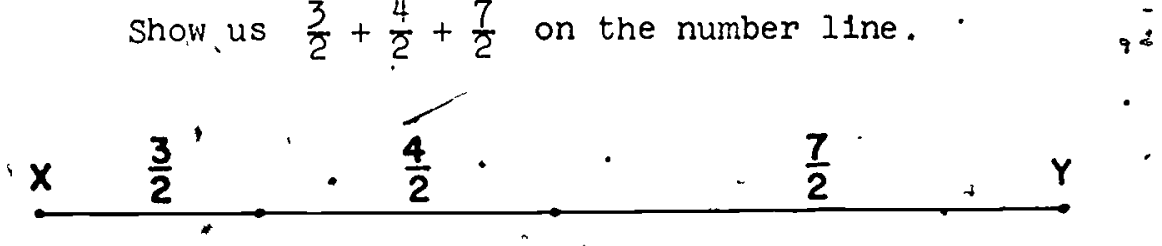

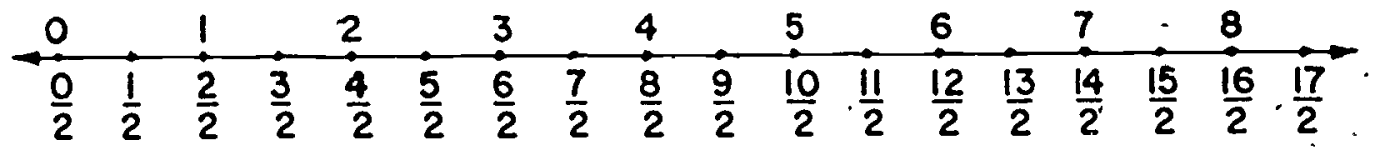

\section{$\gamma$}

Will the measure of $\overline{X Y}$ be $\frac{14}{2}$ if we think $\left(\frac{3}{2}+\frac{4}{2}\right)+\frac{7}{2} ?$ (Yes) W11l, the measure of $\overline{X Y}$ be $\frac{14}{2}$ if we think $\frac{3}{2}+\left(\frac{4}{2}+\frac{7}{2}\right) ?$; (Yes)

Use a number of examples of the assoclat1ve property for addition with rat1onal numbers as addends. This form 1 S suitable for computation.

Is. $\left(\frac{5}{2}+\frac{6}{2}\right)^{\circ}+\frac{3}{2}=\frac{5}{2}+\left(\frac{6}{2}+\frac{3}{2}\right)^{\prime}$ ?

Doles $\frac{7}{2}+\frac{3}{2}=\frac{5}{2}+\frac{6+3}{2}$ ?

Notice Erot

$$
\begin{aligned}
(5+6)+3) & =(5+(6+3) \\
\frac{(5+6)+3}{2} & =\frac{5+(6+3)}{2} \\
\frac{11+3}{2} & =\frac{5+9}{2} \\
\frac{14}{2} & =\frac{14}{2}
\end{aligned}
$$

The addition of rational numbers hastine Associative Property. We see that thisy 1 s

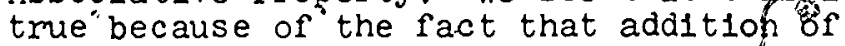
whole numbers has this property.

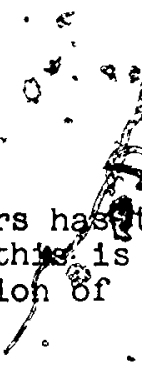




\section{PROPERTIES-OF ADDITION OF RATIONAL NUMBERS}

\section{Exploration}

You know that (1) any fraction, such as $\frac{2}{3}, \frac{8}{4}, \frac{50}{78}, \frac{5}{2}$, ' $\frac{0}{16}$, names a rational number. (2) You can find the polint on a" number line matching a rational number by (a) separating the. unit segment into the number of congruent segments named by the denominator, (b) counting of from 0 the number of segments named by the numerator. (3) Some of these rational numbers, such as $\frac{6}{2}$, are also whole numbers.

1. Which numbers named above are al so whole numbers? $\left(\frac{8}{4}, \frac{\dot{6}}{2}, 0\right)$

2. What whole number can be a numerator of a fraction, but

$\because$ not a denominator? (0)

3. Think of two whole numbers. Find their sum. What kind of $\because$ number is the sum? (whiale number)

4. Think of two rational numbers. Find their sum. What kind of number is the sum?

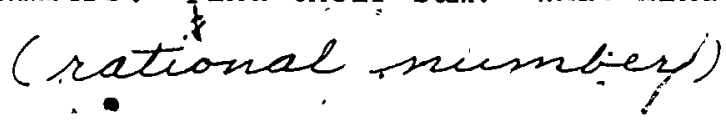

5. Think of two rational numbers. Subtract the smaller from the greater.' What kind of number' is your answer? (National),

6. Try to subtract the "gpeater number in exercise 5 from the - smaller. ' Can you do 1 t? $(\boldsymbol{N o})$ Can you al ways subtract one rational number from another? (no.)

7. Think of a rational number, $n$ named by a fraction with denominator 6 . Add 1 to $\frac{0}{6}$. What do you notice about. the sum?. (At is.the siameras thi ficst 
8. Find thęse sums.
an $\frac{2}{3}+\frac{3}{4} \cdot\left(\frac{17}{12}\right)$
b. $\frac{3}{4}+\frac{2}{3}\left(\frac{17}{12}\right)$

9. Illustrate each part of exercise 8 on the number line.

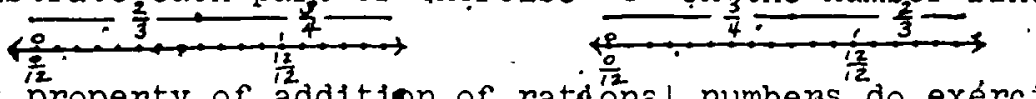

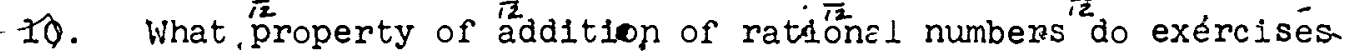

8 and 9 suggest? (Commutative)

11. Find fraction names for the numbers $\mathrm{n}$ and $t$.
a. $\left(\frac{\dot{1}}{2}+\frac{1}{3}\right)+\frac{3}{4}=n\left(n=\frac{19}{12}\right)$
b. $\quad \begin{aligned} & \frac{1}{2}+\left(\frac{1}{3}+\frac{3}{4}\right)=t \\ &\left(t=\frac{19}{12}\right)\end{aligned}$

12. What do you notice about $n$ and $t$ in exercise II? (They ace the same)

13. What property of addition of rational numbers does exercise 11 suggest?

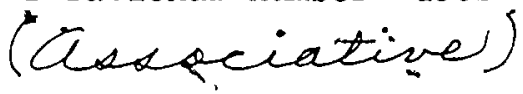

Summary of Properties for Addition of Rational Numbers

1. If two, rational numbers are added, the sum is.a rational number.

2. If, 0 . 1 added to any rational number ' $n$, the sum: is the same rational number, $n$ (Addition Property of Zero).

3. The order of adding two rational numbers may be chainged without changing their sum: (Commutative Property).

4. To find the sum of three rational numbers, you may. .

(1) add the first two and add the third to their sum; or

(2) add the second and third, and add their. sum to, the first (Associative Property). 


\section{Exerc1se Set 21}

Write a mathematical sentence for each problem. Solve it and answer the question in a sentence.

1. One measuring cup contains $\frac{1}{8}$ cup of liquid. A second measuring cup contains $\frac{3}{4}$ cup of liquid. If the liquid in the first cup is poured into the second cup, what amount of liquid will be in the second cup? $\left(\frac{1}{8}+\frac{3}{4}=n ; n=\frac{7}{8}\right.$. There nuel be $\frac{7}{8}$ cup liguid un econd cupa) .

2. Dxrections on a can of concentrated prange juice call for mixing the juice with water. one-half quart water is to be mixed with $\frac{3}{16}$ quart of concentrated juice. What. amount of liquid will result? $\left(\frac{1}{2}+\frac{3}{16}=n ; n=\frac{11}{16}\right.$. There uill be $\frac{11}{16}$ gt. liguid)

3. I have $\frac{2}{3}$ don cookies in one box and $\frac{1}{4}$ dozen in another. You have 1 dozen cookies. Who has more? (yow) many cookies do I, have? $\left(\frac{2}{3}+\frac{1}{4}=m_{1} ; r_{2}=\frac{11}{12}\right.$.

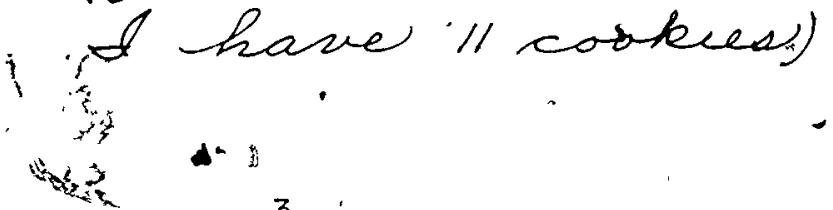

4. A measuring cup is filled to the 3 mark with milk. Enough water is added to bring the level of the mixture to the $\frac{3}{4}$ mark. How much water was added? $\left(\frac{3}{8}+n=\frac{3}{4} ; n=\frac{3}{8}\right.$.

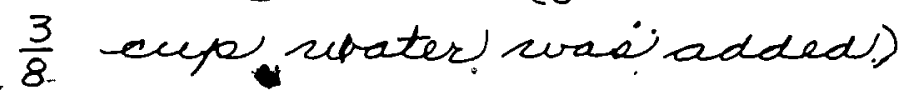


5. Cars have dials which show the quant1ty of gasoline in the tank. The dial might look like this:

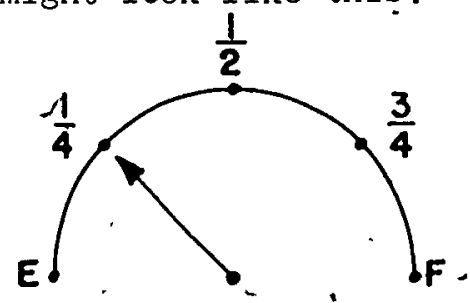

How do the markings on the dial differ from those on our number lines? (They ace not haryontal) What unit of measure is represented on the dial? (Ome tant) Suppose enough gasoline were added to the tank to move the pointer to a position halfway between the $\frac{1}{2}$ mark and the. 3. mark.

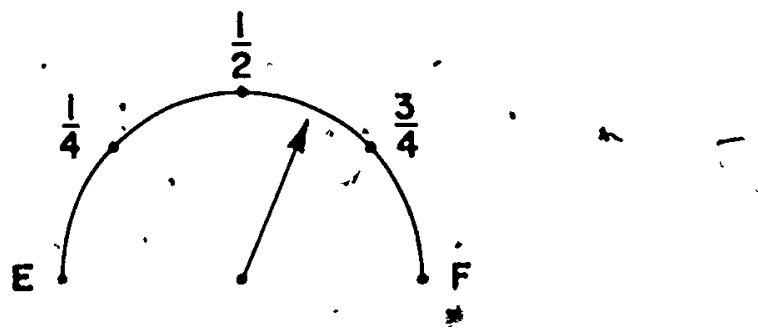

How much gasbline was added?. ( $\frac{3}{8}$ of a tank $)$

6. On the number line below the unit represented fis the inch. What is the measure in inches of each of the six line segments pictured?
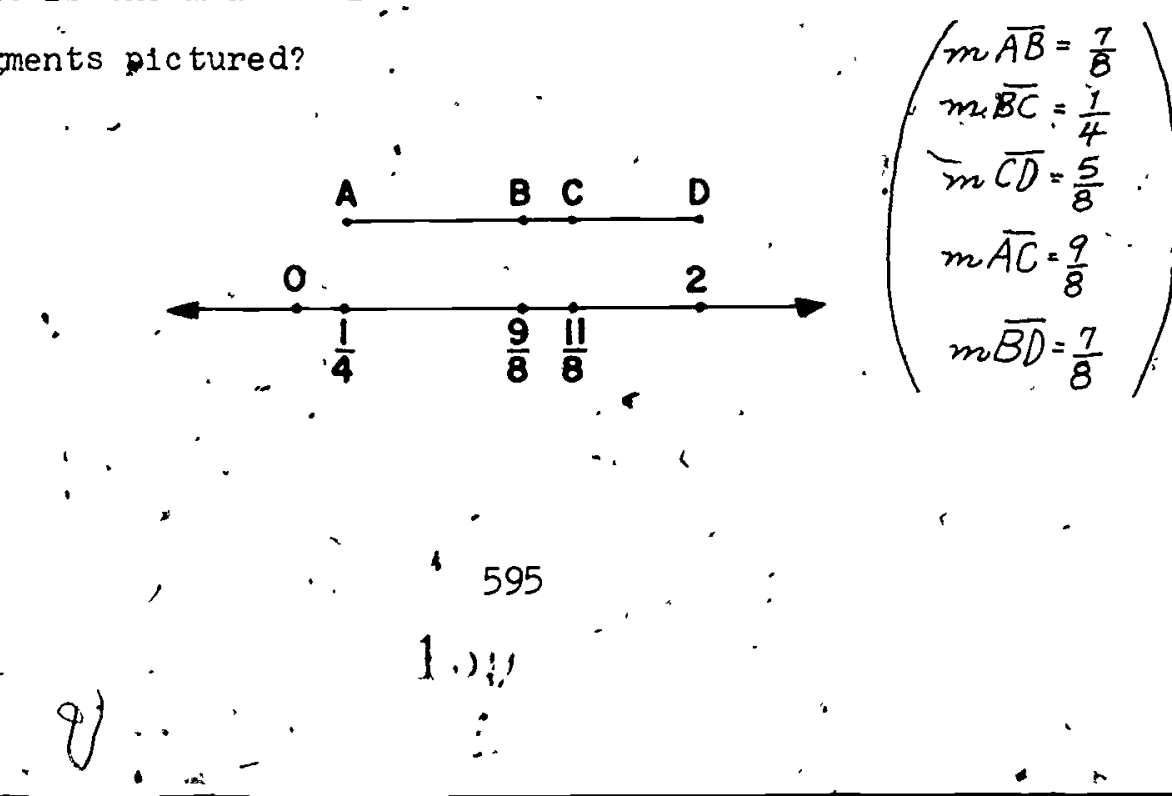

P335

WHOLE NUMBERS AND RATIONAL NUMBERS

Exploration

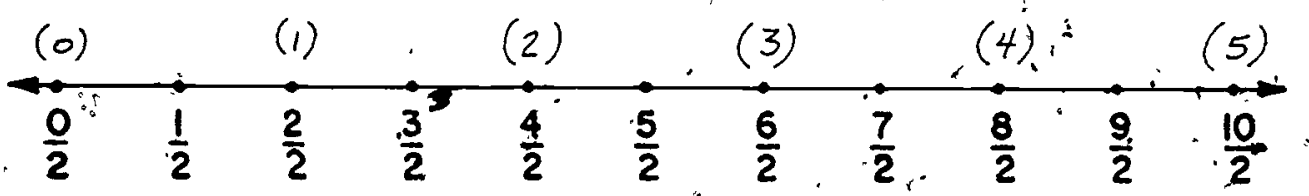

1. Trace the number line and copy the scale of halves.

2. Write a who number scale above the line. Be sure to keep the same unit segment. What number is at $\frac{0}{2}$ ?

(o).

3. The whole number 1 should be written the point labeled *. $:\left(\frac{2}{2}\right)$.

. 4.. The whole number 2 should be written at the point labeled $\frac{\left(\frac{4}{2}\right)^{1}}{r}$

5. List the numerators of the fractions which name counting. numbers. $(2,4,6,8 ; 10)$

6. List the first five multiples of the denominator, $e$. $(2,4,6,8,10)$ 
$8336^{\circ}$

7. Are your answers for exercises 5 and 6 the same? (jues) Q. Trace the number line and copy the scale of fifths.
(0)
(11)
(2)
(3)
$(4)=$
$\frac{0}{5} \quad \frac{1}{5} \quad \frac{2}{5} \cdot \frac{3}{5} \cdot \frac{4}{5} \frac{5}{5} \quad \frac{6}{5} \quad \frac{7}{5} \frac{8}{5} \frac{9}{5} \frac{10}{5} \frac{11}{5} \cdot \frac{12}{5} \cdot \frac{13}{5} \frac{14}{5} \frac{15}{5} \frac{16}{5} \frac{17}{5} \frac{18}{5} \cdot \frac{19}{5} \frac{20}{5} \frac{21}{5}$

2. Write a whole number scale above the line, Keen "the same unit segment.'

"10." I1st. the numerators of fractions which name counting numbers on the whole number scale: $(.5,10,15,20)$

i1. List the first four multiples of the denominator, 5 .

$$
\text { * }(5,10,15,20)
$$

12. Are your answers for exexcises. 20 and 11 the same?(zyes)

I3. Is this a true statement?

If the numerator of a fraction 1s a multiple of the denominator, then the rraction names a whole number. (Gea)

14. Which of these fractions are names for whole numbers? $(b, a, f)$
a. $\frac{18}{7}$
c. $\frac{60}{16}$
b. $\frac{27}{9}$
d. $\frac{90}{10}$

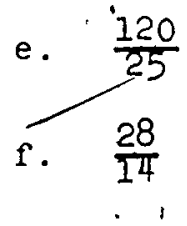


FRACTIONS AND MIXED FORMS

\section{Exploration}

Let ys think of two rational numbers. Their sum is $\frac{9}{2}$. Here is a picture of $\overline{X Y}$ measuring $\frac{9}{2} . d$. What could the addends '. be so their sum is $\frac{9}{2}$ ? (Ancwiere siree vacy).
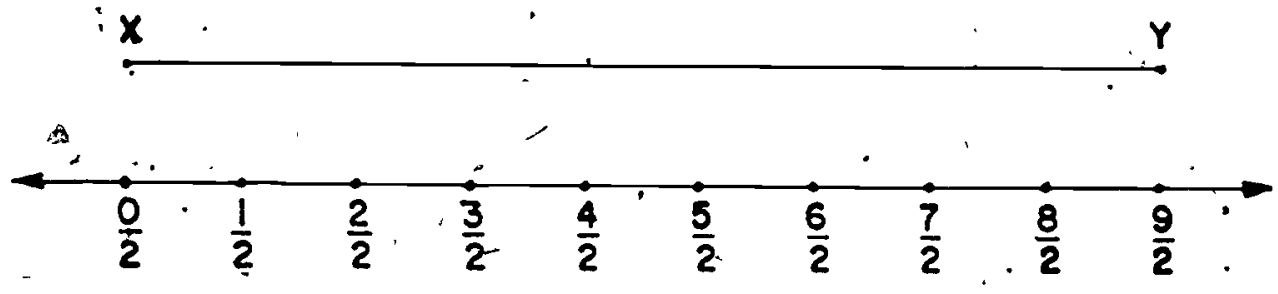

1. 'Iook at these patrs of addends. Is the sum of each pair $\frac{9}{2} ?$ ( $\ddot{\text { quis) }}$

$\therefore \cdot \frac{8}{2}+\frac{1}{2}$.

c. $\frac{6}{2}+\frac{3}{2}$

b. $\frac{2}{2}+\frac{7}{2}$

d: $\quad \frac{4}{2}+\frac{5}{2}$

2. Write each of the sums in exercise. I as the sum of a

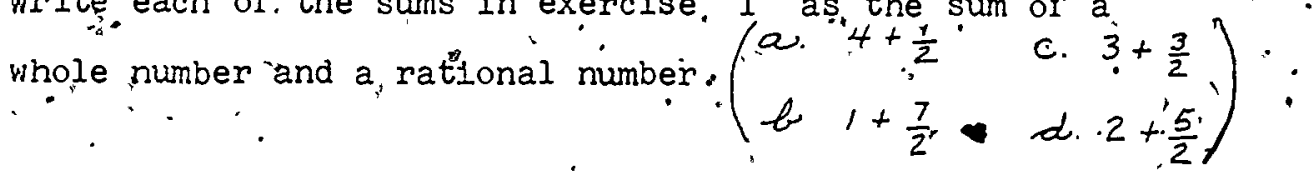

3. Sums like jour answers for exercise 2 are often written without the + sign, in this way: :
a. $-4 \frac{1}{2}$
b. $\quad 1 \frac{7}{2}$
c. $3 \frac{3}{2}$.
d. $2 \frac{5}{2}$

Write these numbers as sums using the + sign.
e. $\cdot \dot{2} \frac{1}{2}$
$\left(2+\frac{1}{2}\right)$
f. $3 \frac{1}{2}$
$\left(3+\frac{1}{2}\right)$
8. $\frac{1}{2} \cdot j: \frac{\dot{h}}{\theta} \cdot 1 \frac{1}{2} \cdot$
$\left(0+\frac{1}{2}\right): \cdot:\left(1+\frac{1}{2}\right)$ 
4. Think of the way you picture $2+\frac{1}{2}$ with segments on the nümber line.

a. What fractions of the halves scale name the endpoints of the segment w1tin measure ? ? $\left(\frac{0}{2} \cdot a d \frac{4}{2}\right)$

b. What fractions name the endpoints of the segment with measure $2 \frac{1}{2}$ ? $\left(\frac{0}{2}\right.$ ind $\left.\frac{5}{2}\right)$

c. What fraction names the same number as $2 \frac{1}{2}$ ? $\left(\frac{5}{2}\right)$

5. Use a number line. Write a whole number scale above 1t - and a scale of thirds below 1t'. Are these sentences true?(qea)

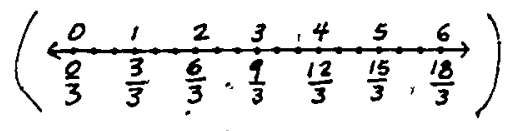

$$
\begin{aligned}
5 \frac{2}{3} & =5+\frac{2}{3} \\
& =\frac{5}{1}+\frac{2}{3} \\
& =\frac{5 \times 3}{1 \times 3}+\frac{2}{3} \\
& =\frac{15}{3}+\frac{2}{3} \\
& =\frac{17}{3}
\end{aligned}
$$

6. Find eraction names for these numbers.

$$
\therefore \text {. a. } 6 \frac{3}{4}\left(\frac{27}{4}\right) \text { b. } 4 \frac{5}{7}\left(\frac{33}{7}\right) \text { c: } 12 \frac{4}{3}\left(\frac{40}{3}\right) \text { d. } 12 \frac{2}{6}\left(\frac{74}{6}\right)
$$

Numerals $x \hat{\mathrm{RSe}} 6 \frac{3}{4}, 4 \frac{5}{7}$, and $12 \frac{4}{3}$. name rational numbers. These numerals, are called mixed forms. In $6 \frac{3}{4}$ and ' $4 \frac{5}{7}$, the fractions $\frac{3}{4}$ and $\frac{5}{7}$ are in simplest form and name numbers less thain 1 . We say $6 \frac{3}{4}$ and $4 \frac{5}{7}$ are "simple'st mixed forms for rational numbers. i2 $\frac{4}{3}$ is not in simplest mixed form, because $\frac{4}{3}>1.12 \frac{2}{6} 1 \mathrm{~s}$ not in simplest mixed form, because $\frac{2}{6}$ is not in simplest form. 
P339

Exercise Set 22

1. Find fraction names for these numbers.

a. $8 \frac{2}{9}\left(\frac{74}{9}\right)$

c. $16 \frac{1}{2} \cdot\left(\frac{3.3}{2}\right)$

e. $1 \frac{7}{9}\left(\frac{16}{9}\right)$

b. $11 \frac{3}{5}\left(\frac{58}{5}\right)$

d. $\because \frac{1}{7}\left(\frac{22}{7}\right)$

. f. $15\left(\frac{15}{1}\right)$

2. Separate these fractions into 3 sets as follows:

Set $M$ is the set of fractions which name whole numbers.

set is the set of reactions which can be expressed as mixed forms.

Set $R$ is the set of fractions which name numbers less than 1 .

a. $\frac{30}{5} \cdot(M)$

d. $\frac{75}{8}(P)$

8. $\frac{32}{5}$

(P)

: $\frac{56}{4},(M)$

e. $\frac{120}{10}(M)$

r. $\frac{4}{9}$

(R)

c. $\frac{72}{8}$

(M)

f. $\frac{127}{10} \cdot(P)$

1. $\frac{54}{9} \cdot(M)$

3. Suppose you have a whole number scale and a scale labeled in fractions on the same number line. Between what two whole 'number points will' a point lie which is labeled with these fractions?

a. $-\frac{7}{3}+(2,3)$

c. $\frac{12}{5}(2,3)$

e. $\frac{38}{7}(5,6)$.

b.: $\frac{9}{4}(2,3)$

d. $\frac{19}{8}(2,3)$.

f. $\frac{83}{4}(20,2 !)$.

601 
$\therefore \quad$ P340

4. Express these mixed forms:as fractions. Then find the Indicated sums and addends.

a.: $\quad \frac{3}{5}+4 \frac{1}{5}=n\left(\frac{24}{5}\right)$

c. $\quad 3 \frac{1}{7}=2 \frac{1}{2}+\mathrm{n} \cdot\left(\frac{9}{14}\right)$

b. $\quad 8 \frac{2}{3}-\frac{7}{4}=n \cdot\left(\frac{83}{12}\right)$

d. $6 \frac{1}{5}+1 \frac{3}{10}=n\left(\frac{15}{2}\right)$

5. Which humber of each pair is greater? Answer in a.. sentence, using $>$ or $<$.

a. $\frac{17}{8}, 2 \frac{3}{8}\left(\frac{17}{18}<.2 \frac{3}{8}\right)$

d. $\frac{7}{10}, \quad \frac{48}{5}\left(9 \frac{7}{10}>\frac{48}{5}\right)$

b. $7 \frac{1}{5}, \quad \frac{31}{4}\left(7 \frac{1}{5}<\frac{31}{4}\right)$

e. $\quad 14 \frac{1}{2}, \frac{42}{3}\left(14 \frac{1}{2}>\frac{42}{3}\right)$

. $1 . \quad 6 \frac{3}{7}, \quad \frac{45}{8} \cdot\left(6 \frac{3}{7}>\frac{45}{8}\right)$

. f. $\quad \frac{39}{13}, \quad 2 \frac{8}{13}\left(\frac{39}{13}>2 \frac{8}{13}\right)$

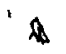

602

ERIC

$15 i$ 
P341

RENAMING -FRACTIONS IN MIXED FORM

Exploration

When a number is named by a mixed form, such as $2 \frac{3}{7}$, you know how to renkme 1 in fraction form.

$$
\begin{aligned}
2 \frac{3}{7} & =\frac{2}{1}+\frac{3}{7} \\
& =\frac{2 \times 7}{1 \times 7}+\frac{3}{7} \\
& =\frac{14}{7}+\frac{3}{7} \\
& =\frac{17}{7} \\
2 \frac{3}{7} & =\frac{17}{7}
\end{aligned}
$$

When a number is named by a fraction, you can easily rename it in mixed form when the numerator and denominator are small enough to use the number faets jou know and to think about the points on the number line.

$$
\begin{aligned}
\frac{15}{4} & =\frac{12+3}{4} \\
& =\frac{12}{4}+\frac{3}{4} \\
& =3+\frac{3}{4} \\
\therefore \quad & =3 \frac{3}{4} \\
\frac{15}{4} & =3 \frac{3}{4}
\end{aligned}
$$

Let us see how you can rename a number when the numerator and genominator are greater; for example, $\frac{437}{16}$.

Since a fraction names. a whole number when the numerator is a multiple of the denominator, think about the set of multiples of. 16 .

$$
\text { Multiples of } 16=(16,32,48,64 \ldots\}
$$


P342

You would have to find a good many multiples of 16 to find a multiple close to .437 . Sa try another way to find a multiple close, to 437 .

Suppose you write 437 in the form

$$
437=\left(16 k^{\prime} n\right)+r \text {, wherè } r<16 .
$$

Yold know you can find, $n$ and $r$ by using division. So 437 can be renamed as follows:

$437=(16 \times 27)^{\circ}+5^{\circ}$

Explain why these sentences

are true:
a) $\frac{437}{16}=\frac{(16 \times 27)+5}{16}$
b) . $=\frac{432+5}{16}$
c). $=\frac{432}{16}+\frac{5}{16}$
d) $=27+\frac{5}{16}$
e). $=27 \frac{5}{16}$
f) $\frac{437}{16}=27 \frac{5}{16}$.

In line b) 1s.1t necessary to write $(16 \times 27)$ as 432 ?

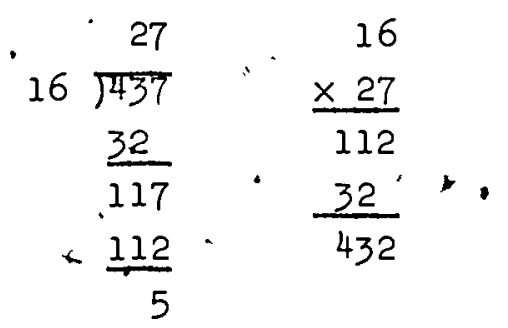

$$
\begin{aligned}
\frac{437}{16} & =\frac{16 \times 27}{16 \times 1}+\frac{5}{16} \\
& =\frac{27}{1}+\frac{5}{16} \quad \text { (Why?) } \\
& =27 \frac{5}{16}
\end{aligned}
$$

Fịnd mixed form names for these numbers. Write your work in the way shown above.

1. $\frac{97}{13}\left(7.7 \frac{6}{13}\right)$ 2. $\frac{147}{23}\left(6 \frac{9}{23}\right)$ 
PB $43^{\circ}$

Exercise set 23

Rename these numbers in simplest mixed form or as whole numbers. Show your work.

1.

$$
\begin{aligned}
& \frac{34}{5}=\frac{(5 \times 6)+4}{5} \\
& =\frac{5 \times 6}{5 \times 1}+\frac{4}{5} \\
& =6+\frac{4}{5} \text {. } \\
& \therefore \quad .=6 \frac{4}{5} \\
& \frac{34}{5}=-6 \frac{4}{5}
\end{aligned}
$$

2. $\frac{79}{8} \cdot\left(9 \frac{7}{8}\right)$

7.. $\frac{238}{10}\left(23 \frac{4}{5}\right)$

$2\} 0$

$=3 \because \frac{.96}{11} \quad\left(8 \frac{8}{11}\right) \quad$

8. $\frac{367}{12} \quad\left(30 \frac{i}{12}\right)$

4. $\quad \frac{157}{13} \quad\left(12 \frac{1}{13}\right)$

9. $\frac{367}{36} \cdot\left(10 \frac{7}{36}\right)$

5. $\frac{241}{15} \quad\left(16 \frac{1}{15}\right)$

$100 \div \frac{451}{100} \quad\left(4 \frac{51}{100}\right)$

6. $\frac{352}{7} \quad\left(50 \cdot \frac{2}{7}\right)$

$11 . \frac{5280}{3},(1760)^{\circ}$

ERIC

605 
P344

Find simplest mixed forms to make these sentences true."

12. 50 ounces $=\frac{50}{16}$ pounds $=\left(3 \frac{1}{8}\right)$ pounds.

13. $=100$ feèt $=\frac{100}{3}$ yards $=\left(33 \frac{1}{3}\right.$. yards.

14., 254 inches $=\frac{254}{12}$ feet $=\left(2 / \frac{1}{6}\right)$ feet.

15. $37 \cdot$ pints $=\frac{37}{8}$ gallons $=\left(4 \frac{5}{8}\right)$ gallons.

16. Fill in the blanks' in the table.

Fraction

a. $\left(\frac{62}{8}\right)$

b. $\quad \frac{49}{10}$

$\therefore \quad \frac{13}{11}$

d. $\frac{720}{25}$

e. $\quad\left(\frac{323}{21}\right)$

f. $\frac{\frac{227}{10}}{-}$

g. $\quad \frac{79}{9}$
Simplest Fraetion

Name

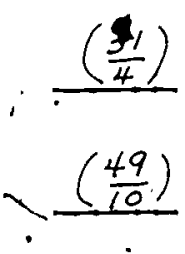

$\frac{\left(\frac{13}{11}\right)}{.}$

$\left(\frac{144}{5}\right) \cdot=$

5

$\left(\frac{323}{2 J}\right)$

$\left(\frac{227}{10}\right)$

$\left(4 \frac{9}{10}\right)$

$\left(1 \frac{2}{11}\right)$

$\left(28 \frac{4}{5}\right)$

$15 \frac{8}{21}$

$22 \frac{7}{10}$

$\left(\frac{79}{9}\right)$

$\left(8 \frac{7}{9}\right)$

606 
COMPUTING WITH MIXED FORMS

objecflive: To use the commutative and Associative Pxóperties to develop computational procedures for adding and subtracting rational numbers named by mixed forms .

$\because:$

- Sugges ted Teaching Procedure

The development in the textbook provides an opportunity to emphasize the way in which the basic properties of rational numbers (Associative and Commutative) are applied in finding simple ways to compute sums.

You may wish'also to have the pupils compute the sum of $4 \frac{1}{2}$ and $7 \frac{3}{4}$ by using the fraction names, $\frac{9}{2}$ and $\frac{31}{4}$, in order to verify that the result is the same as that shown.

Several forms which pupils" may use for recording the steps in adding and subtracting rational numbers named by mixed forms are shown in the text. It is advisable for pupils to use the longer forms at first; and adopt. shorter forms when the process is familiar and they can keep the steps in mind without recording all of them.

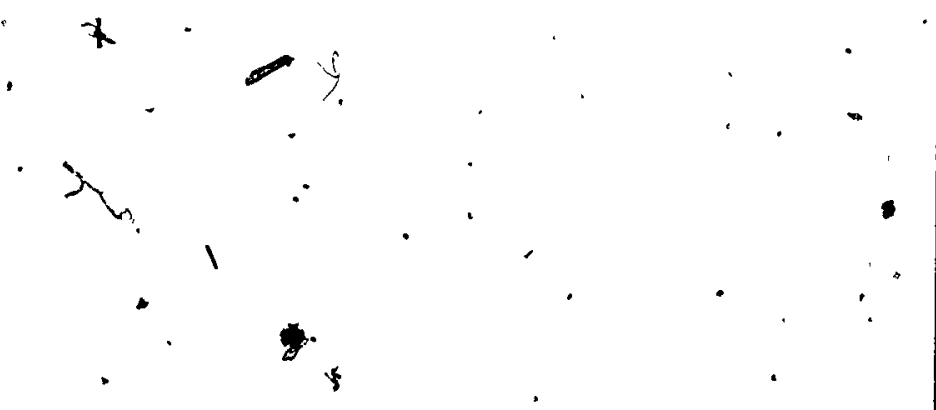


P345

COMPUTING WITH MIXED FORMS

When numbers are expressed in mixed forms, you can add and

for tract them without finding fraction names for them.

j.

- $4 \frac{1}{2}+7 \frac{3}{a}=n$

a) $\quad 4 \frac{1}{2}+7 \frac{3}{4}=\left(4+\frac{1}{2}\right)+\left(7+\frac{3}{4}\right)$

b) $.1=4+\left(\frac{1}{2}+7\right)+\frac{3}{4}($ acosectine $)$.

$=\left(4 .+\left(7 \div \frac{1}{2}\right)+\frac{3}{4}\right.$ (commutating)

$=(4+7)+\left(\frac{1}{2}+\frac{3}{4}\right)$ (associative)

e) .

$=11+\left(\frac{2}{4}+\frac{3}{4}\right)$ Penance $\frac{1}{2}$;

f)

renarne $(4+7)$

$=-11+\frac{5}{4}$

- (addition),

$=11+\left(\frac{4}{4}+\frac{1}{4}\right)$. (restansing $\frac{5}{4}$ )

h) $\quad=11+\left(1+\frac{1}{4}\right) \quad$ (renaming $\frac{4}{4}$ )

1) .

$=(11+1)+\frac{1}{4}$ (associative).

j) -

$=12+\frac{1}{4}$

(renaming "It!)

k)

$=12 \frac{1}{4}$

(renaming $\left(2+\frac{1}{4}\right.$ )

Explain each line.

You do not need to write all this to show your work.

For example, write:

$$
\begin{aligned}
4 \frac{1}{2}+7 \frac{3}{4} & =(4+7)+\left(\frac{1}{2}+\frac{3}{4}\right) \\
& =11+\left(\frac{2}{4}+\frac{3}{4}\right) \\
& =11+\frac{5}{4} \ldots \\
& =11+1+\frac{1}{4} \\
& =12 \frac{1}{4}
\end{aligned}
$$

608

163 
8346

You may prefer this form:

$$
\begin{aligned}
& 4 \frac{1}{2} \doteq 4+\frac{1}{2}=4+\frac{2}{4} . \\
& \frac{7 \frac{3}{4}}{-\cdots} \quad \frac{7+\frac{3}{4}}{11^{\circ}+\frac{5}{4}}=11+1+\frac{1}{4}=12 \frac{1}{4} \\
& \text { - } 4 \frac{1}{2}=4 \frac{2}{4} \\
& +7 \frac{3}{4}=\frac{7 \frac{3}{4}}{11 \frac{5}{4}}=12 \frac{3}{4}
\end{aligned}
$$

$\therefore . . \quad \therefore$

$\theta$.

The vertical form is best for subtraction. Explain these examples.

$-1$

$8 \frac{5}{6}=8 \frac{10}{12}$. $)$ (common denominator)

$-\frac{3 \frac{1}{4}}{\overline{2}} \cdot \frac{3 \frac{3}{12}}{5 \frac{7}{12}}$

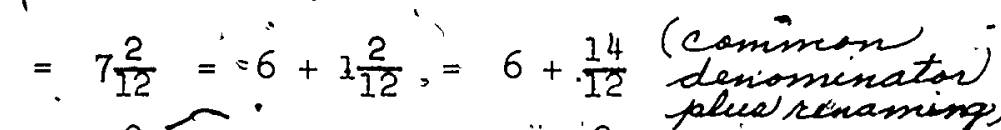

$$
\begin{aligned}
& -2 \underline{\frac{3}{4}}=2 \underline{\frac{9}{12}} \\
& 2+\frac{9}{12} \\
& 15 \div 14+1=14+\frac{8}{8} \\
& -2 \frac{5}{8} \\
& =\frac{2+\frac{5}{8}}{12+\frac{3}{8}}=12 \frac{3}{8}
\end{aligned}
$$

609 , 


\section{Exercise Set24}

Find a fraction name or mixed form for $n$ so each mathematical sentence is true. Show your work in the form your teacher. suggests.

1. a. $4 \frac{2}{3}+2 \frac{2}{3}=n\left(7 \frac{1}{3}\right)$ b. $3 \frac{2}{5}-2 \frac{1}{5}=n\left(1 \frac{1}{5}\right) \therefore=\left(\frac{3}{8}+2 \frac{3}{8}=n\right.$

2. a. $2 \frac{1}{3}+3 \frac{1}{3}=n\left(5 \frac{2}{3}\right)$ b. $43 \frac{5}{6}-1 \frac{5}{6}=n(2) c \cdot 3 \frac{1}{5}+2 \frac{2}{5}=n \frac{n}{\left(5 \frac{3}{5}\right)}$ 3. a: $2 \frac{1}{2} \div 3 \frac{3}{8}=n\left(5 \frac{7}{8}\right) \frac{b}{b} \cdot 3 \frac{1}{2}+2 \frac{2}{5}=n\left(5 \frac{9}{10}\right) c \cdot 1 \frac{3}{4}-1 \frac{1}{6}=n$ 4.. a. $\quad 2 \overline{\frac{2}{5}}-1 \frac{1}{3}=n\left(1 \frac{4}{15}\right)$ b. $\frac{11}{2}-2 \frac{2}{3}=n\left(1 \frac{5}{6}\right)$ c. $1 \frac{7}{8}-1 \frac{1}{5}=n-$

5. a. $\quad 3 \frac{1}{2}+4 \frac{1}{2}=n(8)$ b. $5 \frac{2}{3}-4 \frac{1}{3}=n\left(1 \frac{1}{3}\right)$ c. $\quad 1 \frac{7}{12}-1 \frac{2}{12}=n$ $\left(\frac{5}{12}\right)$

6. a: $\quad 4 \frac{4}{5}-3 \frac{1}{2}=\mathrm{n}\left(1 \frac{3}{10}\right)$ b. $2 \frac{1}{2}+i \frac{7}{16}=\mathrm{n}\left(3 \frac{15}{16}\right)$ c. $\quad 3 \frac{3}{4}+2 \frac{5}{8}\left(\frac{1}{8}\right)^{-}$

7. a. $13-7 \frac{5}{8}=n\left(5 \frac{3}{8}\right)$ b. $8-2 \frac{5}{16}=n\left(5 \frac{11}{16}\right)$ c. $25-\frac{4}{9}=n$

8. a. $12-7 \frac{5}{8}=n\left(4 \frac{3}{8}\right)$ b. $.9-2 \frac{5}{16}=n\left(6 \frac{1}{16}\right)$ c. $18-\frac{5}{4}=n$

9. a: $\quad 15 \frac{7}{9}-8=n\left(7 \frac{7}{9}\right)$ b: $\cdot 15-8 \frac{7}{9}=n\left(6 \frac{2}{9}\right)$ c. $36-\frac{-11}{8}=n$ 
P348

Exercise Set 25.

Copy and subtract. Exercise l (a) is done for you.

1. a. $3 \frac{6}{8}=2+\frac{14}{8}$, ir b. $3 \frac{3}{5}$

c. $4 \frac{1}{6}$

$$
\frac{1 \frac{7}{8}=1+\frac{7}{8}}{1+\frac{7}{8}=1 \frac{7}{8}}=\frac{1 \frac{4}{5}}{\left(1 \frac{4}{5}\right)}
$$

$\therefore \frac{2 \frac{5}{6}}{\left(1 \frac{1}{3}\right)}$

2: a. $8 \bar{z}$

b. $\quad 7 \frac{5}{12}$

c. $8 \frac{1}{3}$

$4 \frac{5}{8}:$

$\frac{6 \frac{7}{12}}{\left(\frac{5}{6}\right)}$

$\frac{5 \frac{2}{3}}{\left(2 \frac{2}{3}\right)}$

3. a: $7 \frac{1}{4}$

b. $\frac{3}{10}$

c. $\quad 12 \frac{2}{6}$

$\therefore \quad \frac{3 \frac{3}{4}}{\left(3 \frac{1}{2}\right)}$

$\frac{3 \frac{7}{10}}{\left(5 . \frac{3}{5}\right)}$

$\frac{3 \frac{5}{6}}{\left(8 \frac{1}{2}\right)}$

4. a. $5 \frac{3}{8}$

b. $9 \frac{1}{6}$

c. $4 \frac{3}{16}$

5.

$1 \frac{7}{8}$
$\left(3 \frac{1}{2}\right)$
$14 \frac{1}{12}$
7

$\frac{2 \frac{7}{12}}{\left(4 \frac{1}{2}\right)}$

6. 'as.

$5 \frac{3}{8}$
$1 \frac{5}{8}$
$\left(3 \frac{3}{4}\right)$ $\frac{3 \frac{5}{6}}{\left(5 \frac{1}{3}\right)}$

$\frac{2 \frac{9}{16}}{\left(1 \frac{5}{8}\right)}$

b $\therefore \quad \frac{3}{10}$

c. $11 \frac{7}{16}$

$\frac{5 \frac{9}{10}}{\left(3 \frac{2}{5}\right)}$

$\frac{8 \frac{9}{16}}{\left(2 \frac{2}{8}\right)}$

b. $4 \frac{1}{12}$

- c. $\frac{43}{10}$

$\frac{2 \frac{5}{12}}{\left(1 \frac{2}{3}\right)}$

$\frac{2 \frac{9}{10}}{\left(1 \frac{2}{5}\right)}$

7. BRAINTWISTER. Find $n$ so each mathematical sentence is true.

a. $\quad\left(11 \frac{7}{8}-4 \frac{2}{8}\right)-2 \frac{1}{8}=n$ $\left(5 \frac{1}{4}\right)$

b. $\quad\left(9 \frac{7}{12}-3 \frac{1}{12}\right)+n=10 \frac{11}{12}$ $\left(4 \frac{5}{12}\right)$

611

160 . 
ESTIMATING SUMS OF RATIONAL NUMBERS

objectives: To reemphasize the fact that any rational number either (a) is a whole number, or (b) lies between two consecútive whole numbers.

To use this fact to determine two whole numbers which "bracket" the sum of two giver' rational numioers.

Vocabular:: >, <, consecutive.

Suggested Teaching Procedure

- BęFore discussing "Estimating Sums of Rational Mubers.", ask the pupils to" tell whether suggested iractions and mixed forms rame whole numbers; if not, to teli between what two conseculive whole numbers they lie. Use the number. In in if necessary.

A number line diagram can al so be ised. to dicture the interval witnin wich the sum must be.. For example, in $3 \frac{1}{5}+7 \frac{2}{3}, \quad 3 \frac{4}{5}>3$ and $7 \frac{2}{3}>7$, so : $\quad 3 \frac{4}{5}+7 \frac{2}{3}>3.7$
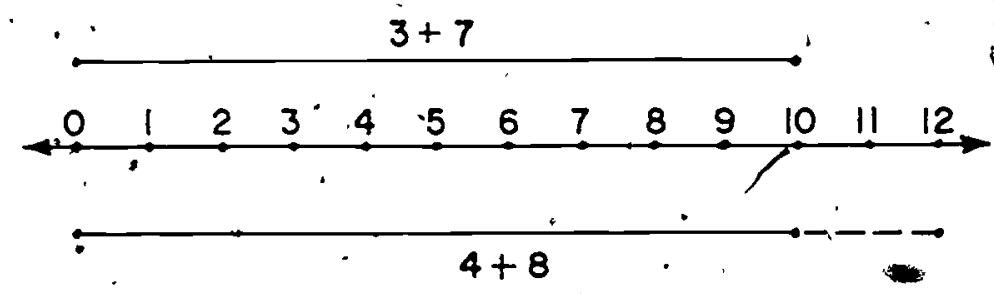

$3 \frac{4}{5}:<4$ and $7 \frac{2}{3}<8$, so $3 \frac{4}{5}+7 \frac{\dot{2}}{3}<4+8$.

The dotied segment represents the interval within which the segment whose measure is. $3 \frac{4}{5}+7 \frac{2}{3}$ mist end.

Puplls should be encourąied to use the" procedure outlined in this section to judge "the probable correctness of any sums or apdends they compute.

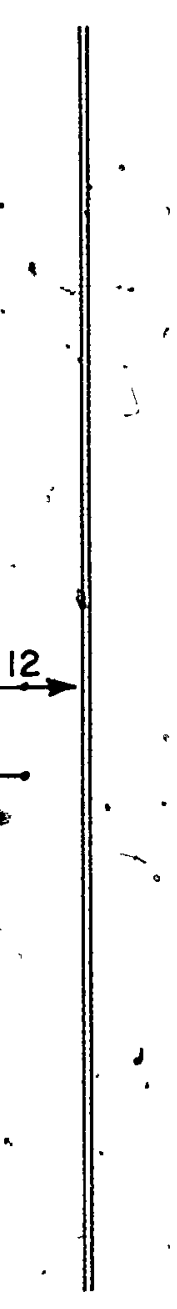


P349.

ESTIMATING SUMS OF RATIONAL NUMBERS

Exploration

. When you are adding rational numbers, it is a good ldea to estimate the sum first.

1) Consider the sentence:

$$
3 \frac{4}{5}+7 \frac{2}{3}=n^{\cdot}
$$

Between what two consecutive whole numbers would each aderd be?"
a. $\quad 3 \frac{4}{5}>(3)^{\prime \prime}$ and $3 \frac{4}{5}<(4)$
b. $\quad 7 \frac{2}{3}>(7)$ and $7 \frac{2}{3}<\frac{x}{(8)}$

Are those "statements' true?

$$
\begin{aligned}
& \text { c. } 3 \frac{4}{5}+7 \frac{2}{3}>3+7 \text {, (yes) } \\
& \text { d. } 3 \frac{4}{5}+7 \frac{2}{3}<4+8 \text { (yess. } \\
& \text { e. The sum of } 3 \frac{4}{5} \text { and } 7 \frac{2}{3}, \text { is a number between } 10 \\
& \text { and } 12 \text { (yes). }
\end{aligned}
$$

2) Between what two conseçutive whole numbers is
a. $\frac{17}{2}$ ?
( 8 , and 9)
b. $\frac{13}{4}$ ?
( 3 and: 4 )

c: The sum of $\frac{1 \dot{7}}{\frac{1}{2}}$ and. $\frac{11}{4}$ must be a number greater than (IX) and less than (13). 
P350

\section{Exercise Set $^{3} 26$}

Which of the answers below may be right? Which ones must be wrong? Answer by'findint two consecutive wholg numbers between which the sum must be.
1. $: \frac{7}{8} \div 1 \frac{3}{4}=\frac{29}{8}$. (must be surong) $4.14 \frac{4}{5}+6 \frac{9}{10}=20 \frac{7}{10}\left(\begin{array}{l}\text { maight } \\ \text { relght }\end{array}\right)$
2. $\frac{11}{3}+\frac{5}{6}=4 \frac{1}{2}$ (may be right) $5 . \quad \frac{25}{7}+\frac{14}{3}=6 \frac{5}{21}$ ( weong)
3. $\frac{13}{2}+7 \frac{3}{4}=15 \frac{1}{2}$ (muat, he weing) $6 . \quad \frac{21}{8}+\frac{19}{4}=\frac{59}{8}$ (Meght)

Between what two consecutive whole numbers must each sum be?
7. $2 \frac{3}{4}+3 \frac{1}{2} \quad(5,7)$
10. $\quad 128 \frac{5}{6}+73 \frac{1}{8} \cdot(201,203)$
8. $7 \frac{8}{9}+14 \frac{2}{3}$
$(21,23)$
11. $6 \frac{43}{8}+19 \frac{3}{4}(83,85)$
9. $10 \frac{7}{10}+12 \frac{4}{5}$
$(22,24)$
12. $89 \frac{1}{7}+15 \frac{9}{10}(104,106)$

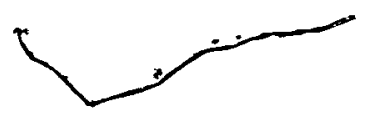

近ht these addends be correct?

13. $5 \frac{3}{7}-2 \frac{1}{2}=1 \frac{5}{14}$ (Think: If $2 \frac{5}{14}+2 \frac{1}{2}=n$, then

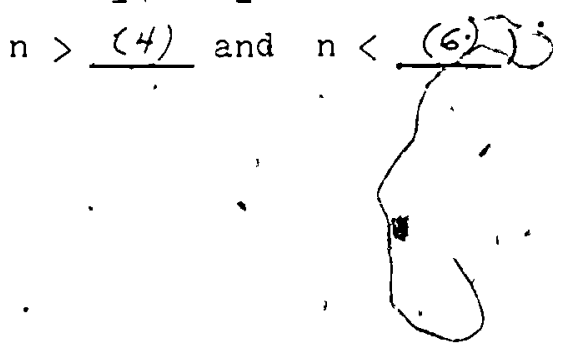

14. $\quad 12 \frac{7}{12}-5 \frac{7}{8}=4 \frac{9}{16}(.20)$

15. $15-6 \frac{3}{8}=\frac{2}{8} \cdot(22 \sigma)$ 
1.

I.1 Robert needs $\frac{22}{3}$ reet of nev'cord to reach from his desk lamp to a wall outlet. The hardware store sells lamp cord in 'no smaller divisions than the foot. How long a piece of lamp cord will Robert have to buy? $\left(\frac{22}{3}=7 \frac{1}{3}\right.$ He wece have to buy 8 fect)

2. Joan's family leaves on a trip at noon. The tipe required for the round trip is $\frac{13}{2}$ hours. At what time will they be back? $\left(\frac{13}{2}=6 \frac{1}{2}\right.$ They wile he back at $\left.6.30 \mathrm{pm}\right)$

3. Suppose a man finds that to paint the outside of his house he will need about 17 quarts of paint. The paint he needs, is. sold only in gallons. How much paint will he need to buy? $\left(\frac{17}{4}=4 \frac{1}{4}\right.$. We will have to buzk 5 giallana!).

4. Driving time from Boston to New York'is $\frac{9}{2}$ hours. Driving from liew York'to Philadelphia requires $\frac{7}{4}$ hours. How long does it take to drive from Boston to Philadelphia by way of New York? $\left(\frac{9}{2}=4 \frac{1}{2}, \frac{7}{4}=1 \frac{3}{4} ; 4 \frac{1}{2}+1 \frac{3}{4}=n\right.$; $n_{1}=6 \frac{1}{4}$ It sucel tared $6 \frac{1}{4}$ hri) 
A magic square is one in which you can perform the operation on the numbers verticallì, horizontally or diagonally and always get the same number for a result.

5. Copy the square below.

a. Add the numbers named by the fractions in each column and record the sum for each coiumn. (10)

b. Add the numbers named the fractions in each row and record the sum for each row. (10) and recing

c. Begin in lower left hand corner. Ad the numbers named by the fractions diagonally. Record their sum. (10)

d. Begin in upper leit hand corner. Ada the numbers named by the fractions diagonally. Record their sum. (10)

e. Is each sum the same rational number? What is the numberf (10)

r. Is the square a magic square? (yes).

\begin{tabular}{|c|c|c|c|c|}
\hline $2 \frac{1}{2}$ & $3 \frac{3}{8}$ & $\frac{1}{2}$ & $1 \frac{3}{8}$ & $2 \frac{1}{4}$ \\
\hline $3 \frac{1}{4}$ & 1 & $1 \frac{1}{4}$ & $2 \frac{1}{8}$ & $2 \frac{3}{8}$ \\
\hline$\frac{7}{8}$ & $1 \frac{1}{8}$ & 2 & $2 \frac{7}{8}$ & $3 \frac{1}{8}$ \\
\hline $1 \frac{5}{8}$ & $1 \frac{7}{8}$ & $2 \frac{3}{4}$ & 3 & $\frac{3}{4}$ \\
\hline $1 \frac{3}{4}$ & $2 \frac{5}{8}$ & $3 \frac{1}{2}$ & $\frac{5}{8}$ & $1 \frac{1}{2}$ \\
\hline
\end{tabular}


P353

6. Copy the square below, write fractions in. A, B, C, D, E, and $F$ to make it a magic square whose sum is 5 , (Recall. that a "magic .square" "Is one in which the sum" of the numbers named in a row, a column, or on a diagonal is the same number. This number is called the "sum" for the square.)

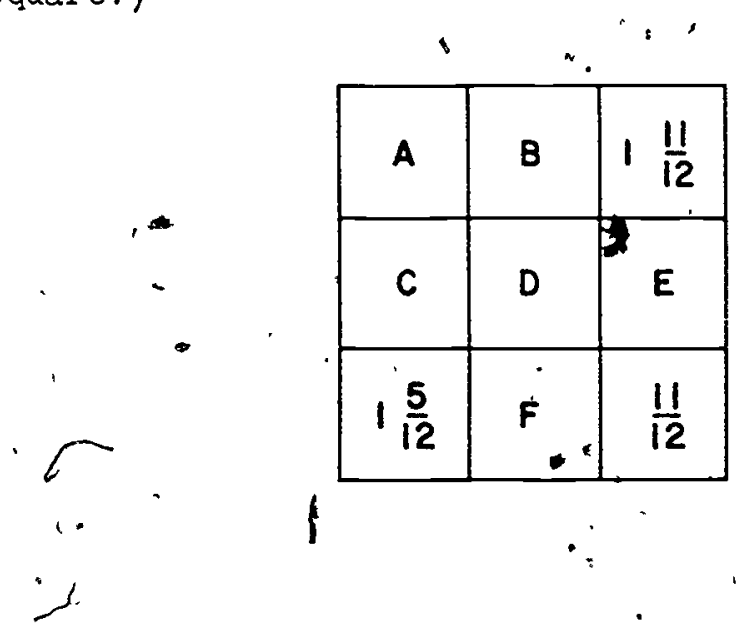

1.

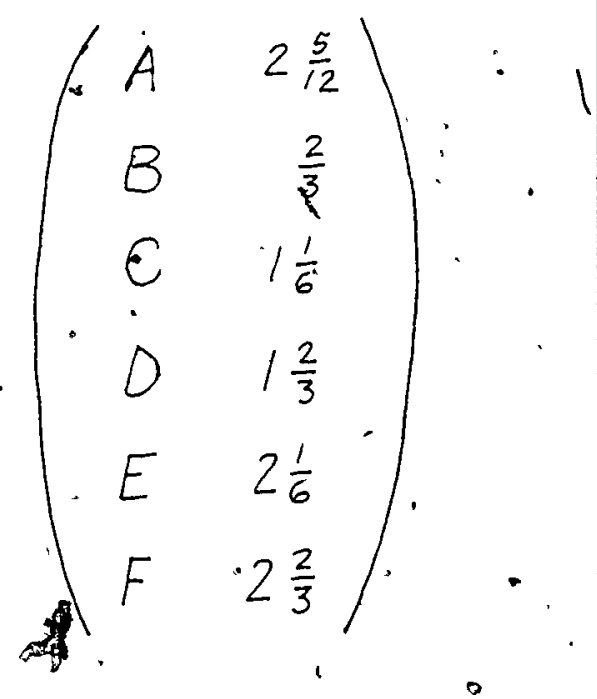

ERIC

$17:$ 


\section{THINKENG ABOUT DECIMALS}

- Objective: To develop understanding of the naming of rational numbers by using decimals, and ability to translate from fraction symbols to decimal symbols.

Materials: Place yalue pocket chart and cards. Eniarged number line as shown in the Exploration

\section{Suggested Teaching Proeedure}

Review ithe system of decimal notation. Use the place value pocket chart as needed. (See Grade 4, Chapter 2; Grade 5, Chapter 1.).

The puplis have studied some properties of rational numbers and have learned to add and subtract them, using fraction names.. They should learn to look on decimals simply as other names for, these familiar numbers.'

Decimal language, has certain advantages for. - computing which fraction language does not have In order to exploit. these advantages, it is 1mportant that the pupils iearn. to think in.more: than one way about the numbers named by decimals. Start with decimal names for whole numbers.

345 is 3 hundreds +.4 tens $+5^{\circ}$ ones

or 34 tens +5 ones

similarly,

3.45 is 3 ones +4 tenths 5 hundredths

- or. 34 tenths +5 hundradths

or $\cdots 2$ ones +14 tenths +5 hundredths, :etc.

- A number line showing tenths and huñdredths, as. shown in the Exploration, may be drawn on the $\therefore$ board and used to advantage.

$\therefore$.. To simplify notition, the 0 , before the decimal point in the decimal numeral for a number less than $\$$. Is sometimes omltted.

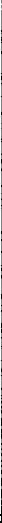


THINKING ABOUT DECIMALS"

Exploration

If you had grown in France, you would say

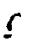
"Mon frere est plus grand que moi,"

Instead of "My brother is tailer than I." $\therefore$

Both sentences express the same relation. A French pupil does not have to know the English language to understand the relation we cakl "taller". We do not have to speak French to..

understand this relation. But to understand the idea, in the French sentence we would have to translate 1 into English. As soon as we learned to understand French well, we would not have to translate French sentences. We would think in French.

our problem with rational numbers is very much ilke this. We know a meaning, for addition $s$ if $s$ and $r$ are rational numbers, then $s+r$ can be pictured as measures in this way:'

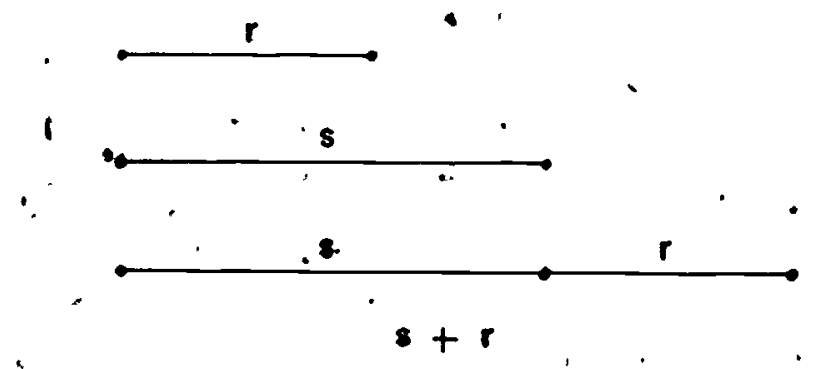

We also know how to express addition relations in the "language" of fractions, and in mixed form "lanbuage".

$$
\begin{aligned}
& \frac{8}{5}+\frac{37}{10}=\frac{16}{10}+\frac{37}{10}=\frac{53}{10} \\
& 1 \frac{3}{5}+3 \frac{7}{10}=1+3+\frac{6}{10}+\frac{7}{10}=4+\frac{13}{10}=5 \frac{3}{10} .
\end{aligned}
$$


- But these are not the only languages we use for ratfonal numbers. A very common language is the decimal numerai sirstem. We already know how to "translate" some fractions into decimals. 'For example:

$$
\frac{6}{10}=.6, \frac{5}{10}=.5
$$

In fraction language we can express this addition relation:

$$
\frac{6}{10}+\frac{5}{10}=\frac{11}{10} .
$$

Can we translate this sentence into decimal language? - We onily have to translate $\frac{11}{10}$. To do this ue first find a mixed form expression.

$$
\frac{11}{10}=\frac{10+1}{10}=\frac{10}{10}+\frac{1}{10}=1+\frac{1}{10}=1 \frac{1}{10}=1.1
$$

Now we can wite the decimal sentence:

$$
.6+.5=1.1
$$

We can always use, this method, but it can beslons. Here is another example:

What is the decimal name for $.38+.75$ ?

First we translate: ${ }_{2} .38=\frac{38}{100} ; \quad .75 \cdot=\frac{75}{100}$.

We compute: $\quad \frac{38}{100}+\frac{75}{100}=\frac{113}{100}$.

$$
\begin{array}{llrl}
\text { We translate to mixed form: } & \frac{113}{100}=1 \frac{13}{100} . \\
\text { We translate back: } & 1 \frac{13}{100}=1.13
\end{array}
$$

In decimaí languàge: .

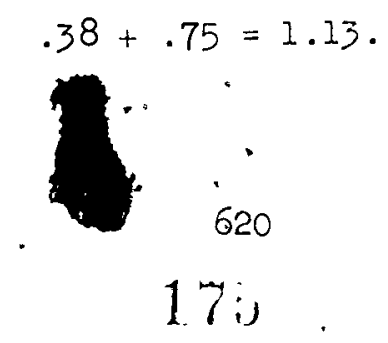


Our probiem is this. Can we learn to express addition relations in decimal language without transiating to fractions and back? Yes, we can. It is really very easy. We can begin to learn how by thinking of the meaning of decimals.

It helped us in thinking about decimal numerals for whiole numbers, to write an expanded form. Now we will write it this. way :

$$
\begin{aligned}
246 & =200+40+6 \\
& =2 \text { hundreds }+4-\text { tens }+6 \text { ones. }
\end{aligned}
$$

Can we think of all decimals in. the same way? Can we think of .25 and 8.4 and 1.06 in this wày? We know

$$
\begin{aligned}
.25=\frac{25}{100} & =\frac{20}{100}+\frac{5}{100} \\
& =\frac{2}{10}+\frac{5}{100} \\
& =.2+.05 \\
& =2 \text { tenths }+5 \text { hundredths }
\end{aligned}
$$

A number line diagram helps us to understand why this is so." Look at this number $11 \bar{n} e$, marked with a tenths scale and a hundredths scabe.

1.
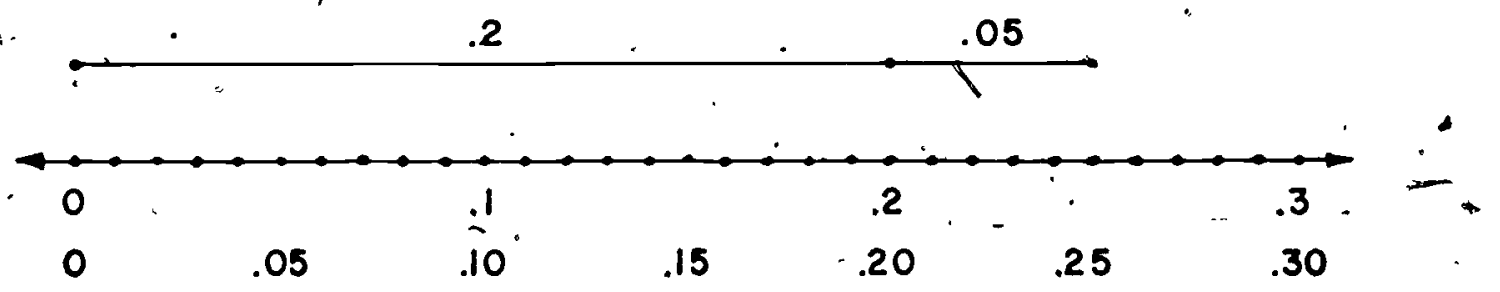

Now consider 8.4. We. know $8.4=8 \frac{4}{10}=8+\frac{4}{10}=8+.4$.

We know

$$
1.06=1 \frac{6}{100}=1+\frac{6}{100}=1+.06 \text {. }
$$

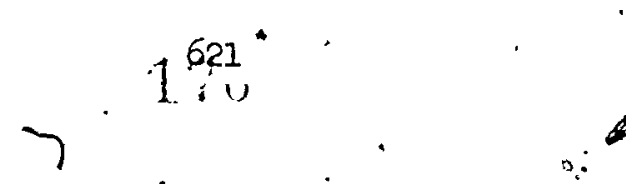


Write these in expanded form:
1.. $38.47 \quad(30+8+.4+.07)$
2. $160.13 \cdot(160+60+.1+03)$
$3 .=57.06(50+7+.06)$
)

We can express the meaning of decimal numerals in many vays.

$27.38=2$ tens +7 ones +3 tenths +8 hundredths

$=27$ ones $+38^{\prime}$ hundredths $\quad$

$=270$ tenths +38 hindredths .

$=2$ tens +7 ones +2 tenths +18 hundredths .

$\simeq 20+7+.3+.08$

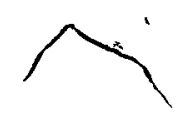

$2738=2$. thousands $+I_{7}$ huridreds +3 tens +8 ones

$=27$ hundreds $+38^{\circ}$ ones

$=272$ tens +18 ones

$=2000+700+30+8$

7

$2.738=2$ ones +7 tenths +3 hundredths 8. trowsandths.

$=27$ tenths +38 thousandths

$=2$ ones +6 tenths +13 hundredths +8 thousandiths

$=2+.7+.03+.008$
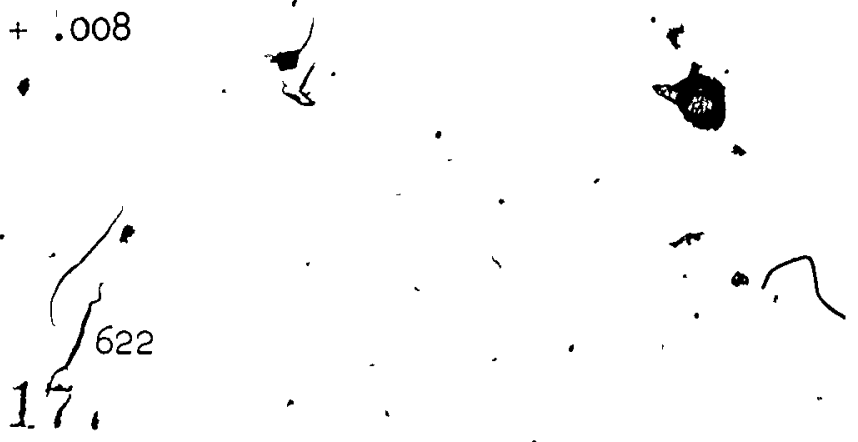
P358

Rename these numbers four ways, as shown on the preceding page.

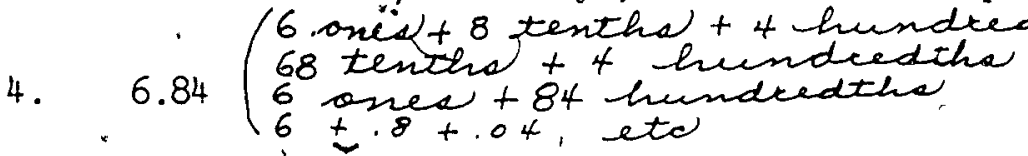

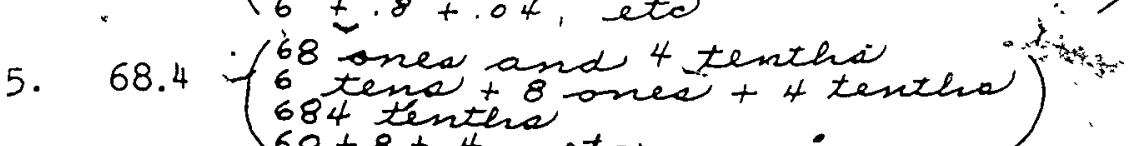

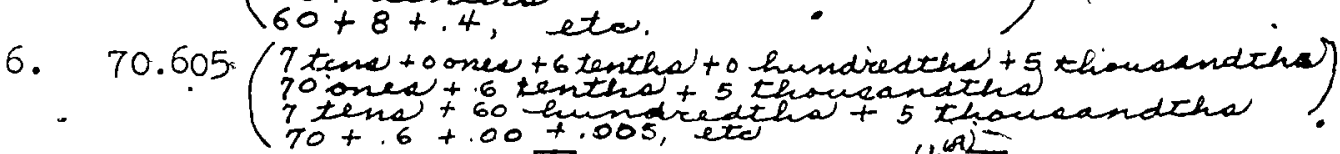

7. What is the measure $\overline{\mathrm{AB}}$ pictured below? ${ }^{(10)} \overline{\mathrm{AC}}$ has measure. $1, \overline{C D}$. has measure .6 and $\overline{D B}$ has measure .09 .

What is the decimal for each of these?

8. -5 tens +6 , ones +4 tenths +3 hundredths? (56 43.

9. 5 tenths +6 tens +4 ones +13 hundredths? (64.63\%)

10. 6 tens +6 hundredths. $-\left(\begin{array}{lll}60 & 06\end{array}\right)$

Translate to decimals:

11. $\frac{567}{1000}(.567\}$

13. $\frac{567}{10}(567)$

15. $40 \frac{6}{1000}$

(40.006)

12. $\frac{567}{100}$

(5.67)

14. $28 \frac{34}{100}(28.34)$

$\cdot 623$

17. 
P359

Exercise set .28

1. Complete:

a. $72.9=\underline{(2)}$ ones and $(9)$ tenths and $(7)$ tens:

b. $.702 .09=(7)$ hundreds $+(2)^{\prime \prime}$ ones $+(0)^{\circ}$ tenths

$\mp$ (d) tens + (9) hundredths.

c) $68.75=(68)$ ones $+(75)$ hundredths.

d. $52 \frac{16}{100}=(52)$ ones $+(16)$ hundredths.

e: $400+5+\frac{15}{100}=(4)$ hundreds $+(0)$ tens $+(5)$ ones $+(1)$ tenths $+(5)$ hundredths.

2... Write the decimal for each of these.

a. 5. hundreds and 5 hundredths $(50005)$

b. 43 tens +16 puinoriedths (430.16)

c. 2 tens +7 'hundreds +6 ones' (726)

d. 14 . hundredths +6 . tenths +3 ones $(374)$

e. 12 hundredths $\div 9$ tenths. (102)"

3. Write the decimal for each of these..

a. $\frac{14}{10}(1.4)$

c. $\frac{5243}{1000}(5.243)$. e. $\frac{6840}{10}(68 \% 0)$

b: $\quad 6 \frac{3}{10} \cdot(6.3)$

a. $\frac{5243}{10}(524.3)$ f. $20 \frac{6}{100}(20.06)$ 
P360

4. Express in dollars the value of:

$(\$ 46,80)$

a. 4 tendollar bills, 8 dimes, and 6 ore dollan, bills:

b. 15 one doldar bills, i2 pennes, and $\div$ dimes. (\$1582)

c. 253 pennis. (253)

d. 8 one dolian bilis and 58 pernes (\$88)

BRAIMT.ISTERS:

5. Transiate to des:mais:

a. $\frac{4}{25}$

(.16)

b. $6 \frac{7}{20}(6+35)$

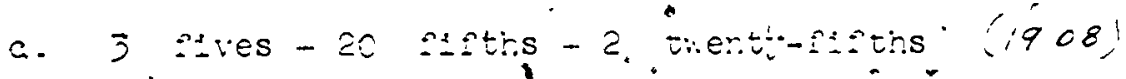

a. $\frac{7}{8}(: 875)$

3 
ADDITION OF RATIOMAE NUMBERS USING PECIMALS

Objective:

To use untierstanding of place vaiue in the decimal system tó ievelop a metrod for computing sums of. rational numbers raned by decimals.

Materials Nimber lines sealed in tenths ard in hundred,ths. - $8:$

Túsosested Teachir:z Procedine:

Since Jecimal notation Sor ratonal numbers has ti.e same properties as decimal rotatiorts for virole rumbers, the disciustion of addition and. . subjractior. es rational rimbers using decimal.

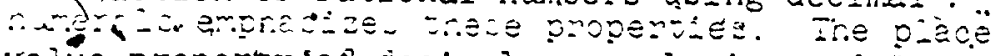
value proper" "o: dec1mal numerals is used to name eact. number in expanded notation." The com mitative: and associative properties of addition are ised to sroup.tentins $x_{1}$ th tenths, hundreaths with hundredths, etc. Place value is used again to regroud hundredirs when a sum is more than $g$ Cundredirs, terths khen a sum is more than 9 certis, etc. This, all. the properties used in computitg sums o: ratsonal numoers usins dec1mal numerals are aireadi famliar.

The use 0 : these properties in addition $c \mathrm{~cm}$. be exilfited on a number I Ine witr scales in.: tertiss and rundredits.

.15

19 $.15+.19$

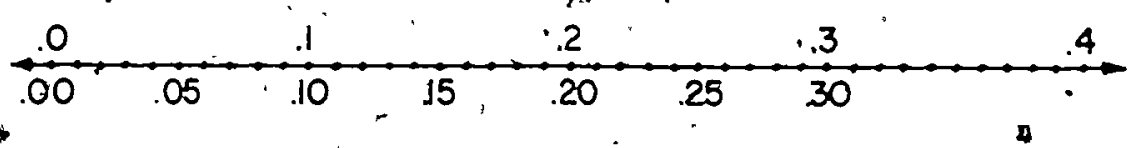

$\theta$
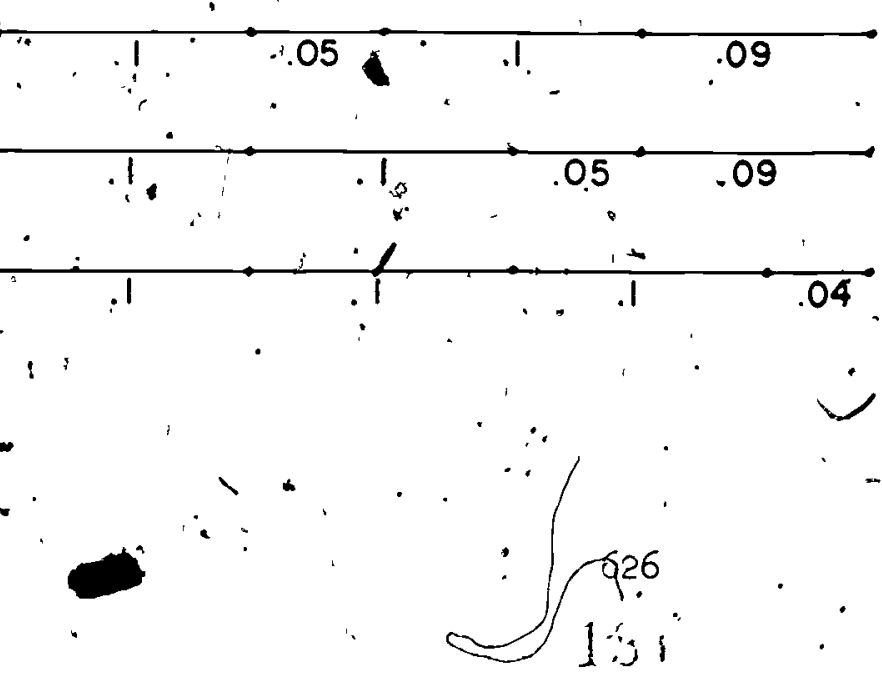


\section{ADDITION OF RATIONAL NUMBERS USING DECIMALS}

\section{Exploration}

- Now we are ready to sind a quick way to ada or subtract rational numbers using decimal names.

suppose we want to add .22 and 34 . We could transiate. to fractions:

$$
\begin{aligned}
.12-.32 & =\frac{12}{100}+\frac{34}{100} . \\
& =\frac{22-34}{100} \\
\therefore \quad & =\frac{46}{100} \\
& =.46
\end{aligned}
$$

Butwe coula anso vemegioer this:" -

$$
\begin{aligned}
.12-.3^{4} & =(.1+.02)-(.3+.04) \\
\because & =(.1+.3) \neq(.02+.04)
\end{aligned}
$$

What properties of adition have we used? (Cococeatine and) Now $.1 \div .3 .1$ tentr. $\div 3$ tenths $=4$ tenths

$$
.02 \div .04=2 \text { hundrediths }+4 \text { hundreaths }=\ddot{6} \text { hundredens }
$$

In deçimais:

$$
\begin{aligned}
& .2+.3=.4 \\
& .02+.04=.06
\end{aligned}
$$

We have, then,

$$
\begin{aligned}
\therefore .12+.34 & =(.1+.02)+(.3+.04): . \\
& =(.2+.3)+(.02+.04) \\
& =.4+.06+. \\
& =.46 \\
. &
\end{aligned}
$$


- P362

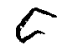

This is not" a new method. The place value idea is, used for tenths' and'hundreaths. We have al ready used this Idea for places to the left of the decimal point'.

Here is ar example with numerals on Doth sides of the decimal point.

$$
\begin{aligned}
16.31+43.52 & =(10-6+.3+.01)+(40+3+.5+.02) \\
& =(10-40)+(5+3)+(.3+.5)+(.01+.02) \\
& =50-9-.8-.03 \\
& =30.33
\end{aligned}
$$

He car use the Vertical form to make the computations easier:

$$
\begin{aligned}
16.31= & 20+6 \div .3+.01 \\
+43.52= & \frac{40.3+.5+.02}{50+9+.8+.03}=59.83 .
\end{aligned}
$$

Ese the vertical form. to compute:

$$
\text { -(1) } 18.5+3.24(49.9)
$$

(2) $72.86+15.5(8.7 .49)$.

How stolid we thy rh of problems like these?

(a) $. .7-.8$

(b) $.06 \div .09$

(a) ie known: $.7-.8=7$ tenths +8 tenths $=15$ tenths

$$
\begin{aligned}
& =20 \text { teritins }-5 \text { tenths } \\
& =1 \text { one }-5 \text { tenths } \\
\therefore & =1.5, . .
\end{aligned}
$$

* You could picture .7. '. 8 on the number line.
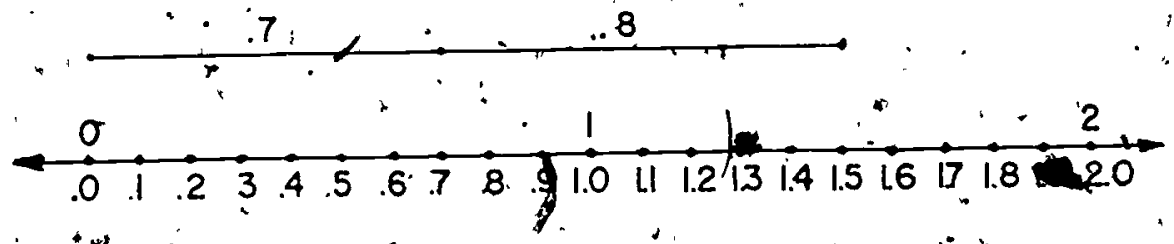

1).

-628 .

15.5 
P363

(b) . We know:, $.06+.09=6$ hundredths +9 hundredths

$$
\begin{aligned}
- & =15 \text { hundredths } \\
& =10 . \text { hundredths }+5 \text { hundredths } \\
& =1 \text { tenth }+5 \text { hundredths } \\
& =.15
\end{aligned}
$$

Th1s is thy same 1dea, regrouping in sets of ten, that we have used many times th problems like:

$$
80+70=150 \text { or . } 600+900=1500 \text { : }
$$

There is one thing new. We must be very careful to locate the decimal point correctly.

Now we can do problems like these:

$$
\begin{aligned}
14.56=10+4+.5+.06 & \\
+27.25=\frac{.00+7+.2+.05}{30+11+.7+.11} & =30+11 .(.7+. .1)+.01 \\
& =(30+10)+1+.08+.01 \\
& =40+1+.8+.01 \ldots \\
& =41.81
\end{aligned}
$$

Try these examples:
(3) .6 .37
(4) 20.08
(5) 0.87
$+3.24$
$+7.39$
$+0.76$
$\left(\begin{array}{l}6+.3+.07 \\ \frac{3+.2+.04}{.9+.5+.01}= \\ 9+.5+.01+.01 x \\ 9+.6+.0(=961\end{array}\right)$
$\left(\begin{array}{l}20+.0+.08 \\ \frac{7+.3+.09}{27+.3+.17=} \\ 27+3+.1+07 . \\ 27+.4+.07=27 \cdot 47\end{array}\right)$
$\left(\begin{array}{l}.8+.07 \\ \frac{.7+.06}{1.5+.13=} \\ 1.5+.1+.03=1.63\end{array}\right)$

- our methọ can be shown In the thertical form you used for whole numbers : 
P364

* "Or course this can be shortened by "remembering." Here are the steps:

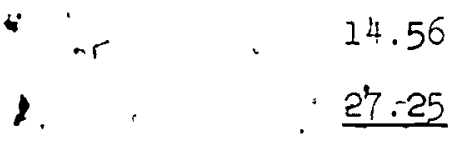

(1) Add hundredths: Write 1 hundreden, remember 1 tentho: 14.56

\subsection{5}

, $\therefore$

(2) Ada,tenths. irite.8. tenths. Mark the decimal point. 14.56

27.25

.81

(3) Adă ones. Write 1 one fand rememier 1 ten.

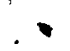

$$
\frac{27.25}{1 . \dot{8} 1}
$$

(4) Adu tens. "urte 4 tens.

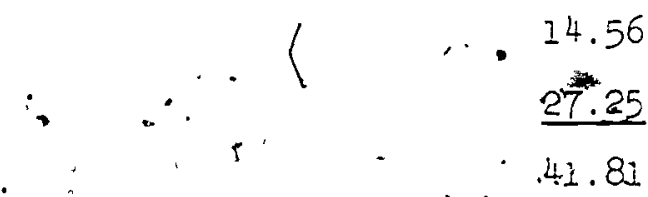

I Here is an example with more "remembering. Only the "long way is shown:
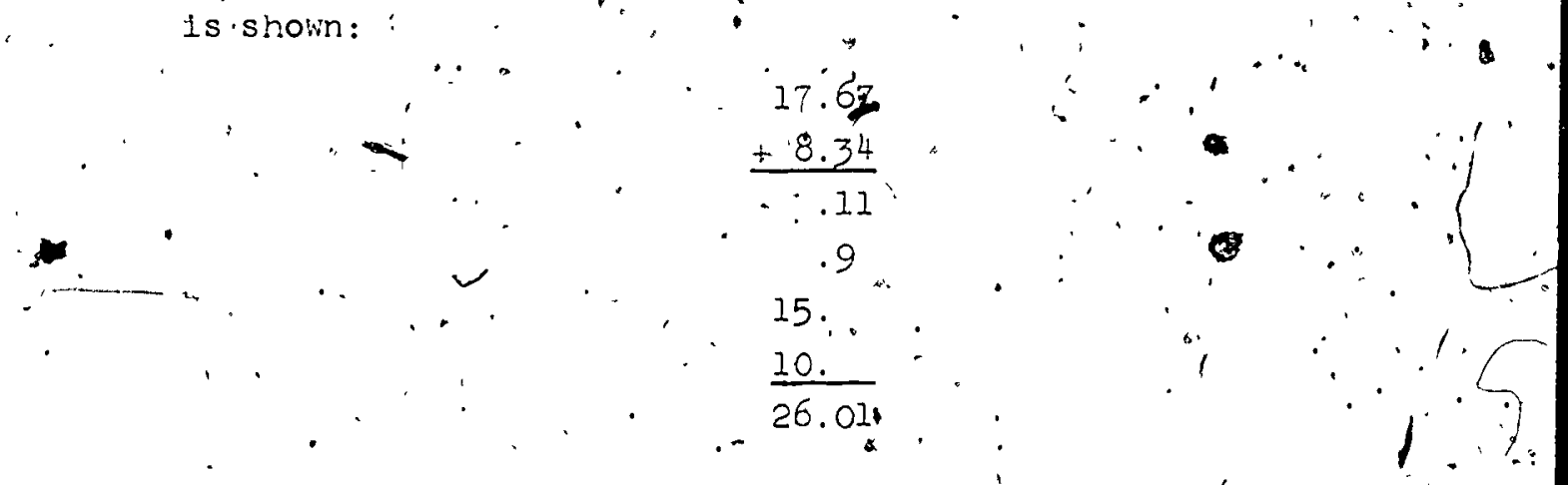


\section{Exercise set 29}

Find fraction or decimal names for the following sums. The forms suggested below may be oused.
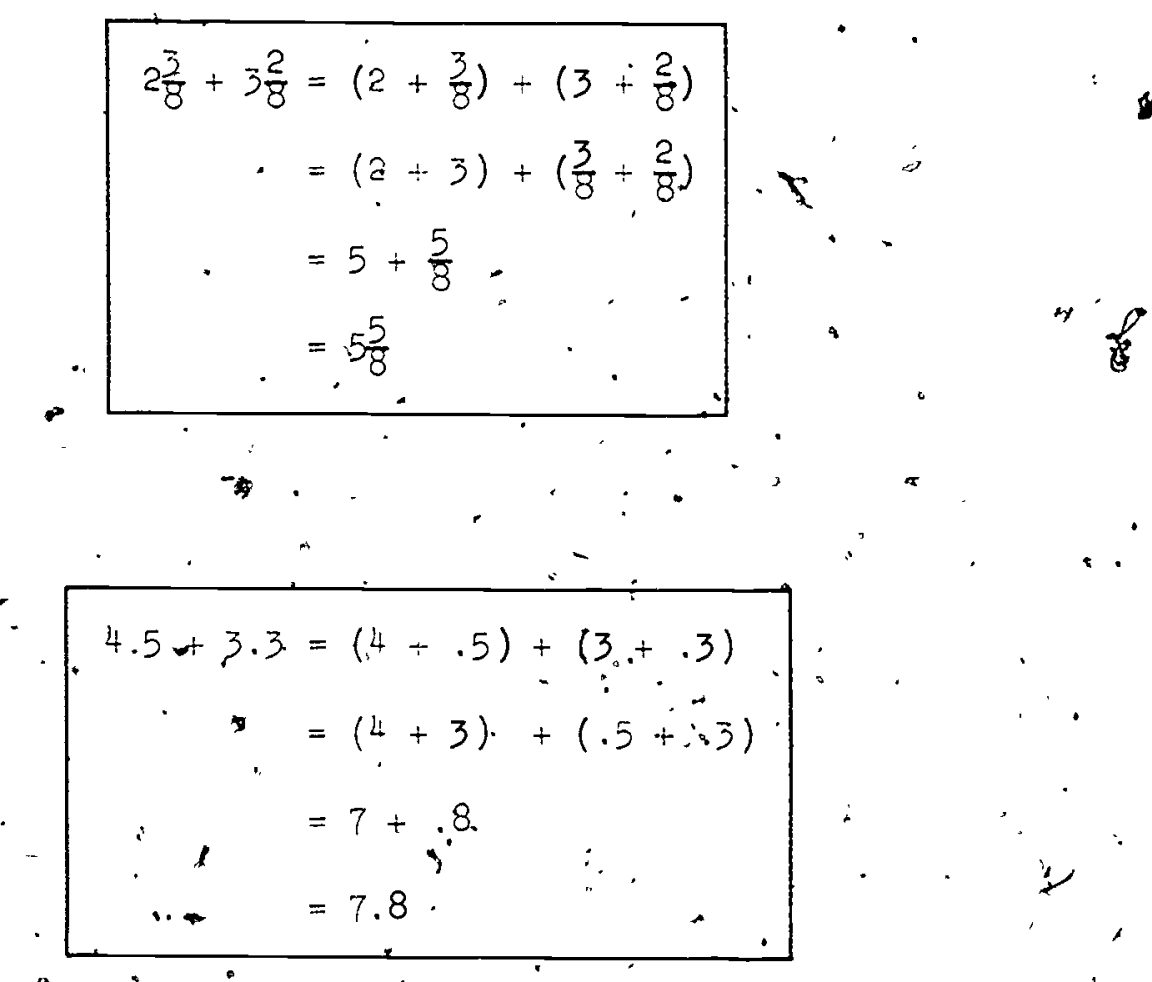

1. (a) $3 \frac{3}{5}+\frac{4 \frac{1}{5}}{3}\left(7 \frac{4}{5}\right)(\mathrm{b})=8 \frac{4}{8}+3 \frac{3}{8}\left(11 \frac{7}{8}\right)(\mathrm{c}) 2 \frac{2}{4}+1 \frac{1}{4} \cdot\left(3 \frac{3}{4}\right)$

2. (a) $1 \frac{1}{6}+6 \frac{4}{6} \cdot\left(7 \frac{5}{6}\right)$ (b) $8 \frac{5}{12}+6 \frac{6}{2}\left(44 \frac{11}{12}\right)$ (c) $4 \frac{\dot{5}}{16}+\frac{0}{16}\left(9 \frac{7}{8}\right)$

$3 ;$ (a) $2.5 \pm 4.2(6.7)(b) 8.6+3.3$ (119) (c) $5.4+38.5(13.9)$

(a) $5.3+6.6(11.9)(\mathrm{b}) 8.4+4.4(128)$ (c) $7.7+3.2(10.9)$ 
Try.to work these mentally. Write the answers onily.

5. (a) $8 \frac{3}{5}+5,\left(13 \frac{3}{5}\right) \therefore(6) \quad 7+6 \frac{2}{8}\left(13 \frac{1}{4}\right)$ (c) $-8 \frac{5}{12}\left(13 \frac{5}{12}\right)$

6.

(a) 8.6
$\therefore \quad$
$\because \quad$

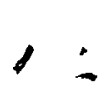

1.
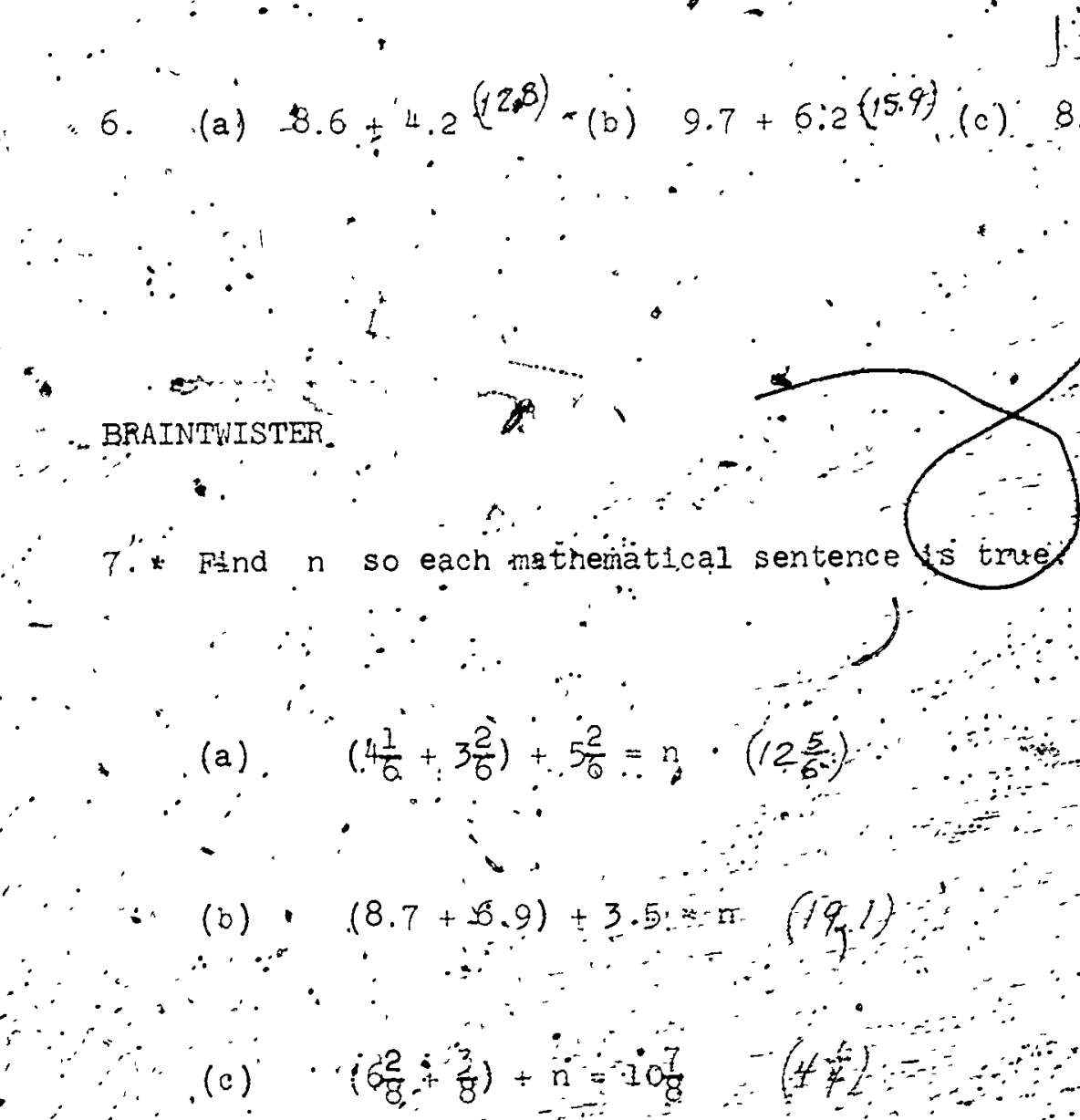

jor (a) $(4)(2)$ 
$367^{\circ}$

Exercise set $30^{\circ}$

Copy and find the sums. The form of exercises $I(a)$ and $l(b)$ may be ușed.

$\cdot 1$.

(a)

$$
\begin{aligned}
& 2 \frac{3}{10}=2+\frac{30}{10} \\
& \frac{8}{10}=\frac{5+\frac{8}{10}}{7+\frac{i 1}{10}}=8 \frac{1}{10}
\end{aligned}
$$$$
\text { (b) } 2.3^{\prime}=2+.3
$$$$
\underline{5.8}=\underline{5+.8}
$$

$$
7+1.1=8.1
$$

$2 a^{2} \cdot(a) \cdot 5 \frac{\overline{3}}{8}$

(b) $4 \frac{5}{6}$

(c) $5 \frac{3}{4}$

(d) $7 \frac{2}{4}$

$$
-\frac{4 \frac{7}{8}}{\left(10 \frac{1}{4}\right)} \cdot \frac{3 \frac{4}{6}}{\left(8 \frac{1}{2}\right)}
$$$$
\frac{4 \frac{7}{8}}{\left(10 \frac{5}{8}\right)}
$$

$\frac{63}{\left(14 \frac{1}{4}\right)}$

(a) 5.8

(b) 6.4

(c) 8,7

(d) 8.5

$$
\frac{6.3}{(12.1)} \because \because \because \frac{8.9}{(15.3)}
$$

$\frac{9.9}{(18.6)}$

$\frac{6.8}{(15.3)}$

4.

(a)

$$
\begin{array}{lll}
8.6 & \therefore(9) & 6 \frac{3}{5} \\
\frac{9.5}{(18.1)} \cdots & \because \frac{74}{5} \\
\left(14 \frac{2}{5}\right)
\end{array}
$$

(c) $8 \frac{7}{12}$

(d) 9.8

$$
\frac{6 \frac{10}{12}}{\left(15 \frac{5}{12}\right)}
$$$$
\frac{8.9}{(18.7)}
$$

Copy these examples andadd. Write only the answers on your paper.

5.

(a) 4.5

$$
\frac{3.3}{(7.8)}
$$

$\because 6: 1$ (a), 4.7 $\frac{4.6}{(9.3)}$ (b) 5.7 .

$\frac{4.2}{(99)}$

(b) 5.4

(.c) 7.6

$\frac{6.9}{(12.3)} \cdot \frac{4.8}{(12.4)}$

(c) 8.3

$\frac{9.5}{(178)}$ (d) 4.5

$\frac{2: 7}{(7.2)}$ (d). 5.6

$\frac{4.9}{(10.5)}$

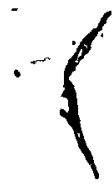

633 
Exercise set $\underline{31}$

$$
\begin{aligned}
& \therefore 1 . j \text {-compute: } \\
& \text { a. } 25.06 \\
& \text { d. } \quad .58 \\
& +\frac{37.84}{(62.90 .)} \\
& \frac{.15 .09}{(15.67)} \\
& \begin{array}{r}
-.847 \\
\therefore .138 \\
\hline .985 \lambda \\
3.707 \\
+2.988 \\
\hline(6.695)
\end{array}
\end{aligned}
$$

b. 108.07

e.

$$
\begin{aligned}
& \frac{46.94}{(576.01)} \\
& \text { c. } 117.6 \\
& \because \frac{38.74}{(156.34)}
\end{aligned}
$$

2. Compute the sum in any language:

Example:

- $4 \frac{1}{2}+6.7$

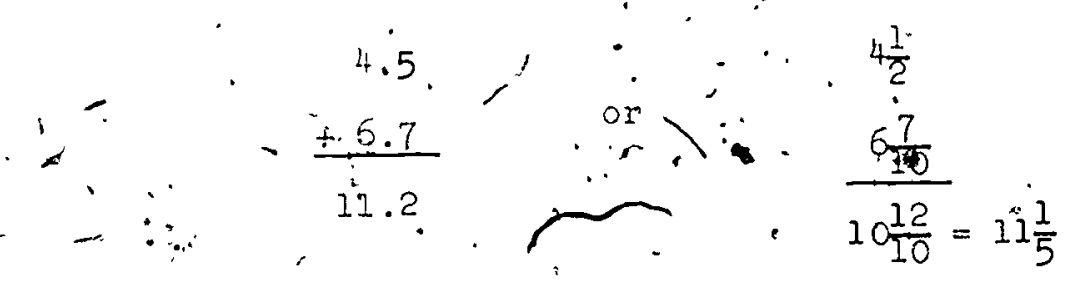

a. $23 \frac{1}{2}+\frac{7}{4} \quad\left(25 \frac{1}{4}\right)$

b. $15 \frac{1}{4}+16: 7 \quad(3195)$

c. $\frac{3}{100}+18.57^{\circ} \quad(18.6)$

d. $\quad 15 \frac{7}{8}+18 \frac{2}{3} \quad\left(3 \dot{4} \frac{5}{24}\right)$

e. $\quad \frac{1}{4}+7.18+\frac{2}{5} \quad(13.83)$

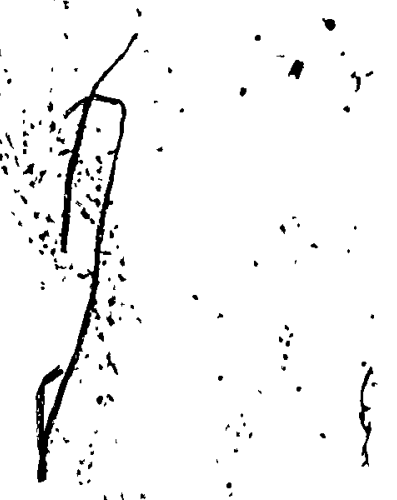


The way we name money values" 10 dollars is réally a

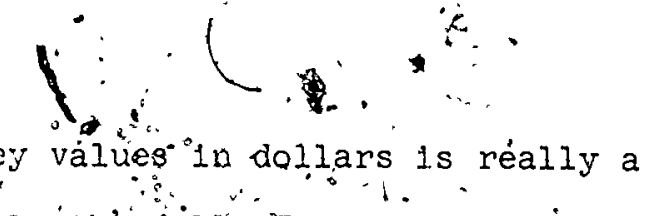

declmal numeral and symbol, the dollaros sgn, whieh indicates the undt:

$\$ 12.98$. is usually read "twelve dollars anglninety-eight. cénts," but it could just as weli bel rege "twellve and "ninetyeight one hundredths dollars."

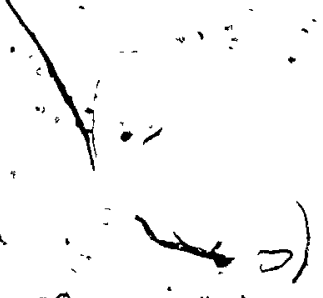

3. Stores often sell things at prices $11 \mathrm{ke} \$ 1.98$ or $\$ .49$.

- If you bought somethigg $\$ 2.98$ and something for

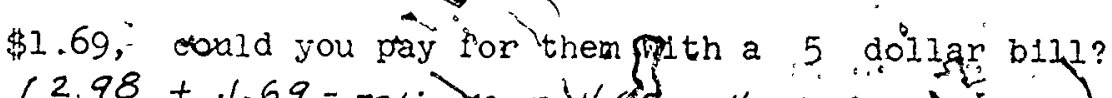

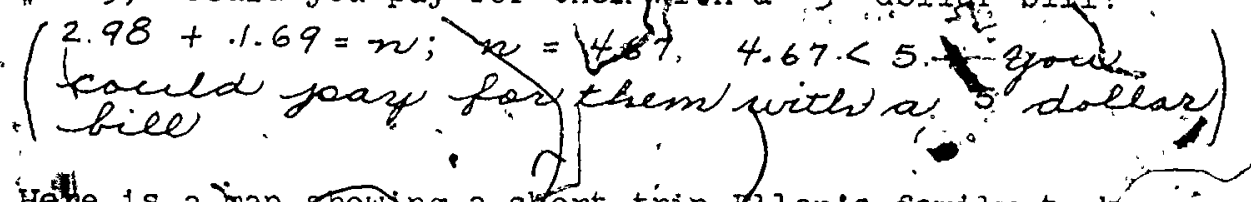
4. Here is a tran showing a short trip flilen's family took in a car. They went from: $A$, (home) to $B$ to $C$. to $D$ to $E$, and back to $A$. How fán did they travël?

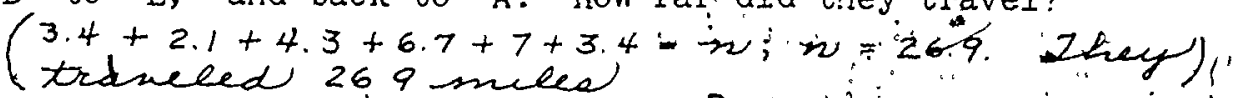

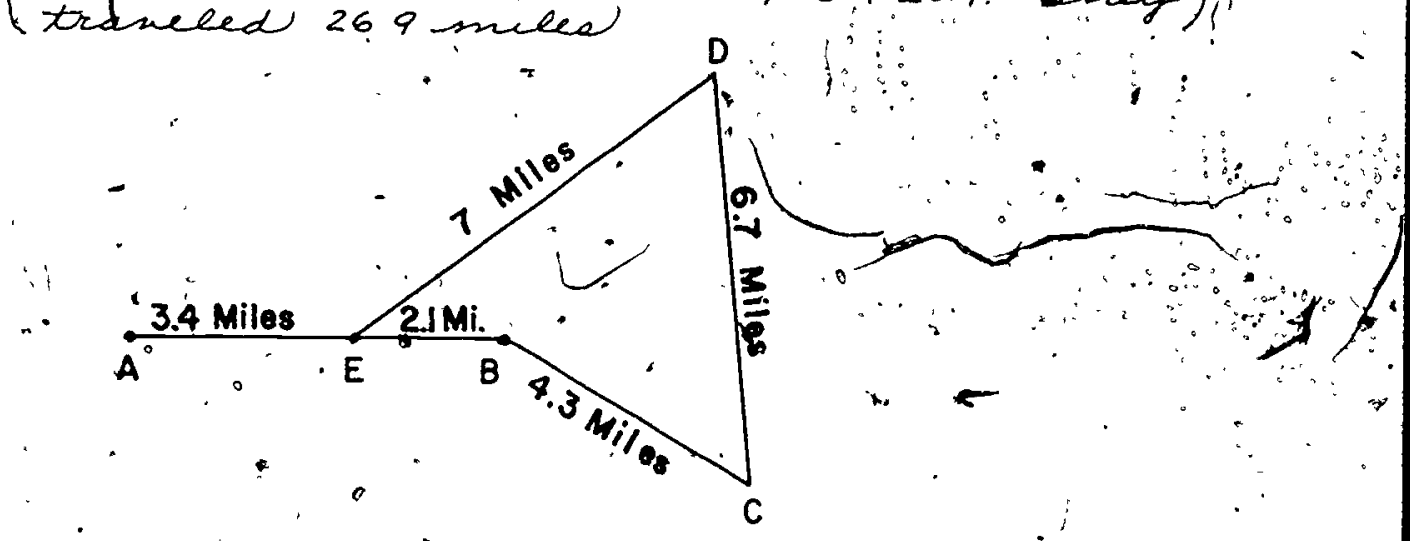




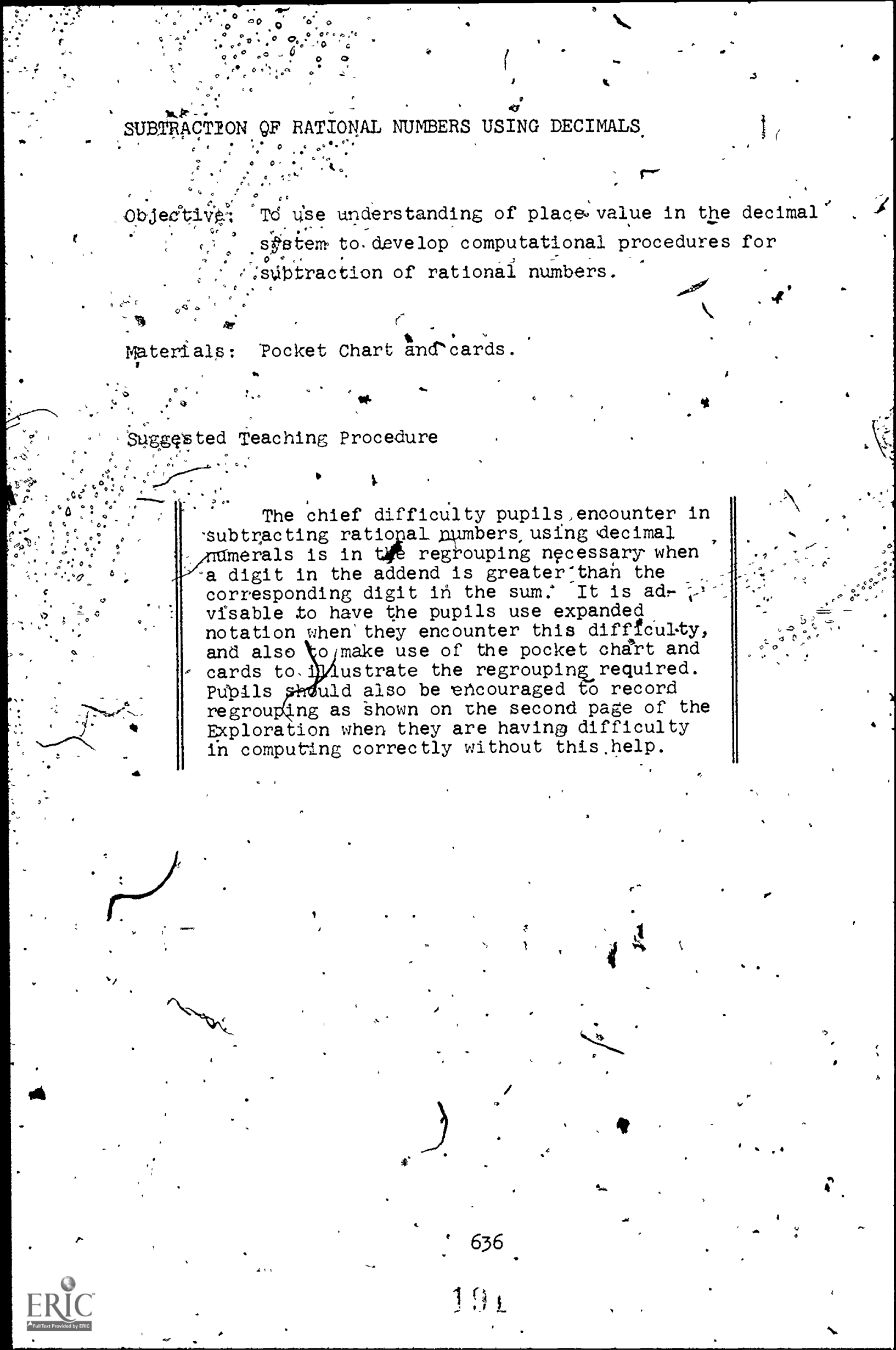


$\mathrm{P} 370$

SOBTRACTION OF RATIONAL NUMBERS. USING DECIMALS

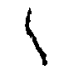

\section{Exploration}

Do. you remember how you found a process for subtracting. whole numbers using decimal notation? (Recall that "decimal" meanis "base .ten.") .

To get the dec1mal numeral for"

you thought this way.

$$
237-145^{\circ}
$$

$$
\begin{aligned}
237 & =200+30+7 \\
-145 & =\frac{100+40+5}{\because} \\
\because \quad & = \\
237 & =100+130+7 \\
-145 & =\frac{100+40+5}{90+2}=92
\end{aligned}
$$

Then you thought

can we think this way our problem is

$$
\begin{aligned}
& 2.37-1.45=1 ? \\
& 2.37=2+.3+.07=1+1.3+.07 \\
& 1.45=1+.4+.05=\frac{1+.4+.05}{. .9+.02}=.92
\end{aligned}
$$

Here we thought of $-1.3^{\circ}$ as. ten s: 13 tenths -4 tenths $=\dot{0}$ 9 ten'ths. He 'see that the 1dea is one more example:

$$
\begin{aligned}
3.08-.1 .9 & =. ? \\
3.08 & =3+.0+.08=2+1.0+.08 \\
1.9 & =1+.9+.00=\frac{1+.9+.00}{1+.1+.08}=1.18
\end{aligned}
$$

Here 1 t helped us to think of $.08^{\circ}$ as $(10+.08)$ and .9 as $(.9+.00)$. 
Try these examples. Write your work as shown on the preceding dage:
1) $9.25-4.13$
$(5.12)$
2) $18.36-2.5$
$(15.86)$

We can shorten this method if we think but do not write all of the steps. Here is one way:
$\begin{array}{r}\quad 3.08 \\ -\quad 1.9 \\ \hline\end{array}$
a) Write this'as:
b) Subtrac $\$$ hundredths
c) Think $\dot{2}+1.0$ for 3.0. Wirte this in tenths if you need to:

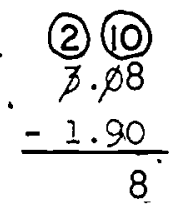
a) Subtract tenths. 10 tenths - $g^{*}$ "tenths $=1$ tenth.

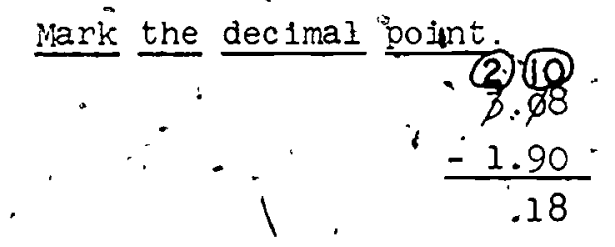
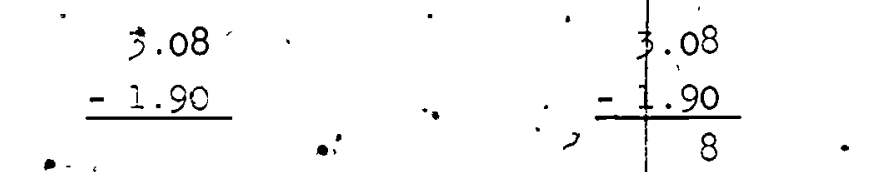

e) Subtract ones;

Try these examples the short way.

4) $7.38-5 . ?(2.18) \cdot 5) \quad 32.49-8.62 \cdot(3.876) \quad 6) 10.37-4.59$ 
Copy and subtract. Exercise $1(2)$ is done for you.
1.
(a) $5.6=4+16$ tenths
(b) 4.7
(c) 5.8
$3.7=\frac{3+7 \text { tenths }}{1+9 \text { tenths }}=1.9$.
$\frac{1.9}{(2.8)}$
$\frac{2.9}{(29)}$
2. (a) 5.6
(b) 8.1
(c) 7.6
(d) 8.5
$\frac{2.9}{(2.7)}$
$\frac{4.7}{(3.4)}$
$\frac{3.7}{(3.9)}$
$\frac{2.9}{(5.6)}$

Try to do these subtractions mentally. Write only the
results for exercises 3 through 5 .
3. (a) 7.1 .
(b) 8.7
(c) 7.4
(d) 8.3
$\frac{3.9}{(3.2)}$
$\frac{2.9}{(5.8)}$
$\frac{3.5}{(3.9)}$
$\frac{4.4}{(3.9)}$
4. (a) 8.2
(b) 9.0
(c) .18 .2
(d) 9.3
$\frac{3.4}{(4.8)}$
$\frac{3.6}{(5.4)}$
$\frac{6.5}{(117)}$
$\frac{5.8}{(3.5)}$
$5 \div$ (a) 9.8
(b) 8.6
(c) 7.5
(a) 8.3
$\frac{7.9}{(19)}$
$\frac{6.8}{(1.8)}$
$\frac{3.8}{(3.7)}$
$\frac{5.6}{(27)}$

'6." BRAINTWISTER. Fill in the squares so the sum of each row and column is the same number.

(a)

\begin{tabular}{|c|c|c|}
\hline 2.4 & $\left(5^{\prime} 4\right)$ & 1.2 \\
\hline$(1: 8)$ & 3.0 & $(4.2)$ \\
\hline 4.8 & $(.6)$ & 3.6 \\
\hline
\end{tabular}

(b)

\begin{tabular}{|c|c|c|}
\hline .8 & .1 & $(.6)$ \\
\hline$(.3)$ & .5 & $(.7)$ \\
\hline$(.4)$ & .9 & $(.2)$ \\
\hline
\end{tabular}


Copy and subtract. Exercise $l(\alpha)$ is done for you.
I.
(a) $7.83=7+.7+.13$
(b) 2.48
$5.35^{\circ}=\frac{5+.3+.05}{2+.4+.08}=2.48$. $\frac{1.09}{(1.37)}$
2. (a) $: 6.85$
(b) 7.7 .4
(c) 9.96
(d) 8.86
$\frac{2.49}{(4.36)}$
$\frac{3.37}{(4.37)}$
$\frac{4.37}{(5.59)}$
$\frac{3.57}{(5.29)}$
$30^{\circ}$ (a) 7.61
(b) .8 .94
(c) 5.50
(d) 9.72
$\frac{3.36:}{(4.25)}$
$\therefore \frac{2.78}{(6.16)}$
$\frac{4.37}{(1.13 .)}$
$\frac{3.69}{(6.03)}$

subtract the mentally./ Write just the answers.
4. (a) 7.34
(b), 8.92
(c) 9.71
(d) 8.54
$\frac{3.28}{(4.06)}$
$\frac{3.47}{(5.45)}$

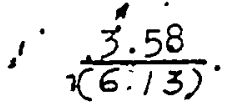
$\frac{6.39}{(2.15)}$

5.

(a) 9.65

(b) 8.47

(c) 9.9

(d) 7.81

$\frac{3.39}{(6.26)}$

$\frac{4: 38}{(4.09)}$

$\frac{4.64}{(3.17)}$

l.

6. Sybtract
(a) 0.34
(b). 9,28
(c) $8.32^{\circ}$.
(d) 9.34
$\frac{4.83}{(3.51)}$
$\frac{7.85}{(1.43)}$
$\frac{4.58}{(3.74)}$
$\frac{5.89}{(3.45)}$

BRAINTWISTER

7. Find $\bar{n}$ so each of the following is a true mathematical sentence.
(a) $\frac{5^{\circ}}{6 \times 2}+\frac{8}{4 \times 3}=n\left(j \frac{1}{12}\right)$.
(c) $\frac{4}{2 \times 8}+\frac{7}{4+4}=n$
(b) $: \frac{4+3}{4 .+1}+\frac{3}{3+2}=n$
(a) $\frac{-5 \times 3}{8+2}+\frac{3+4}{5 \times 5}=n$ 


\section{Exercise Set 34}

You have learned these two methods to express $2.52 .+5.46$ as a decimal numeral
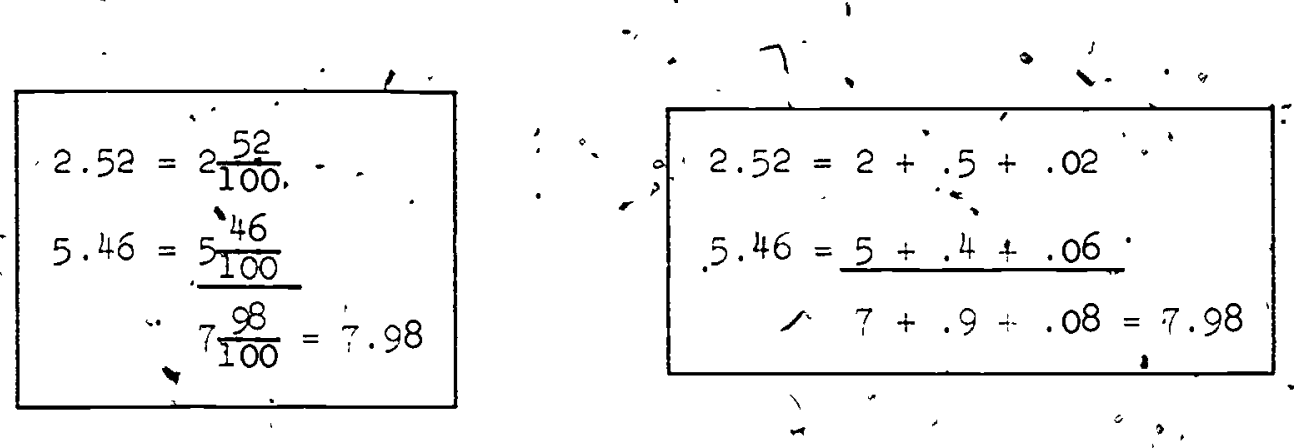

You have learned these two methods to express $5.84-3.32$ as a decimal numeral.

$$
\begin{aligned}
& 5.84=5 \frac{84}{100} \\
& 3.32=\frac{5 \frac{32}{100}}{2 \frac{52}{100}}=2.52
\end{aligned}
$$

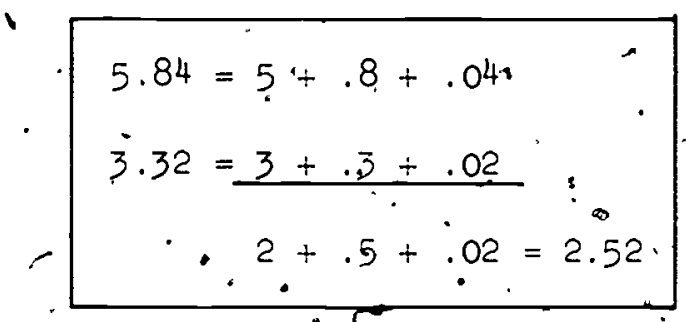

'Use two methods to add in exercise 1 .

1. (a) 5.63 . $\frac{+2.34}{(7.97)}$ (b) 6.35

$\frac{+3.44}{(979)}$ (c) 7.24 $\frac{+8.33}{(15.57)}$ -(d) 5.56 $\frac{+3.33}{(8.89)}$ 
P375:

Use two methods to subtract in exercise 2.
2. (a) 6.24
(b) 8.69
(c) 7.87
(d) $8: 86$
$\frac{-3.12}{(3.12)}$
$\frac{-4.35}{(4.34)}$
$\frac{-5.36}{(2.51)}$
$\frac{-4^{6} .24}{(4.62)}$

0

Write only your answers for exercises 3 and 4 . Do your work mentalily.
3. (a) 4.62
(b) 8.36
(c) 5.21
(d) 6.54
$\frac{+3.26}{(7.88)}$
$\frac{+3.43}{(11.79)}$
$\frac{+3.47}{(8.68)}$
$\frac{+3.35}{(9.89)}$
4 . (a) 6.57
(b) 8.79
(c) 9.68
(d). 8.89 .
$\frac{-3.35}{(3.22)}$
$\frac{-5.34}{(3.45)}$
$\frac{-4.35}{(5.33)}$
$\frac{-3.67}{(5.22)}$

5. BRAINTWISTER: Find $\mathrm{n}$ so each mathematical sentence 1s true.

$$
\begin{gathered}
\text { (a) }(8.97-4.31)+n=11.89 \quad(7.23) \\
\text { (b) } 11.89-n=8.97-4.31 \quad(723)
\end{gathered}
$$

642

$19:$ 


\section{Exercise set 35}

You have shown your work for renaming $6.28 \pm 3^{\circ} .57$ as a - declmal númeral in two ways.

$$
\begin{aligned}
6.28 & =6+.2+.08 \\
3.57 & =3+.5+.07 \\
& =9+.7+.15 \\
& =9+.7+.1+.05+ \\
r \quad & =9.85
\end{aligned}
$$

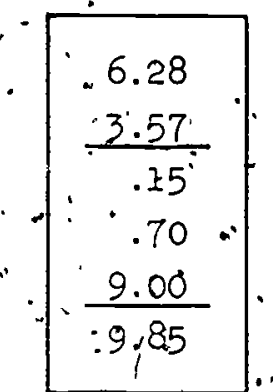

Use both metho,ds to find the' sums in.exercise 'l.
(a) 3.49
(b) 5.64
(c) 6.28
(d) $\because 6.2 \dot{9}$
$\frac{+2.38}{(5.87)}$
$\frac{+3.18}{(8.82)}$
$\frac{+3.47}{(9.75)}$
$\frac{+4.38}{(10.67)}$

Write only' the sums for exercises 2 and $\dot{3}$.

2. (a) 6.29

$\frac{5.38}{(1.67)}$

-3. (a) 7.34

$\frac{5.37}{(12.71)}$ (b) 3.42 .

$\frac{4.48}{(7.90)}$

(b) 8.23

$\therefore \frac{4.39^{\circ}}{(12.62)}$ (c) 7.23

$\frac{3.58}{(10.81)}$ $\because$ (d) 8.25 .

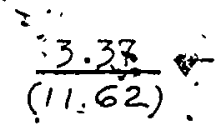

(d) 8.38

(c) 9.34

$\frac{2.59}{(11.93)}$

$\frac{5.52}{(18 \cdot 90)}$

- 46. BRAINTWISTERS:, Find these sums. Write only the decimal numeral for the sum.
(a) 3.24
(b) 4.4 .4
(c) 5.36
(d), 7.88
.3 .56
3.32
$4: 75$
5.31
$\frac{4.16}{(10.96)}$
$\frac{5.51}{(13.29)}=\frac{2.83}{(12.94)}$
$\frac{6.54}{(19.73)}$ 
Exercise set 36

1. Compute. Show your work the long way.

$$
(a)^{i} .25 .27-4.82
$$

(b) $3.75-0.28$

(c)

$$
28.75^{\prime}=13.86
$$

2. Compute:

(a) $\$ 86.23$

(3) $\cdot 50.09$

$$
\frac{-57.70}{(2853)}
$$

$$
\frac{4.91}{(45: 18)}
$$

(b) 862.3

(e)

.$\frac{\square 57.7}{804.6}$

$$
\begin{array}{r}
.725 \\
+.375 \\
\hline(.500)
\end{array}
$$

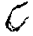

(c) $90-67.86(22.14)$

(ई) $75-7.83$

$(67.17)$

(g) Translate each addend in (e) to a fraction in simplest form. : Compute in fractions: $\left(\frac{1}{8}+\frac{3}{8}=\frac{4}{8}=\frac{1}{2}\right)$

$\therefore$ 3. Compute these repeated sums. Use the long way if you cannot remember.

$$
\begin{aligned}
& \because \quad 7.08 \text {. } \\
& \text { - (a) } 16.67 \\
& \text { - 38.92 } \\
& 3.04 \\
& \because \frac{+16.60}{(62.60)} \\
& \text { 7. } . .08 \\
& \frac{+\dot{6} .20}{(25.99)}
\end{aligned}
$$
38.

$$
\frac{+6.94}{(45.67)}
$$

644

190 
$\mathrm{P} 378$

4. How much change should you get from a 10 dollar bill.. If you bought 'things' costing' $\$ 3.98, \$ 1.49$, $\$ .98$, and

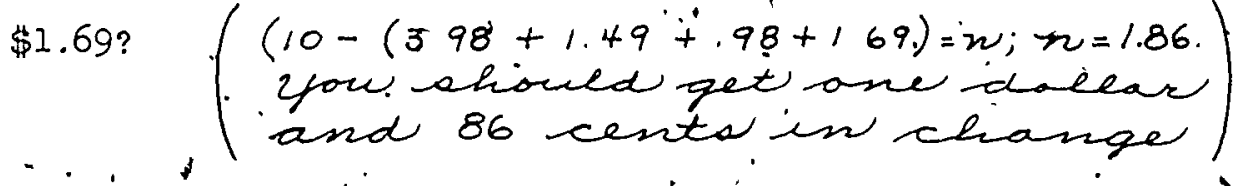

5. The population of trie United States was $131.669^{\circ}$ milizon in 1940. 150.697 million in 1950 and 179.323 milizon in 1960. Did the pupulation increase more between 1940 and 1950 or between $1950^{\circ}$ and 1960 ?

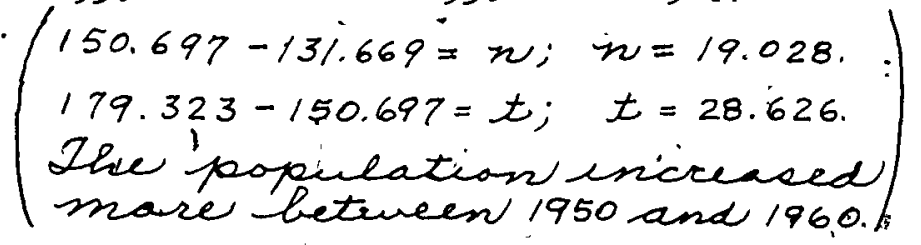

4. . .

6. Here/are the populations of the 5 largest cities in the United States in 1960. Was the part, of the united states population which lives in these cities more or less than $\frac{1}{10}$ of the total population $\left(\begin{array}{l}7.78+3.55+2.48+2.00+1.67=n, n=17.48 \\ 17.48<\frac{1}{10} x / 79.323 \text {. 2he }\end{array}\right)$

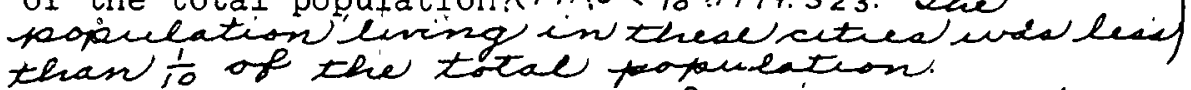
New York

Chicago

.7 .78 milition

,

Los Angeles $.3: 55$ million Philadelphia 2.48 milition Detroit 2.00 milition 1.67 milition

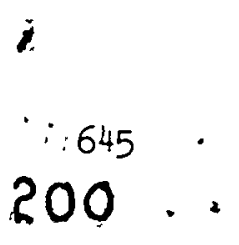




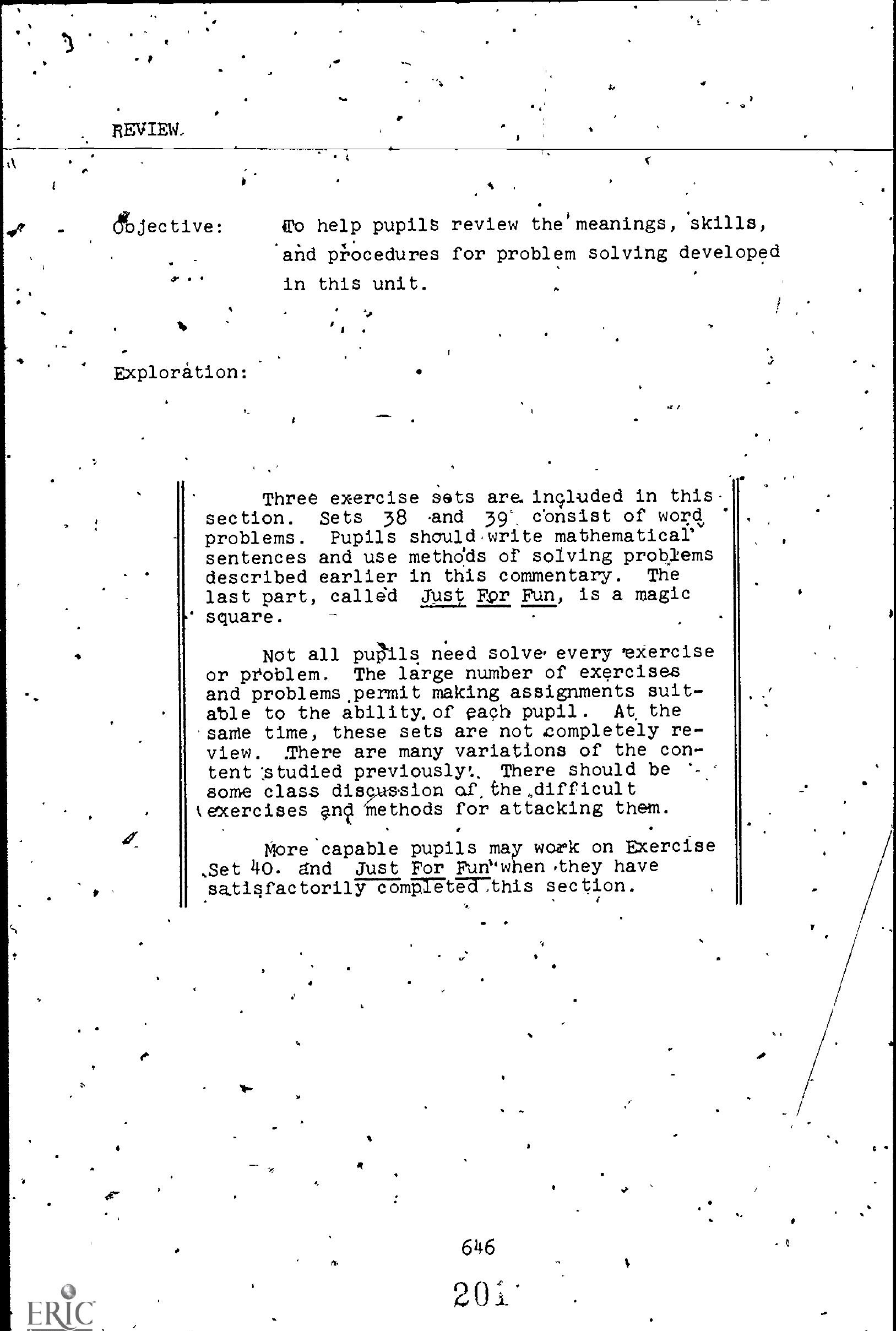


1. What number $\mathrm{n}$. will make eaçh mathematical sentence true?
(a) $\frac{1}{4}=\frac{n}{8} \quad(2)$
(c) $\cdot \frac{3}{4}=\frac{n}{8}$
$(6)$.
(e) $\frac{7}{8}=\frac{14}{n}(16)$
(b) $\frac{1}{2}=\frac{n}{12}(6)^{10}$
(d) $\frac{n}{\frac{n}{d}}=\frac{12}{16}$
(3)
(f) $\frac{5}{n}=\frac{10^{\circ}}{16}$

2. Copy and write " 3 ", " <", or "= " in each blank so - éach mathematical sentẹnce is true.
(a) $\frac{1}{3} \cdot(\geqslant) \frac{1}{8}$
(c) $\frac{1}{9}(<) \frac{1}{5}$
(e) $-\frac{2}{3}(<) \cdot \frac{3}{4}$
(b) $\frac{1}{7}(<\cdot) \frac{1}{5}$
(d) $\frac{2}{3}\left(-\frac{12}{18}\right.$
(f) $\frac{9}{8}(<) \frac{4}{3}$

3. Which would you 'rather have?
(a) $\frac{1}{4}$ or $\frac{1}{8}$ of a pie $\left(\frac{1}{4}\right)$ (c) $\frac{1}{4}$ or $\frac{1}{3}$ of a candy bar $\left(\frac{1}{3}\right)$
(b) $\frac{1}{4}$ or $\frac{1}{5}$ or a dollar $\left(\frac{1}{4}\right)$
(d) $\frac{1}{8}$ or $\frac{1}{6}$ of a watermeion $\left(\frac{1}{6}\right)$

4. Find a fraction name for 'in' so each mathematical sentence - Is true.
(a) $\frac{1}{2}+n=\frac{5}{8}^{\circ} \cdot\left(\frac{1}{8}\right)$
(c) $\frac{1}{4}+n=\frac{3}{12}+\frac{7}{12} \cdot\left(\frac{7}{12}\right)$
(b) $\frac{1}{2}+n=\frac{3}{6}+\frac{2}{6}\left(\frac{1}{3}\right)$
(d) $n+\frac{1}{2}=\frac{3}{6}+\frac{4}{6}$

5. Which fractions name numbers greater than the number i? $\frac{1}{4},-\frac{7}{4}, \frac{3}{8}, \frac{19}{16}, \frac{9}{10}, \frac{12}{10}, \frac{15}{9}, \frac{8}{9}, \frac{4}{6}, \frac{6}{5}$

6. Find the simplest fraction name for each number. $\left(\frac{7}{4}, \frac{19}{6}, \frac{12}{60}, \frac{15}{9}, \frac{6}{5}\right)$
(a) $\cdot \frac{16}{24}\left(\frac{2}{3}\right)$
(b) $\frac{10}{20}\left(\frac{1}{2}\right)$
(c) $\frac{18}{24}\left(\frac{3}{4}\right)$
(e) $\frac{4}{20}\left(\frac{1}{5}\right)$
(8) $\frac{14}{18}\left(\frac{7}{9}\right)$
(1) $\frac{14}{2 I}\left(\frac{2}{3}\right)$
(b) $\frac{10}{20}\left(\frac{1}{2}\right)$ (d) $\frac{12}{15}\left(\frac{4}{5}\right)$
(1) $\frac{16}{20}\left(\frac{4}{5}\right)$
(h) $\frac{24}{30}\left(\frac{4}{5}\right)$
(j) $\frac{10}{16}\left(\frac{5}{8}\right)$ 
P38

7. Rename each number in' inixed form.
(a) $\cdot \frac{16}{2} \quad\left(1 \frac{1}{9}\right)$
(c) $\cdot \frac{8}{3}$
$\left(2 \frac{2}{3}\right)$
(e) $\frac{17}{12} \quad\left(1 \frac{5}{12}\right)$
(b) $\frac{12}{7} \quad\left(4 \frac{5}{7}\right)$
(d) $\frac{19}{5}\left(3 \frac{5}{5}\right)$
(i) $\frac{13}{5} \cdot\left(2 \frac{3}{5}\right)$

8. Copy each statement below. For each missing numerator or denominator write a numeral so each mathemałical sentence is true.

$$
\begin{aligned}
\text { (a) } \frac{8}{12} & =\frac{(2)}{3}=\frac{(4)}{6}=\frac{(12)}{18}=\frac{(16)}{24} \\
\text { (b) } \frac{9}{15} & =\frac{(12)}{20}=\frac{(21)}{35}=\frac{6}{(10)}=\frac{15}{(25)} \\
& \left(\text { c) } \frac{3}{18}=\frac{(2)}{12}=\frac{4}{(24)}=\frac{5}{(30)}=\frac{(6)}{36}\right.
\end{aligned}
$$

9. Which of these fractions are other names for $\frac{1}{2}$ ?
$\frac{6}{18}$
$\frac{10}{25}$
$\frac{4}{5}$
$\frac{5}{10}$
$\frac{7}{5}, \frac{8}{6}$

( $\frac{2}{4}$

- $\frac{7}{8}$
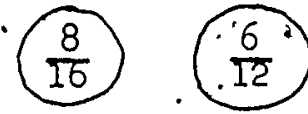

$\frac{13}{21}$ :

$\frac{6}{4}$

$\frac{3}{5}$

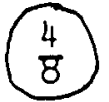

$\frac{14}{28}$

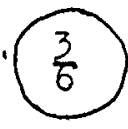

$\frac{8}{5}$
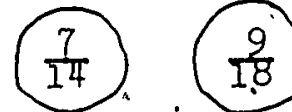

$\frac{12}{.8}$

( $\frac{6}{4}$ and $\left.\frac{12}{8}\right)$

10. Which fractions in exercise 9 are other names for $\frac{9}{6}$ ?

11. Which pairs of numbers below can be named by fractions with a common denominator of $24:(a, d, e)$
(a) $\frac{1}{2}$ ) and $\frac{4}{3}$
(d) $\frac{10}{3} \cdot$ and $\cdot \frac{1}{8}$ ?
(b) $\frac{4}{6}$ and $\frac{4}{7}$ ?
(e) $\frac{7}{6}$ and $\frac{3}{8}$ ?
(c) $\frac{7}{4}$ and $\frac{4}{7}$ ?
(f) $\frac{5}{2}$ and $\frac{7}{9}$ ? 


\section{Exerc1se Set 38}

1. There were $2 \frac{2}{3}$ qt. vanilla 1 cè cream and $3 \frac{1}{2}$ qt. chocolate 1ce cream in the freazer. How much 1ce chan

$\therefore$ was in the freezer? $\left(2 \frac{2}{3}+3 \frac{1}{2}=n ; n=6 \frac{1}{6}\right.$. 2his were

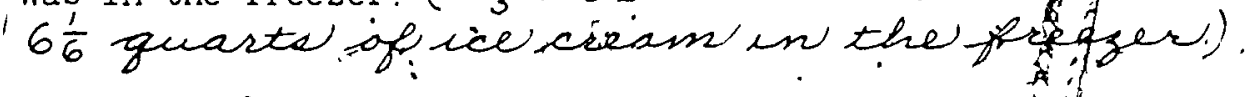

2. Mary's mother made two costumes for a school play "One costume took $3 \frac{2}{3} \because$ ds material and the other took $2 \frac{1}{1}$ yards. How much material did Mary's forther bux? ( $3 \frac{2}{3}+2 \frac{1}{6}=n ; n=5 \frac{5}{6}$. She bought 5

3. Dick's welght was $56 \frac{3}{4} \mathrm{lb}$. In June. At the eng vacation he weighed $59 \frac{1}{2}$ ib: How much velgh a d he gain? $\left(56 \frac{3}{4}+n=59 \frac{1}{2} ; n=2 \frac{3}{4}\right.$ : He qaunedis to

4. Mr. Long roticed thiat the odometer in his capjowed 8523:4 miles when-he bought some ras. "Difing the day he traveled 49.3 miles: What did his. odónteter read at the end of the day? $(8523.4+493=n$ nis $=85.72 .7$. The odometery

5. Bruce wanted to buy a'sleeping bag, for a scout camping trip that was priced $\$ 26.95$. Bince's father gave him-\$5 to help pay the cost. Biruce had saved \$7.35. How much more money does Bruce' need to buy the sleeping bag? $\left(5+7.35+n=26.95 ; m=1460^{\circ}\right.$. Price needed $\not 1460$ miace)! :

6. Gerry was 111 one day and her mother took her temperature In the morning. The thermometer read' $99: 8^{\circ}$. Later in

$\therefore$ the day Gerryis fever increased and the thermometer read $102.6^{\circ}$. How many degrees did" her fever increase?

$(99.8+w=102.6 ; n=2.8$. 2her increased 2.8 edegreen)

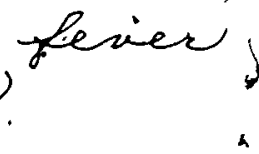


7. - Tell whether each of these mathematical sentences is an example of the commutative or, the associative properties for addition.

$$
\begin{aligned}
& \text { (a) } \frac{2}{3}+\left(\frac{1}{4}+\frac{3}{4}\right)=\left(\frac{2}{3}+\frac{1}{4}\right)+\frac{3}{4} \text { (expaciative). } \\
& \text { (b) } \times \frac{3 *}{12}+\frac{2}{8}=\frac{2}{8}+\frac{3}{\sqrt{2}} . \quad \text { (coisinsutative) } \\
& \text { (c) }\left(\frac{8}{9}+\frac{12}{3}\right)+\frac{1}{6}=\frac{8}{9}+\left(\frac{12}{3}+\frac{1}{6}\right) \text { (ascociatise) } \\
& \text { (d) }\left(\frac{1}{3}+\frac{1}{4}\right)+\frac{2}{3}=\left(\frac{1}{4}+\frac{1}{3}\right)+\frac{2}{3}(\text { commictative }) \text {. } \\
& \text { (e) }\left(\frac{8}{5}+\frac{4}{5}\right)+\frac{1}{2}=\frac{8}{9}+\left(\frac{4}{5}+\frac{1}{2}\right)(\text { pecocuatere) }
\end{aligned}
$$

8. In his butcher shop, Mr. Fisher had some bologna in chunks. On Monday he sold $2 \frac{7}{8} \mathrm{lb}$. The next day he sold $\frac{3}{4} \mathrm{lb}$. - on Wednesday, he sold $3 \frac{1}{2} 3 \mathrm{~b}$.

Use the above information to complete problems (a) $\stackrel{-}{-}$ through (d).

(a) Howl many poulnds of bolögna did $M r$. Fisher' sell in the threde days? $\left(2 \frac{7}{8}+\frac{3}{4}+3 \frac{1}{2}=7, ; 2=7 \frac{1}{8}\right.$. He sold $7 \frac{1}{8}$ tounds.)

(b) How much less than $10 \mathrm{lb}$. iras sold? $\left(10-7 \frac{1}{8}=n\right.$; $n=2 \frac{7}{8}$. Wh sold $2 \frac{7}{8}$ lhw lewe than 10 eba)

(c) How much bologna was sold on Tuesday and Wednesday?

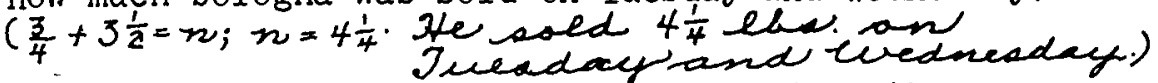

(d) The total number pounds of bologna sold on the last two days is how many more than the number of pounds sold on the rirst two days? Express your
\[ \left(2 \frac{7}{8}+\frac{3}{4}=n ; n=3 \frac{3}{4}+3 \frac{3}{2} \cdot t_{;} t=4 \frac{1}{4} .\right. \] answer in simplest form. $4 \frac{1}{4}-3 \frac{5}{8}=p ; p=\frac{5}{8}$. an, $3 \frac{1}{2}-2 \frac{7}{8}=k ; k=\frac{5}{8}$. The total number of pands sold owthe leat two day

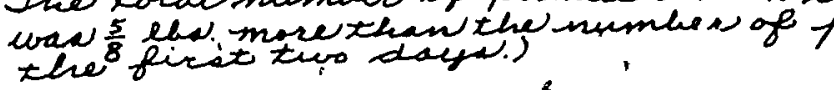

$$
\begin{aligned}
& 650 \\
& 205
\end{aligned}
$$


1. The fastest p1tched ball on record traveled 98.6 m.p.h. Then'a hockey player strikes, a puck, the puck 'travels about 98.0 m.p.h. Which traveledifaster? How much? (98.6-98.0-w; $i=6$. the bill traveled .6. per mile fiater.)

2. A recert census showed that out of every 100 , people in South Carolina. 36.7 lived. In towns and cities, and 63.3 Ilved in rural. communties. Out of every 100 people, how many more lived in miral communtties? (63.3-36.7 $=n ; n=26.6 .26 .6$ space lised in cural comsunetien)

3." "The flight time of Explorer III was 115.87 minutes. That of Explorer I was 114.8. What is the difference In the two flight times? $(115.87-1 / 4.8=\pi ; n=1.07$. Ihe difeference in the two flight timed is 1.07 insincted.).

4. In George Washington's t1me, .90 of the American people, could not read or write. Today only about \$.05 of the American people cannot read and write. What part of the people: could read and write in washington's time? In our time? (1.00-.90 = n; $n=.10 . \quad 10$ of the Amercan people coued read assisurite in esearge tuashingtonis teme $1.00-.05=n$; $n=-95 . .95$ of the Anercon peopec can read and owerte today.)

651 
5. The Simplon Tannel between Italy and Sw1tzerland is 12.3 miles iong. The Cascade Tunnel in Washington is 7.8 miles long. How much longer is the simpion thenel?

$(12.3-7.8=n ; i n=4.5$. The Lemplon Jumsel is 4.5 miles honiger.)

6. 'In 1950, statistics showed that the population"per square mile in Cal1fornta was 66.7 persons. In 1940 it had been 43.7 persons. On the average, how many more people lived on a square mile in 1950? (66.7. $-43.7=n ; p=23$. an the asuerage, 23 mote peopele lined an a squake sailetun 1950)

7. The Moosehead Lake in Maine has an area of 116.98 square miles. The area of Lake Mead in Nevada is 228.83 square (Lake Mead)) miles. Which lake has the greater area?A How much greater? (228.83-116.98 =n; in = 111.85. Lake meadi has an area ! 111.85 greater than Mosaliend have)

8. The length of a day on Mars is 24.5 hours. The length 1. of a day on reptune is 15.7 hours. How muich longer 1s a day on Mars? ( $24.5-15.7=n ; n=8,8$. The day on mave is 8.8 hourd Loniger than a day aw pepane.).

9. The distance from Earth to Cygnus is 10.6 light years. The distange from Earth to Sirius is 8.6 ilght years. Wh1ch star is closer to Earth? How much closer? (10.6-8.6 =n; $n=2$. Airind is 2 lopt. years chaser to Earthi) 
- Make a square 11ke this.

\begin{tabular}{|c|c|c|c|}
\hline (8) & $\begin{array}{l}2 . \\
(3.5)\end{array}$ & 3 & ${ }^{4} \cdot(2)$ \\
\hline (1) & $(5.5)$ & $\begin{array}{l}7 \\
(3.50)\end{array}$ & 8 \\
\hline$(i 50)$ & ${ }^{10}(5)$ & ${ }^{11}(3)$ & $\begin{array}{l}12 \\
(7.50)\end{array}$ \\
\hline $\begin{array}{l}13 \\
(6: 5)\end{array}$ & 14 & ${ }^{15}(7)$ & 16 \\
\hline
\end{tabular}

In each small square, write the answer of the example below having the same numeral as the square. Yf your work is correct, the sum of the numbers of each row and column w1ll be the same number.
$1 . \cdot 6.50+1.50$
9. $.70^{\circ}+.80$
2. $4.75-1.25$
10. $4.85+.35$
3.) $1.8+1.7$
11. $5.37-2.37$
4., $96+48$
12. $6+1.50$
5. $.25+.75$
13. $7.7-1.2$.
6. $8.00-2.50$
14. $\overline{108} \div 36$
7. $1.75+1.75$
15. $13.76 \div 6.76$
8. $63 \div 9$
16. $7.25-6.75$ 
P386

\section{Exercise sét 40}

1. Below are a number of'steps showing $\frac{1}{2}+\frac{2}{3}=\frac{2}{3}+\frac{1}{2}$ Státe a reason for each step. Let $n=\frac{1}{2}+\frac{2}{3}$.

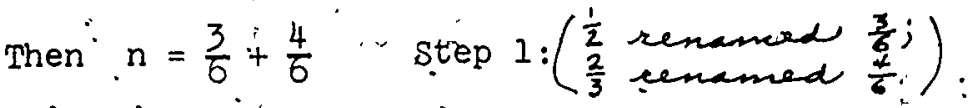

$$
\begin{aligned}
& =\frac{3+4}{6} \text { Step 2:(addition wixh } \\
& =\frac{4+3}{6} \text { Step 3:(Commutative }(\text { property of addetion) }): \\
& =\frac{4}{6}+\frac{3}{6} \text {. Step } 4: \text { (addition urtw). }
\end{aligned}
$$

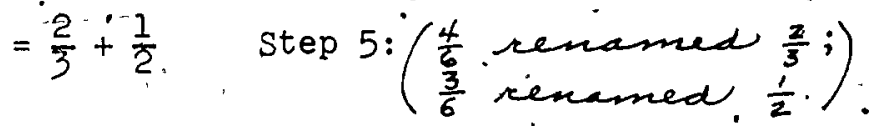

2. Write steps like those in exercise 1 to show that

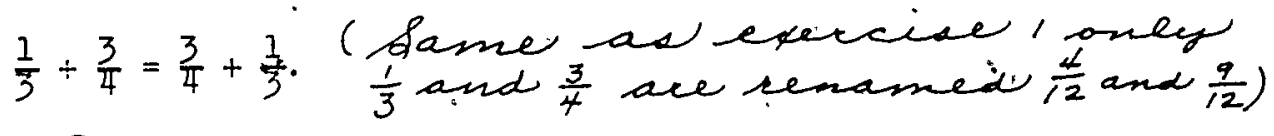

3: Study this sentence: $\left(\frac{7}{4}=n\right)-1=\frac{7}{4}-(n+1)$. Is this sentence true?
(a) if $n=\frac{3}{4}$ ?
(b) if
$n=\frac{1}{4}$ ? (yes)
(c) if $n=\frac{0}{4}$

4. In each sentence what number does $n$ represent the

- mathematical sentence is true? Be carerul. There may be no answer, one answer or more than one answer.
(a) $\frac{3}{4}+n=\frac{3}{4}\left(\frac{2}{4}\right)$
(d) $n=n$ number).
(B) 3.4 (number)
(b) $\frac{7}{4}+n=\frac{21}{10}\left(\frac{7}{20}\right)$
(e) $n+\frac{5}{4}=\frac{5}{4}+n 1$
(h) $n=9.17 p^{2}+n_{2}$
(norationate
(c) $1 \frac{2}{3}+n=3 \frac{1}{4}\left(\frac{19}{12}\right)$
(f.) $11.6+n=11.6^{(0)}(\mathrm{g})$
(norational
$6.432+n=n$ 
5. (a). What number is $n$ if $n+n=\frac{14}{5}$ ?

(b) What number is $n$ if $n+n=3.94 ? .(1.97)$

6. Think of $x, y$ and $-z$ as representing rational numbers. suppose $x+y=z$.

(a) If $x=\frac{15}{4}$ and, $z=\frac{15}{4}$, what number is $y$ ? (a)

(b) (No, thesers) no (b) Can $x$ be greater than, 2 ? Why? rational mumber

(c) If $y=8.94$ and $z=8.94$, what number is " $x$ ? (o)

7. For each of the sertances below, 'n, is a fraction name for a rational number. Make $n$. inave a denominator of 2 . Find $n$ if, each mathematical sentence.is true.
(a) $\mathrm{n}<\frac{1}{2}$
$\left(\frac{0}{2}\right)^{\prime}$.

(b) $\mathrm{n}$ is less than $\frac{9}{2}$ and greater than $\frac{7}{2} \quad\left(\frac{8}{2}\right)$. (c) $\mathrm{n}$ 1s greater than $\frac{13}{4}$ and less than $\frac{15}{4}\left(\frac{7}{2}\right)$ (d) The sum of $\mathrm{n}$ and $\frac{7}{2}$ is less than $\frac{8}{2},\left(\frac{0}{2}\right)$

8. One mathematical sentence showing a'relationship among numbers $n, 7$ 'and 12 is $n+7=12$. Write two mathematical sentences showing different relationships among $\mathrm{n}, 1.75$, and 4.25. Make them so that for each mathematical sentence, $n$ represents a different number. $(n+1.75=4.25 .1 .175+4.25=n)$. 
9. Two numbers to be subtracted are represented by $n$ and $n$. (The two numbers are the same.) John said the result of the subtraction is $\frac{10}{3}$. Was John correct? Why? (nim must be 0 becacuei $n+0=$. )

10. What rational number $n$,will make each mathematical sentence true?

(a) $\left(\frac{3}{2}+\frac{7}{2}\right)+n=\frac{11}{2}\left(\frac{1}{2}\right)$.

(d) $(1.35+6.47)+n=8.92$

(b) $\left(\frac{3}{2}+\frac{7}{2}\right)+\frac{11}{2}=n\left(\frac{21}{2}\right)$

(e) $(1.35+6.47)+8.92=(16.74)$

(c) $n-\left(\frac{3}{2}+\frac{7}{2}\right)=\frac{11}{2}\left(\frac{21}{2}\right)$

(f) $\mathrm{n}-(1.35+6.47)=8.92$

11. Sometimes you have more than two numbers to add. You may be able to make the exercise simpler by changing the order of the addends'. For example; think about

$$
\frac{5}{3}+\frac{1}{4}+\frac{2}{3}+\frac{3}{4}+\frac{5}{3}
$$

You may think $\left(\frac{5}{3}+\frac{2}{3}+\frac{5}{3}\right)+\left(\frac{1}{4}+\frac{3}{4}\right)=4+1=5$.

Find these sums. Change the order of the addends if you think it will make the computation easier.

(Hectare picabably best.)

(a) $2+\frac{2}{3}+3+\frac{2}{3}+\frac{2}{3}\left((2+3)+\left(\frac{2}{3}+\frac{2}{3}+\frac{2}{3}\right)=7\right)$

(b) $\frac{1}{2}+\frac{1}{4}+\frac{7}{4}+\frac{3}{2}+\frac{4}{4}\left(\left(\frac{1}{2}+\frac{3}{2}\right)+\left(\frac{1}{4}+\frac{7}{4}+\frac{4}{4}\right)=5\right)$

(c) $\frac{8}{5}+\frac{7}{6}+\frac{3}{5}+\frac{9}{5}+\frac{11}{6}\left(\left(\frac{8}{5}+\frac{3}{5}+\frac{9}{5}\right)+\left(\frac{7}{6}+\frac{11}{6}\right)=7\right)$

656

211 


\section{Pract1ce Exerc1ses}

I. Solve for, $n$.
a) $54,982+n=80,000$
k) $2 \frac{3}{5}-\frac{4}{5}=n \quad\left(n=1 \frac{4}{5}\right)$
b) $300,678+27,492=n$
1) $32 \times n=5024(n=151)$
c) $658 \times 319=\underset{(n=328,170)}{(n=209,902)} \mathrm{m}) \cdot 2 \frac{2}{5}+\frac{1}{3}+1 \frac{1}{5}=n\left(n=3 \frac{14}{15}\right)$
d). $n \times 85=4,089 n=48)$ n). $77 \times 34618=n(n=2,665,586)$
e) $36 \times n=2,700(n=75)$ o) $2 \frac{1}{6}-\frac{2}{3}=n\left(n=1 \frac{6}{12}\right.$ or $\left.1 \frac{1}{2}\right)$
f) $: 2,340 \div n=36 .(n \div 65)$
g) $6 \frac{5}{8}+7 \frac{1}{8}=n,\left(n=13 \frac{6}{8}\right.$ or $\left.13 \frac{3}{4}\right) \quad n-8 \frac{1}{2}=\frac{5}{12} \quad\left(n=8 \frac{11}{12}\right)$
n) $n+8 \frac{1}{5}=11 \frac{3}{10}\left(n=3 \frac{1}{10}\right)$
1). $\frac{3}{4}+\frac{2}{3}+\frac{5}{6}=n\left(n=\frac{27}{12}\right.$ or $\left.\left.2 \frac{1}{4}\right) / 3\right) \quad 4,006 \times 78=n(n=312,468)$;
r) $n=1 \frac{9}{10}+6 \frac{2}{5}+3 \frac{1}{2}\left(\dot{n}=10 \frac{18}{10}\right.$ or $\left.11 \frac{4}{5}\right)$
j) $185 \times 85=n(n=15.725)$ t) $\frac{7}{8}\left(t+n=4 \frac{1}{2}\left(n=3 \frac{5}{8}\right)\right.$

II. Solve for $n$.
a) $12 \frac{1}{4}-n=8 \frac{1}{2}\left(n=3 \frac{3}{4}\right)$
k) $747.314-288.405=n$
b) $\frac{5}{8}+\frac{1}{2}=\frac{1}{2}+n\left(n=\frac{5}{8}\right)$
1) $\frac{3}{5}+\frac{1}{2}+n=1 \frac{1}{2}\left(n=\frac{4}{10}\right.$ ó $\left.+\frac{2}{5}\right)$.
c) $\frac{7}{8}-\frac{1}{2}=\frac{1}{4}+n\left(n=\frac{1}{8}\right)$
m) $954 \times 384=n_{i}(n=366,336)$
d) $\frac{4}{5}+\frac{3}{10}+\frac{2}{5}=n\left(n: \frac{15}{10}\right.$ or $\left.\left(\frac{1}{2}\right)^{-n}\right) \quad 11 \frac{3}{8}+n=.14 \frac{1}{3}\left(n=2 \frac{23}{24}\right)$
e) $5 \frac{7}{10}-\dot{n}=2 \frac{2}{3}\left(n=3 \frac{1}{30}\right)$
o) $6,400 \div 80=n(n: 80)$
f) $2.45+.7+3.05=n \cdot(n=6.20)^{p)} 52,871 \div n=91 \quad(n=581)$
B) $248.09+n=388.6$
q) $n \times 53=3,498 \quad(n=66)$
h) $\frac{0}{4}+\frac{3}{4}=\frac{3}{4}+n(n=0$;
r) $37,039 \div 46=n \quad(n=805)$
1) $\dot{n}+1 \frac{1}{3}=2 \frac{1}{2}\left(n=1 \frac{1}{6}\right)$
s) $48,369 \times 789=n(n=38,163,141)$
j) $3,354 \div n=39(n \div 86)$
v). $4 \frac{1}{3}+5 \frac{1}{2}+3 \frac{1}{4}=n\left(n=12 \frac{13}{12} \text { or } 13 \frac{1}{12}\right)^{\prime}$ 
$R 390$

III. Add:

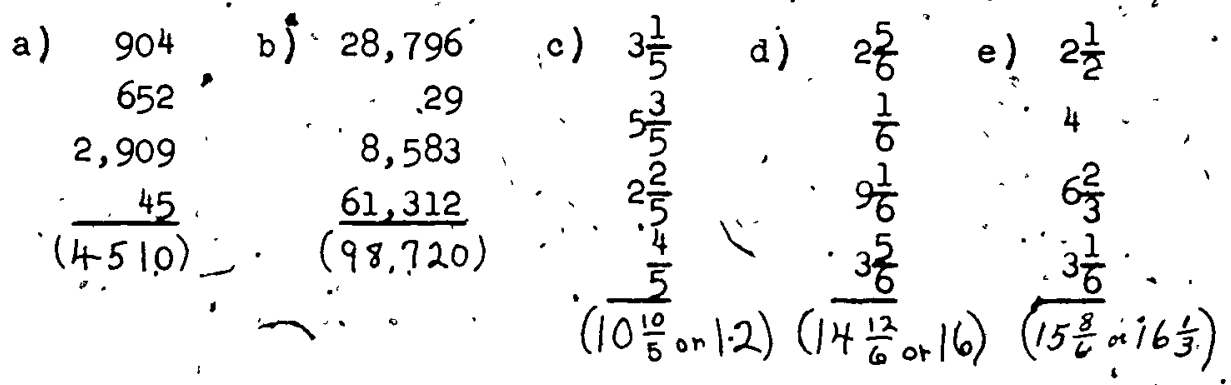

Subtract:

f) 5,934

g) 17,004

h) $11 \frac{7}{10}$

1) $3 \frac{1}{2}$

J) $26 \frac{1}{3}$

$\frac{2,046}{(3888)}$.

$\frac{5,280}{(11,724)}$

$\frac{4 \frac{3}{10}}{\left(7 \frac{4}{10} \text { or } 7 \frac{2}{5}\right)} \frac{1 \frac{1}{3}}{\left(2 \frac{1}{6}\right)}$

$\frac{9 \frac{5}{6}}{\left(16 \frac{3}{6}+16 \frac{1}{2}\right)}$

Multiply:

k) 508

1) 369

m)

n) 957

o) :5;836

$\therefore \quad \frac{67}{(34,036)}$

$\frac{26}{(9,594)}$

$\frac{58}{(20,184)}$

$\frac{39}{(37,323)}$

$\frac{47}{(274,292)}$

Divide:

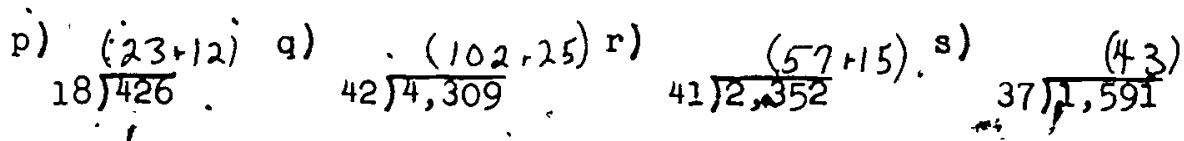

t) $\frac{(93)}{1 8 \longdiv { 1 , 6 7 4 }}$

IV. Find the sum:

a) 264,$829 ; 78,080 ; 1 \ddot{96}, 809919,998 .(559,716)$

b) $\cdot 132 ; 435 ; 412,754 ; 216 ; 734,646^{\circ}(1,280,051)$,

c) $28 \frac{17}{3} ; 8 \frac{1}{2} ; 17 \frac{1}{4} ; 6 \frac{2}{3} ; 19 \quad\left(79 \frac{3}{4}\right)$

d) $4 ; 027.9 ; 617.26 ; 503.07 ; .8(5,149.03)$

e) $\left..219 \frac{5}{8} ; 1,726 \frac{7}{4} ; 63 \frac{2}{3} ; 109 \frac{3}{8}\left(2,118 \frac{11}{12}\right)\right)$. 
P391

V. Subtract:-

a) $678,543,-254,745 \quad(423,79,8)$

b) $800,096-173,295 \quad(626,801)$

c) $128,791-37,782 \quad(91,009)$

. d ) $52,096 \frac{3}{8}-29,636 \frac{3}{4} \cdot\left(22,459 \frac{5}{8}\right)$

e) $212,983-31,006,(181,977)^{\prime}$

VI. -Multiply:

a) $27,465 \times 697^{\circ} \cdot(19,143,105)$

b) $379,865 \times 756-(287,177,940)$

c) $36,492 \times 489 \quad(17,844,588)$

d) $843,476 \times 654:(55 \times, 633 ; 304)$

e) $81,918 \times 248^{\circ}(20,315,664)$

VII. Divide:

a) $\cdot 85,591 \div 95 \cdot(900, r \cdot 91)$.

b) $34,997 \div 34 \quad(1,029+11)$

c) $87,600 \div 67 \quad(1,307 \times 3.1)$

d) $801,356 \div 89(9,004)$

e) $457,267 \div 74(6179+21)$

659

214 
Rev1ew

SET I

Part A

1. Complete each of the following to make 1t a true statement llustrating the distributive property.

- Exanple: $12 \times(20+15)^{\circ}+(12 \times 20)+(12 \times 15)$

a) $"(40+5) \times 22=(40 \times 22)+(5 \times .22)$

b) $=154 \div 7=\left(\frac{140}{6}+14\right) \div 7$

c) $468 \times 15=(424 \times 15)+(44 \times 15)$

(d) $1,824 \div 10^{\circ}=(1000 \div 10)+(800 \div 10)+(20 \div 10)^{\circ}+4$.

e) $: 63 \times 34=(60+\underline{3}) \times(\underline{30}+4)$

2. Answer yes or no" to the questions below.

a) Does $(7 \times 8) \times 3=7 \times(8 \times 3)$ ? (yes).

b). Does $3 \times\left(9, x^{\prime} 5\right)=(3 \times 9) \times 5$ ? (yes).

c) Does $(36 \div 6) \div 3=36 \div(6 \div 3)$ ? (no).

d). Doès $6 Q \div(30 \div 2)=(60 \div 30) \div 2$ ? (no)

e) Does $\left(\frac{5}{4}-\frac{2}{4}\right)-\frac{1}{4}=\frac{3}{4}-\left(\frac{2}{4}-\frac{1}{4}\right) ?($ no $)$ :

s) Does $\frac{6}{12}-\left(\frac{4}{12}-\frac{3}{12}\right)=\left(\frac{6}{12}-\frac{4}{12}\right)-\frac{3}{12} ?($ no $)$

g) Does $(37+1 \cdot 3)+g^{m}=37+(13+9) ?$ (yes)

h) Does $26+(32+10) \div(26+32)+10 ?$ (yes $)$

1) Does $(25-13)-7=25-(13-7)($ no $)$

j) Does, $75=(50-25)=(75-50)-25 ?($ no $)$

k). Does $\frac{3}{4}+\left(\frac{1}{4}+\frac{2}{4}\right)=\left(\frac{3}{4}+\frac{1}{4}\right)+\frac{2}{4}$ ? (yes)

1) Does.. $\left(\frac{2}{6}+\frac{3}{6}\right)+\frac{1}{6}=\frac{2}{6}+\left(\frac{3}{6}+\frac{1}{6}\right) ?$ (yes)

In the exercises above tell which examples 1llustrate the associative property: $(a, b, j, h, k, 1)$ 
'P393

3. Write the following expressions as decimal numerals. - Example a. is done for you.

a) $7+\sqrt{ } .6+.05=8.65$

f): $16+1.6+.16(.17 .76)$

b) $2+.3 \pm .06(=2.36)$

g) $3+.2+-.75(=3.95)$

c) $5+.2+.17(=5.37)$

h) $61+.3+.81(-62.11)$

d) $\cdot 21+.4+.22(-21.62)^{\circ}$

1) $8+2.5+.52(-11.92)$

e) $9+.8+.23(=10.03)$. j) $19+9.7+.36 \cdot(=29.06)$

4. Express each answer in its simplest form.

a) $\frac{3^{\circ}+1}{2+2}+\frac{4+1}{3+5}=n\left(n=1 \frac{5}{8}\right)$

f) $\frac{3}{4}+\frac{3}{8}=n \quad\left(n: 1 \frac{1}{8}\right)$

b) $\frac{2+3}{1+2}-\frac{2+2}{3+3}=n(n=1)$

8) $\frac{3 \times 3}{2 \times 3}-\frac{1 \times 2}{1 \times 3}=n\left(n=\frac{5}{6}\right)$

c) $\frac{5+1}{2+2}-\frac{2+3}{3+3}=n\left(n=\frac{5}{12}\right)$

h) $\frac{4+3}{4+2}+\frac{2+1}{3+1}=n \cdot\left(n-1 \frac{11}{12}\right)$.

d) $\frac{4+0}{1+2}+\frac{5+2}{5+7}=n_{y}\left(n=1 \frac{4}{12}\right)^{1}$

1). $n=\frac{2 \times 1}{3 \times 1}-\frac{3 \times 1}{5 \times 2}$

$\left(n=\frac{16}{30}\right)$

e) $\frac{-4+1}{3+3}-\frac{2+1}{4+4}=n\left(n=\frac{11}{24}\right) j$

f) $\frac{4}{5}+\frac{5}{8}=n \cdot\left(\frac{k}{n}=1 \frac{17}{40}\right)$.

5. Writers base tee numeral 18.

a) 24 five $(14)$

f) 43 eight (35)

b). 312 four $(54)$

g) $4381 x$

(27)

c) $64^{4}$ seven $(46)$

h) 322 four $(58)$

d) $30 i_{\text {five }}(76)$

1) $441_{\text {five }}(121)$

e) 212 three $(2,3)$,

j) 645 seven $(327)$

.661

210 
P394

6. Complete the chart below. Example a 1 s worked for you.

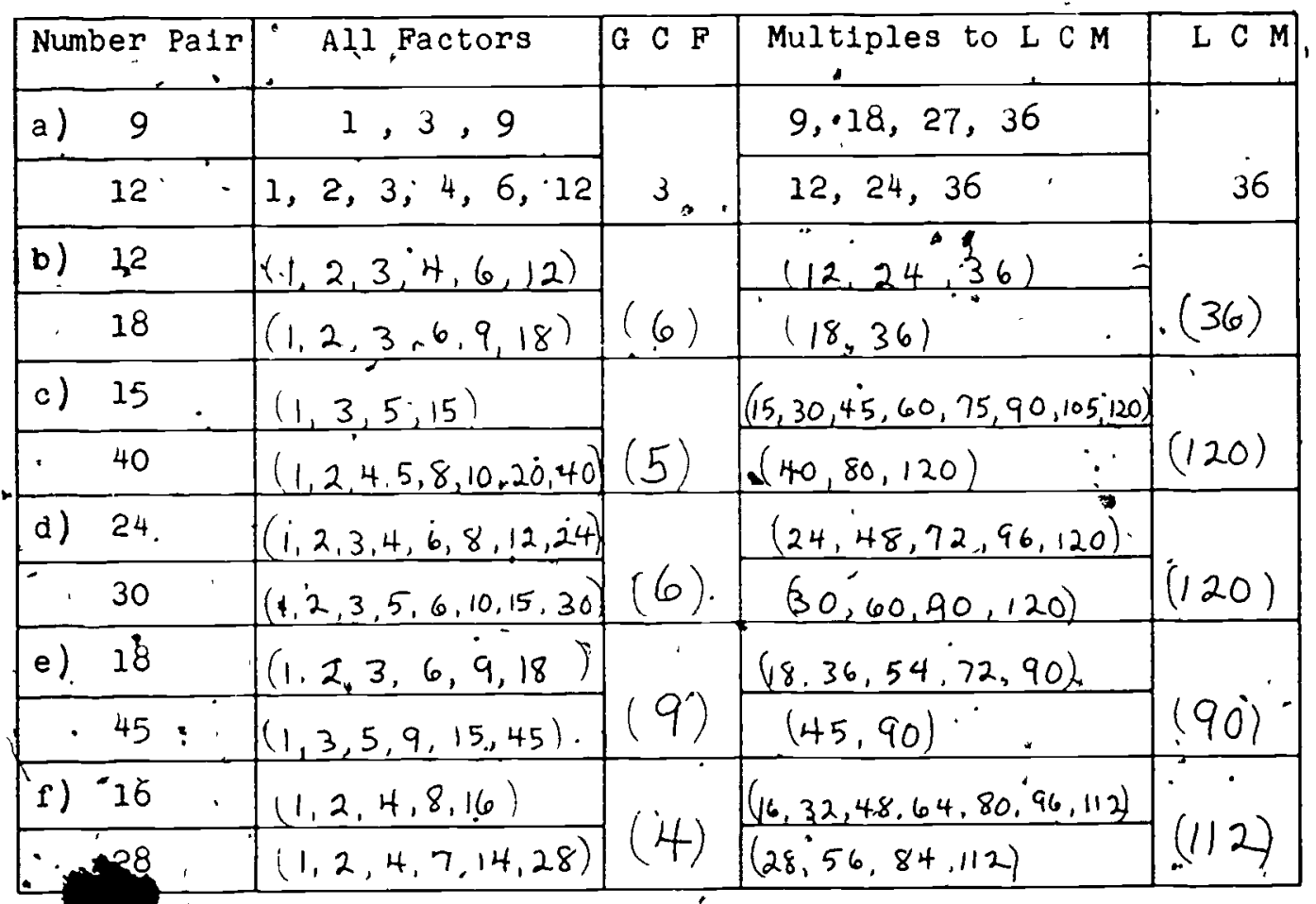

7. For your answers do not depertd upon the appearance of the triangle. Use only the facts given.

a) In these two triangles,

we know that

$$
\begin{aligned}
& \because \angle A \cong \angle B_{A} . \\
& \therefore \overline{A C} \cong \overline{B E} \\
& \therefore \quad \overline{A D} \cong \overline{\mathrm{BF}}
\end{aligned}
$$
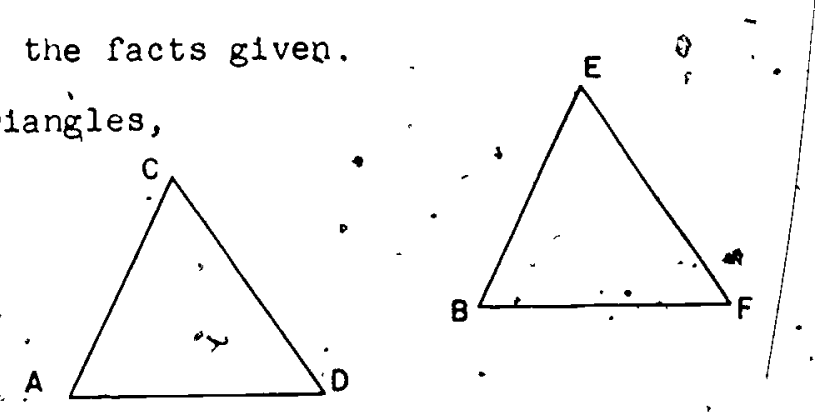

What do we know about $\angle \dot{D}$ and $\angle D, \angle C$ and $\angle E$, also $\overline{C D}$ and $\overline{E F} ?(\angle D \cong L F, \angle C \cong \angle E, \overline{C D} \cong \overline{E F})$

.662

$2\}$ 
"b). $A \dot{B} C$ is a scalene triangle.

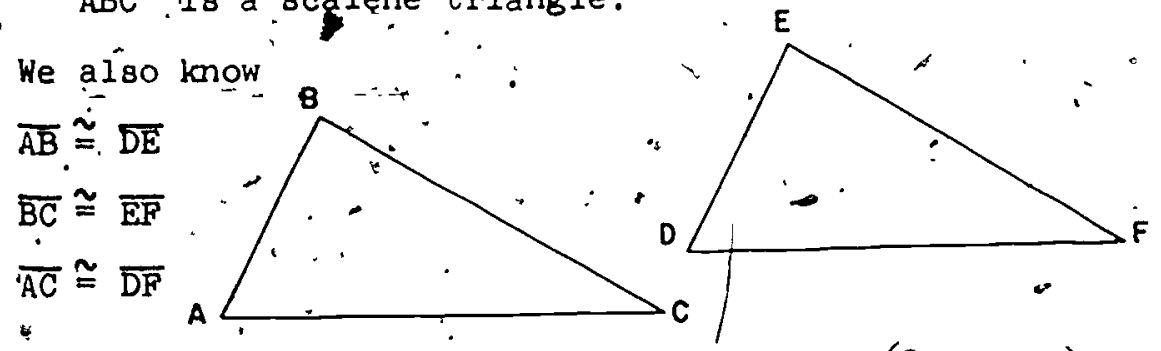

What kind of a triangle would. $\triangle D E F$ be? (Scalene) 7. List the palrs of congment angles. ( $\angle A \cong \angle D, \angle B \cong L E$,

\section{$\sim$ Part B}

Write a mathematical sentencé (ór two senténces if necessary) f for each problem and solve. Write an answer sentence.

1. A hostess made $1 \frac{6}{8}$ gals. of punch for a party. She had

$\therefore \frac{7}{8}$ gal. left. How much punch did the guests drink?

$\left(1 \frac{6}{8}-\frac{1}{8}=n\right.$ or $\frac{7}{8}+n^{\prime}=1 \frac{6}{8}, n=\frac{7}{8}$ The guests drank $\frac{7}{8}$ gal punch.)

2. Wendy lives $8.6, \mathrm{~m} 1$. Lrom her friend's house. One day she rode her horse part of the way to het frlend's house. - She walked the rest of the way. She walked $1.23 \mathrm{ml}$ " How far did she ride before she started to walk to her friend's house? $\cdot(8.6-1.23=n$, or $n+1.23=86, n=7.37$ Wendy rode. $7.37 \mathrm{mi}$.)

3. In 1864 Abraham LIncoln was elected President for a second term. He recelved $2,216,067$ votes. George Mcclellan ran against $\mathrm{h} 1 \mathrm{~m}$ and recelved $1,808,725$ votes. How many fewer votés than Lincoln did McClellan recelve?

$(-2,216,067-1,808,725=n, n=407.342$ Mclellan received 407.342 fewer votes than hincoln.)

4. When Sandra's father welghed her he sa1d, You weigh exactly 58 .1bs. You have gained $1.8^{r}$ lbs. s1nce your. b1rthday " How much did. Sandra we1gh on her b1rthday? $=(58-1.8, n, n=56.2$ or. $1.8+n=58$ Sandira weighed 562165 . On her" birth day.) 
5. The Emplre State Building was sold in 1951 for $\$ 51,000,000$. Th1s is three times the amount pata for the land on which 1t. stands. How much did the land cost? $(51,000,000 \div 3=n$ or $3 \times n=51,000,000 ; n=17,000,000$ "The land $\operatorname{cost} \$ 17,000,000.00.)^{2}$ 6 . One week B1li worked for $\frac{3}{4} \mathrm{hr}$. on Monday, $1 \frac{1}{2}$. $\mathrm{hr}$. on Tuesday, $\frac{1}{2} \mathrm{hr}$. on Wednesday, $1 \frac{2}{8} \mathrm{hr}$. on Thursday, and 2 hrs. on Friday, He is pald 65 cents an hour. How much did he make this week? $\left[\left(\frac{3}{4}+1 \frac{1}{2}+\frac{1}{2}+1 \frac{2}{8}+2\right) \times 65=n\right.$ or $3 / 4+1 \frac{1}{2}+\frac{1}{2}+1 \frac{3}{8}+2=t ;, t=6,6 \times 65=n, n=390$ B.ll made 3.90 .1$]$

Individual Projects

i. You have developed mules for divisibility by the numbers 2, 3 and 5 and have given examples in which they are tested as factors of a number. Here are some rules for. divisibility for you to test. You should try at least five examples to see if the rule is true.

a). A number is divisible by .4 if two times the tens digit plus the units digit is divisible by 4.

b) A number is divisible by 6 if the number is even and is divisible by 3 .

c) A number is divisible by 7 if the difference between. twice the units digit and the number formed by omitting the units digit is divisible by, 7.

d) A number is divisible by 8 .if four times the hundreds digit plus two times the tens digit plus the units digit is divisible by 8 .

e) A number is divisible by 9 . "If the sum of the digits is divisible by 9 . 
P397

Review

SET II

Part A

1. Write the numerator and denominator as the product of primes. Example a is done for you.

a) $\frac{4}{16}=\frac{2 \times 2}{2 \times 2 \times 2 \times 2}$

d) $\frac{15}{24}\left(\frac{3 \times 5}{2 \times 2 \times 2 \times 3}\right)$

b) $\frac{12}{42}\left(\frac{2 \times 2 \times 3}{2 \times 3 \times 1}\right)$ :

e) $\frac{10}{25}\left(\frac{2 \times 5}{5 \times 5}\right)$

c) $\frac{63}{81}\left(\frac{3 \times 3 \times 7}{3 \times 3 \times 3 \times 3}\right)$

f) $\frac{12}{8} \cdot\left(\frac{2 \times 2 \times 3}{2 \times 2 \times 2}\right)$

2. Select the symbols that represent zero from each row.

a) $\frac{3}{5}-\frac{6}{10} ; 42 i+0 \% 0 \div 6$

$\frac{6-6}{3} 12 \times 0$.

b) $\frac{8}{8}, \frac{4-4}{2-2}, 0 \times 364, \frac{12}{8}-\frac{3}{4}, 75-0$

c) $0 . \times 6,5-\frac{10}{2},(12 \times 3) \times 0, \frac{3}{3}, \frac{8-8}{6}$

d) $10.6-10.60,0+982,7 \times 0, \frac{6+2}{8}, 0 \div 12$

3. Copy and place parentheses to make each a true statement, then solve. Example a is shown.

a) $(3 \times 5)+7=20+2,22=22$

b) $\left.18, x(3+3)=108(108=108)_{r}\right) \quad 48+(10 \div 2)=(6 \times 10)-77$ '

c) $(32 \div 8)+4=6+2(8=8)$

g) $7+(49 \div 7)=(12-2)+4$

d) $44=50-(12 \div 2)(44=44) h) \quad 15 \times(12 \div 6)=(24 \div 4) \times(30=30)$

e) $72 \div(4 \times 2)=(6-2)+5$

1) $45 \times(24 \div 6)=(54 \times 3)+18$ $(9=9)$

j) $(31 \times 22)+18=(35 \times 40) \div 2$ $(700=700)$

665

220 
P398

4. Rename the following in simplest mixed form.

a) $\frac{7}{2} \quad\left(3 \frac{1}{2}\right)$

e) $\frac{14}{4}\left(3 \frac{1}{2}\right)$

b) $\frac{14}{3}\left(4 \frac{2}{3}\right)$

f) $\frac{21}{9}\left(2 \frac{1}{3}\right)$

c) $\frac{17}{4} \cdot\left(4 \frac{1}{4}\right)^{\circ}$

в) $\frac{17}{5}\left(3 \frac{2}{5}\right)$

d) $\frac{15}{6}\left(2 \frac{1}{2}\right)$

n) $\frac{18}{4} \quad 4 \frac{1}{2}$

5. Copy and replace $n$ with the number $n$ represents. An example is shown.

a) $\frac{3}{4}=\frac{n}{16}, \frac{3}{4}=\frac{12}{16}$

e) $\frac{5}{n}=\frac{15}{24},\left(\frac{5}{8}=\frac{15}{24}\right)$

b) $\frac{2}{3}=\frac{n}{15},\left(\frac{2}{3}=\frac{10}{15}\right)$

f) $\frac{n}{3} \cdot \frac{12}{9},\left(\frac{4}{3}=\frac{12}{9}\right)$

c) $\frac{7}{5}=\frac{21}{n}-\left(\frac{7}{5}=\frac{21}{15}\right)$

8) $\frac{3}{6}=\frac{n}{18} \cdot\left(\frac{3}{6}=\frac{9}{18}\right)$

d) $\frac{n}{4}=\frac{20}{16} \cdot\left(\frac{5}{4}=\frac{20}{16}\right)$

n) $\frac{8}{7}=\frac{32}{n},\left(\frac{8}{7}=\frac{32}{28}\right)$

6. Use the largest multiple of 10,100 , or 1,000 to make each of these true sentences. Example a 18 shown.

a) $9 \times 7000<63,801$

f) $53,871>6000 \times 8$

b) $20 \times 90<1,848$

в) $80 \times 9000<764,892$

c) $4,328>600 \times 7$

h) $70 \times 7<535$

d) $300 \times 30 \cdot<10,380$

1) $38,462>90 \times 400$

e) $5,161>6 \times 800$

j) $3000 \times 30<96,483$.

666

221 
P399

7. Which of these numbers are equal to $1 \frac{1}{2}$ ?

$$
1.5, \frac{7}{5}, 1.05, \frac{9}{6}, \frac{72}{8},\left(1.5, \frac{6}{8}, \frac{12}{8}\right)
$$

- Which of these numbers are equal to 3 ?

$$
\frac{12}{3}, 2+\frac{5}{5}, 1 \frac{8}{4}, \frac{24}{6}, 2.10 \quad\left(2+\frac{5}{5},-1 \frac{8}{4}\right)
$$

Which of these numbers are equal to $\frac{3}{4}$ ?

$$
\frac{9}{12}, 8-7 \frac{1}{2}, .75, \frac{15}{20}, \frac{8}{12}\left(\frac{9}{12}, 75, \frac{15}{20}\right) \text {. }
$$

Which of these numbers are greater than $\frac{2}{3}$ ?

$\frac{9}{12}, \frac{9}{15},: 7, .285, \frac{5}{9}\left(\frac{9}{12}, .7\right)$

Which of these numbers are less than $\frac{5}{6}$ ?

$$
.7, \frac{11}{12}, .90, \frac{3}{4}, .065 \quad\left(7, \frac{3}{4}, .065\right)
$$

8. Use the word plane, line, line segment, ray, circle or quadrilateral to complete these statements.

a) A sheet of paper could be thought of as a model of a (plane).

b) A clothes line stretched tightly between two poles could be thought of as a model of a (line segment).

c) A wedding ring could be thought of as a model of " at (circle).

d) A window frame could be thought of as a model of a (quadrilateral).

e) The beam of light from a spotlight could be thought of as a model of a (ray). 
9. Construct two congruent triangles with sides whỡ in inches are 3,2 , and 4 and whose intersection is one vertex. The interior of one triangle should be in the exterior of the other.

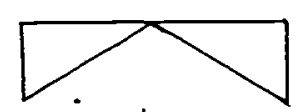
(not to scale, also, answers may vary)

10. Construct two triangles with one common side. One triangle i has sides whose length in inches are 5,3 and 3 . The second triangle has sides whose lengths in inches are 2,2 and 3 . The interior of one will be in the interior of the other.

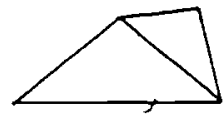
(not to scale, answers will vary)

11. Construct two triangles with one common side. One triangle has sides whose lengths in inches are 4,4 and 6 . The second triangle has sides whose lengths in inches are 3 , 3 and 4 . The interior of the second triangle w111 " be in the exterior of the first

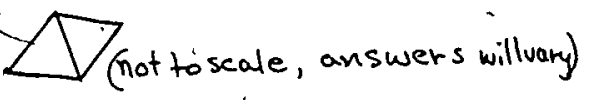

Part B

Write a mathematical sentence (or two sentences if necessary) for each problem and solve. Write an answer sentence.

1. Mary has $\frac{1}{2}$ c. flour. She looked at three recipes for cupcakes, one uses $\frac{7}{24}$ c. flour, another uses $\frac{2}{6}$ c. flour, and the third uses $\frac{4}{8}$ c. flour. Which recipe, will use all of her flour? $\left(\frac{1}{2}=\frac{12}{24} ; \frac{7}{24} ; \frac{3}{6}=\frac{8}{24}, \frac{4}{8}=\frac{12}{24}, \frac{4}{8}=\frac{1}{2}=\frac{12}{24}\right.$

The third recipe uses $\frac{4}{8} c$. or $\frac{1}{2} c$ flour, all of her flour.)

2. The German bobsledding team made a trial run in 5 minutes, 07.84 , seconds. The United States bobsledding team made their trial run in 5 "minutes, 20.1 seconds. Which team $(20.1-07.84=n ; n=12.26$
made the faster time? How much faster? The German team made better time, 1226 seconds faster.) 
P 401

3. A parking lot has 24 rows for cars. Each row holds 32 cars. How many cars are in the lot when the rows are filled? $(24 \times 32=n, n=768$ There are 768 cars in the lot.)

4: In the Soap Box Derby John finished h1s run in $2.7 \mathrm{~min}$. Mac finished in $2.68 \mathrm{~min}$, and Terry finished in $2.07 \mathrm{~min}$. What was the difference in time between the fastest and slowest runs? $(2.70-2.07=n, n=.63$
Terry's time was .63 min. faster than John's)

5. If the speed of a meterold moving through space averages 30 miles per second, what will be 1 ts average speed per hour? $\left(30 \times 60 \times 60=n\right.$ or $30 \times 60=t, t \times 60=n, n=108,000^{\circ}$ The meteoroid w.ll have an average peed of $108,000 \mathrm{~m} . \mathrm{p} . \mathrm{k} .$.

6. A gallon of water welghs $8.33 \mathrm{lbs}$. It is carried in a bucket weighing $1.8 \mathrm{lbs}$. What $1 \mathrm{~s}$ the total weight of the galion of water and the bucket? $(1.8+8.33=n \quad n=10.13$ The total weight will be $10.13165 .:)$

Puzzles

1. What number base 18 used in each of these?

a) Jan sa1d, "My fat weighs 2 pounds, or 112 founces."

b) My little sister 1s, 100 two years old. In one year she w11l be entering the first grade. (Base Two)

- c) The teacher 18 five feet $81 x$ inches or 73 "s inches tall.

2. Cross out query dot with four ine segmenta. Do not iff the pencil from the paper until all nine dots are crossed. Do not retrace a liṇe or, cross any dot morè than once.

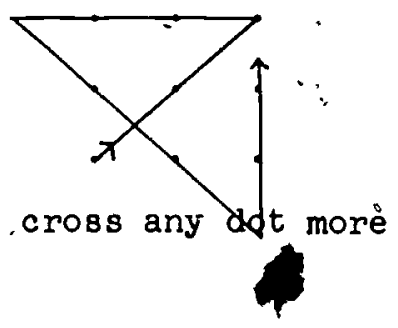


P402

Review

SET III.

Part A

I. Arrange the following numbers in order from least to greatest.

a) $\frac{1}{2}, \frac{1}{3}, \frac{1}{6}, \frac{1}{4}, \frac{1}{5} \cdot\left(\frac{1}{6}, \frac{1}{5}, \frac{1}{4}, \frac{1}{3}, \frac{1}{2}\right)$

b) $\frac{3}{8}, \frac{7}{8}, \frac{4}{8}, \frac{1}{8}, \frac{5}{8}\left(\frac{1}{8}, \frac{3}{8}, \frac{4}{8}, \frac{5}{8}, \frac{7}{8}\right)$

c) $.7,1.4, .73, .29, .4(.29, .4, .7, .73,1.4)$

d) $\frac{1}{2}, \frac{2}{3}, \frac{3}{8}, \frac{1}{6}, \frac{1}{4}\left(\frac{1}{6}, \frac{1}{4}, \frac{3}{8}, \frac{1}{2}, \frac{2}{3}\right)$

e) $\frac{5}{12}, \frac{3}{8}, \frac{11}{24}, \frac{7}{12}, \frac{5}{6}\left(\frac{3}{8}, \frac{5}{12}, \frac{11}{24}, \frac{7}{12}, \frac{5}{6}\right)$

f) $.29, .029,2.9,29, .5(.029, .29, .5,2.9,29)$.

g) $: 8, .46,2, .059, .4(.059, .4, .46, .8,2)$.

n) $51 ., .5,5.01,5.1, .51(.5, .51,5.01,5.1,51)$

2. Using the symbol >, =, or < make the following true sentences.

a) $28 \times 2 \leq 154+88$

b) $24 \times(24 \times 6)=6 \times(24 \times 24)$

c) $32 . \times 127 \leq 4,060+.24$

d) $\frac{4}{10}=.40$

e) $\frac{3}{5} \geq \frac{6}{12}$

f) $31,106 \geq 74 \times 419$

в) $47 \times 608=28,576$

h) $\frac{2}{5}+\frac{1}{2}+\frac{2}{5} \leq 1.4$

1) $.6+2.15+.25>2.9$

j) $2,605 \div 56=46 \times 29$

670

225 
P403

3. Write fraction names for these numbers.- Example a is done for you.
a) $5 \frac{1}{3}=\frac{16}{3}$
r. . . $73\left(\frac{23}{100}\right)$
b) $1.1 \cdot\left(\frac{11}{10}\right)$
g) $7 \frac{1}{4}\left(\frac{29}{4}\right)$
c) $8 \frac{3}{5} \quad\left(\frac{43}{5}\right)$
h) $25 \frac{1}{2}\left(\frac{51}{2}\right)$
d) $7 \frac{7}{9} \cdot\left(\frac{70}{9}\right)$
1) $4.8\left(\frac{48}{10}\right)$
e) $2.53\left(\frac{253}{100}\right)$
j) $12 \frac{4}{8}\left(\frac{100}{8}\right)$

4. Without working the problem, tell which expression in each row represents the largest number.
a) $253 \div 15,253 \div 26,253 \div 19,253 \div 39$
b) $341 \times 23,314 \times 23,336 \times 23,364 \times 23$
c) $\frac{4}{8}+\frac{1}{3},: \frac{4}{8}+\frac{3}{3}, \frac{4}{8}+\frac{4}{3}, \frac{4}{8}+\frac{2}{3}$
d) $2,192 \div 28,1,301 \div 28,1,099 \div 28$,
$1,900 \div 28$
e) $\frac{2}{3}-\frac{1}{2}, \frac{2}{3}-\frac{1}{4}, \frac{2}{3}-\frac{4}{6}, \frac{2}{3}-\frac{5}{8}$

5. In the above examples find the expression in each row that names the smallest number. (a) $253 \div 39$; b) $314 \times 23$;
c) $\frac{4}{8}+\frac{1}{3}$
d) $1099 \div 28$
e) $\left.\frac{2}{3}-\frac{4}{6}\right)$

6. Write the greatest common factor for each pair.
a) 28,35
(?).
f). 72,30
(6)
b) 40,54
g) 12,84
c) 27,54
(27)
h) 42,70
d) 18,60
e) 25, 120
1) 225,45 .
j) 33, 363 
7. Copy and place parentheses to make each à true statement.

a) $18 \times 23 \div 9=9 \times 92 \div 18 \cdot[(18 \times 23) \div 9(9 \times 42) \div 18]$

b) $31 \times 50 \div 2=24 \times 33-17[31 \times(50-2)=(24 \times 33)-17]$

c) $64 \times 23: 4>26+17 \times 43[(64 \times 23)-4>26+(17 \times 43)]$

d) $42 \times 24+3 \neq 21 \times 54[(42 \times 24)+3 \neq 21 \times 54]$.

e) $43=9 \times 16=4+27 \times 20 \cdot[(43-9) \times 16=4+(27 \times 20)]$

f) $36+18 \times 47<48 \times 12+8[36+(18 \times 47)<48 \times(12+8)]$

g) $27 \times 96+8-13 \times 196+4[(27 \times 96)+8=13 \times(1 \overline{96}+4)]$

h) $65^{\prime \prime} \times 64+30>36 \times 113+\dot{8}[(65 \times 64)+30>(36 \times 113)+8]$.

8. Use closed or not closed to complete and make these true sentences.

a) The set of whole numbers is (closed) under addition.

b) The set of odd numbers is (not closed) under subtraction.

c) The set of counting numbers is (closed) under addition. -

d) The set of whole numbers is (dosed) under multiplication.

* e) The set of whole numbers is (not closed). under division.

r) The set of counting numbers from, 23 to 75 is (not closed) under addition.

8) The set of whole numbers is (not closed) under subtraction:

h) The sat of counting numbers less than 43 is (not closed) under muitiplication.

9. Copy and compare the sizes of : $\angle$ RST and - $\angle \dot{C} A B$, $\angle T R S$, and $\angle B C A, \angle R T S$ and $\angle C B A$.

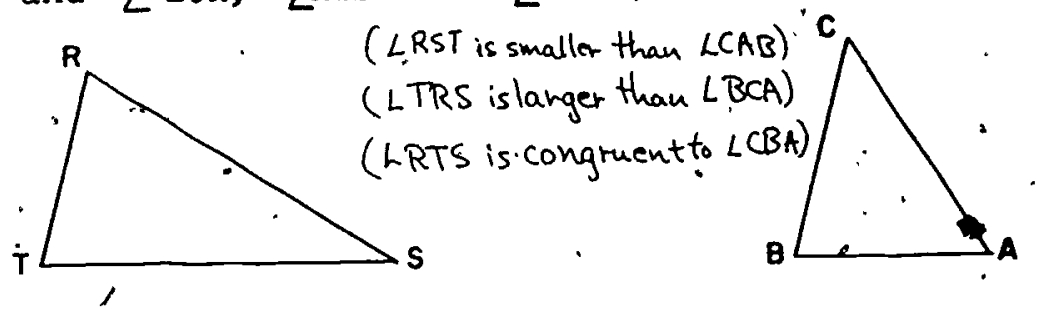


10. Use your compass and straightedge to copy $\angle \mathrm{BAC}$ on $\overleftrightarrow{\mathrm{RS}}$ so. that point $S$ corresponds to $A$ and $\overrightarrow{A B}$ falls on $\dot{S R}$.

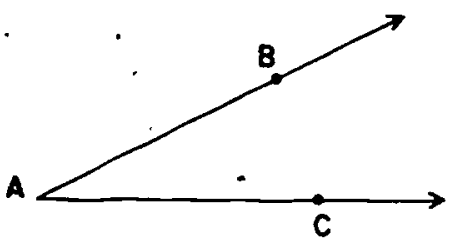

Part $\mathrm{B}$.

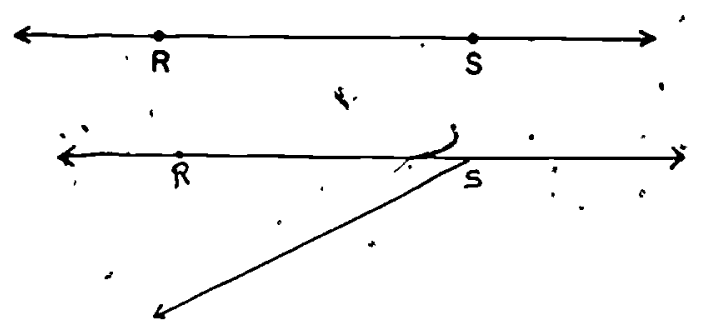

Write a mathematical sentence (or two sentences if necessary) for each problem and solvé. Write an answer sentence.

1. There are 40 pages in each,Orange Trading Stamp Book. Each page holds 35 stamps. How many stamp's will be, reeded to fill one book? $(35 \times 40=n, n=1400$ 1400 stamps will be needed to fill one book.

2. Smith's Department Stọe ordered 1608 Christmas tree ornaments from Japan. They arrived in 67 boxes with the same number of ormaments in each box.' How many ornaments " were in each box? $(1608 \div 67=n, n=24$ There are 24 ornaments in each box.)

3. The food committee for the class picnic ordared fiamburgers." . Five-eighths of the class wanted hamburgers with onions. What part of the class wanted theins w1 thout onions? ( $1-\frac{5}{8}=n, n=\frac{3}{8} \quad \frac{3}{8}$ of the class wanted theirs withent onions.) 4. On its picnic, the class took $2 \frac{1}{3}$ gals. 1ce cream. They used $1 \frac{2}{5}$ gals. for sundaes. How much lce cream was left for cones? $\left(2 \frac{1}{3}-1 \frac{2}{5}=n, n=\frac{14}{15}\right.$

They had $\frac{14}{15}$ gal, ice creami left for cones.). 
5. The Campire Girls in one town sold 426 boxes of carty the

- first week of their sale, 281 boxes the second week; and 469 boxes the third 'week. What was the average number of boxes sold each day? $\{(426 \div+281+469) \div 21=n$ or $426+281+469=p$

(The girls sold an average of 56 boxes a day.)
6. Three frying chickens weigh $1 \frac{5}{4}$ pounds, $2 \frac{1}{2}$ ppunds and. $2 \frac{1}{4}$ pounds. What is their total weight? $\left(1 \frac{3}{4}+2 \frac{1}{2}+2 \frac{1}{4}=w, \omega=6 \frac{1}{2}\right.$ ( Their total weight is $\left.6 \frac{1}{2} / 65 ..\right)$

7. The speedometer of a car shows $74,286.1$ miles at the end of the month. The car had gone $3,729.4$ miles that month.

What had the speedometer shown at the beginning of the month? $(74,286.1-3,729.4=r, r=70,556.7$

'The speedometer had shown 70,556.7 miles.)

8. A box factor makes 2,940 soap boxes in one hour. How many. dozen boxes are made in one continuous eight hour shift? $[(2940 \times 8) \div 12=t$ or $(2940 \div 12) \times 8=t$ or $2940 \times 8=d, d \div 12=t, t=1960]$ (There are 1960 dozen boxes made in eight hours.)

Individual Projects

1. Mfere model of a geometric prism. The measure of the - shortest edge should be no less than 3 inches. Color the faces so that none of the faces with a common edgé are the same color.. Display it for your'class.

2. Make a model of a polygon. Use wire for the line segments.. The measure of the shortest. segment should be no less than 4 inches. You'could use two or more of these. to. make an interesting mobile for your class.

3. Many great men have made important contributions to mathematics. Make a report about one of these famous mathematiclans and his contributions.. 


\section{Chapter 7}

MEASUREMENT OF ANGLES

PURPOSE OE UNIT

- The purpose of this unit is to develop understanding and skill in the measurement of angles. Like the study of inear measurement the study of angular measurement may be divided into four major considerations which parallel the historical development :

I. Intuitive awareness of difference in size.

- 2. Choice of an arbitrary unit with the understanding that the unit must be of the same nature as the 'thing to be measured-a line segment to measure a line segment, an angle to measure an angle, etc."s

3. Selection of a standard unit for purposes of.

- comiunication.

4. Designing a suitable scale for convenience in measuring. 
It' is of Anterest to 'note that in the development of the ldea" of measurement for the pupils, we are actually following the historical development of this concept. The counting of discrete. or separate objects (like finding the number of sheep in a' herd) was not a technique applicable to the measure of a continuous curve (like determining the length of the boundaries of a wheat field). At first the notion of size was realized intuitively. one boundary was longer than another; one, plece of iand was larger than another. This surficed until fields bordered more closely on eàch other and more rerined measures were necessary. Then a unit of measure (e.g. that part of a rope between two knots) was agkeed. upon. Now it was possible to designate a piece of property as having a length of "50 units of rope" and having a width of "30 units of rope." With the increase of travel and communication, it became obvious that "50 units of rope," did not represent the same length to all people unless they were ramiliar with the unit. The need for a standard unit arose. Once a standard unit was agreed upon, a scale was devised for greater convenience in measuring.

Basing the development of the concept of angle measurement upon these four considerations, appeal is first made to the "pupilis intustion in making comparisons of the sizes of angles. Recall from. Ch. 4 (Congruence of Common Geometric Figures) that an angle is a set of points consisting of two rays with $p$ common endpoint, but not both" on the same ilne. Consider any two angles, say $\angle A B C$ and $\angle D E F$, as pictured below.
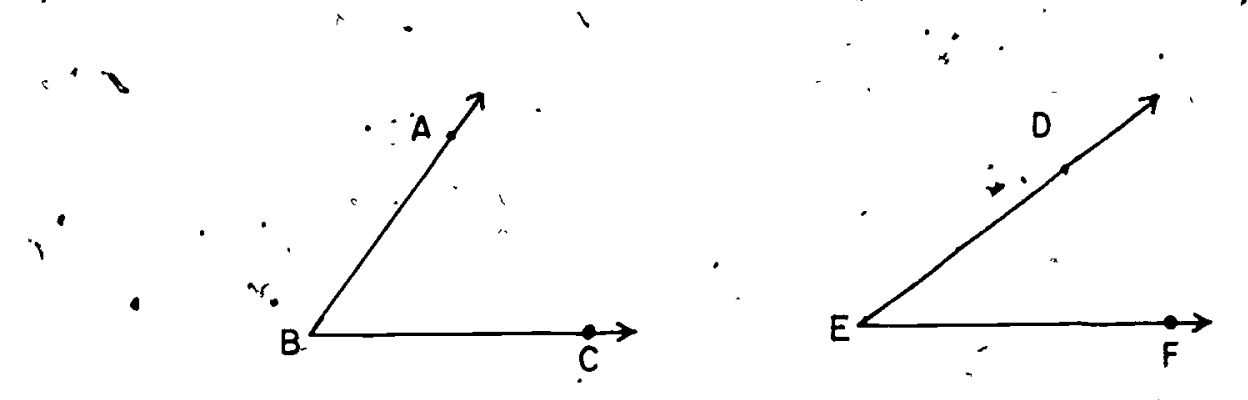
We can conceive of placing these angles one on top of the other in such a way that $\overrightarrow{B C}$ and $\overrightarrow{E F}$ coincide while $\overrightarrow{B A}$ ante $\overrightarrow{E D}$ both extend above $\overrightarrow{B C}$, thus.

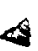

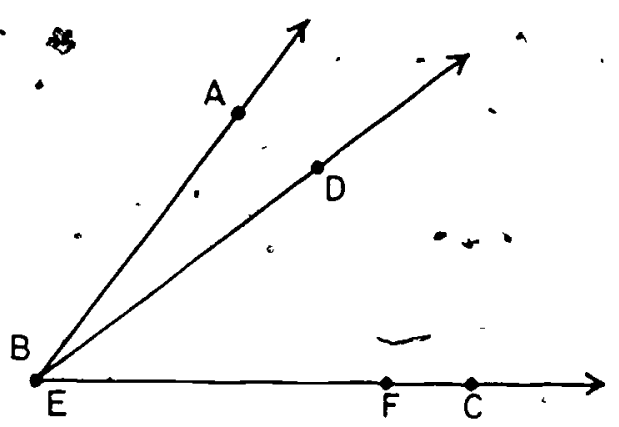

In the example illustrated above, $\overrightarrow{E D}$ extends into the interior of $\angle A B C$. We say that $\angle D E F$ is smaller than $\angle A B C$. If instead it happens that $\overrightarrow{E D}$ coincides with $\overrightarrow{B A}$, we say that $\angle D E F$ is of the same size as $\angle A B C$. (Recall that in this case we also say that $\angle A E C$ and $\angle \mathrm{DEF}$ are congruent.) If $\overrightarrow{\mathrm{ED}}$ extends into the exterior of $\angle A B C$, as shown below, we say. $\angle D E F$ is greater than $\angle A B C$.

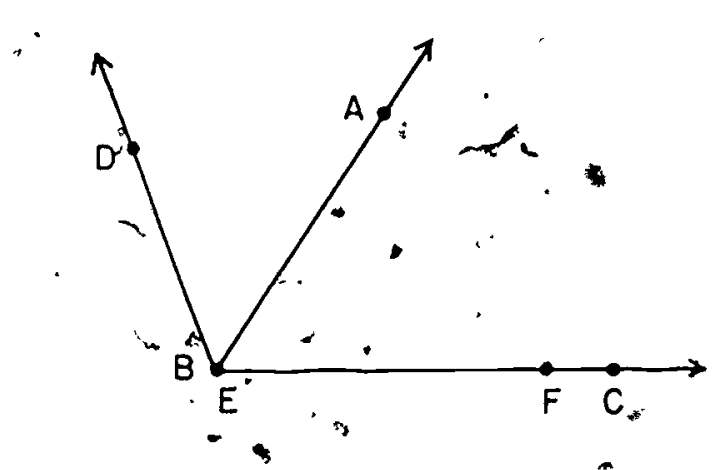


Thus, given $\angle A B C$ and $\angle D E F$, exactly one of the following three statements is true:

1. $\angle D E F$, is of smaller size than $\angle A B C$.

2. $\angle D E F$ is of the same size as $\angle A B C$.

13. $\angle \mathrm{DEF}$ is of greater size than $\angle \mathrm{ABC}$.

Just as we thtnk of every, ilne segment as having a certain.. exact length, so too we think of every angle as having a certain exact size, even though this size can be determined only approximately by measuring a chalk or penc1l drawing representing 1 t.

Let us examine this process of angular measurement more, closely. As in the case of linear measurement, the first step is to choose'a certain angle to serve as unit. Th1s means that we select an angle and agree to consider its size to be described or measured, exactly, by the number 1 . Cail this LRST: .

A

Unit Angle.

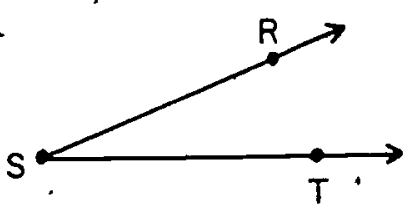

Now we can concleive of forming an angle DEF by laying off the unit $\angle \mathrm{R} g \mathrm{~T}$, twice about a common vertex $\mathrm{E}$ as suggested in the picture beIow.

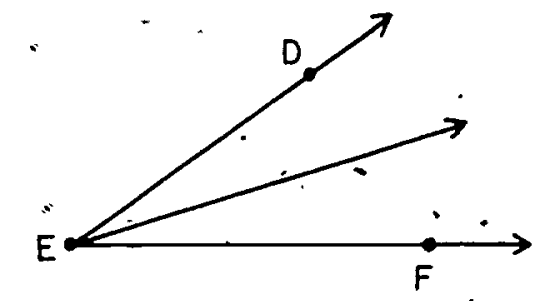

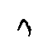


We say that $\angle \mathrm{DEF}$ has size exactly 2 units, although $\angle \mathrm{DEF}$ can be represented oniy approximately by a drawing.

In similar fashion we can concelve of forming an angle of size exactly 3 units, or exactly 4 units, and so forth, unt1l we have drawy an angle whose interior is nearly half' a plane, as shown below.

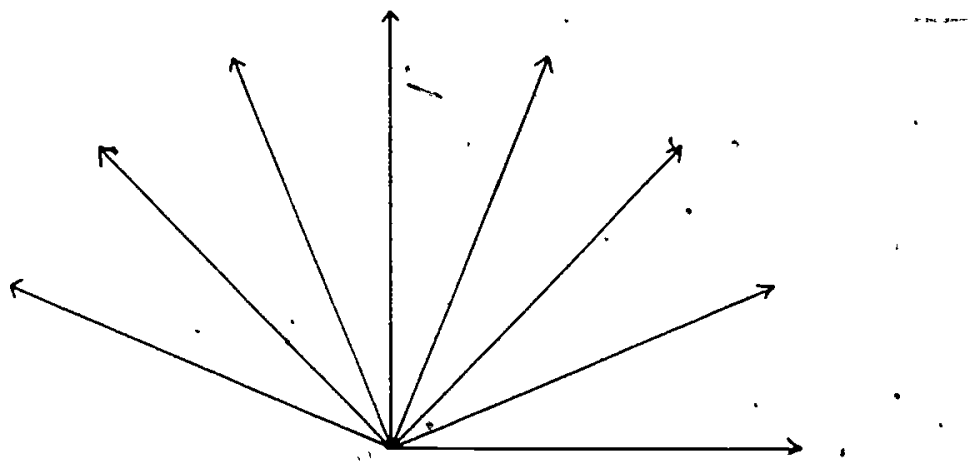

We 'also can conceive of an angle, call it $\angle A B C$; such that the unit. $\angle$ RST will not fit into $\angle A B C$ a whole number of times. In the picture below we have shown an $\angle A B C$ such that, starting at $\overrightarrow{B C}$ the unit $\angle \underset{R S T}{\rightarrow}$ can be la1d off 2 times about $B$ without quite reaching $\overrightarrow{B A}$, though if we were to lay off the unit 3 times we would arrive at a ray, call 1 t $\overrightarrow{B D}$, which is well beyond'
$\overrightarrow{B A}$.

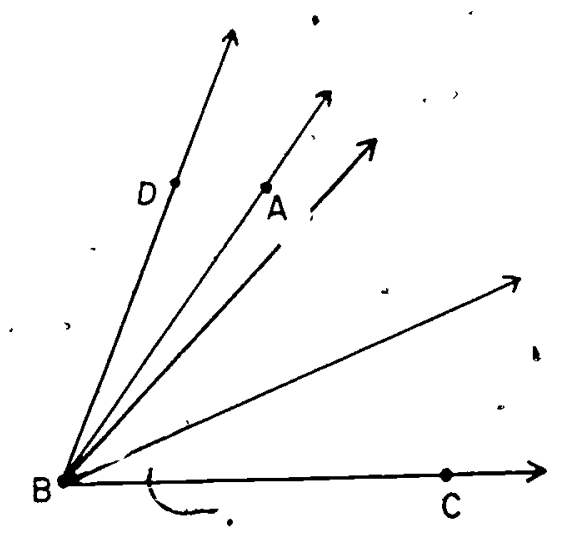

w. 
What can we say about the size of $\angle A B C$ ? Well, we can surely -say that $\angle A B C$ has size greater than 2 units and less than 3 units. In the particular case pictured we can also estimate by eye that, the size of $\angle A B C$ is nearer to '2 units than to 3 . units, so we can say that to the nearest unit $\angle A B C$ has size 2 units. Th1s is the best we gan do without considering fractional parts of units, or else shifting to a smaller unit.

When a unit is agreed upon, then a scale may be devised to facilitate measurement. If we decide to use a segment of one inch as a ufit of linear measure, then a straightedge may have successive congruerle segments of unit length la1d off on 1t (each segment intersecting adjacent segments in one endpoint only). If we further assoclate each endpoint from the first to the -last on the stralghtedge, with the whole numbers taken in order, $(0,1,2,3$, - $4,5,6, \ldots$ ) then we have established a scale for linear measure.

We follow the same procedure in setting up a device for angle' measurement - a protractor. Fo the pupil's first introduction to the use of a protractor, the unif chosen is a large one. Thus, attention, properly directed to the correct use of the protractor, Is not diverted by problems due th difficulty in reading a closely marked scale.' Since this first sepection of a unit angle to be la1d of successively on the half plane is not a standard unit, we may name 1t whatever, we choose, even an "octon".

The cholce of the octon as a unit angle is arb1trary but not accidental. It was selected so that if eight congruent ángles, each 1 octon in size, are la1d-out successively with a common vertex then they together with their interiors will exactly.xover a half-plane. The following demonstration shows how to use a paper folding to determine the size-of the octon. 
Select any plece of paper (1t might even be 1rregularly shaped):

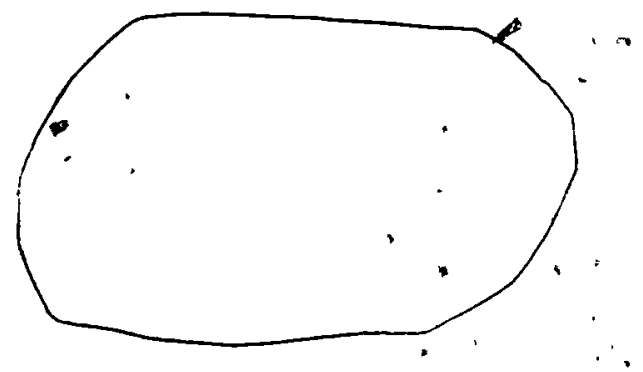

Fold it once to make a model of a line separating two halfplanes: Call it $\overleftrightarrow{\mathrm{AB}}$.

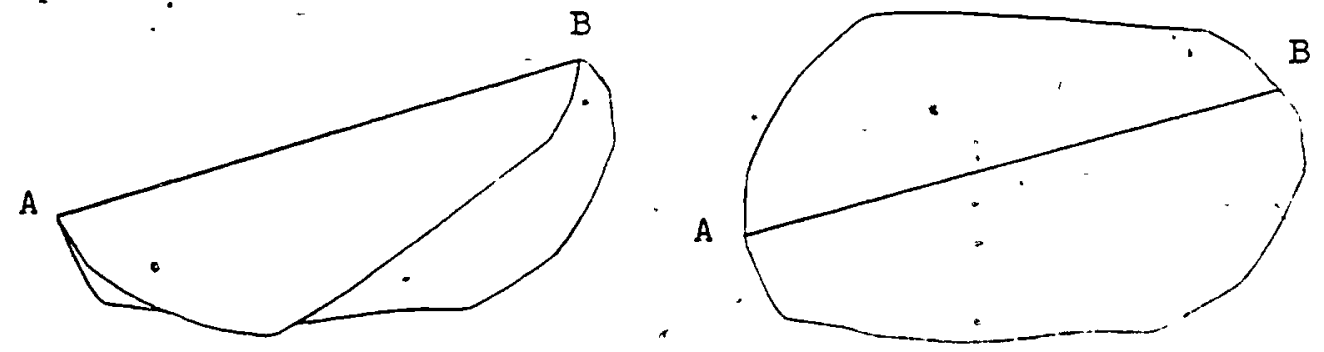

Choose a point $M$ on $\overleftrightarrow{A B}$ and fold through $M$ so that $\rightarrow \vec{A}$ ralls on $\overleftrightarrow{\mathrm{MB}}$.

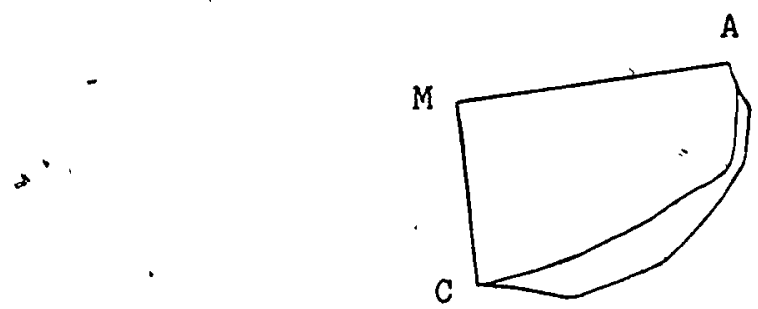

The $\angle$ AMC is a model of a right angle.

If you unfold the paper, it w1ll appear like this.

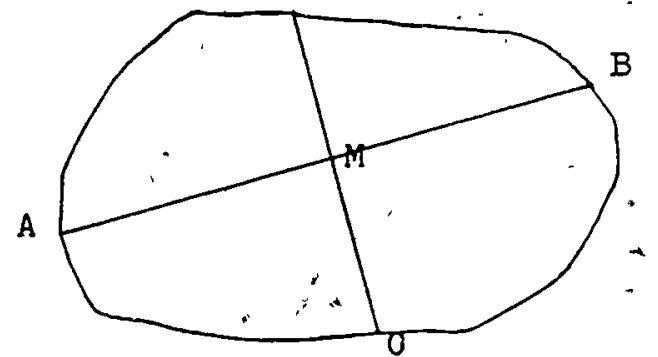

This shows four models of angles, all congruent, which together with their interiors, f1ll the plane. 681 
Refold the paper so that you again have a modell of a single right angle. Now fold so that the rays represented by $\overline{A M}$ and CM colncide.

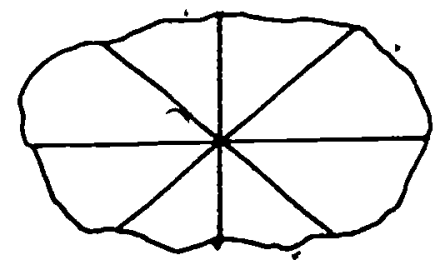

This provides us with a model of an. angle such that any four successive angles with a common vertex will exactly fit in the haIf-plane.

Refold your paper. Proceed to make one more fold as before. You now have a model of an angle of one octon, eight of which, successively placed with a common vertex, will exactly fit on the half-plane and its edge.

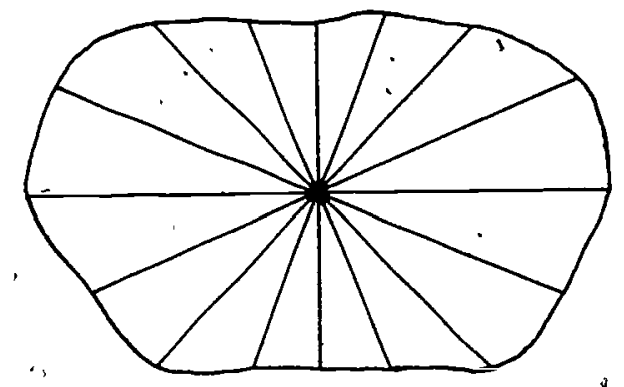

Each ray of the successively marked-off octons-is associated with a whole number, taken in order from 0 to 8 . We row have. a protractor, with a scale on $1 t$ suitable for use in measuring. angles. It should be emphasized that the measure of an angle is a number. We read $\mathrm{m} \angle \mathrm{ABC}=7$ as "The measure of angle $A B C$ is seven." If the unit is the octon, then we understand the statement to mean: "The measure of $\angle A B C$,. In octons, is seven." We cannot say, " $\angle A B C=7 "$ because $\angle A B C$ is a set of points and 7 is a numeral. But the measure of $\angle A B C$ is a number, so that a statement like: " $\mathrm{L} / \mathrm{ABC}=7^{\prime \prime}$. is permissible, since we have numerals on 
both sides of the " = "' symbol.

Eventually the pupil recognizes, that approximate readings of angle measure "to the nearest octon" leads him into a situation in which both $\angle A$ and. $\angle B$ (clearly not the same size) have a measure of 2 to the nearest acton.
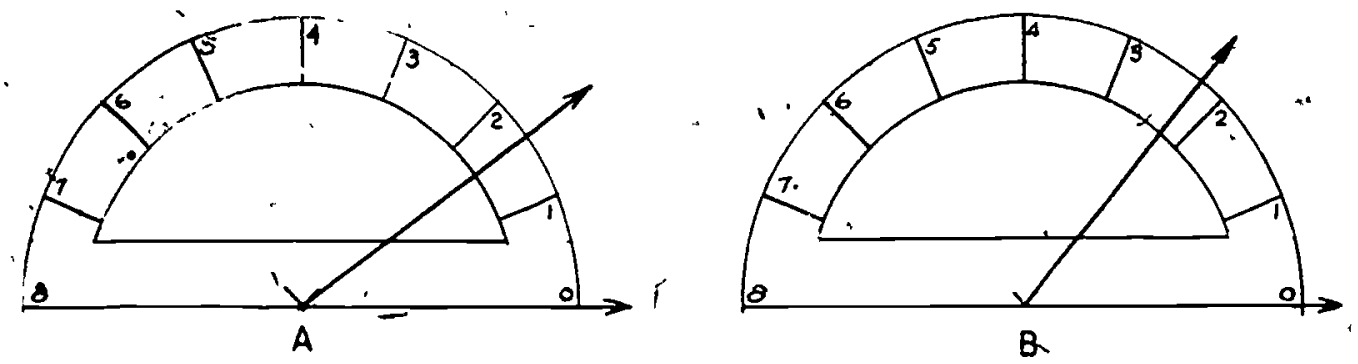

The need for a smaller unit is appreciated. For purpose of commnication, a standard unit has been agreed upon. We call the size of the standard unit of angle measure, one degree, and write $1 t$ in symbols as $1^{\circ}$. When we speak of the size of an angle we may say its size is $45^{\circ}$, but if we wish to indicate its measure we must keep in mind that a measure is a number, and say that 1 ts measure, in degrees, is 45. If we lay off 360 of these unit angles using a single point as a common vertex, then these angles together with their' interiors cover the entire plane.

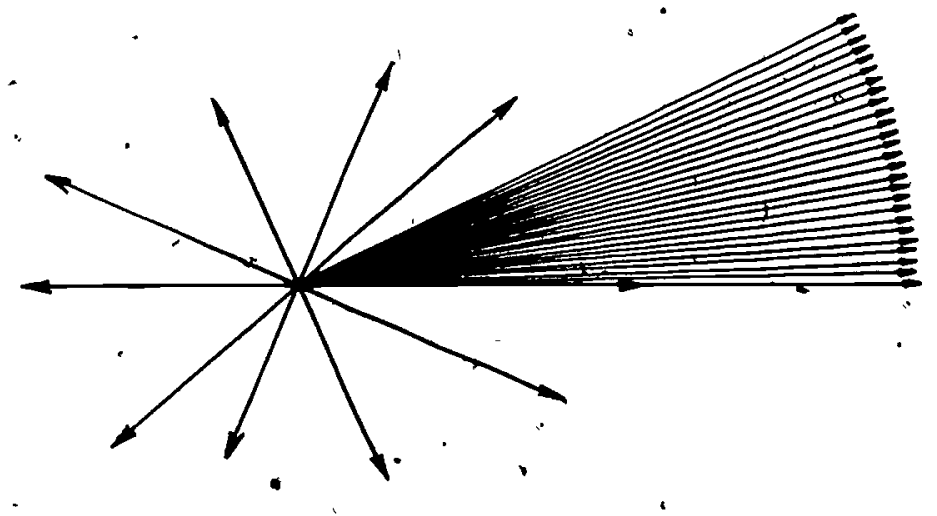

683
$2 \therefore 8$ 
Even in ancient Mesopotamian civilization the angle of $1^{0}$ 'as the angle of unit measure, was used. The selection of a unt angle which could be fitted 1nto the plane (as above) just $360^{-}$times was probably influenced by their calculation of the number of days in a year as 360 .

In this unit' we concern ourselves only 'With angles whose measures are between, 0 and 180. Because of our definition of an angle and its interior it is not possible to have an angle whose rays colncide or extend in a straight line: Subsequent extension in later grades of the definition of angle will make $1 t$ possible to discuss an angle of any size. 


\section{TAACHING TIE UNIT}

The Lessons in tinis unit vary in their composition. Some have three parts which are: first, Suggested Teacining Procedure,

- secona, Exploration, ana thira, Exercises which the cirildren should do independently. - In some lessons the Exploration and Exercises are sulticient to develop the lesson. Some lessons need only the Exploration to clarify the concepts for the children.

The first part Suggested. Teaching Procedure provides an. overview of the lesson. It is here that the teacher will find suggestions for providing the background the children will need for the understandings and skills to be developed.

Some teachers may prefer to have the children's books ciosed during this introduction of the concepts. During the second part of the lesion, the Exploration in the pupil's book, the pupils and teacher w1ll read and answer the questions together. She may say, fon example, "Now tum to page - and look at the" " Exploration. Is this what we did? Is this what we found to be true?" A sourceful teacher willbe sensitive to the mood of her class and will not extend this part of the lesson beyond the point of interest.

Other teachers may gaimmediately, into the Explorations. The Exploration then sarves as a guide for the lesson. Still others may wish to have the pupil's book closed during the presentation and then have the pupils read the Exploration independently for review.

The tinird part of the lesson is the Independent Exercises. These are designed for the pupil to work independently. They are provided for maintenance and establishment of skill but

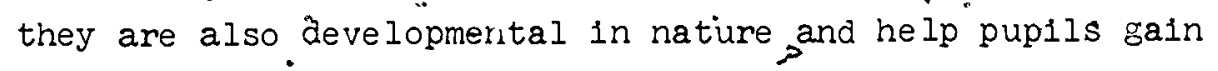
additional understandings and skills: "

Each teacher should feel free to adapt these ideas in a way that will suit her mëthod of teaching and in a way that meets the particular needs of her class. 
F The first section of this unit is a review of material covered in the SMSG ikext for the fourth grade. If the pupils have not studied this material, you will need, to spend more time on this section. In either casc, you should have a copy of the SMSG text for grade four.

"References: *4. School Mathematics study Group, Text for Grade Four.

2. Natiematics for Junior High School, Volume I* Chapter IV, School llathematics Study Group.

3. Freeman, Mae and Ira, Fun with Figures, New York: Random House, 1946.

4. Ravielli, A., An Adventure in Geometry, New York: Vilking Press. .

5. Bassetti, F., Solid Shapes Lab, New'York Science Waterial Center.

6. R. D. Anderson, Concepts of Informal geometry, Volume $V$, Studies in Mathematics, School Mathematics study Group. 
,UNIT SEGMENTS AND UNIT ANGLES

Objective: To develop the following understandings and skills.

1. The unft used for measuring an angle is an arte.

2. The unit apgle is chosen arbitrarily.

3. The exact measure of a given angle in,terms of a unit is the number (not neçessar1ly a whole number) of times the unit angle will fit into the given angle.

4. The approximate measure of a given angle in tèms of a: unit is the nearest whole number of times the unit angle will fit Into the given angle.

Materials :

Teacher: Chalkboard or string compass, stralghtedge (meter stick or yard stick), sheet of plastic for tracing, colored chalk.

Pup11: Compass, stra1ghtedge; trácing paper.-

Vocabulary: measure, unit

If there is an interval between the study of EB-114 and this unit, review the definitions of line, ray, line sgement, angle, and congmence. Work at - the chalkboafd while the puplis work on paper at - thelr.seats. Follow the Exploration as closely as possible. Exe 1 ise 1 on Page. 1, be sure the pup1l choose a segment. MN smatis enough so that it can be tald of at least three times on the rays as 4 Exercise 3. Stress that the measure is a number. Hente $\mathrm{m} \overrightarrow{\mathrm{AB}} 3$ and not $m \cdot \overline{A B}=3$ units. "3) tunits." not anumber but a description of a physical quantit to similasly. $m \angle R=5$ and not $m \angle R=5$ undts Use colored chalk to help puplis in cexer c1ses 7-10 of the Exercises to visualizing overlapping angles whose interlors intersect 
You have studied congmuent angles, and you know that congruent angles have the same size. YYou have jeamed also how to tell which of two angles has the larger size. But we need to have a yay to describe the size of an angle more exactly, that 1s, to measure an angle. Let us see hoi this could be done,

Recall how you found a method to measure a line segment: See if what you did to measure a segment sugges.ts how an angle might be measured. Read the instructions of examples 1,2 , and 3 , before you start the drawing requested in example 1 .

1. Draw a ray on your paper. Call 1 ts endpoint. $P$. Also draw a short segment not on the ray. Call it

2: On your ray 'construct a segment congruent. to $\overline{M N}$, with one endpoint $\cdot P$. Call it $\overline{\mathrm{PA}}$. 
3. On' the ray, construct a second segment congruent to $\overline{M N}$, with $A$ as endpoint. Call it $-\overline{\mathrm{AB}}$. On the ray "construct" a third" segment, congruent to $\overline{\mathrm{MN}}$. Call it $\overrightarrow{B C}$. Your drawing. should look like this:
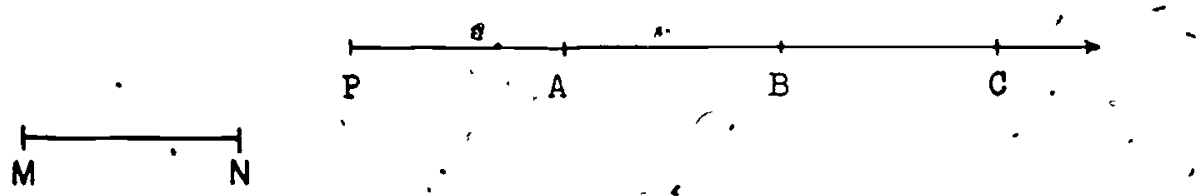

4. Copy and complete the following statements. Look at $\overline{M N}$ and $\overrightarrow{P C}$ youlhave drawn on your paper. Call the

$\therefore$ length of $\overline{\overline{M N}}$ one unit. Then

a) the length of $\overline{\mathrm{PA}}$ is

$(1)$ unit.

b) the length of $\overline{A B}$ is $(1)$ unit.

c) the length of $\overline{\mathrm{BC}}$ is unit.

d) the length of $\overline{\mathrm{PB}}$ is (2). units.

e) the length of. $\overline{\mathrm{PC}}$ is. units.

f) the length of $\overline{\mathrm{AC}} \cdot$ is $\therefore(2)$ units.

The number $a$ is called the measure of $\overline{\mathrm{PB}}$. $\because$

5. What is the measure of $\overline{\mathrm{PC}}$ ? (3) of $\overline{\mathrm{AC}}$ ? (2) of $\overline{\mathrm{AB}}$ ? (1).

6. Did the pup1l next to you make $\overline{\text { M }}$ "the same length?

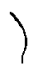


7. If you are told only the measure of a segment can your know how long it.1s? ( $\left.\boldsymbol{W}_{0}\right)$ What else must you know?

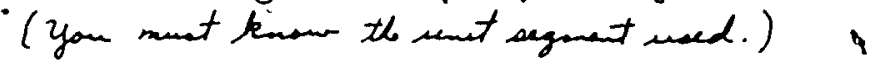

8. Choose a new segment, different from $\overline{M N}$, as your unit. Construct a segment. whose measure, using this new unit, is 4. Construct another segment whose measure is 3.

9. You used a line segment as a unit to measure line spgments. What should you use as a unit to measure an angle? ( an angle)

10. Use $\angle P$ as a unit angle. Draw $\overrightarrow{R T}$ on a sheet of kaper. Make a tracıng of $L . A$ on thin paper. Place the tracing with

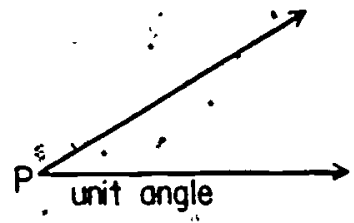
$P$ on $R$ and one side of $\angle P$ on $\overrightarrow{R T}$. Then use the sharp end of your compass to mark a point $A$ through. the tracing to your. drawing. Remove the tracing and draw $\overrightarrow{R A}$. Is $\angle A R T \cong \angle P$ ? (yee $)$

11. What is the measure of $\angle$ ART? (The as the unt aigh, in 1.)

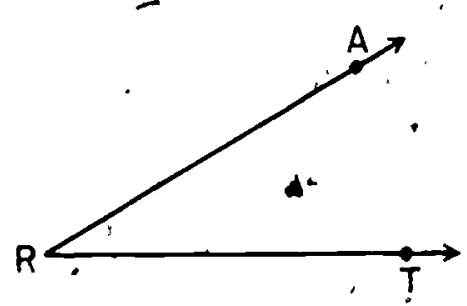


P410

12. On your drawing of " $\angle A R T$, place the tracing of the unit $\angle \mathrm{P}$ so ${ }^{\circ} \overline{\mathrm{P}}$ is on $R$ and one side of $\angle \vec{P}$ is on $\overrightarrow{R A}$ and the other $\mathcal{A}$ ide of $\angle P$ is in

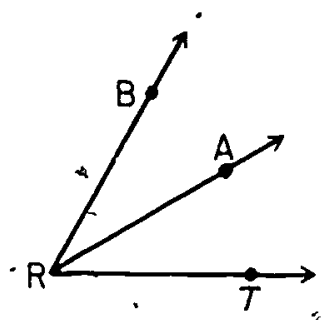
the exterior of $\angle$ ART. Use the sharp end of your. compass to mark a point $B$, and draw $\overrightarrow{R B}$. Is $\angle A R B \cong \angle P ?$ (yes)

13. Using $\angle P^{\prime}$ as the unit angle, what is the measure of $\angle A R B ?(i)$. What is the measure of $\angle$ BRT? (2)

14. Continue as in problem '12.

a) Place the tracing on the unit $\angle P,{ }_{0}$ with $P$ on $(R)$. and one side of $\angle P$ on $\underline{(\overrightarrow{R B})}$. Be sure to place - the tracing so the other side of. $\angle P$. is in the (exterior) of $\angle$ BRT. Use the sharp end of your - compass to mark a point $C$, through your tracing to your drawing. "Remove the tracing and draw $\overrightarrow{R C}$.

b) Repeat this process one more time in order to draw $\angle C R D$. Your drawing should now look like this:

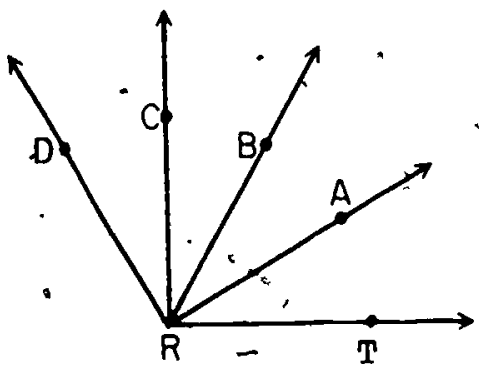

15. What is the measure in unit angles ${ }_{4}$ or $\angle \mathrm{CRT} ?(3) \angle \mathrm{CRA}$ ?(2) $\angle \mathrm{CRB} ?(1) \angle \mathrm{CRD} ?(1)$ 
P411

- 16. Since you, and all your classmates used the same unit angle, ' $\angle \mathrm{P}$, should your $\angle \mathrm{DRT}$ be congruent to theirs? ( Yer) Work with a classmate and test to see whether his angle and your angle seem to be congruent. Place your paper over a classmate's paper and hold them up to the light.

17. Choose a new unit angle smaller. In size than a right angle. Then use your compass to construct an angle whose measure is 1. Call. it $\angle$ ABC. (Pupilawill probably. chore unit angles whet are of different size.)

18. Or the drawing you made for Exercise 17. construct with compass an angle whose measure is 2 .

To state the measure of an. angle we write:

$-m \angle D B A=2$. The small " $m$ " is read "measure of". We also write $" m \overline{\mathrm{AB}}=5$ " to state the measure of a segment equals 5 . " $m \overrightarrow{\mathrm{AB}}=5$ " is read "the measure of $\overline{\mathrm{AB}}$ equals $5 . "$.

Remember that. a measure is a number.

692 
P412

\section{Exercise Set 1}

- On a sheet of paper write the answers to the following exercises. - Be sure to number each exercise..

1. State the measure of each segment named. The unit segment Is shown at the right.

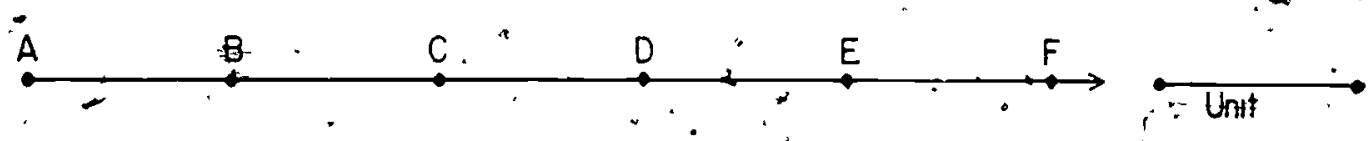

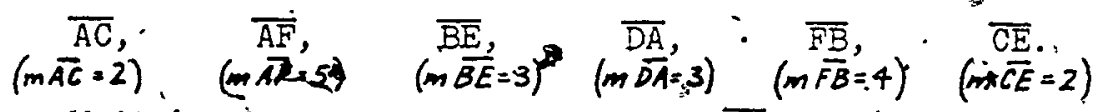

Write your answer like this: $m \overline{A C}=2$ ?

2. In the sketch below; name

a) Four segments' èach of whose heasure is 2 .

- Write your answer like this: $m$ HJ $=2$. $(\overline{G I}, \bar{H}, \overline{I K}$, and $J L)$.

b). Three segments each of whgse measure is $3:$

$*$

c) Two segments each of, whose measure is 4 .

(GK and $\overline{H L})$.

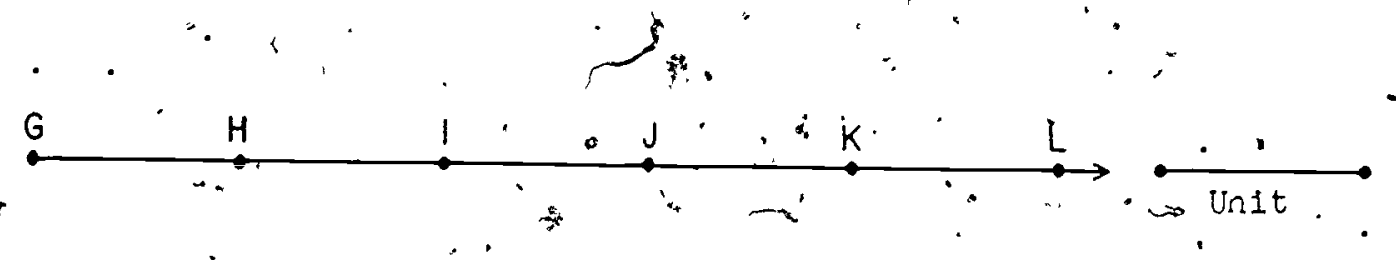

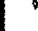

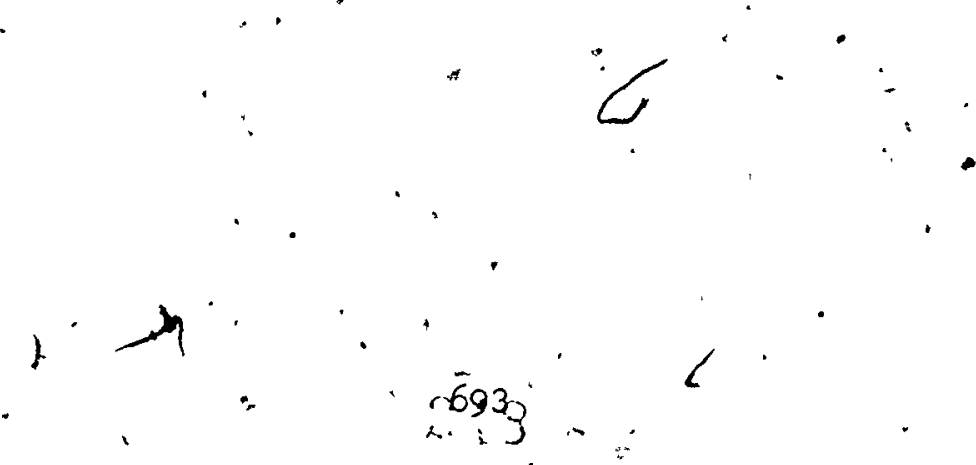


P413

3. The small angles in the sketches are all congruent to the unit angle shown. - State the measure angles named. Write your answer like this: $\mathrm{m} \angle \mathrm{ABC}=$ ?
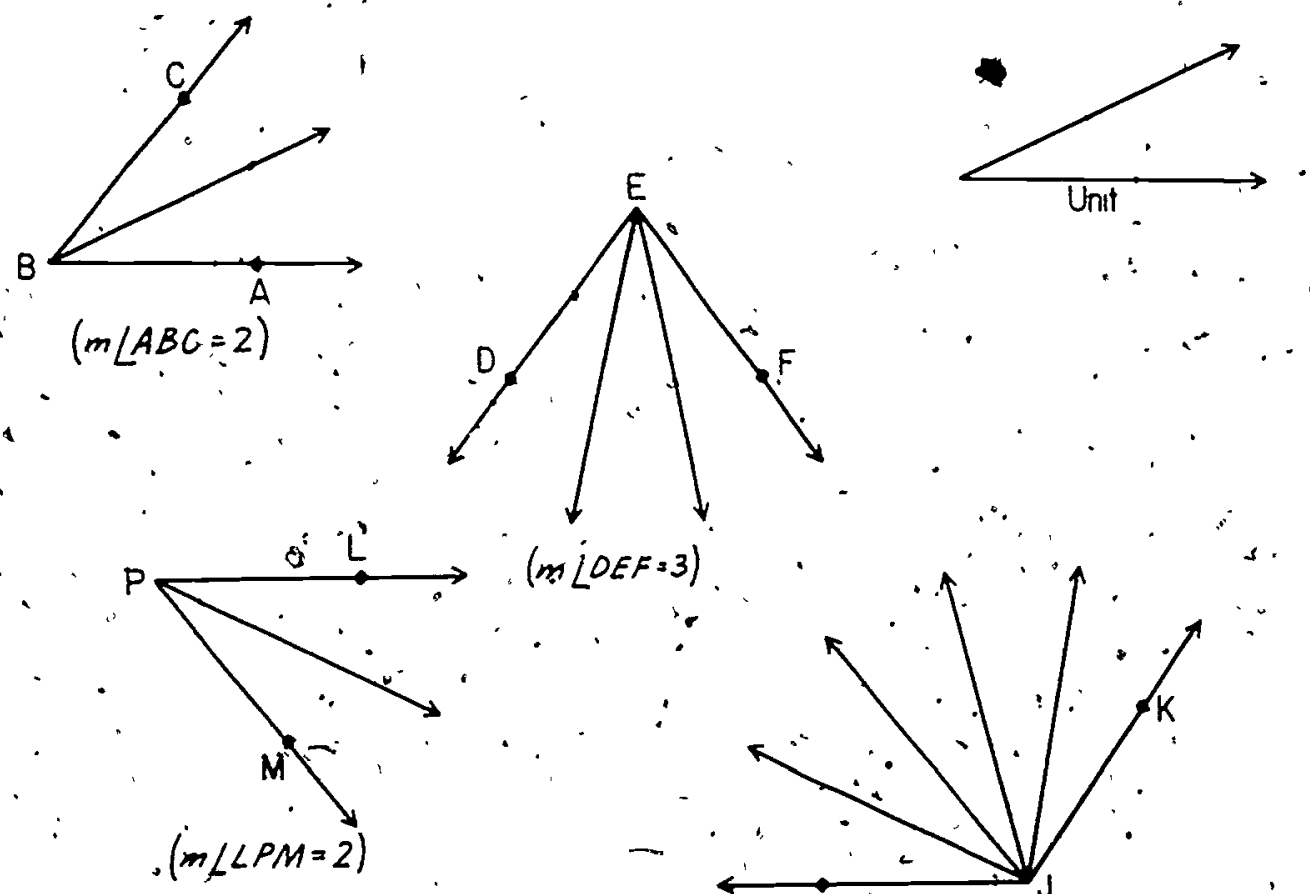

$(m[O E F=3)$

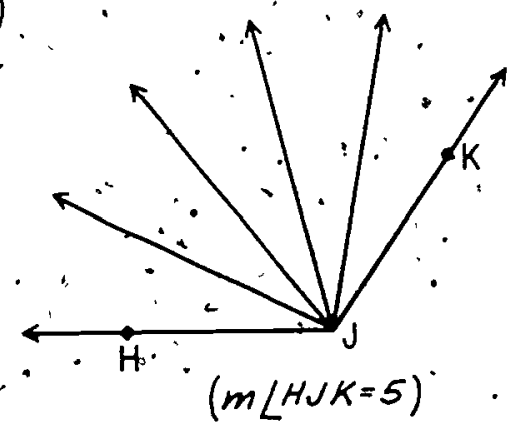

4. Each of the small-angles in the sketch is congruent to $\because$ the unit angle. State the measure of each angle named.

$\therefore \angle \ddot{G A C}, \angle \overrightarrow{B A E}, \angle C A \vec{F}, \angle D A G, \angle B A C, \angle F A G$
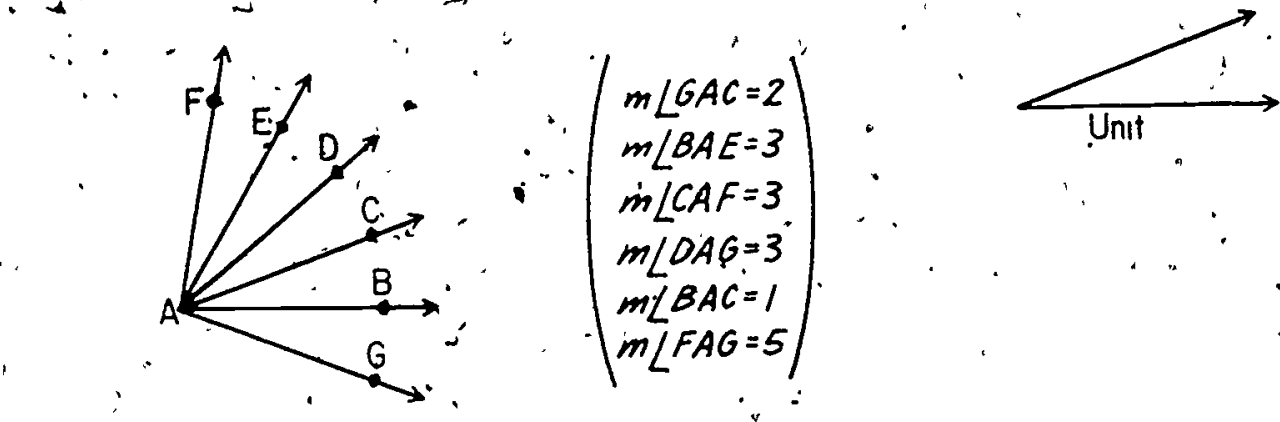

249 
5. Each the small angles in each figure below is congruent to the unit angle. Using only the points which are marked, name:

a) An angle with measure $2 \cdot(\angle H B I) d$

b) An angle "with measure $4 .(\angle \angle O M)$

c) An angle with measure $7 \cdot(L J C K)$

d) Two angles, the sum of whose measures is 7 . ( $\angle F A G$ and $\angle L D M$ or $\angle H B I$ and $\angle N E P$ )

e) Two arigles, the sum of whose measures is $\dot{9}$. (LNEP and LLOM or LJCK and LHBI)

f) Three angles, the sum of whose measures is 16 . - ( $\angle J C K$ and $\angle N E P$ and $\angle L D M$ )
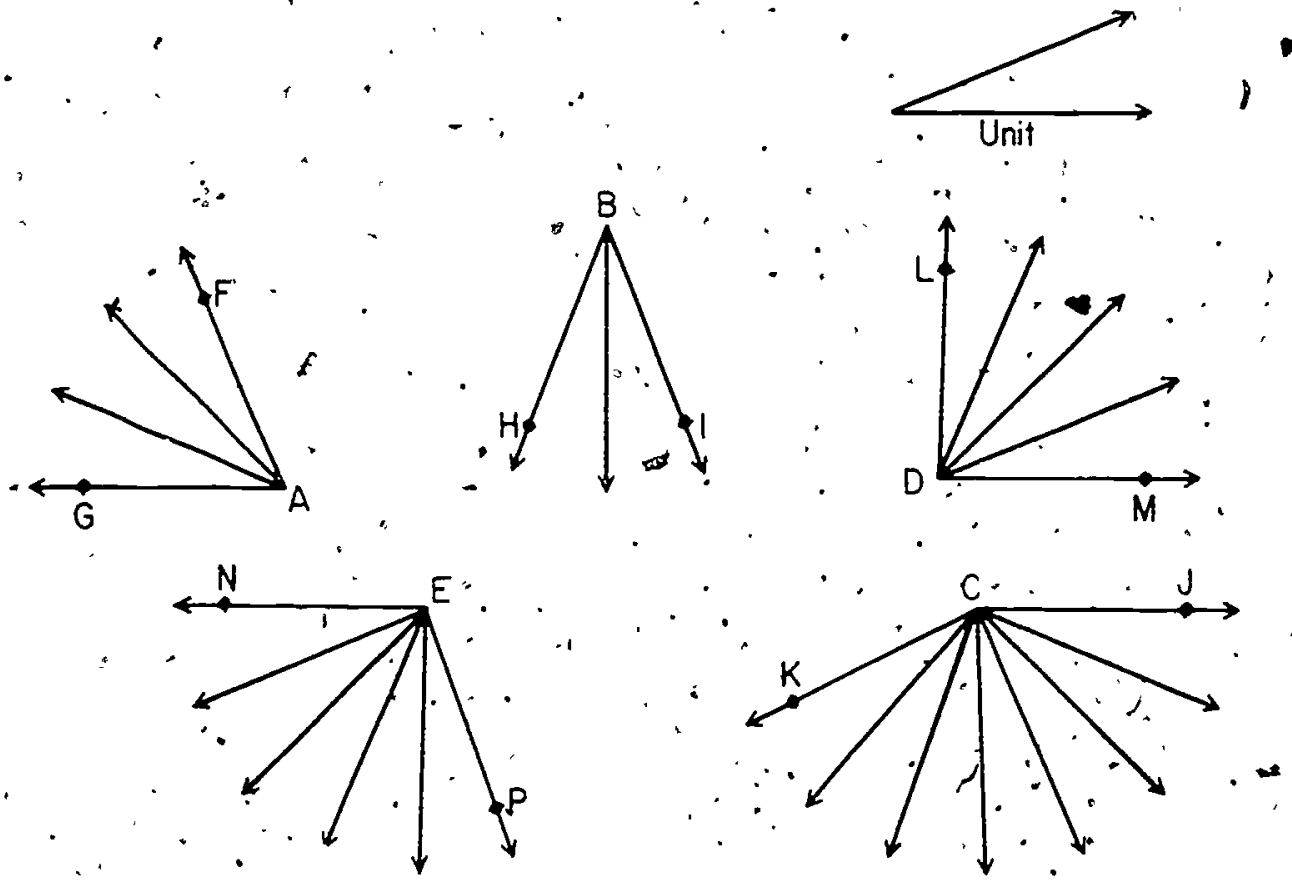
6. Each of the small angles in the rigure below is congruent." to the unit angle. Name:

a) Three angles with meașure 2. ( $L H P K, \angle J P L, \angle K P M, \angle L P R)$.

'b) Three angles with' measuré 3. (LHPL, LUPM, LKPR)

c) Two angles with measure 4. (LHPM, LJPR)

d) Four angles with measure $1 .(\angle H P J, \angle J P K, \angle K P L, \angle L P M, \angle M P \hat{R})$
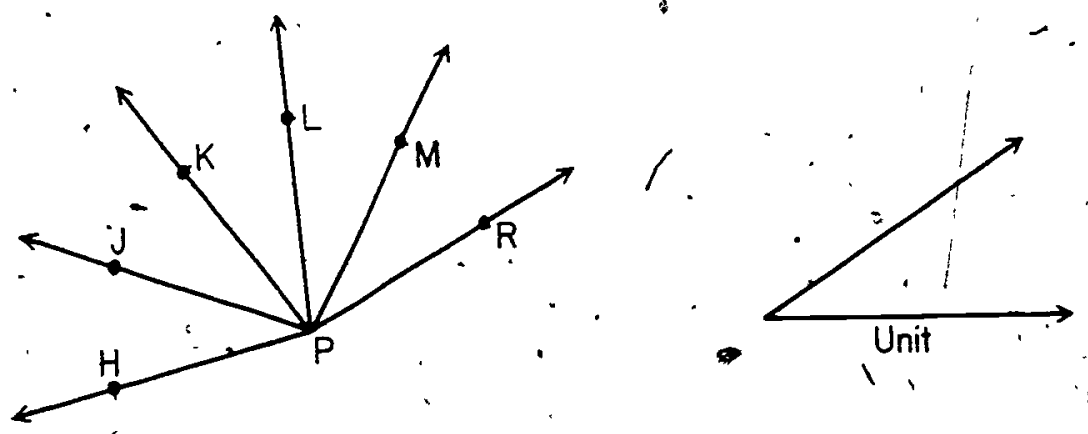

\section{j}

7. In the flgure of Exercise 6,
a) $\mathrm{m} \angle \mathrm{RPL}=(2)$
d) $\mathrm{m} \angle \mathrm{MPK}=(2)$
b) $\mathrm{m} \angle \mathrm{IPH}=(3)$
e) $\mathrm{m} \angle L P M=(1)$
$\therefore$ c). im $\angle R P H=Y_{(5)}$
f) $\mathrm{m} \angle \mathrm{MPH}=(4)$

- Look at your answers to Ex. $7 a, b$, añd c.

8. Is this true?: $m \angle R P L+m \angle I P H=m \angle R P H ?$ (Yfer).

9. "Now look' at your answers for Exercises $7 d, b$, and $\dot{f}$. Is this true? $m \angle M P K+m \angle L P H=m . \angle M P H ?\left(n_{0}\right)$, . 
- $\mathbf{P} 416$

10. A boy wished to construct an angle of measure 4. He chose the unit angle shown below. He used his compass and straightedge to construct the $\angle A F E$. A picture of his work is shown below. Look at the picture and answer the following:

a). What ray can you draw to complete an angle whose

- measure is 3 ? ( Nair $\overrightarrow{F D}$, to complete $\angle A F D$ whore measure is 3 os draw $\overrightarrow{F B}$ to complete are angle $E F B$ whore measure in 3.)

b) What ray can you draw to complete an angle whose measure is. 1? (Draw one i of the rays Fo on FB to complete $\therefore$ angle whose measure in 1.)

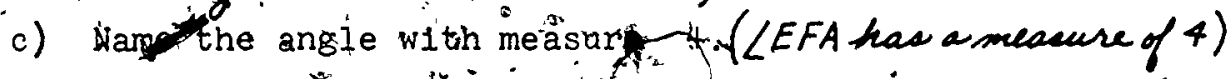
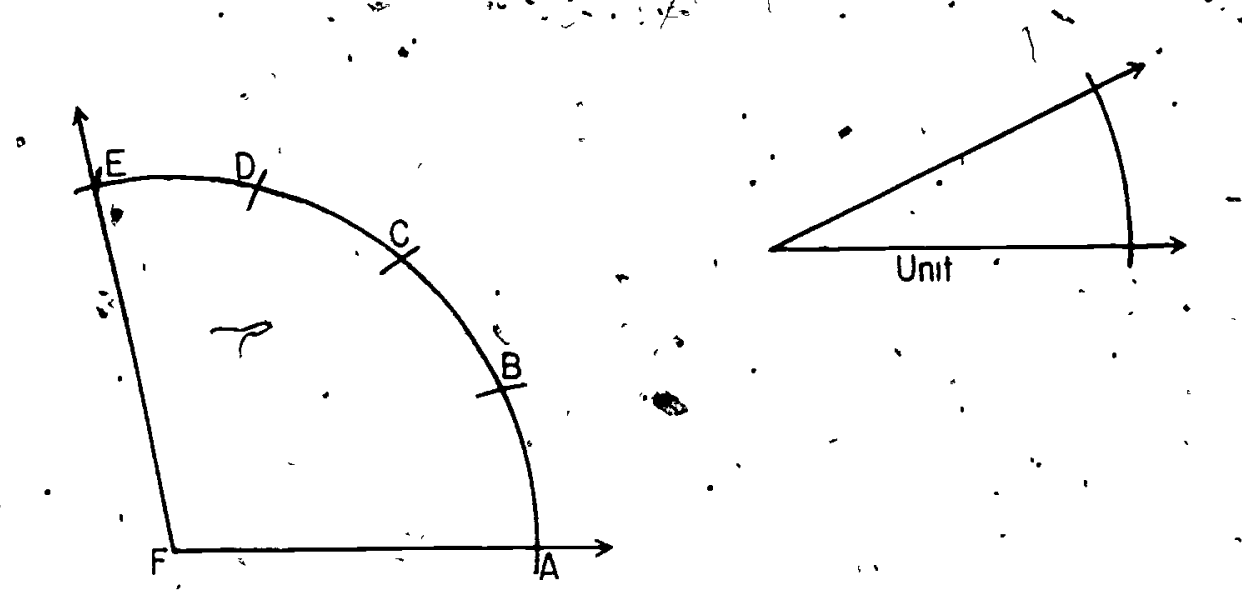

11. Use the method shown in the sketch, for Exercise 10 to construct an angle who
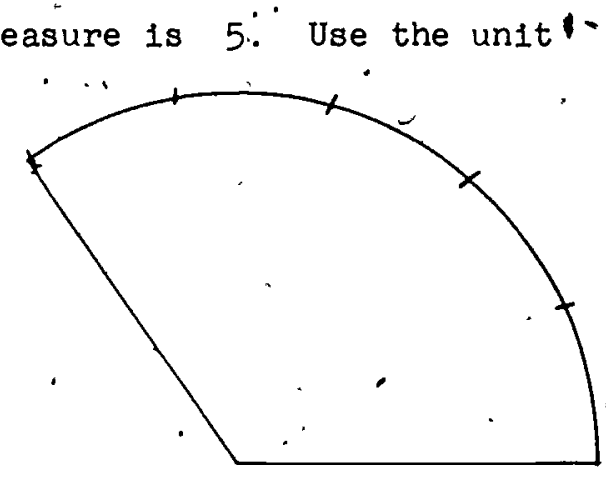

697

252 
USE OF UNIT ANGLE IN MEASURING ANGLES

Objective: To develop the following understandings and skills:

1. We measure an angle by counting the number of congruent unit angles we may place successively in the interior of this given angle.

2. A compass may be used for laying off the unit angle in the interior of.the given angle. The procedure is essentially that used in copying an angle.

3. At best, our meásurements are approximattions.

4. While $\mathrm{m} \angle \mathrm{A}=4$, the slze of $\angle A$ is 4 units.

5. If the size of an angle is closer to 7 units than It $i s$ to 6 units or 8 units, then we say $m \angle K=7$ to the nearest unit.

Materlals Needed:

Teacher: Chalkboard or string compass, stralghtedge 'Pupil': Compass, stralghtedge

Vocabuiary: To the nearest unit
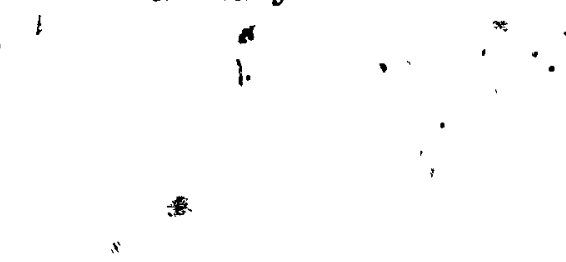
USE OF UNIT ANGLE IN MEASURING A ANGLES

\section{- Exploration}

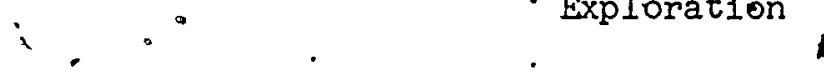

You have used your compass to construct a line segment of a given measure and an angle of a given measure. Now suppose you wish to find the measure of , $\overline{A B}$, using $\overline{M N}$ as unit. Trace $\overrightarrow{A C}$ and point. $B$ on a sheet of paper.
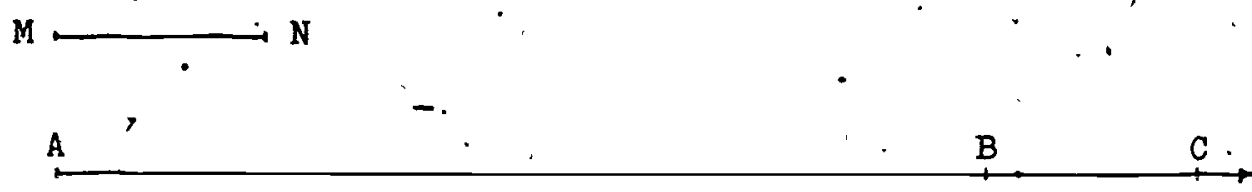

i. Now.copy $\overline{\mathrm{MN}}$ on $\overline{\mathrm{AB}}$ with $A$ as the left endpoint. Call the right endpoint, $H$. Repeat the process 4 mure times to get line segments $F D, D E, E F$, and FG. Make each Iine segment congruent to $\overline{\mathrm{MN}}$. How many such coples can you make on $\overline{\mathrm{AB}}$ ? (4)

Your drawing should look like this:

M $-\mathrm{N}$

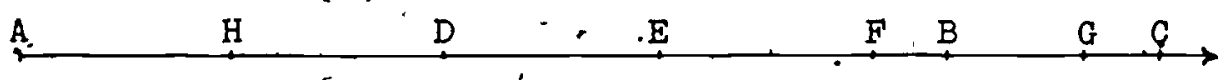

In the sketch, $\overline{\bar{M} N}$ was copied 4 times on $\overline{A B}$ so

$\mathrm{m} \overline{\mathrm{AF}}=4^{\circ}$. When $\overline{\mathrm{MN}}$ is copled the last time, $30^{\circ} \overline{\mathrm{FG}} \cong \overline{\mathrm{MN}}$, you see that $m \overline{\mathrm{AG}}=.5^{\circ}$.

- Since point $B$ is between point $F$ and point $G$, $\cdots \mathrm{m} \cdot \overline{\mathrm{AB}}>4$, and also $\mathrm{m} \overline{\mathrm{AB}}<5$. Since $\bar{B}$ is nearer $F$ than $G$, we say that $m \overline{\mathrm{AB}}=4$, to the nearest unit: If $B$ were nearer. $G$. than . F, then we would write $m, \overline{A B}=5$, to the nearest unit. 
$\mathrm{P} 418$

2. Suppose you are to ind the measure of $\angle$ DEF, using $\angle A$ as unit.
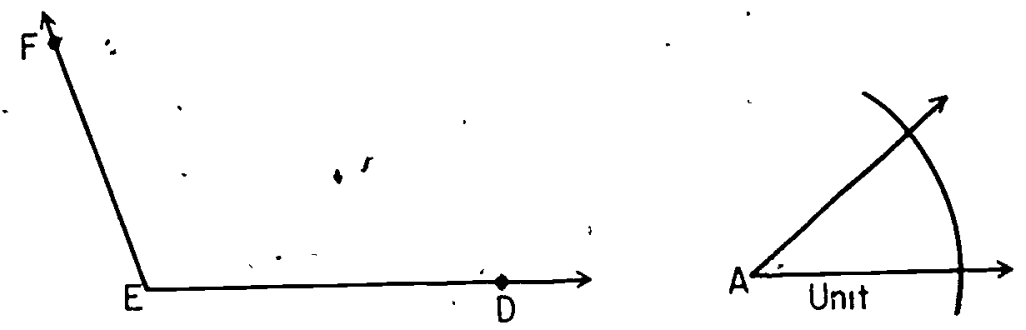

Make a tracing of $\angle D E F$ and $\angle A$ on your paper. can you use your tracing to estimate the measure, of $\angle$ DEF? (yea) $\mathrm{m} \angle \mathrm{DEF}=(3)$, to the nearest unit.

3. Now instead of tracing', use your compass as you did in Exercise 11, Set 1. Does your drawing look like this?

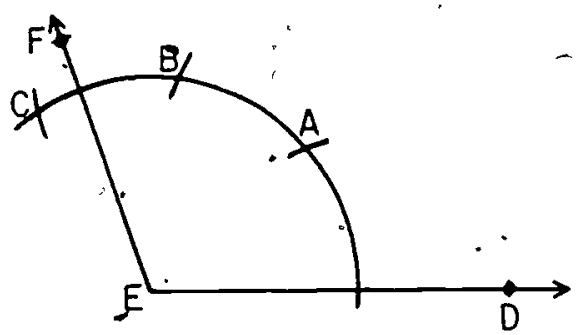

4. Draw $\overrightarrow{E A}, \stackrel{!}{\overrightarrow{E B}}$, and $\overrightarrow{E C}$.

Copy and complete the following statements.

5. $\mathrm{m} \angle \dot{D E F *}\rangle \mathrm{m} L(\underline{D E B})$, and $\mathrm{m} \angle \mathrm{DEF}<\mathrm{m} \angle$ (DEC)

6. $m \angle D E B=(2)$ and $\mathrm{m} \angle \mathrm{DEC}=\underline{(3)}$

so. $\dot{\mathrm{m}} \angle \mathrm{DEF}>$ (2) and $\mathrm{m} \angle \mathrm{DEF}<$ (3)

7. $\mathrm{m} \angle \mathrm{DEF}$ is nearer (3) thar (2).

$\mathrm{m} \angle \mathrm{DEF}=(3)$ to the nearest unit. 


\section{Exercise Set 2}

1. Make $a$ copy of the following figures. Use the unit segment. shown to find, to the nearest unit, the measure of each of the segments below. Use your compass.

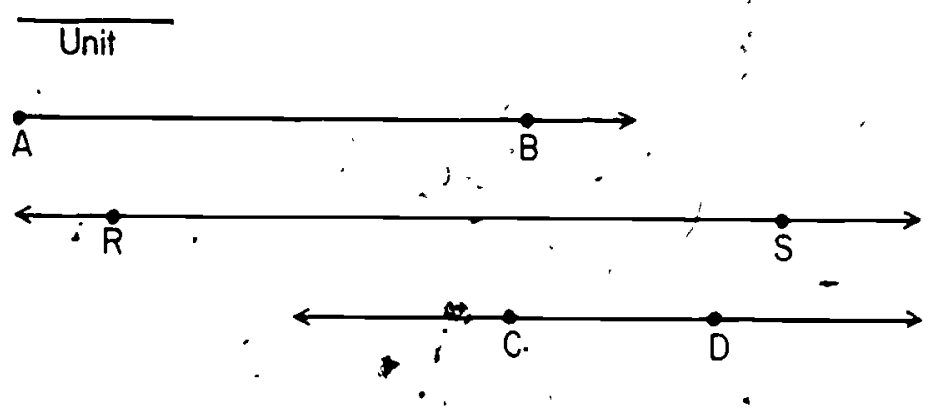

Copy and complete the following statements
$\therefore$
$\mathrm{m} \overline{\mathrm{AB}}=(3)$
$\mathrm{m} \overline{\mathrm{RS}}=$
(4) $\cdot \mathrm{m} \overline{\mathrm{CD}}=$

2. Trace the figures below on your paper. Use your compass and straightedge to find the measure, to the nearest.unit, of each angle below. Use the unit angle $K$ as the unit

. of measure.
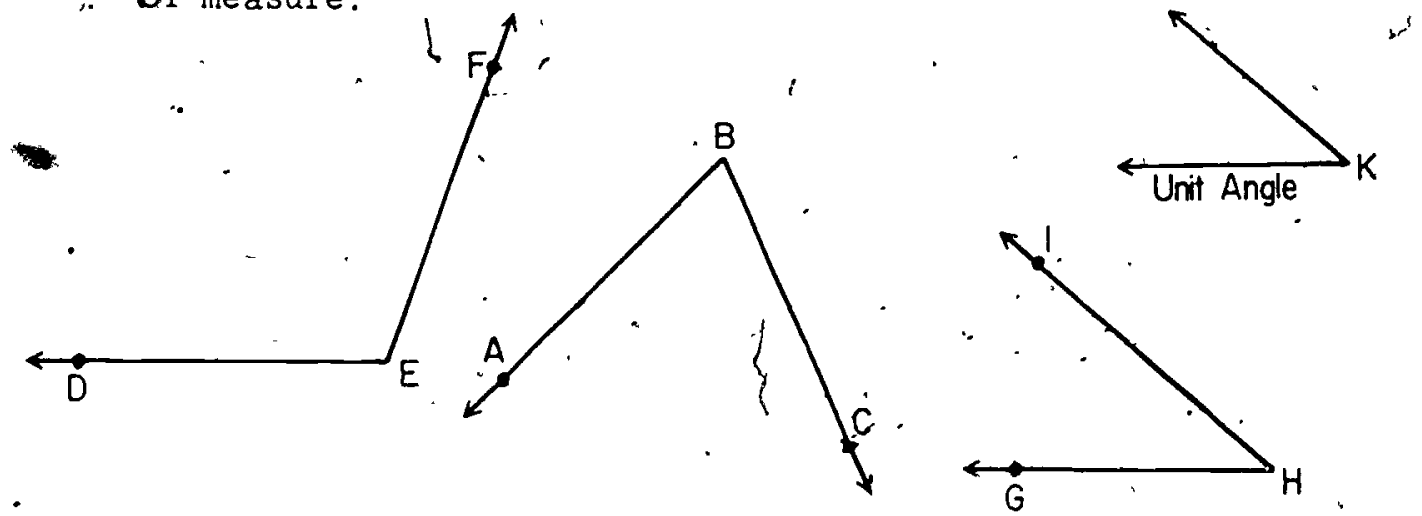

$\dot{\mathrm{m}} \angle \mathrm{ABC}=$ (2), $\mathrm{m} \angle \mathrm{DEF}=$ (3), $\mathrm{m} \angle \mathrm{GHI}=$ (1). to the nearest - to the nearest unit. unt:. to the nearrest inft. 


\section{A SCALE FOR MEASURING ANGLES}

Objective: To" develop the following understandings and skills:

1. We can create a device for convęience in making measurements. The ruler'is one such instrument.

2. The measuring device is marked with whole numbers in consecutive order, so that to each successive "copy of the unit laid off on the device, there corresponds a number.

3. For convenience, we choose a unit angle frich when. ' laid off successively will fit into the half plane

- a whole number of times.

4. The instrument we. will use for angle heasure is a protractor. When the protractor is properly placed on the angle the measure of the angle can be read on the sciale.

5. The two scales on a protractor are mereliy a convenience for measuring angles in either direction çlockwise or couniter-clockwise.

Materials we eded:

Tea hier: straightedge, chalkboard protractor, one half-disc, (semi-circular 'region), plece of tag' board for each pupil to make an octon scale. A diameter of 4 inches is àbout right.

Pupil: Stra1ghtedge, protractor (to be made)

Vocabulary: Protractor, scale, octon

In order to carry through the development. in items 1-7 of this Exploration the pupil will need. his book open. Exeralises 2,3 and 4 call attention to three common errors in use of the protractor, namely

(a) faliure to place the zero iay of the. 'protractor along a ray of the angle to be measured,

(b) fallure to place the $V$ mark of the protractor (intersection of rays) on the vertex of the angle to be measured.

(c) fallure to read the correct scale. 
P420.

A SCALE FOR MEASURING ANGLES

Exploration

As you know, when you measure a line segment, youk usually use a linear scale or muler, with the endpoints of - the unit segments marked with numerals. You place the ruler beside the segment and find the measure of the segment from the numerals on the ruler, at the endpoints of the segment.

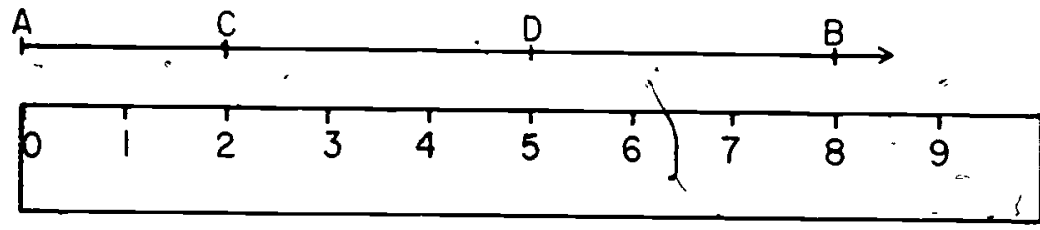

1. $m \overline{A C}^{-}=(-2), m \overline{A D}=$, (5), $m \overline{C D}=$ (3), $m \overline{A B}=$ (8)

2. Must-you' place the zero on the sciale at the endpoint of the segment in order to find the measure of the segment?

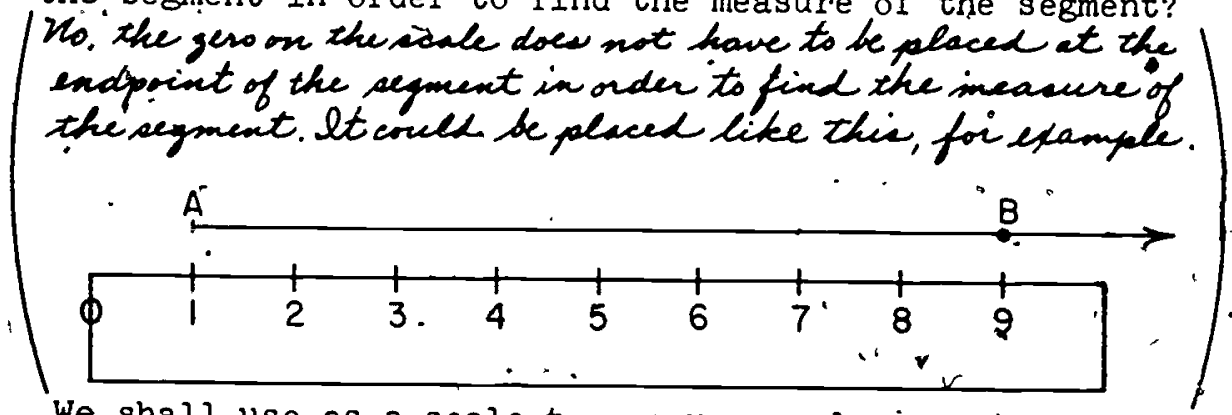

We shall use as a scale to measure angles a set of rays which are the sides of angles congruent to anit angle. Any unit angle can be used but for convenience, we shall choose one so that eight of them whth their interiors will exactily cover a half plane. We may name it whatever we want to. "We will name our unit angle an "octon." Two of the rays $\overrightarrow{V A}$ and $\overrightarrow{V B}$, ax'e on the same straight line and, extend in opposite firections from $V$. 
P) 21

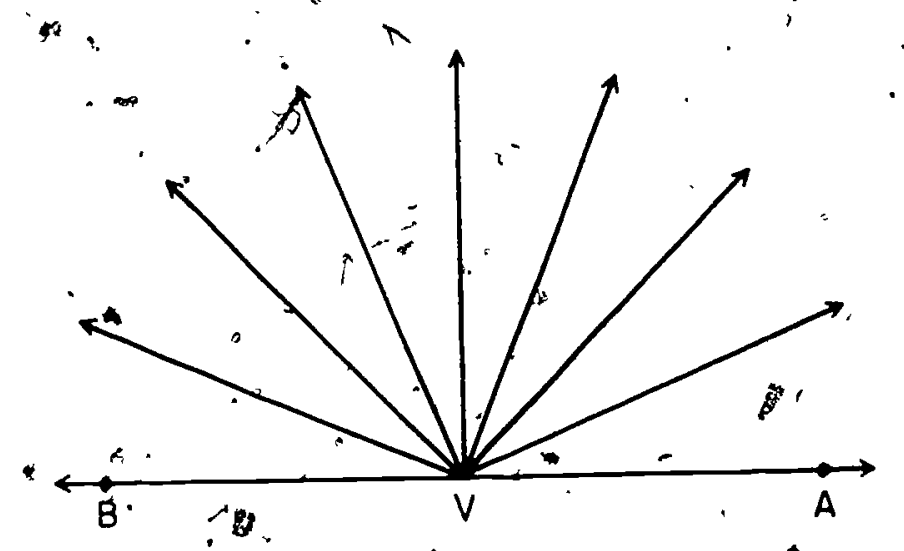

Then we will number the rays in order, putting 0 on the ray. to the ring $(\overrightarrow{V A})$ and ending, the scale when we reach the ray on the same straight line as the zero ray $(\overrightarrow{V B})$.

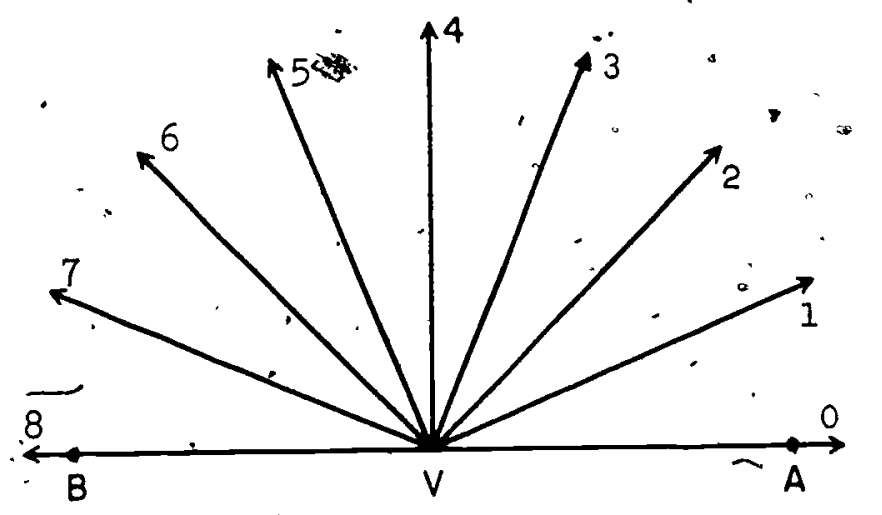

3. Wake a tracing of $\angle D C E$. To. measure $\angle D C E$, how should the tracing be placed on the scale? Put $\mathrm{C}$ on $\mathrm{V}$ and $\overrightarrow{C E}$ on the zero ray. number cf the ray on which $\overrightarrow{D P}$ falls, $\mathrm{m} \angle \mathrm{DCE}=$ (3).

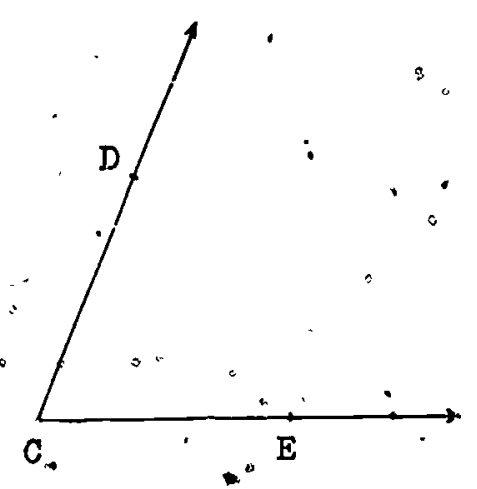

$$
259
$$


422

4. Trace the angles below and use the angle scale to find the measure of each, to the nearest unit.

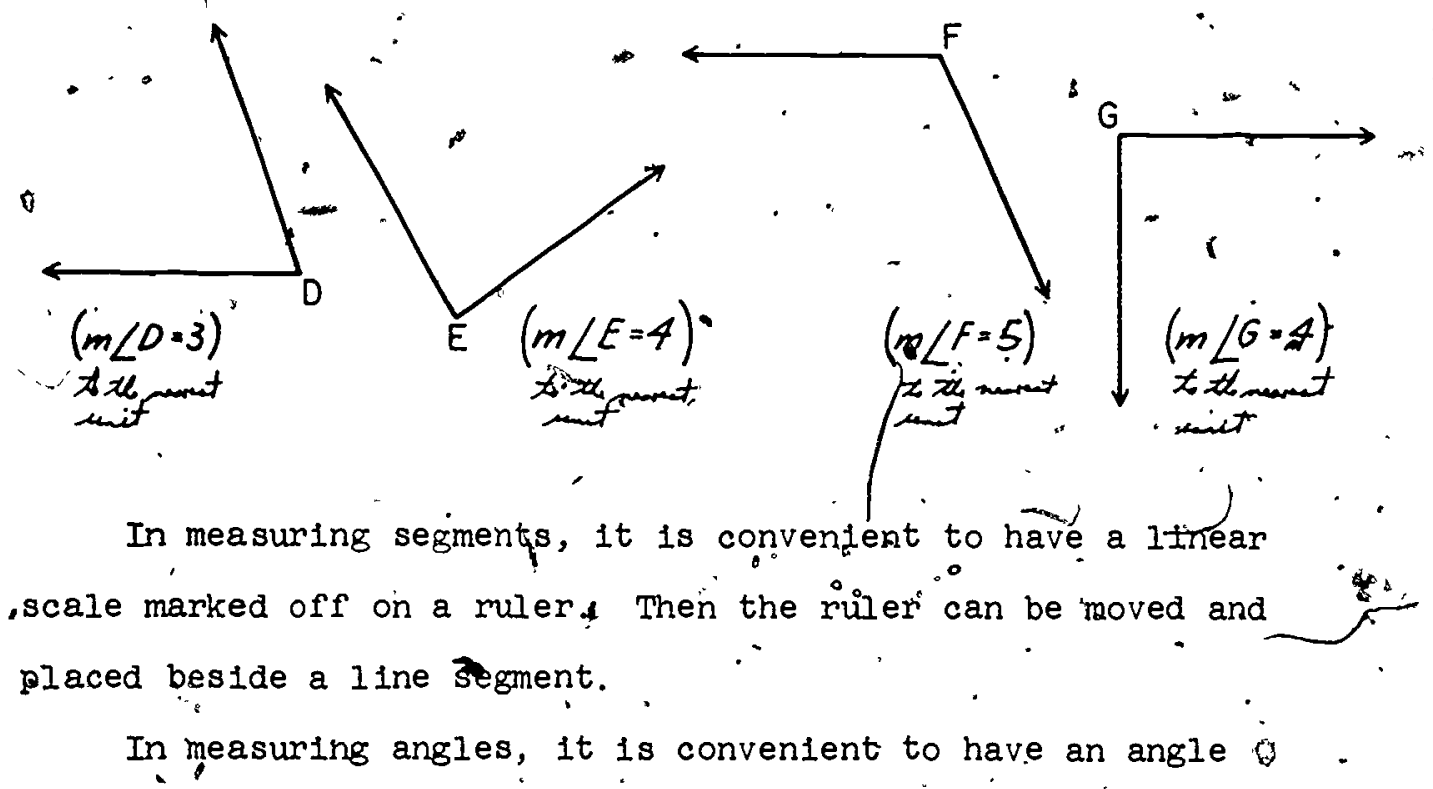
scale marked off on a protractor: Then the protractor can be moved and placed on an angle. Your teacher will show you how to make a protractor. 
At this time there is value in a teacher demonstration.lesson showing how to make a protractor and mark. it off with an octon scale. The teacher should have a half disc of tag board about the size of the chalkboard protractor with the middpoint of the diameter of the disc clearly marked. On the chalkboard she should have'a scale like the one on page $1 / 21$ of the pupils' text. Two of the rays of the scale,, $\mathrm{VA}$ and $y \mathrm{C}$ are on the same straight line and extend in opposite directions from $V$. Place the midpoint of the $r$ disc on $V$ (the common endpoint of all the rays) on the scale of the chalkboard, making sure that the diameter of the disc falls along the nays VA and $V C$. The rays of the scale on the chalkboard should extend beyond the disc. Mark the point... where each ray falls on the tag board disc. Then connect each of these points with the midpoint of the diameter of the disc.

The teacher should have a tas boand hal $f^{2}$ disc about the size of a standard protractor; for edch child. After the demonstration she can have each child make bis own protractor, marked off in, octons; using the scale of rays provided in his text on page ${ }^{\prime} 2 I$. 
P423

Here is a picture of a cardboard protractor with a smaller unit angle than we used before. Only parts of the rays are shown.

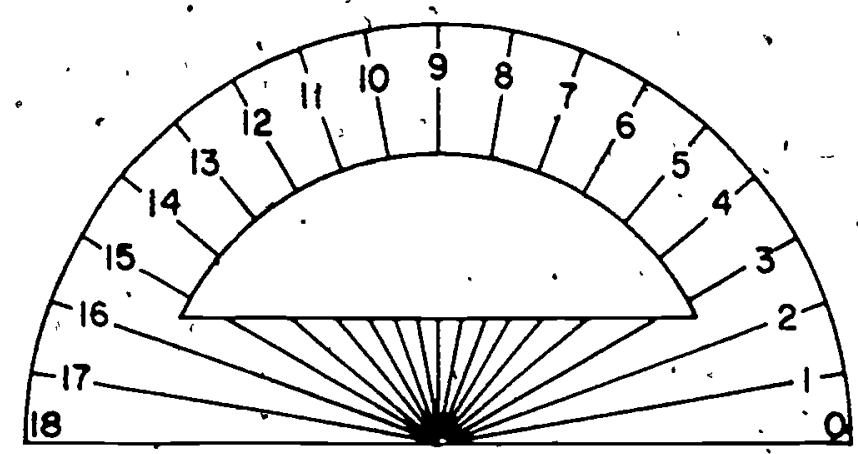

The rays are broken because part of the cardboard is cut out so you can see the ray of the angle you are measuring.

5. Below is a sketch showing the protractor placed on a set of rays. The rays have the same endpoint, $A$, and the V-point of the protractor is on. A. Find the measures of the angles named.

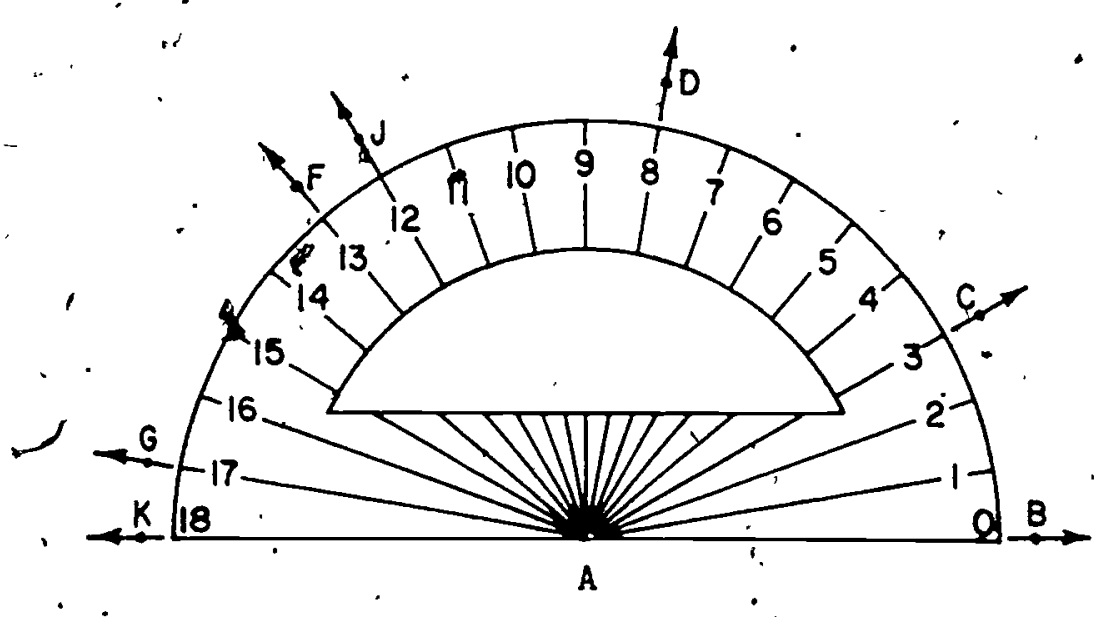

707

262 
* Exploration
a) im $\angle B A C=(3)$
f) $\mathrm{m} \angle \mathrm{GAF}=(4)$
b) $\mathrm{m} \angle \mathrm{BAD}=(8)$
s) $\mathrm{m} \angle \mathrm{CAD}=(5)$
c) $\mathrm{m} \angle \mathrm{BAJ}=(12)$
h) $m, \angle D A F=(5)$
d) $\mathrm{m} \angle \mathrm{BAF}=(13)$
1) $\mathrm{m} \angle \mathrm{DAG}=$ (9)
e) $\mathrm{m} \angle \mathrm{BAG}=(17)$
j) $\mathrm{m} \angle \mathrm{CAJ}=(9)$

In addition to the scale with the zero ray at the right, many protractors also have another scale with the zèro ray at the left. This scale is placed on the finner rim.

6. Look at the second scale on the protractor shown in the plcture below. This scale, is written on the inner rim. Zero is put on the ray the lest $(\overrightarrow{R S})$ and rays have

- been numbered in order unt1l the ray on the same line with zero is reached ' $(\vec{R})$. Write these numerals on the sketch you made for Exercise 5. Find the measures of the angles named in Exercise 5-using the new scale. Are the measures the same? (Jhe measures are thes same:)

* This may be used as an independent exercise.

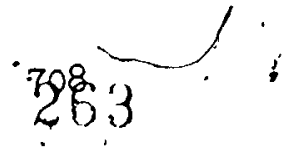


The advantages inhaving the two scales can be seen from the. following sketcheș:

To measure $\angle S \dot{R} T$, the zero ray at the leit is placed on $\overrightarrow{R S}$. You use the Ianer scale to find the measure. $m \angle \mathrm{SRT}=\frac{(7)}{*}$

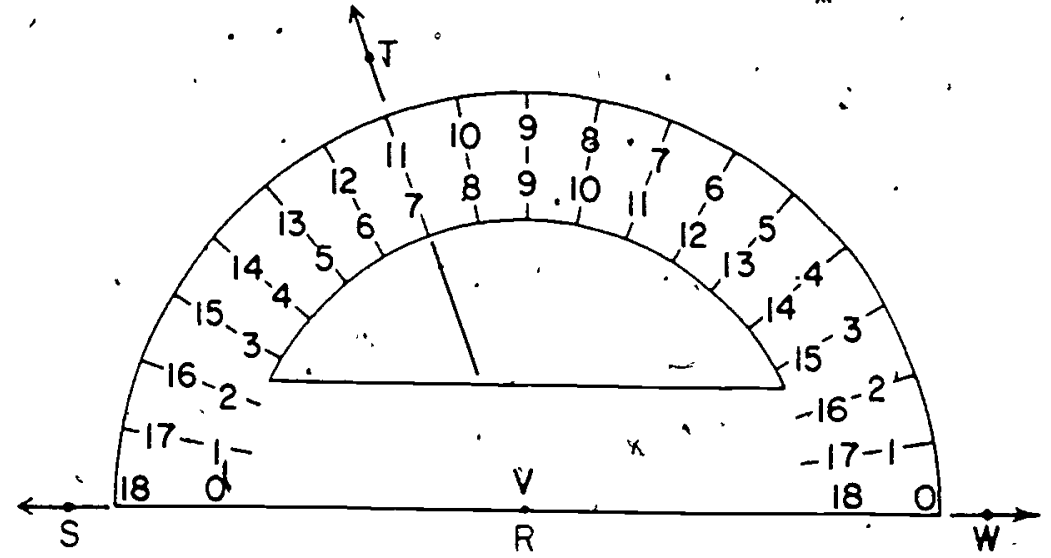

- To measure $\angle$ DEF, the zero ray at the right is placed on $\overrightarrow{B D}$. You use the outer scale to find the measure. $m \angle D E F=(8)$.

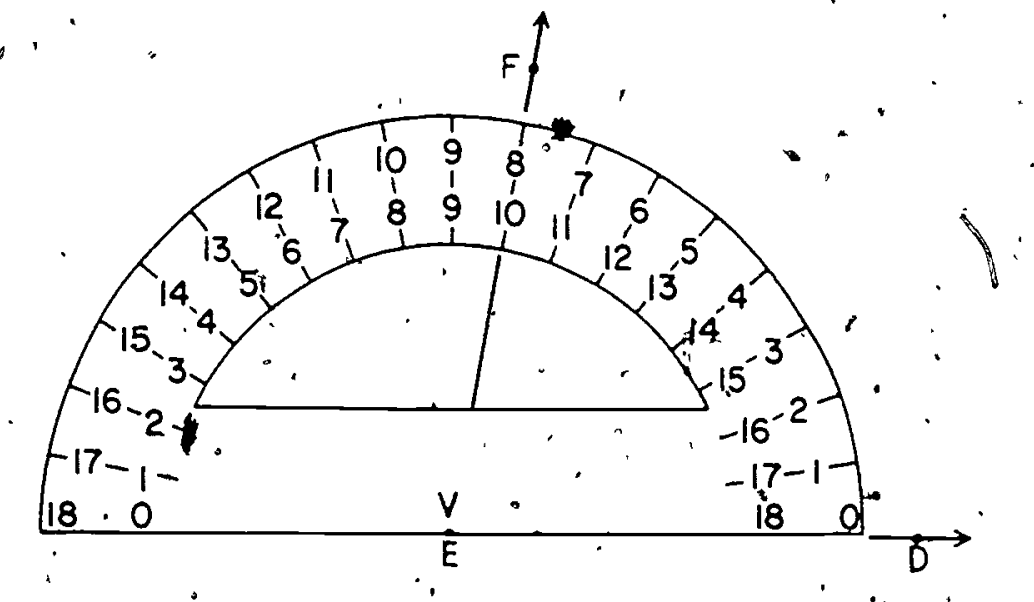

It Is very easy-to read' the wrong scale by mistake. "You 't1 prevent most such errors by estimating of the angle as a check of your measurement. Of course measure the same angle, by movirg the phractor. 
7. The following two sketches are coples of the same angle, $\angle A B C$., In the first sketch, the protractor is placed so the zero ray on the left of the protractor is on $(\overrightarrow{B A})$." Which scale would you use to find the measure of the angle? (thminamale) In the'second sketch, the zero, ray on the right of the protractor is placed on $(\overrightarrow{B C})$. Now which sfale would you use. to find the measure of the angle? Is the measure of the angle the same either way? (yes)

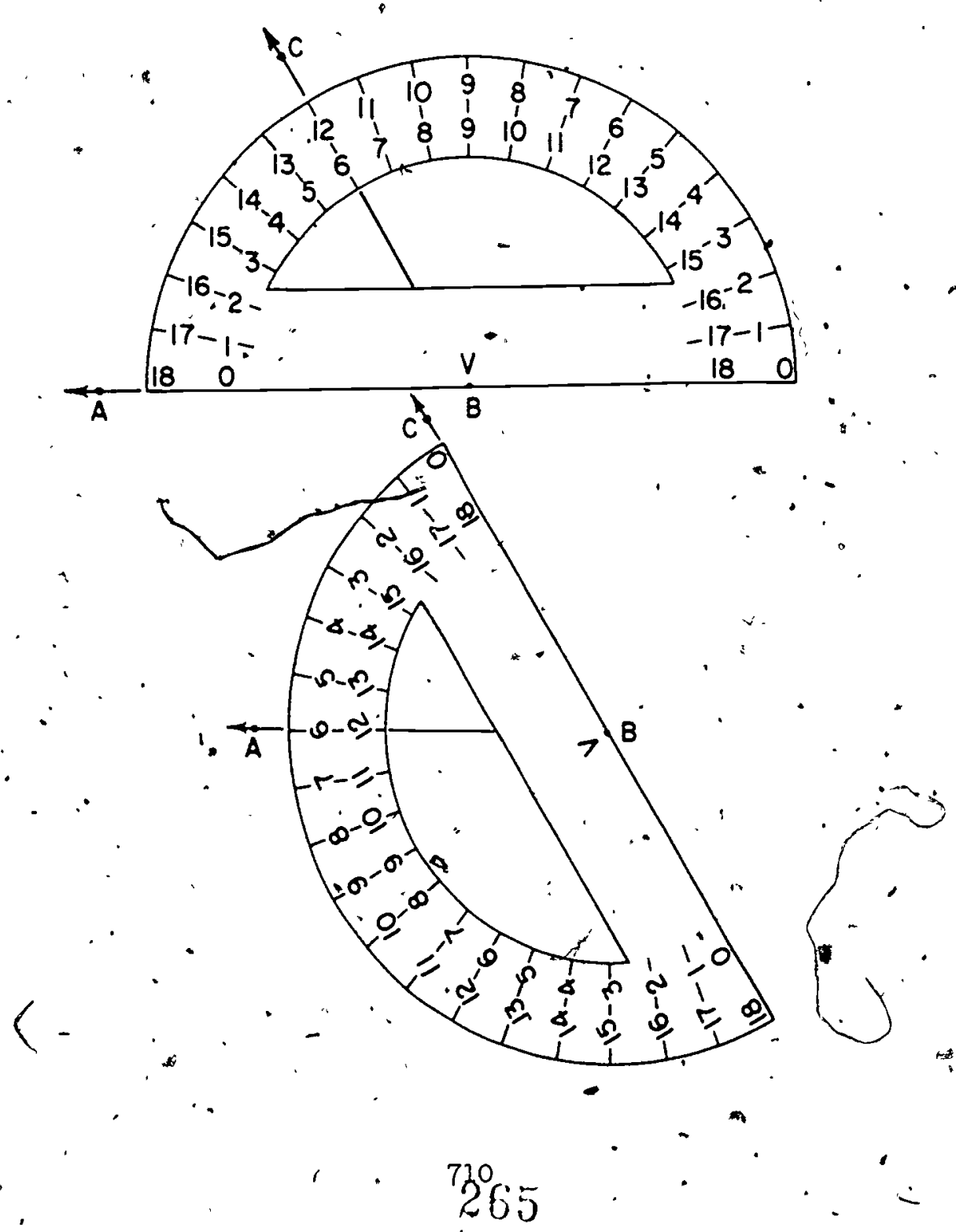


P427

Exercise set 3

1. Use the "acton" scale on your protractor to find the measure of each of the angles below (to the nearest acton). After you have measured an angle, check your measure by placing the protraction with a zero ray on the other side of the angle. Write your answer like this:

. $m \angle B=3$, to the nearest acton.
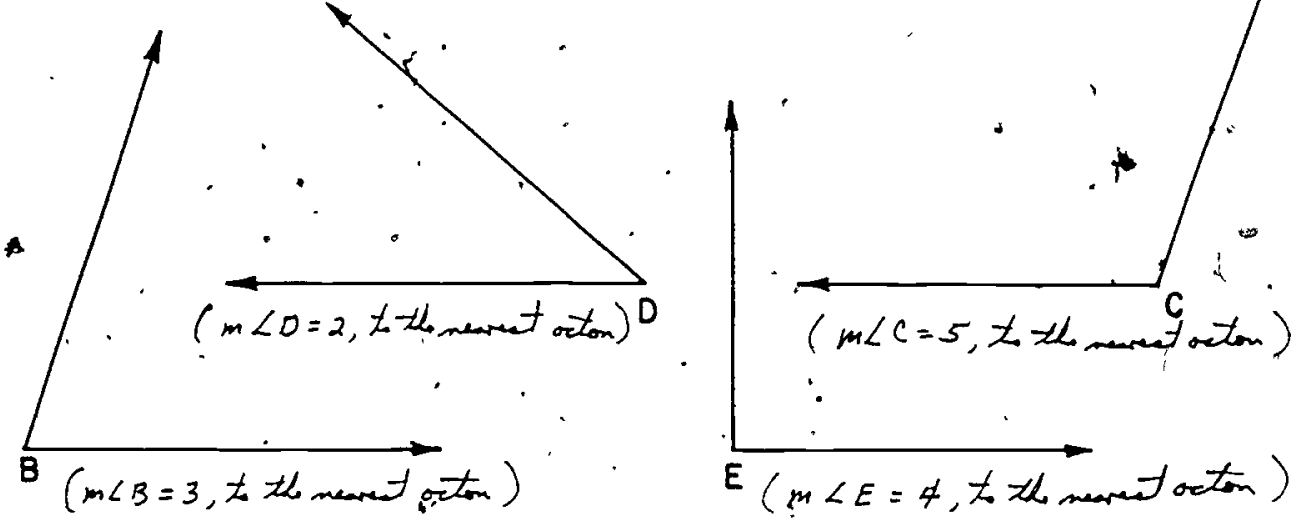

2. Which of these sketches shows the correct way to place the protractor to find the measure of $\angle$ DEF? Why?

$\mathrm{m} \angle \mathrm{DEF}=(2)$, to the nearest acton.
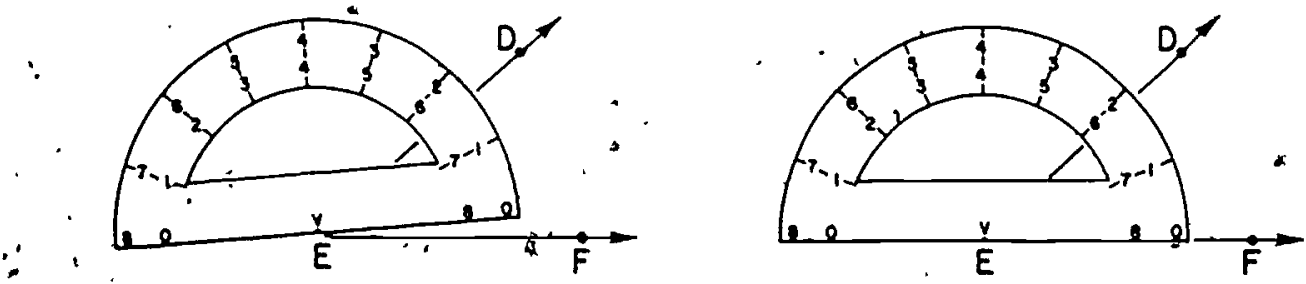

(The arete' on th sight a hive tho correct arg to place the)

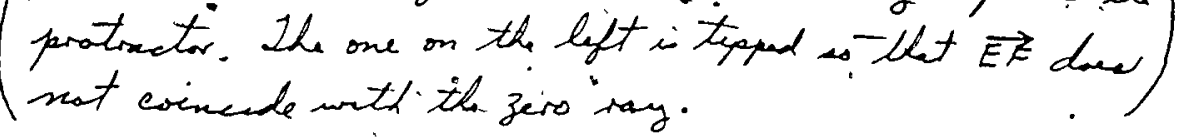

${ }_{2}^{8} 66$ 
3. Which of these sketches shows the correct way to find the measure of. $\angle \mathrm{GHI}$ ? Why?

$\mathrm{m} \angle \mathrm{GHI} \cdot=3$, th tho nearect oiton

(the akitel on the left alume the correct way to fendthe measure $)$ palacid on the wartex of the angle.
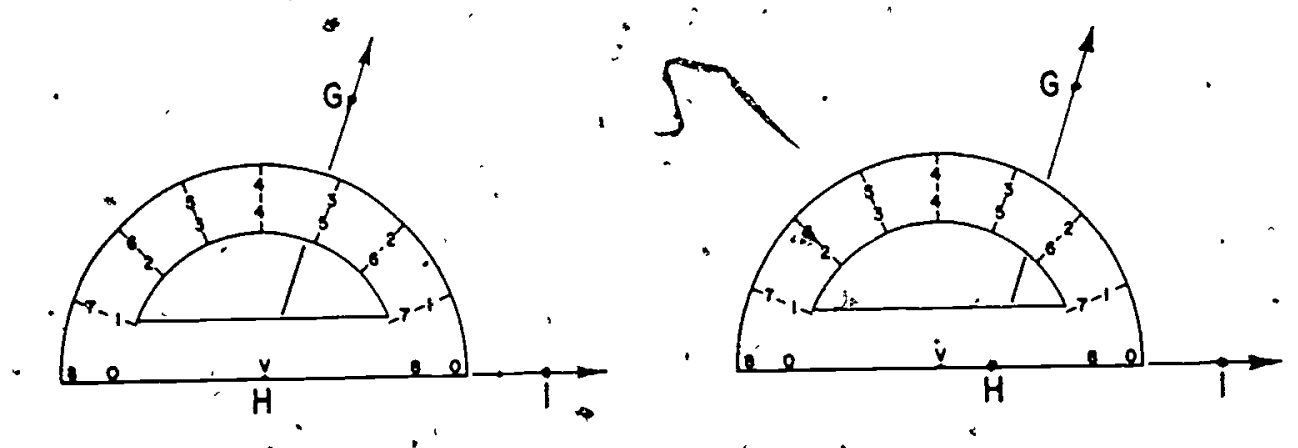

4. A boy said that the measure of the $\angle J K I$ in octons. is 5. Whot was his mistake?. What is $m L j K L$ ? (The bay reffl from the worng acele. He read fromi the enmer scile" instead the that scale. $m<J \dot{K} L=3$ ).

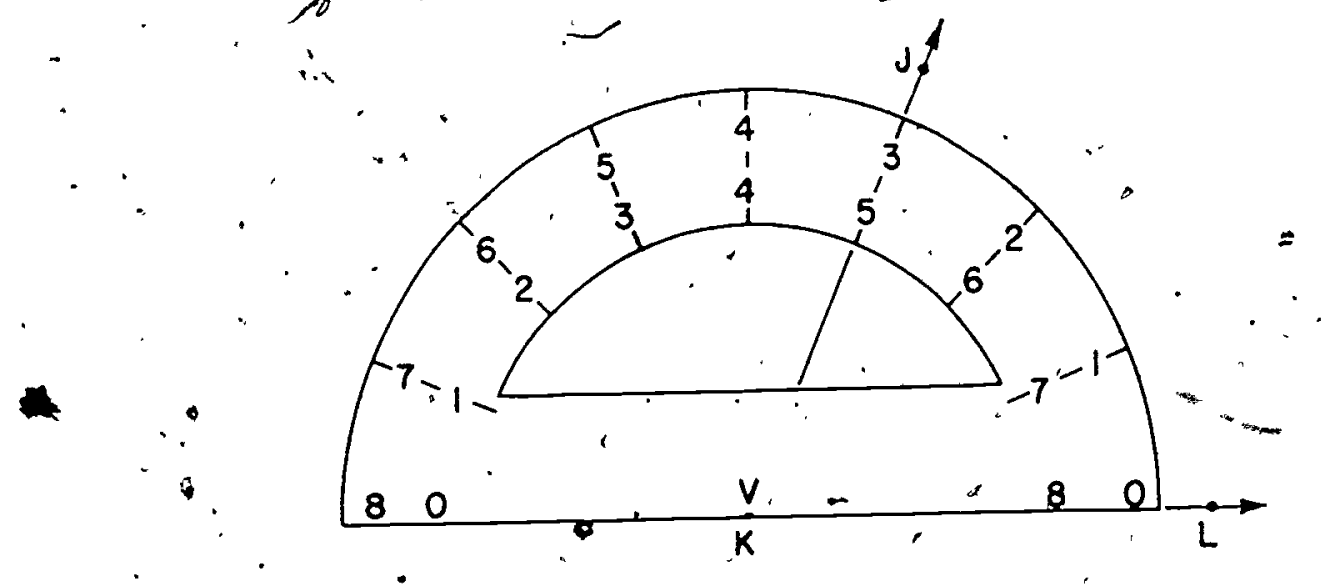


DRAWING AN ANGLE OF GIVEN MEASURE

Objective: To develop skill in making drawings with a protractor of an angle whose measure is . a given whole number of units.

Materials Needed:

Teacher: Straightedge, acton protractor Pupil: Straightedge, acton protractor

Vocabulary: No new words in this section. . 


\section{2 \\ P429}

DRAWING AN ANGLE OF “GIVEN MEASURE.

\section{Exploration}

You can use your protractor to draw an angle whose measure, in octons, is to be a given. Whole number. Do you see how to i. use the protractor in this way?

Draw $\angle \dot{B}$ so that $m \angle B$, in octons, is 6 . Since the vertex mast be point $(B)$, draw $\overrightarrow{B A}$. Place the protractor with the V-point on the vertex and the zero ray of one scale on $\overrightarrow{B A}$. Mark a point ' $C$ at the number. 6 on the same scale. Remove the protractor and draw $\overrightarrow{\mathrm{BC}}$. Each of these angles has a measure of 6 , in octons. Does your angle look." itke one of them?' (yes)

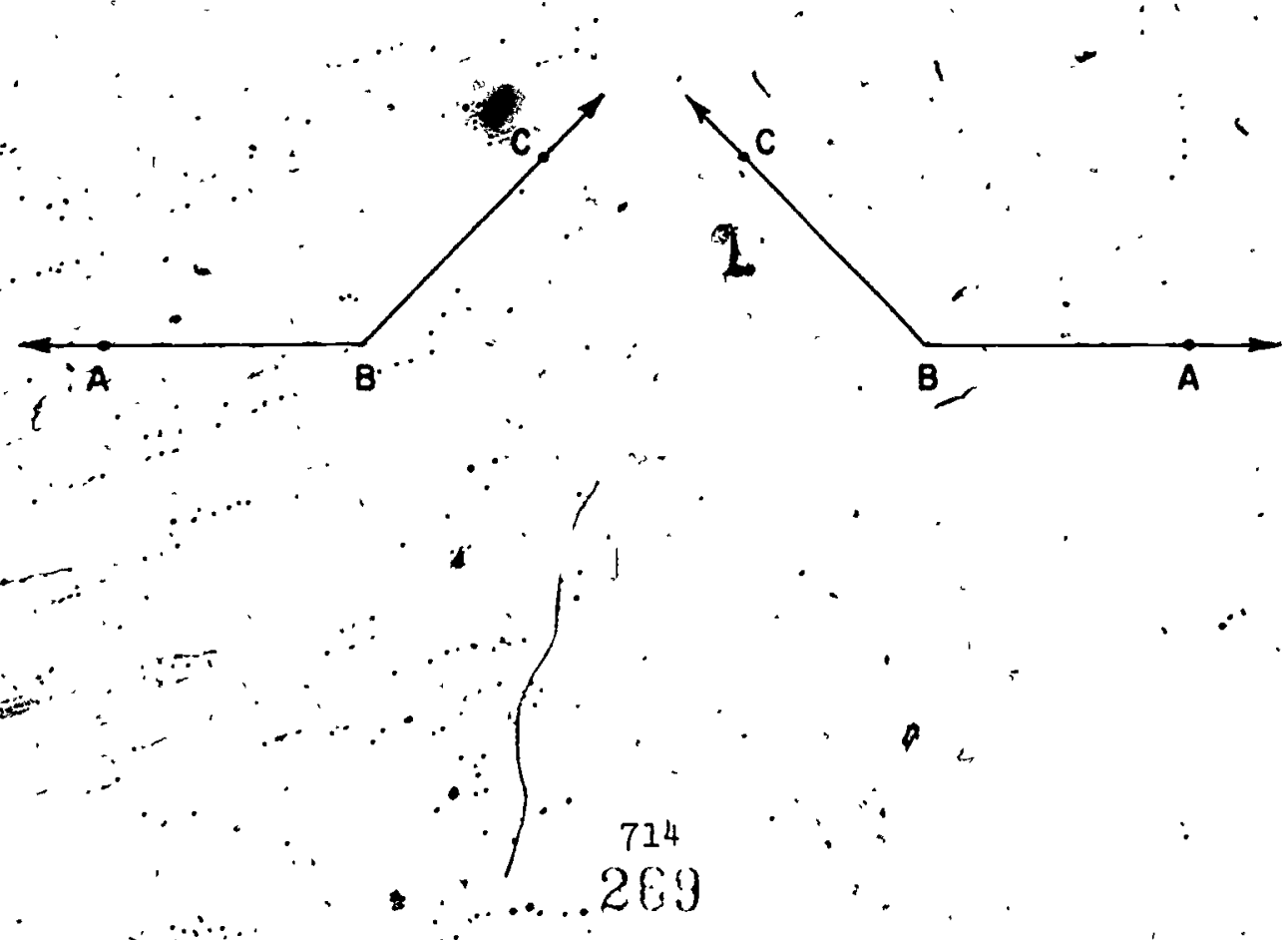


P430

\section{Exercise Set 4}

In these exercises, draw rays and label points as in the sketches.

1. Copy the figure below on your paper.

Draw on $\overrightarrow{A B}$ an angle with a measure of 5 , in octons.

Label 1t " $\angle$. BAC. Draw the angle so that $\overrightarrow{A C}$ is above $\overrightarrow{A B}$. 2

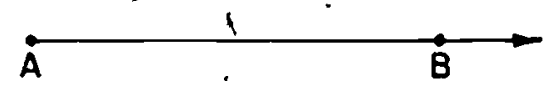

2. Copy the figure below on your paper. Draw an angle with a méasure of 3 octons, using $\overrightarrow{D E}$ as one ray. Label it $\angle E D F$. . Draw the angle so that $\overrightarrow{D F}$ is above $\overrightarrow{D E}$.

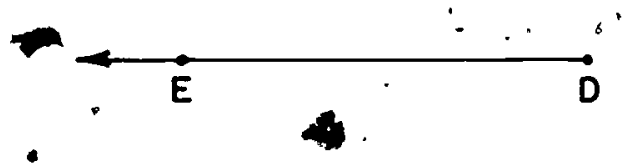

3. Copy $\overrightarrow{J K}$ on Jour paper. Draw an angle with a measure of 2 using $\overrightarrow{J K}$ as one ray. Label it $\angle K J L$. Draw the angle so that $\vec{J}$ is below $\overrightarrow{J K}$.

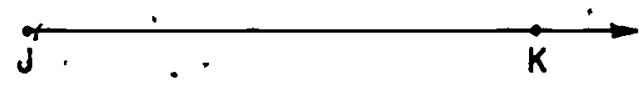

4. Copy $\overrightarrow{\mathrm{RS}}$ on your paper. Draw $\angle$ SRT whose measure is 7 using $\overrightarrow{R S}$ as one ray. Draw the angie so that $\overrightarrow{R T}$ Is below $\overrightarrow{R S}$.

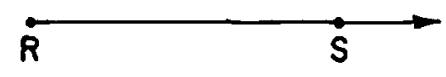




\section{PRACTICE IN MEASURING :ANGLES}

Objective: - To develop the following understandings and skills:

1. Not every angle has one ray drawn horizontally.

2. Regardless of the position of the rays of an angle, we use the same procedure to measure the angle with a protractor. .

3. We may need to extend our representations of one ray of an angle in order to read 1 ts measure on the scale.

4. Extending our representation of the rays of an angle does not change the measure.

Materials Needed:

Teacher: Straightedge, octon protractor

- Pup11: Straightedge, octon protractop

volabulary: No new/words in this section

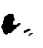

If the exploration is followed closely al1 the understandings w111 be developed. The Exercises provide opportunity to practice the skilis. 
P 431

$\sqrt{n+2}$

PRACTICE IN MEASURTNG ANGLES

Exploration

In most of the angles you have measured, one ray was horizontal, as in $\angle R$ and $\angle S$ below.

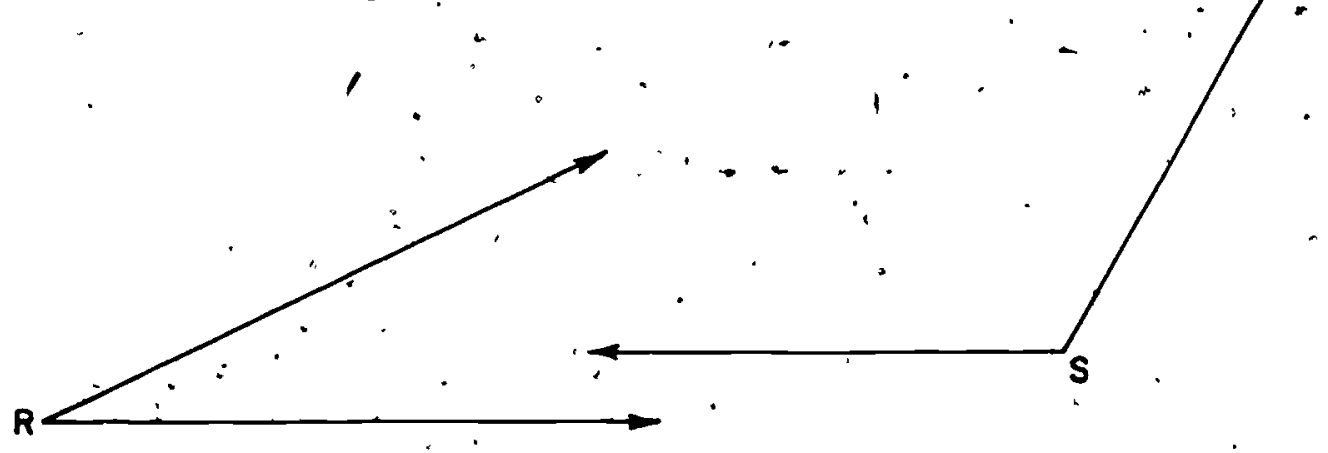

1. How weuld you find the measure of $\angle A$ ? This angle is in

a dfferent position from others you have measured. Its measure is found in the same way. Place your protractor so a zero ray falls on either $\overrightarrow{A B}$ or $\overrightarrow{A C}$. Be sure. the $V$ of the protractor. is exactly on, vertex $A$. The other ray of the angle can then be matched with the part of a ray marked on the protractor.

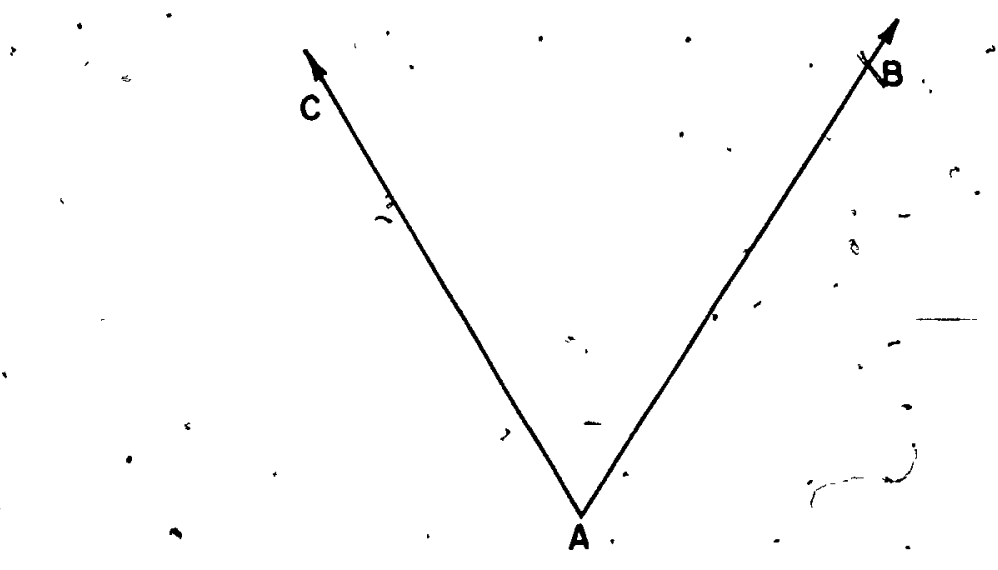

717

272 
2. These sketches show the two ways to place the protractor.
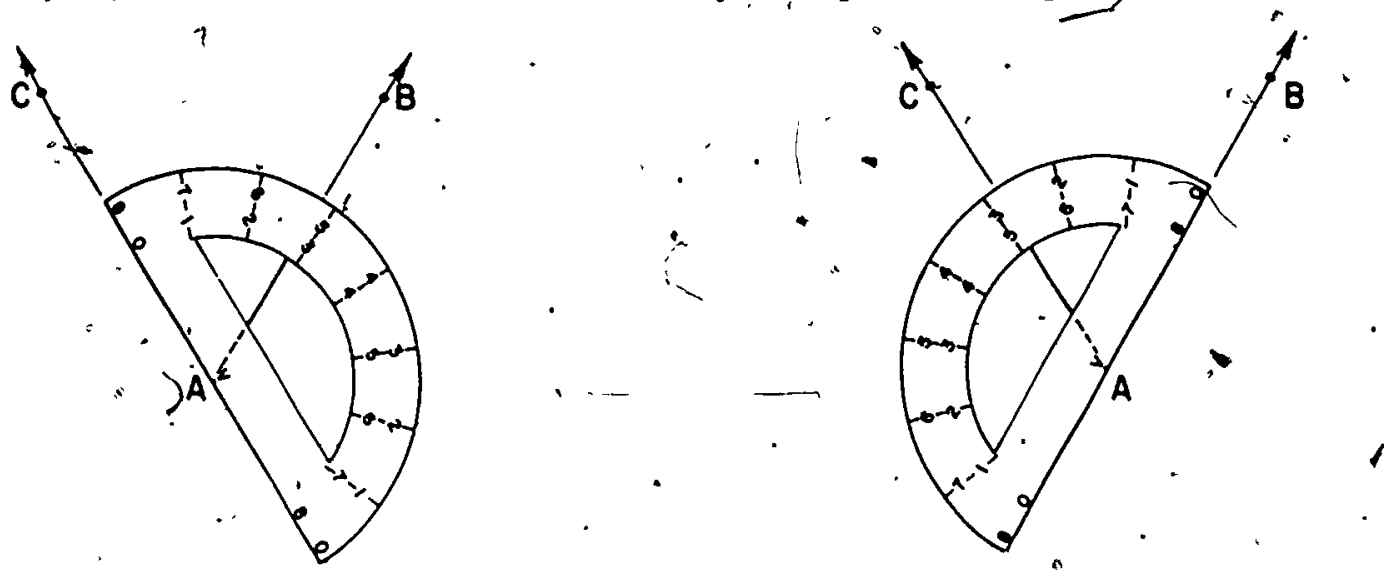

Put the zero ray on $\overrightarrow{A C}$ or put the zero ray on $\overrightarrow{A B}$.

Does it make any difference in the measure whether the zepo ray is on $\overrightarrow{A B}$ or on $\overrightarrow{A C}$ ? In each sketch, we see the measure of $\angle A$ to be about 3 . Why. Is its measure 3 rather than' 5 , to the nearést octofi? (It mekee mo defference y the zaro ray.is on $\overline{A B}$ or on $\overline{A C}$. He measure is:3 rather then 5 becauce the rey whet. felle under th protructor ecalo is neareat ti 3 from the zaro ray if eech caes.)

3. Find the measure of $\angle R$ and $\angle E$.

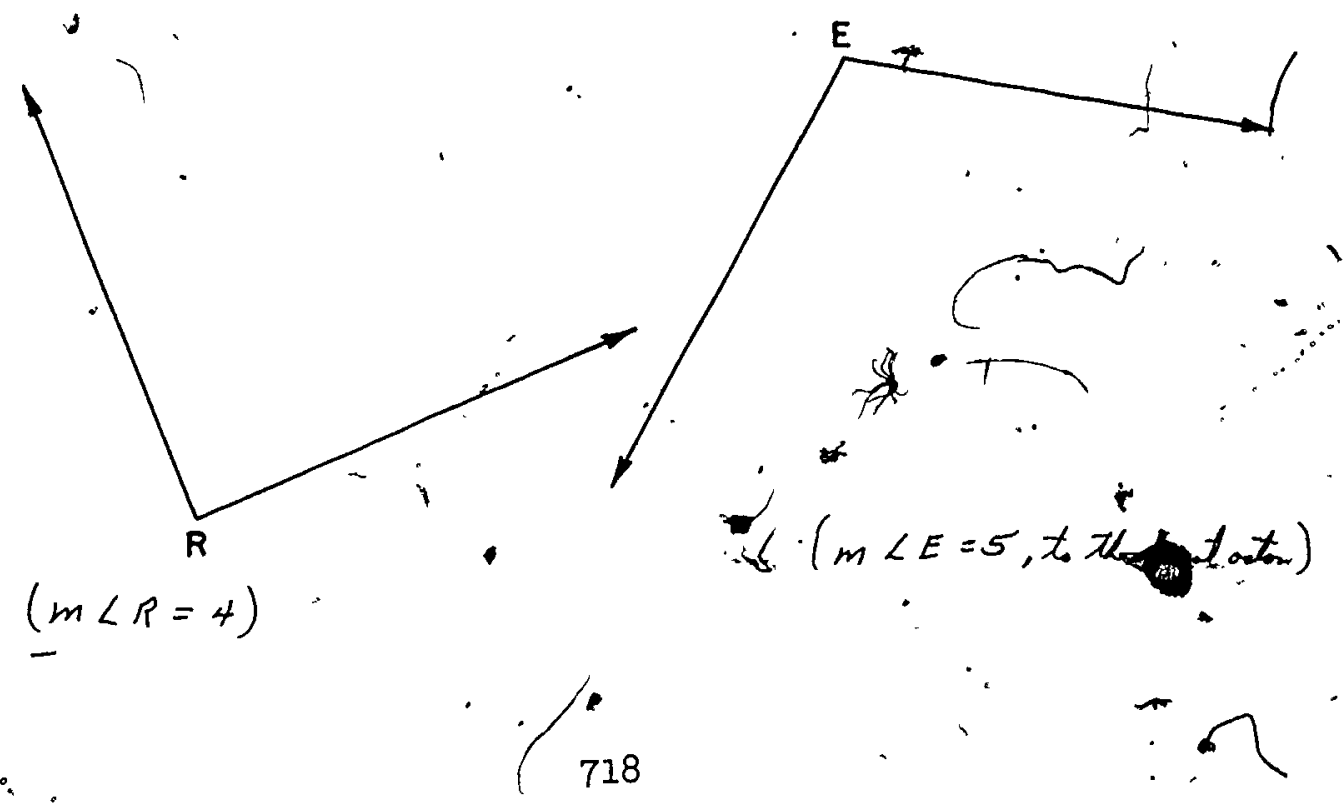


$\mathrm{P} 433$

4: How do you think we can firn the measure of $\angle L$ ? Can a protractor be "placed on $L L$ so that you can read its measure? Do $\overrightarrow{\mathrm{IN}}$ and have a definite length? $\left(\eta_{0}\right)$ (a protractor canst he placed on $L L$ as thetis measure can.

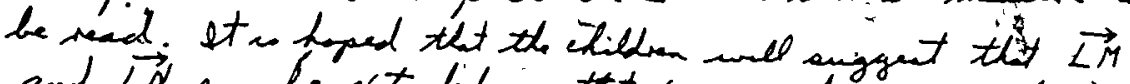

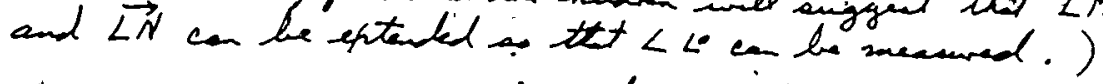

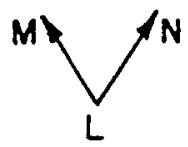

5. If $\overrightarrow{L M}$ and $\overrightarrow{L N}$ are not long enough to extend beyond the protractor, can they be extended without changing the size of the angle? What is its measure? Check you measure by putting the zero-ray of your protractor of other ray of the angle. Could you repress.. the rays in some way without drawing them?

- (Clue: Try using a sheet of paper or some other kind of. straightèdge.) (mm $L L=3$, th th nearest acton. Il ray coned be reverenced without drawing the by placing a pence of pepper on the ray so that the edge of th paper is a ecterien of the part of the ray alow.)
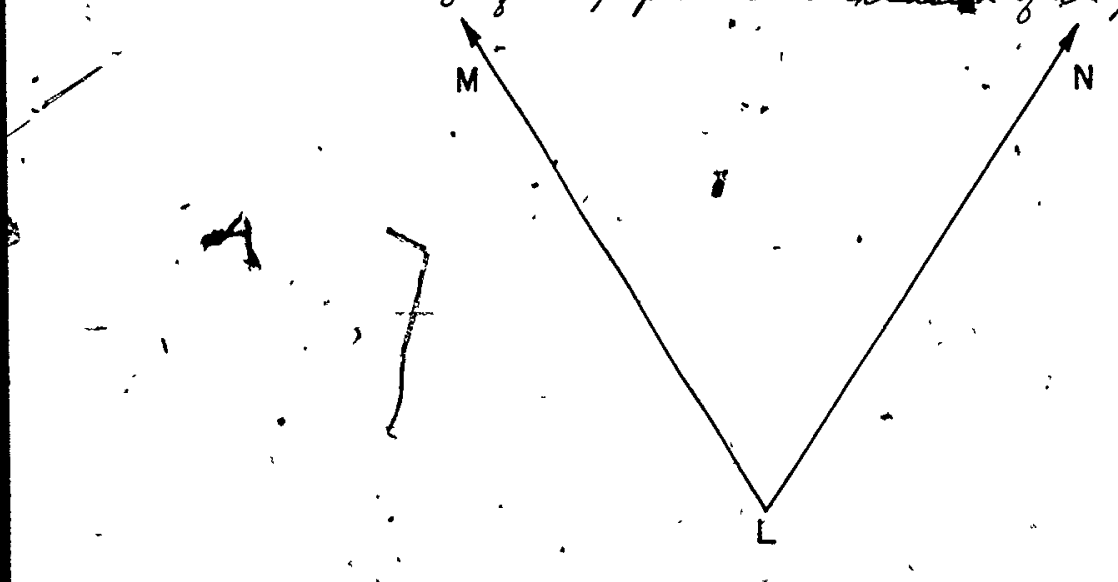

$-719$

$$
\therefore \quad \therefore \quad 27
$$

ERIC 
$P 434$

6. Find the measures of $\angle R$ and $\angle T$. You will need to show more of one or both rays.
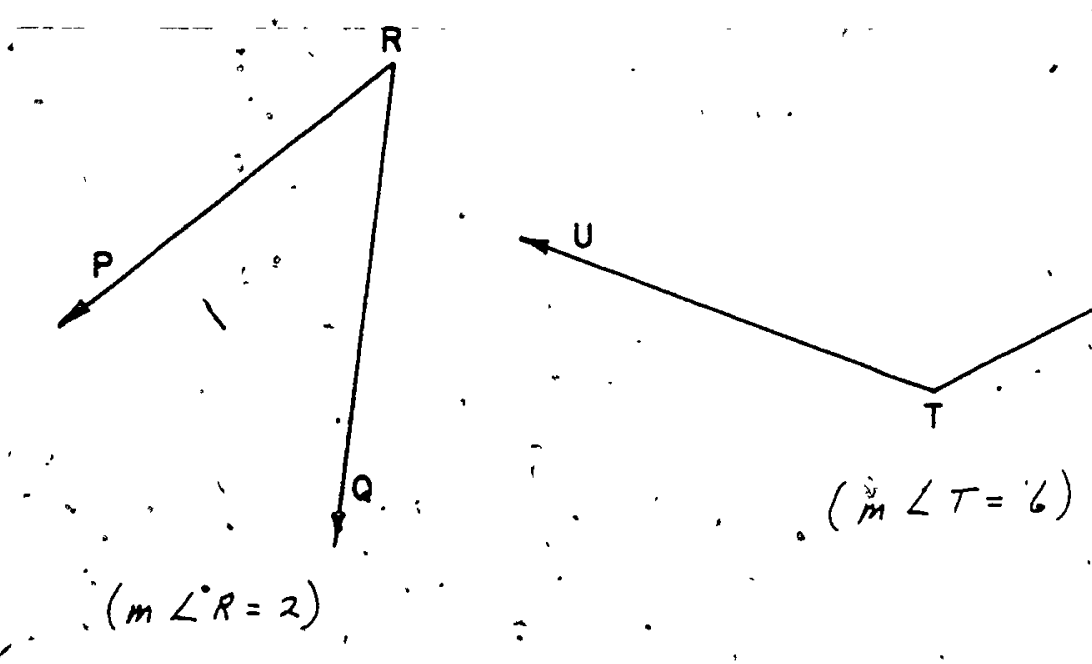

7. a) Which angle has the larger measure, $\angle A$ or $\angle B$ ? $(\angle A)$.
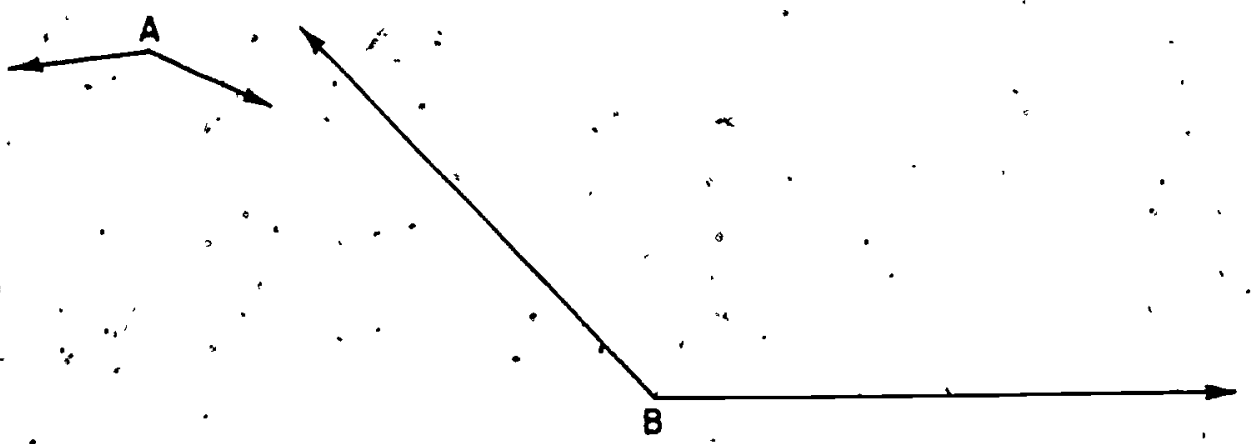

b) Is the measure of $\angle A$ changed if you extend the part of 1 ts rays which are shown on this page? $\left(\eta_{0}\right)$

c) $\angle A=(7)$, to the nearest acton:

d) $/ m \angle B=/(6)$, to the nearest acton. 
P435

Exercise Set $\underline{5}$

Find to the nearest octan the measures of the angles below. Use your acton protractor.

1.
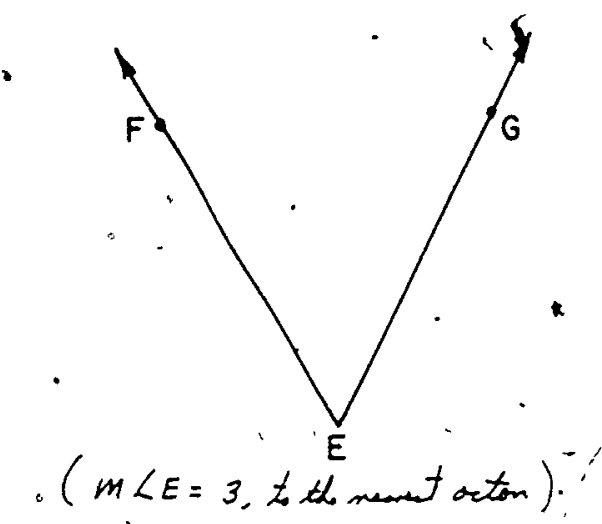

2.

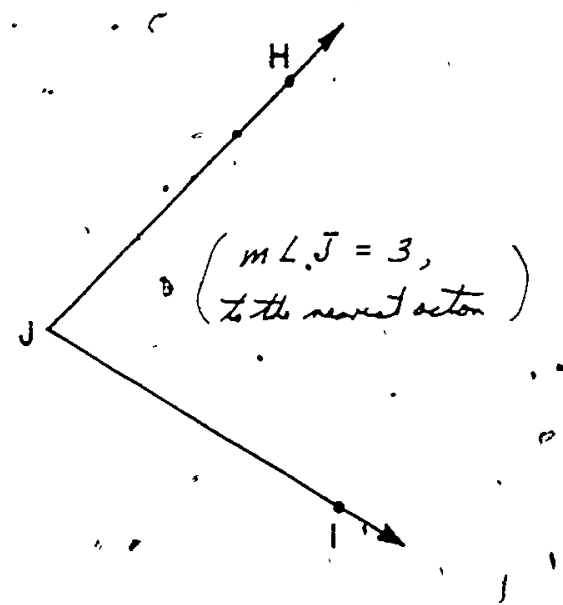

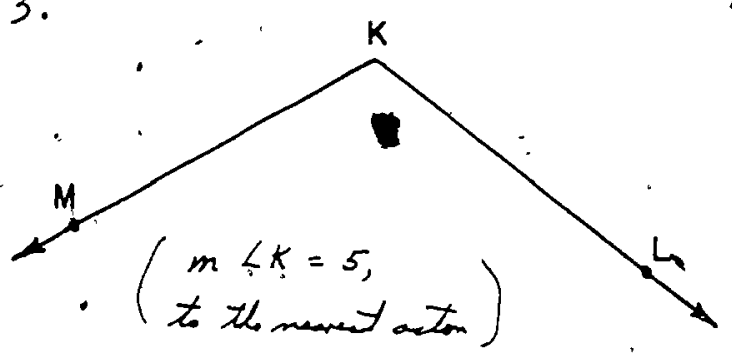

4.

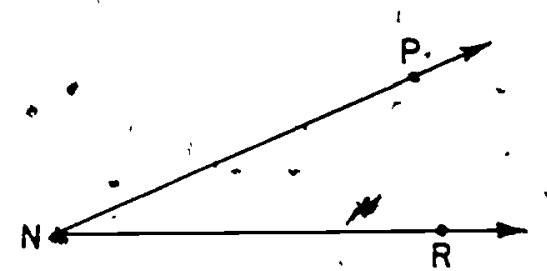

$\left(\begin{array}{l}m \angle N=1, t \\ \text { te nevers ox tom }\end{array}\right)$.

5. In Exercises $I$ and 2, was $m \angle E=m \angle J$ ? (yes) Is $\angle \dot{\mathrm{E}} \cong \angle \mathrm{J}$ ? $\left(x_{0}\right) \quad$ (Use a tracing)

$\therefore$ Although $\angle E$ is noticongruent to $\angle \mathrm{J}$, the measures, to the nearest acton, were the same' number. 'If the unit angle, were much smaller, then the fact that, the angles are not congruent would be shown clearly in the, measures.

721

ERIC :

$\therefore 276$ 
A STANDARD UNTT FOR MEASURING ANGLES

Objective: To develop the following understandings and skllls:

1. The desire to communicate gives rise to the need for a standard unit of measure.

2: The standard unit of angle measure' is the degree. Its symbol is.a raised "o", 1.e. " $42^{\circ}$ "is read". t2 degriees.

3. One hundred elght $t^{5}$ unit angles of 1 degree each may be laid off successively and represented on a" semi-clrcular protractor.

- The measure of an angle is a number. The size of an angle 'is given by 'naming its measure and also" the unit used:

5. We concern ourselves only with anglès whose measure 1 in degrees are more than 0 and less than .180 .

Materials Needed:

Teacher: Straightedge, standard protractor for chalkboard (with the numbers represented-on the outer scale. increasing in the counter-clockwise direction)

'Pupil: Stralghtedge, standard protractor (1f protractors are purchased, try to obtain ones in which the . numbers represented on the outer scaje increase In the counter-clockwise direction)

Vocabulary: Standard unit, size of an angle, degree.

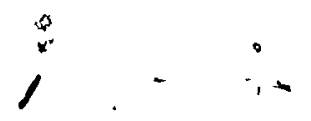

Follow.the ExpIoration. 
A STANDARD UNIT FOR MEASURING ANGLES

\section{Exploration}

As you know, the Iinear scale on a muler is usually marked off usins a, standard unit such as the inch or the centimeter. A staridard unit is one whose size has been determined by agredment among people. We would foind it difficult to commicate witr" people or to carry on business yf everyone made up his orm units. What other standards of measure can you name?

There are also s'tandard untts for meatsuring angles, so i that people throughout the world can communicate easily. The tandard upit for measuring angles is the jegree. The undt, angle of one degree is smaller than the octon, the urit angle we used on the preceding pages. In fact, the octon is $22 \frac{l}{2}$ times as large as an ang of one degree. Its measure in degrees is, $22 \frac{1}{2}$. The symbol for degree is $\because$, An angle of $+5_{0}^{Q}$ means an angle whose measure, in degrees, is i5. As you work with your protractor you will discover that it takes 360 of these unit angles usingra single point as a common veritex and their. interiors, to cover the entire plane. Even'in ancient Mesopotamia the angle ol $1^{\circ}$ was used as the angle of unit meașure. The selection of ir unit which could be fitted into a plane just 36,0 times ras probablý influencéd. by the 'fact that their year had ' 360 days. 
P437

1. Look at the side of your protractor on which the standard unit is the degree.

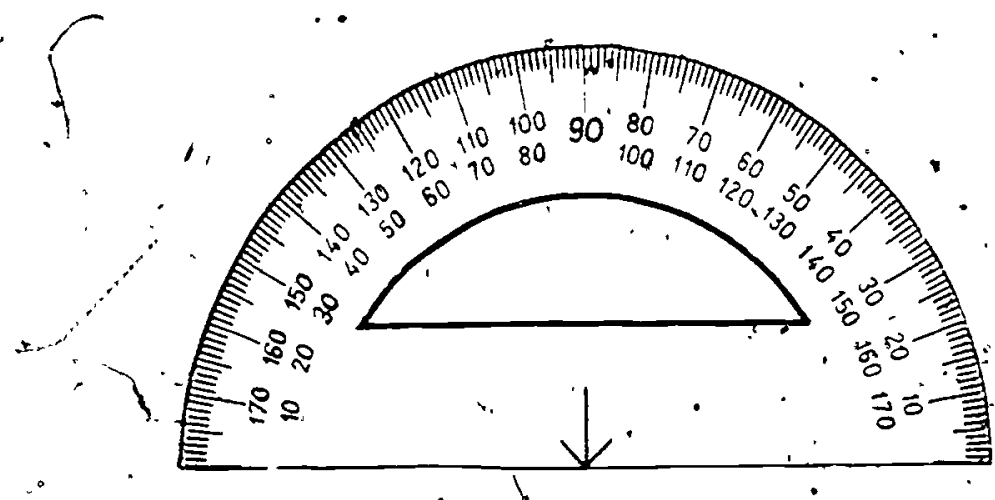

An angle of I degree is formed by rays, with endpoint.. $\mathrm{V}$, through two of the marked points next to each other: Does this seem like a very small angle? (yen) Would it. seem so small if the segment of the ray shown were 'extended to 15 feet?

- (An angle of on degree "would probably seen to be a small angle th th pung. It mill mot seem so suable if the

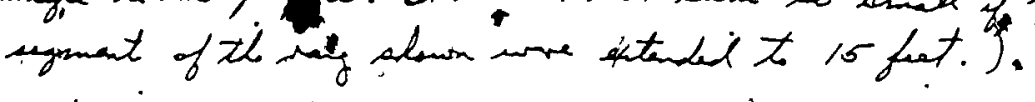

2. Since ' $I$ degree is so small, only every tenth degree is numbered on the scale, What other numbers are mil sing?

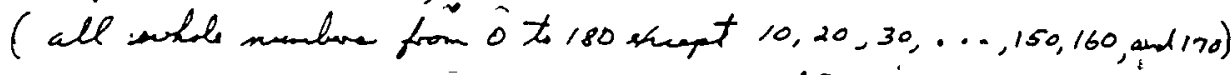
Why is ' 0 not printed on' the scale? What is the largest number represented on the scale? Is 1 ts humeral

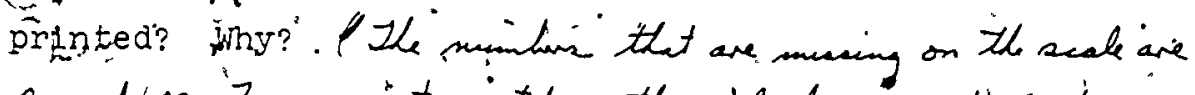

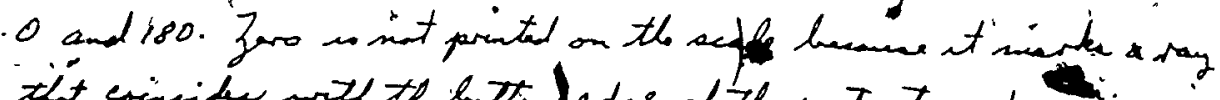

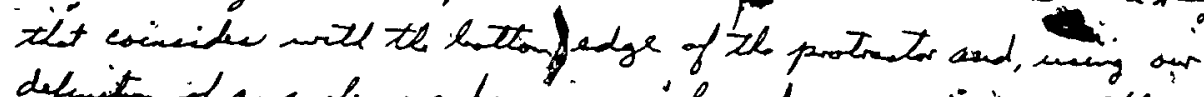

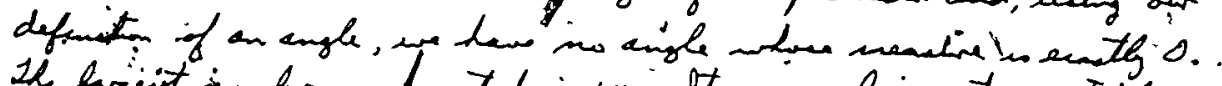

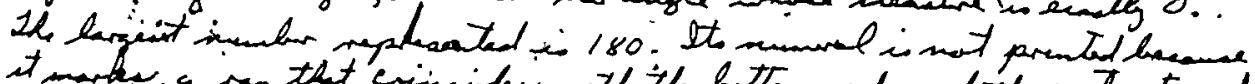

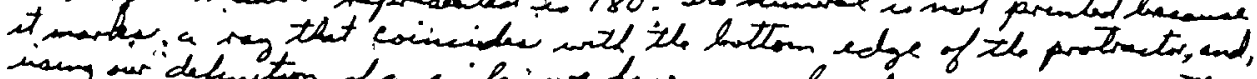

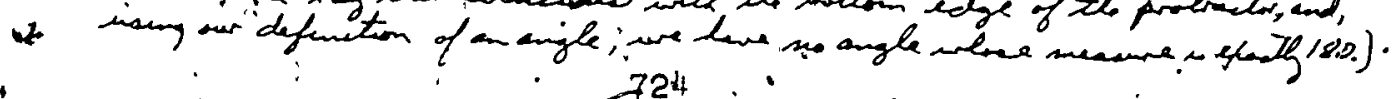
273 
3. Look at the side of the protractor on which the standard unit is the degree.

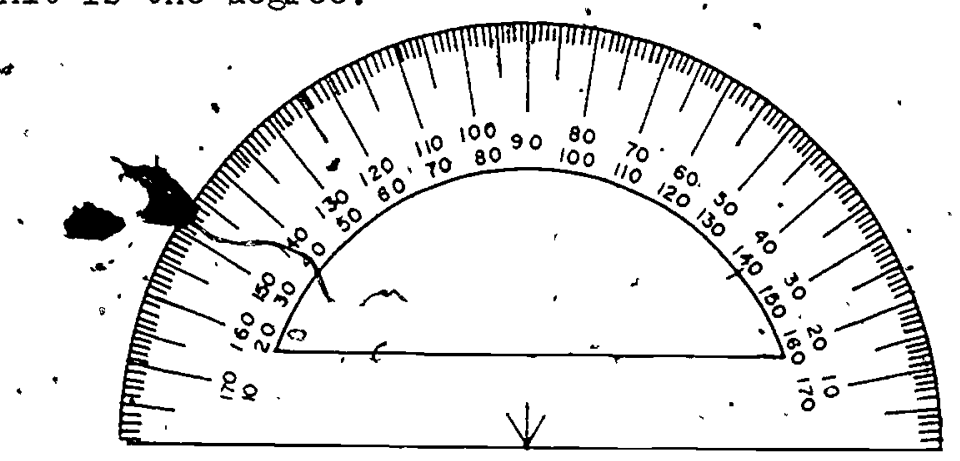

You use "thi's standard protractor to" measure an angle in degrees in the same way you used the scale on the other side. to measure an angle in octons. 'You must be eareful'. about the rollowing things:

a. Place the $V$ polnt of the protractor on the vertex of the angle. Be sure the protractor cpiers part of the interior of the angle.,

b. Place the protactor with one of the zero rays exactly on one side or" the angle. Notice whether this zero is a number on the Inner scale or the "outer scale. This is the scale you must use. 'c. Find the polnt where'the other side' of the angle. N intersects the rim of the protractor. If not enough of the rayi is, shown to intersect the rim, - can the raýs of the angle be extendeqd without.

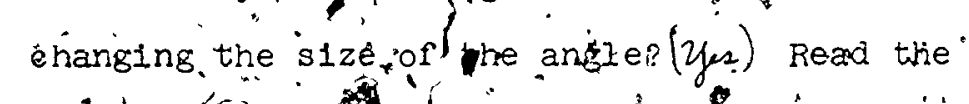

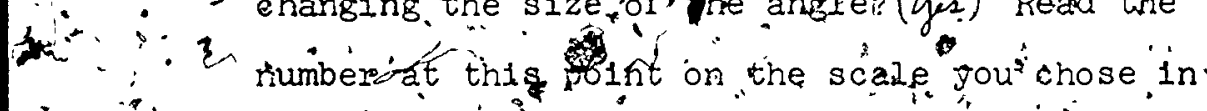

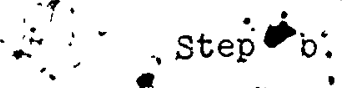

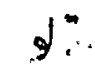

is 
p439

$\therefore$

Exercise Set

1. The sketch shows a protractor placed on a set "or rays from", point, K. Their point of the protractor is on $K$ : Find the measure', in degrees, of each angle named.

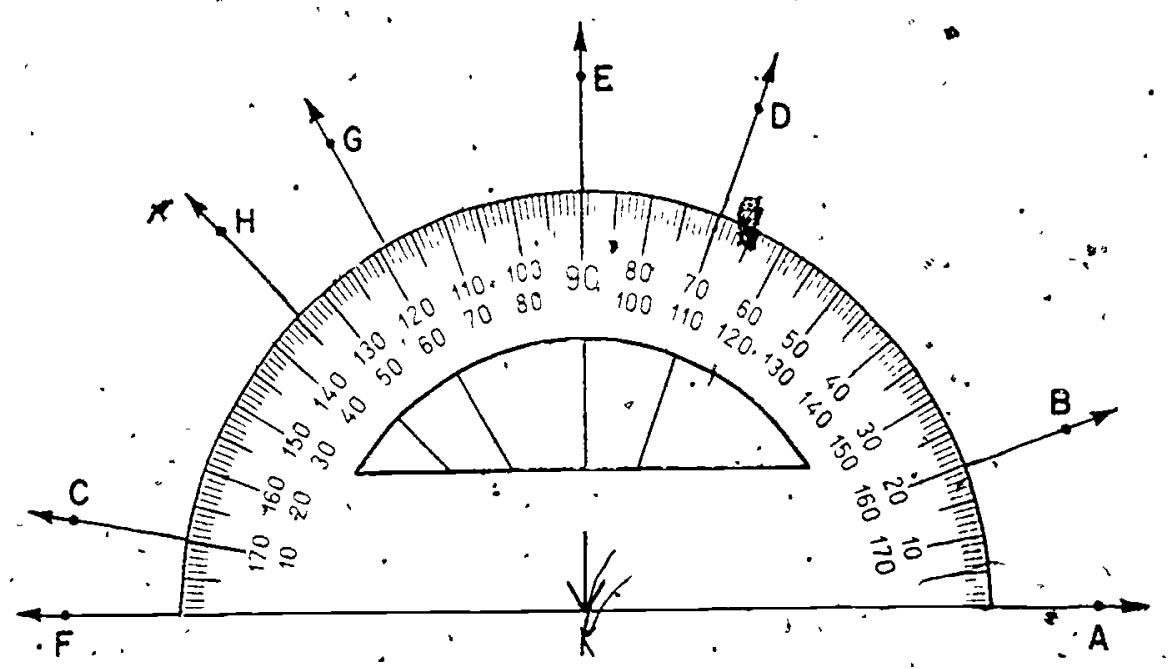

a), $\dot{m} \mathrm{AKB}=(20)$

b) $m \angle F K E=(90)$

c) $\mathrm{m} \angle \mathrm{AKC}=\frac{(170)}{.}$

d) $m^{\prime} \angle F K G \circ=(61)$

e) $\dot{m} \angle A K D=(70)$.

f) $m \angle \mathrm{BKE}=(70)$ h) $m \angle H K D=.(65)$

Imagine that the protractor has been moved so that the zero ray lies along $\overrightarrow{\mathrm{KH}}$ (or $\overrightarrow{\mathrm{KO}}$ )

1) $m \angle D K B=(50)$

Imagine that the protractor has been moved so that the zero ray lies along, $\overrightarrow{\mathrm{KD}}$ (or $\overrightarrow{\mathrm{KB}}$ )

Imagine that the protractor has been moved so that: the zero rax.Iles along $\overrightarrow{\mathrm{KB}}$ (or Ka)

8)

$m \angle C K D=(100)$

Imagine that the protractor has been moved so that the zero ray lies along. $\overrightarrow{\mathrm{KC}}$ (or. $\overrightarrow{\mathrm{KD}}$ )

ie) $m<$ HiC $\doteq \frac{(135)}{\square}$

Imagine that the protractor has been moved so that the zero ray lies along $\overline{\mathrm{KH}}$ (or $\overrightarrow{\mathrm{KC}})$

726 
2. Use your protractor to find the measures, in degrees, of $\therefore$ the ofollowing angles.
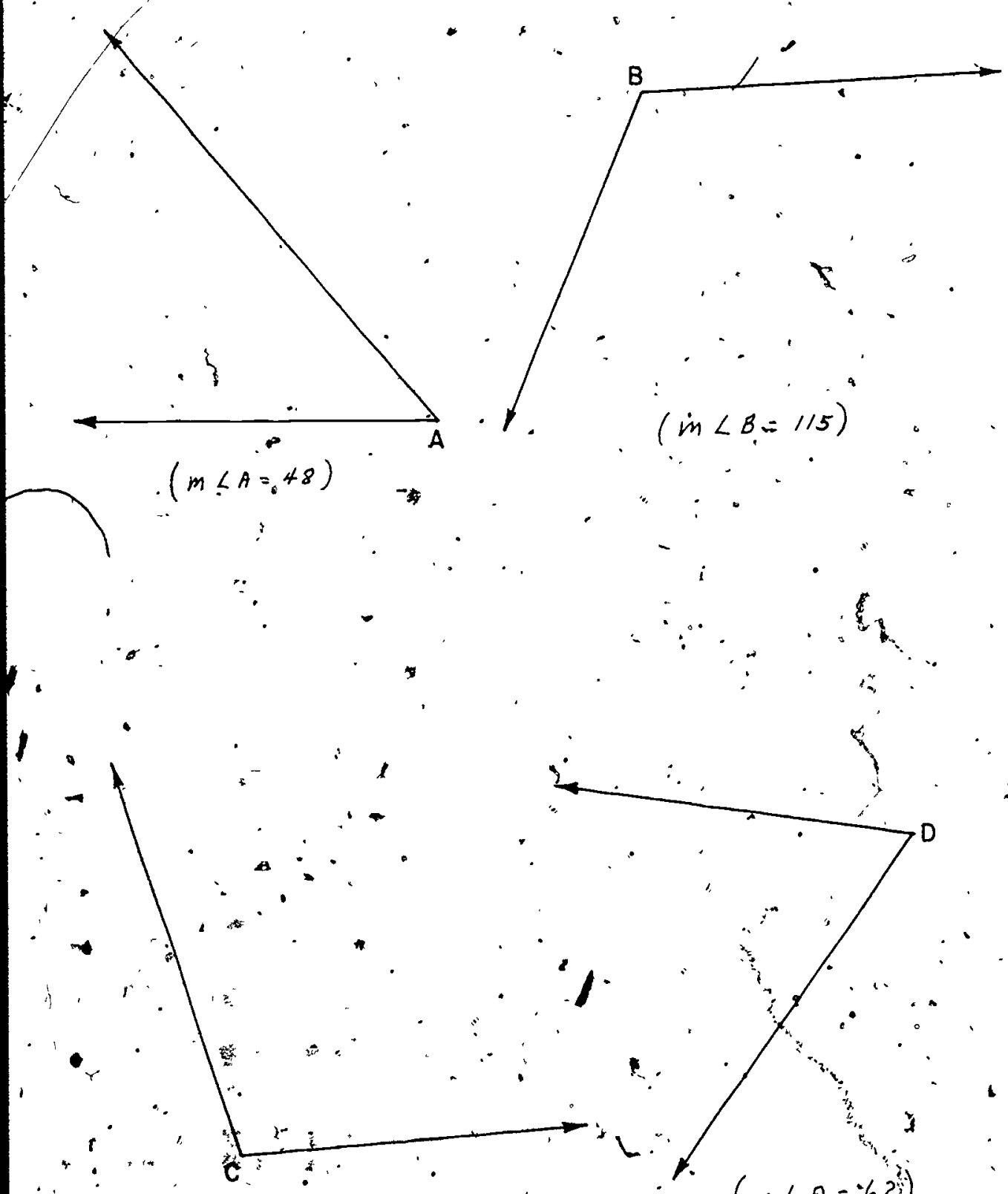

.

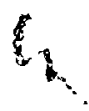


ESTIMATIXG THE MEASURE OF AN ANGLE

Objeetive:" To develop the followang understandings an skilis:

1. Estrmating the measure of an angle proviaes a..

: 'check when reading the measure on a protractor ...: $:=$

- scale.

2. Visualizing angles whose sizes are $45^{\circ}$, Visualizing angles whose sizes are $45^{\circ}, 90^{\circ}$,
and $35^{\circ}$ is nelpful in estrating the size
of a given angle. .

Materiais reeded:

Teacher: Straightedge, châkboard protractor

. Rup1l: . Straightedge, städard

Vocabulary:" Estimate
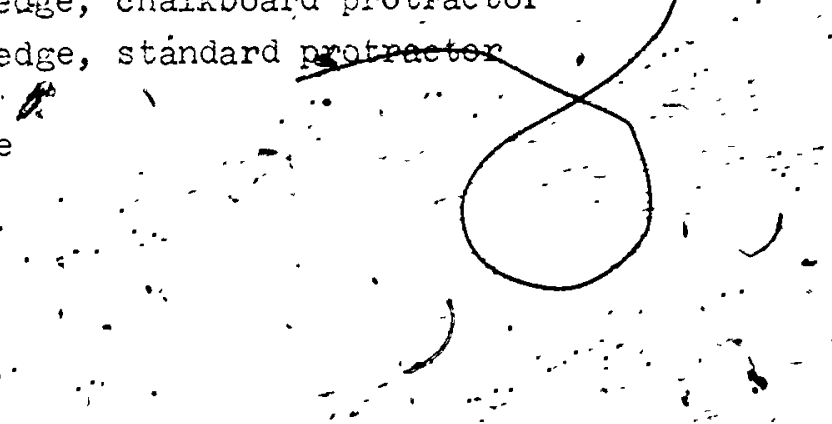

- . The exploration in this section ts verit - readable." The -teacher might try haviris some broup. of puptis go through.this Explóration as an independent actikity and then check to be sure that understandings and skilis are achleved. Keep in ming that onejsoal at this unit is to help the pupti Improve his ability. - to read mathementes.

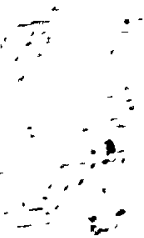


$-\mathrm{p} 44 \hat{1}$ $\therefore \therefore$

ESTIMATING THE MEASURE OF .ANGLES

\section{Fxploration}

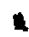

.

Helen used her protraçtor

to flind the measurë. of ' $\angle A$.

She made a mistake andread the -

wrong, scale of her protractor,"

so she wrote for her answer

$B \angle A=130$. Max was asked to

check hèr paper to see whetrer

"her answer was correct. Max

sald, "I donnot have my

pratractor to find the measure

of $\angle A$, but I know that, Helen's

answer is wrong." How did Max know that Helen's answer was not correct?

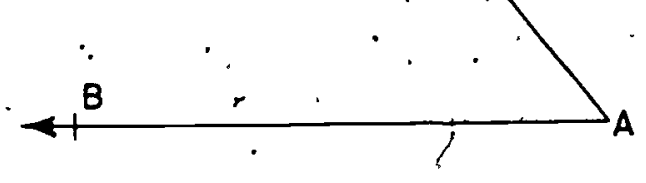

Whenever you can, you should make an estimate of an answer. to a problén. Then $1 f^{\prime}$ your answer is not close to this estimate you w1ll suspect you may have made a mistake.

A good angle to $y$ se as a gulde in estimating the measure of angles $1 \mathrm{~s}$ a right angle.

1. Do you remember how to fold a pap̈er to make a right angle? Just two rol'ds are needed. (Io make a model of a 'reght, angle, fold a ahect of paper once, in any ung. Ihan fold agam so that tha fold hien on italf. The edgea of the dabblifold and the angle fold make a modil of a right anghe.)

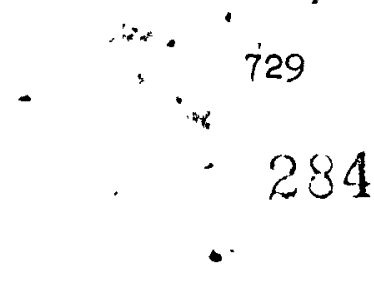


P442

2. What is the measure of a right angle? Use your protractor if you need to. (Ihe measure if a rybt angle, in degreen, is 90.)

3. Which of these angles has a mêasure greater than the measure of a.right angle? Do not us your protractor: (For $\angle B$, imagine $\overrightarrow{B D}$, which would make $\angle A B D$ a right angie. Place your pencli on the figure to represent $\overrightarrow{\mathrm{BD}}$. would $\overrightarrow{B D}$ be in the interior of $\angle \overrightarrow{A B C}$ ?) ( $N_{0}$, thenefine $\angle B$ han ret hlavi is mieasure greater the that of a inght angle.)

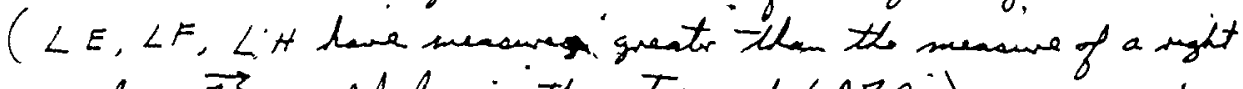
Angle. $\overrightarrow{B D}$ womed be in the extevior of $\angle A B C$.

11
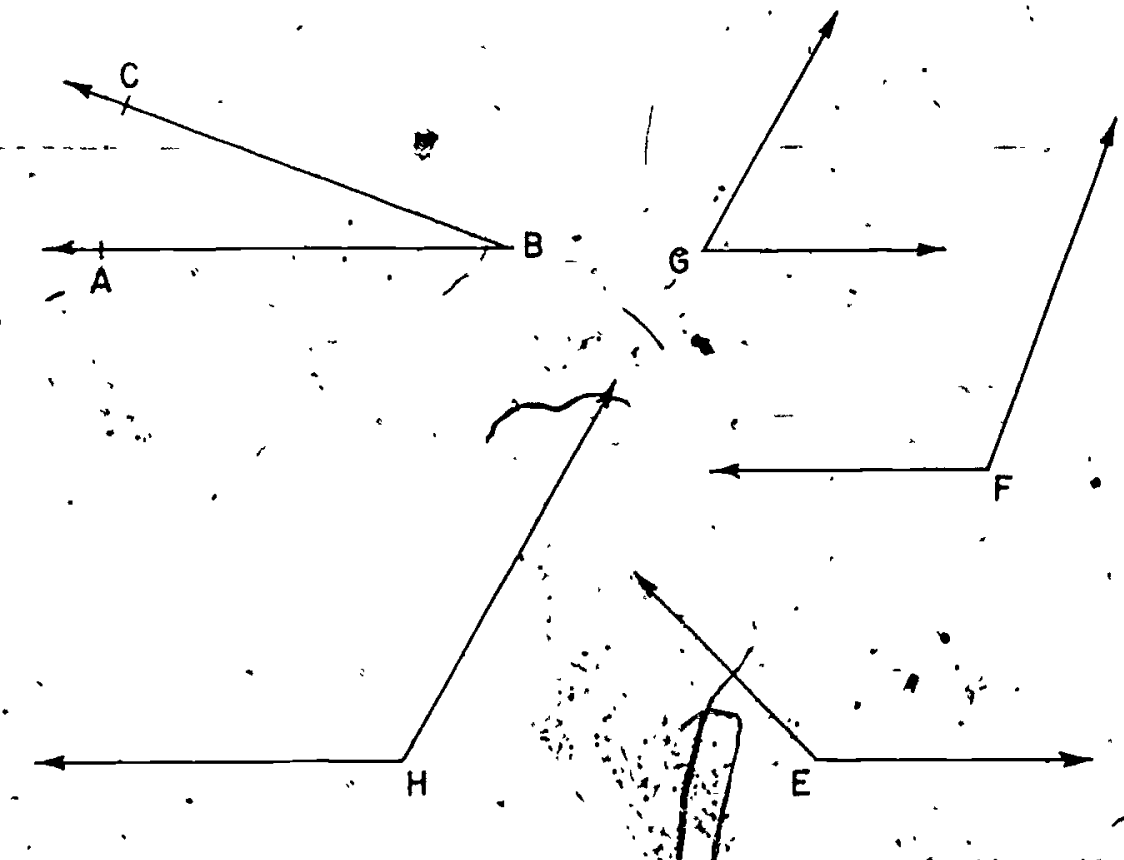

4. Which of the angles above havelfasures less than the measure of a right angle? ( $L B$ and $\angle G$ have

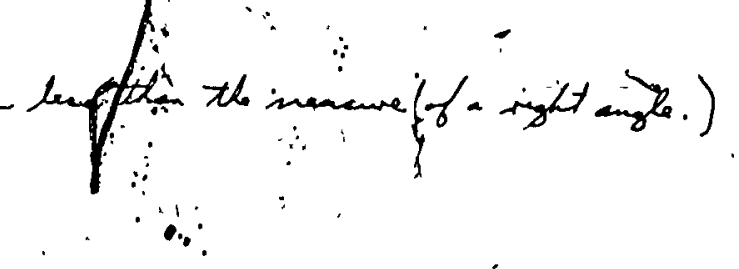


P 443

5. Draw $\overleftrightarrow{W Z}$ on a piece of paper: on, $\overleftrightarrow{\leftrightarrow Z}$, choóse a point $x$. Your drawing should lookilike "Finis.

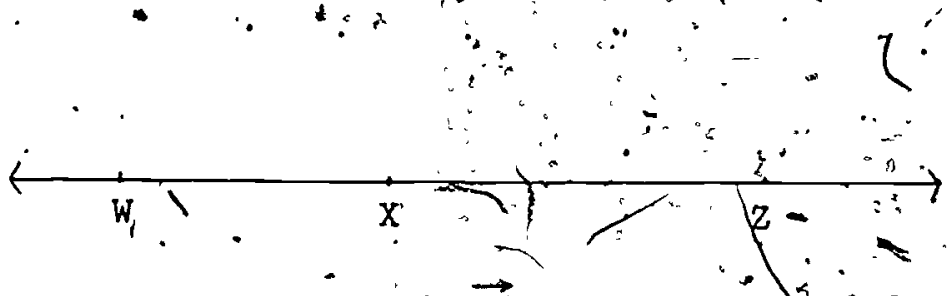

Uśe potnt $X$ as vertex and $\overrightarrow{X Z}$ as one ray, and draw with your protractor an angle with a measure, Ap degrees, of .90. call this $\measuredangle Z X T$.

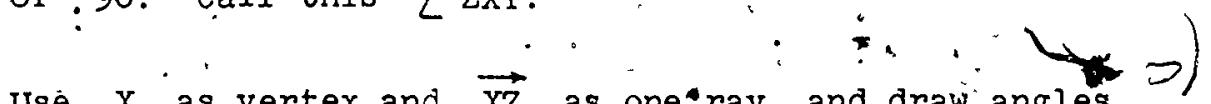
Use $X$ as vertex and $\overrightarrow{X Z}$ as one ray, and draw angles with measures, in degrees of $4 \dot{5}$ and 135. 'Draw all three rays on the same stap of $\overrightarrow{y z}$. Label' them so that $m \angle Z X Y=45$ and $m \angle Z X R=135$.

in

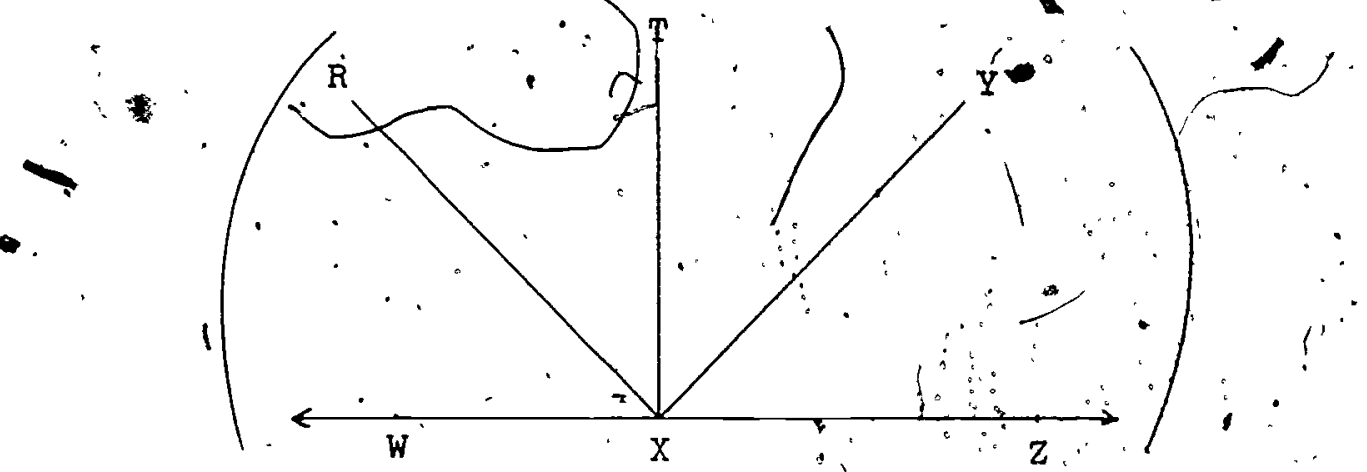

6. What other angles in the rigure, have a measure of 15 ?

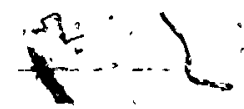


7. Hoo at each angle below and estimate its size. Use an angie of one degree as the unit. Now compare each angle with an angle in the drawing you made for Exercise 5 .

a) Is $\mathrm{m} \mathrm{K}$ nearer 0 or $45 ?(45)$

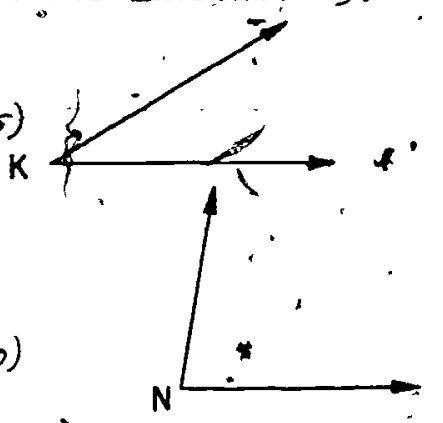

(j) Is " $\mathrm{m} \angle \mathrm{N}$ nearer $4 \overline{5}$ or $9 c ?(90)$

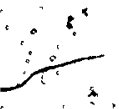

c) Is $m \angle R$ nearer 90 or $135 ?(135)$
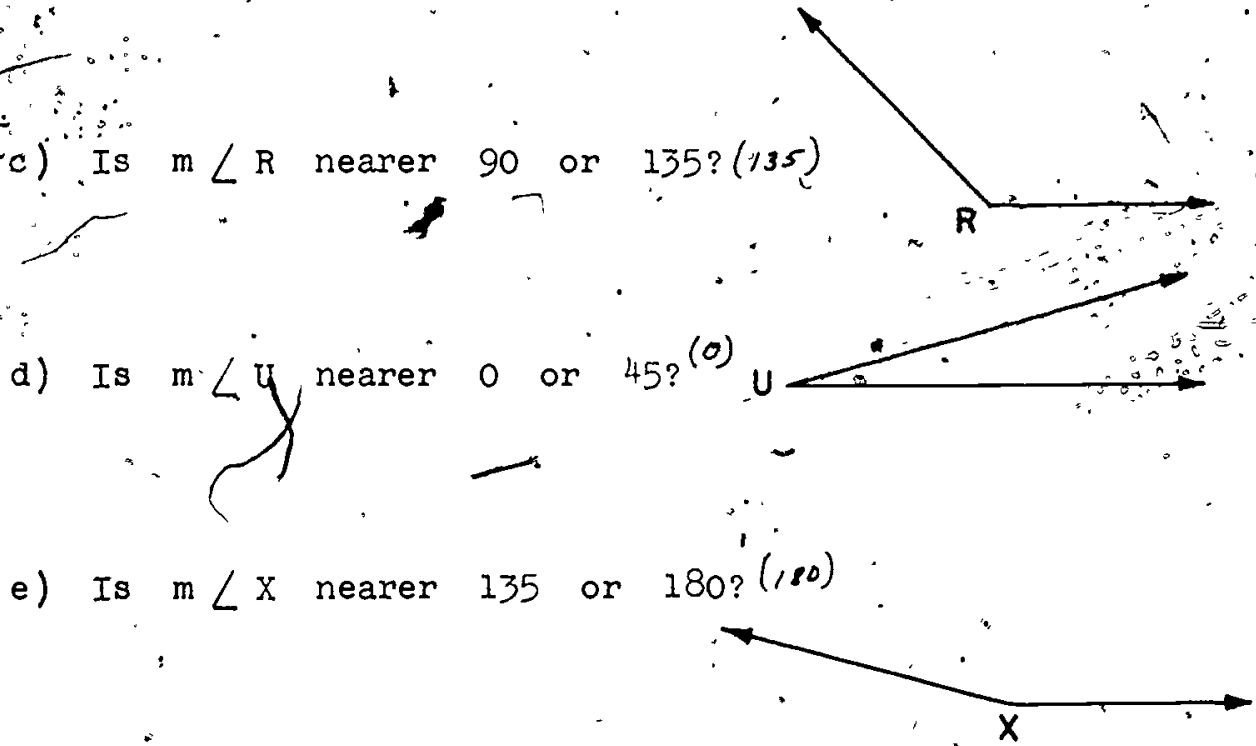

ff) Is $\mathrm{m} L \mathrm{~A}$ nearer 90 or $135 ?(90)$

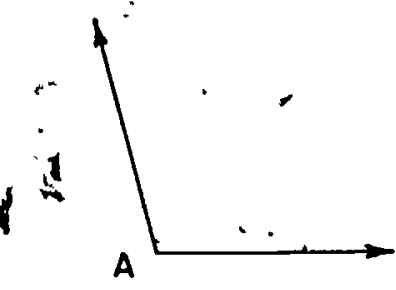

$\Upsilon \cdot$

8. Now measure, in degrees, each angle in Exercise 7 with your protractor and write the measure you find. (a. $m \angle K=30$ $m \angle U=15$ e. $m \angle x=165$ f. $m \angle A=105$.

732

287

ERIC 


\section{Exercise Set 7}

f. Which of these angles has a measure less than the measure of a iright angie? $(\angle A, \angle D, \leq E, Z G)$
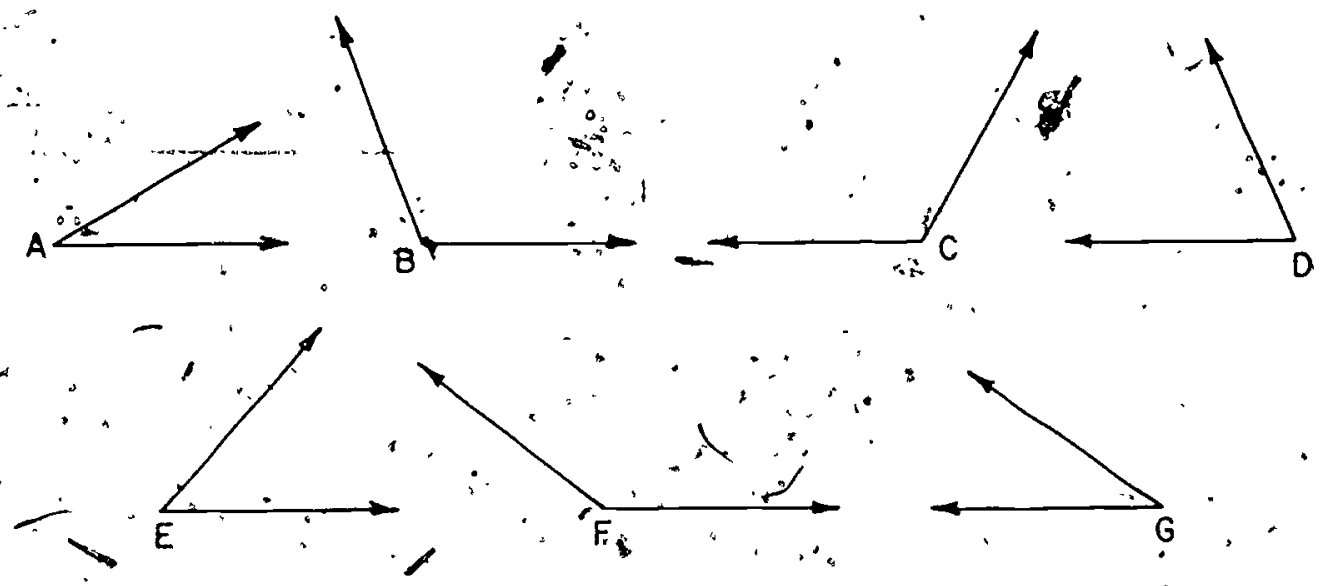

2. Which of the angles above" have a measure greater than 90 ? $(\angle B, \angle C, Z F)$

3. Look carefully at each angle. Choose the better estimate of Its measure in degrees. $\mathrm{m} \angle \mathrm{H} ;-5$ or $45 \quad(45)$ $\mathrm{m} L \mathrm{I} ; 90$ or $135(135)$

2. mL $5 ; " 45$ or $90 \%$ $\mathrm{m} L \mathrm{~K} ; 135$ or 17,5 (1,75) . . . . . $\mathrm{m} \cdot \mathrm{LL} ;^{2} 45$ or $90(90)$
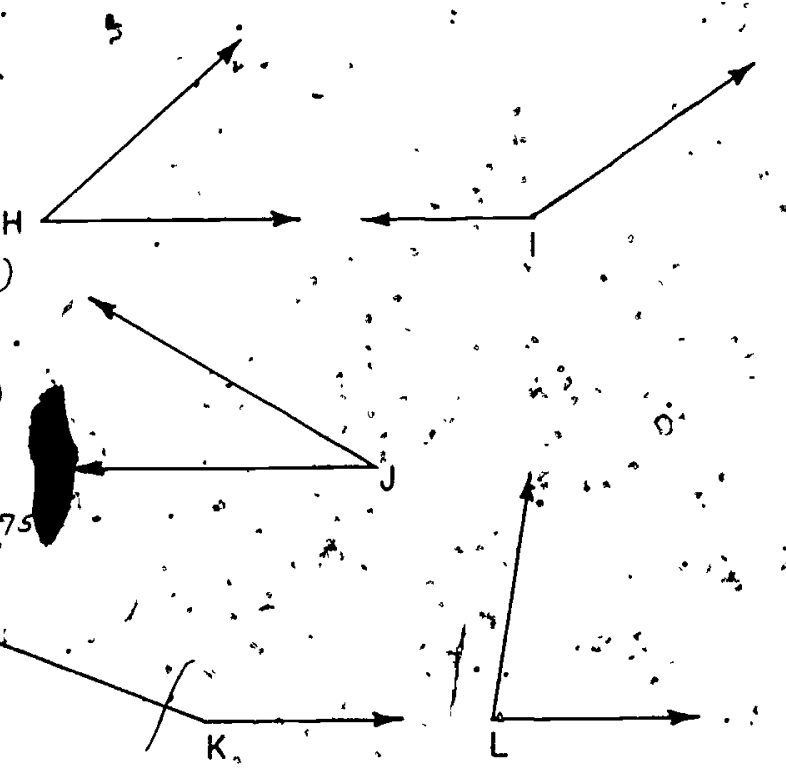

4. Measure each of the angles In Exercise 3 in degrees.. $\left(\begin{array}{l}m \angle H=40 \\ m \angle I+146 \\ m \angle J=30\end{array} \quad \begin{array}{c}m \angle K=160 \\ m \angle L=80\end{array}\right)$

$$
283^{734}
$$
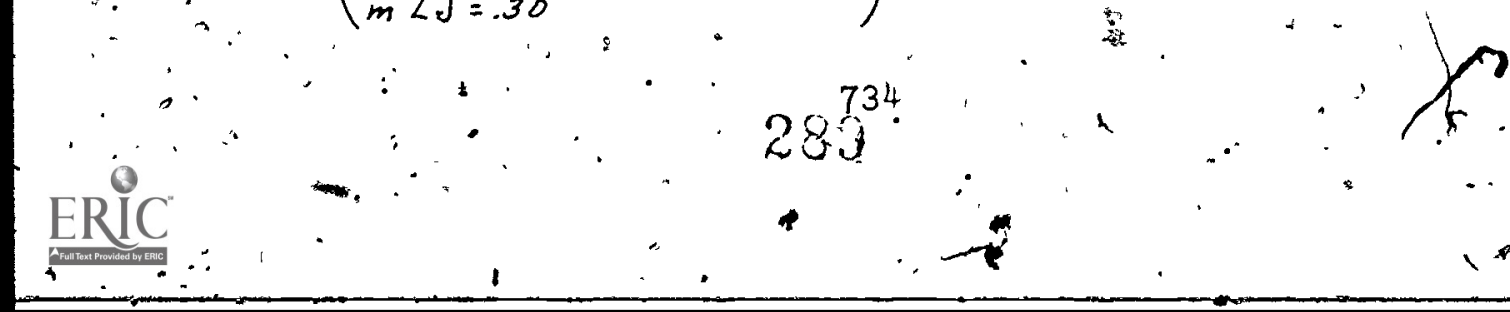
P $446^{-}$

\section{$\therefore$ Exercise Set 1}

- "Which of these angles has a' measure less than the measure of a rigint angle? $(\angle \dot{A}, \angle O ; \angle E, \angle G)$
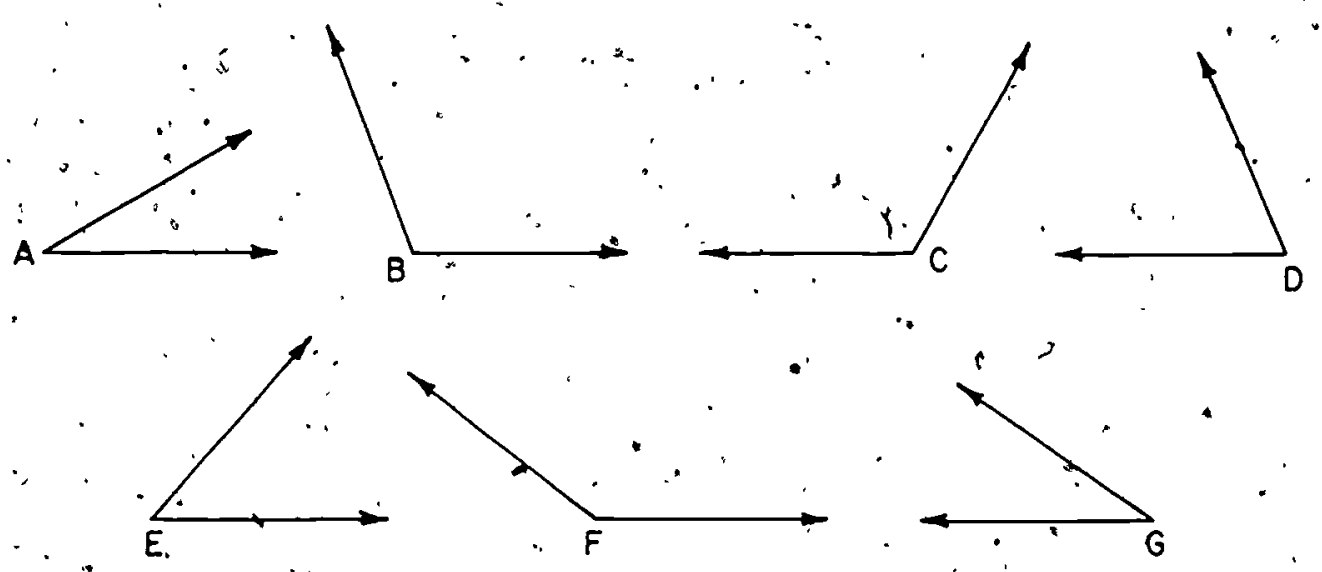

2. Which of the angles above have a measure greater than 90 ? $(\angle B, \angle C, \angle F)$.

3. Look carefuliz at each angle. Choose the better estimate of its measure in degrees!

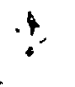

4

$\mathrm{m} \angle \mathrm{H} ; \quad 5$ or, $45 \quad\left(45^{\circ}\right.$

$m \angle I ; 90$ or $\$ 135(135)$

$\mathrm{H}$

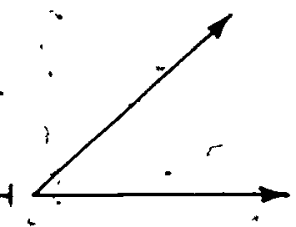

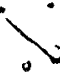

$m \angle J ; \cdot 45$ or $90(45)$

$\mathrm{m} \angle \mathrm{K} ; \quad 135^{\circ}$ or $175(175)$
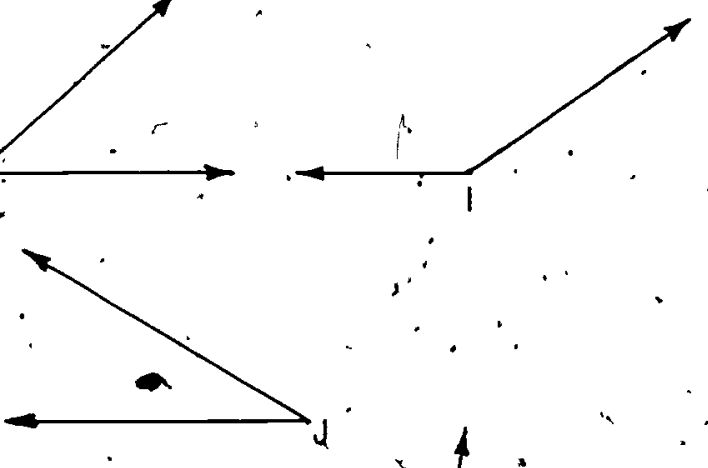

m $\angle . L ; 4: 5$ or $90(90)^{x}$

$\therefore$
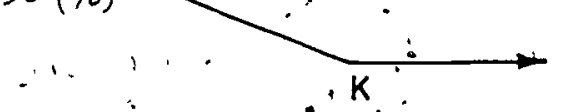

4. Measure each of the angles in Exercise, $z$ in degrees.

$$
\left(\begin{array}{ll}
m \angle H=40 \\
m \angle L=146 \\
m \angle J=30
\end{array}, \quad \begin{array}{l}
m \angle K=160 \\
m \angle L=80
\end{array}\right), 1
$$

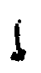


- 5. Estimate the measure of each angle in degrees. Write your

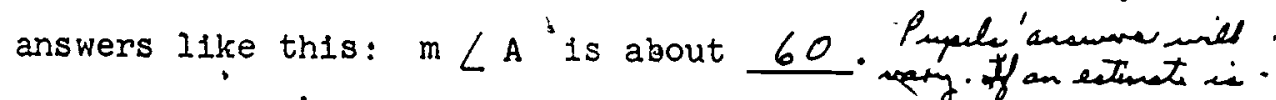
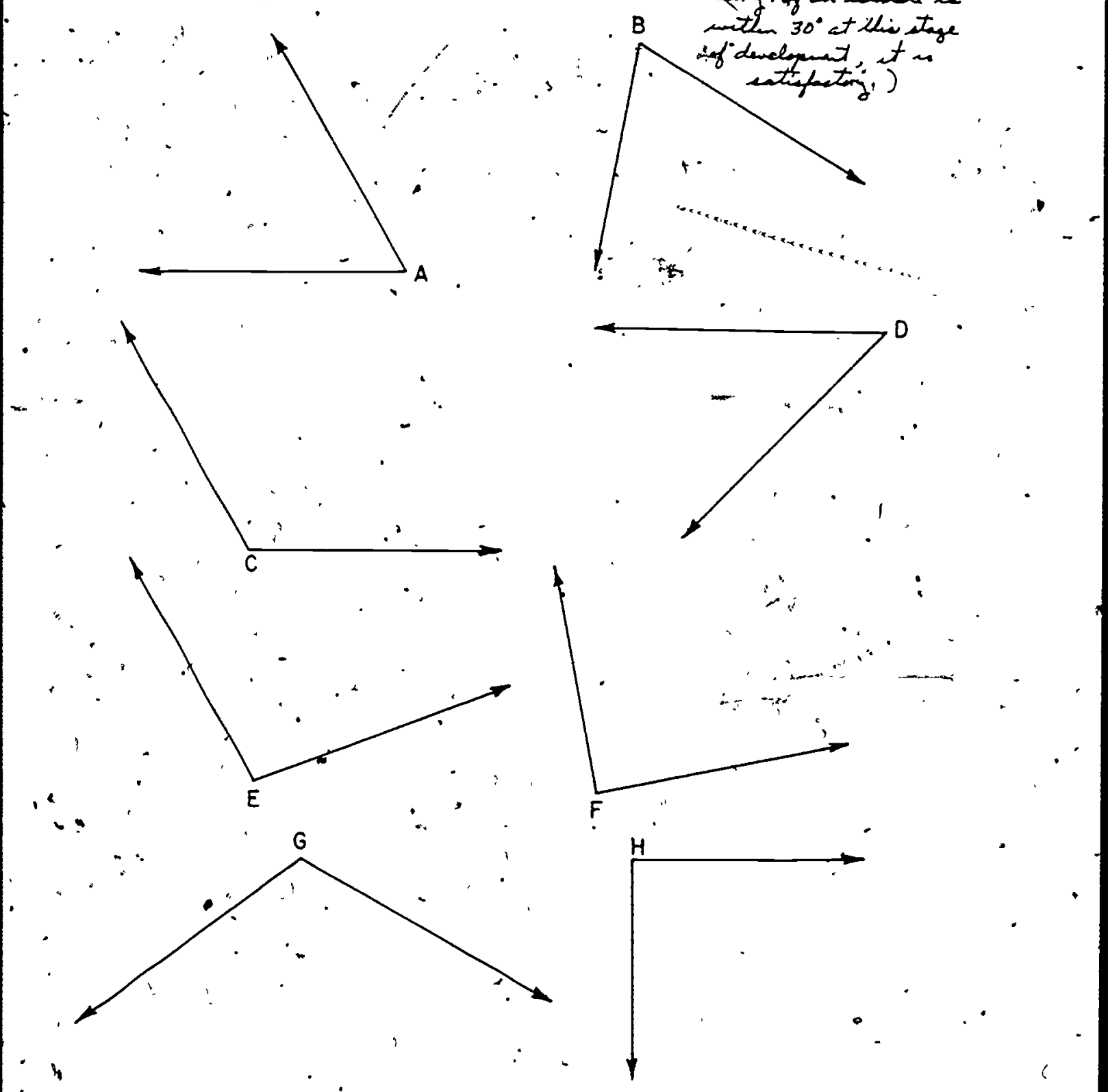

6. Weasure, in degrees, each angle in Exercise 5 with your protractor. $(m \angle A=60$ $m \angle B=7 P$ $m \angle C=120$ $m \angle D=45$ $\left.\begin{array}{l}m \angle E=100 \\ m \angle F=80 \\ m \angle G=115 \\ m \angle H=90\end{array}\right)$ 
7. Use your stralghtedge to draw an angle which you think: has the size given below. Then measure the angle. with a protractor to see how closely the measures agree w1th your estimates.

\section{AN'GLE \\ $\underline{\text { SIZE }}$}

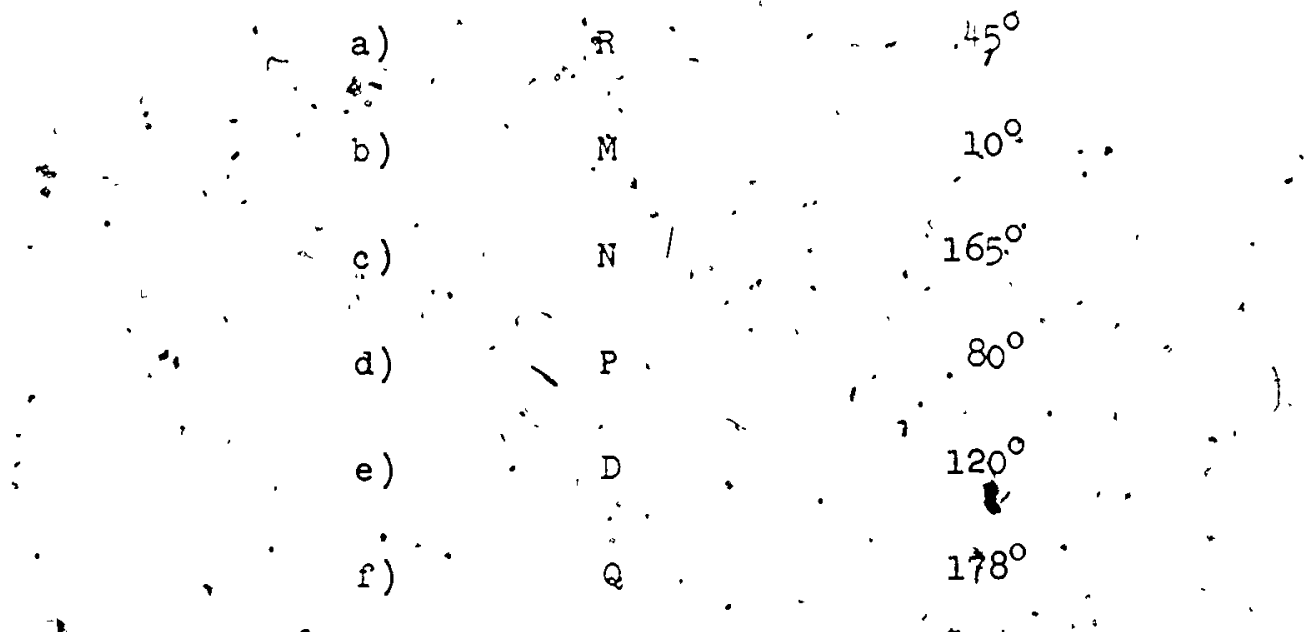

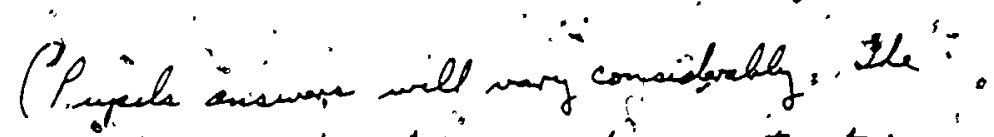
goal is to geve childen prazteris estimating th azer of anghe.) 
SUM OF THE MEASURES OF ANGLES

objective: fo develop the following understandings and skills:

i. If two angles can be placed so that.

a. "they hàve a cömmon vartex

b. they have a common ray

c: - their interiors do not intersect

- d. the other two rays of the angles lie on the same ifre,

then the sum of the measurts, in degrees, of the two angles is 180 .

2. If, In addition to the four conditions above, the. two angles are congruent, each hạs a measure, in degrees, of 90 .

3. If a set of angles is laid off successively and the union of the angles and their interiors covers a half-plane and its edge, then the sum of the measures, in degrees, of these angles is 180 .

Materials Needed:-

Teacher: Straightedge, chalkboard protractor

Pup11: Straightedge, protractor

Vocabulary: No new words in this, section

This exploration should be carried through" with pup1l texts open because the questions asked depend upon the drawing provided. The reading matertal in Exercises, set 8 may well, be a challenge to some pupils, 'because it is written largely in the symbolic language of mathematics. If necessary, the teacher might read through the Exercises with the pupils before they attempt them alone. 
5449

SUM OF, THE MEASURES OF ANGLES

1. Copy and complete the following table. Find the measures. of the angles from the sketch. "When the unit angle is not mentioned, use the measure in degrees.).

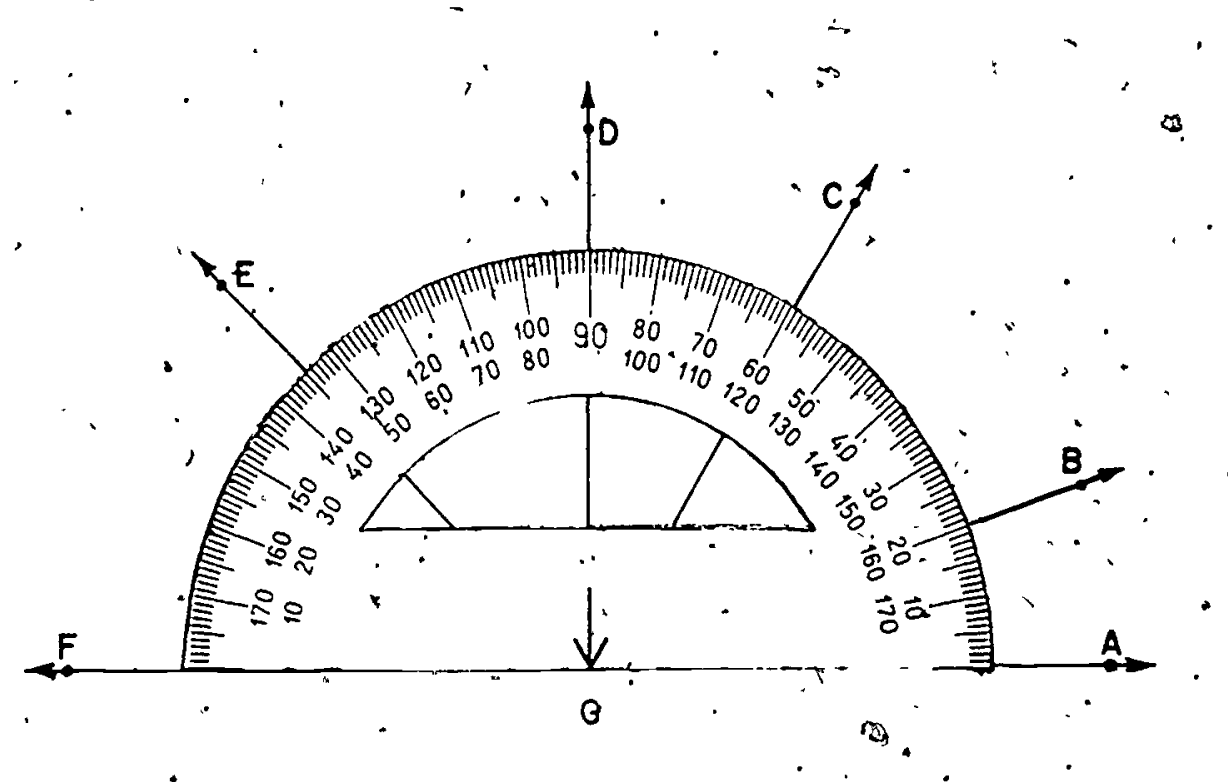

NAME OF ANGLE

$\underline{\text { SIDES }}$

MEASURES

$\angle \mathrm{AGB}$

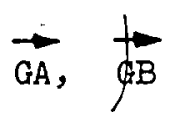

Pair 1

$\angle B G F$

$\overrightarrow{\mathrm{GF}}, \overrightarrow{\mathrm{GB}} \quad(160)$

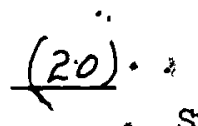

sum of measures $(/ 80)$

Pair 2

$\angle A ́ G C$

$(\overrightarrow{G A}, \overrightarrow{G C}) \quad(60)$

$\angle \mathrm{CGF}$

$(\overrightarrow{G C}, \overrightarrow{G F})$

(120) 
2. "Trace wl th your finger the rays" which form the angle pair, Palr 1, In Exercise 1.

a) What ráy:1s a s1de of both angles? $(\overrightarrow{G B})$

b). What can you say about the other two rays? What is

the1r intersection? What 1s their union? (Jhe oth tw

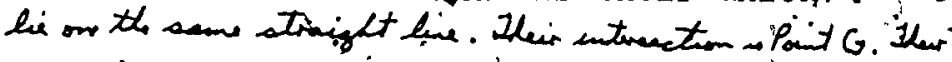

c) Do the interiors of the angles intersect? $\left(x_{0}\right)$..

d) What is the sum of their meatsures?"(180)

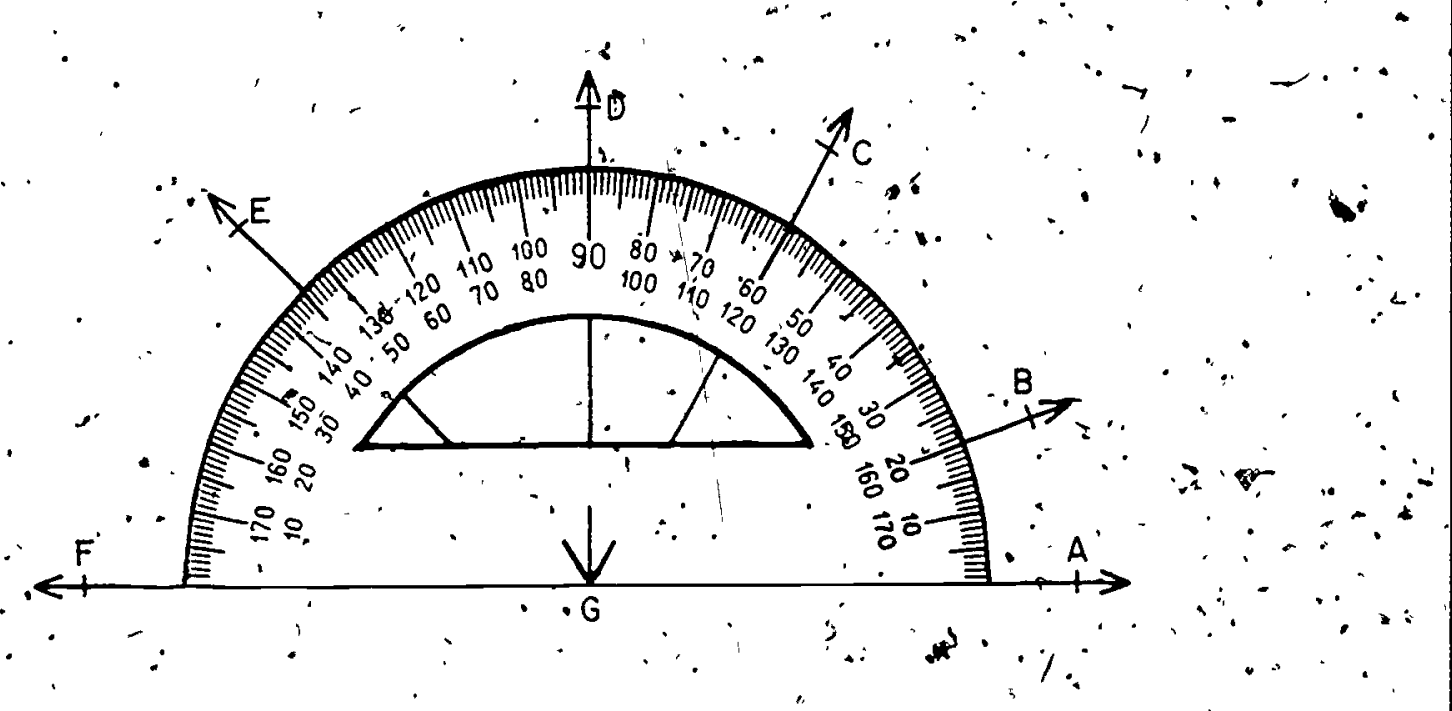

13. Trace with your finger the rays which ferm angle pair, , $\because$

- S Pair 2. Answer the questions as in Exencise 2 about this pair. Are your answers. to questions, $b, c$; and $d$ the same as for Pair I?

$\because\left(\begin{array}{l}a \overrightarrow{G C} \cdot \\ b . \text { semes above }\end{array}\right.$

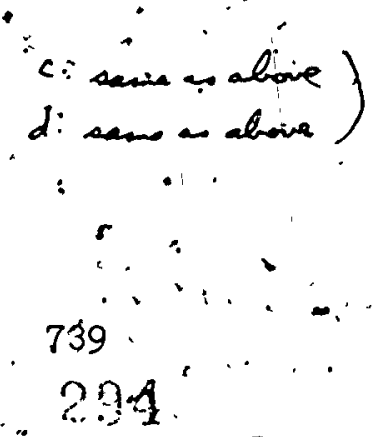


.: 4. Find a third pair of angles in the sketch for which the answers to questions $b, c$, and $d$,are the same as for Pair 1 and Pair 2. Trace their rays w1th your finger.

$$
\text { ( } \angle A G D \text { and } \angle O G F \text { or } \angle A G E \text { land } \angle E G F \text { ). }
$$

5. When these rays intersect at the same point and two of the rays form a lipe, what can you expect will be the 'sum of the measures of the two angles formed? (/80)

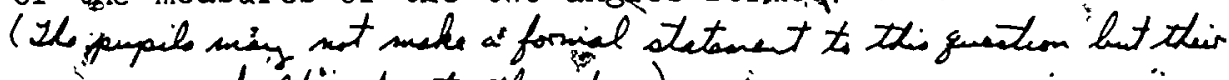
responseres shed "indecte the idea.)

6. I1st the names of ally of the angles in the sketch whose. 1 Interiors do not intersect. (There are five.)

$\left(\angle A G B, \angle B G C, \angle C G O, \angle D G E, \angle E G F^{\circ}\right)$

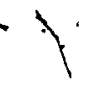

7. Find the measure of each angle in.your list.

( $m \angle A G B=20, m \angle B G C=.40 \times m \angle C G D=30 .$, in $\angle O C E=45, m \angle E G F=45$

8. Find the sum of the measures of the five angles:

(The sum, in degreen, is 180 .)

9. What conclusion can you reach from Exerc1se 6-8? (14 idea beng

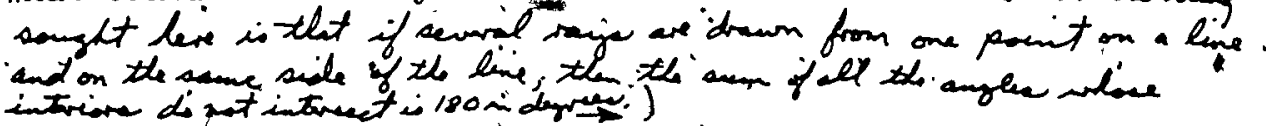

- 10. Name'a pait of angles with $\overrightarrow{G F}$ a side of one angle, $\overrightarrow{G A}$ a side of the other, and $\overrightarrow{G D}$ a side of both angles. $\because 1 \therefore . . \quad \therefore D G F$ and $\angle A G O)$

11. What is the measure of $\angle A G D$ ? $\angle$ DGF? What kind of angle is $\angle$ AGD? $\angle$ DGF?

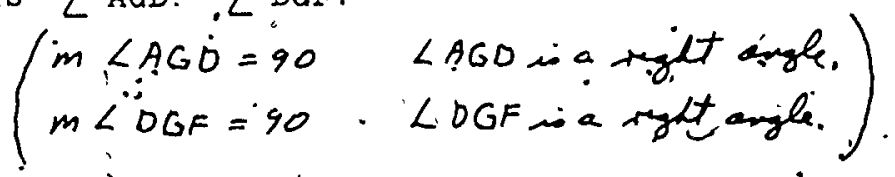


$\mathrm{P} 452^{\circ}$

Exercise set 8

Use this figure for:

- Exercises $1^{\circ}$ and 2 . $\overrightarrow{\mathrm{BA}^{\prime}}$ ' and

$\overrightarrow{\mathrm{BD}}$ are on the same line.

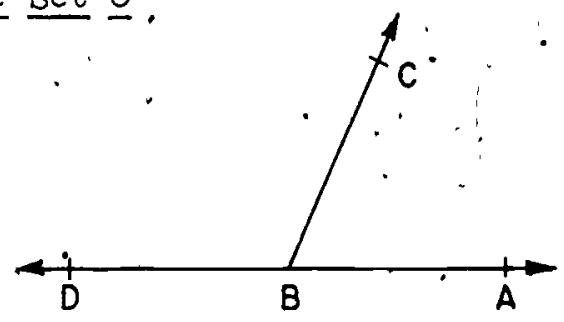

1. If $-\widehat{D A}$ is a straight line, $m \angle A B C+m \angle C B D=(180)$

2. If $m \angle A B C=65$, then $m \angle C B D=(115)$

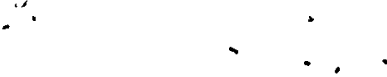

Use this figure for,

Exercises 3 and $4 . \overrightarrow{F E}$.

and FJ are on the

same line.

$$
.1
$$

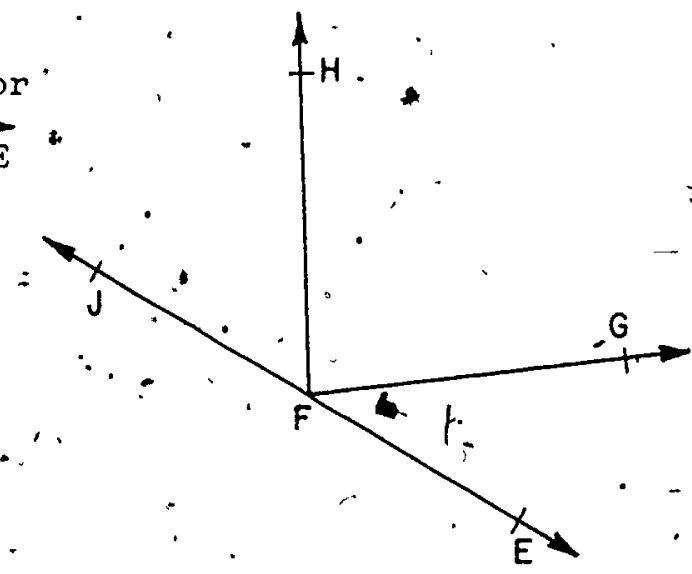

1

3. Is it true that $m \angle E F H+m \angle J F G+m \angle H F G=180$ ? (mo)

If not, what true statement can you make?

$\left(m \angle E F G^{\prime}+m, \angle G F H+m \angle H F J=180\right)$

4. If $\mathrm{m} \angle \mathrm{JFH}=58$ and $\mathrm{m} \angle \mathrm{EFG}=36$, then $\mathrm{m} \angle \mathrm{HFG}=\frac{(86)}{\cdot}$

* 5: If $\overrightarrow{\mathrm{BA}}$ and $\overrightarrow{\mathrm{BC}}$ are on a

straight .line, and $\angle A B D \cong \angle D B C$,

then $m: \angle A B D=(90)$

and $\mathrm{m} \angle \mathrm{DBC}=\underline{(90)}$.

$\angle A B D$ and $\angle D B C$ are $\frac{(\mu i L t)}{r}$ angles.

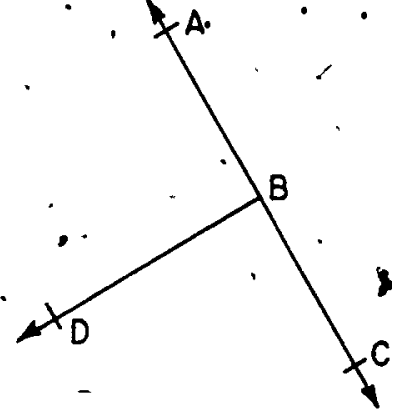


SUMMARY

- 1. If three rays have the same endpoint, and two of the ray's form a line, then the sum of the measures, In degrees, of the two angles formed is 180 .

2. If several rays are drawn from a point on a' line, all on the same side of the line, the sum of the measures, in degrees, of all the angles formed whose interiors do not intersect is 180 .

3. If one ray is drawn from a point on a line and the two angles formed are congment, each angle is a right angle and its measure, in degrees, $\because \therefore$ is 90 .

742

297

1 
AREA,

PURPOSE OF UNIT

This unit is one of a series intended to constitute the study of measurement in the elementary grades. It is intendead that the study of measurement as it applies to line segments, angles, area, and volume provide the child with a valuable experience in a branch of geometry which is a poweprui taol in the pitysical world. These units form a continuous and coordinated treatment of the topic of measurement.. The sequence of topics in each unit parallels the historical development of this body of knowledge. 'The Teachers: Commentarles for the units on Linear Measurement and Measurement of Angless contain discussions on $(l)$ the difference between comparisons of the sizes of sets of discrete objects. and comparisons of the sizes of sets of points which form continuous curves, (2) the need for intultive awareness of comparisons of size between sets of points which form continuous chirves, before any formal procedure for measuremerit is set up, (3) the concept of an arbitrary unit of measure, and the need to select as a unit a thing of the same sort as the thing whose measure we wish to" - find (a line segment as a unit for measuring length, a region as a' unit for measuring area, etc.). (4) the creation of a scale for convenience, and (5) the final step of selecting a standard unit to meet the needs of our, society. 


\section{MATHEMATICAL BACKGROUND}

\section{'Comparing Areas}

Lèt us recall how the subject of linear measurement was approached 1 in Chapter 9, Grade. 4, since area will be approached in a simfiar manner in the present unt t. Flrst we encountered the intuitive concept of comparative length for line segments: any two line segments can be compared to see whether the first of them is -of smaller length; "or the same length, on greater length than the second. Corresponding to this we have in the present unit the $1 \dot{d e a}$ of comparative area for plane regions. (Recall that by definition a plane region is the union of $a$. simple closed curve and 1 ts interior.) Even when they arenather complicated in shape, two regions 'can, In principle'at least, be compared/to see whether the first of them is of smaller area, or the same area, or greater area than the second.

$\because$ In the case of line segments, this comparison is conceptually very simple: we think of the two segments to be compared; say $\overline{A B}$ and $\overline{C D}$, as beling placed one on top of the other in such a manner that $A$ and $C$ coincide: then elther $B$ 'is between $C$ and $D$, or $B$ coincides with $D$, or $B$ is beyond $D$ from. $C$, etc. Th1s conceptual comparison of line segments is also easy to carrỳ out approximately using approximate physical model's (drawings and tracings, etc.). of the line segments involved.

In the case of plane refions, this comparison 1s more com.plicated both conceptually and in practice. This is because the shapes of the two plane regions to be compared may be such that neither will "f1t into" the other. How, for example, do we compare in size. (area) the two plane regions pictured below?

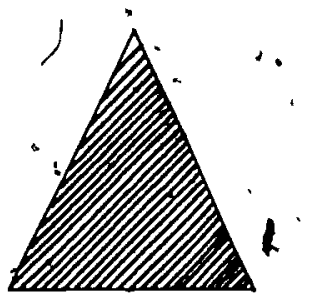

3
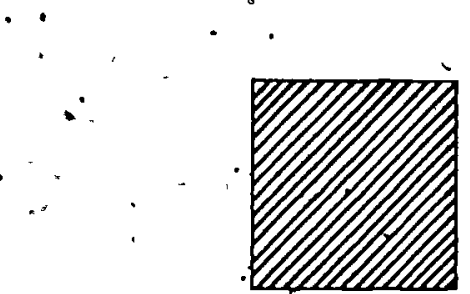
'If we think of these regions as placed, one on top of the other, - neither of them will fit into the other.

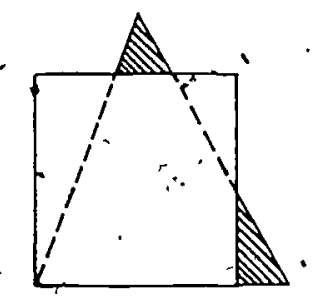

In this "particular case, however, we can think of the two - pleces of the triangular region which are shown shaded in the figure above as snipped off and fitted into the square region thus :
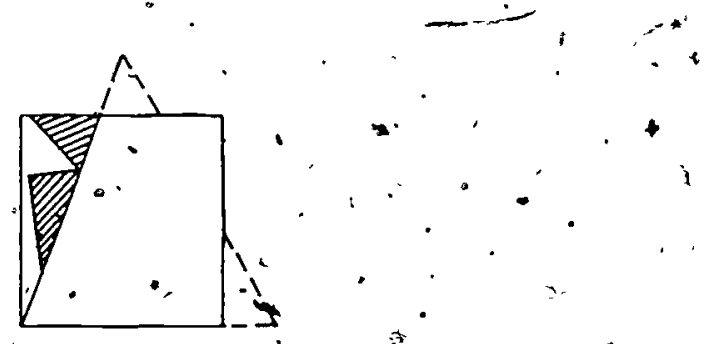

Thts. shows that the triangular regton is of smaller area than the squape region. Eay. in this un1t, ithe pup1l is asked to carry out approximately some simple comparisons of this sort;" using paper models of , the', plane regions to be compared. The pupil actualiy cuts up the paper model of one region and fits the pieces, w1 thout overlapping, on the model of "the otther region. Here we rely on-the pupilis, intuition to "see" that a plane region can be thought of as "cut up into pieces" and even "reassembled" to form, a figure of different shape, without

? changing its area. (See the "robot exerclse" in the pupilis!" book.). As the rigures involved become more complicated in shape, this sort of comparison becomes inereasingly difficult in practice. We need a betțer way of estimating the $a^{*}$ regiton. 
Let us recall what we did next in the case of linear measurement. We chose a unit of length. That is, we selected a certain line segment and agreed to consider 1 ts length to be described or measured; exactly, by the number ?. In terms of this unit we could then concelve of line segments of lengths exactly 2 units, 3 units, 4 units, etc, as being con-structed by laying of $f$ this unit "successively along a line 2 times, ' 3 times, 4 times; etc. The process of laying of the unit successively along a given line segment also yielded

<. (under-and-over-) estimates for the length of the given iine segment in terms of the unit. For example, the length of a Given'line segment might have turned out to be, greater than 3 units (underestimate.) but less than 4 units (overestimate).

We now proceed similarly in the measurement of area. The rirst step is to choose a unit of area,. that is, a region whose. area we shall agree is measured exactly by the number 1 .

Regions of many siapes, as well as many sizes might be considered. An important thing about a line segment as a urit of length was that enough unit line segments placed end to end (so that they touch but do not overlap) would together cover any glven Ine segment. Similarly, we need a unit plane region such that enough of them placed so that they touch but dp not overlap w1ll together cover any given plane region. Circular regions do not in general have this property. For example, if we try to cover a triangular region with small non-overlapping congruent c1rcular regions, there are always parts of the triangular region left uncóvered.
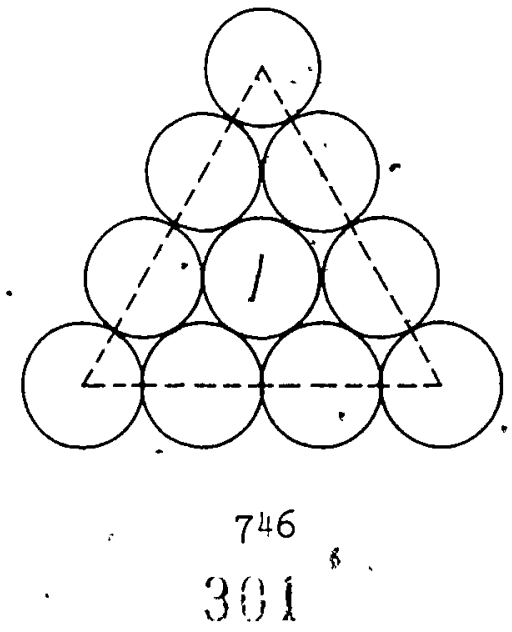
On' the other hand we can always completely cover a triangular. - region, or any region, by using enough non-overlapping congruent squiare regions.

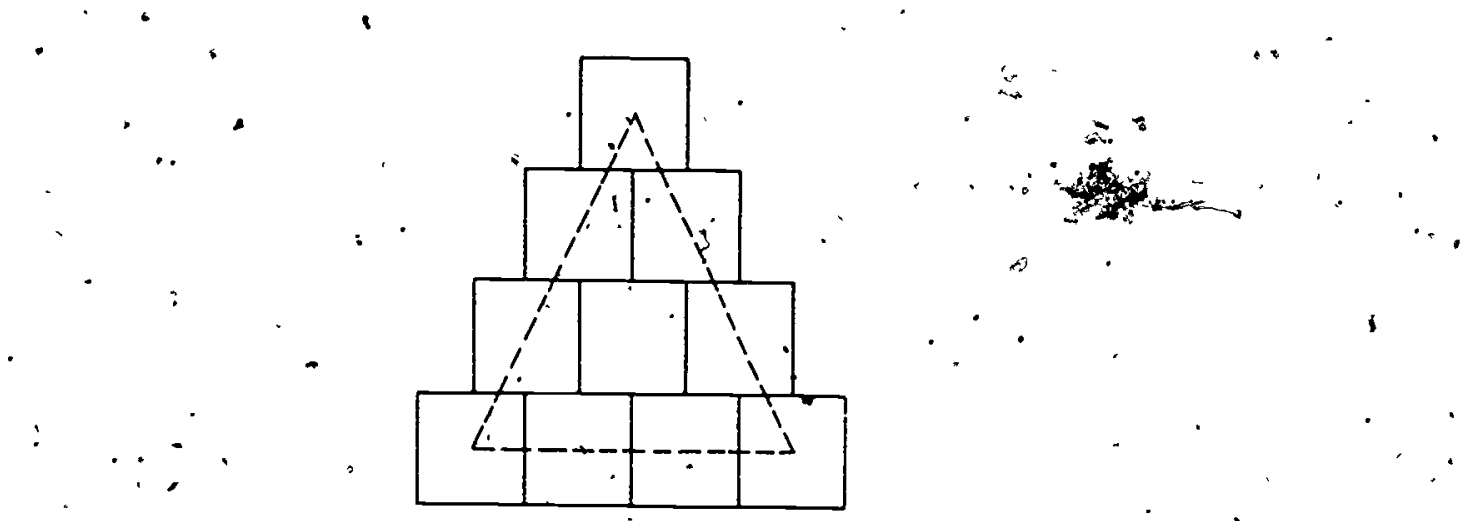

While a square region is not the only kind of region with this. covertng property, 1t has thè advantage of being amsimply. , shaped region whose size can be conventently chosertisy letting 1 ts side be of length 1 unit. More 1mportantly, 1t. thent turns. out that the use of such'a square region as the unit'dforarea makes 1t easy to compute the area of a rectangle by folming the product of the numbers measuring the lengths of 1ts sides, a matter which we shall discuss in the unit.

\section{Estimating Areas}

Just as we used the unit of length to fintd underestimates and overestimates for the length of a given line segment, so can now use the unit of area to find underestimates and overestimates for the area of a given region. A convenient tool for this purpose is a regular arrangement or grid of square unit reglons as shown on the following page. 


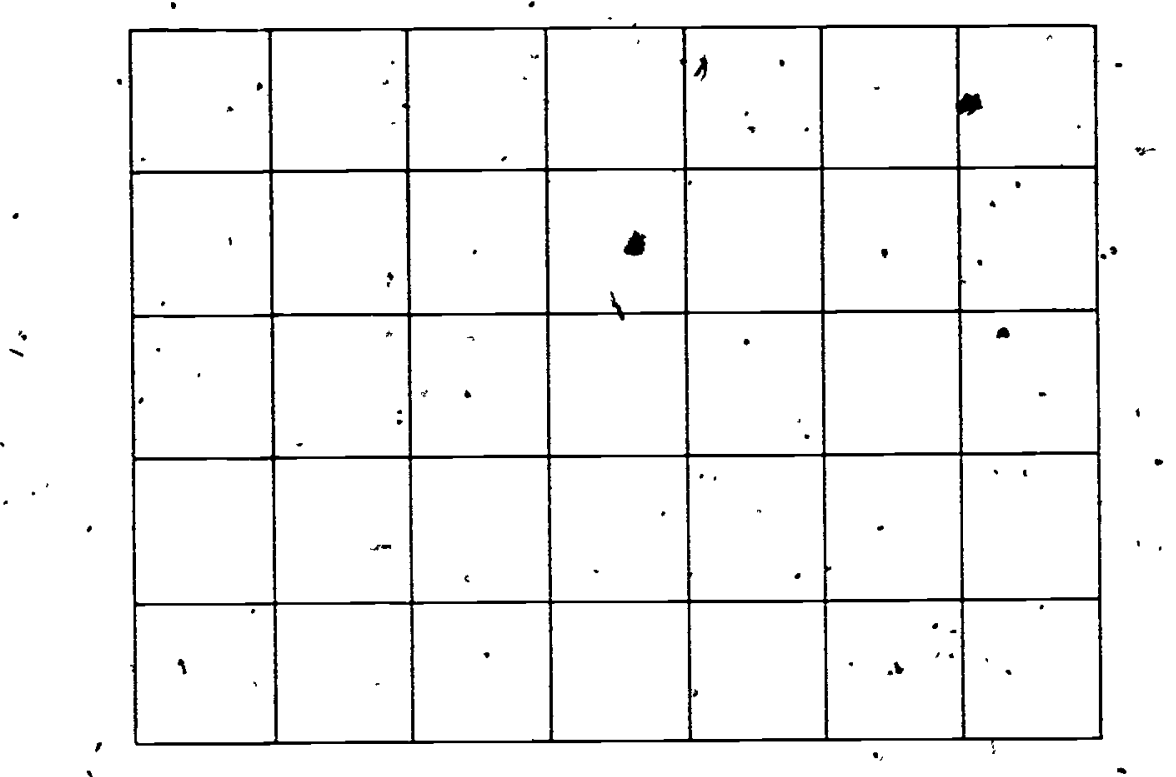

To use such a grid in estimating the area of a given region, we think of it as superimposed on the region. This is. iliustrated below for an oval region. 
We can verify by counting that 12 of the unit regions pictured are contained entirely in the given oval region. This shows that the area of this region is at least 12 units. In fact, we can see that the area is more than i2 units. We can also verify by counting that there are 20 additional unit regions pictured which together cover the rest of the region. Thus; the entire region is covered by $12+20$ or 32 units. This shows that the area of this region is at most 32 units. In fact, we can see that the area is less than. 32 units. That is, we now know that the area of the region is somewhere between 12 units and 32 units.

In Chapter 9, arade 4, on Linear Measurement, we saw that more accurate estimates of lengths could be achieved by using a smaller unit. The same is true with area. To 1llustrate this fact, let us re-estimate the area of the same oval region considered on the previous page, using this time the uhit of area determined by a unit of i ength just half as long as before.

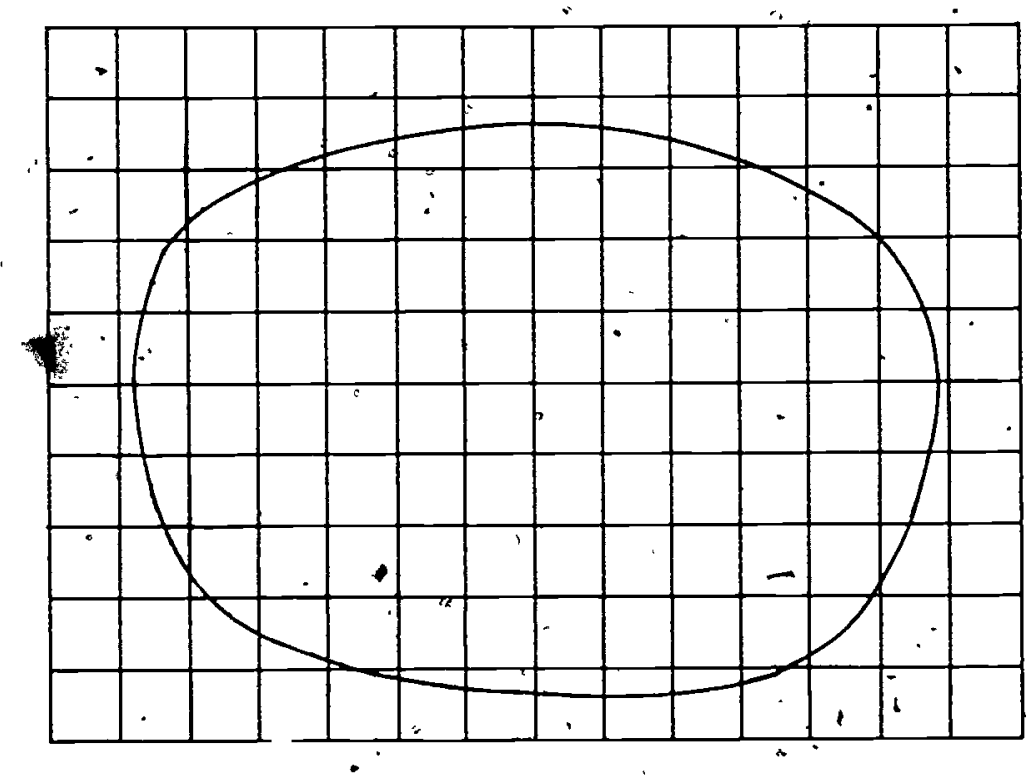


As before, we can verify by counting that there are 59 of the new unit regions pictured which are gontained entirely in the given oval region. This shows that the area of the region is at least 59 . (new) units. We can also verify by counting. that there are $37^{\circ}$ additional unit regions pictured which together cover the rest of the region. Thus, the entire region is covered by $59+37$ or 96 of the new units. This shows that the area of this region is at most 96 (new) units. That is, we now know that the area of the region is somewhere between 59 . (new) units abd 96 (new) units.

Let us compare these new estimates of the area with the old ones. Each old unit contains exactly it of the new units, as is clear from the figure below.

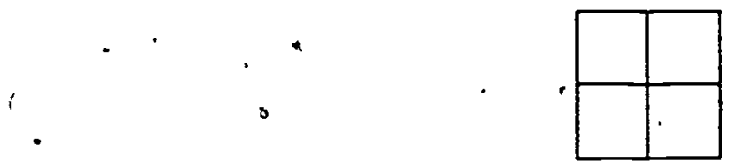

"Thus, the old estimate of i2 units becomes $4 \times 12$, or 48 , new units; and the old estimate of .32 units becomes $4 \times 32$, or 128, new 'units.: Thus, in terms of the new unit, the old" estimates tell us that the area of the region lies somewhere between $48^{\prime}$ units and 132 units, whereas the new estimates tell us that this area lies somewhere between 59 units and 96 units. Plainly, the new est1mates based on the smaller unit are the more accurate ones.

In principle it would be possible to estimate the area of region of quite general shape to ary desired degree of accuracy by using a grid of sufficiently small units in this vay. In practice, the counting involved would quickly become very tedious. Furthermore, where approximate drawings are used to represent the region and grid irvolved, we would, of course, also be limited by the accuracy of these drawings. 
Actually, the emphasis here is not so much on accurate. estimates as it is on simply leading youngsters to grasp the following basic sequence of 1deas.

1.. Area is a feature of a region (and not of its

- boundary).

2. Reglons can be compared in area (smalier, same, gréater), and regions of different shapes may have the, same area.

3. Like a length, an area should be describable or measurable, exactly, by some appropriate number (not necessarlly a whole number).

4.' For this purpose, we need to have chosen a unit of area just as we earlier needed a unit of length.

5.- The number of units which measures exactly the area of a region can be estimated approximately, from below and from above, by whole numbers of units.

6. In general, smaller units yield more precise estimates of an area.

\section{Computing the Area of a Rectangular Region}

First we consider the case of a rectangle whose length and width are glven exactly by whole numbers of units of length; for example, a rectangle of length exactly 5 unsts and width exactly 4 units. The region bounded by such a. regtangle would naturally be placed on a grid of unit regions thyo, Where we are using as unit of area a square region whose side is of unit length. 

" " Although, It is not treated in the pupil's book, the teacher might wish to extend the treatment. to include the case of a rectangle whose length and width are measured only "to the -nearest un lt"; for example, a rectangle of length slightly more than 5 units and width nearly 4 units, as shown below.

$\because *$

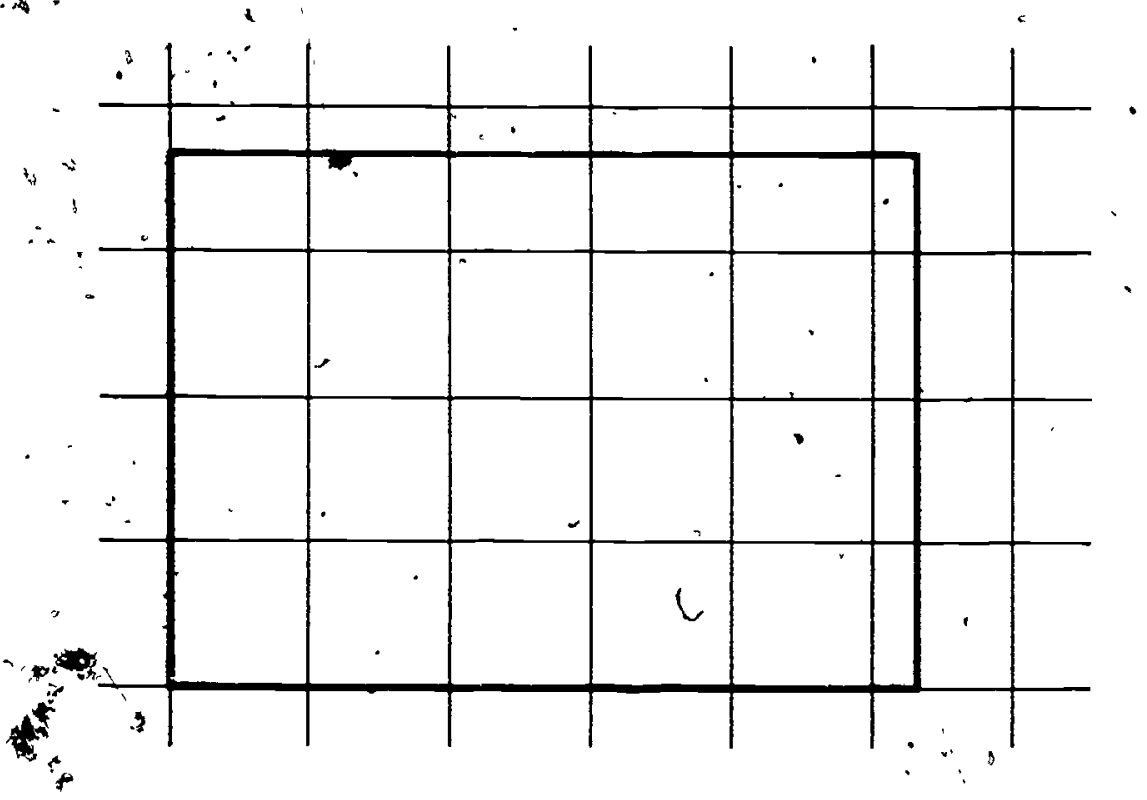

By counting unit regions in the superimposed grid (just as in our earlier example of the egg-shaped region) we see that an underestimate for the area of the region bounded by this rectangle is .15 units, and that an, overestimate for this area is 24 units. Now the actual length of the rectangle les between 5 and 6 units of length and is 5 units, to the nearest unit. The width of the rectangle lies between $3^{\circ}$ and 4 units of length and $1 s^{\prime} 4$ units, to the nearest unit. Therefore, if in, the formula

$$
\text { 2. } \quad \text { area }=\text { length } \times \text { width }
$$

we use length and width as measured to the nearest unit, we obtain $4 \times 5$, or 20 , units of area. This is exactly' the '

753

308 
area of a slightly, different rectangular region, namely the one shown shaded in the figure below.

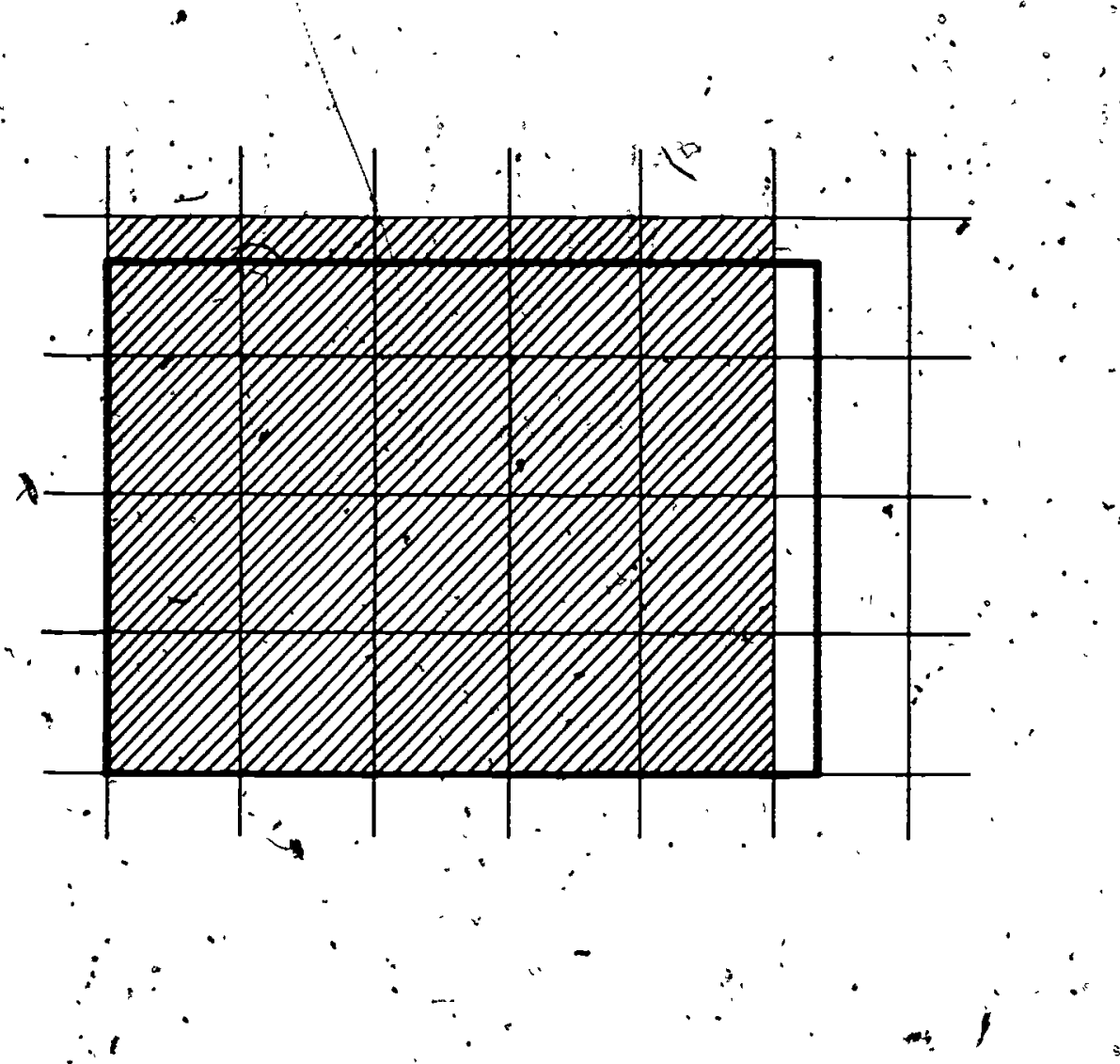

It is "plain from this figure that the shaded rectangular region contains the same unit regions, and is covered by the same unit regions, as the original rectangular region (whose" - rectangular boundary is shown in hejavy outline in the fy/gure). . This illustrates the fact that when we apply the formula

$$
\text { arga }=\text { lenghs } \times \text { width }
$$

to a rectangular 'region whose length and width are measured only to the nearest unit, we do not in general get the exact area of this region. What we do get is an estimate for this area. This estimate (20 units of area in the example) is ireasonalbe one in that" it necessarily lies between our underestimate ( 15 units) and our overestimate ( $24^{k}$ units) for this area:

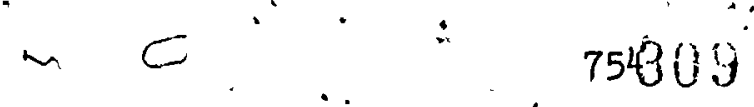


Once we have learned to calculate the area of a rectangular region, we can easily calculate the area of a right-triangular $\$$ region. Given a right-triangle, we fis locate the fourth-vertex of the rectangle whose other three vertices are the vertices, of the triangle. (The pupil learns to do this approximately. using a drawing of the triangle and a compass.)
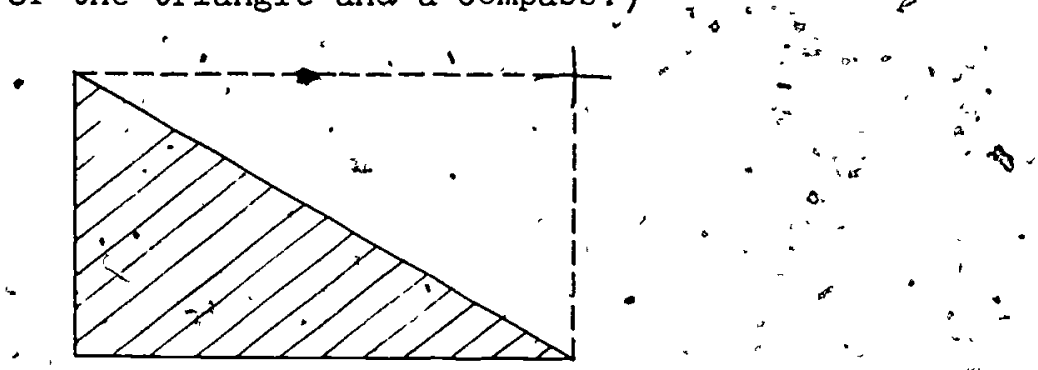

The resulting rectangular region is seen, to consist. two congruent non-overlapping triangular regions. The measure of each triangular region is one-half, the measure of the rectangular region.

Now the measure of the rectangular region is the product - of the measures of two of 1 ts adjacent sides." It follows that the measure of the right-triangular region is one-half the : proluct of the measures of the sides forming the right angle. Wher one, of these sides is taken as an altitude of the iright triangle; the other becomes the basq. In these terms the. -measure of the right-triangular region is one-half the product of the measures pr its base and its altitude. $\&+\infty$

TMS is extended to the mote general case of a triangle $M P Q$, with altitude ' $\overline{P R}$ ' and base $\overline{M Q}$, as pictured bejow: 
As a review, the argument'presented in the pupils' book - makes use of associative and distributive laws from arithmetic. Alternatively this can'be argued as outlined below.

1. The measure of rectangular region MQTS is the product of the measures of $\overline{M Q}$ and $\overline{\mathrm{PR}}$.

2. The measure of tri angulph regton $\mathbb{M P Q}$ is one-haif : the measure of rectanghiar region MQTS.

3. Therefore, the measure of triangular region $\mathbb{M P Q}$ is one-half the product of the measure of 1 ts base $\overline{\mathrm{MQ}}$ and the measure of its altitude $\overline{\mathrm{PR}}$.

An alternate figure, which holds only for the isosceles triangal-ar region, is sometimes useful in clarifying the ideas. It can be used-with the children as a different applroach after they have worked through the material given.

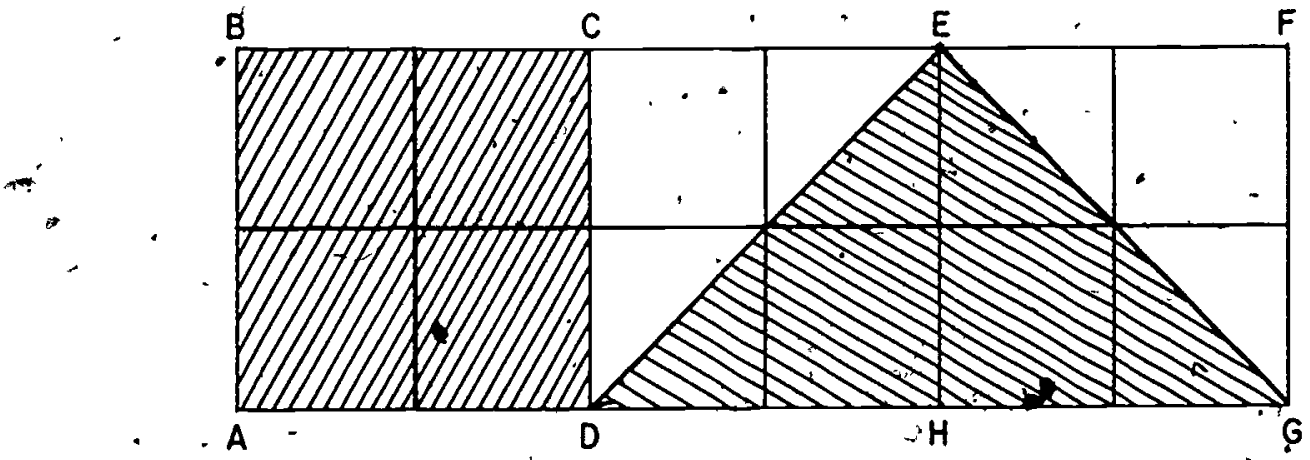

Observe that the measure of rectangle $A B C D$ is the same as the measure of $\triangle D E G$, whereas, $m \overline{\mathrm{HE}}=. \mathrm{m} \overline{\mathrm{AB}}$ and $m \overline{D G}=2 \mathrm{~m} \overline{\mathrm{AD}}$.

The charts on the next three pages tabulate rconcepts of measurement in connection with length and ârea. The first two pages of this tabulation have already appeared in Chapter 9, Grade $4^{*}$, on Inear Measurement. A fourth page, concerning Volume, will be alded in the approppiate latep unit.

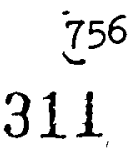


The unit for measuring must be of the same nature as the thing to be measurfa: a line segment as a unit for measuring line. segments, an angle as a unit for meaburing angles, fetc. For. convenience in communication, standard unlts (foot, meter, degree, square foot, square, meter, etc.) are-used.

The measure of a geometric object (11ne segment, angle, plane region, space region) in tems of a unit is the number (not. necessarily a whole number) of times the unit w111 $\mathrm{r}$. the object.

Measurements yield underestimates and giverestimates of measure in terms of whole numbers of units. In the case of ing segments and angles, they also yield approximations to the nearest whole number of unitg.

Segments and regions can be thought of as mathematical models of physical objects. Physical terms are used to describe the physical objects and" the physical terms are also used in discussing mathematical models. This is acceptable provided the correct mathematical interpretation of the physical terms is understood.

A curve in space may have length.

Some measures of a ffgure may be calculated from other measures of that rigure.

A set consisting of disjolnt segments (several separate pieces) may: also have the property of length.

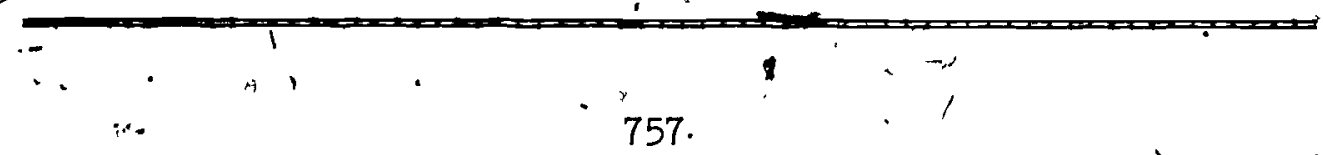




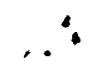

A line segment is a set of points consisting of two different points - $A \cdot$ and $\cdot B$ "and all points between" $A$ and $B$ on the line containing $A$ and B. Sometimes we say "segment" when $i$ is clear that we mean "ilne segment."

We use a linẹ seoment as a unit for measuring life segments. We use the word "meter" to name the segment which" is accepted. as the standard umt for ilrear"measurement. We use "Inch,": "root," and" "yard" to name certain other units which are. defined with relation to the standard unit.

- The measure of a line segment in terms of a unit is the number (not necessarily a whole number) of.times the unit will fit' "into the line segment". The unit segments may have common endpoints but must not overlap.

In measuring a line sejmèt, "as the unit becomes smaller, the In measuring a line segment, as the unit becomes smaller, the
interval within which the approximate length may vary, decreases in size. The precision of a meașurement depends upori the size fothis interval. The smaller"the unit, the smailer the intervali and the more precise the "measurement.

The length of a line segment in terms of a given.unit confsists of (1) the measure of this segment in terms of this unit together $\dot{1} 1 \mathrm{th}(\dot{2})$ the unit used. Examplo: if. the measure (in. inches) of a line segment is 5, then lats length is 5 inches. Many of the familiar curves in a plane or in space also have Many of the familiar curves In a plane or in space also have length. We can bend a wire to the shape of the curve and then. istraighten the wire to represent a' segment.

,

We calculate the perimeter of a triangle or other polygon. If . the measures of the sides of a triangle (where the unit of measurement is the 1 nch) are 4,5 , and 6 , then the perimeter. of the triangle is measured by the number $4+5+6$ or 15 . We say that the perimeter of the triangle is 15 inches.

A figure consisting of several segments that do not touch may have length. The measure of the figure in.terms of a given unit is the sum of the measures of the separate segments in terms of that unit. 
. 


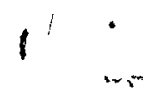

TEACHING THE UNIT

Each section of the chapter is divided into Explorations, and Exercises.. It is intended that, unless otherwise indicated in the teacner's commentary, the Exploration be a teacher-directed activity with the fullest possible pupil participation. Each teacher will decide whether the Exploration can best be directed with opened or closed books. If the books are closed, you may or may not. wish to go over the Exploration again when the books are opened. The pupil book contains the Exploration as a written record of the activity in which the class ras engaged. The pupils should work independently on the : Exercises.' Since the Exercises serve not only for maintenance and drill, but also are sometimes developmental in character, it is suggested that class discussion of the Exercises follow their completion by the pupils. The answers which are included in the oommentary may prove helpful in these. discussions.

It is recommended that throughout this unit, wherever pertinent, the teacher have the pupils shade or color the plane regions triey will use. In this way, the pupil will. not always just see a simple closed curve when he is really working with a plane region. 
objective: To develop the following understandings and skills :

1. Sometimes we compare the sizes of objects by comparing their lengths.

2. Sometimes we compare the sizes of objects by comparing the sizes of flat surfaces they cover.

3. The size of a representation of a boụnded. flat surface does not change when, we-bend or fold it:"

4. To establish a, unit for measuring a. boundea flat surface, wैe need. the concept that a simple closed curve separates the "plane into three sets of points: the set. of points of the aurve, the sgt if points.. of the interior of the curve, and "the set of points of the exterior of the curve.

5:. The union of a simple closed curve and its interior is called a plane region. This is fust one kind of region.

6. We use a plane region as a unit for. measuring plane regions.

7. The area of $\dot{a}$ plane region is the measure of the plane region and the unit used to. make the measure. We measune a plane region to flith $1 \mathrm{ts}$ area. 
Materials:

Teacher: Any objects considered necessary for discussion of gross comparison of size in the first Exploration.

Pupils: Crayon, any objects necessary for answers to Exercise sets $i$ and 2.

Vocabulary: Surface, bounded, flat surfacé, plane region, area, triangular regiıon, polygonal (pó 11 ğ' t năl) region, rectangular region.

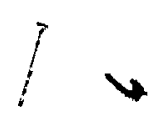

4

By' the end of this section, the' pup11 should be able to differentiate between a rigure which has length and a rigure which has area. 
P455

Chapter, 8

AREA .

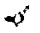

WHAT IS AREA?

Comparing Sizes of Regions

Exploration )

You have had experience in comparing the sizes of line segments and the sizes of angles: Look around your classroom. Find representations of two line segments which are not the same length, can you tell without making any measurement which/ is - longer? Find representations of two angles, which are not the same'size. Can you tell

'which' is larger without using the: compass or (. prot $-$

-

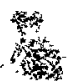

1 . , 
P456

Exercise set 1

In each of the following, tell which is larger:

1. A sheet of typing paper or a stamp. (a sheet of tyreripapar).

2. A pin head or a dinner plate. (a dining plate)

3: A pillow case or a bed sheet. (a bed sleet)

4: A television screen or a motion picture screen. ( ámotion pustersenem)

5. A nickel or a dime. (a nickel)

6. A wash cloth or a handkerchief. (depends on abject)

7. A window or 1 ts window-shade. (fivendan means th pare, th shade shall be larger.)

8. Your classroom floor or your classroom ceiling. (publ the ne)

9. The sole of your shoe or the sole of your friends shoe, (depends on digests)

10. A sheet of your notebook paper or this page of your text. (Lependomoljest.)

11. Did you know the answer to the above exercises immediately? Were there some cases where you were not certain, at once, which was larger? How did you decide? ( wee st int busing which

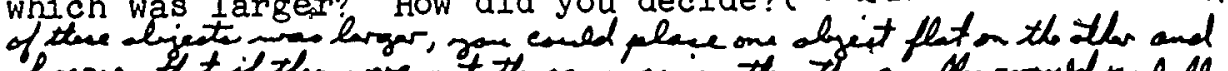

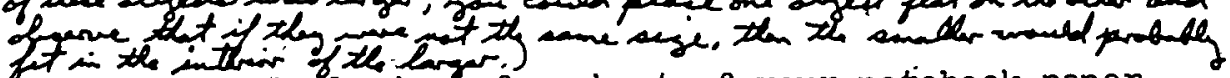

12. Will the original size of a sheet of your notebook paper change if you, fold it into four parts? How will you test - to see if it has remained the game size? (Place the fold shat ow ere unfold one.)

13. Does the size of your bath towel change when it is wrapped around your body? $\left(x_{0}\right)$

14. What happens to the size of a map when 'you roll it up?

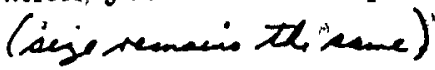

$$
\therefore \quad 313 \ldots \quad+\cdots
$$


Length of a Curve or Size of the Surface Enolosed by It

\section{Expioration}

Sometimes we compare sizes of objects by comparing lengths and sometimes' we compare sizes by comparing the sizes of flàt surfaces. Suppose we have pictures of two rectangular fields:

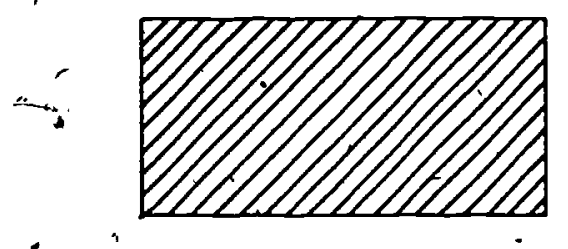

1.

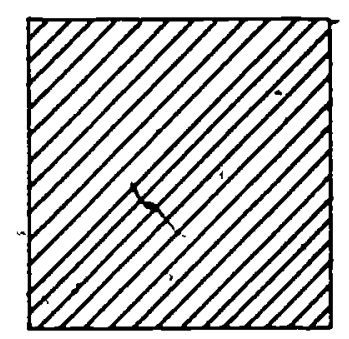

- If we wish to cdmpare the amounts of fencing we need to enclose these fields, yhat property of the rectangles will we compare? (hemember that a rectangle is a simple, closed curve: * and if we measure a simple closed curve we are finding its length.

- we might, however, be interested in dividing one of the flelds so that half would be planted in com and half in beans. Would we need to know the length of the rectangle? $(x)$ be helpful to know the size of the surface of the flefu?(ya) 


\section{Exercise set $?$}

Tell whether you are interested in the length of a-simple closed curtve or the size of the surface in 1.ts interior, or both:

1. To trim the edge of a handkerchief with lace. (length of

2. To buy a rug to cover the living room fioor. (both)

3. To buy a desk blotter for your desk. (both)

4. To put a book cover on your text. (both)

5. To string enough beads for a necklace. (lingth $\%$ curve)

6. Can you give 3 other examples of situations in which. you would need to know the size of the

- surface enclosed by a simple closed curvet rather than just the length of the cuirve? (tabuy materal to.

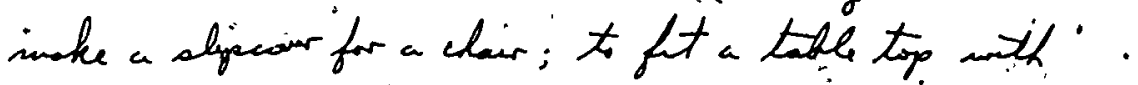
a glase cour; to bny linokmin for a kitch flow, ete.)

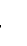


1. Recall that a simple closed curve by our definition is a path having the following properties:

a. All of 1 ts points lie in a plane.

. b. If one traces the path, he eventually feturns to the starting point.

c. The path never intersects itșelf; 1.e., in proceeding once around the path, any point is encountered just once (except for the starting point).

It also has the property that it separates the plane linto three sets of points: the set of points of the curve, the set of points of the interior of the curve, and the set of points of the exterior of the curve.

2. Which ones are simple Rosed curves? $(a ; c, g, l)$

a.

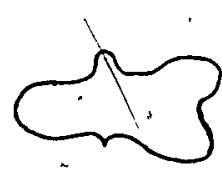

d.

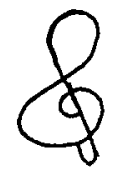

b.

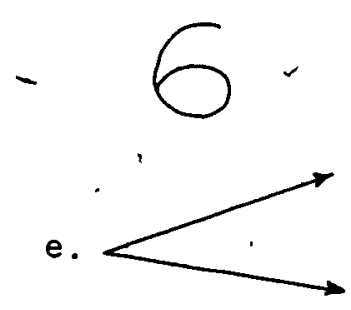

c. .

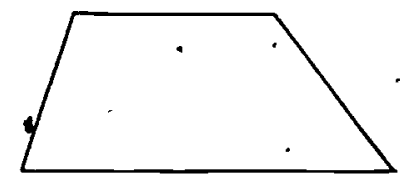

f.

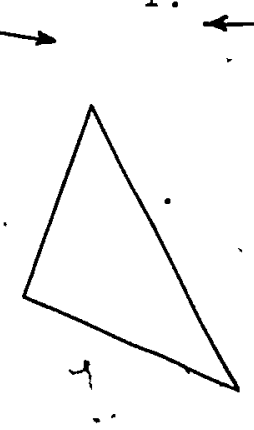

2.

h.

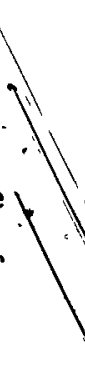


The unton of $\dot{a}$ simple closed curve and its interior is called a plane region. If the curve is a triangle, the plane region is calied a triangular region. The union of a $*$ poljgon and 1ts interior is called a polygonal region. Itels . the region that we meàsure when we. want to know the size of . part of a rlat surface.

If the curve is a simple closed curve, trace the curve (the curver of $a ; c, d, e, f$ shat he taned and colori the plane region: and thir intwini cabore.)

a.

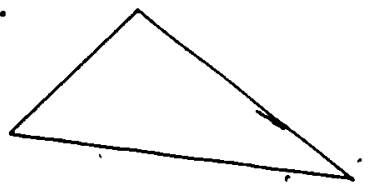

b.

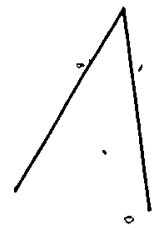

e.

d.

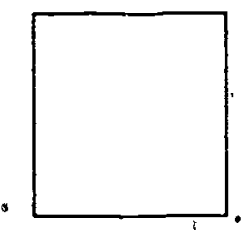

g. ,

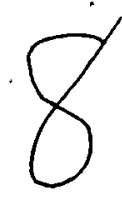

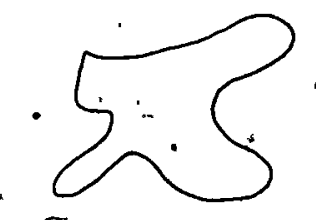

h.

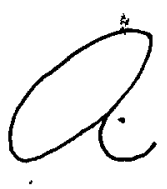

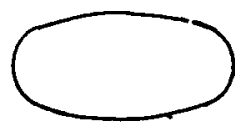

f.
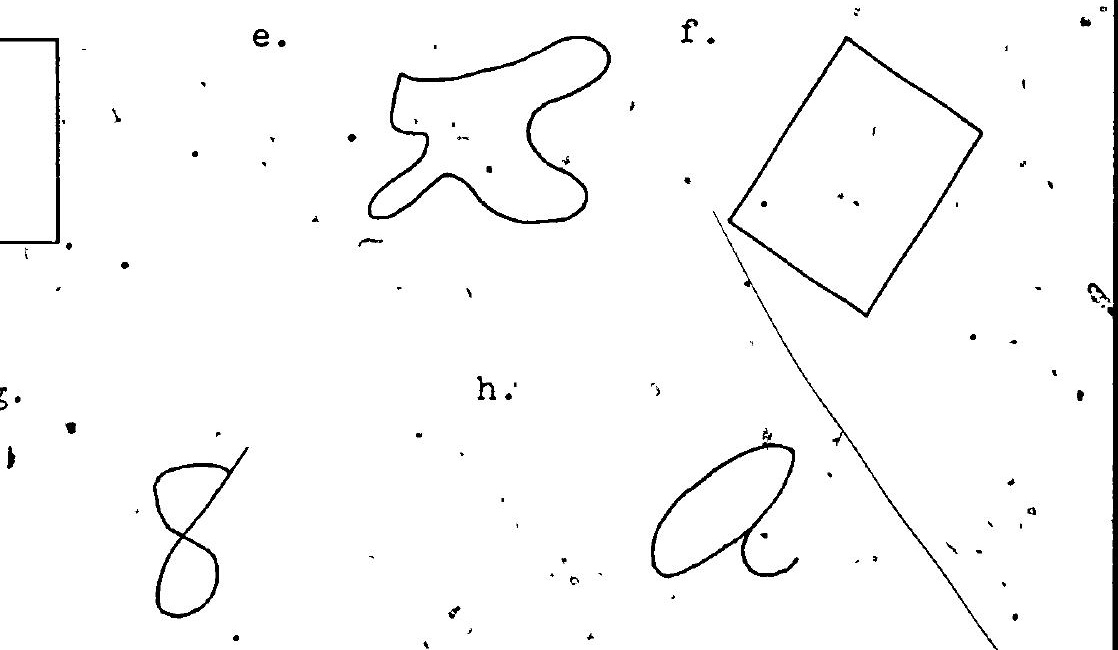

I

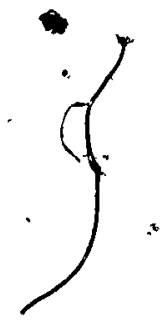


Tell whether jou would be interested in area or length, or both, in each of the following:

1. To, buy enough wrapping paper for a package. (area-chorahipe) '

2: To decide on the amount of twine needed to wrap

a package. (lengtl)

3. To decide ơn the size of a belt. (length)

4. To buy a piece of land. (areat-abas elape)

5. To mow a lawn. (area)

6. To mun around a closed track. (.lengtt).

7. To sa1I around an island. (length)

8. To tile a basement floor. (area)

9. To measure a triangle. (lengt/)

10. To measure a triangular region. (area)

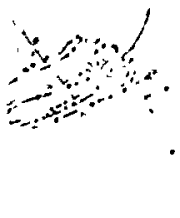


Objective: To develop the following understandings and skills:

1. We can compare the areas of fwo plane regions by seeing whether one region can be fitted into the other region.

2. In making a comparison of two plane regions by inclusion, we may dissect one region and see if the smaller regions can be made to fit in the other region.

Materials:

Teacher: Scissors, large cardboard models of -figures to be dissected, paper clips, scotch tape.

Pupils: Scissors, tracing paper, paper clips, scotch tape.

.1

,

Vocabulary: Dissect 
COMPARING AREAS

The areas of two plane regions usually can be compared by seeing whether one region may be Included in the other. That is, if one plane region can be placed entirely in the interior of the other, then the area of the -first region is smaller than the area of the second region.:

A plane region can be cut up into smaller

-.. plane regions. When we cut up a plane region, we say we are dissecting it. To dissect something means to cut it into parts or pieces. Suppose you can dissect one plane region and place all' the pieces, without overlapping, entirety on a second plane region; What would this show about the

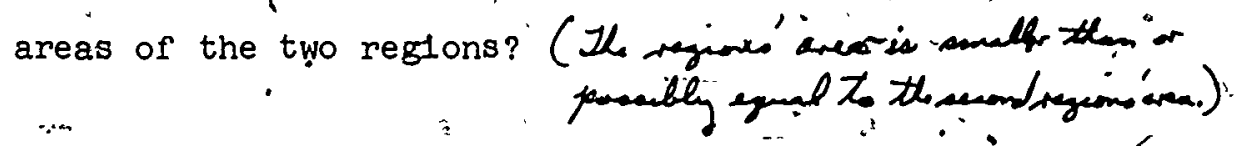

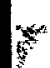

$\therefore$ 
P463

\section{Exploration}

Which fectangular region has the smallest area? Will a.tracing of one of them fit into the interior of each of the others? (Yen, region IJKL will.)
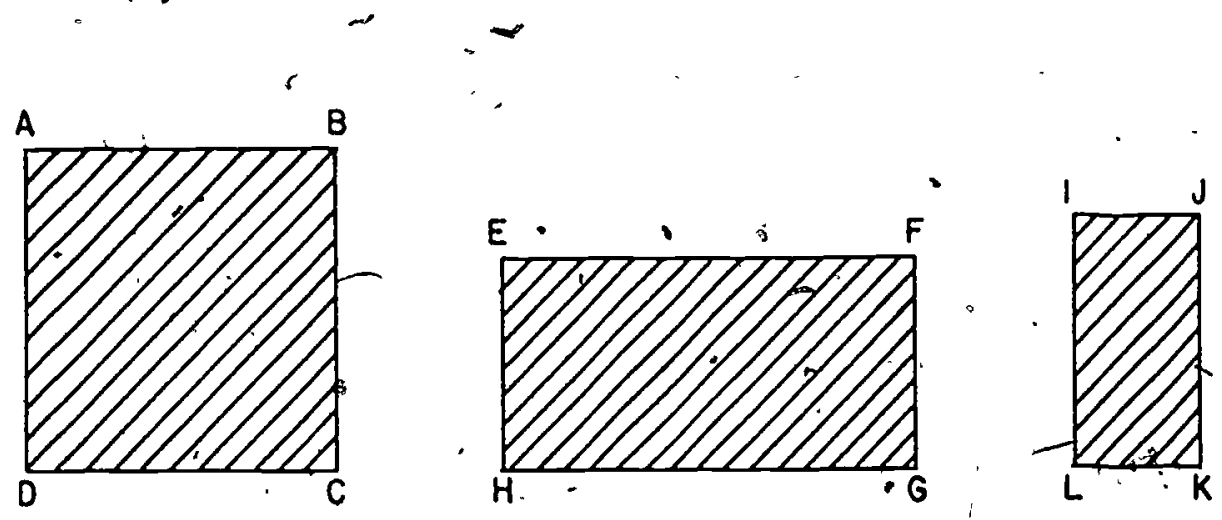

Which reptangular region has the greatest area? 1 . WIIl a tracing of either figure'fit into the interior of the other? ( $w$ )

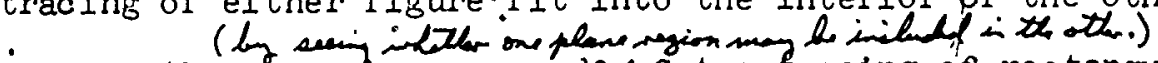
How can the areas be compared? 1 qut a tracing of rectangular region EFGH Into mall pleces. Can alr of these small pleces be placed, without overlapping, in the interiop of rectangle $A B C D ?(y e x)$.

$r$

Is the area of triangular region WXY less than the area of rettangular région $\mathrm{PQRS}$ ? (yea)
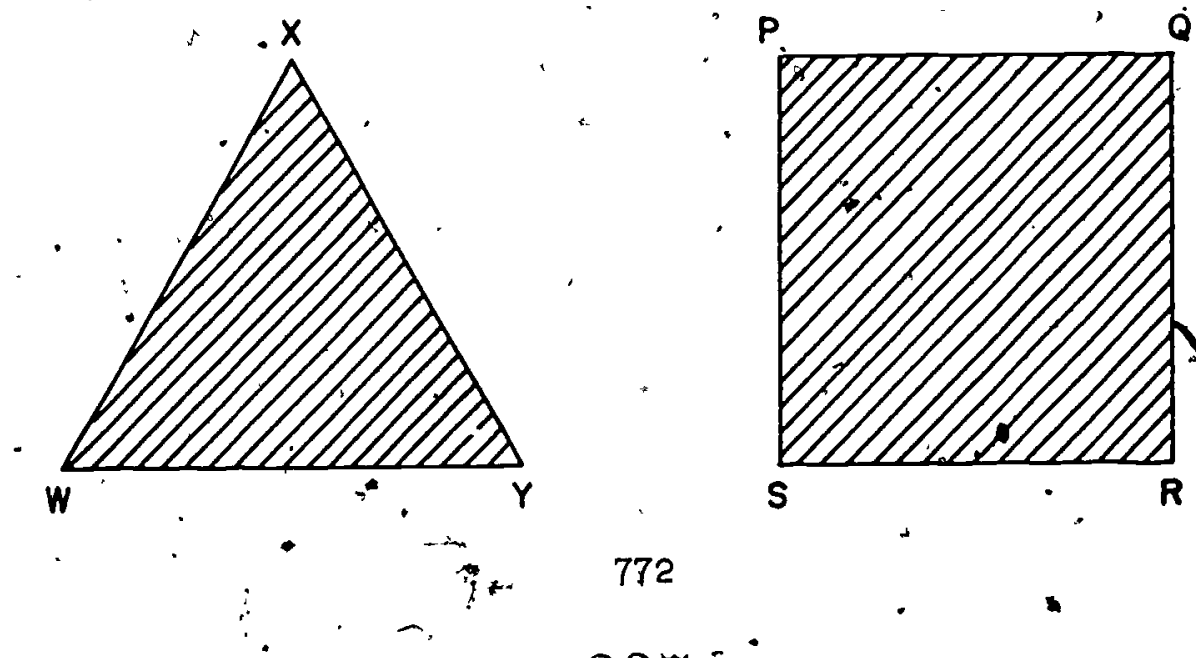

772 
In Exercises ' $1-3$ tell which region of each pair has the ireater area (You may make a paper model of one of these regions and "cut it to see if the pleces can be placed, without overlapping, on the other region.)

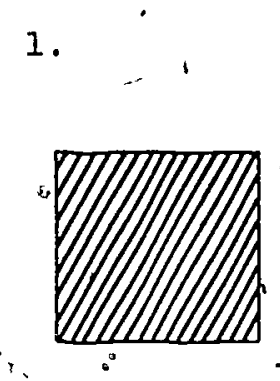

(a)

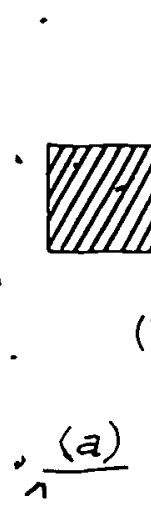

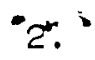

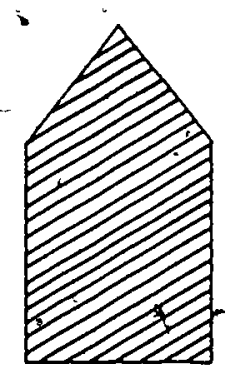

(a)

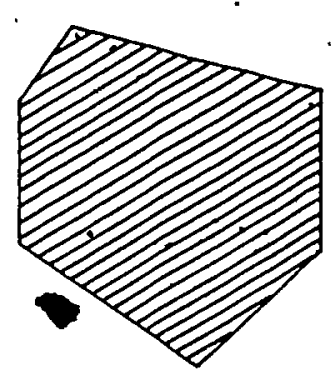

(b)

$(b)$

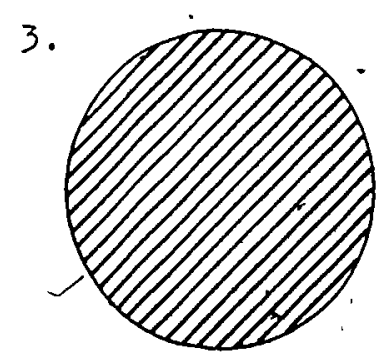

(a) (b)

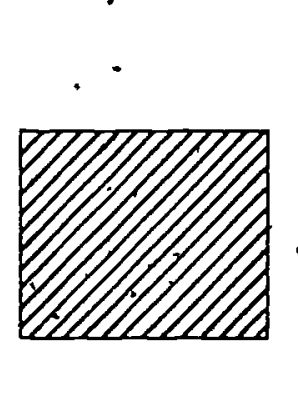

(a)
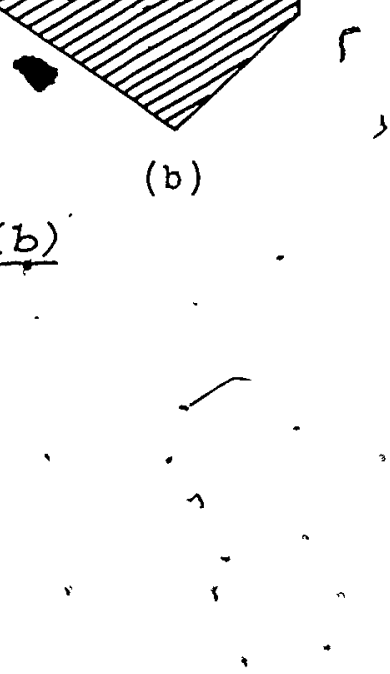

(1)
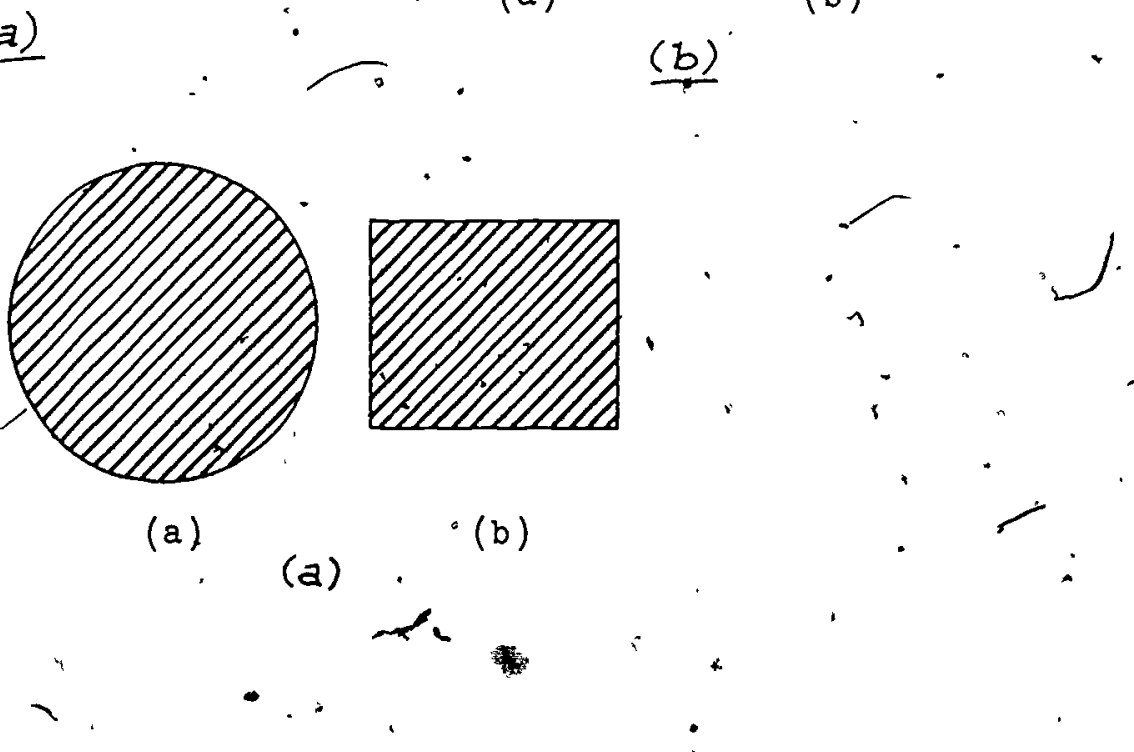

\section{u}

4. Which plane region has the greater arsea - a region bounded - "by a square with a side whose length is 3 inches or a " region bounded by an equilateral triangle. with a side whose length is 4 inches? ( $\mathrm{X}$ You w1ll need models of these reglops 
P465

BRAINTWISTER.

Trace "Robert Robot."

Can you arrange the parts fof the "robat" in such a I way that they form a . rectangular region? The rectangle will have sides whose lengths are $2 \frac{1}{8}$ inches and ' $4 \frac{5}{8}$ inches. (answer below) (answer below)
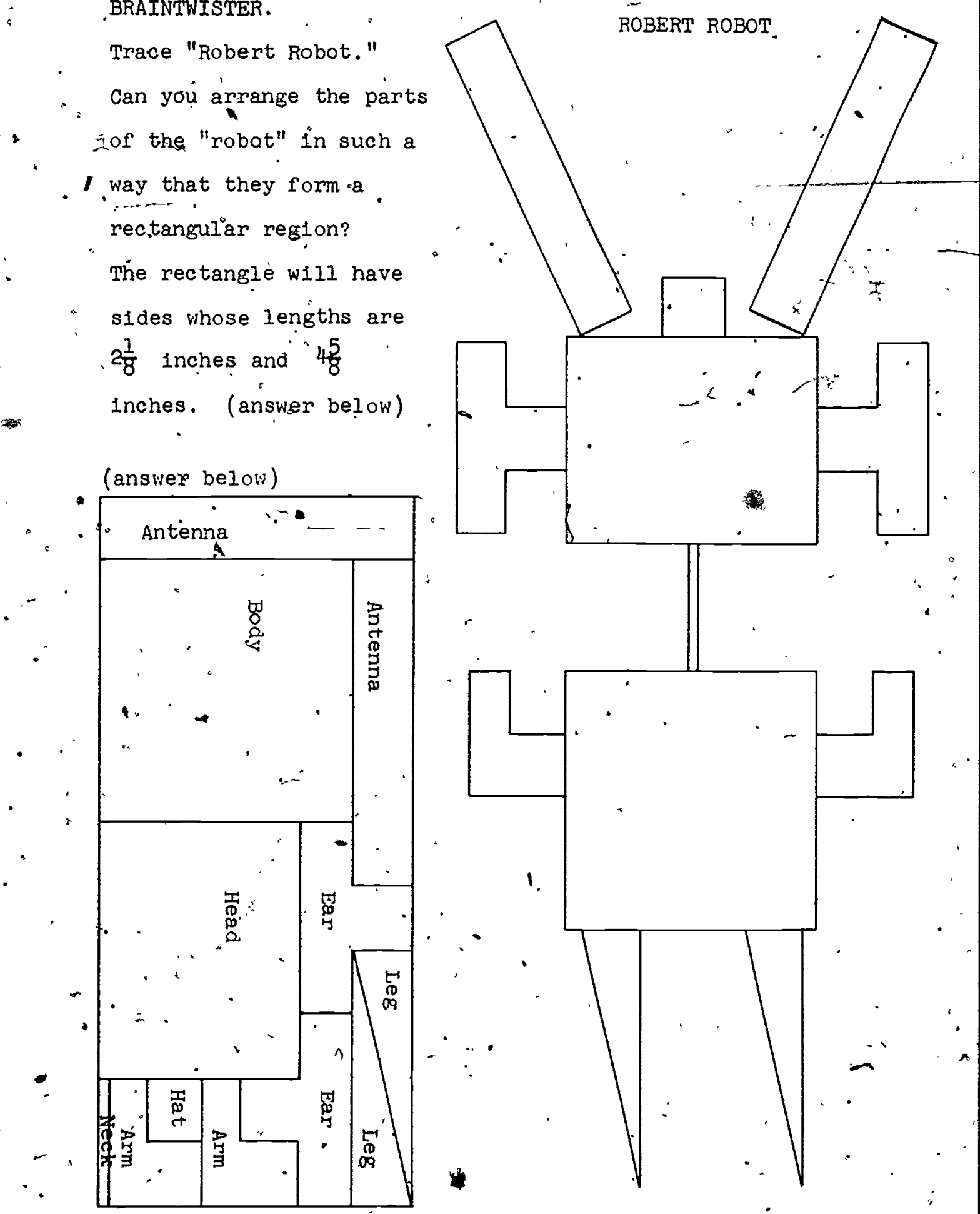
UNITS OF AREA

objective: To develop the foliowing understandings and skills :

1. In measuring areas a unit ṕlane region is used, as in measuring lengths a unit line segment is used.

2. We need as unit a plane regibn such that any given plane region can be covered completely by placing these units on $1 t^{\prime \prime}$ without ovierlapping.

3. Circular regions (and regions of many other shapes) do not have the covering property needed, but square regions do.

4. As a unit of area we can use a square region whose size is determined by making each side of length 1 unit.

Materials Needed:

Teacher:

1. Large triangiular piece of flannel,' whose sides have, lengths of about 20", 15", and $10^{\prime \prime}$. Alternatives: a triangular piece of paper of the same size that can be used on a bulletin board, or a chalkboard drawing.

2. Nine or ten pieces of flannel cut in the form of regions of diameter about four inches. Nine or ten pieces of flannel cut in the form of square regions of side about 4 inches.

3. Straightedge, pieces of string or pieces of wire.

Pupil: For the exploration and for the flesst part of the next section; each pupil w1 II 'need'about! 24 square pieces of construction paper, $1 \frac{1}{2} " \times 1 \frac{1}{2} "$ in size. 
Exploration

This Exploration does dot appear in the pupils' book. The following questions and outline of procedure may be used as a basis for class discussion. The discussion is, summarized in thề pupilst book. As you begin the discussion, place the triangular plece of flannel on the flannel board.

This represents a triangular region. What do the edges of the region form? What does each side of the triangle form? Who can tell how to measure the length of a line, segment such as the side of a triangle? Will you show the class how you would do this?
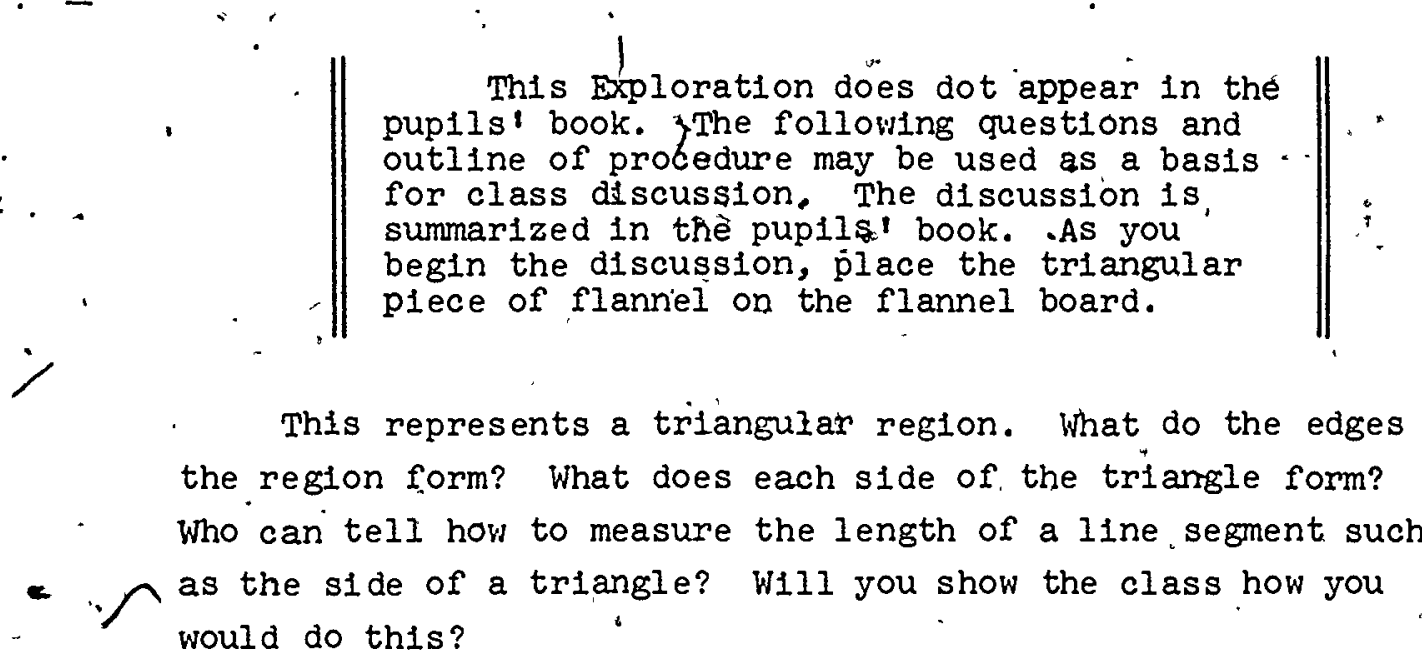
*.

Allow time for child to do this part
of demonstration before class. Then draw
the following summary from the discussion.
It may be well to add the following
for emphasis.

We first choose a line segment as unit. Then we measure this side of the triangle by placing units end to end along " It so that they touch but do not overlap. Enough units placed in this way will completely cover the whole side. By counting we find that it takes more than (for example) 4 . units but less than 5 units to cover the side exactly. Therefore, we say that the length of this side is greater than 4 units but less than. 5 units.

We can measure the area of a plane region such as this triangular region in much the same way. Finst we choose a plane regton to serve as unit. 
At this time ask the chlldren for suggestions how an area of a plane region might be measured by using a plane region as unit. Encourage the children to experiment and demonstrate their 1deas at the flannel board: You may wish to give guidance by saying, "Let us think about what sort of plane region would serve best as a unit. We-need to cover the whole triangular region by placing enough units next to one another so that they rtouch but do noteverlap."

How would a small circular region do as' a unit? Can we cover the whole triangular region by placing these circular regtons so that they touch but do not overlap?

Why can we not cover the whole triangular region with

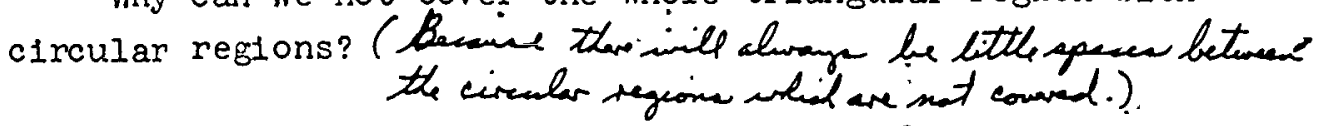

Can you think of a plane region that is, better than a circular one to use as a unit? (yew, a genare region)

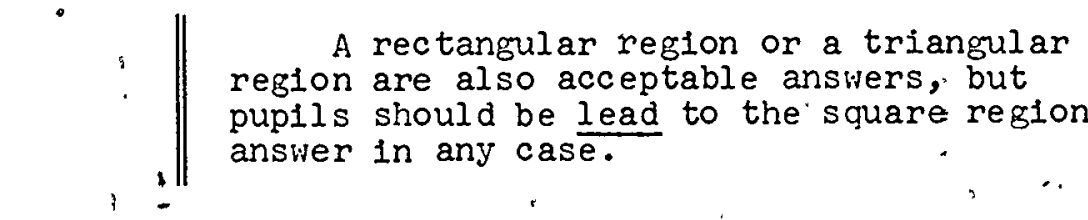

Why is a square region better than a cireular region? (recoune

[ill someone use these square regions to cover this

Let us agree to use a square region as unit of area. Suppose we have already decided on a unit of length. Can we use this unit of length to determine the size of a square region to be used as unit of area? ( yee, chace as went of area a semare whace ande is 1 mit of length.):

this at the flannel board by using the triangular region and the small square regions:

Encourase children to illustrate 
P466

UNITS OF AREA

- Choosing a Unit of Area

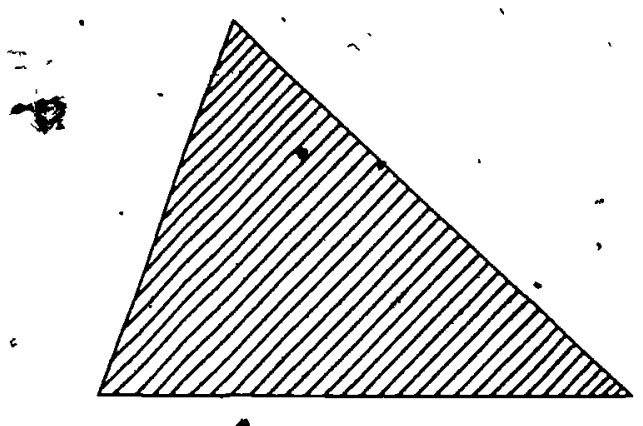

This is a picture of a triangular region. Suppose we wish to measure 1 ts area. When we 'measured the length of a line segment, we needed a unit of length. To measure the area of a region, like this triangular region, we need a unit of area, a unit region.

We need to cover the whole region to be measured by placing unit regions on it so that they, touch but do not overlap. Is it possible to cover a whole triangular region with circular regions in this way? Why not?

Fin

$\infty$

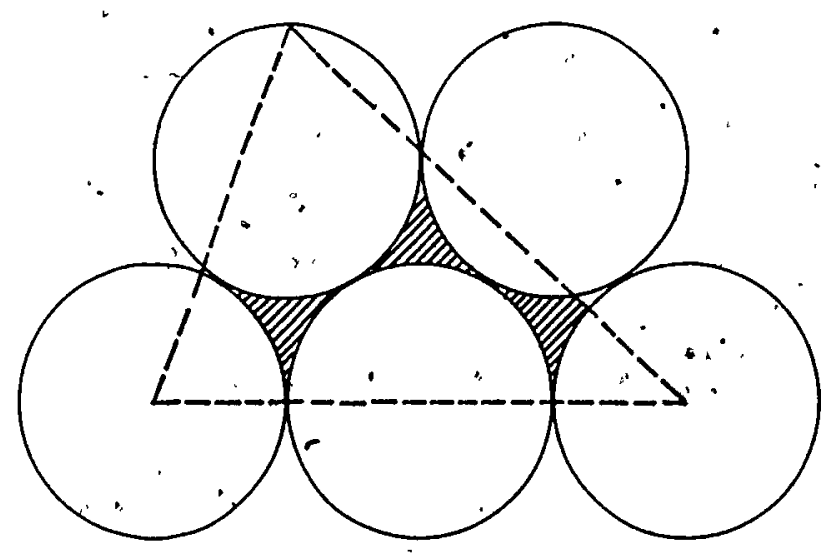


$\$ 467$

- Is 1 possible to cover the triangular region with square regions? Why

1.

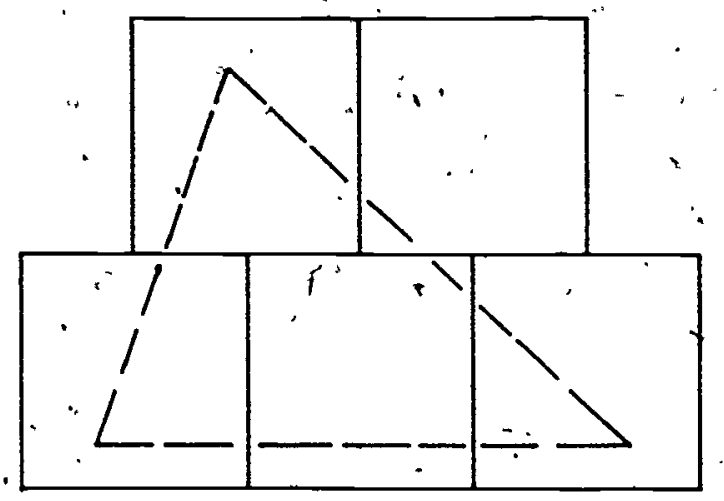

Left us choose a square region as unit of area! We choose a square region whose side is Just one unit of length.

unit of length . . unit of area

.779

334 
$\mathrm{P} 468$

Differently Shaped Regions of Same Àrea.

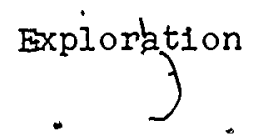

Each of you has some square pleces of paper all of the same gize. Each plece represents $I$ ' unit of area. 'Place two

- pieces side by side on à sheet of paper, so that they touch but do not overlap. Trace around the region. formed by these pleces. Does your picture look like this?
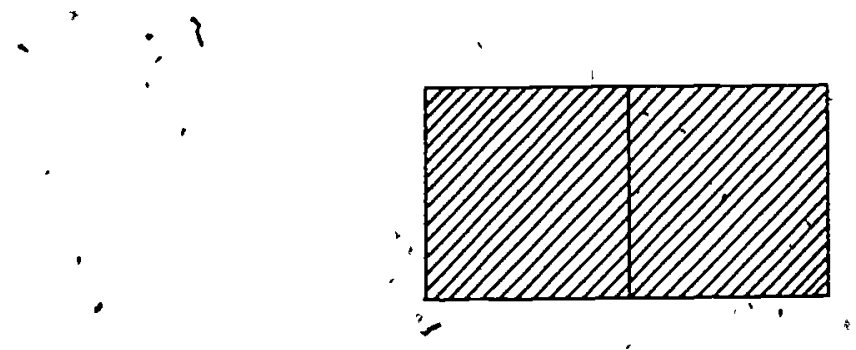

$$
\text { (a rectergle) }
$$

What is the figure you have drawn? Color the rectangle and 1 ts interior. What is the figure you have colored? What is the area of this region? (2 2 irte)

- Draw and color a rectangular region of area 3 units. Does your picture look like this?
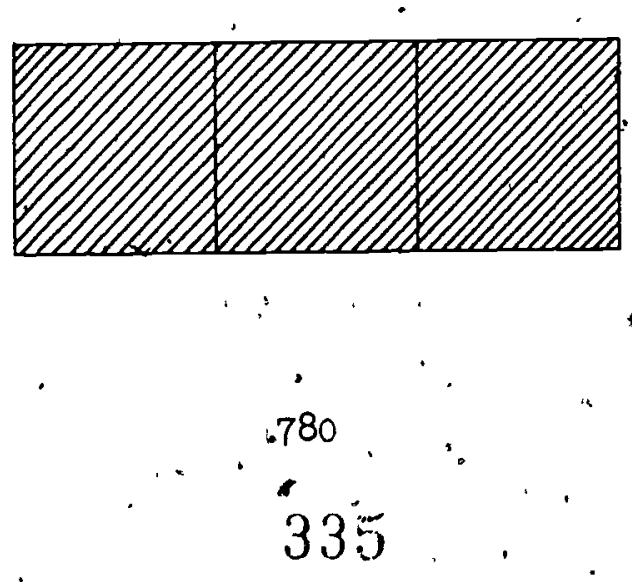
$w$

$\mathrm{P} 469$

Here are some regions of different shapes, each with area 3 units. Can you think of some others?
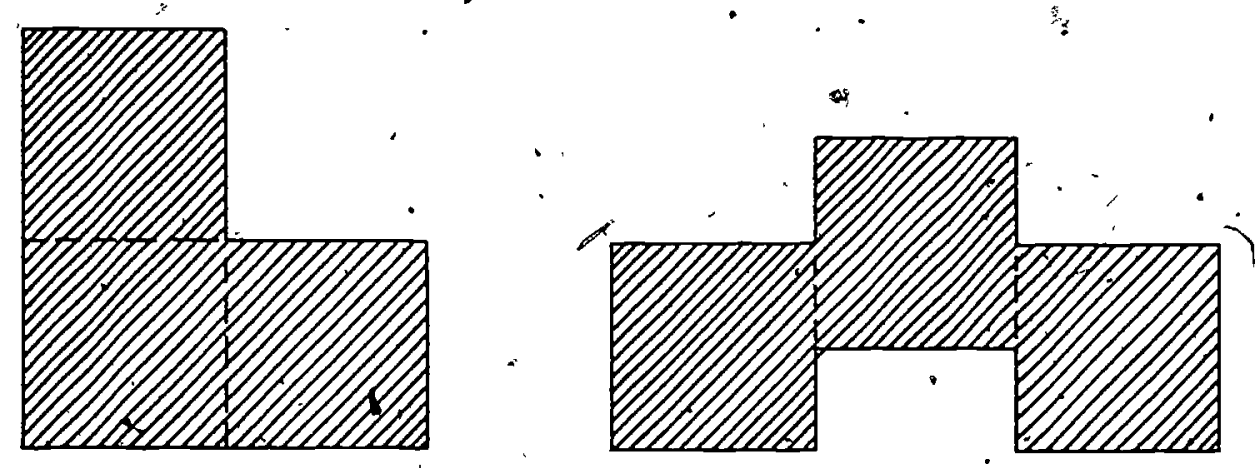

Here are some regions of different shapes, each th area of $1 \frac{1}{2}$ units. Can you think of some others?
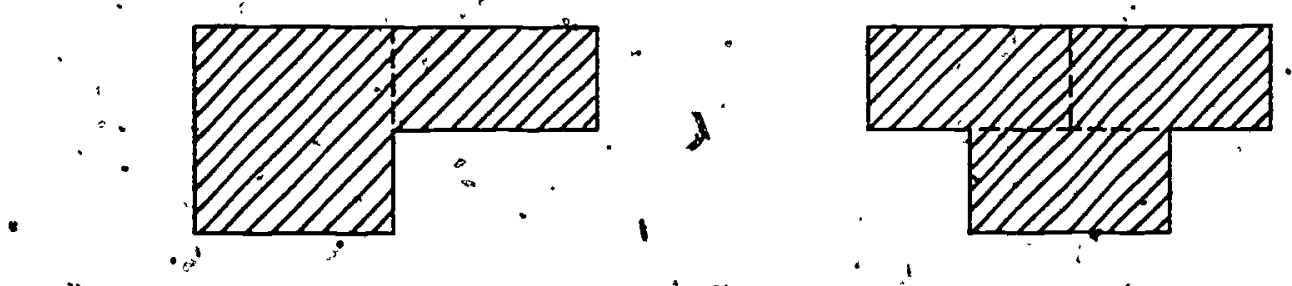

$\because \quad \cdots, \quad y$

$\therefore r \cdot \cdot 781$

ERIC

336 


\section{$1 \quad \therefore \quad$ Exercise Set 5 :}

1. Use your square region of paper to trace out and tolor. a region of area 5 .units. Make' the region, any shape you wish:"

$2 . \%$ Use your squares of paper (you may want to fold one of them) to trace out and color a region of area $2 \frac{1}{2}$ units. Make the region, any shape you wish.

3. Take two of your unit square gions of paper and cut eách of them into at leas't. three polygonal regions. Now make a new region of different shape, using all your pieces. What is the area of this new region? ( 2 genore regeomic $)$

$\therefore$ We teacher may wish to assign additional exercises of this sort. ,

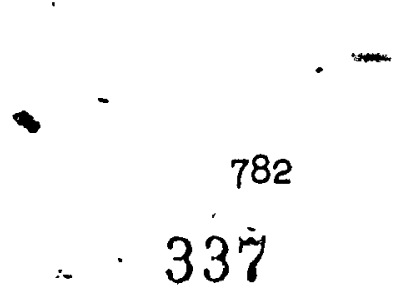




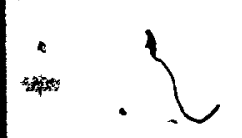

ESTIMATING AREAS

Understanding and Skills:

1. If a ceatain number of units can be fitted"into a given region without overlapping, then the area of

* this region is at least this certain number of units.

2. If a certain number of units together cover.a given region entirely, then the area of this region is at most this number of units.

3. Just as scale is useful for measuring lengths; a grid is useful for measuring areas.

4. Smaller units result in more precise estimates of." areas.

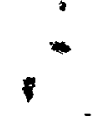

Materials: 1 . Teacher: Large blackboafo drawing of gures from the
text. :

Pupil: The same square pieces of constritanger. used in the preceding section, sheetsiof: paper ruled with 1 inch squares, ts of paper ruled with half-inch squares (ththt is, $\ldots$ - squares with sides of length onefrelf an
inch). 
P471

ESTIMATING AREAS

Uṣing Unit Regions to Estimate Areas

\section{Exploration -}

Suppose we wish" to estimate the area of a region with $\mathrm{q}^{*}$ curved boundary along the top, l1ke. a church window, in terms of the unit shown.
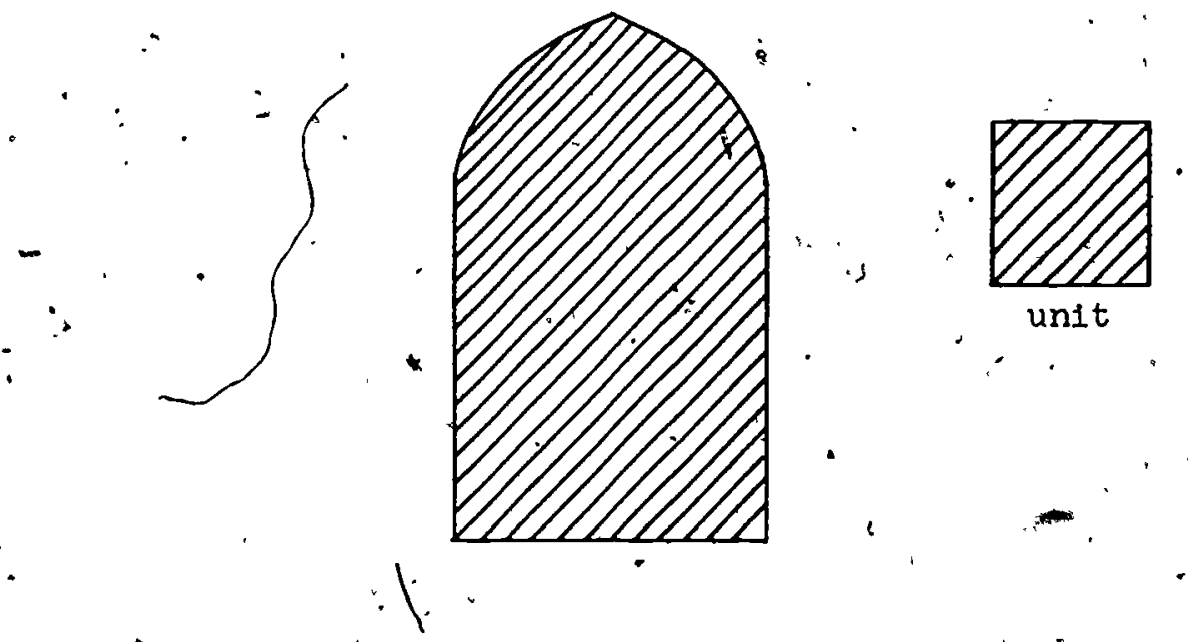

We can fit units into this region as suggested by the picture below.
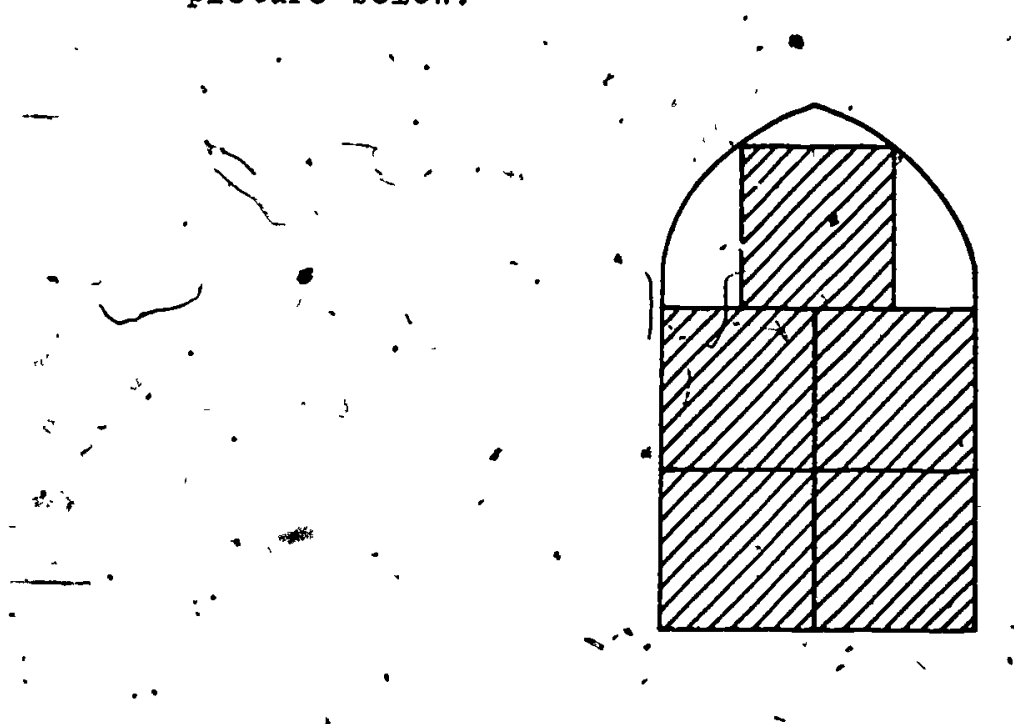
$\triangle P 42 \dot{2}$

What does this"shori about the area of the region? (lt is at least 5 mitt.)

We dan also cover this region with unit regions, as shown below.

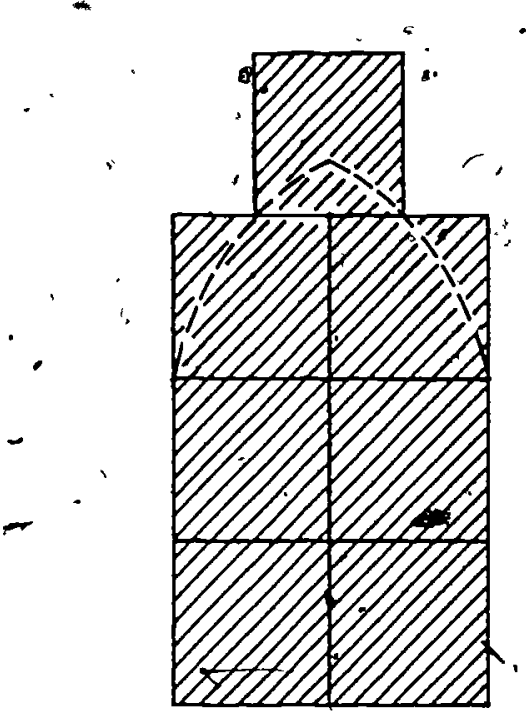

What does this show about the area of the region? (I tiv fen than

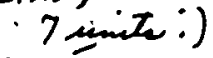

We have not found the exact area of this region, but we now know. it is a number of units, (not necessarily a - whole number) somewhere between $\dot{5}$ and 7 .

"Can you guess from the picture about what the area is?

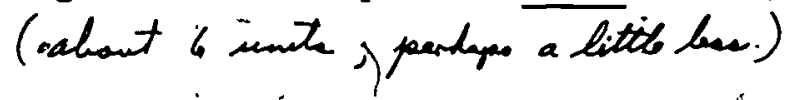

ERIC

785. 


\section{Exercise set $\underline{6}$}

- 1. On the next page is a quadrilateral region. See how many of: your square pieces of paper. you can place entirely on this region. Be sure that no piece goes outside the region and that no piece overlaps another piece. : How many pleces are you able to place on

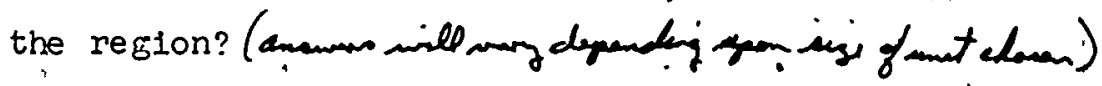
What does this tell you about the area of the region? (ftia at lent_inest:

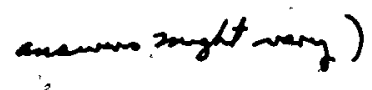

1. Next, see how many of your square pieces of paper you need to cover the region

completely. No plece.should overlap anothex piece:" How many pieces do you

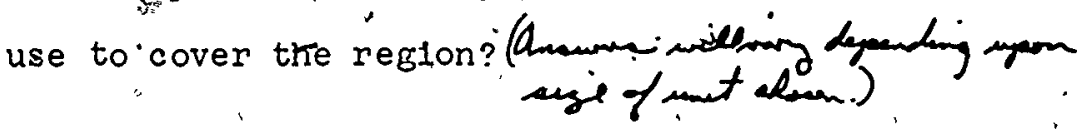
What does, this tell you about the area of the racion? (It is at int mit.)

Can you estima'te about what the area might be? (Anamers will wary) 


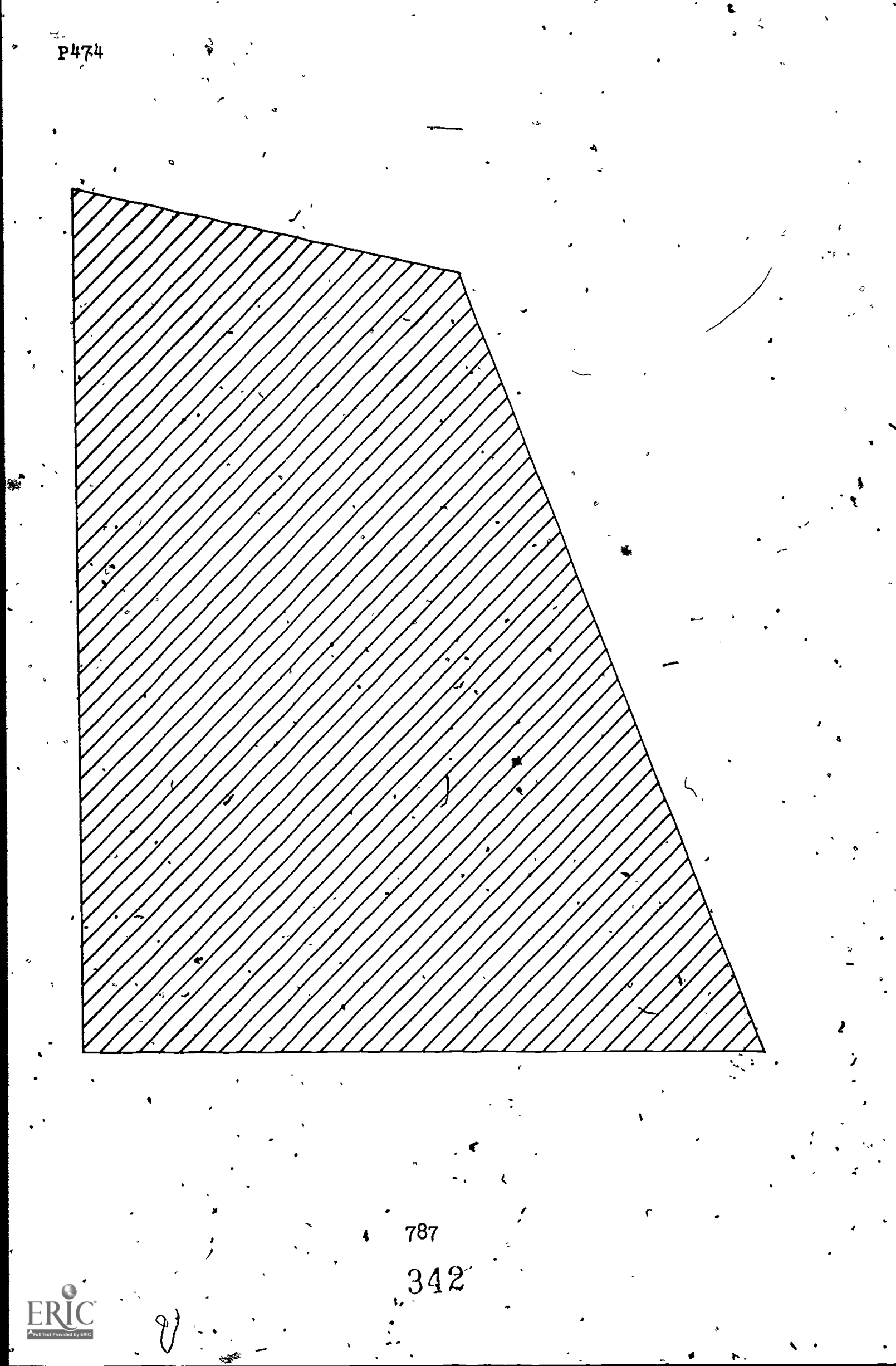


3. On the next page is a picture of an oval region. See how many of your square pieces you can place entirely on this region.: Be careful that no piece goes outside the region and that no piece. overlaps another plece. "How many pieçes are you able to place on the region?

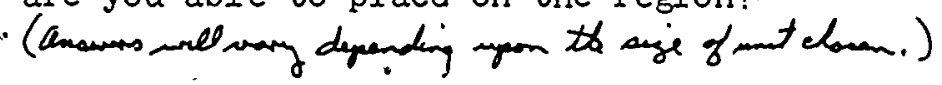
What does this teil you about the area

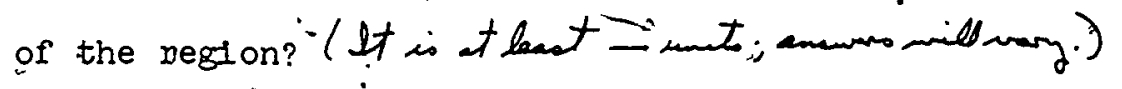

4. How many of your square pieces of paper do you need to cover the region compl e tel y? (ancmon trill wary depanding yaon the aye fract chan.) What does this' tell you about the area. - of this region? (ltis atmont- mut; -

\section{Can you estimate about what the area} might be?

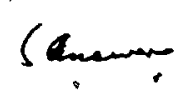

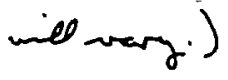

Your class may need-möre exercises similar to these to establish and maintălin skill. 


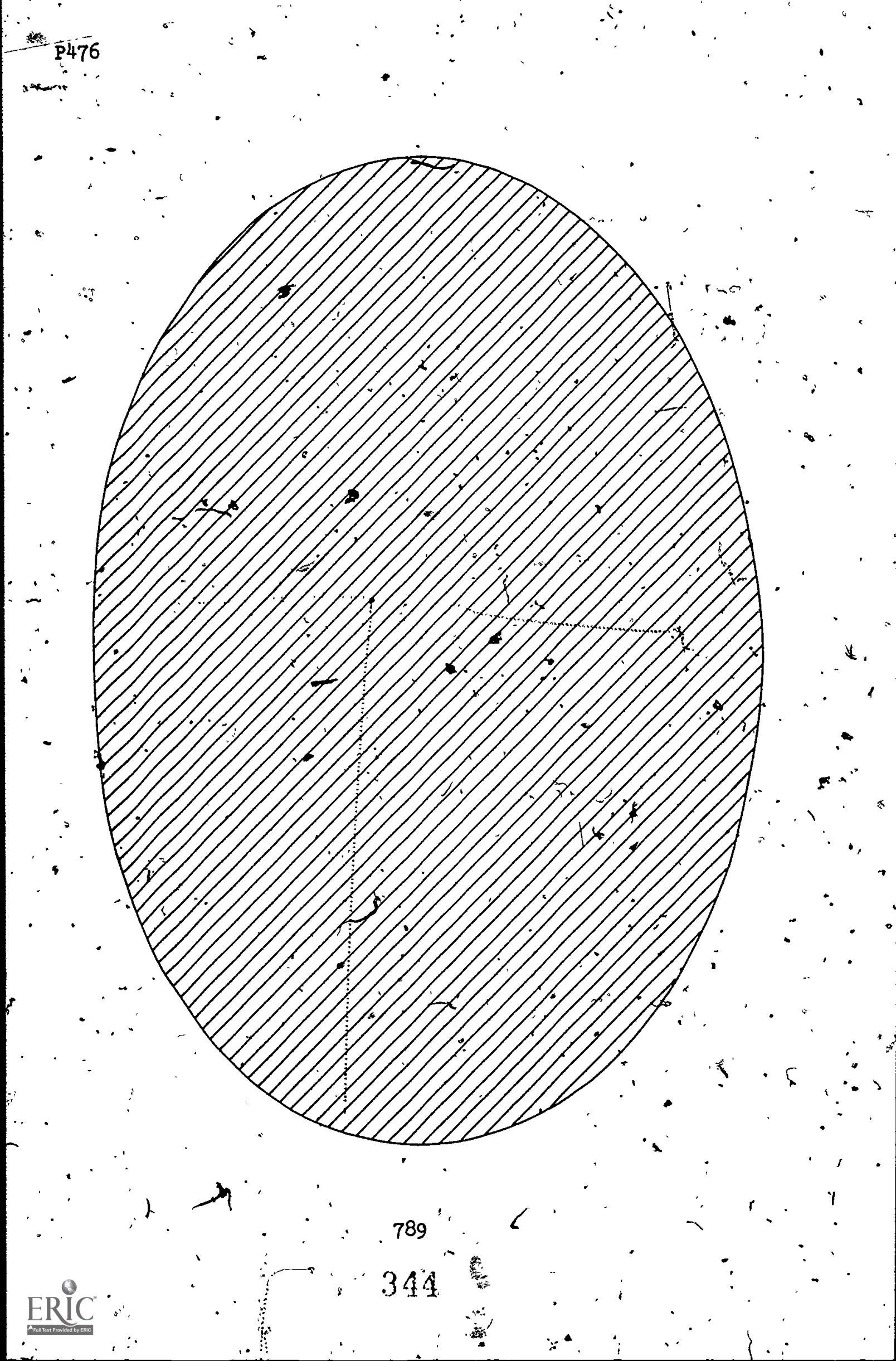


Pi 477

Using Grids too Estimate Areas,

Exploration

Suppose we wish to measure the area of the oval region * below. An terms of the unit shown.

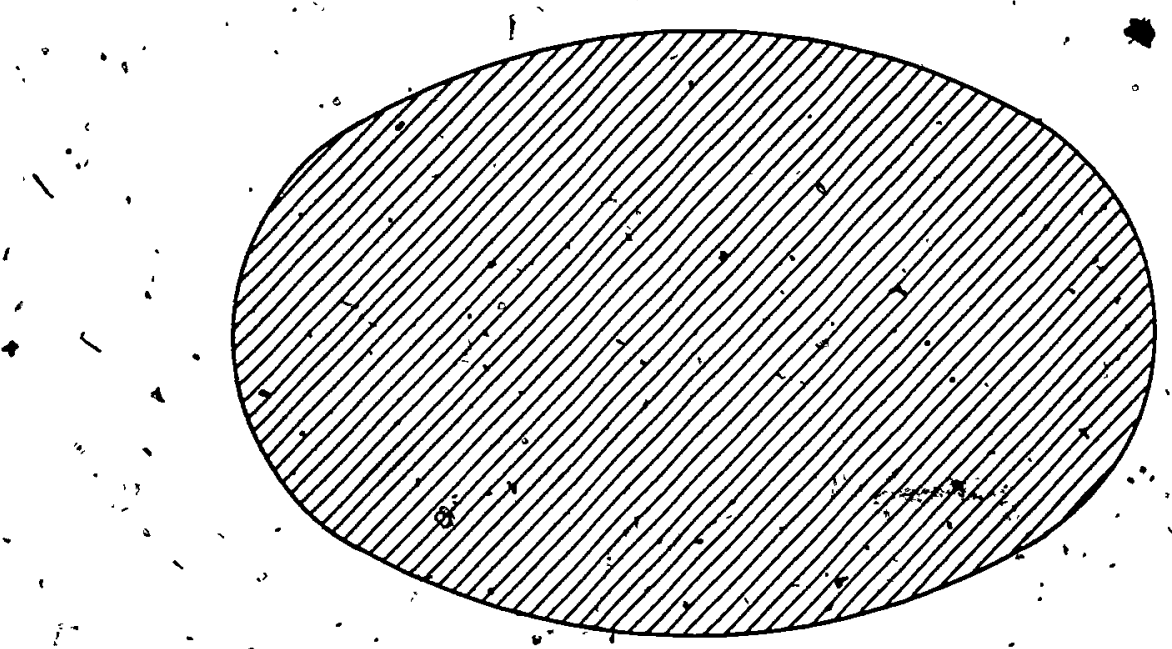

We do not have to use square pieces of paper. Instead we can draw this oval on a grid of units as show below.

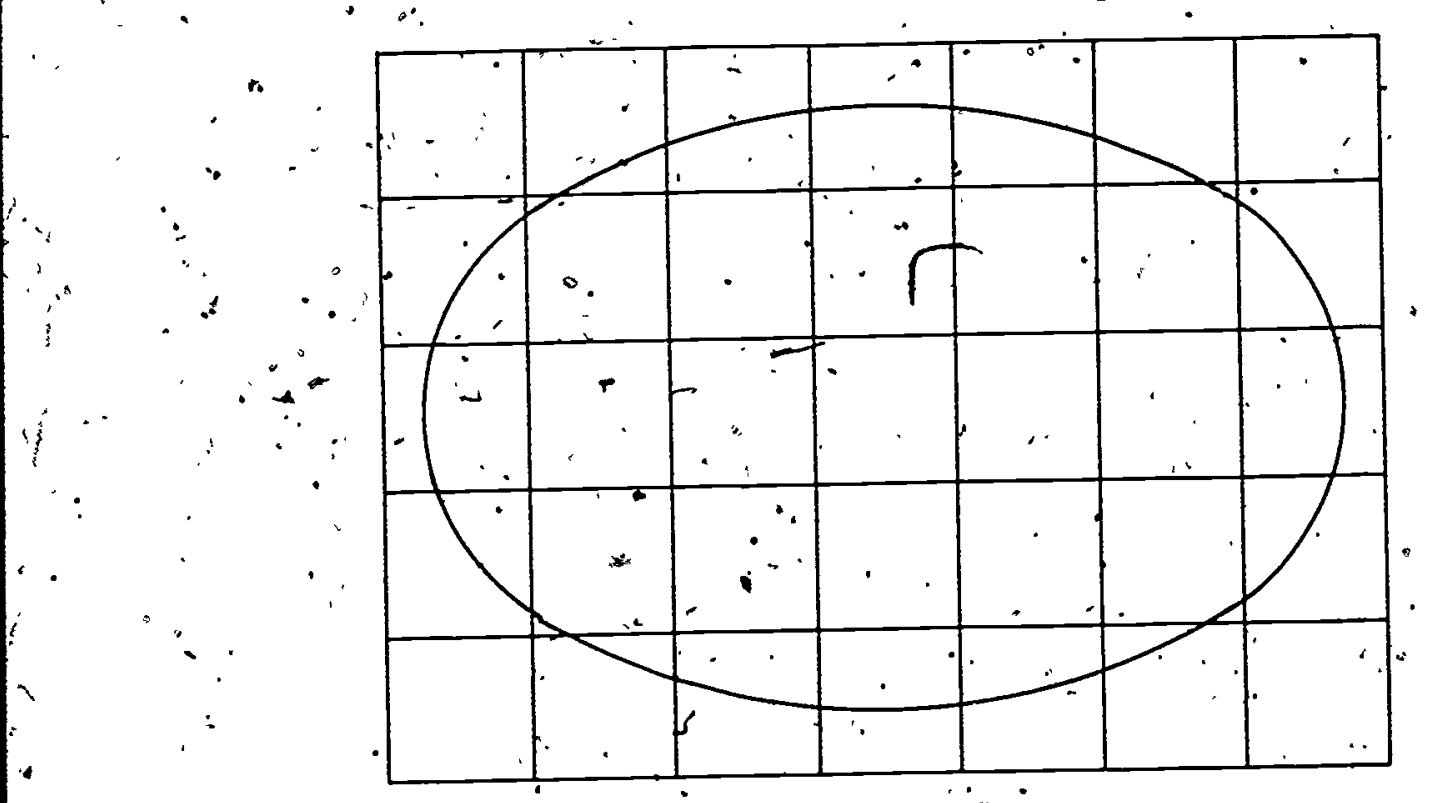


P.478,

Count the units that are contained entirely in the oval region. How many are there? (II) What does this tell about the area of the region? 1 Count the units needed to cover the oval region completely. How many are there? $\wedge$ what does this tell about the area of the region? 1 The area of the oval region is somewhere. between. (11) "units and (31) units. Looking at the figure, car you guess about what the area would be? (alout 18 mats; anarere inill mary.)

* We can get a better estimate of this area by using a smaller unit. Suppose we use a new unt of length just haif as long as the old one. The resulting old and new units of area lobk like this:

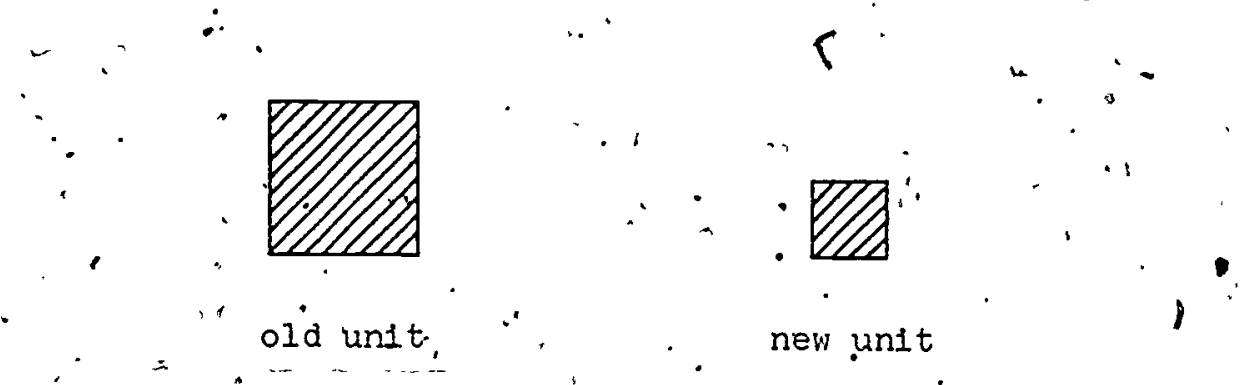

How many new units does each bld unit contain? $(4)$

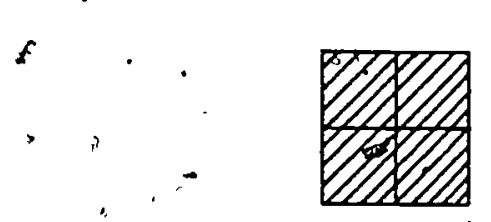

We have" already found that the area of the oval region is somewhere between. $11 \cdots$ old units and 31 old units. In terms of, the new unit, "what does this tell us about the area

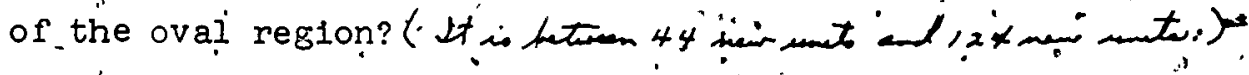

Hoin do you know? ( Ead eld init eontenis th and $\left._{G},+31=12.4.\right)$. 
- Now let us use, a grid of the new smaller unts to get $\dot{a}_{w}$. better estfmate of this area.

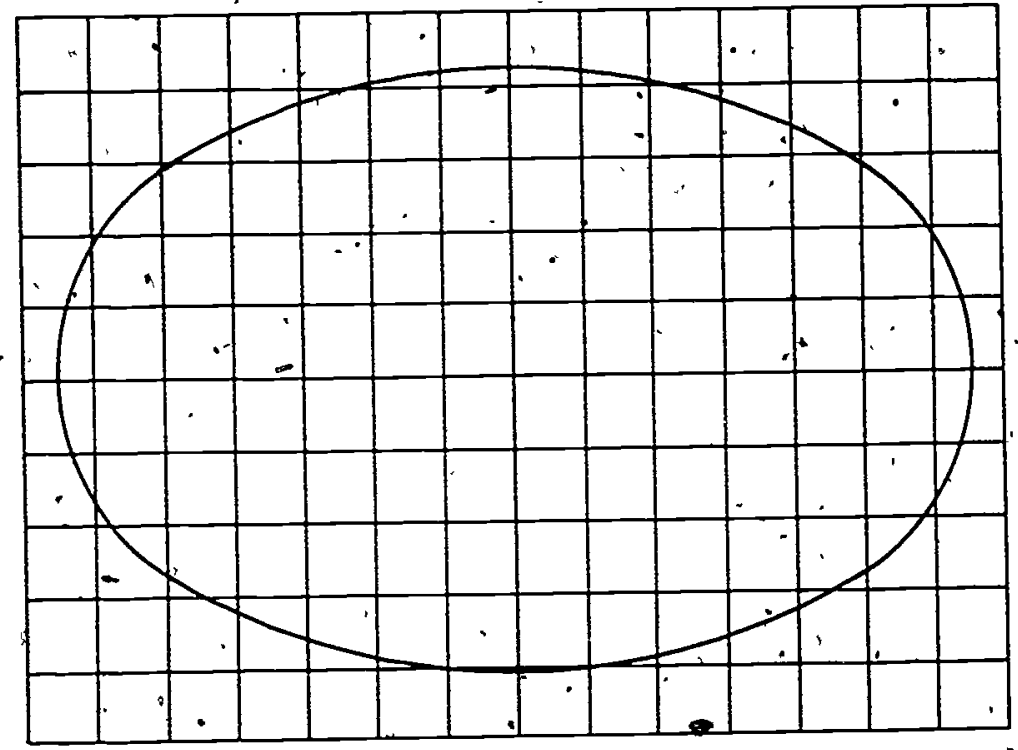

Count the units that are contained entirely in the oval region. How many are there?

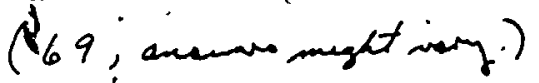

Counting by rows and pointing with the eraser end of his pencil as hej counts will help keep, the pupil from makthg errors in counting, and at the' seme time he 'will not be writing in his book. It would be an advantase if the pupil oould have his own dittoed copy, of. such figures. Then he could color all the units contained in. the oval region, etc. coloring would help emphasize, that area is a property of a region, and not of its boundary.

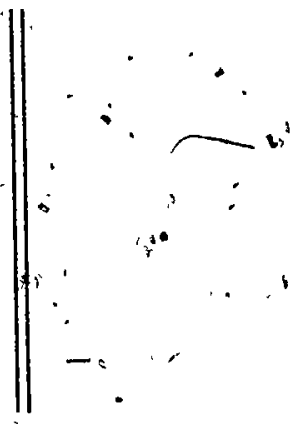

count the units that are needed to cover the oval region (108, insmane mesht wary) - completely: How many are there? What does this tell about the area of the region? (1tis ct rat 108 und.)

"Thus, we now know that the area of" the region is "somewhere

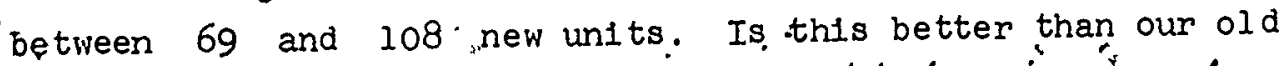

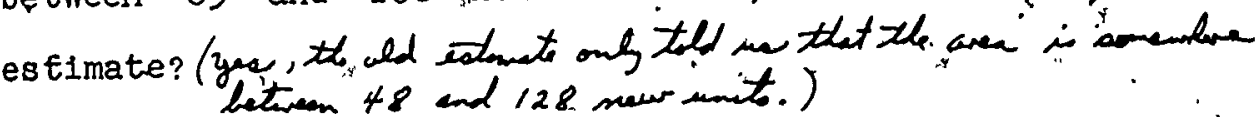


$\mathrm{P} 480$

\section{Exercise Set 1}

1. 'a.' Consider the region pictured below on a grid of units.

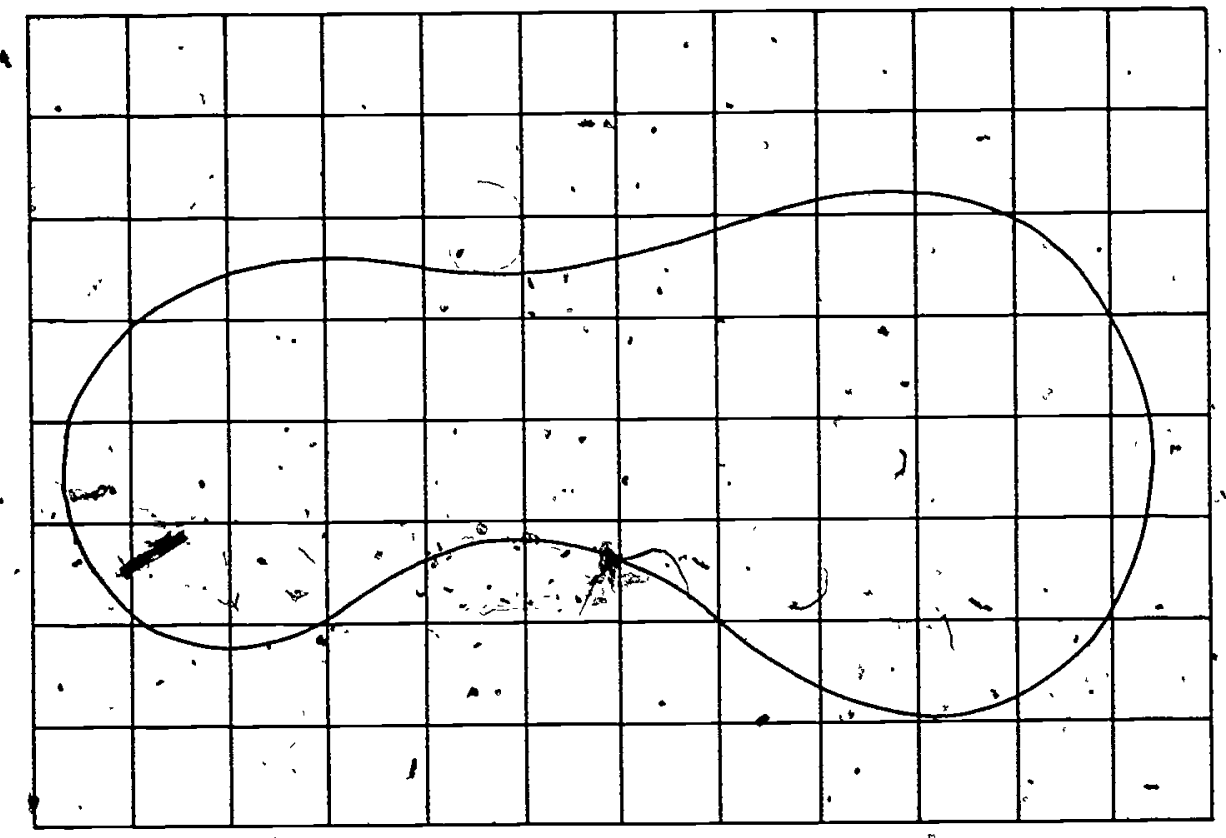

: Fill in the blanks:

There are (23) und contained entirely in the region. There are $(55)$ units neaded to.cover the region. completely.

The area of the region is at least (23) units and at most (55) units.

$f$

Let us choose a new unit of area a square region has as its side, a segment just half as long as before. For every old unit of area," we "wll then have 4 , new units of area. $\cdots$ 
P481

In terms of the new unit, we could say that the area of the region shown on the previous pagelis at least $(92)$ new units and at most $(\underline{220)}$ new units. (

b. Consider the same region pictured below on a grid of new units.

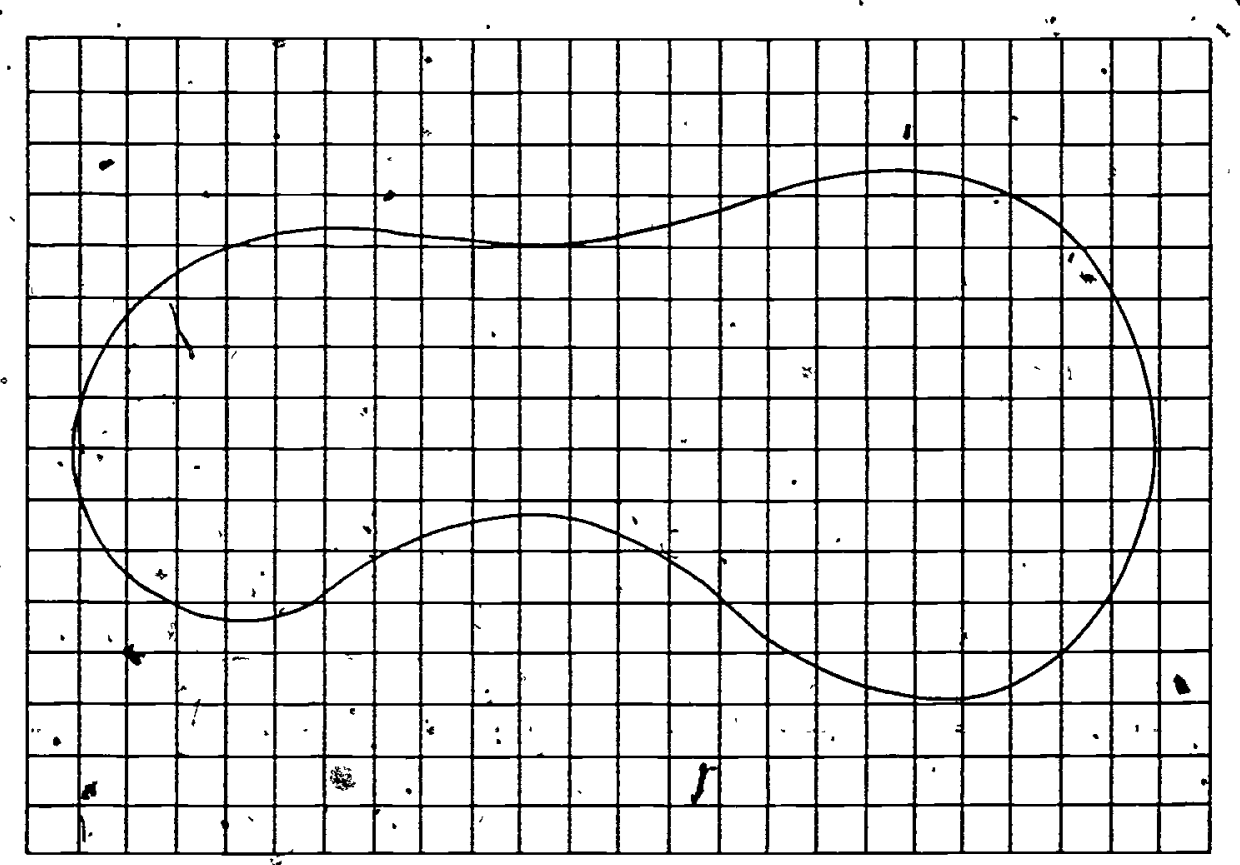

Fill in the blanks:

There are (i28, enconits contained entirely in the region. There are (191 manch units needed to cover the region completely.

The area of the region is at least $(128)$ units and at most (191) units.

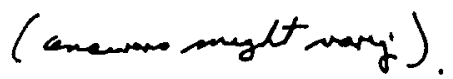

Is this estimate better than the estimate you made using the larger unit? (ijes, the eld istimate only told we that th area

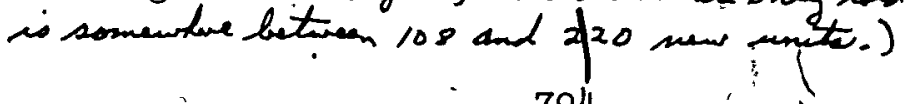


$\mathrm{P} 482$

2. a. On a sheet of paper "ruled with 1 inch squares, draw a representation 'or a simple closed' curve. 'Estimate the area of the region formed by the simple closed curve and its interior.

b. On a sheet of paper mauled with $\frac{1}{2}$ Inch squares, trace the simple closed curve you drew in part (a) of this exercise. Estimate the area of the region formed $\mathrm{by}_{\boldsymbol{k}}$ the simple closed curve and 1 ti s interior.

c. Which estimate, the one in part "(a) or the one in part $\left(b^{\prime}\right)$, is the more precise?. (Ilo estimate in pant $(b)$ is morepreciar.)

- 795

350 


\section{STANDARD UNITS OF AREA}

Objectives: To develop the following understandings and skills:

1. ' One standard unit of area -is a square region with 1-inch sides; this unit is called the square inch.

2. Other unt obtained simflarly are the square centimeter, the square foot, the square yard, and the square mile.

3. An area of 1 square yard is the same as an area of 9 square feet.

4. An area of 1 square foot is the same as $a n_{i}$ area of 144 square inches:

5. An area of 1 square inch is about the same as an area of 6 or 7 square centimeters.

Materials Needed:

Teacher: Yardstick, coliored chalk

Pupil: Sheets of paper (say $8^{\prime \prime} \times 10^{\prime \prime}$ ) marked with grid of 1 -inch squares

Before beginning the Exploration, ask pupils to summarize what they learned about standard units of linear measurement, biringing out the following points:

1: Standard units of measurement are needed for convenience and for ease of com-. munication.

2. A unit for measuring the length of a line segment is itself a line segment.

3. The meter is the basic standard unit of length in most countries and in all. scientific work.

4. Other standard units of length include the centimeter, the inch, the yard, and the mile.

5. Smaller units permit more accurate measurements. 
STANDARD UNITS OF AREA

The Basic Britiah-American Units

\section{Exploration}

To measure the area of a region, we first have to choosed a unit of area. The most convenient unit of area is a region square in shape. Can you think how hard it would be to talk about areas ": if each of us chose his own dfférent unit of area? People have found it is simpler if everyone agrees to use the same few units of area. We call these standard units. one standard unit is a square regton with sides 1 inch long like this.

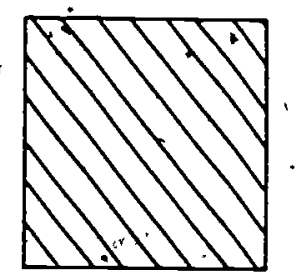

We call this unit of area the square inch," Would the square* 'Inch be a conventent unit for measuring the area of a sheet of. $r$ writing paper? (y.-)

At this point the teacher might pass out to each
pupil a sheet of paper marked with a grid of
square inches (such sheets are commercially
avallable) and ask the pupil to determine by
counting what area of the sheet is in.square
inches. Use sheets whose edges are themselves
lines of the grid so that the area.-1s cleariy
a whole number of grid units.

Would the square inch be a convenient unit for measuring

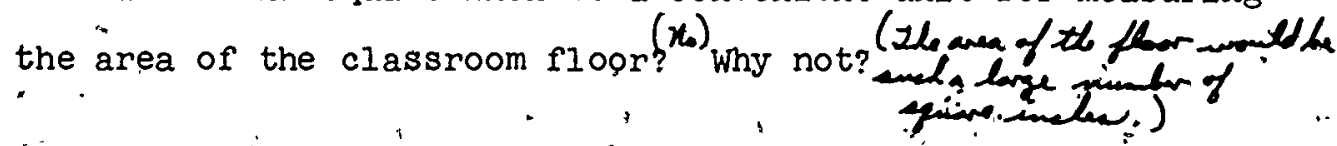

Can fou suggest a better'untt for measuring the area of the classroom floor? (yen, th givere fort of th agnore jand.)

Can you explain what a square foot is? (yea; tis

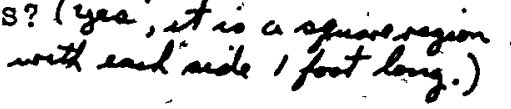



At the right is a small picture of a square. Your.. teacher will use a model whose side is actually one foot long. Let us pretend that the length of the side of square .EFGH is 1 foot. How many squares of side 1 in length could you place, touching but not overlapping, with one side on $\overline{E F}$ as shown in the figure? (12)

that is the area of region $\quad \therefore$ EFJK? ( 12 sgure incle.)

How many regions the size of region EFJK could you place in region. EFGH? (12)

Stince you can place 12 regions the size of EFJK in the region EFGH", and since the area of, region-EFJK is, 12 square inches, then, the measure of region EFGH (where the unit is the regtion whose area is one square inch) is . $12 \times 12$ or 144 
F486

\section{- Exercise set 8}

1.7. Make a table showing the number of units required to cover these regions:

$0 \div$

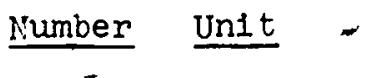

Squảre, side 1 inch long:

square, side 1 foot iong:

$\frac{(1)}{l^{*}} ;$ square inch.

(1) square foot,

or

square inches.. .

- Square, side 1 . yard 1ong: .

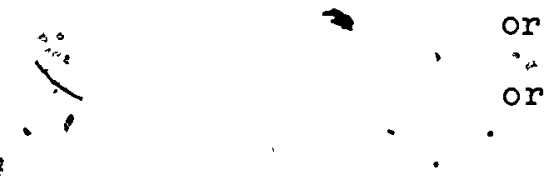

(9) squàre fét,

(1246) square inchesig

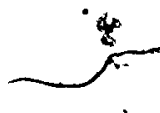

2. Here are 1isted areas of some regions. Write each area , in at least ơne other way, using different units.
a. 6 square reet
.1. 7 square feet.
(1008 y.in)

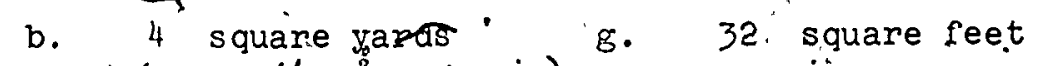

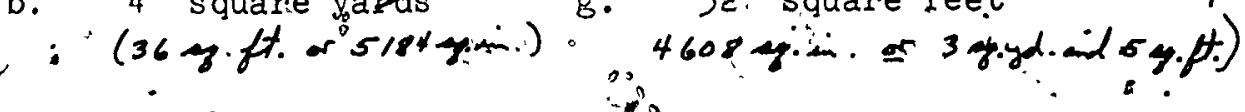

c. Lo square reet $\rightarrow$ h. 1296 square inches

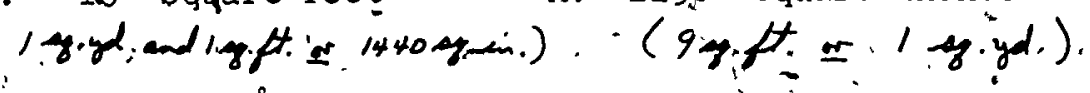

d. 288 square inches $1 . \quad 5$ square yards

$$
\begin{aligned}
& (2, y \cdot f t) \text {. } \\
& \text { (45 g. pt. } \approx 6480 \mathrm{~g} . \mathrm{m}^{\circ} \text { ). }
\end{aligned}
$$

e. 300 square inches,$j . \ldots 16$ square feet

(1 gy.yd.end 7y.ft. of 2304 zy. in.)

801

$-3.76$ 


\section{BRAINTWISTER}

Find different measures for the area listed below, changing the units of measure as no'ted:

a. I" square yards: (36) دsquare fêtt

b. 5 square feet:- (720) square inches

c. 2 square yards 18 square inches: (18), square reet (18) square inches

d. Z square" inches. A

$\therefore \quad$ e. 20 square feet: $(2)$ squareyards $(2)$
$\because \quad$ square reet
$\because \quad$ f. 324 square inches: $(2)$, square feet $(36)$ square inches

ig. 2000 square inches: (1) square yärds (704) square inches.

h. 36 square inches: $\frac{1}{4}$ square foot

1. 2 square feet: $\frac{2}{9}$ square yard

j. 18 square inchès: $\frac{1}{8}$ square foot 
P488

Area in Square centimeters

\section{Exploration}

All these units--, the square 1nch, the square foot, and the square mile--are units of area, in the British-American, System. of measures. Do you know what system of measures is used in (yes, th metricarth.)
most countries? What unit of length in the Metric system corresponds most closely to the yard in the British-American system? (What unit of length in' the Metric System corresponds most closely to the inch in cantemets) most closely to the inch in the British-American System? Do you.

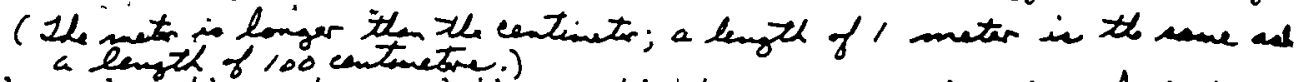
'know how the meter and the centimeter compare in size? 1 what unit of area in the Metric, System would you get by taking a

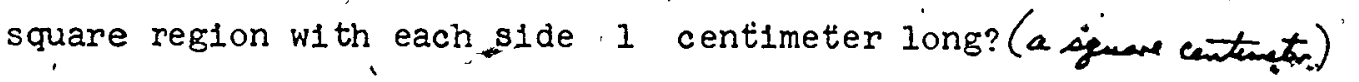

Even in Britain and America it is the Metric System that 1s used for scientific measurements. Therefore, we sometimes need to compare units. of area in the British-American System. with units 'of area in the Metric System. Here is a picture of the square inch and the square centimeter.

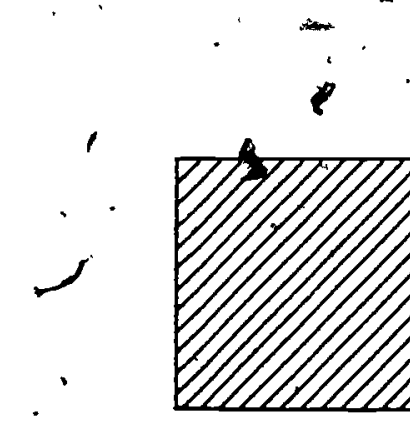

square-1nch

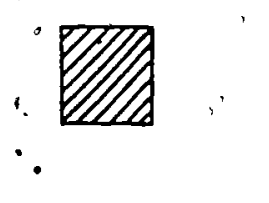

square centimeter

803 
3489

Which is larger, the square inch or the square centimeter? What would you estimate is tharea in square centimeters of the square inch region pictured? (dow woll wary) (B plaing the is a square inch shown on a grid of square. centimeter regions.

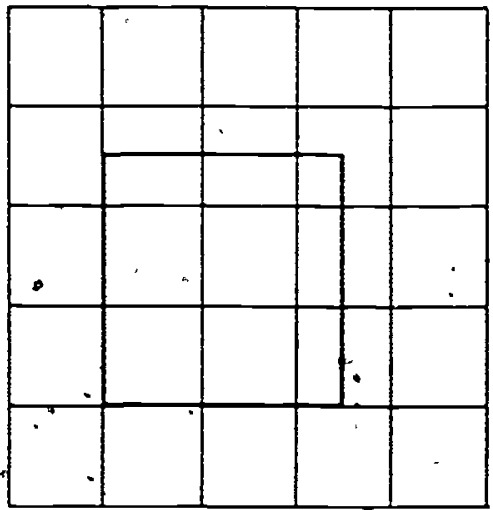

Foy many of the square regions of the grid are,contained entirely in the square inch region? (4)

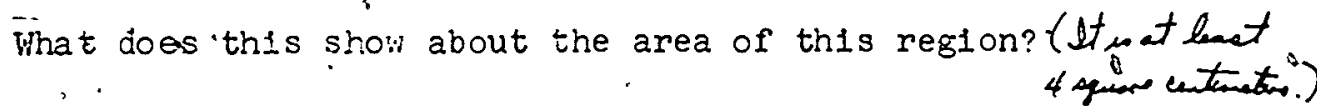
How many, of the square regions of the gri.i are needed to cover the square inch region completely? (9).

What does this show about the area of the region? (Ht is at mant 9 . yenentinat.) Gen you now guess this area more accurately? (It mabat o or 7 grave centinetre) 
P490

\section{Exercise Set $\underline{9}$}

I. Suppose, we have a rectangular region with adjacent sides of 'iength' 2 inches and 3 inches...

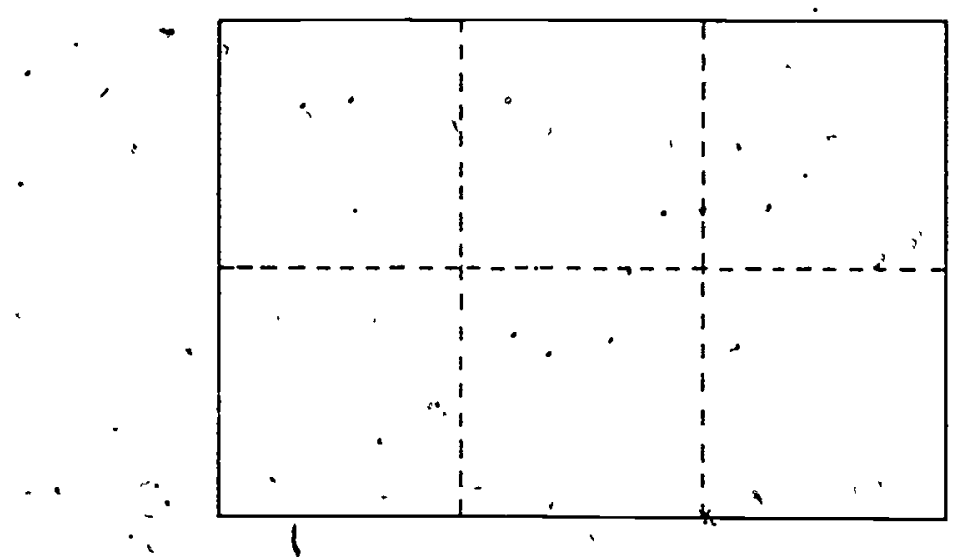

What is the area of the region in square inches? (6 a sich.)

Below'is a picture of this same rectangular region on a grid of square centimeter regions: Use this picture to estimate the area of, the rectangular region in square centimeters. If : you need questions to guide you, look on the next page.

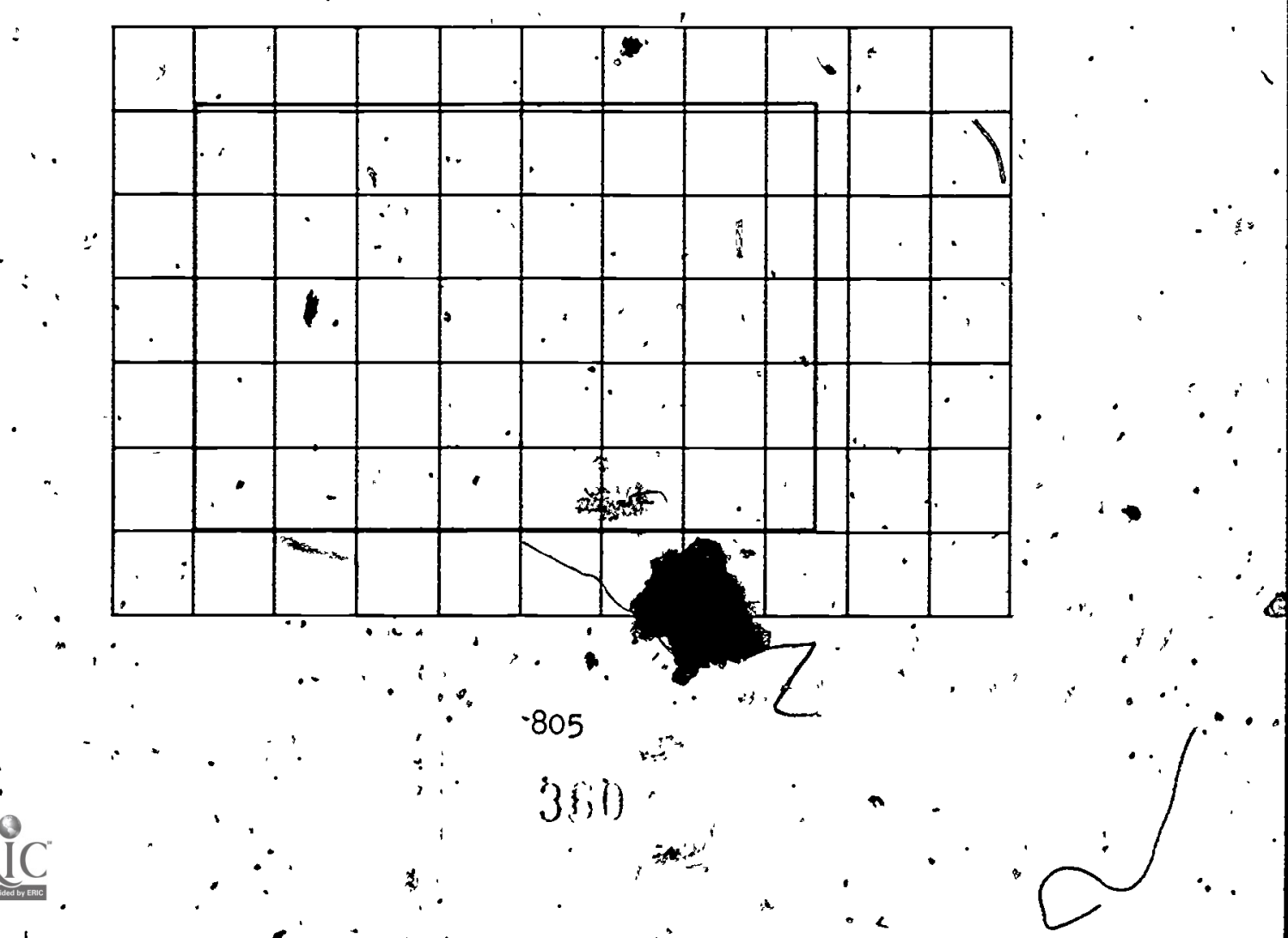


P491

The following questions should help you to find ain estimate:

a. How many square regions of the grid' are contained $(35)$ entirely in the rectangular region? What does this' tell about the area of the region? (It is at lent' is square centenetivi.)

b. How many square regions of the grid are needed. "to cover the rectangular region completely? (48) What does this tell about the area of the region?. (lt is at mat 48 genome centurato.).

c. Can you look at the rectangular region and estimate 'about what the area would be?

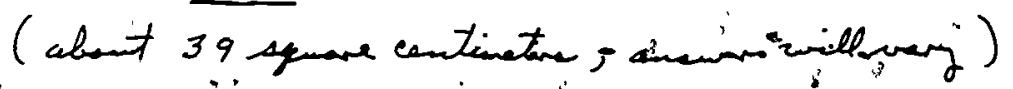

- 4. FIll in the blank: If the area of a rectangular region is 6 square inches, its area is about (39) square centimeters. (amman.willary)

$\therefore \quad . \quad 36 \hat{1}$ 
2. Below pictured a right triangular region on a grid of square chtimeter regions:- The sides adjacent to the right angle have lengths 2 inches and 3 inches. $\because$ Find an estimate for the area of the triangular region in square centimeters.

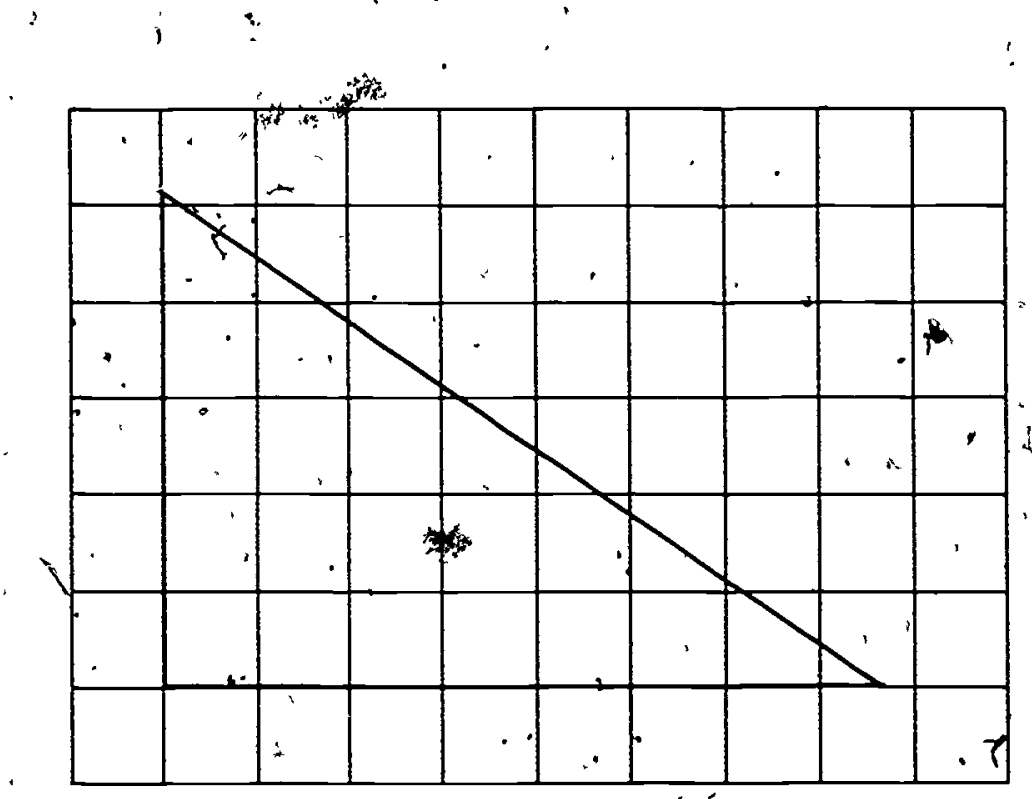

.

a. The; area of the triangular region is at ieast (14) square centimeters, and at most. (27) square ceńtimeters.

$\therefore$ b. What would you estimate the area would be? (abut 20 pare centinetion; answers will ivary.) 
3. Below is plcturéd a clrcular region of radius.?2 inches on a grid of square centimeter regions. Find an estimate for the area of the circular region in square centimeters.
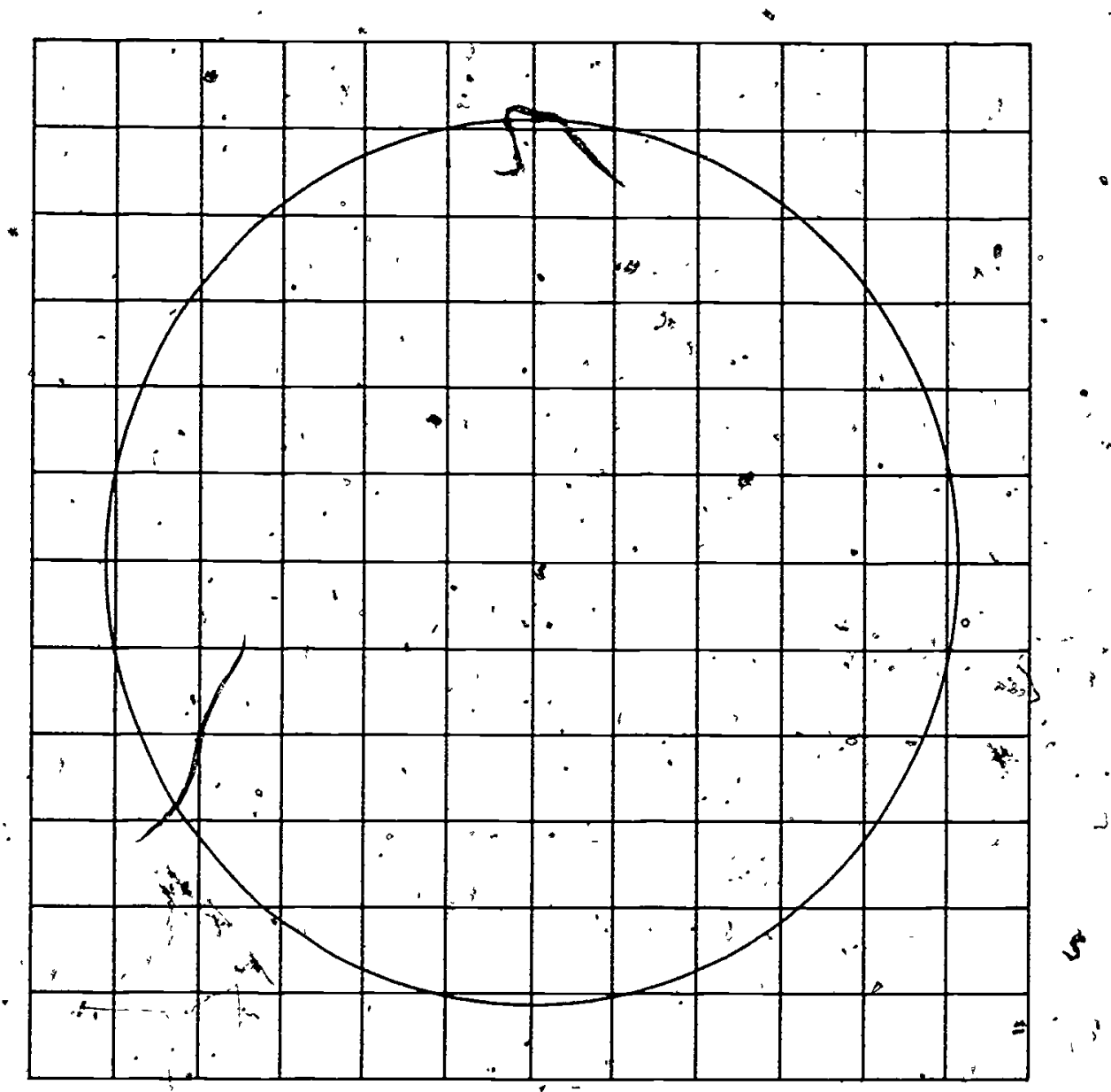

a. The area of the crroular region is at least

(68) square centimetẹrs and at most (1/2) squàre centimeters.

b. What, wọidd you estimate the area would be? (ábat 82

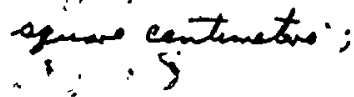

808 


\section{AREA OF RECTANCULAR REGIONS BY CALCULATION}

Objective:. To develop the following understandings frid skills

1. For each unit of lergth there is an associated unit which is the square region each of whose sides has length 1 unit.

2. Several differentiy shaped rectangular regions of the same area can sometimes be formed using a given number of unit square regions.

3.- Suppose that in terms of the same unit of. length, the measures of the sides of a rectangulan region are given whole numbers. Then the product of these numbers is the measyre of the region in terms of the. associated unit of area. (See note below)

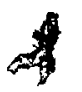

In simpler terms, 1 tem 3 above just . says "area is length times width." This - simpler formulation 1s, however, both, Inexact and incomplete. It is inexact because we don't really multiply, lengths and widths; we only multiply numbers. It is incomplete because it does not specify. that botbolength and width must be measured in the same linear unit and that area must be measured in the associated unit of farea.

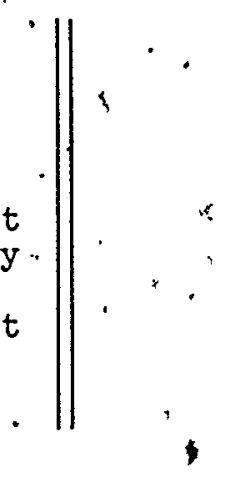

Materiáls Needed;:

Teacher: None

Pupil:. Twenty 1-inch squares of construction paper'for each pupli', sheets of paper "muled with" l-inch squares. 
P494-

$\therefore$ AREA OF RECTANGULAR REEGIONS BY CALCULATION

Building a Rectangular Regtion

管

\section{Exploration}

You remember that a rectangle has four sides. If we know the measure of' any two sides that form (hight angle, then we know the measure of all four sides. Why? 1 . When we speak of "the adjacent sides, of a rectangle," we will mean two sides which form part of 'right angle.

Earlier in this Untt you found the area of a plane regian by covering the region with models of a unt regipn. What is the shape of "a staridard untt region? (equare)

Your t'eaeher whll give you some 'models of unit regions," each wh an area 'i square 1nch. Count out twelve of these unit regions. F1t these i2 regions together, without. overlapping, so that their bouna ary is a rectangle. How long' are the sides of the rectangle?/ See how many differert rectangutar reglons you can form from the 12 square regions and IIst the information in a chart like the one below:

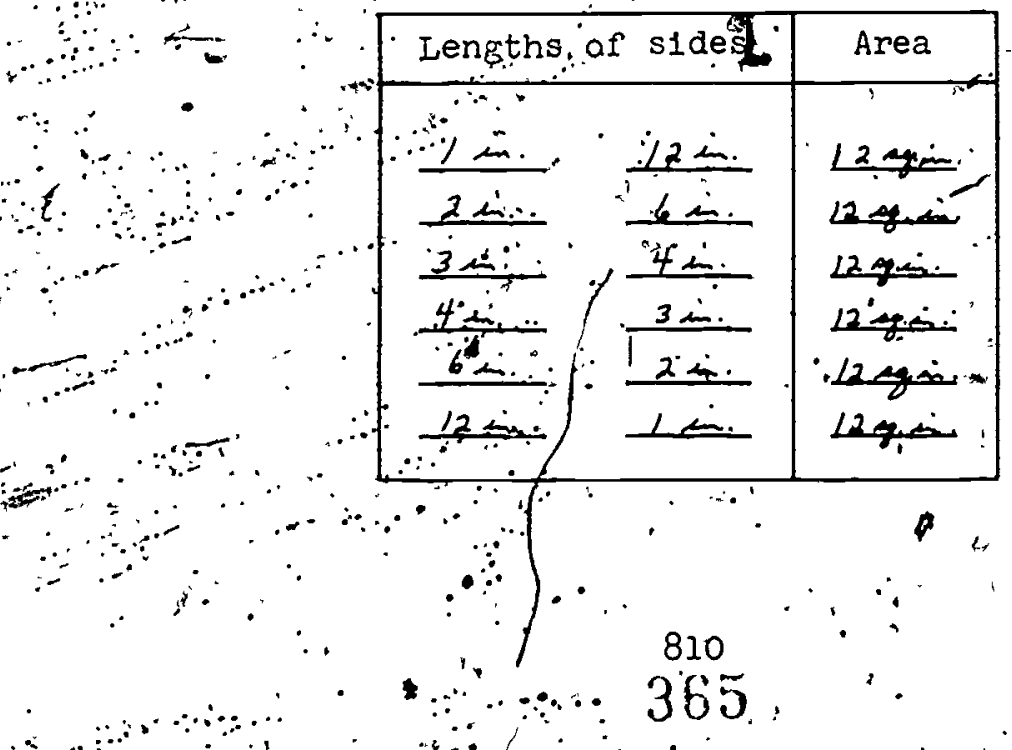


P.495

Now use sheets of paper ruled with' "l-inch squares. Draw : two rectangles of such size that the area of each of the rectangular regions is 20 square inches. (Keep each square inch unit ail in one piece.) List the information in a chart as before.

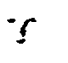

\begin{tabular}{|c|c|c|}
\hline Length & of 'śldes & Area \\
\hline $1 \dot{m}$ & $20 \mathrm{in}$. & 20 axi \\
\hline $2 \dot{\pi}$ & $10 \mathrm{~m}$. & نش 20 \\
\hline $4-\infty$ & $5 \dot{\text { in }}$ & 20 a 20 \\
\hline$\sigma_{\min }$ & 42 & 20 og \\
\hline $10 \mathrm{~m}$ & $2 \therefore$ & نذ \\
\hline $20 \dot{~}$ & 1 in. & $20 \mathrm{gen}$ \\
\hline
\end{tabular}

What, do you notice about the number's which are the measures

of the sides of a rectangle and the measure of 1ts region?

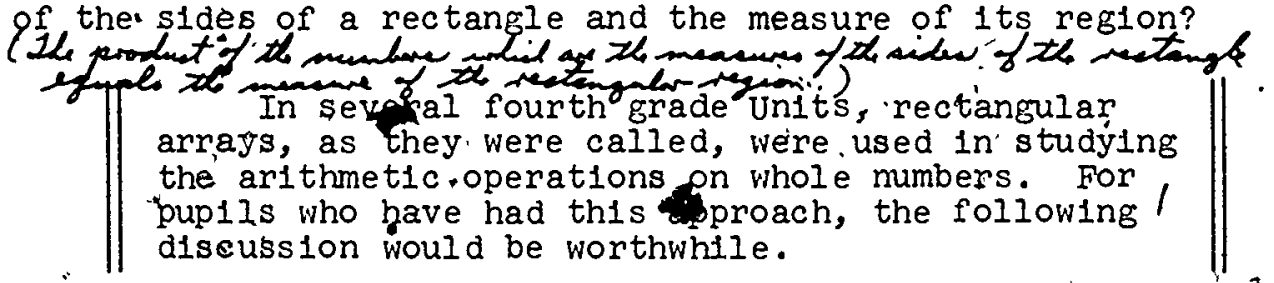

Whe re have we already used rectangular arrangements of square regions, quite a while ago? 1 what were these rectangular

arrangements of square regions called? $A$ what were the square regions " (th elenent of th array)

In an arráy 'call ed?. $\mathrm{H}^{\mathrm{w}} \mathrm{d} 1 \mathrm{~d}$ you. learn to calculate the number of elements in an array? 1 . What are we now calling the number of

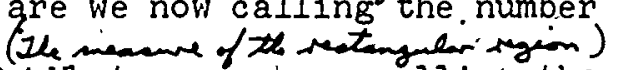
elements in, the whole array? 1 what are, wey calling the numbers of elements in a row and in a column of the array? If the number of elements in an array is the product of the number of elements In one row and the number of elements in one column, what does this tell us about the measure of a rectangular region? (It 2 the

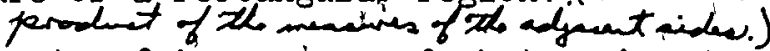

Is the following statement a falr summary of what we have: been saying? Two, adjacent sides of the rectangle have measures whose product is the measure of the rectangular region. (yar) 
P496

Exerc1se Set 10

1. Suppose a rectangular region has a measure in square inches of 10. What pair of numbers could be the measures in inches of 1ts sides? $(5,2)$ Can you think of another pair? $(1,10)$

2. Draw on 1-inch squared paper two rectangular regions having a measure of 10 in square inches. On each side write its measure in inches. On the interior of each rectangular region write, its measure in square inches.
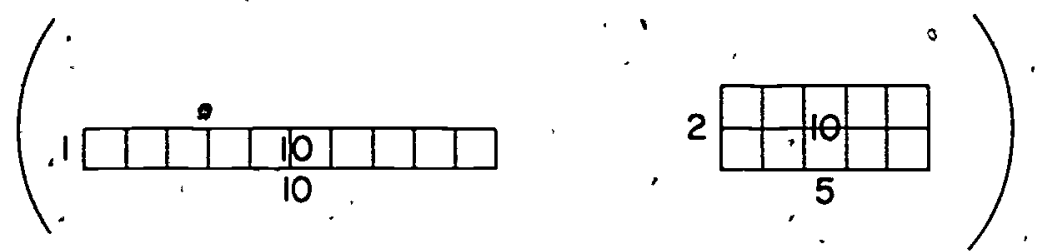

For each of the next three exercisès draw rectangles on: squared paper and urite the measures of the rectangular regions and their.sides as in Exercise 2.

3. Draw three rectangles such that the measure of each rectangular refion is 16 .
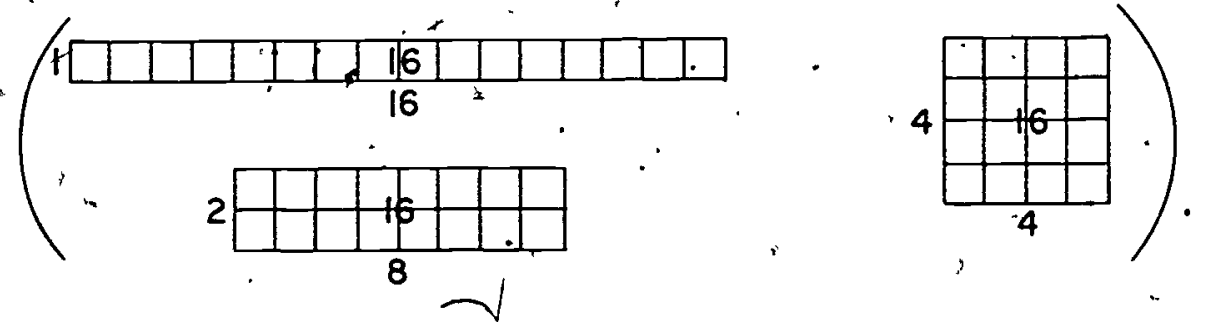

4.' Draw three rectangles such that the measure of each." rectangular region is 18 .
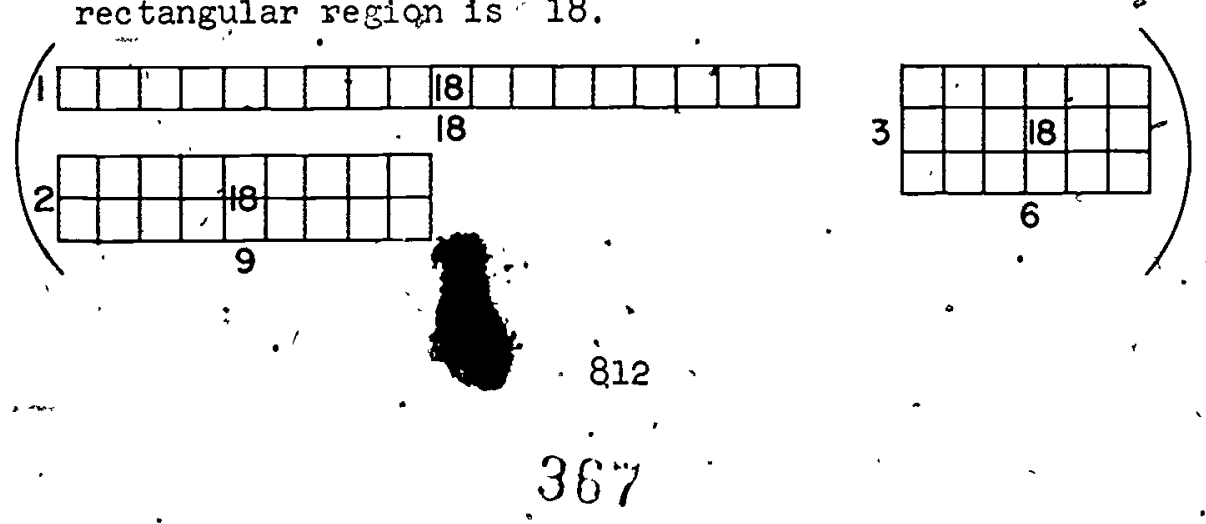
5. Draw four rectangles such that the meanure of each
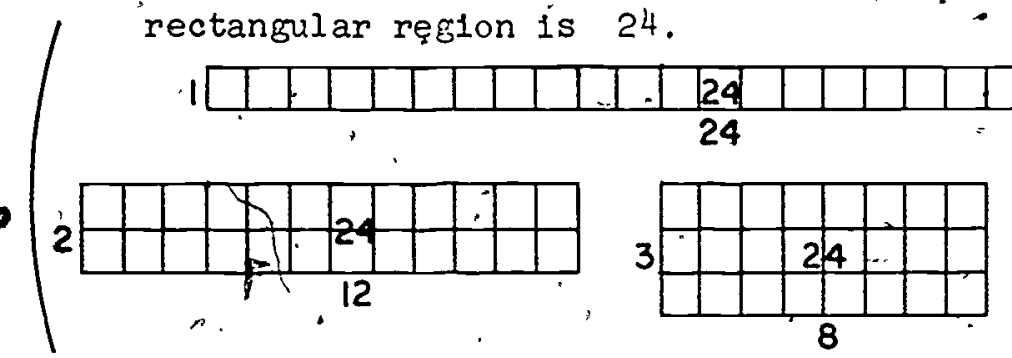

6. Make and filf in a table like the one below. Get the

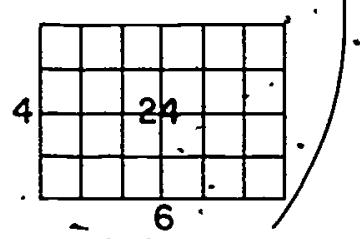

information you.need from your drawings in Exercise 3.

\begin{tabular}{|l|c|}
\hline $\begin{array}{l}\text { Measures in-Inches of } \\
\text { s1des of rectangle }\end{array}$ & $\begin{array}{l}\text { Measure in square inches } \\
\text { of rectangular region }\end{array}$ \\
\hline$\frac{1}{2} \cdot \frac{16}{8}$ & $\frac{16}{16}$ \\
\hline$\frac{4}{4} \cdot \frac{1}{4}$ & $\frac{16}{4}$ \\
\hline
\end{tabular}

What do you notice about the product of the measures of the sides in each case? (It is the meare f the rajion and is the

7. Make and fill in a table similar to that in Exercise 6. Get the iffiormation you need from your drawings in Exiercise 4.

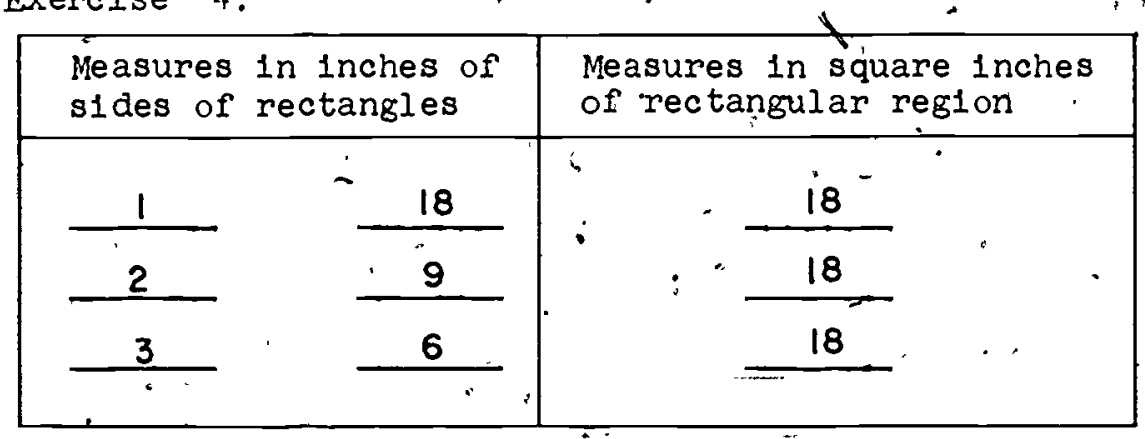

What do you notice about the product of the measure of the

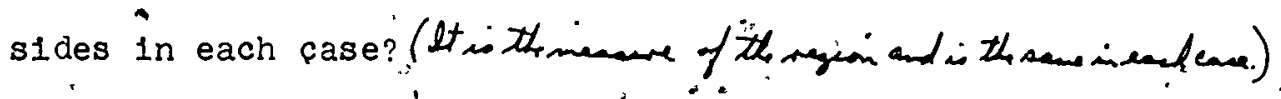


8. Make 7 and fill in a table similar 'to that in Exerdise 6.r. Get the information you need from your drawings in

- Exercise 5 .

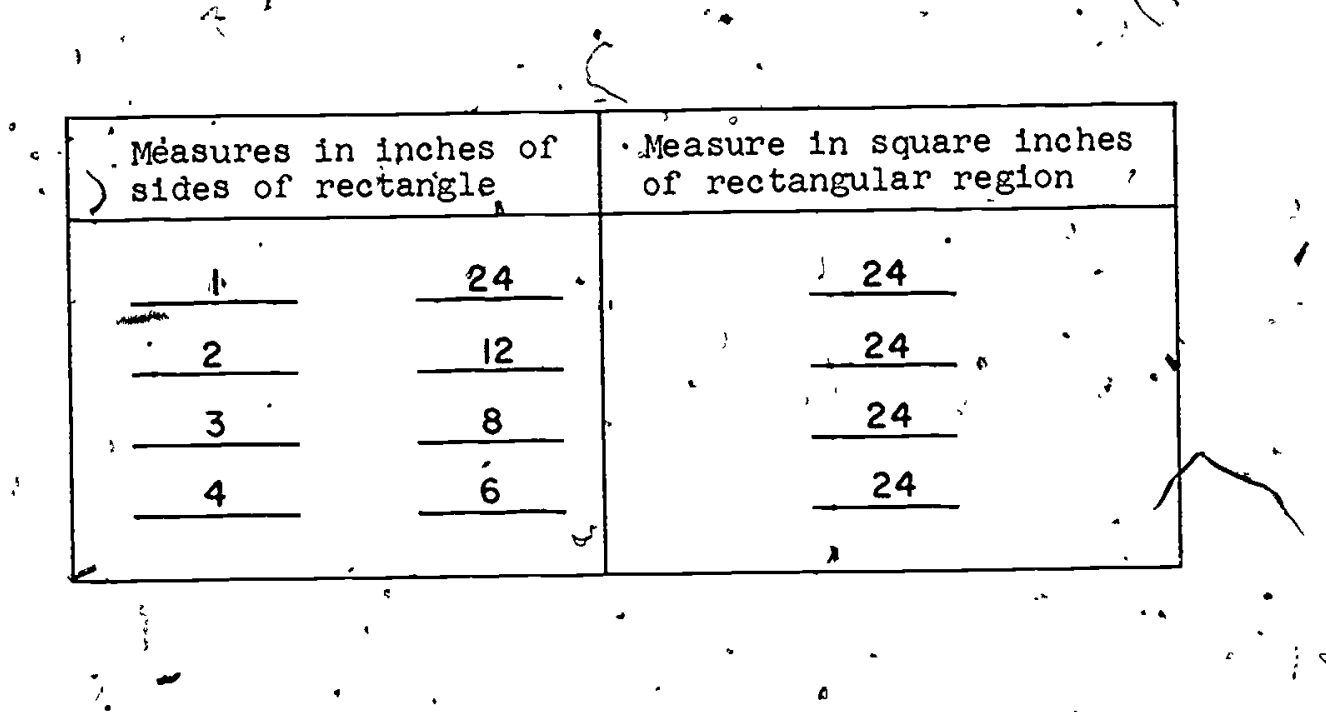

What do you notice about the product of the measure of the sides in each case? (If is th meanue of th regeon

$$
1 \text { and is the seare in ead case.) }
$$

9. Suppose you are given the measures in inches of the sides of a rectangular, region. "In terms of these gtoin-riearures, what is the measure 1n. square inches of the rectangular region? ( lt is the jerolust if ther
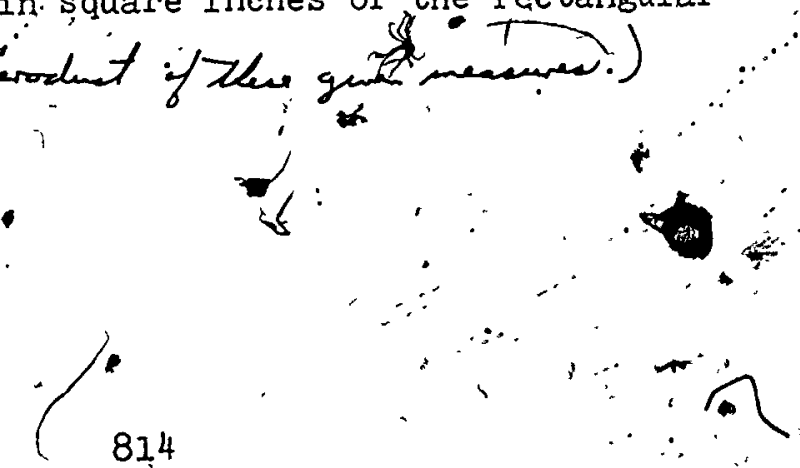

369 


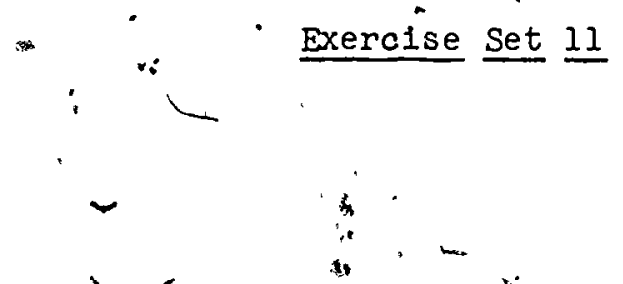

1. Susan made a rectangular. doll blanket whose sides were 12 inches and 10 inches long. Find the area of the blanket.

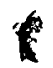

2. Peter made a plýwood shelf for his model collection. The

- shelfawas 30 1nchés long and 8 inches wide. What was. tits aréa? $(j+0$ s. in.) tas

3. Suppose the edges of a brick have the lengths as shown in

- this picture.

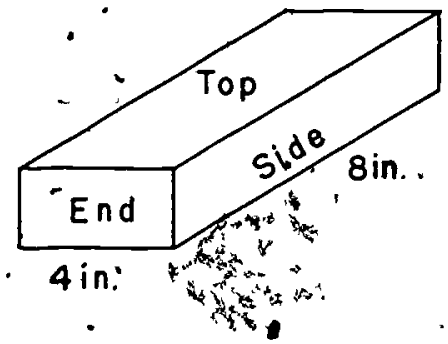

2 in.

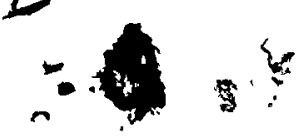

\section{,}

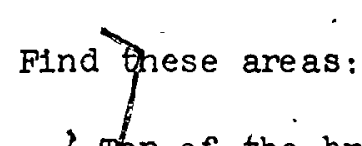

Thp of the brick. ( 32 agim)

Side of the brick. (16 igri.)

End of the brick. ( 8 gy.in.)

Total surface of the brtck. (1/2. An. $)$ 
4. Here is a picture of a kitchen floor, with the lengths of the . edges shown. Find the area ofs the floor: "Hint: Can you. draw a segment which divides the region into twe rectanguiar.

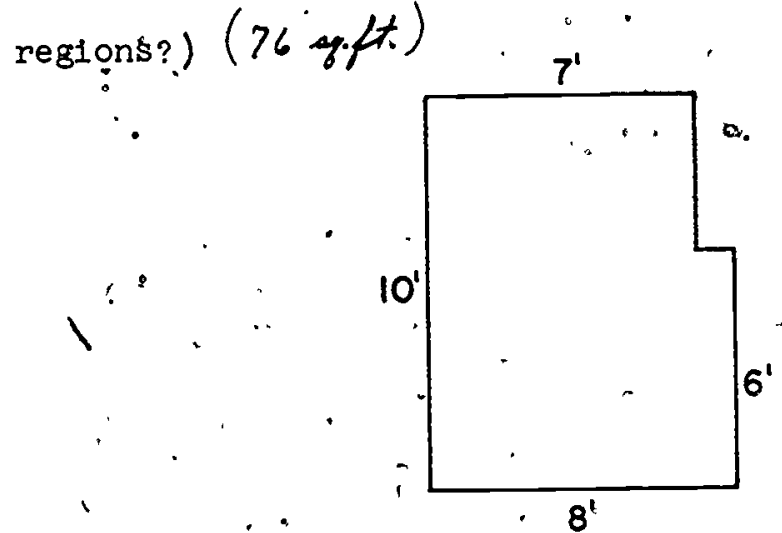

5: Here is a picture of a floor of a house, with lengths of the edges shown.

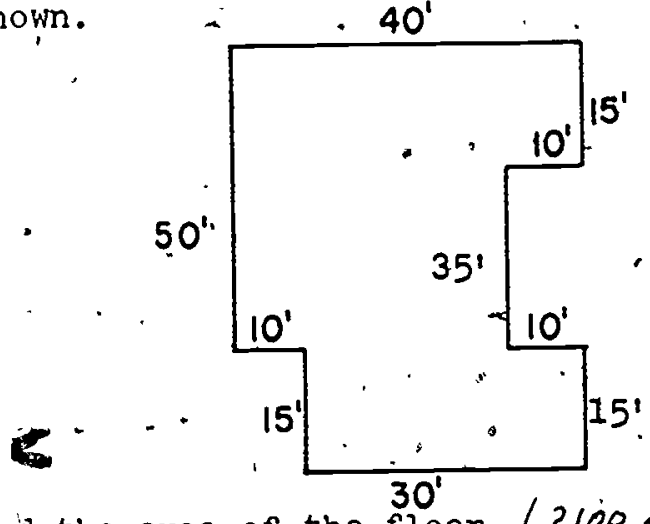

a. Find the area of the flopr. (2100 of.ft.)

b. Can you find the area another way? (yos) How? (2 Way will wary)

6. Suppose a high pressure salesman tries to sell yoù a rectanguiar lot for your home. After many questions, he reluctantily admits that he has two rectangular lots. One is $3^{\circ}$ feet wide' and 2000 . feet long. 'The other is ' 60 feet wide and lop feet long.

a.' What is the arta of each 'lot? (6,000 g.f.f.) b. .. Which lot would you prefer? $A$ why?
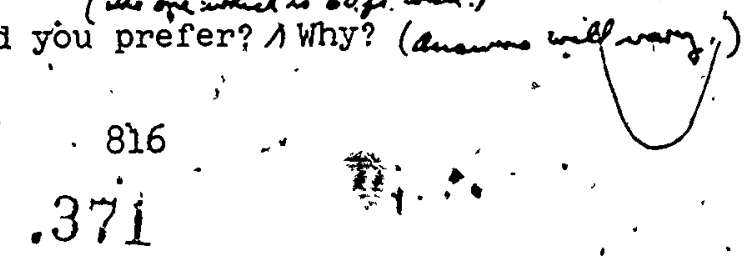
AREA OF. A TRIANGULAR REGION

objectiye: To. develop the following understandings and skills:

1: From every right triangle a rectangle may be formed properly locating a fourth vertex. The region bounded by the triangle has an area which is one-half that.of the region bounded by the rectangle.

2. The measure of a region bounded by a right triangle 1s, found iy calculating the product of the measures of the sides of the triangle" which determine the right angle, and dividing the product by two.

3. An altitude of a triangle is a segment drayn' from a vertex to the opposite side so as to form right. angles with this side.

4. Whe "opposite side" to which the artitude is dram is called the pase of the trianile associated with that aititude.: -

5. We calculate the measure of a triangular regton by taking one-half the product of the measures of an altitude of the triangle and.1ts associated base. .

Vocabiuary: Alt1tude, base.

\section{a}

This section challenges the pupil's ability to do some deductive thinking. He needs the skills/ and understandings of - many of the sections in this unit to be able to conclude with a mule for calculating the measure of triangular region. 
P501

AREA OF A TRIANGULAR REGION

"Area of a Region Bounded by a Rlght Triangle"

Exploration

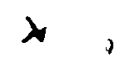

Let us think about how we would find the area of a region. . : agunded by a right triangle.

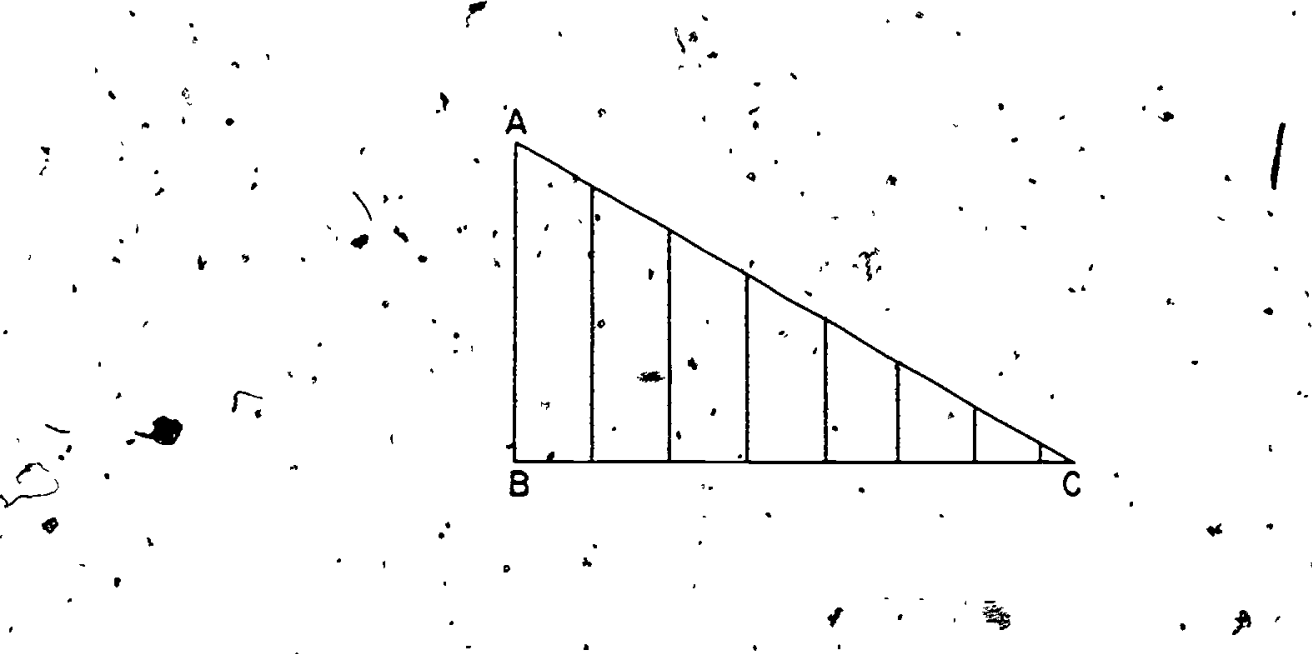

Using a compass draw a 'rectangle by making $\overline{\mathrm{AD}} \cong \overline{\mathrm{BC}}$ and

- $\overrightarrow{\mathrm{CD}_{3}}: \cong \overline{\mathrm{AB}}$.
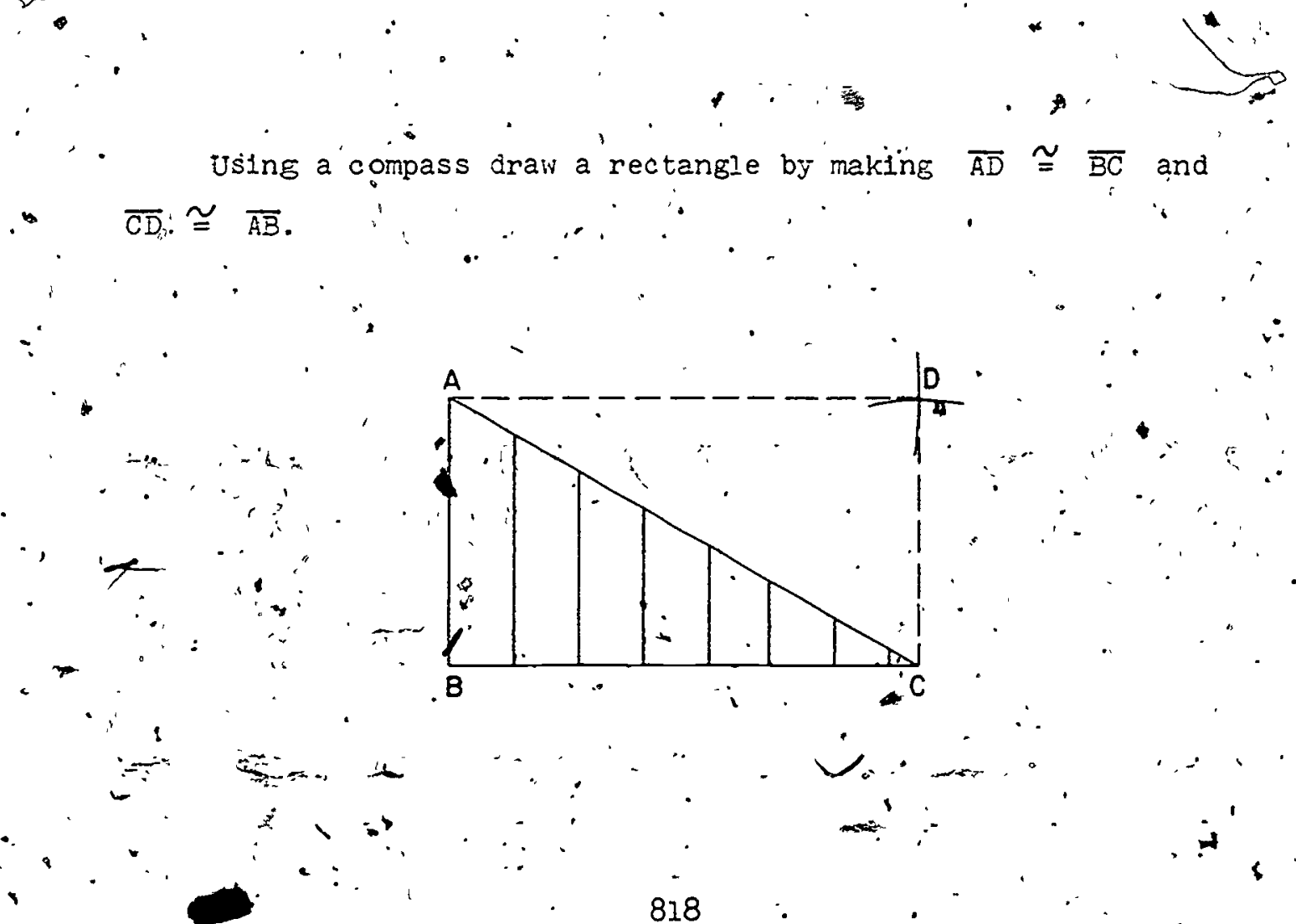

818

373 
The resulting rectangular region is divided into 2 triangular regions in the following way:

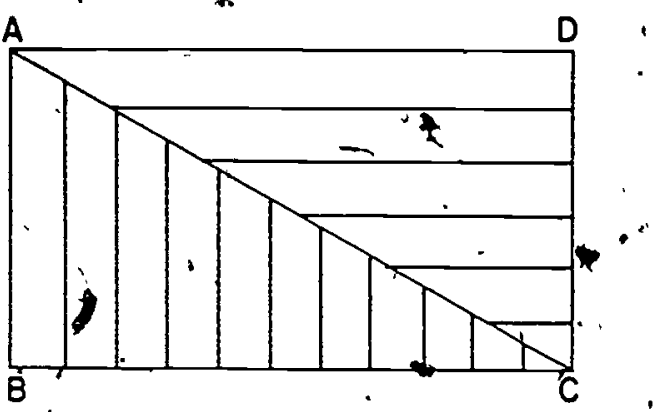

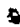

How do the lengtins of the opposite sides of the rectanglea. compare? $A$ Do you have enough information to be sure that $\triangle B B C \cong \triangle C D A ?^{-}$( We thens thet $\triangle A B C \cong \triangle C O A$ semier $\overline{A B} \cong \overline{C O}, \overline{B C} \cong \overline{D A}, \overline{A C} \cong \overline{C A}$ and we, then hive there sidies of one thingli the saine length

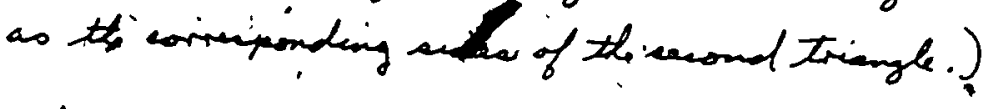

If two line segments are congrient, then they have the same length. Similarly, if two triangles are congruent to each other, then the regions assisoclated with them have the' same' area. Therefore, the area of 'triangular. région $A B C$, is 'the same as the area of triangular region CDA. The measure of region $A B C$ is what fractional part of the measure of region aBCp? (one-haf) What fractional part of the measure of region $A B C D$ is the measure of region cDA? (one-hy) 


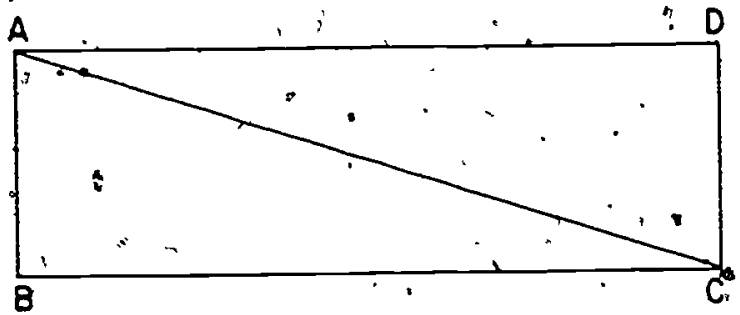

Suppose $\overline{B C}$. hàs length 10 . Inches. What is the measure - of $\cdot \overline{\mathrm{BC}}$ in inches? $(10)$ Supposel $\overline{\mathrm{AB}}$ has length' 3 inches. "What." is the measure of $\overline{A B}$ in inches? (3) What is the measure, in square inches, of rectangular region $A B C D$ ? What ( 15 s the measure,

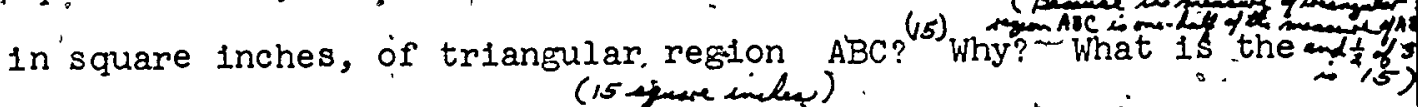
area of triangular region $A B C$ ? $A$ what is the measure, in square inches, of triangular region $A D C$ ? ${ }^{(15)}$ What is the area of triangular region "ẢDC?
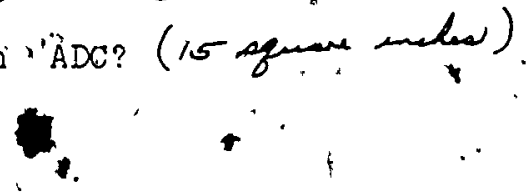

$\theta$

\section{Summán'y}

From èvery right triangle a rectangle may be follnd by properly locating a fourth vertex. The region bounded by the triangle has an area whlch is orie-half that of the region. bounded by the rectangle.

The measure, in square units, of a region bounded by a "right tridnge is found by calculating the ploduct of the mesures, in. units, of the sides of the trlangle which determine the right angle, and dividing the product by two. 
$\$$

P50年 $\because j$

Arez- of al Region Bounded by a General Tryangle

\section{Exploration}

$\therefore$ Not every triangular región is bounded by a right trianglè. We made the area of a region bounded by a right angle depend Apon our knowledge of the area of a rectangular region. Naw - we will make our study of the area of any triangular region depend upof what ve have íearned, about he area of a pegion bounded by a right trianglè. "Again we use the concept that area is unchanged when a region is digsected. '.

If we.start with a general triangIe such as $\triangle \mathrm{APQ}$ Q

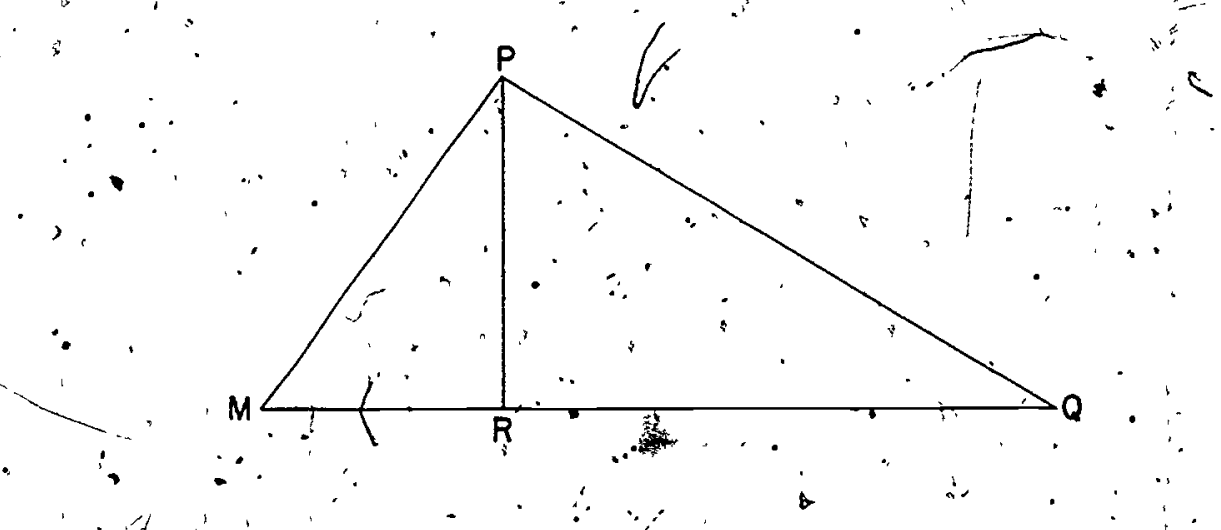

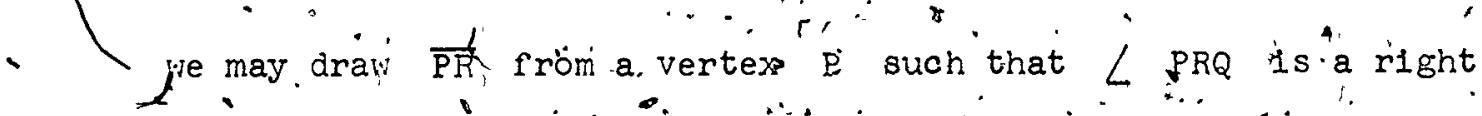
angle and $\angle P R M$. is aright angle. $\overline{P R}$ is referred to as the height or the ailt tude of. $\triangle$ MPQ. The side:or the triangle." opposite trivertex ' $P$ is calle'd the base of the thangle. If:I the altitude I' 'drawn from vertex $\grave{M}$, then $\overline{\mathrm{PQ}}$ 'is, called the base of $\triangle \mathrm{MPQ}$. If: the altitude is drawn from $Q$; then is the base. "Every triangle has three altitudes and thrẹe" corresponding bases. 


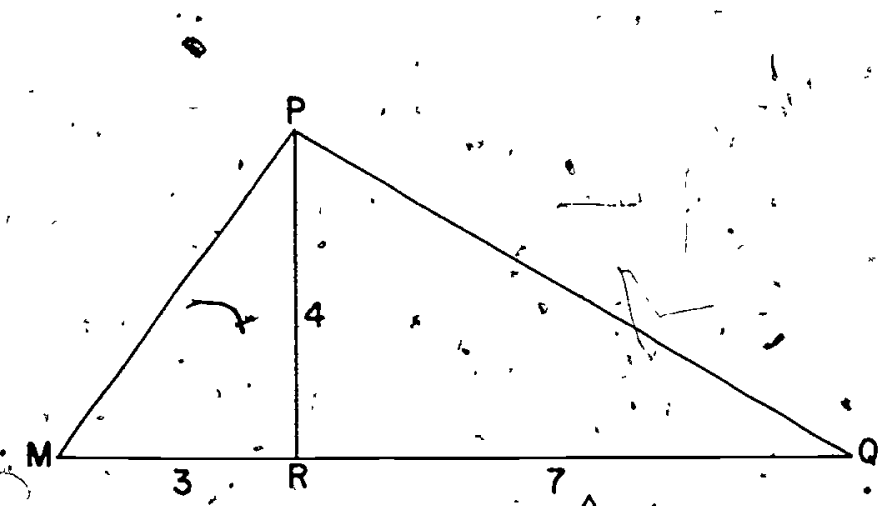

Is $\triangle$ I.PR a right triangle? is $\triangle R Q P$. a right triangle?

Suppose the length of $\bar{R}$ is 3 incheg,' the length of '/ $\overline{R Q}$ is 7 inches, and the length of $\overline{P R}$ is 4 'inches as shown above. What is the area of the region bounged by, right triangle (MPR? 1 .) What is the area of the region bounded by right triangle (14agni) What is the area of the region, bounded by. $\triangle M \ddot{M Q}$ ? $(20$ so

Orserve:

Measure of region MP $\tilde{R}$ is $\frac{1}{2} \times\left(4 \times \frac{3}{2}\right)$

- Measure or region QPR is $\frac{1}{2} \times\left(4 x_{0} .7\right)$.

Measure of region ${ }^{*} \mathrm{PQ}$ is $\left(\frac{1}{2} \times(4 \times 3)\right)+\left(\frac{1}{2} \times(4 \cdot \times 7)\right)$

Using the associative, and distribjitive property:

$$
\begin{aligned}
\left(\frac{1}{2} \times(4 \times 3)\right)+\left(\frac{1}{2} \times(4 \times 7)\right) & =\left(\left(\frac{1}{2} \times 4\right) \times 3\right)+\left(\left(\frac{1}{2} \times 4\right) \times 2\right)= \\
& =\left(\frac{1}{2} \times 4\right) \times(3+7) \\
& =\left(\frac{1}{2} \times 4\right) \times 10 \\
\therefore & =\frac{1}{2} \times(4 \times 10
\end{aligned}
$$

- This tèlls us" that we may, calculate the measure or" the regtón. $\mathbb{N P Q}$, if we take one-half the product,of the measure of the al'titude and the measure of the base.

Do we get the same measure of the region. $M P Q$ if we add the measures of the regions $M P R$, and $Q P R$ as we get if we divide the product of the measures of the base and the altitude by 2 ? (yea) 823

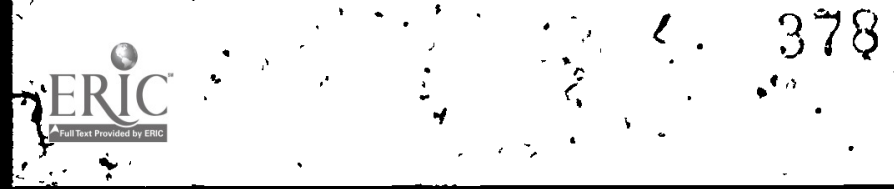




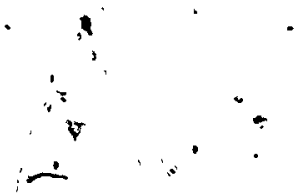

3. $\widehat{C D}$ is an altitude Which

-Iine segment is the base? $(\dot{A B})$

Suppose $m$ m $\ddot{C D}=6$,

m $\frac{\dot{A} \bar{B}}{A}=12$, In Inchés.

$\therefore$ Find the area of region

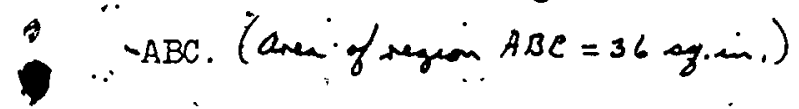

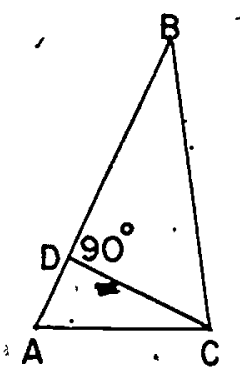

4. WS 1s:an altudè.

'Which Iine: segmènt' is

the base? $(\overline{R T})$ Suppose

$\mathrm{m}$ जS $=16, \quad \mathrm{~m} \overline{\mathrm{RT}}=7$,

In Inches. Flnd the

area of regton 7 RST.

(area of regeon RsT $=56$ ag.in.)
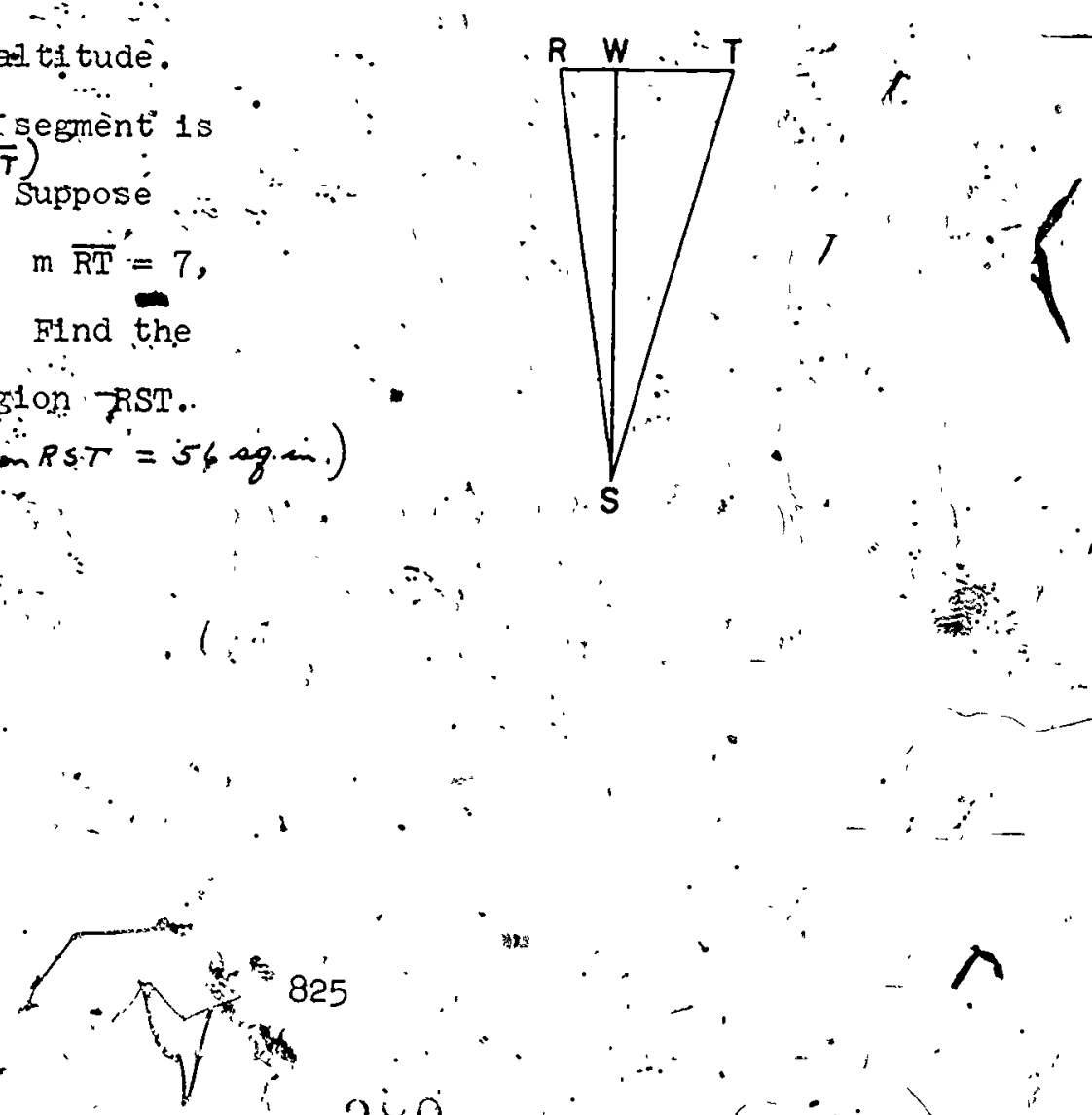

380

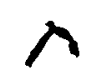


PURPOSE OF UNIT

The purpose of this" unit is to: build the understanding that one use

- of ratio is to indicate'how certain physical situations are alfxe in some - respects.

Pupils will learn to extract. certain propeties from physicat situations, and then to express these propertles usingjpairs of numerals. " $\because$ " chidaren can use the concept of $\because$ ratio when comparing two sets by Foting the correspondence or matching. of members of one set with the members of the other set. 
In other units we have dévelo mathed matics which has proved useful in desorrbing, situations in the physloal world: The concept of natural number enabled us to - Ind cate one way In which certain sets were calike: he lonjow that a set of 5.5

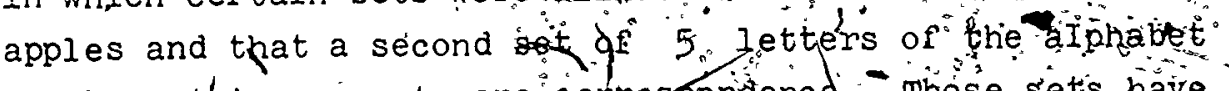
can be put' in a one-to-one correspondence. These sats have something in common: We denote the fundental propertitin which.we are interested by the numera. 5 . We have also. studied congruence and slmflarity, concepts Nich grew out of our destre to compare the istze and shape of models of : geometric figures. The concept of ratio which the will develop in this unit will give us stlll one more way of indicating how certair physical. situations are alike.

- consider the following problem. I cañ buy 2 candy bars for $6 \notin$ while yoù can gft -6 of the same candy bars for 20\%. We find ourelus wondering who \&gettibs the better "buy." we shall àssume that there tis no specials. *1scount for large purchises. Sinde I know that I must $y$ present to for exyo candy fars, I can visual 1 ze my candy purchastng ab1l1ty as plcturedibelow. Every candy bars that I buy must, correspond to 6 peninies.
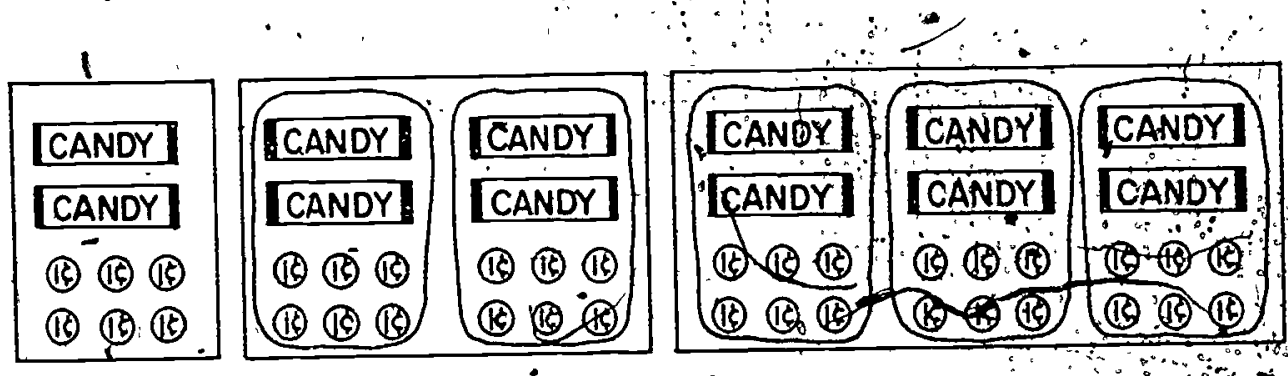

The last, frame clearly indicates that I doing better than you are under the given arrangements, for I am getting. 6 candy bans for 18 cents but you áre p'aying 20 for

6 candỹ bass. 
Extcity, how did we reach" this conclusion? At first we asked ourselves inat sort of purchase would be like the purchase of 20 andy bars for $6 \notin$. The situations represented abou standing of how these situations are alike, iet us summarize the essentiols ofech situation in a table.

\begin{tabular}{|c|c|c|c|c|c|c|}
\hline $\begin{array}{ll} & \ddots \infty \\
\because & \therefore\end{array}$ & $\begin{array}{c}\text { CANDY BARS } \\
\therefore \quad\end{array}$ & ฯ & $n^{4}$ & .6 & 8 & 3 \\
\hline 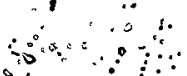 & $\because$ PENNTIES. & 6 & 12 & 18 & - & - \\
\hline
\end{tabular}

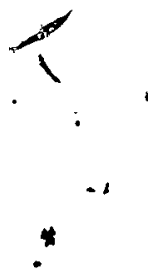

can we fures entries in our table? If we are able to

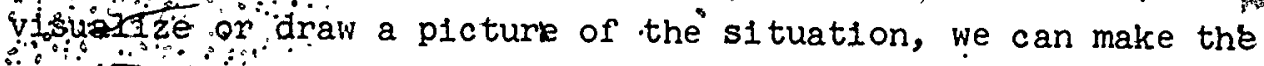
copresponding table entry with ease.

We notice that an essential-aspect of each situation we have desciphed can be represented by using a pair of numeris: $(2,6)$ for the first frame, $(4,12)$ for the second, and (6) for the third. These palrs can be used to represent a property common to $\mathrm{Xall}$ of these situations. Using the pais. $(2,: 6)$ we introduce) the symbol $.2: 6$ (read -2 to .6)! At this point (as suggested by, the above
tabile) you may wish to. write the symbol

- $\frac{2}{6}$ instead of $2: 6$. But the first emphasis is on seeing ratio as a property as another way' of comparing two sets of objects and not as a number. Later the correspondence between Ifratio and rational numbers will be established.

In tering of the above model this can be interpreted as telling us that there are 2 candy bars for every 6 penpies. This same Correspondence could have been described usting the pair (4, 'iz) and the associated symbol - 4:12. For the above model; this would tell us that there are. 4 candy. bars for" every, set of 12 peanies. "Clearly, $4: 12$ and $2: 6$ are different symbols which. we cann use to indicate the same' kind of correspondénce and we write $2: 6=4: 12$. Once more, as in the case of numerals for fractiont numbers, we wave an unlimited 'choice of pairs of numerals to represent the same" iproperty. The common property is called a ratio. In the preceding : 
example, the ratio of candy bars to pepnnies is'said to be .2 to 6 , 4 to 12 , or 8 to 24 .

\{. Can. We télp how much I wlll have to pay for one caray. bar? If fwe take another look at: the flist frame, as indicated below, we see that one candy bar should cost. me. $3 \xi$.

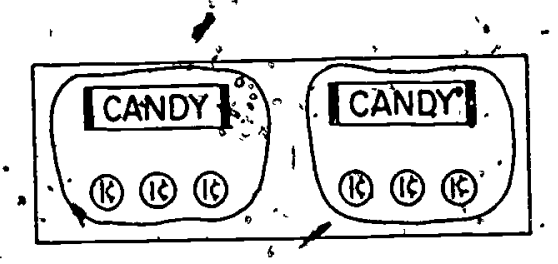

Then, 1,3 is another name for this ratio..

How múch candy can I buy for akr. "In trying to answer. this question we' find ourselves incapable of describing the sttuation by a.sultable paif of numerals unless we constder the candy bars to be divisibit. In fact the candy bars are - divisible although the store owner is not likely to sell us part of a candy bar. If he would, we would expect to get $\frac{1}{3}$ of a candy bar for a penny. Hênce, $\frac{1}{3}: 1$ is another name for - the ratio we have been studying. However if the candy store owner won't cut the candy bar into 3 pieces so that each piece is $\frac{1}{3}$, of the bar, this particular pair of numerais doesn't describe a situation that wili actually occur at the

$\because \quad$ candy store. $\quad$ We have seen that $1: 3,2: 6,4: 12,6: 18,3 \frac{1}{3}: 1$ can all be used to describe the basic property that each el ement. of "the first set, the set of candy-bars, always corresponds to 3 ." elements of the secon set, the set of pennies. We nht now ask. If we can decide which $p$ is of numerals farf be used to describe this 'ratio without awing pictures'. clearly, 'any :" pair of the form $(n, 3 n)$, where in $1 s^{\circ}$ a punting number will. do. Or course, 'if the storekeeper wiliznot subdivide the cands bars and if the penny is the smallest unit of moxey avatlaible, only pairs of the form $(n, 3 n)$ where" $n$, is a dounting number. will represent actual transactions at the candy counter. "That" Is, $5: 15$ and $\frac{1}{5}: \frac{3}{5}$ both represent the same ratio. 5:15. telels us that 5 : candy bàrs" wilp cost us $15 \notin$, whereas $, \frac{2}{5}: \frac{3}{5}$. 
tellg us that $\frac{1}{5}$ candy bars would cost 3 . Since the dealer w1li "not sell us $\frac{1}{5}$ icandy par, $\frac{1}{5}: \frac{3}{5}$ does not actually describe a posstble exchange of money far candy bars as $5: 15$ does.

The property described by $2: 6^{\prime}$ ts exhịbited in a wide varlety of situations arie is not restricted to sets of candy. bars and pennies. consider each of. the following:

1. :There are 2 texts for every 6 , students:

2. "There:are 2 boys for every 6 girls in class.

3.' The kart goes. 2 miles in 6 minutes.

4. : inve'stment earns .\$2 interest för every $\$ 6$ Invested.

After a brief consideration you ,will conclude that the table and the associated plctures which we developed for our example of candy bars and pennles would serve equally well to. describe each of the above situations. 'For example, in 1 , we have texts instead of candy bars and student's instead of pennies.

- Consider the statement 1. It describes a situation Involving 2 sets: a set of texts and a set of students. "The situation in question, exhibits a property described by $2: 6$. "We can say that the ratio or number of texts to number of studènts is 2 to 6. In short, theréare .2 texts for every 6 studẹts (Another name-for this ratio is $3: 9$. This Indicates that there are 3 texts for every" 9, students. $1: 3$ also describes. the ratio of the number of texts to the number iof" students. 'However, the rat1o of the number of students to the number of texts is $3: 1,1 . e ., 3$ members of the set of stadents correspond to each member of the set of iexts. clearly in máking comparisons between numbers of. texts and ' numbers of students $1 t$, w1ll not.be clear. that the ratio is $1: 3$ unless we understand that the first number indicated refers.to the set of texts. The order, in which the numbers are names is important. Anj pair of the form" ( $A, 3 n$ ) when interpreted as $n: 3 n$ could be used to describeritions relationshtp 
betifen the 'set of texts and the set of students. That is, since there are $n$ texts for every $3 n$ students, we have a situation exhibiting the ratio property $1: 3$. "Some pairs of this type are given in the following table. Spaces are provided for further entries.

\begin{tabular}{|c|c|c|c|c|c|c|c|}
\hline$\therefore^{\text {Texts }} \cdot$ & 1 & 3 & -5 & 12 & & $"$ & $\vdots$ \\
\hline Students & 3 & 9 & 15 & 36 & & & \\
\hline
\end{tabular}

9

- We can, of course, never hope to list all possible entries. The pairs indicated in throwtable are sometimes called rate pairs since they indicate how many texts per. student - a distribution rate of texts over the set. of students. He can visualize what the table fintries tell us about our model sets as shown below.

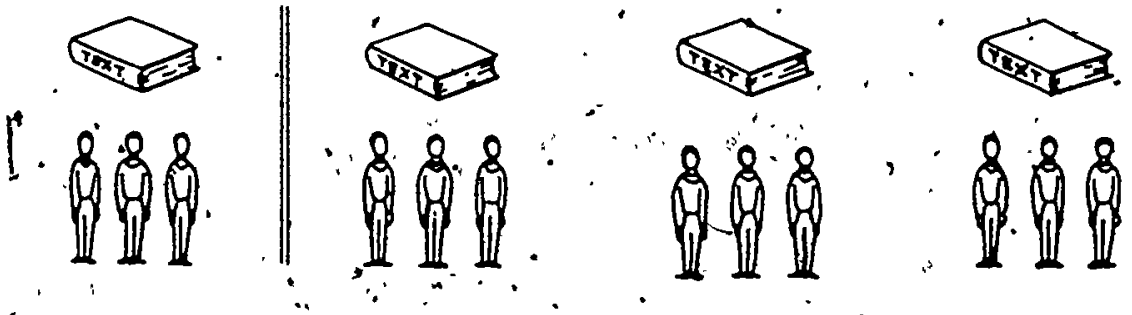

- We might ask how to determine one of our table entries without drawing a ptcture. In the examples we've been. considering the pairs we enter. In our table are alf of the type $(n, 3 n)^{\prime}$ and we see at once that $(9,27)$ will represent a table entry while $(4, " 17)$ will not.

Consider the ratio described by $2: 3$. This symbol telis us that there are 21 tems of the first set for every Items of the second. It follows. that $4: 6,6 ; 9,1: \frac{3}{2}$; 100:150, and, in general, $2 k^{2}: 3 k$ : would all be other ways of 
representing, this same property: If the first set-referred to. Is the set of boys in ischool and the second set is the set br glrls, we. say that there are 2 boys for every 3 girls'in. school. The symbol $2: 3$ can'also be used to describe a... fundamental aspect of what happens when. we have a kart which travels at the rate of 2 , miles every 3 minutes. The kart travils 2 miles every 3 minutes. In other words, corresponding to every 2 miles stretch covered by the kart, there fis a time interval' of 3 -minutes. The symbol $2: 3$. will doscribe the correspondence exhibited here if we choose the elements. of the first set to 'be. alstances of one mile and the elements of the second to be time intervals of one minute. The aspect of the-movement. of the kart is equally well described by any" symbol. of the form $2 \mathrm{kr} 3 \mathrm{k}$. Sgme such pal'rs are-indiçáted below. The sympol 40:60 represents. "40. mtles for each 60. minutes". of "40" mizes per.hour."

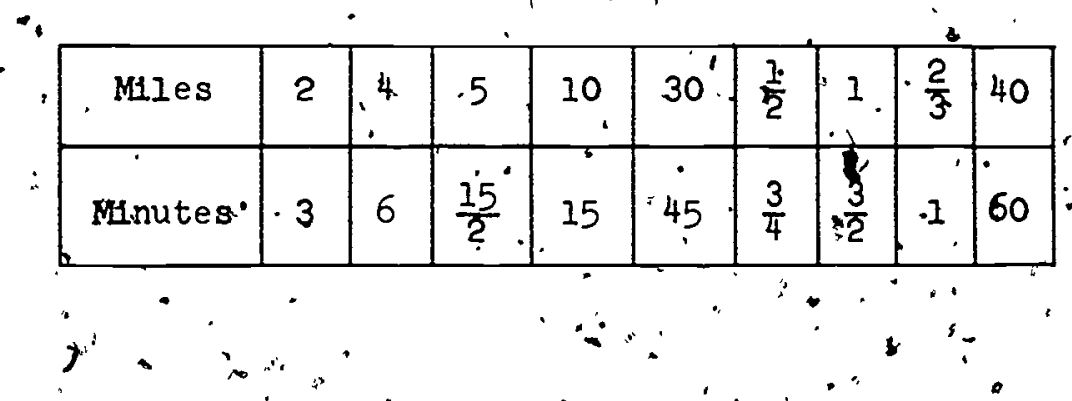

Situations in which the "correspondence of two se'ts can - be described as above by means of, pairs. of numerals of the type . (ka, kb) or $k a: k b$ all possess a property called. the ratio a:b. - To' each collection of $\underline{a}^{*}$ member's of the first.set, there corresponds a collection of $\underline{b}$ 'members . of the second. If two pairs of numerals represent the same ratio, we use an equals sign to show that they are different names for..the same ratio. For example;

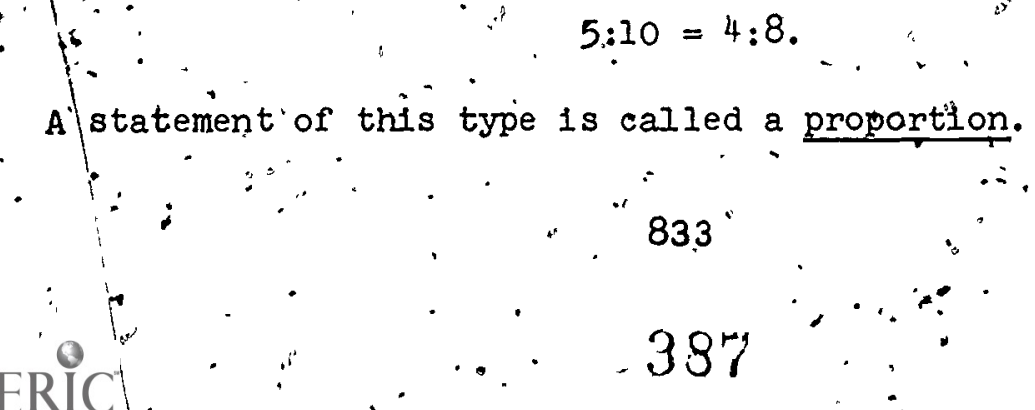


How can we tell if two symbols, for example $6: 18$ and ¿ $8: 32$, represent the same rat1o? The symbol' $6: 18$ tells us that there are 6" members of the first set for every 18 members, of the second. This is the same as il member of the first set for every. $3^{\circ}$ members of the second set. That 1s, $6: 18$ and, $1: 3$ are different names for the same ratio. - Similarly $8: 32$ and 1:4. Zpe different nemes for the same rat1o. These symbols $1: 3$ and $1: 4$ ćlearly desscribè . different correspondençes and we conclude that' $6: 18$ ' and $8: 32$ do not represent the same ratio.".

In general, $a: b \quad(a \neq 0)$ and $(b \neq 0)$ represents the same ratio as $1: \frac{b}{a}$ while $c: d$ represents the same ratio as $1: \frac{d}{c} \cdot(c \neq 0)$ and $(a \neq 0 \%$. It rolfows that $a: b$ and $c ! d$ can represent the same ratio if and only if $\frac{b}{a}=\frac{d}{c}$. That 1s, $a ; b={ }^{\prime} c: \dot{d}$ if and only $1 \mathrm{~b}$ ad $=b c$. Using this test we see 1mmediately that $6: 18 \neq 8: 32$ for $6 \times 32 \neq 18 \times 8$. Consider a situation in whych over a fixediperiod of time I can earn $\$ 1.50$ on a $\$ 50^{\prime \prime}$ inyestment. From what I know about simple interèst. I would expect to get $\$ 0^{\circ} .75$ on a $\$ 25$. Investment, \$0.03 on a \$l inystment; etc. If, as before, we use a table to exhibit, these results, we would have

\begin{tabular}{|l|c|c|c|c|c|}
\hline Dollars of 1nterest & 0.30 & 1.50 & 0.75 & $\because 3$ & 0.03 \\
\hline Dollars Invested; & $i 0^{\circ}$ & 50 & -25 & 100 & $i$ \\
\hline
\end{tabular}

The property common to ạll of these palrs 1s the ratio , $0.30: 10$. In particular note the pair $(3,100):$ "This can be" interpreted to tell us that we recelve $\$ 3$ of interest for every, $\$ 100$ 1nvested: If $k$. se this pair to despribe the ratio property, we write $3: 100$ and indicate that we get a return of 3 per 100 or. 3 per cent. Here cent is used to indicate 100 As $1 t$ is in the words centennial, century," : centipede, etc. We use the symbol $3 \%$ (-pead. 3 per cent). to describe how our interest compares dollar. for dollar. with ".s our investment. 
In studying correspondences between two sets, we were. led, to the concept of ratio. Think about the following statements and you should begin to appreciate the wide applicability of this new idea.".

1. "The population" is 280 "people per square mile. S"

2: The car traveled 100 yards in 6 seconds.

3. The recipe calls for 3 cups of sugar for every cup of water..

4. The 'scale on this floor plan is $1 \frac{1}{2}$ centimeters per 10. fieet.

5. I can buy 2 , sweaters for $\$ 7$.

6. My investment is eaming $4 \%$ interest.

It will, be recognized here that ratio is not presented as a number. On the second page of the Mathematical Background ratio is called a property belonging to two sets and the symbol used for ratio describes this property. The similarity "of "the words "ratio" and "rational" may suggest some, close relation between ratios and rational numbers, and such reliation does exist. But the foll significance of, this relationship is not possiple until after the study of multiplication and division of rational numbers. The final section.of this chapter is intended to establish an awareness in the pupil of some similarity between ratios and rational. numbers. 


\section{"TEACHİNG THE"UNIT}

The lessons in this unit are divided in two parts. The first pait is of an exploratory niature arid is to be developed. by the teacher and the . pupils 'working t toge ther.

- The second párt is cothposed of an axercise set for children to work Independently: Each exercise in the set should be discussed with/the pupils after the s'et has been completed. Many' of the: exercises are designed to carn a bit further the Ideas presented in the teachermpupif exploratoryperiod. Thèse exercises also provide for more clarification. of the concepts that are being developed and for piractice and drill work:

Each teachen shquld feel free to adapt the suggestions presented to fit her methot of teaching and her group of pupils.. As this unit, is an. introduction to ratio; it is not-expectid, that pupils, will develop polished skill's, for example, in rinding different namesrór the same ratio. However, many children will develop conslderable skil? if given an opportunity" to do so. The primary purpose is to "help alf of the pupils-develop an understanding of rato. 
$a^{3} 5^{2}$

Suggested Teaching Procedure;

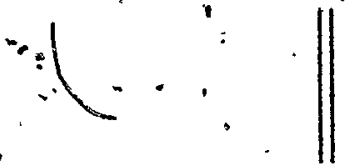

Pup1l books can be closed for this work.

present a number of physical situations which exhib1t the ratio of $2: 3$. For example, you might start by saying:

"Here are two sets - a set of chalkboard erasers and a set of pieces of chalk. What number is associated with the set of eraisers? Yes, 1t is 2 . What number is associated: with the set of chalk? Yes, 1t 1s 3. How many sets do we have?" (?)

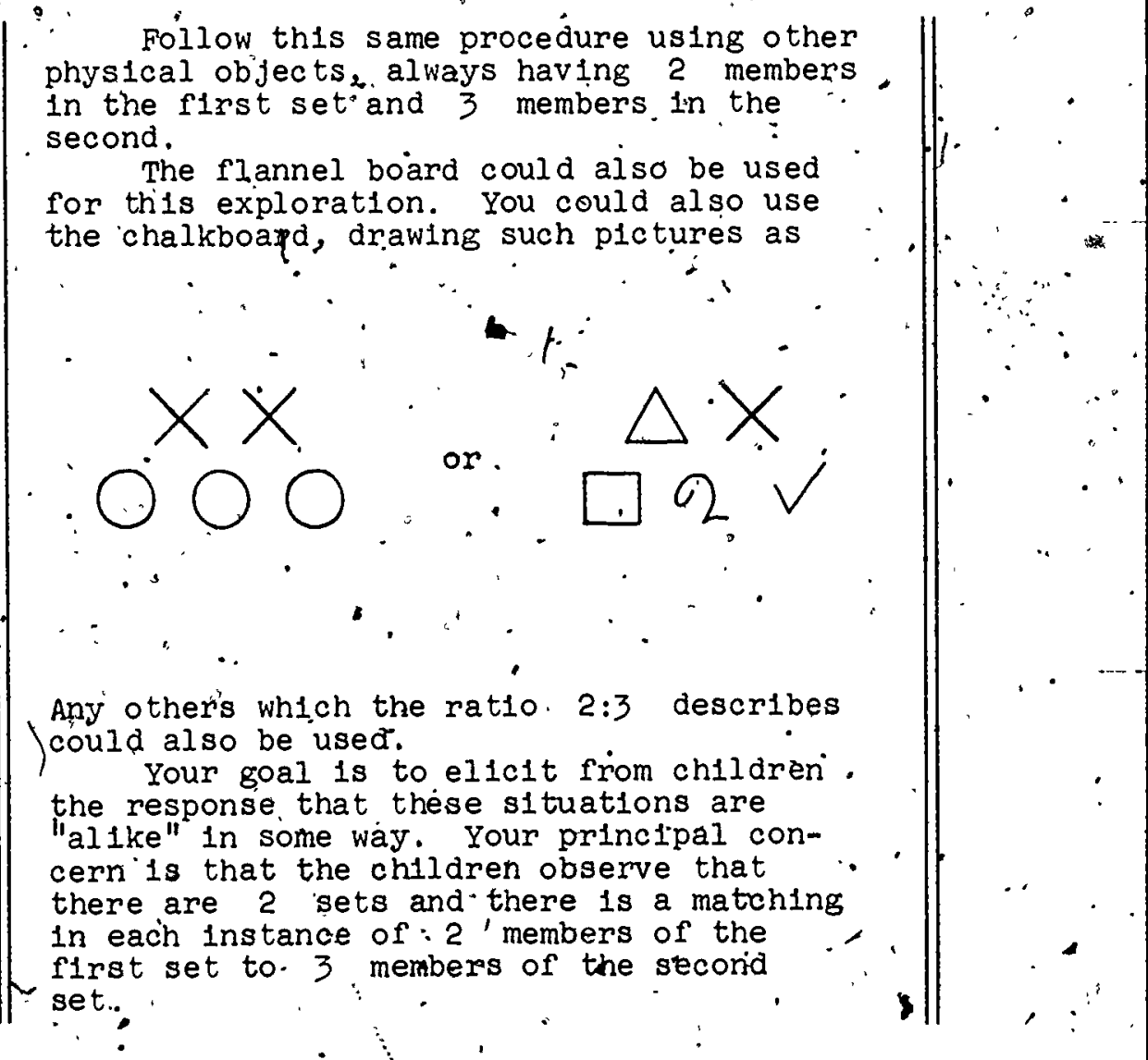

the response that these situations are

"alike" in some way. Your princtpal concern is that the children observe that there are 2 sets and there is a matching in each instance of:2 'members of the first set to. 3 members of the second set. 
Emphas 1ze that the property of the first set having 2 members and the second set having 3 members is called a rat1o. The symbol which represents the . ratio is written $2: 3$. Note that $2^{4}: 3$ is as symbol and not the rat1o, just as. 8 is a numeral and not the number. The symbol $2: 3$ is read as "two for three". or "two per three" or "two to three." Show other matchings using the objects, - rlannelboard, or plctures you draw on the board. Such ratios as $1: 3,5: 2$, and $3: 2$ might be used. Children should first - identify the two .sets being compared, such as "A set of red checkers is the first set and $a^{\prime}$ set of black checkers is the second -set". or "A set of pencils is the first set and a set of erasers is the second set." This is to help children be aware of the fact that there are two sets and to: be able to 1dentify the two sets. Th1s is necessary because when a ratio is expresised by" the 'symbol.; 1t.1s. necessary to know the set to which each numeral refers.

children could.wrt te the symbol.s on the board which express the ratio in. each case where yqu are using objects, the rlannel board, or platures on the chalkboafd. They should then "read". the symbol, noting es-; peclally to what each numeral in the symbol refers. For example, if the two sets are pencils and pleeces of chalk and the symbol showing the matching is $1: 3$, the pup1l. should say, "The ratio is one per three," (or "one to three" or "one for three") In this case, it means 1 pencil to 3 "pleces of chalk,

This ratio is the relationship expressed by "one per three" and it is interpreted in a physical situation as "one pencil per three.pleces of chalk" or as "one pencll for each 3 pleces of chalk." Give children opportunities to read. the symbolis for ratios such as $8: 1$ (elght per one, $3: 5$ : (three per five), $7: 6$ (seven per six). Then ask them to think of a physical situation to go with each of these ratios. A. child might say for 8:1, "Th1s 1s.read eight for one and it could stand for elght boys to one girl."

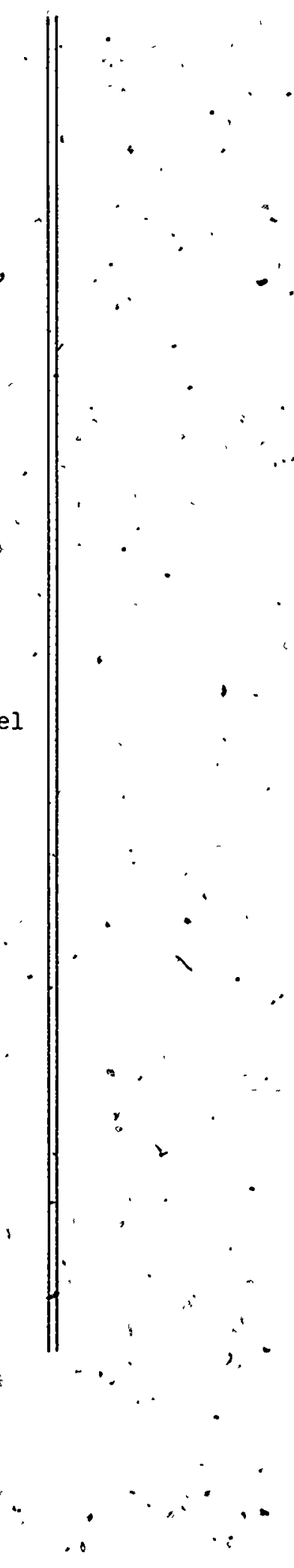


It would be well, to go from this symbol - of $8: 1$ to $1: 8$. Both of these ratios could describe the same physical situation but not in the same way. The first is interpreted as 8 boys to ' 81 rl while the second is interpreted as 1 girl to 8 boys. In each case, there is a -set of 8 boys and a set of i girl. Thus it is important that we know the situation from which we have extracted the ratio of $8: 1$ and $1: 8$. Exercise 6 in, the Working. Together sectionsis designed to develop this ided.

After the above development, the popils might open their books. You could quickl.y go over this section with them; asking, "Does this tell ápproximately what we have "learned?" In this Working Together or. Exploratory section, Examples 1 and 2 focus on the ldea of twa sets being compared.

Example 3 of the Exploratory section develops the idea of ratio and how the symbol is read.

Examples 4 and 5 give pupils opportunities to study situations that occurs in iffe (physical situation). and to note from them the ratio. Then they write a symbol which expresipes this ratio.

In Examples 6 and 7 we develop the idea that it is necessary to know the physical sttuation. "You mis ht now go back to Example. 5 and connect, for example, that the ratio, symbol for (a) can be either, 1:12 or 12:1. But we must know to what the 1 and the 12 refer. This same reasoning. applies. to Example 8. Thus, all four of these situations can be associated with the symbol $3: 5$. Ail that is necessary is that we know to what sets the 3 and the 5 refer.

Exercise set 1 provides for further clarification of the concepts introduced. For example, Exercise 4 of this set is involved with the idea we've just discussed. 
INTRODUCTION TO RATIO

Every day you hear statemen̈ts like these:

$\therefore$ (a) Bill said, "I bought two pieces of candy for four pennies."

(b) "I made two dolls in" four days," remarked Mary.

(c) "Jack'made two hits in? four times at bat," stated Mike:" (d) "My father drove two miles in four minutes, sald

These statements are alike in several way. given in each of them. In the first statement, one of the sets is a set of pieces of candf. The other set is "a set" of pennific.

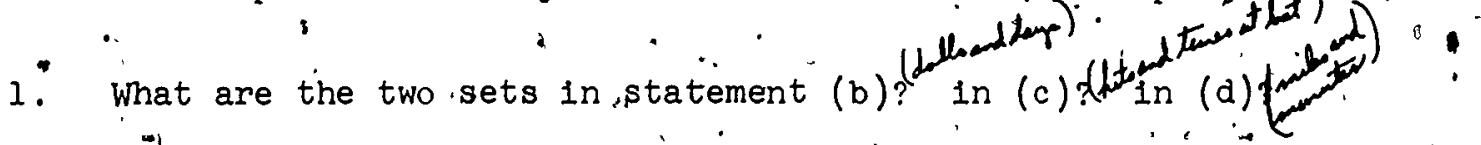
$-1$

In each statement, the tivo sets are matched. In

statement (a), 2 candies áre matched with 4 pennies: A pisture might show'it this: way:

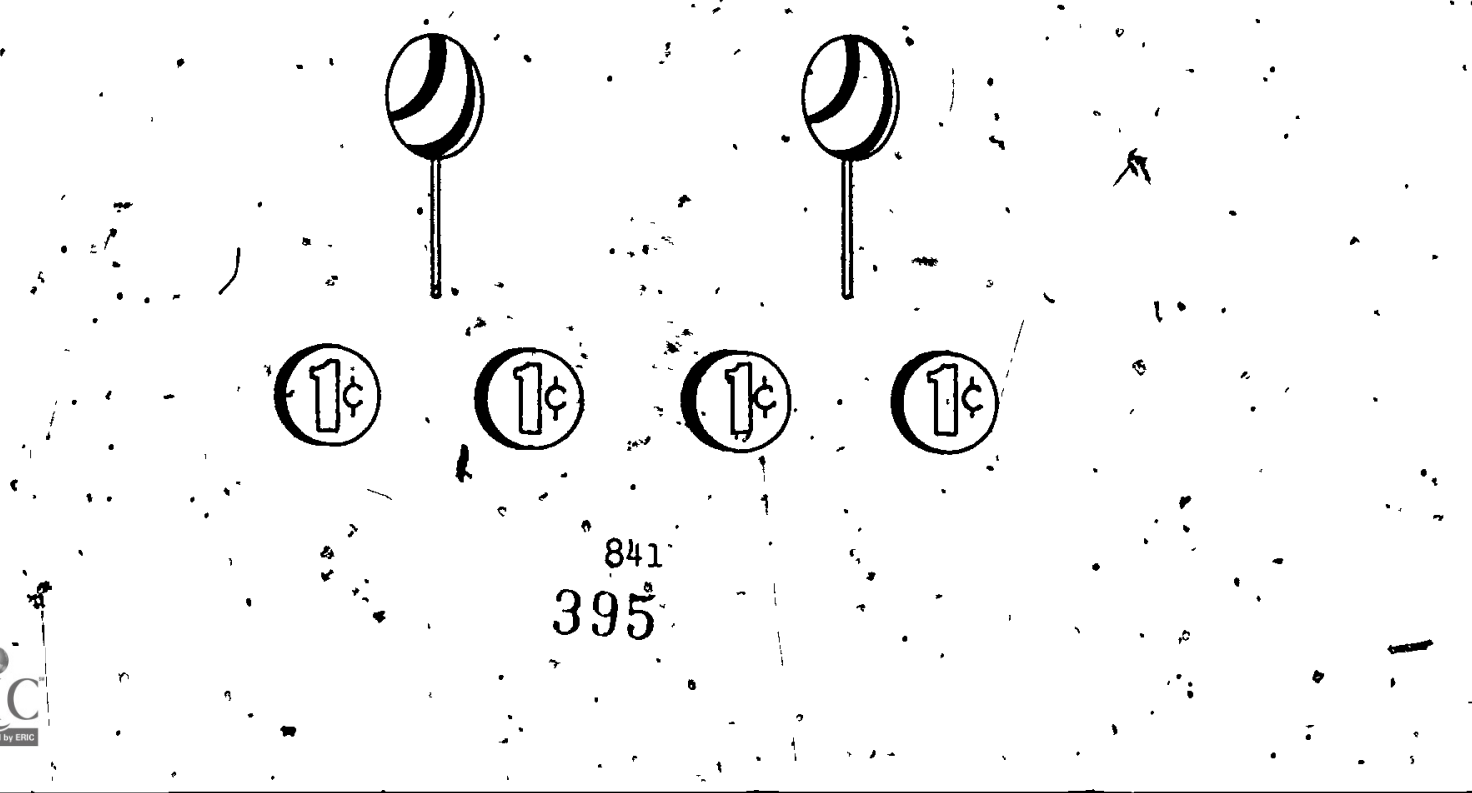


2. In statement (b), 2 dolls are matched with 4. days.

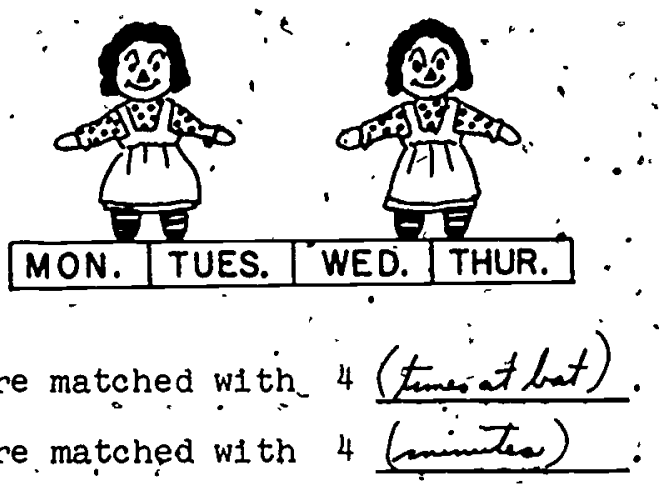

3. In each of the statements 2 members of the first set are matched with 4 mémbers of the secand set. This is the idea of.? to $4^{\prime}$ or 2 per $4^{\prime}$ ' Statement (a) matches. 2 . candies to 4 , pennies. Statement (b) mátches 2 dózlis to 4 days.

In all the statements two things are matched withw: four things. In statement $(c)$, we say the ratio of the number of hits to the number of times at bat. is 2 to 4.

Ratio is a new, word' to use. It is a symbol which contáins two numerais. It is a way of comparing the numbers of two sets of objeçts.

The way that we express the ratio, 2 fór 4 1s 2:4. "Th1s symbol is read "two for four" or "two per four:" Tho númeral's are néeded to express.'a' ratio.

Read these ratios

$3: 10^{\circ} \quad 4: 15,2: 5 \quad 1: 3 \quad 5: 2 \quad-1: 2$


4. Tom can work two problems in four minutes. What sets are being compared? (problems and mimatea) What humerals would you write to express this ratio? (2:4)

Jean can wark fivê problems in four minutes.

How would you express' this ratio? ( $5: 4)$.

$\bullet$

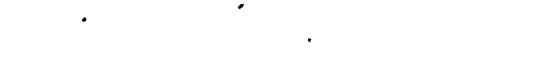

5. In each of the following, name the two sets that are being compared. Write the symbol for the ratio that compares the "two sets.

(a) "I can travel one mile in twelve minutes by using the Boy scout pace," said lee. (mib andminto $i 1: 12$ )

(b) The speed limit on the highway is sixty miles per nour. (mik and hines, 60:t)

(c): Jöhn ate three peaches to Perry's two peaches. $3: 2)$

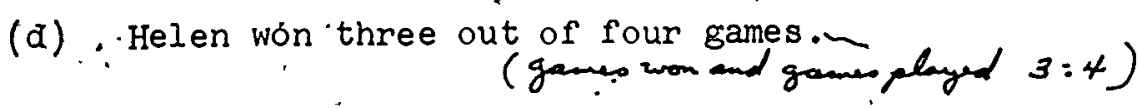

(e) Charles rode his bike to school eighteen times in tiventy days. (Timen and daye, 18:20)

(f) Dickerate Iunch at school four of the last five days. (lumeh daye and achol days", 4:5). 
6. The cook at the Boy scout camp sa1d: "I'w1II bake four doughnuts for each two boys:"

What would you write to express this ratio? If the

(a) (O) (O) 绝 cook said, "I witi. bake some" doughnuts so that there are. two boys for. every four doughnuts," the ratio would be 2.4. When the cook said "four doughnuts for each two. boys" theiratip was $4: 2$. When he said "two boys for every four doughnuts" the ratio was $2: 4$.

To undenstand the symbol 4:2, we need to know that the firist number (4) represents the doughnuts and that the second number (2) represents the boys.. To. interpret $15: 2$ then, we think "four doughnuts to two boys." This means there will be 4 members of the first set (doughnuts) to 2 members of, the second set (boýs). The symbol $2: 4$ means two boys to four doughnuts. 'It means that there wili be 2 members of the irst set (boys) for 4 members of the secand set". (doughnuts).

In order to know, what a symbiol such as. $\cdot 2: 4$ could mean, it helps us to know the situation which gives us $2: 4$. It might be 2 boysoto 4 'girls, 2 snakes to 4 frogs, 2 1deas to 4 plans. Name somé other situations which are $2: 4$ : 
6: The picture shown below shows 4 dogs and 6 cats: We can say, "There are four dogs to s1x cats."

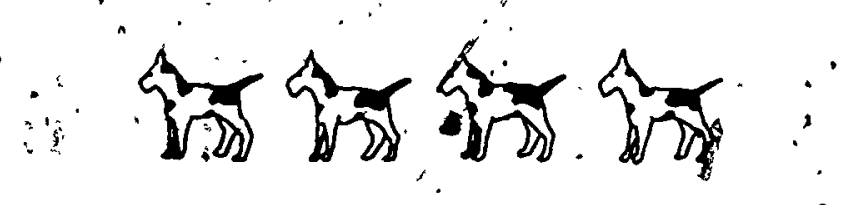

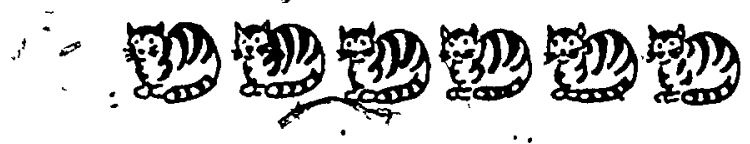

(a) "What ratio expresses how the set of dogs compares to the set of cats? $(4 ; 6)$

We could also say, "There are six cats to four dogs."

(b) What ratio describès the matching of cats to dogs?

"We can usè. a pair of numeral's in two different ways to "describe the same matching." When these are interpreted correctly, they still tell us the same

; "thing - "There are six cats to four dogs." or "There are four dogs to six cats.".

8. Which of the following are " 3 - to 5 " matchings?" $(a, c)$ Which of the following are "5 to 3 " matchings? $(b, d)$

(a). There are three bicycles for five children.

(b) For every five boys in Susan's class there are three gIrls.

(c) Tom has three marbles for every five that Dick has.

(d). The train trayeled five miles" in three minutes. 
$\mathrm{P} 5 \mathrm{i} 4$

\section{Exercise Set 1}

1. In what way are these situations alike? (Evid atars a sath 3:1.)

(a) Henry walks 3 miles an hour.

(b) In our fifth orade room, we have 3 social studies bools for each pupiI.

(c) That big truck can get orily 3 "miles. for each gallon of gasoline.

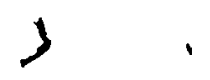

2. In Exercise. 1 name the two sets'that are peing. compared in (a), in '(b), and in (c).

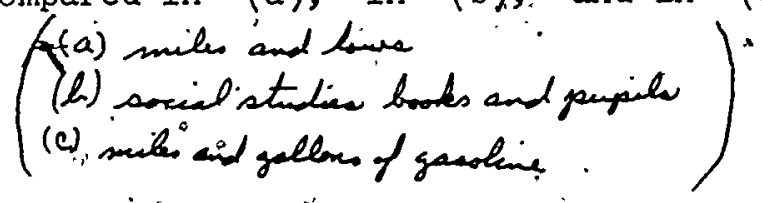

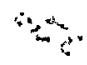

Write, in words, how you read each of these symbols:

(a).. 4:1. (four for on or four par one).

(b) 3:5. (thee for feive there por fine).

(c). 1:6 (one for eif or one par icifi).

.t

846 .

400 . 
P515.

4. Stuidy these pictures. Write a. symbol which describes. the comparison. "Then write a sentence to tell what this symbol means :

(a) (by

Candy Candy Candy

(1) (1) (1) $-(1),($ (b)

(3:5, you can hy the

(1) (1)

$\therefore$ cendy bare forfin cents.

(1:2 jere is one teming richet par tur temin ball)

(c)

(d)

$\therefore \rightarrow$ 真

(3:2 Itre are there

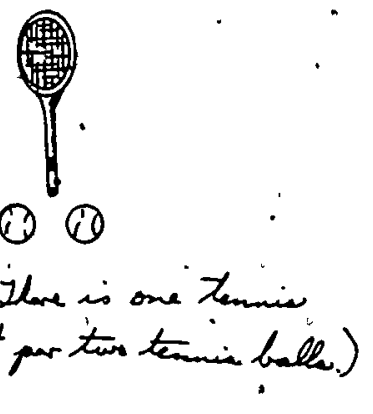

2.

conibage partis lones.)

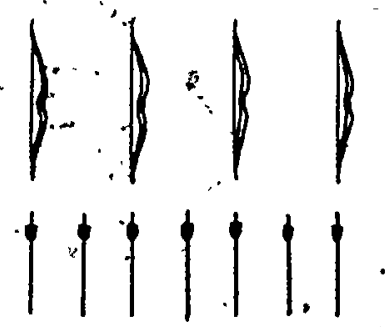

(4:7. The are four boure (e). Jor sevar ciromat.

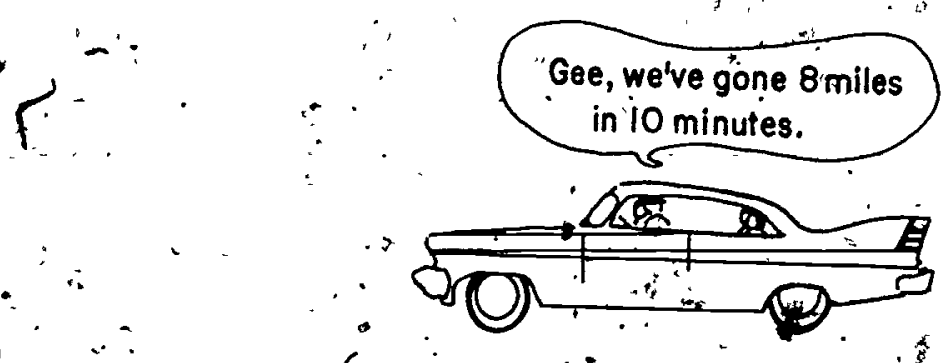

(8:10. Welcan dring eight. miles is te minutas. o o

$$
\begin{aligned}
& 847 \\
& 101
\end{aligned}
$$


5. For each of these sityations", write a symbol which expreseses the ratio.

(a) Ned had 2, bee' stings for one that Dicki had. (2:i)

(b) For our Halloweén party" we had 5 sheets of orange paper for" $3 \cdot$ sheets of black paper. (5:3)

(c) The speedometer on Steven's bike showed this: $(20: 1)$ 广

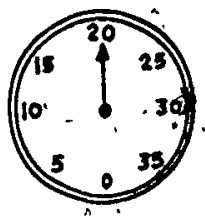

(d) Two bags of potato chips cost twenty-five cents. $(2: 25)^{\circ}$

(e) Jean can work four probiems in five minutes. $(4: 5)$

6. Draw p1ctures which could represent comparisons

- described by these symbols: (The pucturos iniliner.)
(a) $6: 1$
(b) $5: 2$
(c) $2: 3$

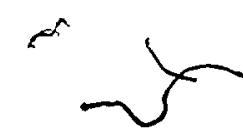

7. Sandra and Mark read this sentence:

on John's farm there are 5 lambs for 3 mother sheep. Sandra wrote $5: 3$ ' to show this comparison. Shersaid, "I know there are 5. lambs for 3 mothers."

Mark wrote $3: 5$ to show this matching. He sald "I know there are 3 mother sheep for 5 , lambs." , ino was correct - Sandra or Mark? (Thy are b-ticorrect.)

4

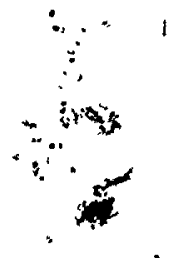




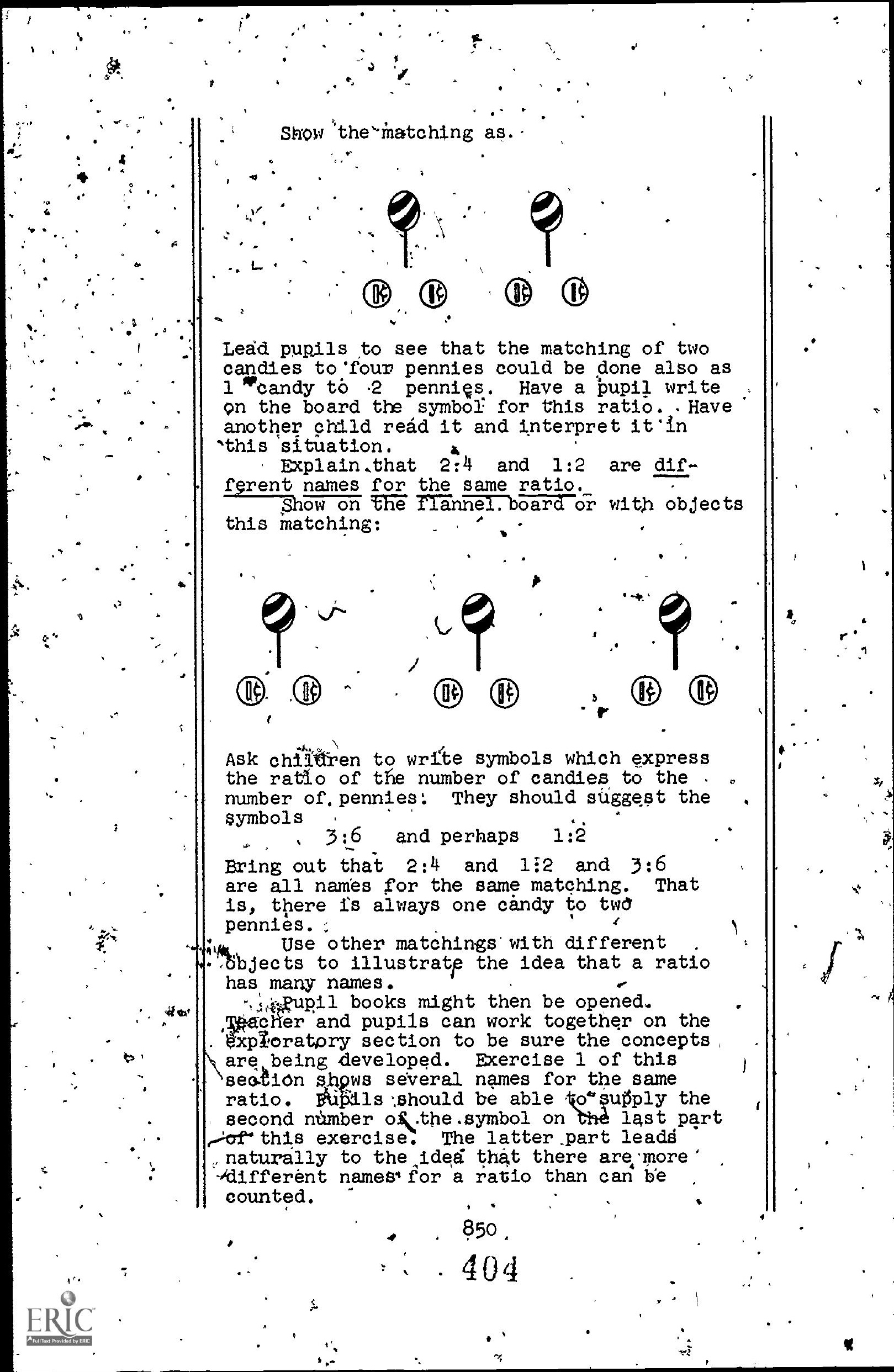


Exercise 2 of the exploratory section 1 designed to emphasize the idea of having many names to describe tre same rat1o." In part (a) there are, of course, many ways of describing the ratio of the number of boys to the number of girls. The name sought here is $10: 20$. In (b) the name sought is $2: 4$; in. (c). It is 5:10. Part (a) asks for still more names for this "same ratio.

- Exercise. 3. of the exploratory section gives practice in witing symbols for ratios., The symbol for the rirst ratio might be $8: 6$ or $6: 8$, but we must know to which. set each numeral refers.' There woul be many sultable. names for this ratio, such as $8: 6,14: 3$, $16: 12,40: 30$, and $12: 9$.

It may be necessary 'to work' with a number of sets of objects or. with a number of illustrations with the flannel board or on the chalkbóard. to develop the 1dea that there are many names for the same ratio. arcise set 2 gives further opportunity to develop this concept. In each of these exercises, the symbol describing the ratio can be either of two, depending on how the matching of sets is done. For Extercise 1 (a), for example, the symbol can be $1: 3$ or $3: 1$ but each of these. refo the same two

sets. In Exercise 2 of this Exercise set different names for the same ratio are desiped....

Exercises 3 and 4 lead to putting different names for the same ratio in tabular form.

Exercise 7 gives the children an opportunity to be original and creative in their thinking. "The basic concept of ratio WIlI be needed here - 2'-sets - and a matching of the members of one set with the. members of the ather." The-children's drawings can be three different pictures, each showing the ratio $4: 1$ or they can use the same sets to show "aifferent names for the ratio $4: 1$. 
P517.

\section{DIFFERENT NAMES FOR THE SAME RATIO}

When BIII bought 2 candy for 4 pennies, we debcribed

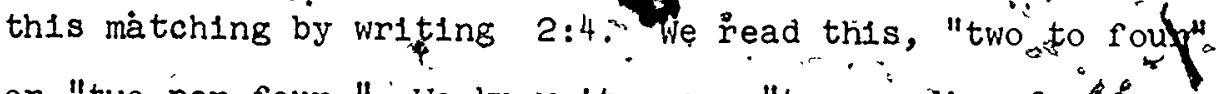
or "two per four." We know 1t means "two candles for four pennies." "We drew a picture to representifhis.."
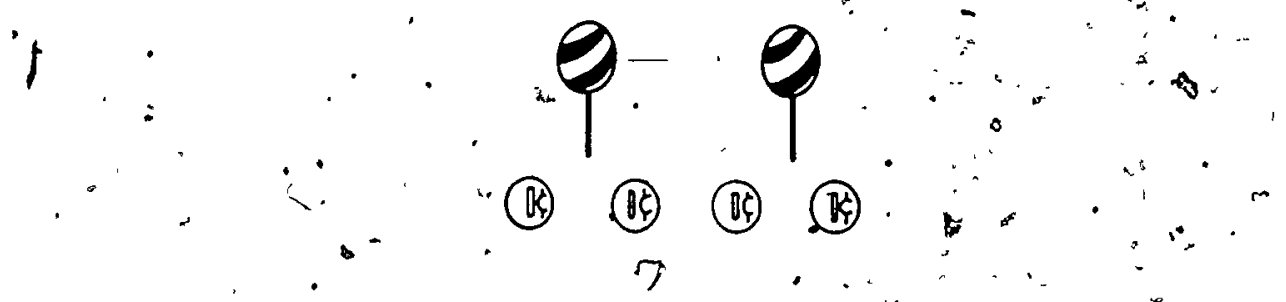

Can we show this in another way? Look-at thes picture.
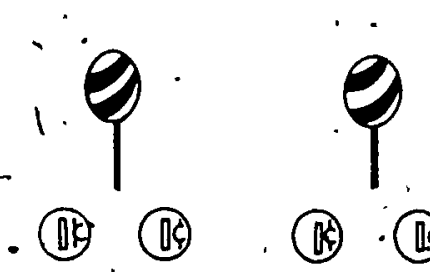

(10) (사)

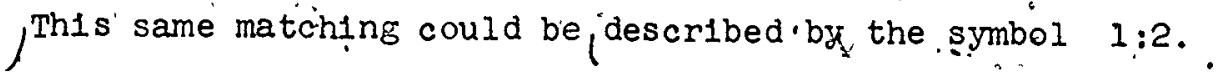
This means 1 candy for 12 pennies. Study this picturè.
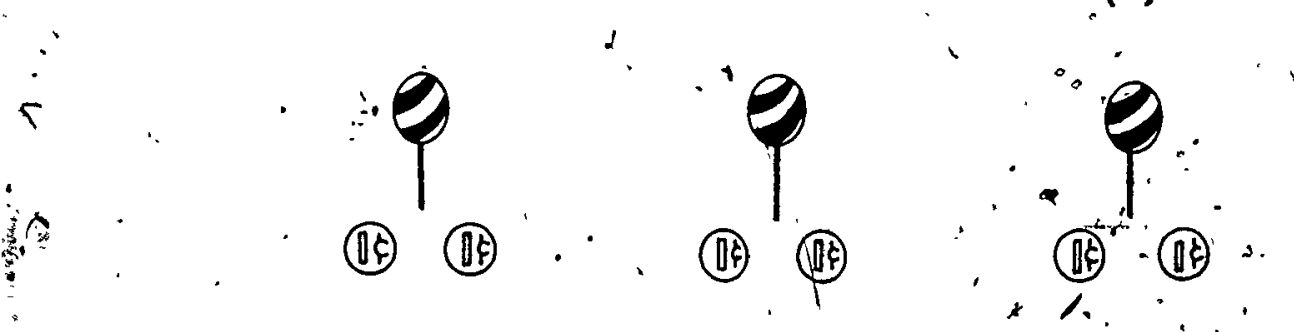

We could also use the symbol $3: 6.1$ Th1s means 3. candies for 6 pennies. 
- Look at this picture.

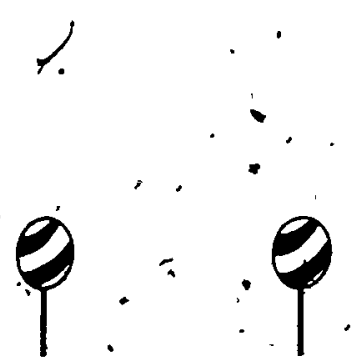
(10) (19)
(B) (B) .
(10) (19)

(19) (19) (19)

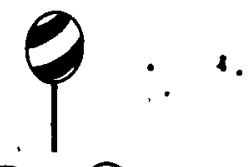

$+1$

$1 \therefore$ We can describe this another way by writing $4: 8$. What does this symbol mean?

The symbols $2: 4$ and $1: 2$ and $3: 6$, and $4: 8$. are all correct ways of, expressing the same cómparison. There are many symbols which describe matching. We can . refer to 1twas two per four, or one per two, or three per six, or four pele elght. Give other names for this same matching.

Write the second numeral to show other names for the ratio of number of candtes to the number of pennies,

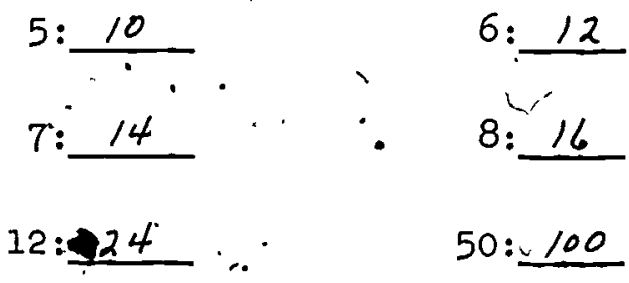

$\therefore$ How many different names will there be? (xin tham yodesint.) 

(c) This picture shows still, another way of lining up. this same class. What symbol expresses this ratio, of boys' to girls? ( $5: 10)$
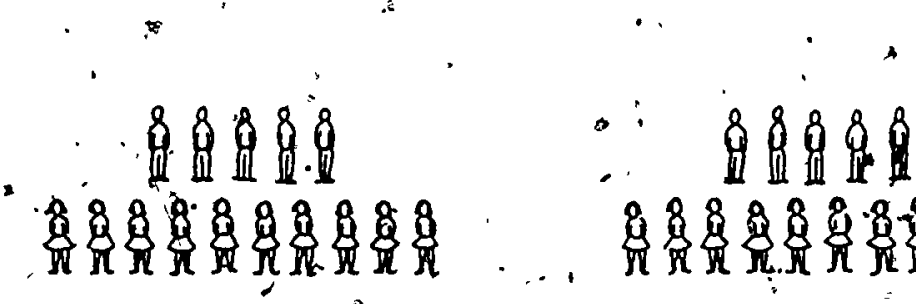

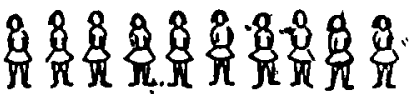

(d) "All of these are names for the same ratio. $A$ Complete these symbols to show they rare names "for the same ratio:

$$
\begin{aligned}
& \text { 10:20, } 2: \div 4: \\
& 5: 10: 2 \cdot 1: 2 . \\
& 50: 100 \quad 2^{4 k}: 4.8 \\
& 234: 428
\end{aligned}
$$

When we are matching one boy to two girils, there are moref-names to show this matching than. we can count. Whenever we are matching one of a set to two of another set, we usualiy' write? $1: 2$ to express thịs ratio. 
. 3.. For each of these pictures, tell four names for the ratio. (angina will wary.)

(a) 8 saddles to 6 horses. (b) $4^{-2}$ flowers to 1.0 bees

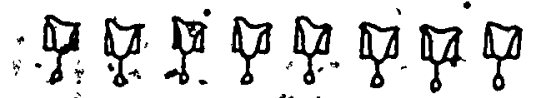

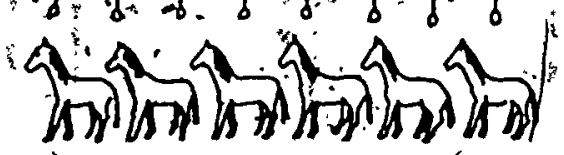

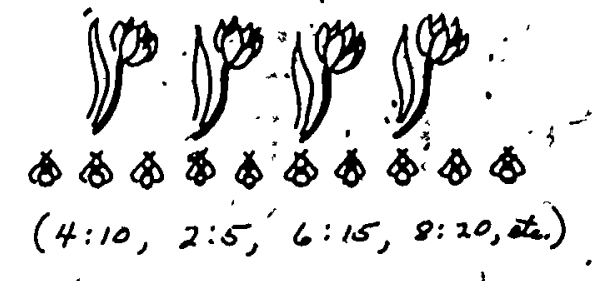

$(8: 6 ; 4: 3 ; 12: 9,16: 12 ;$;.t. $)$ $(4: 10,2: 5,6: 15,8: 20,2)$

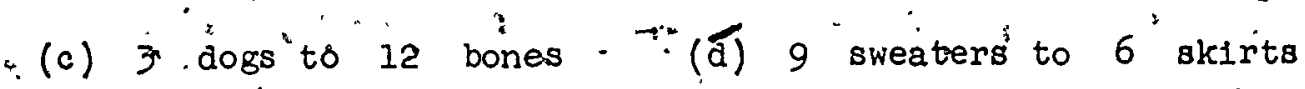

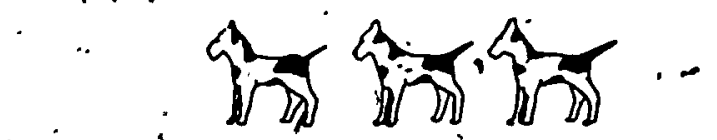

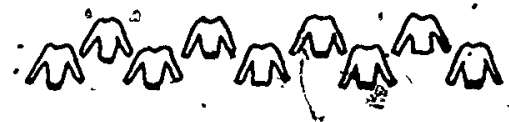

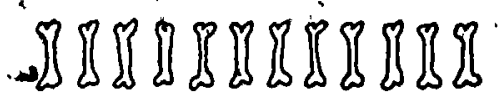

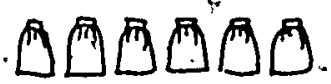

$$
\because(3: 12,1: 4,2: 8 ; 4: 16, \text { at. })
$$$$
(9: 6,3: 2,12: 8,15: 10, \text { etc.) }
$$

(e) is beavers to 6 beaver houses

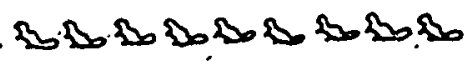

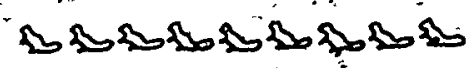

- (18:6, 9:3, 3:1, 6:2, et. $)$

$\therefore$ (s) 6 squares to 4 circles $\square \square \square \square \square \square$,
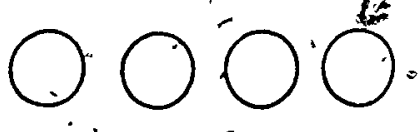

$\because(6: 4,3: 2,9: 6,12: 8$, et. $)$

*

¿ ${ }^{856} 4 i j$ 
1. You know that a ratịa has more names than we can count. Each picture has two sets. Compare the first set to the second set. Write four names for the ratio suggested by the picture. (Ansmars will wary.)

(a)

$$
\text { of } \sum_{10}^{\infty} \sum_{1}^{\infty}(1: 3,2: 6,3: 9,4: 12, \text { at. })
$$

(b)

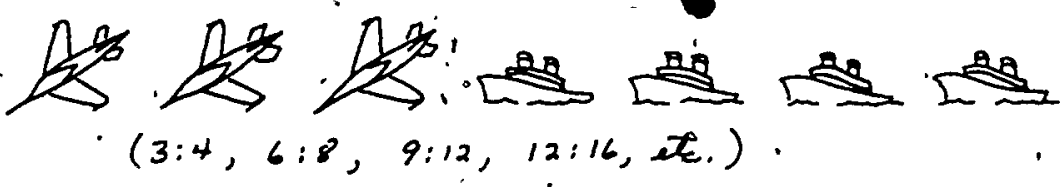

(c)

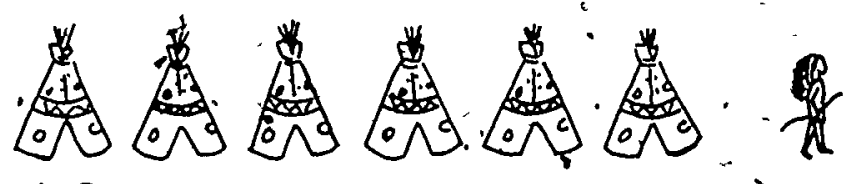
$(6: 1,12: 2,18: 3,24: 4$, et. $)$

(d)

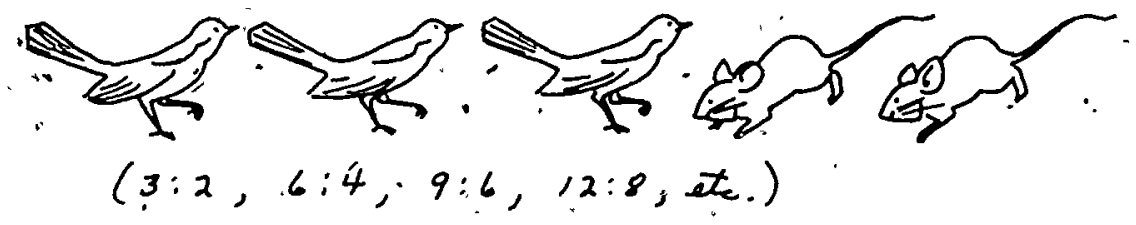




\section{P523}

2. For each sentefrce write two names for the ratio suggested by the sentence. Tell what the'names mean. (anamovillary.)

(a) George was going 5 miles per hour on his b1cycile.

(b) In the baseball game, Ne1l was getting 2 hits for every 5. times at bat. 4 lite for eny 10 then setting $)$

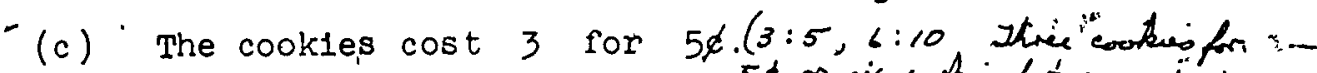

$f(d)$ In Franklin school there are 5, girls for every

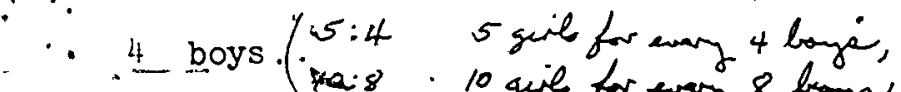

(e) $8 \cdot 10$ give for every 8 brow, et.

(e) The train was golng 4 miles in 3 minutes. $4: 34$ ma 3 in minitio,

(f) The airplane was. going 10 miles in 1 minute.

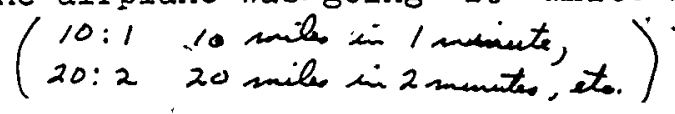

3. This table shows several names for the same ratio. Copy 1t and rill eäch blank space with the proper numerai.

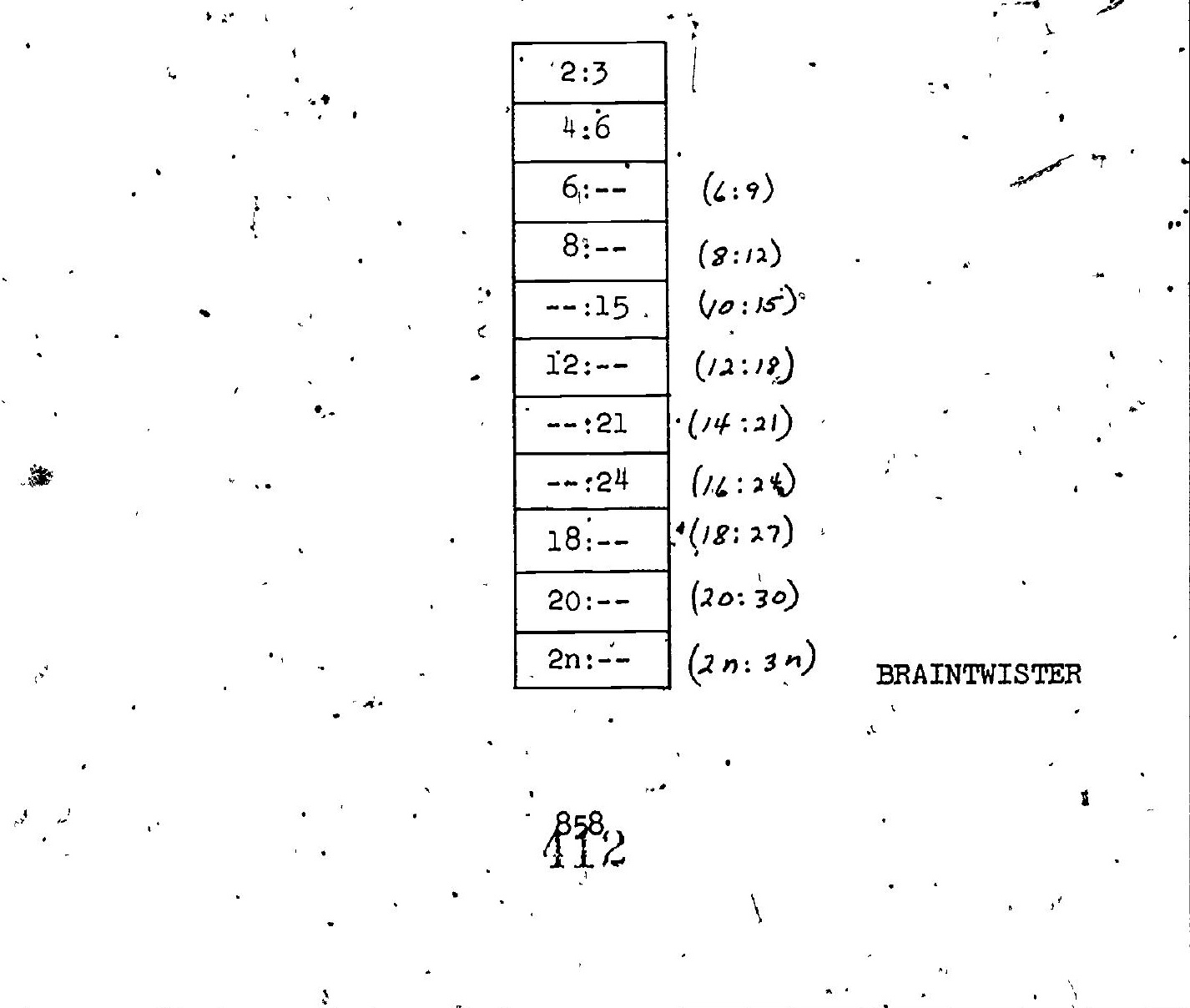


524

4. Th1s table shows sevfral names for the same ratio. "Copy it and complete ite

\begin{tabular}{|l|l|l|l|l|l|l|l|l|}
\hline $5: 4$ & $10: 8$ & $15::^{(12)}$ & $20:(16)$ & $(25)$ & $(30)$ & $(30$ & $(32)$ & $(45)$ \\
\hdashline
\end{tabular}

5. Write the letter of each symbol which is another name for the ratio $8: 16$. (a,b, $(a, e, f)$
(a) $4: 8$
(c) $1: 4$
(b) $\longdiv { 2 : 4 }$
(d) $3: 6$
(e) $16: 32$
(f) $9: 18$

6. Draw two pictures of cowboys and Indians like this to illustrate the ratio 3 "per 9. (Pictures vill wary.)"

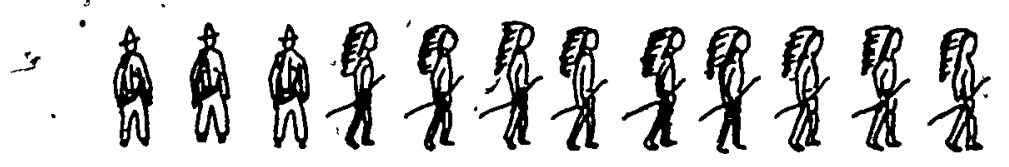

7. Use any pictures you like. Illustrate with 3 drawings the ratio shown by the symbol $4: 1$. (Pictives-ielarary.)

8. What symbol could you write to show the matching of one member from the first set to one member from the second set? $(1: 1)$

9. Express each of these matchings as a number palr using the word "for" or "per."

(a) Jean can work 5 problems in 4 . minutes. ( 5 par 4 )

(b). John ate 3 grapes for every' 2 that Perry ate.

(c) The speed limit is 60 miles per hour. (60 par 1). 
Objective: To further develop the chila's concept of ratio: To develop some arithmetic way of finding. diffepent names for the same ratio

Materials: Flannel board and cut-outs, a variety of objects such as those previously used; $30^{\circ}$ slips of blue paper and 30 slips of red paper for each pupil. (or any other matertal; such as colored sticks, so that each pupil might have 2 sets with about 30 members in each)

Suggested Teaching Procedure:

I.: The procedure as presented, in the pupil rtext is in sufficient detail to follow. This. cann be done with the texts open, teacher, and. pupils working together.

Comments regarding Exampless 1-9, Exploratory section, Pupil Text.

Example $l$ is of a revi'ew nature:

'Example 2 ' is designed' to develop the idea of finding new names for the same. ratio. In Exerci'se 2 (a), (b), (c), because. $6: 18$ and $1: 3$ are names. for the same ratio, we can write

$$
6: 18=1: 3 \text {. }
$$

We do not call this a "proportion," bot merely indicate that the equal sign tells us that symbol $6: 18$ and $1: 3$ are name's fors the same ratio. Exercise 3 carries a bit further the 1dea of different names for the same ratio. Exercise 5 gives the first hint of a way of mathematically determining names for the same ratio: It, is here that we will probably need to move slowly so that a good foundation is laid upon which to build in the last part of this unit. 
Example 6 has the children working with slips of paper (or other objects) to set up a varlety of matching of the two sets. Past experience indicates that children can grasp the concept of ratio quite readily from this type of activity. It would be well to have many exercises based on the matching of these slips of paper. Develop these exercises.as is done in the text for Exfrcise. 6.

Example 7 of this exploratory section provides practice in grouping in matching. . All children are not expected to be able'to solve these without the use of manipulative material. Thésè 10 problems can be solved by using sticks, as a "helper."

Examples 8 and 9 give the child an opportunity to demonstrate his understanding of different names for the same ratio."
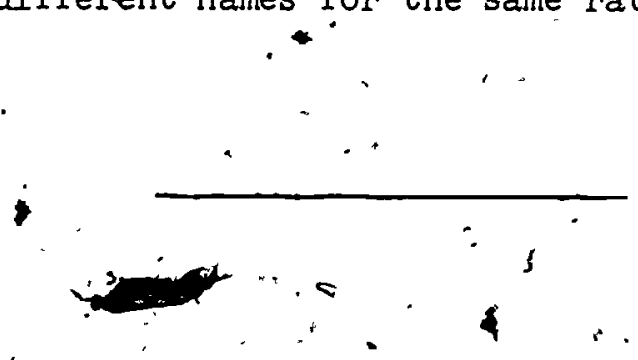

Exercise set 3 demands even more abstract thinking from the child. Exercise 3 of set contains some symbols which cannot eașily be determined with sticks or slips of other.
object.

$\because \quad$ Exercises 4 and 6 of set 3 give practice in working with mathematical sentences concerned with ratio. 
MORE ABOUT NAMES FOR THE SAME RATIO

When we speak of ratio, we Immediately think of sets. We know that members of the first set are matched with members of the second set.

1. Name the two sets in each of these-situations:

(a) A fifth grade girl had 8 envelopess for every 12 sheets of paper. (envelyen and alut of papar)

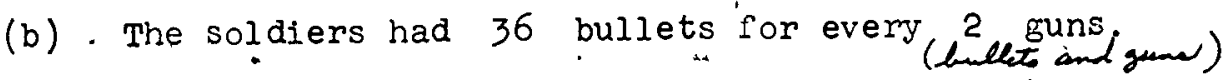

(c) The boys rode their bicycles 4 miles in $2^{4}$ minutes.

(d) At the fifth grade party there were 12 cookies for every. 4 children. (cookus elilhan)

(e) What symbol names the ratio in $(a) ?^{(0: 12)}$ in $(b)$ ? $(36: 2)$ in $(c) ?^{(4: 24)}$ in $(d) ?(12: 4)$

2. Some boys went $\delta$ a camping trip: There were 6 'tents for 18 boys. The same number of boys, slept in each tent. Stfoy these pictures.
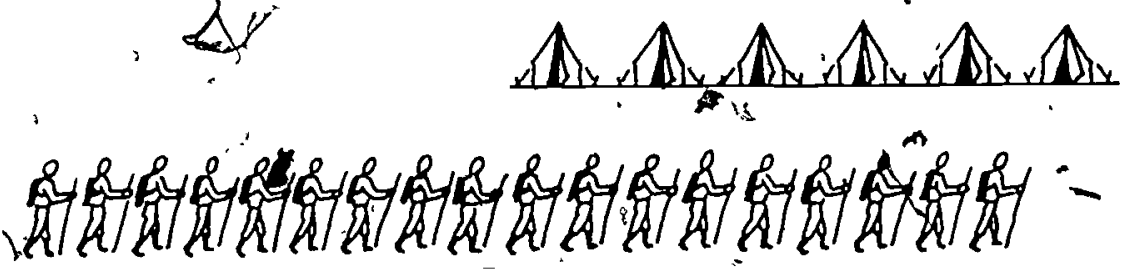

(a) What are the two sets? (tant and bage

one name for this ratio-1s- $6: 18$.

It tells us there are 6 tents per 18 boys.

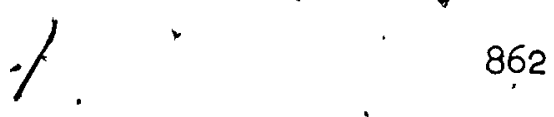


P526.

(b) This picture shows us another name for this same (ratio is $1: 3$. What does this symbol tell us?

An
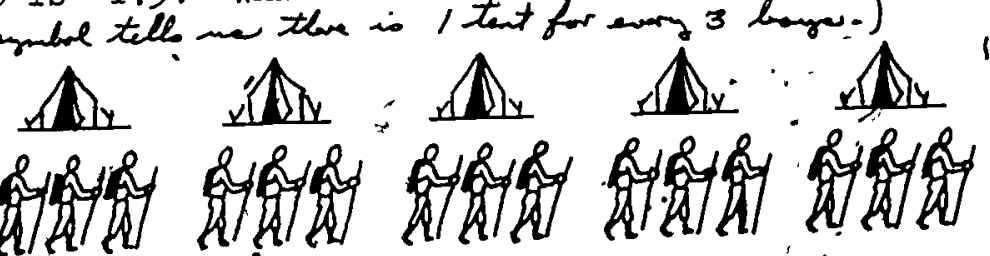

Because $6: 18$ and $1: 3$ are both names for the - same ratio, we can write:

$$
6: 18=1: 3
$$
Hin<smiles>[CH]C</smiles>

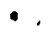

4

We can read this mathematical sentence, "Six to eighteen equals one to three" or "Six to eighteen is the same ratio as one to three." In this problem that means "6 tents to 18 boys."

(c). Is this' a true mathematical sentence? (yer)

$$
6: 18=3: 9
$$

Because $6: 18$ and $1: 3$ and $3: 9$ are ali names for the same ratio, we can write

$$
6: 18=1: 3=3: 9
$$

This tells us that 6 to 18 and 1 to 3 and - 3 to 9 , are names for the same ratio. What is another name for this ratio? $(2: 6,4: 12,5: 15)$. 
P527

3. These pictures also illustrate this same ratio between numbers of tents and numbers of. boys.

(a) What symbol can we use to express this ratio? $(10$ or $2: 6)$

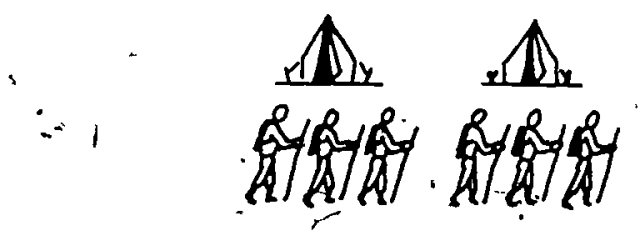

(b) Using the picture to help us, what new name can we write for this same ratio? $\left(4: i_{2}\right)$ 
(c) If there were 7 tents, this picture shows how' many boss could' go camping. What new name' expresses this ratio? $(7 \% 21)$. .

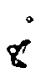

A...
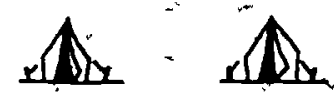

Mr

3

P528

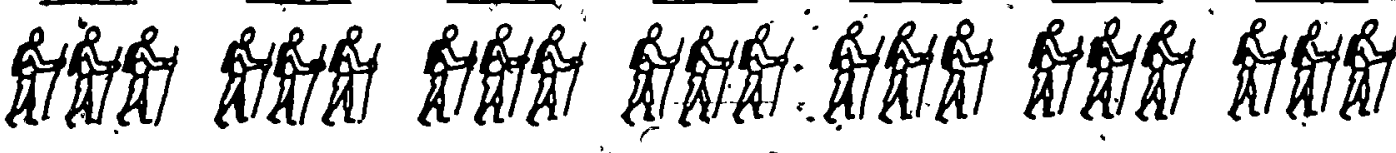

You see that in every case the ratio is the same.

We still match 1 tent to every 3 boys.

4. Draw a picture to' show how many boys could go camping if there were. 8 "tents of this size: (Aram miffuman. There will be eight that and therty-four boy. 8:24) 
5. Draw a picture to show how many tents would be needed for 27 boys. (Jhe pistare will alow 9tanto for 27 by : $9: 27$ ):

Here is a table which shows this information:

\begin{tabular}{|l|l|l|l|l|l|l|l|l|l|l|}
\hline Tents & 1 & 2 & 3 & $\stackrel{(4)}{1}$ & 5 & 6 & $(2)$ & 8 & $\stackrel{(9)}{(10)}$ &.-0 \\
\hline Boys & 3 & $(5)$ & 9 & 12 & $(13)$ & 18 & 21 & $(24)$ & 27 & 30 \\
\hline
\end{tabular}

(a) For this same ratio, how many boys would sleep in 2 tents? (6)

(b) Tell what numbers should be used to fill the spaces" in the table. We can use these pairs from the table to write other names for this ratio: (For example: $1: 3=2: 6=3: 9=4: 12=5: 15=6: 18=7: 21=8: 24$ $\therefore$ and so on.

(c) It is not necessary to draw pictures or to make" a. table to find how many boys could go camping if there were 8 tents. We know that 1 tent w111 house 3 boys. The members of our sets are tents. and boys. We "write 1:3.

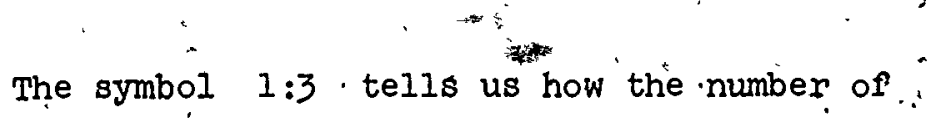
tents compares with thè number of boys. 
We want to find another name for this ratio which. has 8 as its first numeral. We write

$$
1: 3=8: N
$$

In this problem we interpret this as "l tent for 3 boys is the same ratio as " 8 tents for how many boys." Instead of 1 tent, we now have 8 tents. Therefore, instead of being able to house only 2 group of 3

- boys, we can house 8 groups of 3 boys or 24 boys. $(8 \times 3=24)$

The symbol $1: 3$ and $8: 24$ are different ways of naming the sama ratio. We can' write

$$
.1: 3=.8: 24 \text {. }
$$

(d) The symbol 2:6 is another way of describing the ratio of number of tents to the number of boys.

$$
2: 6=8: \mathrm{N} \text {. }
$$

This says that 2 per 6 is the same as 8 per how many. "If 2 tents will house $6 \cdot 6$ boys, then 8 tents, or. 4 groups of 2 tents each, should sleep 24 boys. We write 1 .

$$
2: 6=8: 24 \text {. }
$$

(e) Find the number represented by the letters in the following séntences:

$$
1: 3=5: x, \quad+3: 9=12: y, \quad 1: 3=15: 25
$$


6. Place in two separate piles the 24 , slips of paper (red) and the 12 slips of paper (blue) that your teacher has given you. The blue slips are members of one set and the red slips are members of the other set. We will match $\rightarrow$ blue slips to red slips.

( (a) Arrange the slips'like this:

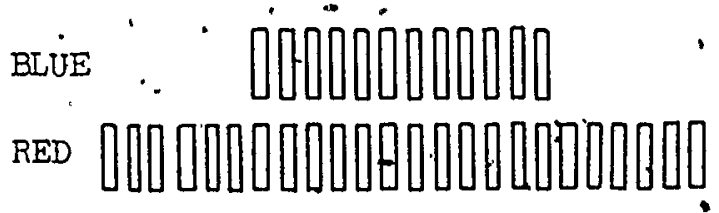

Write a symbol which describes the ratio of blue slips to red slips. $(12 .: 24)$

(b) Now arrange the slips like this:

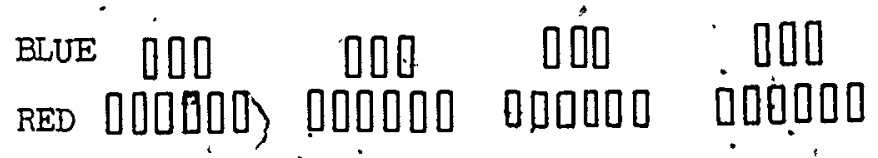

Write the symbol which you think best describes the fatching of blue slips to, red slips. $(3: .6)$.

(c) Arrange the sslips like"this:r

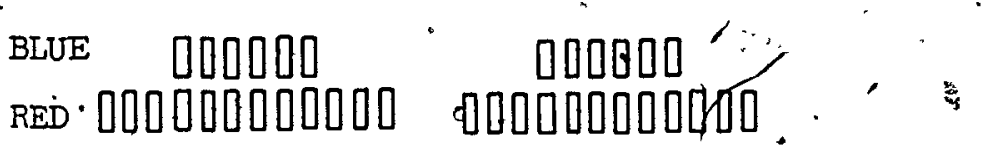

Now what symbol would you use to show the matching of blue slips to red slips?. $(6: 12)$.

(d) Arrange the blue slips so that they are in sets of - 4 . Hów many red slips ${ }^{\circ}$ would be matched with each, set of 4 blue slips? Write the symbol which would best" describe this.matching. (4:8) 
(e) Arrange the blue silps so that they are in sets of 2 . How many red slips would be matched with each set of , blye slips? ${ }^{(4)}$ What symbol best describes this matching?

(f) Arrange the blue slips so there is just i blue slip to a set. 'How manyy red slips would be matched with each $I^{r}$ blue slip? What symbol expresses the ratio of .blue slips to red slips? $(1: 2)$

(g) Complete this table:

\begin{tabular}{|l|c|c|c|c|c|c|}
\hline Blue slips & 12 & $(6)$ & 3 & $?$ & 2 & 1 \\
\hline Red slips & $? ?+4)$ & 12 & $?$ & 8 & $?$ & $?$ \\
\hline
\end{tabular}

(h) Replace each question mark so this mathematical 'sentence is true.
(6)
(6) (4)

$12: 24=?: 12=3: ?=?: 8=2: ?={ }^{\prime} 1: ? "$

(1) Hon many red slips would be-matched with 15 blue slips? "We know that the matching is 1 blue slip for 2 redisilips. So we can write $1: 2=15^{*}: \mathrm{N}$. * We have expressed the idea that 1 blue slip per 2 red slips is the same ratio as 15 blue slips per how many red slips? Instead of one set of 1 biue slip we have 15 sets of 3 slip. "Instead of one set of 2 red slips, we have 15 sets of 2

- slips or 30 slips. So

$$
1: 2=15: 30 \text {. }
$$

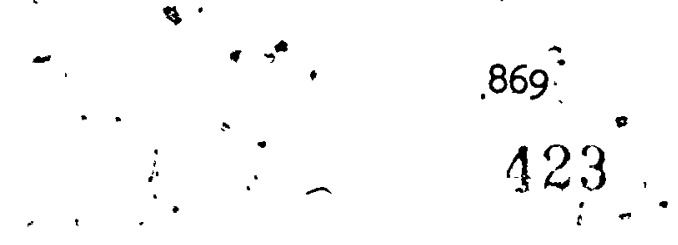


7. Find the number represented by $*$ in each of the $\cdots$ following sentences. Then write the lsentence on your paper. Example: (a) $n=27, \quad$ (a) $1: 3=9: 27$

$$
\begin{aligned}
& \text { (a). } 1: 3=.9: n \\
& \text { (b) } \quad \overrightarrow{l^{\prime}}: 2=8: n \quad(n=16,1: 2=8: 16) \text {; } \\
& \text { (c) } \ldots 1: 4=5: n \quad(n=20,1: 4: 55: 20) \\
& \text { (d) } 2: 5=4: n \quad\left(n=10^{\circ}, 2: 5=4: 10\right) \\
& \text { (e) } 3: 6=9: \dot{n} \quad(n=18, \quad 3: 6=9: 18) \\
& \text { * (f) } 3: 4=15: n \quad(n=20,3: 4=15: 20) \\
& \text { (g) } 1: 4=2: n \quad(n=8,1: 4=2: 8) \text {, } \\
& \text { (i) } 2: 9=10: n \quad(n=45,2: 9=10: 45) \\
& \text { (1) } 4: 5=24: n \quad(n=30, \quad t: 5=24 ; 30) \\
& \text { (J) } 2: 1=24: n-(n=12,2,7=24: 12)
\end{aligned}
$$

.

8. Draw a picfure to show, that this mathematical sentence. is

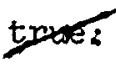

i

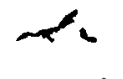

$1: 5=3: 15$

9. Draw a picture to illustrate this:

For every 8 pieces of candy, there were 16 pennies.

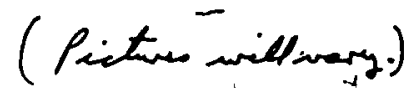




\section{Exercise Set $3+$}

(a). Write two symbols which express the ratio of the number of fish to the numbers of bọ̃s. (io:4.; $5: 2$ )

(b) Write, stwo symbols which express the ratio of the

- I number of boys to

- the number of fish: $(4: 10,2: 5)$

A. . '

(c) Write two symbols which describe ther ratig of the

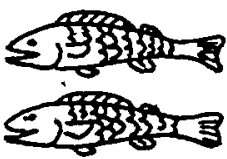

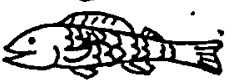

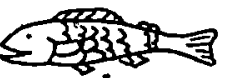
5,3 से का
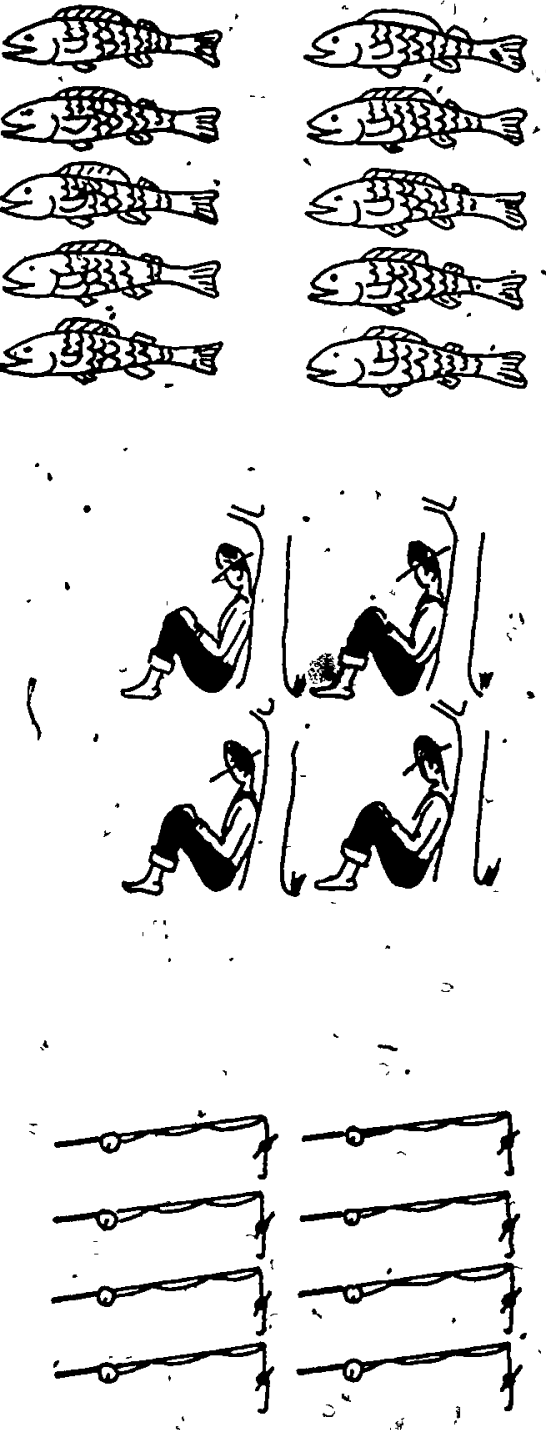
ratio of fishpoles to boys. $(8: 4,2: 1)$.

(d) Write two symbols which describe the $\dot{2}$ $+$

Copy and complete this table.

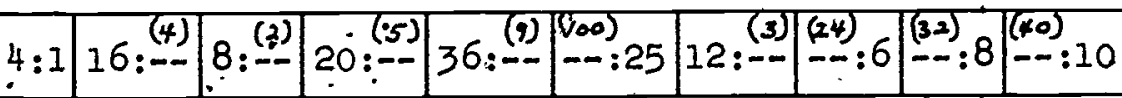


3. Copy and complete each of these three tables. The last 2 names in each table are braintwisters.

(a).

\begin{tabular}{|c|}
\hline $4: 8$ \\
\hline $1:-(2)$ \\
\hline $8:-(x 6)$ \\
\hline$(12)-: 32$ \\
\hline$(2)-: 4$ \\
\hline $12:-(24)$ \\
\hline$(24)-: 48$ \\
\hline$(20)-40$ \\
\hline$(36): 72$ \\
\hline $32:-(64)$ \\
\hline $3:-(6)$ \\
\hline$(52): 1$ \\
\hline
\end{tabular}

(b)

\begin{tabular}{|c|}
\hline $10: 4$ \\
\hline $30:-(12)$ \\
\hline $5:-(2)$ \\
\hline$(20)-: 8$ \\
\hline$(50)-: 20$ \\
\hline$(60)-: 24$ \\
\hline $100:-\div(40)$ \\
\hline $40:-(16)$ \\
\hline$(80): 32$ \\
\hline $1,000:-$ \\
\hline $15:-(6)$ \\
\hline$(25)-: 10$ \\
\hline
\end{tabular}

(c)

\begin{tabular}{|c|}
\hline $6: 10$ \\
\hline$(12):: 20$ \\
\hline$(18): 30$ \\
\hline $30:-50)$ \\
\hline $24:-(60)$ \\
\hline$(48): 80$ \\
\hline$(60): 100$ \\
\hline $54:-(90)$ \\
\hline $36:-60)$ \\
\hline $42:-(70)$ \\
\hline $3:-(5)$ \\
\hline$(92:: 15$ \\
\hline
\end{tabular}

4. Write a mathemätical sentence for each of these situations. Then find the answer.

(a) A car will go 20 mtles on one gallon of gas. How far wifl it go on 5 gallons of gas? $\left(\begin{array}{c}20: 1=5: n \\ n=100 \text { mite }\end{array}\right)$

(b) Elmer threw a basketball through the hoop 3 out of 4 times. If he kept this same record, how. many times would he need to throw to make 24 baskets? 
P536.

5. (a) Write 4 symbols which express the ratio of the number of rabbits to the number of carrots. (amaranow vary. 3:4, 6:8, 9:12, 12:16, it:.)

(b) Write, 4 symbols which exhibit the matching of carrots to rabbits. (an die
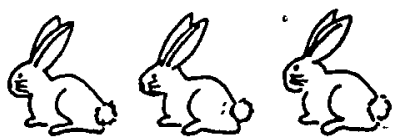

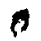
vary. $4: 3,8: 6,12: \lambda$ 16:12, st.)

6. Write a mathematical sentence for each of these.

(a) 6 per 9 is the same ratio as 2 per $\overline{3} .(6: 9=2: 3)$

(b) 6 dinosaurs for 4 cavemen is the same ratio as 3 dinosaurs for 2 cavemen. $(6: 4=3: 2)$

(c) 8 per 3 and 16 per 6 and 24 per, 9 are ail names for the same ratio. $(8: 3 ;=16: 6=24: 9)$

(d) 4 flashlights for 9 boys is the same ratio as to flashlights for how many boys? $(4: 9=20: n)$

7. Write symbols for ratios suggested by the pictures.'.

(a)

$$
\because \text { I. I. I. I. }
$$

(bs)

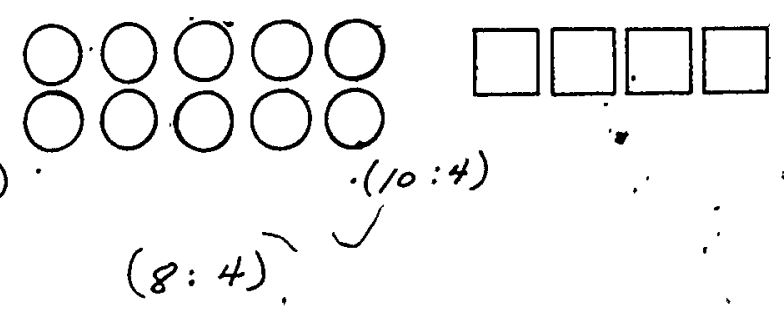

873 
Objective: To develop an art thmetic method of detérmining different names for" the same ratio To give pupils an òportunity to use ratio in the solution of problems

Materials: Flannel board and cut-outs, a variety of objects such as those previously used in this unit

Suggested Teaching Procedure:

The presentation in the pupil text is in sufficient detall to be followed.

Examples $1,2,3$, and 4 i of the Working Together or Exploratory section are concerned with the groupins of members of the two sets. If pupils understand this, they will progress rather easily over the latter part, so be certain they comprehend the 1dea of grouping. The drawing of pictures, encircling the sets, regrouping so the new sets are seen as a separate set, are all helprul devices.

Example 2 presents a method of determining different names for the same ratio without using any manipulative materials. By recalling what they know about factors and primes, children will find this idea a little more understandable. This Example 2 is really. the key to the mathematical solution of proportions. It may take many examples to help children understand the 1dea. Extensive use may need to be made of materials.

Exercise set 4 is long and contains several Braintwisters. AfEer children have worked independently on the exercises, plan to spend considerable time in working with them in checking their answers. Many of the problems are quite difficult bu't should serve as' a good learning situation. You may want to devise problems of your own to check children's understanding if you work with the pupils on this exercise set as a class activi ty.

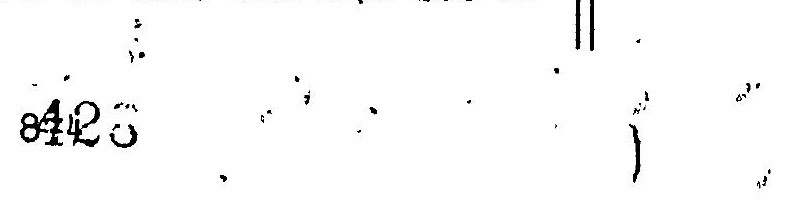




\section{USING RATIOS}

1.: Allce bought" 2 " pencilis for 5 cents. Write the symbol which expresses the ratio of pencils to cents. If Alice

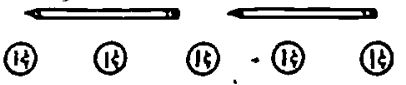
had 10 pennies instead of 5 pennies, how many pencils could she buy? This problem can be solved by 'drawing a plcture such as this:

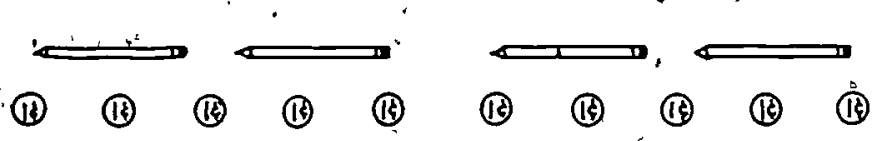

It can be solved by finding another name, for the same ratio, like this:

$.2: 5=n: 10$

$$
\begin{aligned}
& \text { This tells us that } 2 \text { pencils } \\
& \text { per } 5 \text { pennies is the same } \\
& \text { matching as n'pencils for } \\
& 10 \text { pennies. }
\end{aligned}
$$

The symbol, n:lo suggests we have 10 pennfes and that we want to know the number of pencils to match these. We know that $a$ set of 2 pencils matches a "set of " 5 " pennies and that ten pennies are 2 sets of , 5 pennies. So we must have two sets of 2 "pencils each to match the 10 , pennies. We know, then, that Alice could buy 4 pencils for 10 penites. "You could have solved this problem quite easily "in your head", couldn't you? 
2. Could you solve this one "In your head?

Tom was shooting at a target. He made 5 hits out of 7 shots. If, the ratio of the number of hits to the number of shots stays the same, how many hits will he'set in $63^{*}$ shots?

This is a.Iittle more difficult to answer. "It can be written as:

$$
\begin{array}{ll}
: \text { This means that five hits } \\
5: 7=n ! 63 \quad \text { per. seven shots is the same } \\
\text { matchingias. n hits per } \\
63 \text { shots. }
\end{array}
$$

We know we had one set of 7 . shots the first time and

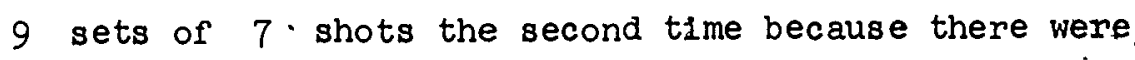
63 shots the second time. So we have. 9 sets of 5 hitsxor' 45 hits per 63 shots.

3. Now let's think about Alice and her penc1ls. How manyi could she buy for 25 pennies? You could figure this out "In your head." You.also can write a mathematical sentence. The members of the two sets are pencils and pennies. The matching is ' 2 pencils for ' 5 pennies. The ratio 2:5 shows how the number of pencils compare with the number of the pennies, We want to know how many. pencils Alice can buy for 25 pennies. So we must find another name for the same ratfo. Wè write $2: \dot{5}=n: 25$. This means 2 pencils - per 5 pennies is the same matching, as $n$ pencils per. .25 " peninites, In our second case, instead of 5 . pennies , 
we have 25 pennies or 5 set's of -5 pennies. Therefore, instead of 2 pencils, we will, have 5 sets of 2 .pencils or 10 pencils. Two different ways of describing the same ratio are 2 " per 5 and 10 per 25 . The mathematical sentence that says thi's is $2: 5=10: 25$. Since $10: 25$ telis us how the number' of pencils compare with the number of pennies; we see that we can buy 10 pencils for. 25 pennies. To find how many pencils we can buy for 15 pennies, we use the mathematical senterice $2: 5=n: 15$. We are asking, "2 per 5 is how mary per 15?"
(a) How many sets of 5 are there in 15? (3)
(b) How many sets of 2 pencils should we have? What should $n$ be? ${ }^{(6)}$ If the first numeral, refers to pencils and the second to pennies," we see that we should get 6 pencils for 15 : cents.

4. Jake bought 6 tharbles for io cents. How mach would 9 marbles cost? Here we have two sets. The members of the first set is marbles and the member's of the second 'set 1s cents. The ratio of the number of marbles to the number of pennies 1s :6:10. our mathematical sentence is $6: 10=9: n$. . In this problem this is interpreted, "Six marbles per 10 pennies is the same ratio as 9 marbles per in pennies." We know'that. 9 jis not a multiple' of 6. - That is, a set of 9 mémbers cannot be separated into sets of 6 members each. 
P540

So let's find some other name for the ratio ' $6: 20$. A name which uses smaller numbers might be found. Think of 6. per 10 as shown in this picture.

(16) (1F) (16)

(IC) (IF)

(IC)

(5)

Q.

We' see that .6 per 10 is also 2 sets of $3^{\circ}$ marbles for 2 sets of 5 pennies .s. Therefore, 1 set of 3 marbles can be matched with ' 1 set of 5 pennies. 
1.- Complete these symbols so that each is a name for the 'rat1o' 2 :

$\begin{array}{llll} & \text { (a) } & 4: ?(6) & \\ \Rightarrow & \text { (b) } & ?: 12 \text { (8) } & \\ & \text { (c) } & 6: ? & \text { (9) }\end{array}$

(d) $12: ?(18)$

(e) 100:? (150)

(f), ?:15 (10)

2." This picture is of wagons and-pioneers.
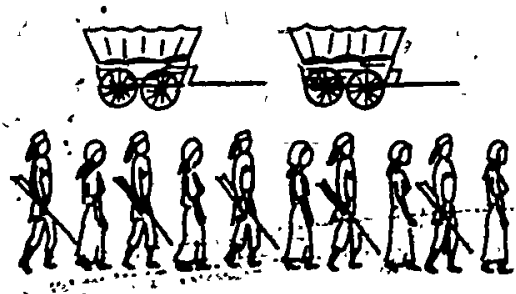

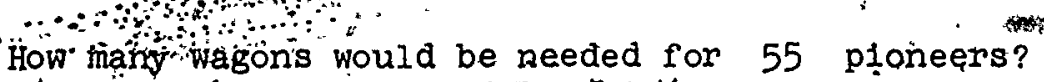

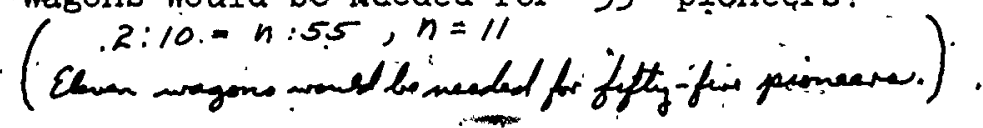


P542

3. Write a mathematical sentence for each of these situations. Let $n$ name the unknown number. Then find the value of $n$.

(a) Druid can run 50 yards in 8 seconds. If he could keep going at this same speed, how long would it take him to mun 300 yards?

* $(-50: 8=300: n, n=48$ it wed to h him forty -eight

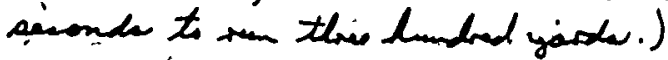

BRAINTHISTER: How long would it take to run 175 yards?

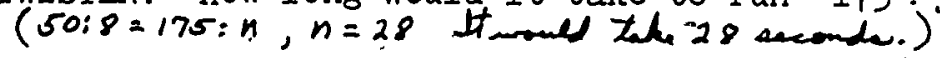

-(b) 18 birds live in 10 birdhouses. If the ratio of. - birds to birdhouses stays the same, how many birds could live in 30 birdhouses? ( $18: 10=n: 30^{\circ}, n=54$

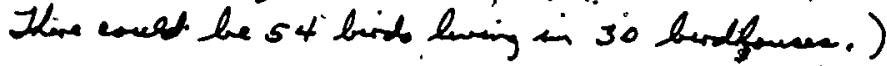

(c) Study this picture. How many boats would be needed for. 30 people? $(6 / 10=n: 30, \underline{n}=1.8$ the womble be 18 but sealed for 38 parole.)

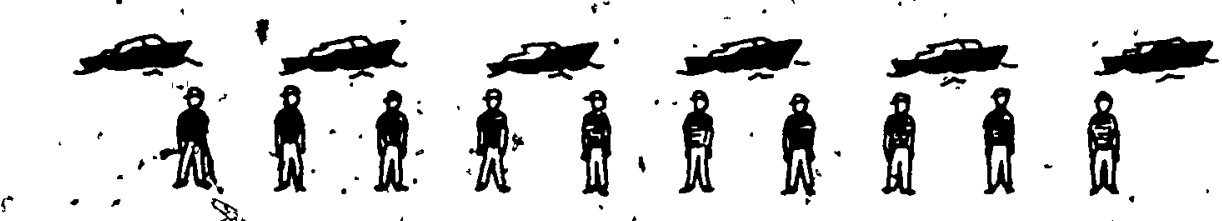

t.

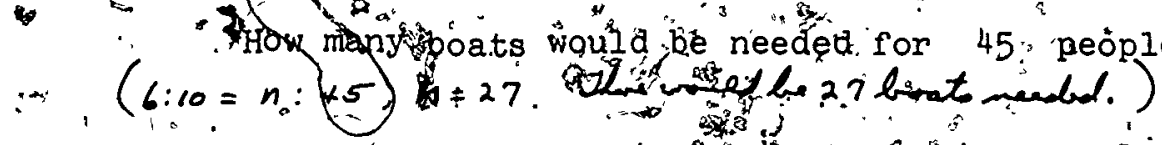

(d) Glen had 7 ideas in 2 minutes for food for a fifth grade parts If he jeeps

this same ratio, how many ideas wi l the have in 8 minutes? $(7: 2=n: 8,28$, the mimed $=$ hue 28 .
$\therefore \quad$
$\quad 880^{\circ}$

434 
' TRYING SOMETHING NEW

Do you think you could solve some ratio problems like thits" without using pictures? Using the idea, we know that $6=2 \times 3$ and $10=2 \times 5$. We see that 6 and 10 have a common, factor. 2. We can divide both 6 and 10 by 2. When we divide by 2 we get 3 and 5 . We can write $6: 10=3: 5$, we know these' are names for the samé ratio. Let's use this second name for the ratio and write: $3: 5=9: \mathrm{N}$. We are asicing, "3 per 5 . is the sàme ratio as 9 per how many̆g" Now we can see that we have 3 grobus of 3 marbles so live need 3 groups of 5 pennies or 15 pennies. Thus, 9 marbles would cost $15^{\circ}$ pernies.

I. Look at the mathomatical sentence wich describes this si tuation.

Mr. Smith can drive 50 miles in 1 hour. If he drives at the same speed, how many hours 111 it" take to drive 250 miles? $(50: 1=250: n ; n=5$

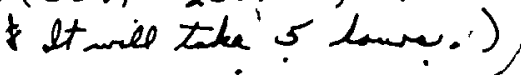

2. Try this one on your own

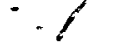

- Set A is a set of names for, the same ratió. Find

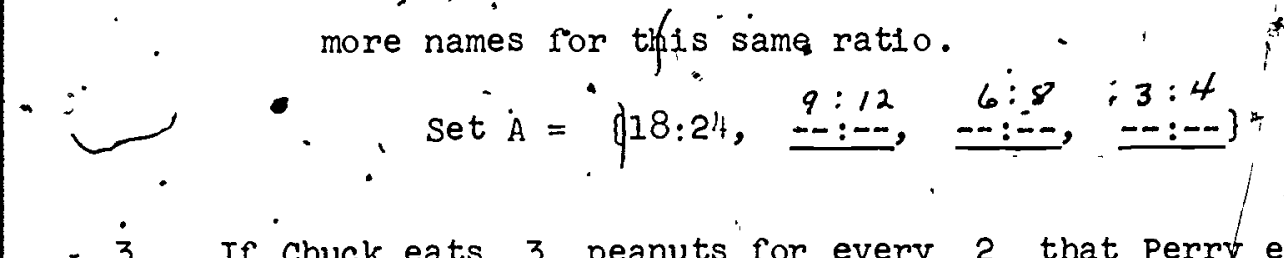

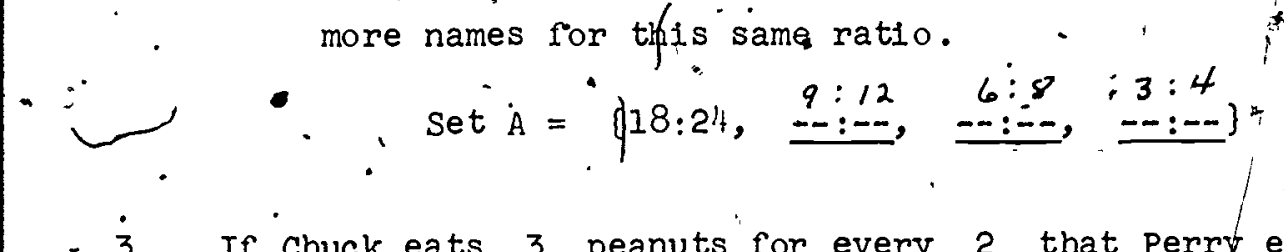

- 3. If Chuck eats 3 peanuts for every. 2 that perry eats, how many peanuts will chuck eat if Perry eats 10? ( $3: 02=. n: 10, n=15$ cluck will eat is paranta)

$8 \overline{81}$

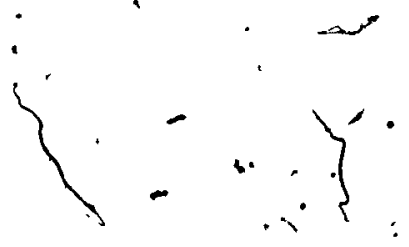


544<smiles>CCCC(C)CC</smiles>

4. "If a car travels 10 miles in 25 minutes, how far will. the car travel in, 75 minutes? ( i0:25 = n:75, $n=30$ Lh ear will travel 30 miles. )

5. Tell which of those sets are names for the same ratio: (Tone)

$$
\begin{aligned}
& \text { Set } A=\{20: 16, \quad 5: 4, \quad 10: 8\} \\
& \text { Set } B=\{12: 18, \quad 6: 9, \quad 2: 3\} \\
& \text { Set } C=\{18: 24, \quad 3: 4, \quad 9: 12\} \\
& \text { Set } D=\{32: 1 \cdot 6, \quad 4: 2, \quad 16: 8,-2: 1, \quad 8: 4\} \\
& \text { Set } E=\{48: 32, \quad 6: 4, \quad 24: 16,12: 8,3: 2\}
\end{aligned}
$$

6. If a bank charges 4 dollars for the use of 100. dollars, how much would It charge for the use of 50 dollars? ( 4:100 =n:50, n=2 th hank would charge tore dollars.)

7. How much would the bank in exercise 6 charge for the $\because \quad$ use of 250 dollars? (4,100 $=n: 250, n=10$ Ityobank Wrong charge ten dollars.)

8. If an airplane flies 570 miles in 1 hour, how far would rit fly in $i \frac{1}{2}$ hours? ( $2 \frac{5}{2}$ lam is 5 hag hame, - thar is 2 tiff-hine. 570:2 = 285:1, $285: 1=n: 5$,

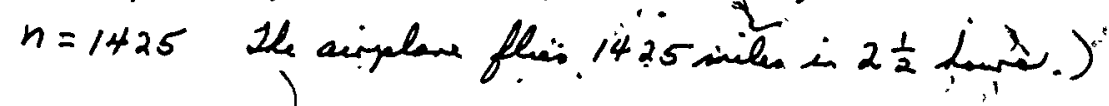

$\therefore$.

882

$\because$ 
You have seen that a ratio such as $2: 3$ is used to describe a property of two sets. It means that there are 2 objects in one set for 3 objects in another set.

(x)

Some other names for the ratio $2: 3$ are $1: 6,10: 15,120: 30,10: 60$, and $2000: 3000$. . You could write māny more.

In finding other names for the ratio $2: 3$ you can multiply the numbers 2 -rand 3 . by the same number, if the number is not, zers.

The pairs of numerals in $2: 3,4: 6$, 20:30 represent the same ratio. We can write $2: 3=4: 6$ and $2: 3=-20^{0}: 30^{-}$and $4: 6=20: 30^{\circ}$. If we know that $4: 5=n: 15$, we can find the number represented by $\mathrm{n}$. It is 12 . Now le us șeê how some of these things we have just said about ratios are. similar to things we can say about rational numbers. The symbol for the ration 2 to 3 is 2:3. The symbol for the number two-thirds is $\frac{2}{3} m$ 
Both symbels use the numerals -2 and 3 . other names for the rational number $\frac{2}{3}$ are $\frac{10}{15}, \frac{20}{30}, \frac{40}{60}, \frac{2000}{3000}$

If we know that $\frac{2}{3}$ and $\frac{4}{6}$ and $\frac{20}{30}$ are. names for the same rational number, we can vrlte

$$
\text { - } \frac{2}{3}=\frac{4}{6} \quad \text { and } \frac{2}{3}=\frac{20}{30} \text { and } \frac{4}{6}=\frac{20}{30} .
$$

In finding other names for the rational. - number $\frac{2}{3}$ you can multiply 2 and 3 by the same number, if the number is not zero.

If we know that $\frac{4}{5}=\frac{n}{15}$, we can find the number represented by $n$. It is 12 .

You can see that ratios and rational numbers are al1ke) in some ways. After you have studied more about rational numbers, you can see other way, in whisch they are al1ke. 
P547,

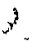

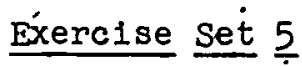

1. $\therefore$ Write the symbols for two ratios using the numerals 3. and $5 . \quad(3: 5,5: 3)$ :

2. Write the symbols for two rational. numbers using the $\because$ numerals 3 and $5 .\left(\frac{3}{6} ; \frac{5}{3}\right)$

3. Is 9:10 the name for a number or a ratio?. (a rato)

4. Is $\frac{7}{8}$ the name for a number or a ratio? (a number)

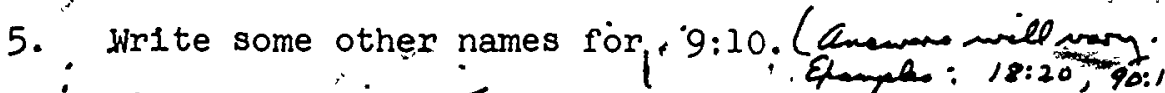

6. Write some other names for 7\% (Annamese will wain.

Etargan: $\left.\frac{14}{16}, \frac{15}{40}, \frac{20}{80}, 5 t.\right)$

7. If ' $n: 25$ ' is another name for $6: 5$, what number does noppresent? (30)

8. If $3: 10=18: n$, then $n$ represents what number? (60).

9. ' If $\frac{6}{9}$. is another name for $\frac{\mathrm{n}}{81}$, what number does $n$ represent? $(54)$

$$
\therefore
$$

10. 'If $\frac{11}{6}=\frac{10}{n}$, then ${ }^{\prime} n$ represents what number? $(60)^{\prime}$ 
Chapter 10

NFIEW

PURPOSE OF UNIT

The purpose of. this unit. is to provide a review of some of the concepts and techniques which the pupils have learned in Mathematics in grades' Four and Five. It is rot necessary to postpone "the review pro=; vied by this unit until the completion of i. Chapter 9: Parts of it maybe used at appropriate places during the year. 'For example; the -review sections that pertain' to the first five chapters of the Fourth Grade might be used after completion of Chapter 5. "The optimum method of use can be determined. best by the teacher. It is suggested, however, that this, entire unit be used as review at 'the end of Grade 5 al though it may have been used "piecemeal" prior to that time.

7 


\section{TEACHING \$ROCEDURES}

'The teaching procedure will depend upon

- the amount of review that 1s reeded. Some. pupils may be able to answer all trie questions and work all the problems with. very little or no assistance from the teaoher. Other pupils may. have șome difficulty: In general, it is " suggested that the puplls be glveh the review on a particular unit without any other preparation than that they had while studying the undt. After the pup17s have responded to the review questions in accord with the given directions, the teacher can determine, whether there needs, to be some instruction to the entire class and to Individuaj pupils. Pupils. who have" difficulty with items in the review should be encouraged, to turn to their copy of the unit which was reviewed in order to correct the1r. own errors. If the puplls have done, well enough to indicate no réview of a particular unit is needed, they pase well undertake the next one.

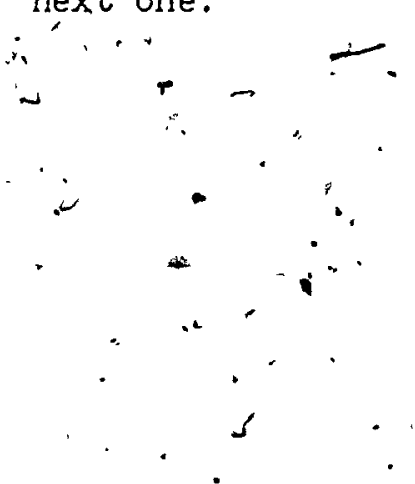


P549

Chapter 10

REVIEW

CONCEPT OF SETS

s.

.

Number the exercises as they are numbered here and write. the answers on your paper. If you do not know the answer to an exercise, write the number of the exercise and leave the

* space beside 1t blank. Later you may. be able to f1ll in the answers that you did not know.

$\because 1$. Set $A$ is the set'of whole numbers greater than io $\therefore$ and less than 20: Write the members of A.

( Let $A=\{11,12,13,14,16,16,17,18,19\})$

$\therefore$ Write a sentence that describes this set:

$$
\mathrm{c}=\{\mathrm{u}, \mathrm{v}, \mathrm{w}, \mathrm{x}, \mathrm{y}, \mathrm{z}\}
$$

(Set $C$ is th set of th lat sid lettre of thalplibet)

$\therefore 3$. If ${ }^{\prime} A=\{3,6 ; 2,12,15\}$ and $\cdot B=\{0,6,12,18\}$

what is $A \cap B$ ? $(A \cap B)=\{6,12\})$

- $\therefore$ what is $A \cap$.

$\because 4 .^{\circ}$ Using the sets $A$ and $B$ in the preceding exercise, what is. $A \cup B$ ? $\{A \cup B=\{\{0,3,6,9,12,15,18\})$.

5. If $R$ is the set.of: the "states of the U..S.A. that" are east of the Mississippl River and $S_{j}$ is the." set of states that touch the 'Pacfifie ocerin,

$\because \because$ what is in $\cap \mathrm{s}$ ?

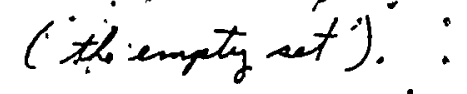

889 
P550

NUMERATTION.

1. Write the letter of each part of this- exercise on your paper. Then to the right of the letter write the words, or word, that you would use to.fill the blank.spaces, or space.

a) $19=\cdot($ thee $)$ fives and (four) ones.

b) $27=$ (fine) rives and (taro) ones:

c) (for) nines and (four) ones $=40$ :

d) (five) elghts and (no) ones $=.40$.

e) (fine) sevens and (fire) ones $=-40$.

r) (sif) s ses and (four) ones 40 .

g) 546 is 5 hundreds, (four) tens, and 6 . ones.

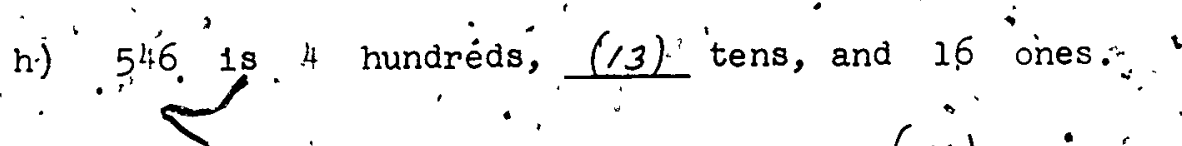
和 546 is 5 . nundreds, 3 tens, and $\frac{(16)}{2}$ ones: $\therefore 1 \%$

2. Express $B, 47$ in 3 different ways as in 1,8$)$, (h), 1). Letter the three ways a), b), and a $^{\circ}$ c). (Amanare will wery.)

890 
P551

3. Write the letter for each part on your paper. If the - statement is right, then write yes after. the letter. If it is wrong, write no.

a) 3729 is 37 tens plus is ones. (no) :

b) $734=600+120+2 \dot{4} \cdot(220)$

c) ten hundreds plus forty tens plus nine ones is the same as 'one thousand forty-nine. ( 20 ; its 1409).

d) $10,129=10$ thousands plus ten hundreds plus nine

$\lim _{\rightarrow \infty}$ - ones. (no)

4. Write the letter for each part on your paper. Then beside it write, $\dot{<,}>$, or $=$, whichever makes each" a tire sentence.

a) $8+4 \Rightarrow 1 i$.

b) $(1+3) \dot{j}<(9+11)$ :

a) $(3+4)+4>1+(5+4)$.

d) $\cdot(15+14)=(7+5)+17$.

e) $(7+4)+2 \leq(7+5)+2$.

f) $(6+5)-2 i<(13-7)+6$.

8) $1 \cdot(3 \times 7)+9 \leq 31$.

n) $.(60+3)+(10-3)=(11-2)$.

891

444. 
P552

- PROPERTIES AND TECHNIQUES OF SUBTRACTION, I.

1. Write the letter of each part on. your paper. Then beside it write the number represented by $n$ in that part.

a). $8+3=n \cdot(n=11)$

b) $144-n=29 . \quad(n=115)$

c). $n=1001-2 \cdot \quad(n=999)$

d) $3-3=n \cdot \quad(n=8)$

e) $\theta+0=n . \quad(n=0)$

f) $99+2=n . \quad(n=101)$

2. Write on your paper the letter for each mathematical sentence that is true. ( $a, d)$

a) $9+4 .=13$.

b) $17-9=9$.

c) $88-64=34.0$

d) $45+5=50$.

e) $: 36+37=.83$.

3. Are some of "the mathematical sentences in Exercise 2 false? If a sentence is false, rewrite it and change one number in $1 t$ so that it will be true. Letter them the . same as in Exercise 2.

(b) $18-9=9$ or $17-9=8$ or $17-8=$

(c) $88-64=24$ ar. $88-54=34$ or $98-64=34$

(e) $36+37=73$ of $36+47=83$ or $46+37=83$ 
4. Write the letter of each part on your paper. Then beside .It write the number that you would use to fill the blank.

a) If $19-10=9$, then $19-9=(10)$.

b). If $23-11=12$, then $11+12=(23)$.

c) If $13-10=3$, then $13^{\circ}-9=$ (4).

5. Write the letter for each part on your paper, Then beside It write the one of these, $>$ or $<$, that you wotald use to make a), b), and c) triè sentences.

a) $(65+42)<(65+43)$.

b) $(300+700) \leq(400+700)$.

c) $(1300+2000)<(1300+3000)$.

6. Write the letter for each part on your paper. Then" beside it write the answer to the question.

How many untts must be marked on "a number line to finḍ $z, s, m, p$, or ' $n$ in eachoof these mathematical. sentences?

a) $14+17=2 z . \quad(z=31)$

b) $139-s=40, \quad(s=99)$

c) $m=20+40 . \quad(m=60)$

d) $p \dot{p}+17=30 . \quad(p=13)$

e) $n-2 \dot{8}=13 \dot{0} \quad(n=41)$ 
7. Write the letter for each part on your paper. Then bestde it write the number you would use for the $p$, $q$, or $r$ in the mathematical sentence.
a) $p-8=24 \quad(32)$.
f) $r=18-18(0) \cdots$
b) $q=13-4+29)$
8) $p-40=30 \quad(70)$
c) $\dot{7}-: 5=\dot{r}$
h) $p+r=0 \quad(p=0, r=0)$
d) $20-p=12$
e) $14-.9: 14^{\circ}(0)$
1) $. p+q+r=0(p=0, q=0, r=0)$
j) $: 10-p=10$

8. on your paper write a mathematical sentence for each prdblem. Then solve to find $n$. Write an answer, sentence.

a) There were 37. cows in a pasture. Eight of them were black. How many cows whte not black?

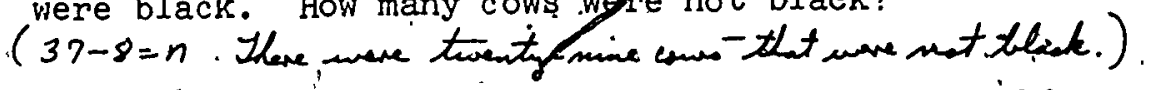

b) Jim has 92 coins in a coin folder. It will hold 150 coins. How many more coins will the folder hold? ( $92+v_{1}=150$ the foldor will hald fifty-eight mand coines.)

c). Margy practiced her flute lésson for 35 minutes mon Monday, 30 minutes on Tuesday, and 45 minutes on Wednesday. How many minutes dia she practice on all these days? $(35+30+45=n$, Thargy thentent one hundad'.

d) A school library had 488 books. The next year 205 books were added. How many books were then in tine

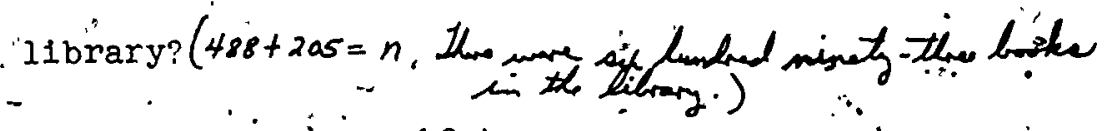


9. Write a different mathematical sentence for eàch of the." - following which iliustrates how the numbers in each. sentence are rélated.

a), $7+2=9(9-2=7$ or $9-7=2)$

b) $10-4=6^{\circ}(10-6=4$ of $6+4=10)$.

c) $30+30=60 \quad(60-30=30)$

d) $x-s=z$ (' $z+s=x$ or $x-z=5)$

ie) $n-5=2 \cdot(2+5=n$ or $n-2=5)$

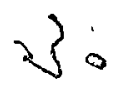

10. Write on your paper the letter for each sentence that is true. $\left(a^{*}, b, c, e^{*}\right)$

a) $.20+11=11+20$.

b) , $103+301=100+304$.

c) $((6+5)+4=4+(6+5)$.

d) $1,207+2,011=1,102,+7,021$.

e) $n+p=p \cdot n$.

11. Some of the statements are true because of the associative property fod some becalise of the commutative property. Write the letter for each part on your paper. Then beside it write associative or commutative to show that you low which property is used.

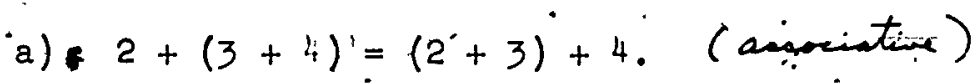

i) $(18+19)+(39+12)=(39+12)+(18+19)$.

c) $(8+9)+6=(9+8)+8 .($ commetion $)$

d) $"(8+9)+6=8+(9+6)$. 
P556

PROPERTIES OF INLTIPLICATION AND DIVISION

1. Copy the letter for each part on your paper. Then beside it write the one of $j,\langle\dot{\rangle}=$ that you would use to fill the blank space so that a) through n) will be true sentences.

a) $5 \times 5 \leq 4 \times 8$.

b) $6 \times \dot{8}=8 \times 6$.

c) $9 \times 5<6 \times 8$.

d) $94 \leq 40^{4}=6 \times 9$.

e) $7 \times 9 \longleftarrow 8 \times 8$.

f) $8 \times 7 \leq .6 \times 10$.

g) $5 \times .9>7 \times 6$. h) $8 \times p>n \times 7$.

1) $140660<9 \times 9$.

j) $9 \times 4=6 \times 6$.

k) $8 \times 7 \geq 9 \times 6$.

1) $\times 4^{*}=4 \times \mathrm{p}$.

m) $7 \times 7 \geq 6 \times 8 \geq 7 \times 6$.

n). $4 \times 8 \leq 7 \times 5 \leq 6 \times 6$.

2. Copy the letter for each part on your paper. Then beside each letter write the number that you would put in the blank to make a) through $h$ ) true sentences.

a) $72 \div 9=(8) \times$

b) $32^{\circ}+(8)^{\circ}=4$.

c) $(56)^{\prime}+8=7$.

d) $63 \div(7)^{2}=9$.

e) $28 \div 7=$ (4).

f) $(8 \times 3)+(3)=8$.

g) $(12+3) \times 3=(12)$

h) $(9 \times(4))+4=9$.

896

449 
P557.

3. We want you to use the Distributive property of Mulipiceation. Study this example to see how we rename, 17, then how we use the Distributive propery of Multiplication.

$4 \times 37=4 \times(10+7)=(4 \times 10)+(4 \times 7)=\cdot 40+28^{\circ}=68 . . \therefore$ Now write each part on your paper and use the method shown

- In the example. Part d) is begun for you but it is not f'inished.
a) $7 \times 12=4 \times\left(10^{\circ}+2\right)=(4 \times 10)+(4 \times 2)=40+8=48$.
b) $6 \times 19=6 \times(10+9)=(6 \times 10)+(6 \times 9)=60+54=114$
c) $7 \times 26=7 \times(20+6)=(7 \times 20)+(7 \times 6)=140+42=182$
d) $4 \times 153=.4 \times(100+50+3)=(4 \times 100)+(4 \times 50)+(4 \times 3)$
e) $\quad 5 \times 34=5 \times(30+4)=(5 \times 30)+(5 \times 4)=150+20=170$
f) $9 \times 22^{\circ}=9 \times(20+2)=(9 \times 20)+(0 \times 2)=180+18=198^{\circ}$

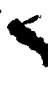


P558

I $\therefore$ it write the number that you would put in the blank space, - or use for the letter in the sentence, so that each of the following will be a true sentence.

a) $\because 6 \times(9)^{\circ}=54$

g) $8 \times q=48$.

(6)

b), $8 \times 8=(64)^{\circ}$.

n) $7 \times n=0$.

(0).

c) $7 \times 9=(63)$.

1) $n \times n=9 .(3 \times 3=9)$

d) $8 \times(9)=72$.

j) $(n \times n) \times 4=36\{(3 \times 3) \times 4=$

e) (0) $\times 7=0$.

k) $24 \div 6=q$.

(4)

f) $9 \times 9=(8.1)$.

6. Write the letter for each part on your paper. Then beside it write the one of $>,\left\langle,^{\circ}=\right.$ that you would put in the blank space so that each of the following $w 111$ be a true sentence.

a) $7 \times 4^{\circ} \geq 9 \times 3$.

b) $9 \times 5 \leq 6 \times 8$.

c) $94-.40^{5}=6 \times 9$.

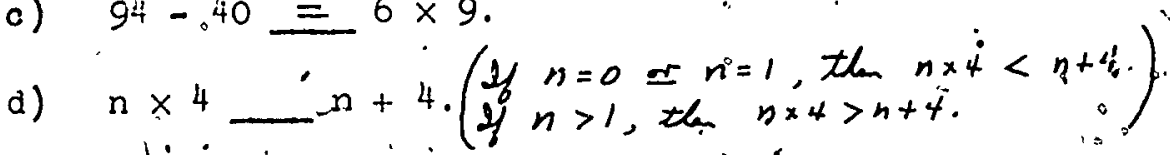

e) $6 \times 1<5 \times 7 .>8 \times 4 \%$

f) $8 \times n \geq 7 \times n$.

898

8

451 
7. Find the missing number. Write your answer on your paper beside the letter for each part.
a) $36 \div 4=(9)$
b) $81+1=(9) \cdot \because$
c) $28+\underline{(4)}=7$
d) $(72) \div 9=8$.
e) $(16) \div 4=40^{\circ}$
f) $36+6=\frac{(6)}{2}$

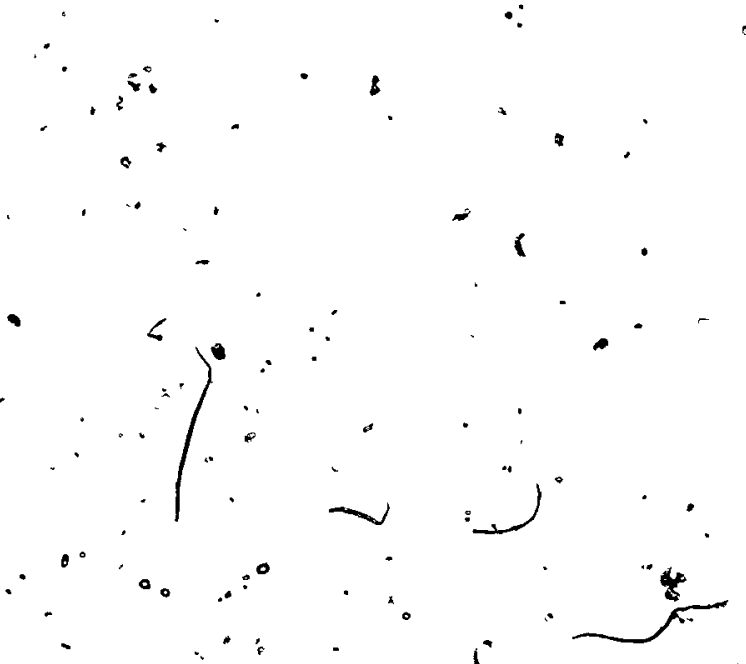

8.1 Are the following statements timue? Write yes or on your paper beside the letter for each part.
a) $7 \times 4=4 \times 7$. (yea).
b) $12=5=5-12$. (no)
a) $10-5=5 \div 10 .(\mathrm{mo})$
d) $61+9=9+6$ (

e) $5 \times 34=5 \times x^{\circ} \cdot(2 \times 17)^{\circ}$. (yea)

f). $(2 \times 5)^{0} \times 3=2 \times(5, \times 3)$. (yea)

g) $25 x^{2} \times 8=(20+10+5) \times 8$ (yin)

h) $51 \times .49=(50 \times 50)-1$.

1) $80^{\circ}+5=(80 \div 10)+2 .(2 n)$. 
2. Write the letter for each part on your paper. Then beside the letter urite true if the statement is true; if the statement is not true, write false:

$\therefore$ :

a) Space is a set of points. (thue)

b) A curve is a set of points. (twe)

c) This is a model of a simple closed curve

d) 'A' ray has one endpoint. (the).

e). A Iine segment has one eadpoint. (false) (thes)

f) A Iine hasn't any endpoints. (the)

g) There is only one plane in space. (foli)

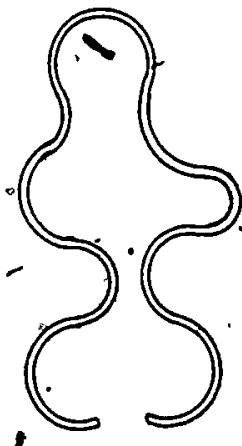

h) A plane may contain man̆y lines. (toue)

i) Two points in space may bé contained in more planes than ean be counted. (true)

j) Three points not on a straight line are in one and only.one plane. (thine)

k) Ail the radil of a circle have the same length. (trie)

1) The union of two rays 'with a common endpoint is called an angle. ('trui')

m) A triangle does not contain its angles. (fabe) 
P561

PROPERTIES AND TECHNIQUES OF ADDTTIQN AND SUBTRACTION, II

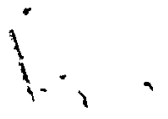

1. Copy each of these on your paper." Then find the sum in each.
a)
b)
c)
d)
e)

79

327

3287

17289

46060 .

42

648

4925

42716

36

905

. 6776

88
$\left.\frac{75}{320}\right)$

$\frac{36}{(1916)}$

$\frac{83475}{(143,480)}$

25349

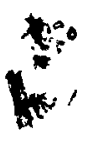

2. Copy each of these on your. paper. Then subtract. After the subtraction, undo each one to show that your, ianswer to the subtradtion was correct.
a): "undo"
b)
"undo"
$\frac{724}{912} \because \frac{724}{1636}$

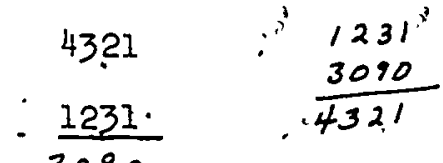

c) "undo"

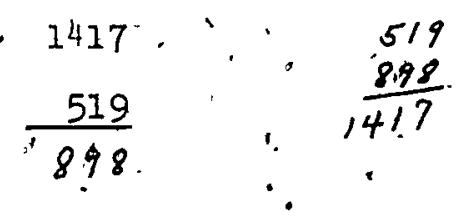


3. ion your paper write a mathemátical sentence for each part: Then solve to find the fanswer to the proplem. Write an answer sentence.

a) Don has 600 stamps. He pasted" 342 in h1s album. How many are left to be put in the album? $(600-34 k=n$ Don hav 258 stampe left to be put in thi allem.)

b) A sehool's stadium has 12320 seats. The schóol has sold 6il80 tickets for a game. How many tickets are

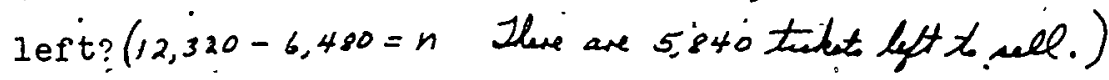

c) John wanted to collect 500 shells. He had 188. His uncle gave him 123. How many more did he need to complete his collection? $(188+123+n=500$

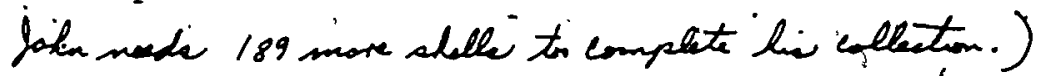

d) Suppose you are going on an automobile trip of 1260 miles. "You travel " $418^{\prime \prime}$ miles the first day and 390 miles the second day. How many miles must you travei on the third day to complete the trip? $(4 / 8+390+n=1260$ You munt tranel 452 mibe to complete the tap.)

i). The earth is $92,900,000$ miles from the sun. Mars 1s $141,000,000$ 'miles from the sun. How much closer to the sun is the earth than Mars is? (141,000,000-92,900,000 =n' Earth is 48,100,0000miles elocer th the sem than mare.) 


\section{'P563}

TECHNIQUES OF MULTI LICATION AND DIVISION

1. Stuidy the exarple in part a). Then copy on your paper the exercises in $b), c)$, d) and multipiy as we do in,a):

$$
\begin{aligned}
& \text { a) }: \quad \text { b) } \\
& \text { c) } \\
& \text { d) } \\
& 38 \quad 95 \\
& 76 \\
& \frac{24}{32}=4 \times 8 \quad \cdot \frac{24}{20} \pm 4 \times 5 \\
& 120^{\circ}=4 \times 30.360 .=4 \times 90 \\
& 160=20 \times 8 \\
& 100=20 \times 5 \\
& \underline{600}=20 \times 30 \frac{18.00}{2280}=20 \times 90 \\
& 32 . \\
& 85 \\
& \text { sum } 912=24 \cdot \times 38 \\
& 12=2 \times 6 \\
& 79 \\
& 140 .=2 \times 70 \\
& 180^{\circ}=30 \times 6
\end{aligned}
$$

2. Copy' each of these problems in multiplication on your paper and then find the product in each.
a) 432
b) 516
c) 237
d) 489 .'
. $\left(\frac{26^{\circ}}{11,232)}\right.$
$(24,252)$
$\frac{6 \dot{9}}{(16,35,3)}$
$(27,384)$

3. The parts of this exercise are division problems: In Each one. there will be. a remainder. Perform each one of the divisions on your paper. Then, write a mathematical sentence as in.
a) to show thé remainder
Lettẹr each part as shown here.
a). $\dot{6} 21 \div 15$;
Sentence: $" 621=(15 \times 41)+6$
b) $\left(\begin{array}{l}983+-24 \div ; \\ (983 \equiv(2+x 40)+23)\end{array}\right.$
d) $1934 \div 21 \cdot(1934=(21 \times 92)+2)$
(c) $i \quad 671 \div 61$

$$
(67 i=(6 ; \pm i 11)+0)
$$


4. A school building has 40 rooms. The school ordered 28 new chalrs for each room. When the chalrs were delivered there wère 1128 chairs, Were there: too many or not enough? On your paper show how you 'would find the answer to the question: $(40 \times 28=1120 \quad 1120<1128$ The ware to. . chavo delinared.)

5. Show on your paper how you, find the answers to the question's in this problem, The Parent Teacher Association of a school' had 324 memberst These were divided into teams of 8 members each.

How many teams couid there be with 8 members?

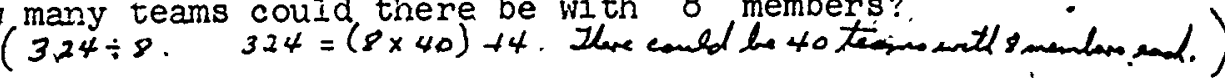
Were any members of thè Association "left oyer"? (yex) What is the largest number of teams, that could have just 8 members and how many, teams would there be that have less $\Rightarrow$ than 8 members so that all 324 persons would be in a

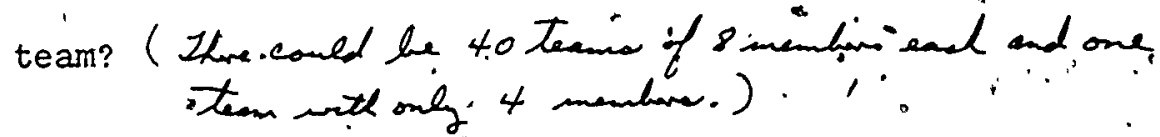

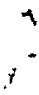

6. Show on your paper how you find the arswers to the question in this problem.

There are 16 piles of blocks. In each pile there are, 144 blocks. How many blocks are there in the 16 plles?

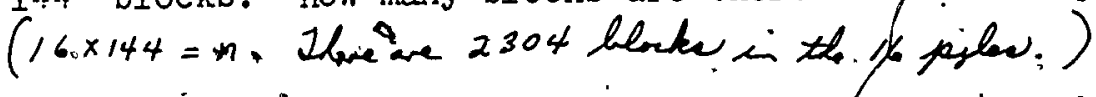
Hoin many more blocks would be needed to have 2400 blocks? $(-2304+n=2400$. The womel" need 96 have 2400 blate. )
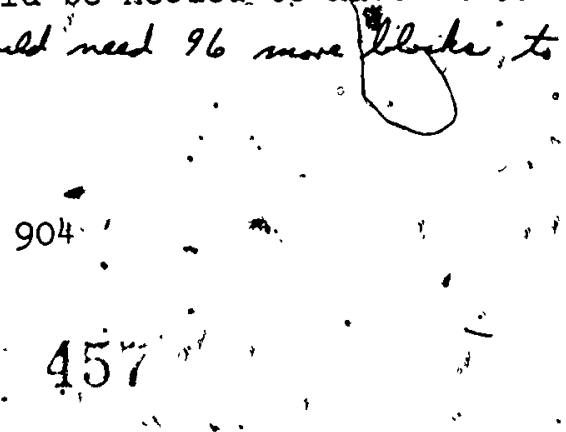
REĊOGITTION OF COHHON GEOIETTRIC FIGURES

1. Write the letter for each part of this exercise on your paper. Then beside it write the words or ward that you would use to f1ll the blank spaces or space.

a) A polyogon which is the union of three ine segments is called a (trimgle).

b) A polygon which is the (umion) of (four) line segmęrits is called a quadrilateral.

c) The endpoints of the line segments in the poilygons in
a) an'd
b) are called
(wantices).

2. Here'are some line segments. The segment $A B$ is congruent to some of them. Write the names of the segments to which $\overrightarrow{A B}{ }^{\prime}$ is con̈riment. ( $\left.\overline{C F}, \overline{O P}, \overline{O M}, \overline{S T}\right)$.

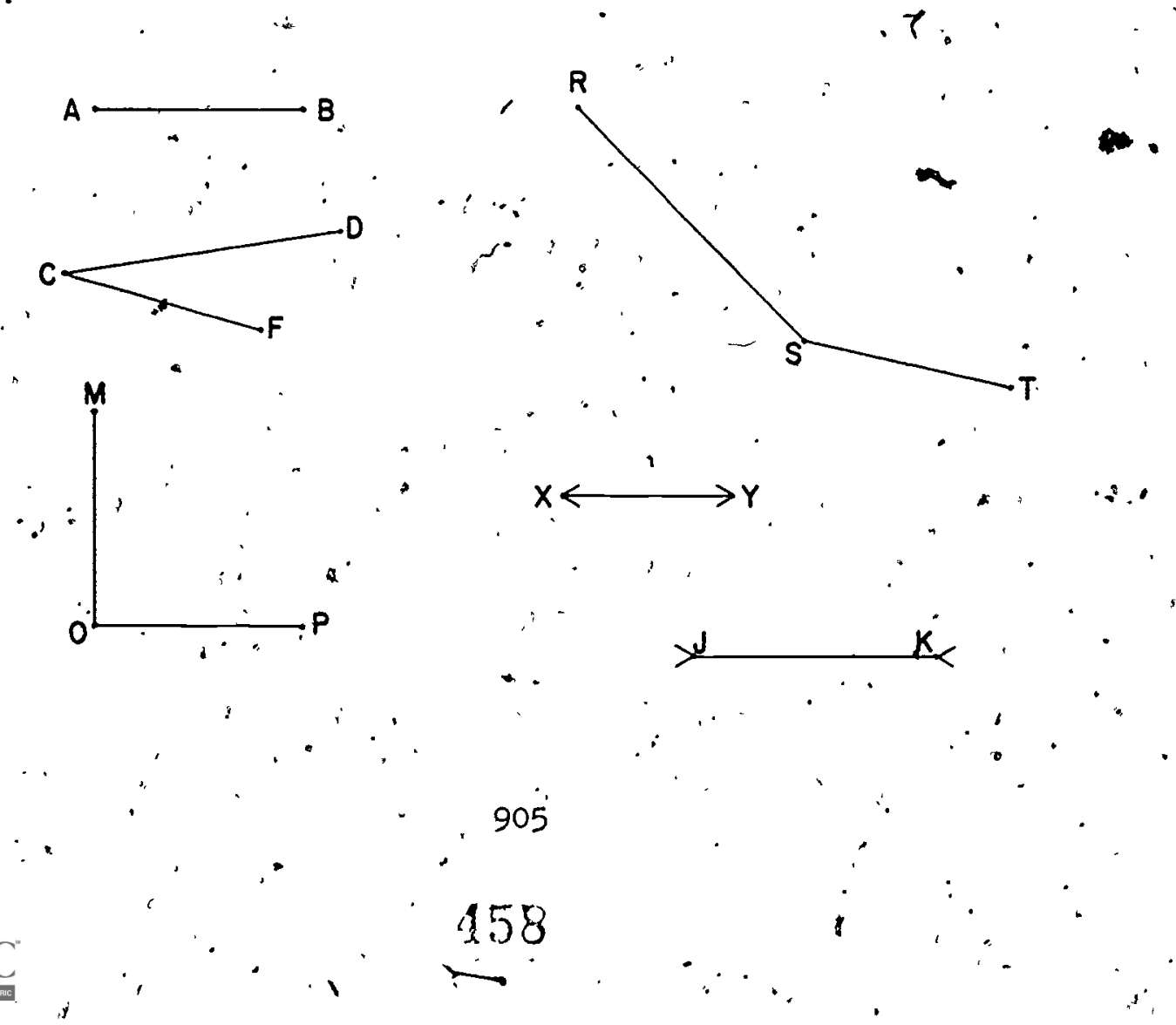


P566:

3. A triangle which has at least two sides congruent to each other is called an isosceles triangle. A tritangle which has all three sides congmient to each other is called an equilateral triangle. Answer these questions on your paper.

a) is an equild teral trianigle an isosceles triangle?(yea) b) Are all of the trfandes drawn below isosceles
triangleș? (yes)
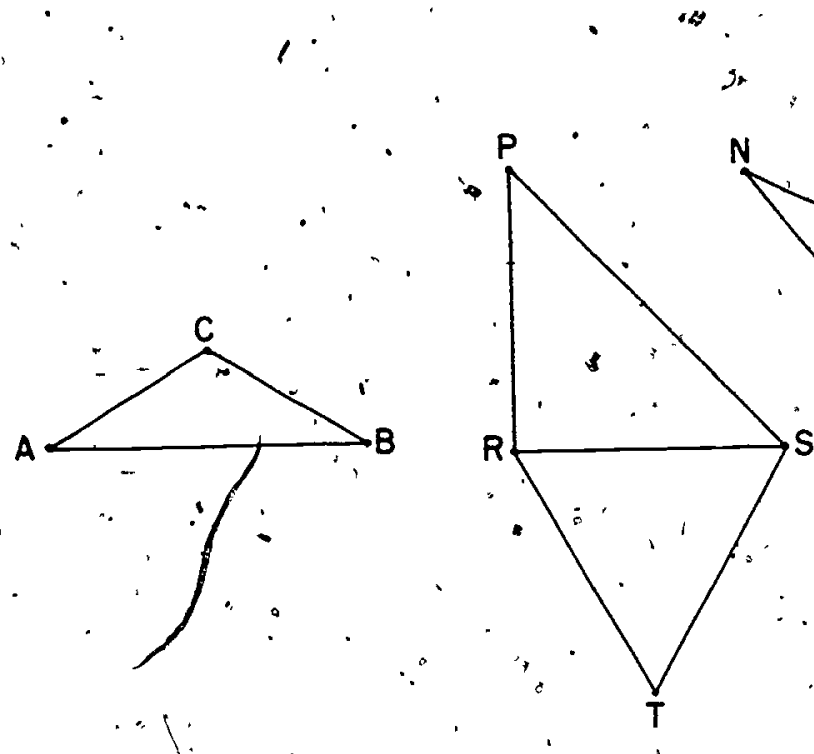

c) Write the names of the ones that are-equilateral triangles. ( $\triangle R S T)$

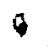

a) inite the pnames of 'the ones that are not equilateral Eriangles. ( $\triangle A B C$, $\triangle R S P^{\prime}, \triangle L M N$ ).

906 
P567

4. Make. a model of a right angle, by folding a sheet of paper.

How use your model to find.which of the angles below are right angles.

Which angles are less than right angle

- Which angles are greater than right angles?

LIst the angles on your paper: Put the name of each angle under the proper heading:

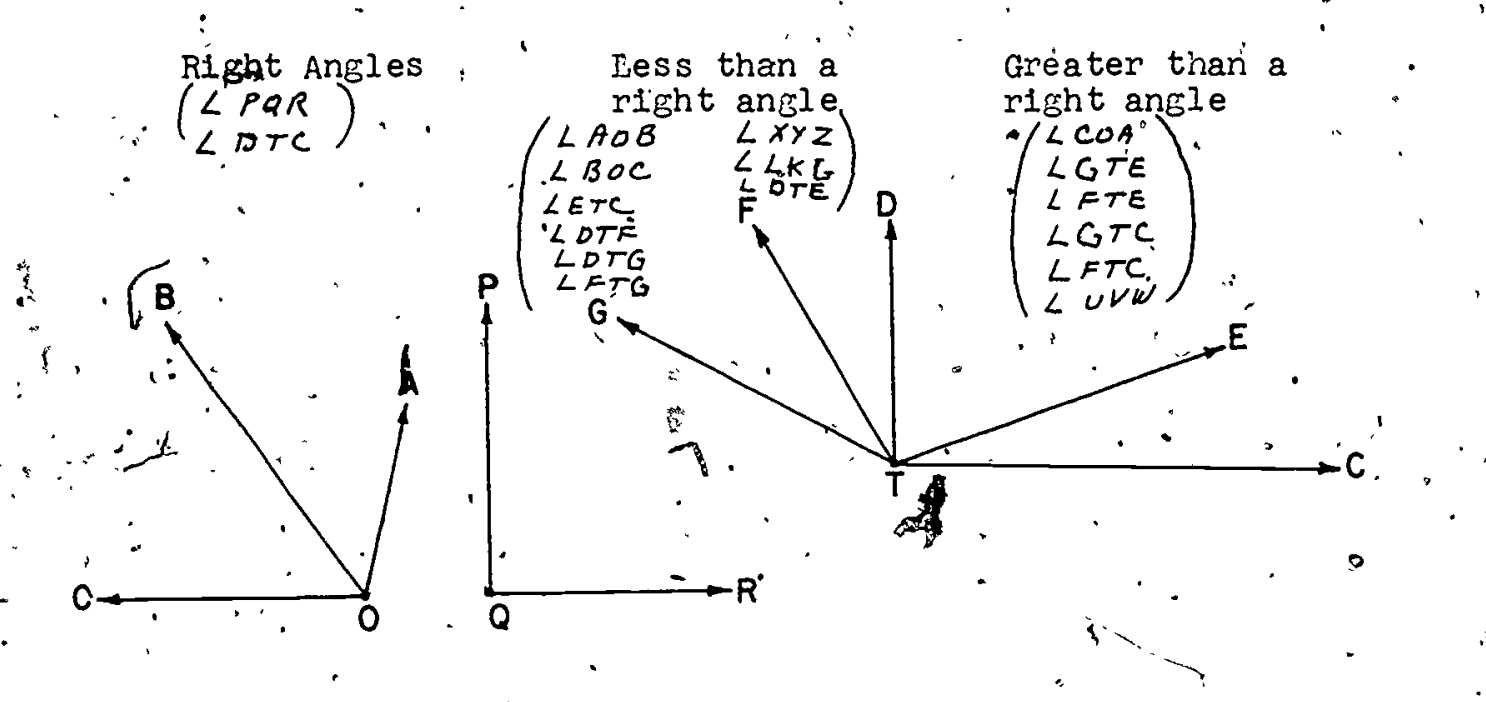




\section{LINEAR MEASURERENT}

In exercises $1,2,3,4$ write the letter for each part". on yoyp paper. Then beste 1t.write what you would write to rilf the blanks.

1. A family drinks 5 quarts of milk each day.

a) the unit of measure, is

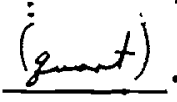

b) The measure is.

c) The amount of milk is ( 5 gintont)

2. in automobile welghs 2860 pounds.

a). The init of measure is (pound)

$\because$ b) The measure is (2860)

c). The automobile!s.weight is (2860 pand).

The tercher's.desk is 42 inches long.

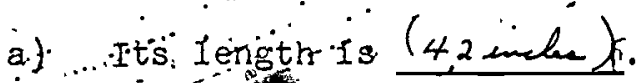

b). Its measurire is

c).: The unit of measure is si-

Aisatifife's distançe from the earth was 450 miles. a) The distanoe from the earth is (450mile)!

b) The tmasure-is (mile)

c) The measure of the distance is
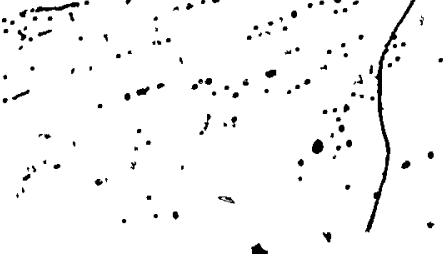

$$
\begin{aligned}
& 908 \%: " \\
& 461=
\end{aligned}
$$


5. The unit to be u'sed in this exercise is shown. Roints. are named on the ray. Use your compass to find the measure of the segments. On your paper write the measure of the segment beside the letter "for each part. .

Unit . .

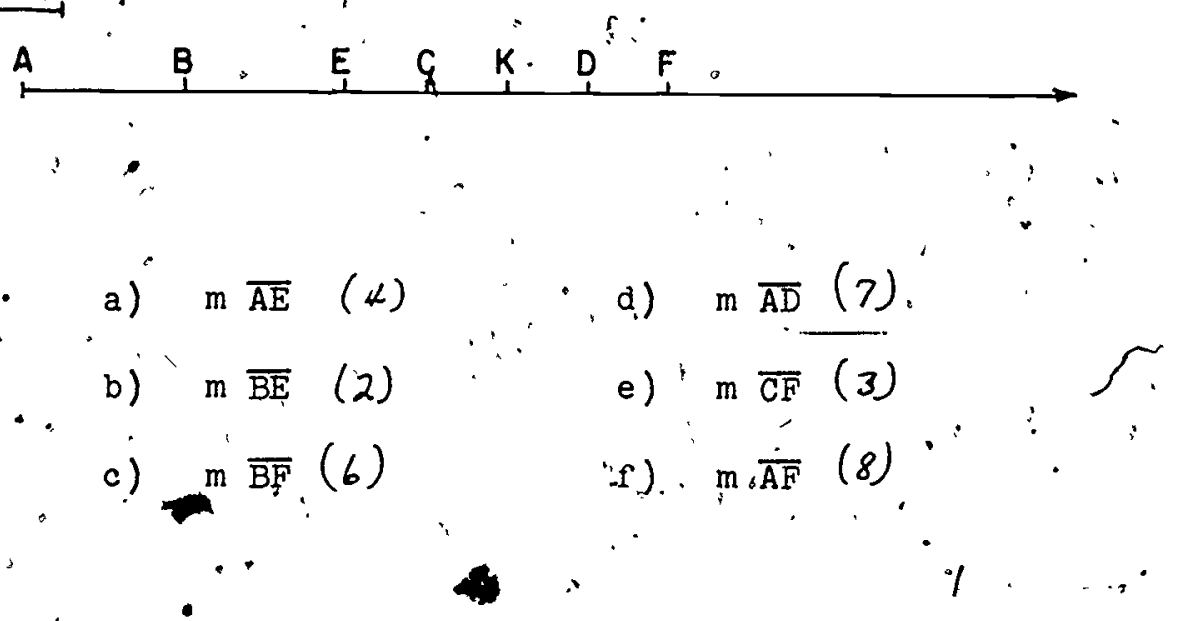

6. Using the unit and the segments in 5 write on your paper what you would write to fill the blank in each part below. May you choose more than one answerfor

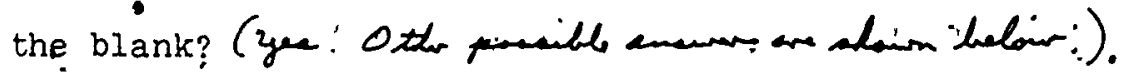

$$
\begin{aligned}
& \text { - a) } \mathrm{m} \underline{\overline{B F}}=6 . \quad \overline{A K} \text {. } \\
& \text { 々). } \mathrm{m} \overline{\overline{B E}}=2 . \quad \overline{A B}, \overline{E K}, \overline{C D}, \overline{K F} \\
& \text { c) } \mathrm{m}, \overline{C F}=3 . \overline{B C}, \overline{E D} \text {. } \\
& \text { d) } \mathrm{m} \overline{\overline{D F}}=1 . \quad \overline{E C}, \overline{C K}, \overline{K D}
\end{aligned}
$$

1909 
P570

7. On your paper make the table like the one bel fow these line segments. Then use your ruler to help you pill in the number which belongs in each blank.

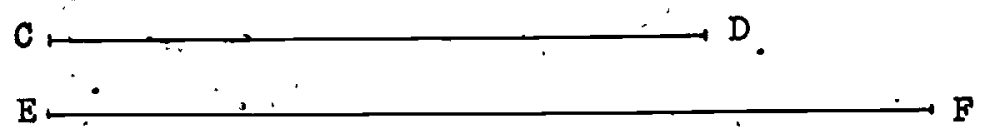

$G$

To the nearest inç

To the nearest To the nearest half-inch fourth-inch
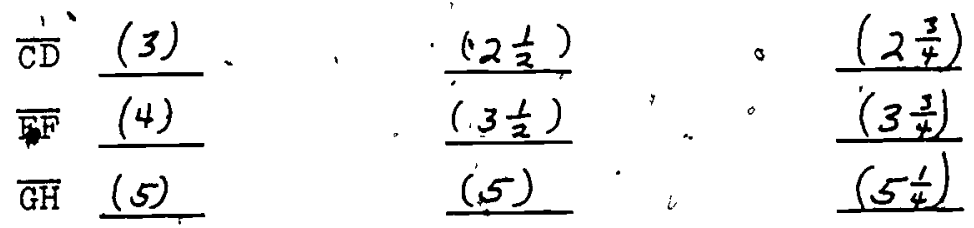

8. On your paper write the letter for each part of this exercise." Then beside it'write what you would write to filil in the blanks for each part'.

If segment have these lengths, which one is longer? How much

Which is How much Longer . Longer

a) 23 inches or $\cdot 1$ foot?

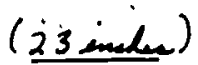

(ilinahes)

b) 2 feet or 11 yard?

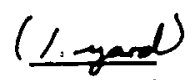

c) .l ft. Elin. or 2 . ft.?

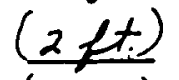

d) 1 yd. 2 .ft. or $6 \mathrm{ft.?}$

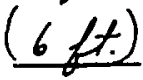

- e) 1 imile or 5300 ft.?

(5300/t.)

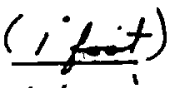

f) 16 in. or 2 ft. 2 in.?.(2ft.2i.)

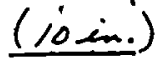

8.) 130 in. or ll ft. 2 in.?
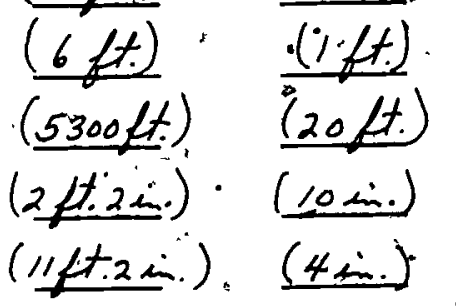

(10 in.

$(4 \dot{i})$ 
P571

9. Add these measures. Write the answers on your paper beside the letter which names each part. Write each answer 2 ways.

a) 6 yd: $2 \mathrm{ft.}$. b) $12 \mathrm{ft}: 11 \mathrm{in.}$ " 5 yd. 1 rt. $16 \mathrm{ft.} 5 \mathrm{in.}$

$\frac{2 y \dot{d} \cdot 2 r t}{\left.13 y^{2} \cdot 5 f^{t}\right)}$

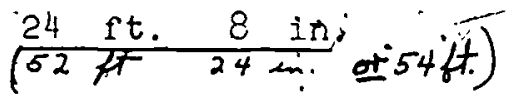
( 14 igd a ft $\%) 2$ yd. 2 ft. 3 in.

$$
\begin{aligned}
& 6 \text { yd. } .1 \text { ft. } 10 \text { in. }
\end{aligned}
$$

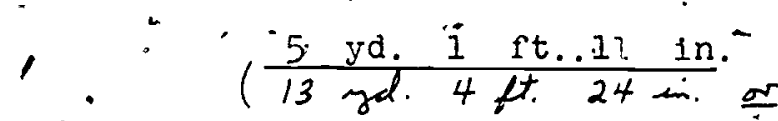

10. Subtract these measures: Write the answers on your paper. beside theletter which names each part.
.a) 5 yd. $2, \mathrm{it}$.
b) $6 \mathrm{yd} .2 \mathrm{ft} .11$ in.
$\therefore\left(\frac{3 \cdot \text { yd. } 2 \text { rt. }}{8 \text { yd. } 3 \text { ft } 9 \text { od. })}\right.$

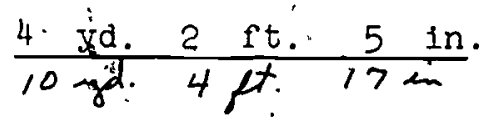

c) 7 ft. 8 . in.

X.

$$
4 \text { ft. } 10 \text { in. }
$$

1i. Find the perimeter of each polygon. On your paper write.

the answer beside the letter which names each polygon.
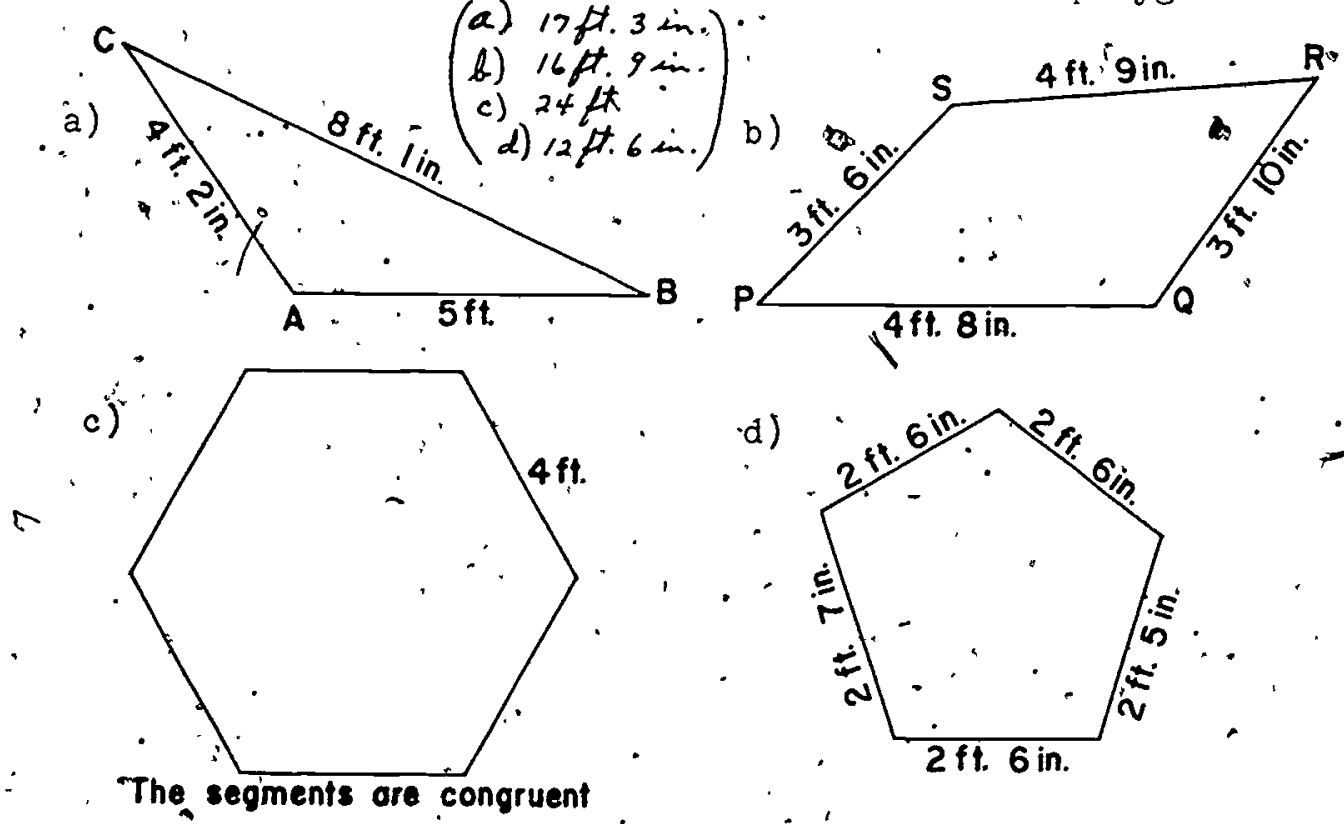

d)

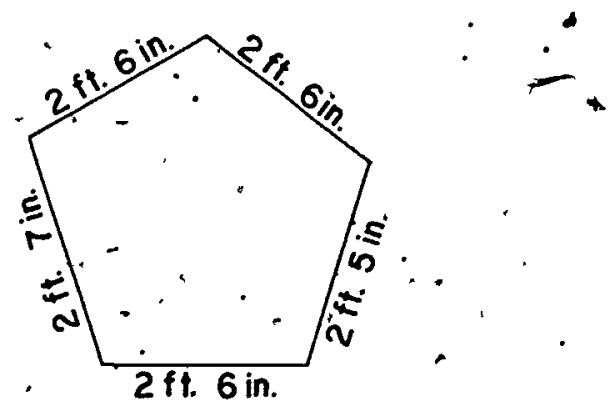


P572

EXTENDING SYSTEMS OF NUMERATION

1. 'On your paper write the word names for' the' following numbers.. Letter the parts of this exercise as they are lettered here.

a) 2,536 (tum themed. fin hind red thity-sit)

b) 45,269 (fort y-fine thanand tors hunched eisty-mine)

c) 40,204 (forty thane two hundred four).

-d.) "60,066 (sipty'thomand sisty-six)

e) 66,066 (sitty-sif thaneand sisty-sic).

f) 66,000 (sisty-sit thomend)

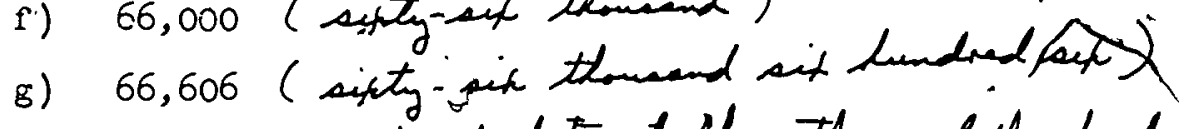

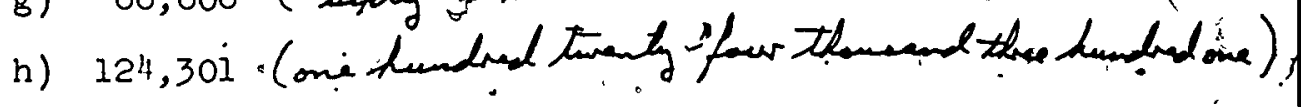

2. 'On your paper write the numerals for the following numbers. Letter the parts of this exercise as they are lettered here,

a) Two thousand five hundred twenty. $(2,520)$

b) 'Three thousand three hundred thirty. $(3,330)^{\circ}$

c) Fifty-five thousand five hundred fifty-fives $(55,(555)$

d) Nine thousand seventy-six. $(9,0,76)$

e) One thousand seven hundred seventy -six. $(\%, 776)$.

r) Twenty thousand two hundred to $(\$ 0,202)$

8)' One thousand two. $(1,002 \%$

h) Eleven thousand one hundred eleven. (11, 1:

912

465 
$4+4$

: 573

\section{FACTORS AND PRIMES}

1. Ori your paper write the letter for each part of this exercise $d$ Then beside it complete each statement, Complete the statement so that each number is a product of 3 ractors. Part a) is done for you.

a) $24=2 \times 3 \times 4$

-b) $18=(2 \times 3 \times 3)$,

c). $36=(4 \times 3 \times 3$ or $6 \times 2 \times 3$ or $2 \times 2 \times 9)$.

d) $-12=(2 \times 2 \times 3)$

e) ${ }^{n}-8=(2 \times 2 \times 2)$

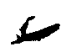

W

2:. For each set of 3 numbers write on your. paper smallest number which has each of trind, 3 numbers"as a factor. Letter each part as lettered here.
a) $2,5, .7$ (70)
d) $4,6,8 \cdot(24)$
$\therefore$
b) $2,3,4(12)$
e) $2,4,8,(8)$
c) $5,7,1$ (35)
f) $3,6,9^{\circ}(18)$

1) $\because, 2,:$

3. Some of the following numbers are prime numbers. Write
the prime numbers on your paper. $\therefore>$
a) 27
e) 310
b) 31 (prime)
f) : 143
c) 55
8) 37 (preine)
d). 53 (prine)
h) 101 (preinit)
,

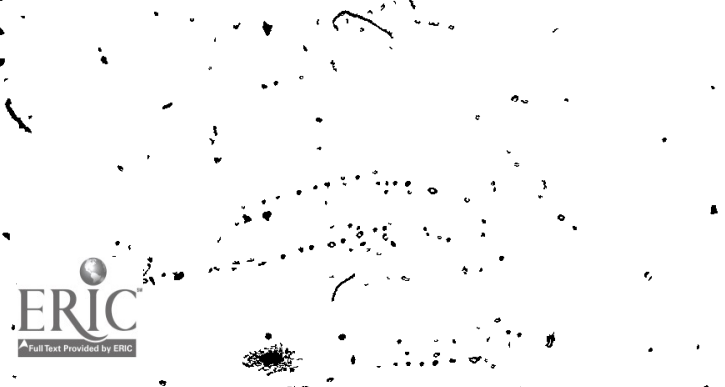

913

$466^{\circ}$ 
4. Find two different prime factors of each of these numbers. Write the prime factors on your paper. Letter the parts as they aroptiered there.
a) $785(5,157)$
d) 6,060 (2, 3,5$)$
b) $3,042(2,3)$.
e) $4,314,(2,3)$

c) $5,055 \quad(5,3)$

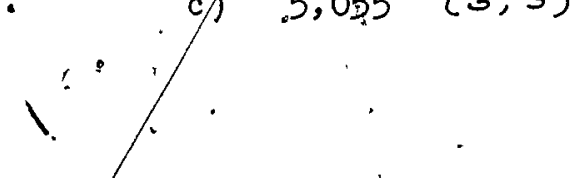

5. On your paper write the get of all factors of each numger: Letter the paints as they are lettered here.
a.).
$96(1,2,3,4,6,8,12,16,24,32,48,96)$
b)
$225(\%, 3,5,9,15,25,45,25,225)$.
c)
$363^{\prime}(1,3,11,3.3,121,363)$
a)
189
$(1,3 ; 7,9,21,27,63,189)$ 6. Find the greatest common factor or the following palrs or
numbers. Use same letters for your answers that are usedihere.
a) 90,84
(6)
b) $90,50^{\circ}(r 0)$
a.) $72,60 \quad(12)$

$\Delta$
d) $48 ; 30$
(6)
e) 12 
7. Find the lowest contmon miltiple of the numbers in each set. Write the lowest common multiple on your paper. Letter the parts as lettered here.
a) $6,8,12 \cdot(2.4)$
b) $6, \$ 5$
c) $3,5,9,15 \quad(45)$
d) $4,8,12,(24), 3$
e) $3,6,5, \dot{9}(90) ;$
f) $3 ; 4,5,10,12(60)$

8. We say that a number is "factored completely" if It is the product of numbers which are all prime numbers. Facto completely each of the following numbers and write them on your paper as in, a) which is done for you.

,

a) $63=3^{\prime} \times 3 \times 7$

b) $126=(2 \times 3 \times 3 \times 7)$

c) $49^{\prime}=(7 \times 7)$

d) $98=3(2 \times 7 \times 7)$

e) $35=(5 \times 7)$

f) $-105:(3 \times 5 \times 7)$ :

g) $45=(3 \times 3 \times 5)$

h) $135=(3 \times 3 \times 3 \times 5) /$

1) $1001=(7 \times 11 \times 13)$. 
EXTENDING MUTIPLICATION AND DIVISION

1. Fnd the product for each produet expression..
a) $3 \times 46=n \quad(138)$
f) $.12 \times 34=n(408)$.
b) $7 \times 83=\mathrm{n} \quad(58: 1)$
g). $23 \times 67 .=\mathrm{n}(1541)$
c) $5 \times 125=n$
(6.5)
id) $6 \times 321=n(1926)$
h), $52 \times 48^{\circ}=\mathrm{n} \quad(2496)$.
e). $\because \times 1269=3(5076)$.
1) $76 \times 94=n(7144)$
3) $38 \times 83=n \cdot(3 / 54)$. in.

2. Find the rumber represented by $n$. to make each sentence trué.
a) $7 \times n=3: 02$
$(4.8 .6)$
f) $58 \times 131$ I $(7598)$
b) $\mathbf{x} \dot{x} 21 \cdot=.966$
$(46)$
c) $n x+18=\$ 26$
$(27)^{\circ}$
d) $24 \times n=1335^{\circ}(64)$
ह) $\because \mathrm{n}, \times 81 "=8667$ (107)
e) $43 \times 267=n(1,1,481)$
h) $\ddot{14} \times 463 \doteq n(6482)$.
if $37 \times 1249=\mathrm{n}(46,213)$
j.) $n \times 125=9250 \cdot(7,4)$

3. Find the numbers represented by $\frac{n}{*}$ and $r$. for each of foliowing so that they are true mathematical senterices.

a) $1487=(n \cdot x \cdot 43)+r \cdot[(11 \times 43)+1.4]$

b) $396=(n \times 63)+r[(6 \times 61)+30]$

c) $1292=(34 \times n)+r[(34 \times 38)+0] \therefore$

d) $3415=(53 \times n)+r \cdot[(53 \times 64)+23]$.

e). $8645=(\mathrm{n} \times 65)+\dot{r}\left[\left(433 \times 45^{4}\right)+0.\right]$

f) $9772=(n \times .73)+r \quad[(733 \times 73)+63]$

5) $12,443=(\mathrm{n} \times 120)+\mathrm{r}[(103 \times 120)+83]$.

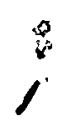

h) $24,8 i 1=(151 \times n)+r[(15 i \times i 64)+47]$

In which of these does $n$ represent a racton of the number given? ( $c$ and $c_{i}$ )

\section{6} 469 
11. The width of a playground 1s, 55 yards. Its length is. 120 yards. Find the area of the plajground. $\left(\right.$ Crea $_{\text {rea }}=55 \times 120$ : Ihe area of theplayground is 6600 sizuar yardo.?

12. Tim sold 45 papers each day. How many papers a1d he

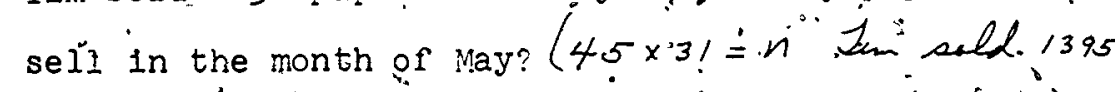
in the month of ningi).

13. Three giris divided 47 pictures equäliy among them." How many pictures did each 'girl get? (Hhy cannot be dended

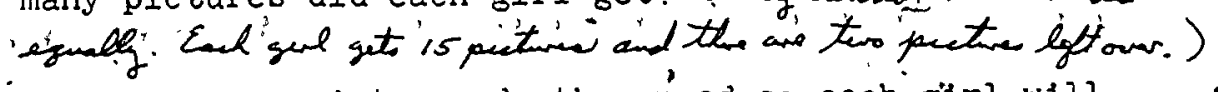
How many more pictures do they need so each girl will. have 25 pictures? $(4+n=75$ they will meed 28 mini pactures.)

\section{$\theta$}

14. There were 79. cookies on a tray. How many dozen

. - cookies were there on the tray? $(79 \div 12=n$ thereme 6 dozen and 7 conkies left oner.).

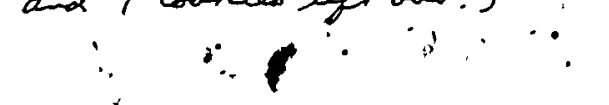

$\therefore$ 15.. On another tray there were 5 . times, as many cookles ias on the tray in Problem 14. How many coakies are on that" tray? How mainy dozen cookies are on that tray?

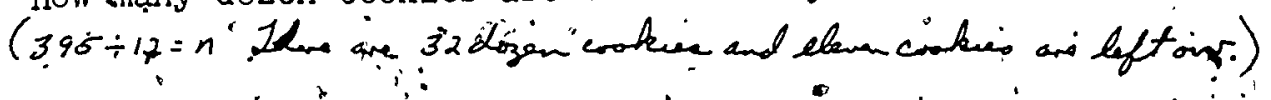

i6. How many dozen cookiestare on both trays?

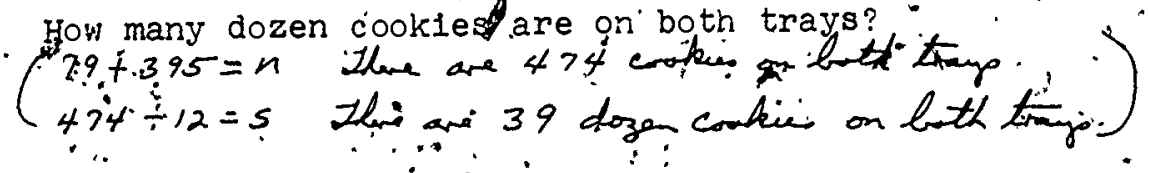
918 . 
580

1

3. Use your compasș and straightedge to draw a trianglé using the given segmen's.
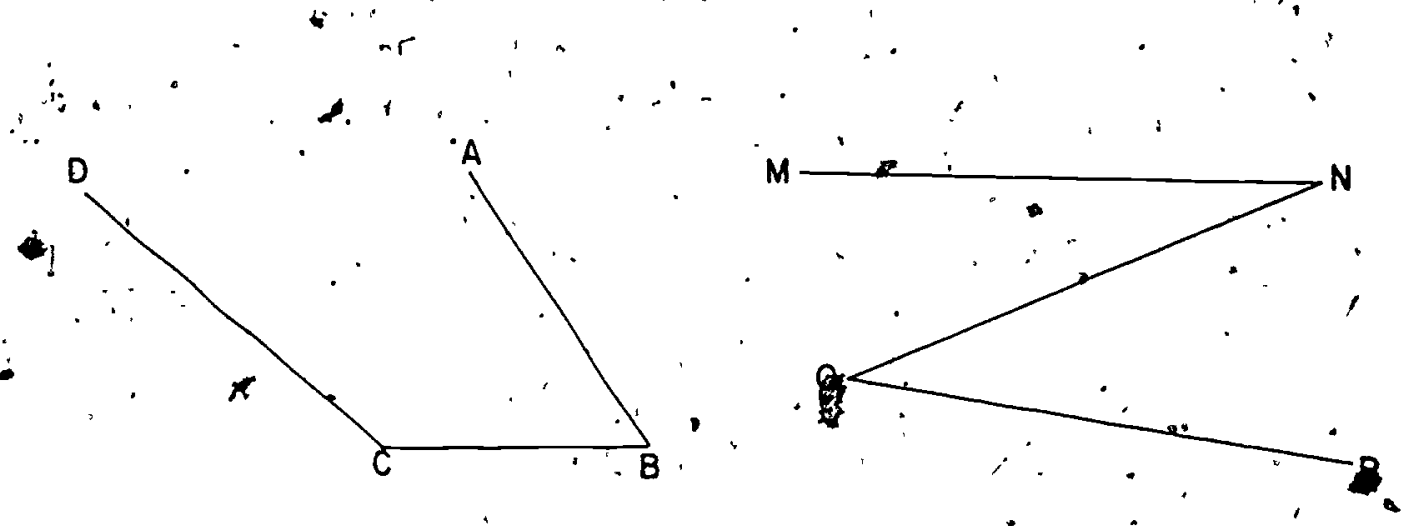

$\therefore \quad$ Can you'construct a triangle, using these three line

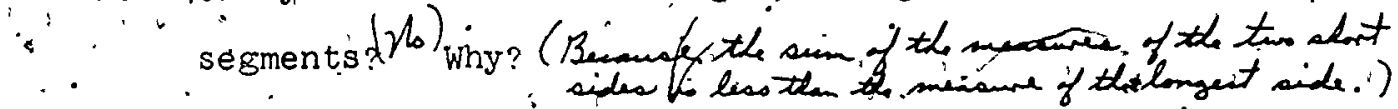

5. What would be true about the triangles constmucted from

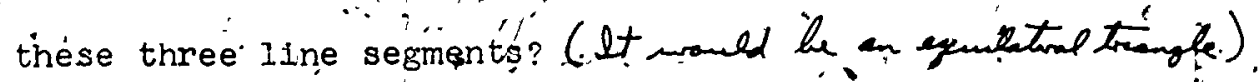
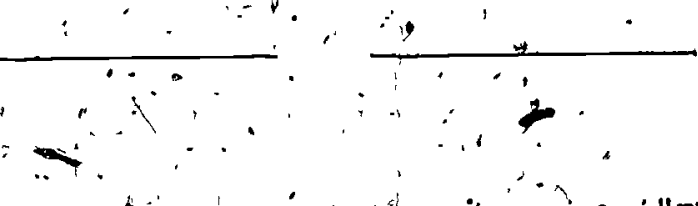

What dós this's tatêment meàn? "Three sides detếrmine a triangle." 


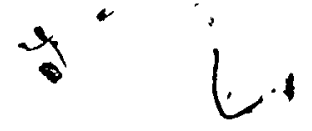

6. Use your compass and straightedge to copy the check mark made by Bill's-teacher.
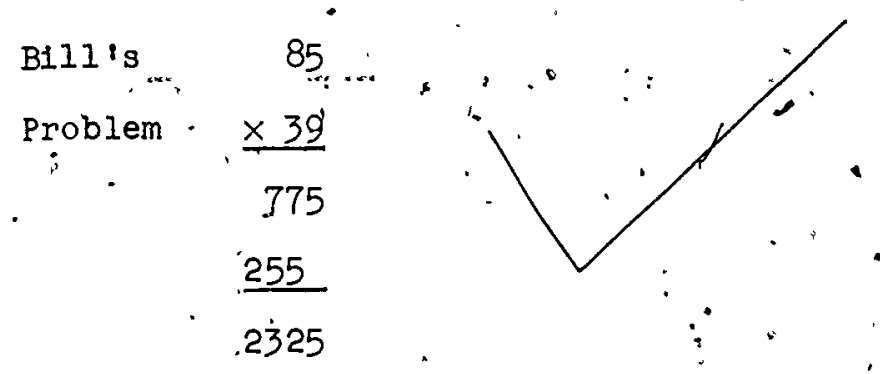

Does the check mark' mean that Bijl's prdbiem has the right answer or the wrong ariswer? (It chack mark meanis Bills problem . $\{$. has the trong a answer.)

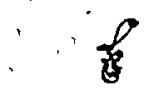

$\mathrm{N}_{7}+7$
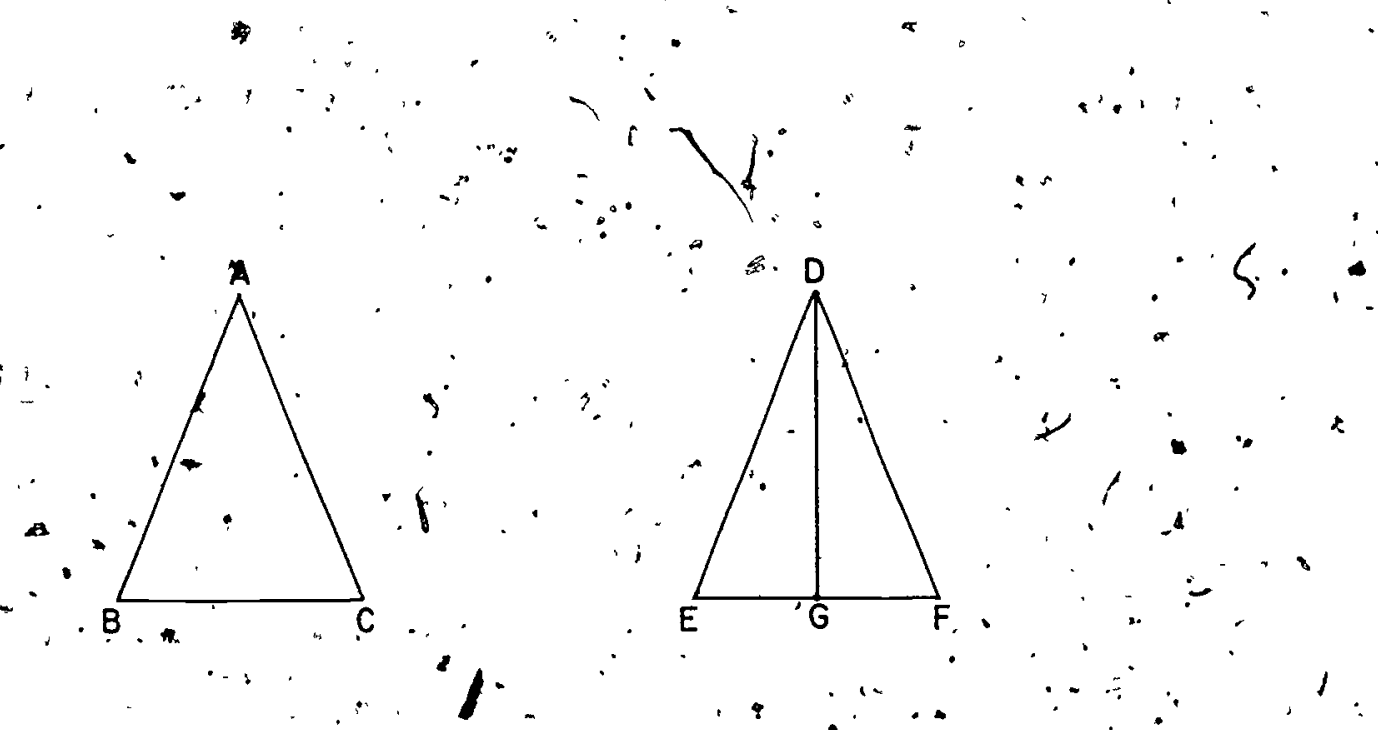

Write, in words, these mathematical sentences.
a) $\triangle A B C Q \cong \triangle D E F "$ "Land
$\overline{\mathrm{AB}}>-\overline{\mathrm{BC}}$. 'Arangli $A B C:$

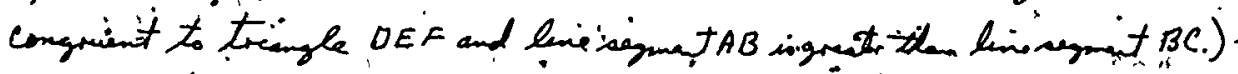

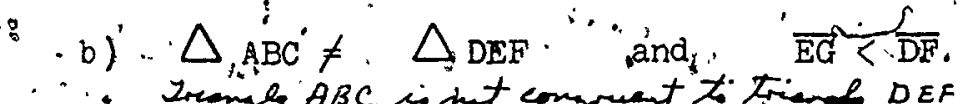
Ireange $A B C$ is hat compruant to trieng $D E F$ is have than hini siegnent of.

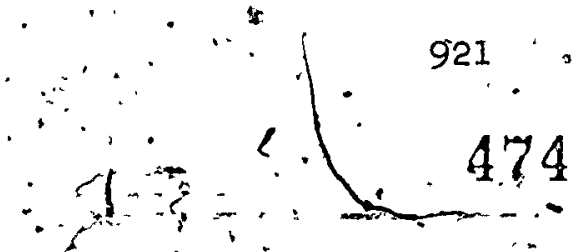


ADDITION AND SUBTRACTION OF RATIONAL NUMBERS

i. "op your paper write the letter for each part of this exerefoe Then beside it write the one of,$>, \quad=,<$ that yourouid use to.fili the blank so Each of the following would ta true mathematical sentence.

a) $\because \frac{1}{2} \div \frac{1}{3}$

e) $\frac{3}{6} \geq \frac{2}{5}$

b) $\leq \frac{1}{4}: \leq \frac{1}{3}$

f) $\frac{0}{\pi} \equiv 0$

c) $\frac{2}{3} \leq \frac{3}{4}$

gi) $\frac{3}{6} \neq \frac{i 7}{34}$

a) $\frac{4}{16}=\frac{1}{4}$

a.) $\frac{7}{4} \leq \frac{6}{3}$

ni $\frac{i}{5} \geq \frac{12}{70}$

$85<\frac{11}{18}$

$\int$

$\stackrel{\circ}{\circ}$

$\cdot A$

$+2$

k) $\frac{3}{7} \leq \frac{3}{6}$

2. On your paper wry te the letter for och part of this exercise.

- Then beside it wite, the lowest conto denominate for the " rational' numbers in the set.

a) $\therefore \frac{3}{4}, \because \frac{5}{6} \cdot(60)$

$c 10 \frac{7}{10}=0$

b) $\therefore \frac{5}{8} ; \frac{3}{4}, \therefore$

$(2: 4)$

(1) $\frac{5}{12},-\frac{3}{16} ;$

$(48)$

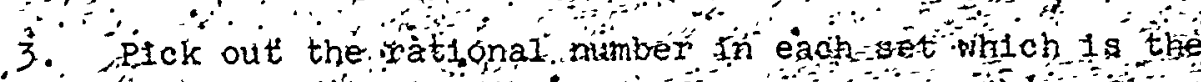

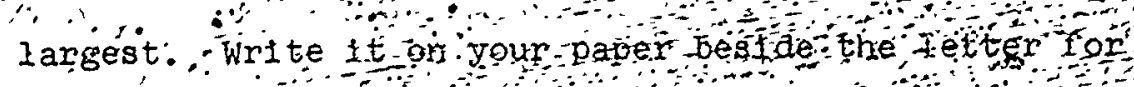
that se set io

$\therefore$ a)

e)

(1)

ax

920 
4. On your paper write the letter for each part of this exercise. Then beside it write the number you would use for In to make the mathemalcal sentence trie."
a) $n=\frac{5}{8}+\frac{7}{8} \quad\left(n=\frac{12}{8}\right)$
d) $\frac{3}{5}+\frac{1}{5}=n-\left(n=\frac{4}{5}\right)$
b). $\frac{2}{3}+\frac{21}{12}=\mathrm{n}:\left(n=\frac{19}{12}\right)$
e) $n=\frac{3}{5}+\frac{5}{5} \quad\left(n=\frac{43}{30}\right)$
c) $\frac{3}{4}+\frac{27}{20}=n \quad\left(n=\frac{42}{20}\right)$
f) $\frac{1}{2}+\frac{2}{3}=n \cdot\left(n=\frac{\sqrt{3}}{6}\right)$

$55^{-}:$on your paper write the letter for each part of this exercise. Then beside it wirite the number you would use for $x$ to make the mathematical. sentence true.
a) $\quad x=\frac{7}{8}-\frac{1}{2} \quad\left(x=\frac{3}{8}\right)$
d): $\frac{3}{4} \div \frac{1}{6}=x \quad\left(x=\frac{7}{12}\right)$
b) $\therefore \frac{11}{12} \div \frac{5}{6}=x \quad\left(x=\frac{1}{12}\right)$
e). $\frac{7}{8}-\frac{9}{16}=x-\frac{\left(x:=\frac{5}{16}\right)}{x}$
c) $\frac{2}{2}+\frac{2}{5}=x \quad\left(x=\frac{9}{10}\right)$
f) $\dot{x}=\frac{2}{5}-\frac{1}{4}:\left(x=\frac{3}{20}\right)$.

6. On your paper wnite the leter for each part of this exercise. Then beside it write the number you would use. for $p$ :
a) $\left.-\frac{1}{3}+\frac{1}{2}+\frac{1}{4}=(p \therefore)=\frac{13}{12}\right)$.
c) $\left.\frac{5}{8}+\frac{2}{3}+\frac{1}{2}=p(\rho)=\frac{1-3}{24}\right)$
$\therefore$ b) $\frac{3}{5}+\frac{3}{4}+\frac{1}{2}=p \quad\left(p=\frac{37}{2 q}\right)$
a) $\frac{3}{8}+\frac{1}{4}+\frac{5}{6}=p\left(p=\frac{35}{24}\right)$ 
P584.

7. Copy each part of this exercise on your paper. Then fill each blank with + or , - so that 'a), b), "c), and d) will be true mathematical sentences:

a) $\frac{4}{3} \frac{(t)}{4} \frac{2}{3}(-) \frac{5}{3}=\frac{1}{3}$

c) $\frac{9}{8}(t)=\frac{7}{8}(-x) \frac{12}{8}=\frac{4}{8}$

b) $\frac{12}{7}(+) \frac{3}{7} \stackrel{(+)}{7} \frac{8}{7}=\frac{23}{7}$

d) $\frac{10}{3}(-) \frac{5}{4}(t) \frac{1}{6} \leqslant \frac{9}{4}$

8. On your paper write the letter for each pate of this exercise. "Then beside $f^{t}$."Write the "number for in" so that" the sentence will be trip.

a) $\left(4 \frac{1}{6}+3 \frac{2}{6}\right)+5 \frac{2}{6}=n \cdot\left(12 \frac{5}{6}\right)$

b) $\left(6 \frac{2}{8}+\frac{3}{8}\right)+n_{-}=10 \frac{7}{8} ;\left(4 \frac{2}{8}\right.$ ar $\left.4 \frac{1}{4}\right)$,

c) $\left(3 \frac{3}{4}+2 \frac{1}{2}\right)+6 \frac{1}{3}=n\left(19 \frac{19}{12} \cdot 12 \frac{7}{12}\right)$

d) $\left(8 \frac{2}{5}-2 \frac{7}{10} 5+3 \frac{1}{4}=\left(8 \frac{19}{20}\right)\right.$

9. On your paper write the letter for each part of this exercise. Then beside 1 t write the number for $n$ so that the sentence will be true.

a). $(8.97-4.31)+40(n=7.23)$

b). $3.24+3.56+4.16 \% 3 .(10.96=n)$

c) $7.88+5.31+6.54+(19.73=n)$ :

a) $-6+.3+n \stackrel{\circ}{=} 6.36$. $(p=0.06)$.

e) $8.34-4.83=n \quad(3 f f y=n)$

r) $n=9.34-5.89 \quad(n+34)$ 
MEASUREMENT' OF ANGLES

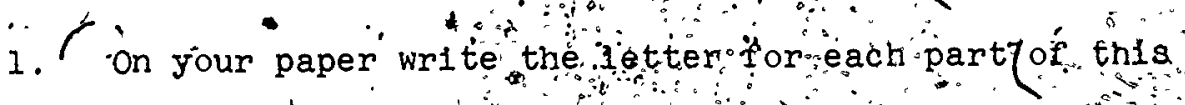
exercise. Beside the lotter writhe the wo true 10 the

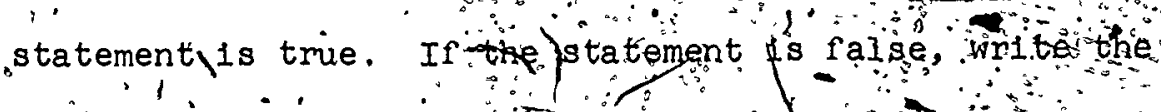
word faise.

a) A-measure of an angle is a number. (- (as)

b) The unit used for measuring angles is angle (t.

c) A measure is not ânumber. (falace)

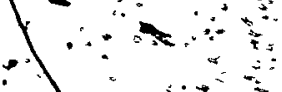

d) The measure 1 socurate, but is only approximately so. (thei) ? ?

e) The instrument tod for angle megsure is a protractor.

f) The sides of an fongle are tays (true) 8) The common endpaint of the two rays forming an ange
is the vertex. (the)

h) The measure of the angle depends upon the "lengths of the rays. (filles)

1) Every anğle has one ray dralw horizontaly

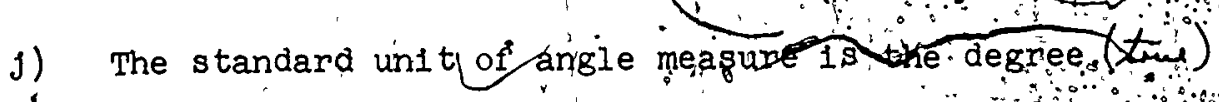

k) The measure in degrees of tach anglè of ân equiliatida triangle is, 60. (the)

1) Angles may be of the same measure but be different in positions. (tane). 


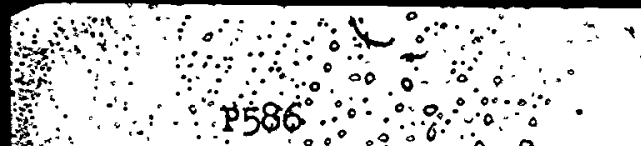

$\therefore$ In each fogure bêlow the measure in degrees of certain $\because$ angles are shown. on your paper wryte the letter that names each angle whose measure in apgrees 1 is-not shom. oftien beside the letter writie the meksure of the angle in
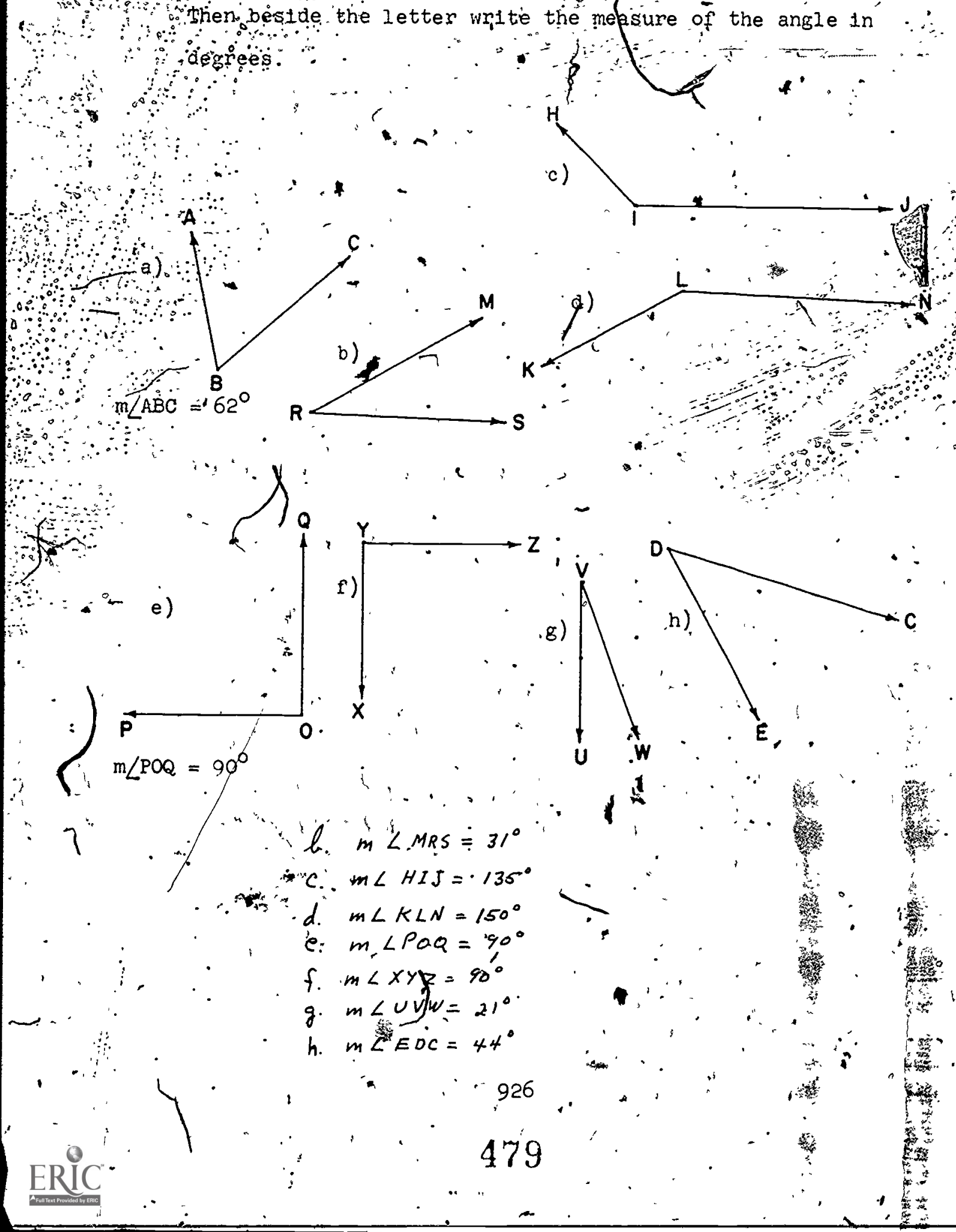
$\mathrm{P} 587$

AREA

1. on your paper write the letter for each part. or" this exercise. Then beside it write the word, on words; that". you would use to fill blanks.

a) A (ogre unit) of used as a un at for measuring plane regions:

b) A simple closed curve separates ap plane into (thee) sets of points.

The union of a simple closed curve and its interior. is called a (plane region).

d) To measuremarea of a region we need a uni of (meme).

e) One standard unit of area is a square region with l-inch sides; this unit is called the (square inch)"

f) An area of 1 square yard is the same as an "area of. (nice) square $\overrightarrow{f e e t}$.

$\therefore$ 8) 4 square, yards $=\frac{(36)}{(29 u a r e: ~ f e g t . ~}$

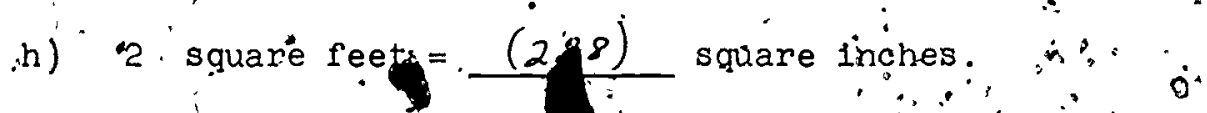

1) 7 square feet and 2 square inches $=(1032) 2^{\circ}$. square inches. j) 2000 square inches $=\frac{(y)}{\%}$ square yards and $\frac{(.704)}{.}$

927

480. 
The polygons show below are.either rectangles or triangles. The numbers are the measures. Find the measure in square unitstof the area of each rectangular and triangular region and write it on your paper beside the name of the rectargle: or triangle.

a)
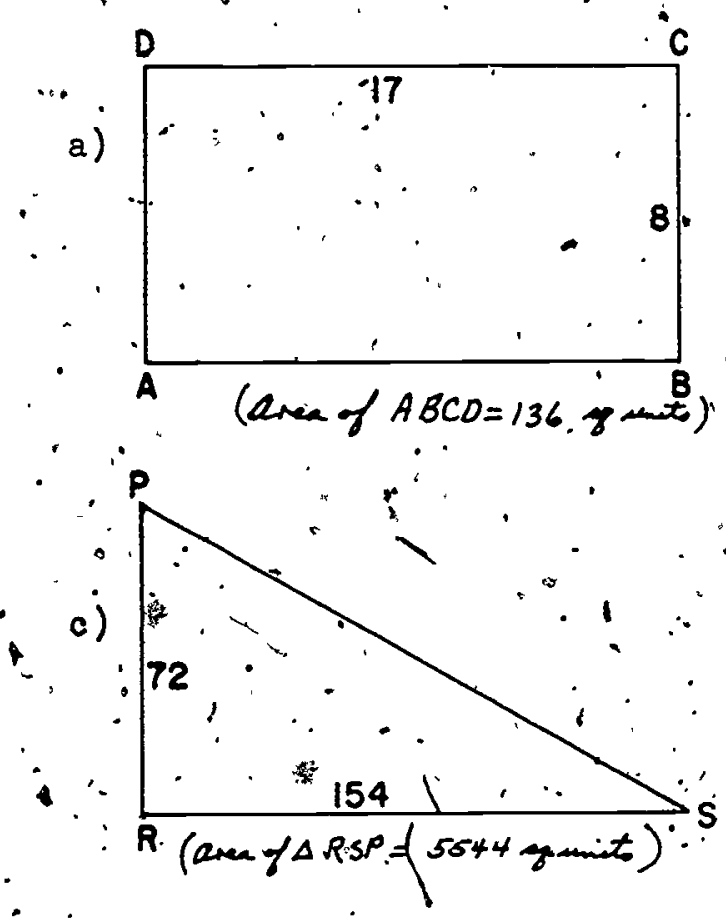

$c^{i}$

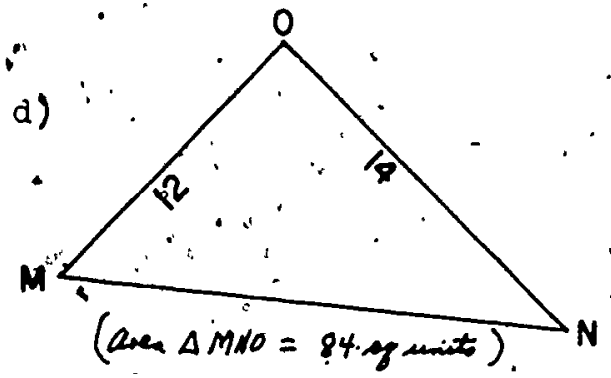

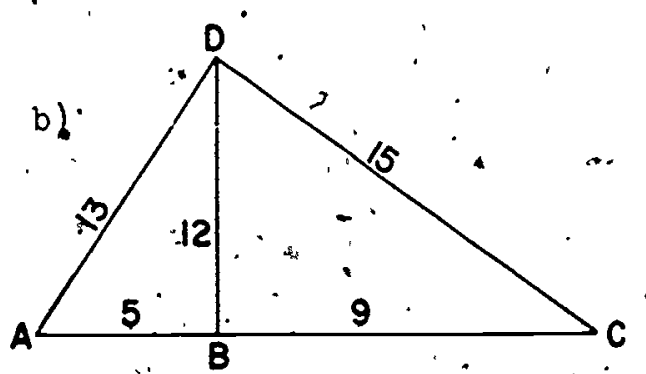

Area of $\triangle A B D=$ (30,pent)

Area of $\triangle \mathrm{CBD}=$ (54-2) Area of $\triangle A C D$ : $(94,2 x)$

$\checkmark$

e)

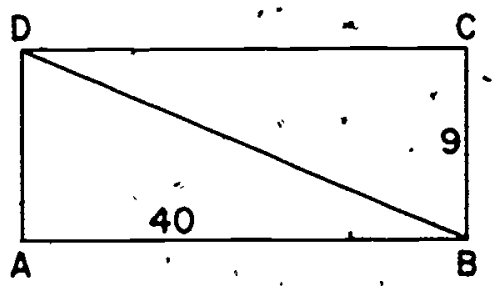

Area of $\triangle A B D=(1$ joequit $)$ - Area of $\triangle B C D=(180$ prentit) -Area of $A B C D=$ (360yent) $\because$ 
The preliminary edition of this volume yas prepared at a writing session held at Stanford University during the summer of 1960 , based in part, on an. outline prepared in Chicago in March of 1960; The first revision of the preliminary edition was prepared at Yale University' in the sumer of 196z." This revision was prepared at. Stanford University in the surmer of 1962 , taking into account the classroom experience with the first two editions during the academic years 1960-1962:

The following is : preparation of this volume.

- Truman A: Botts, Univerșity of Virginia.

Jameș A; Cooley, UniveAsity of Tennessee

Helen L."Curran, Glenview School, Oakland, California

Felen L. Garstens, . University of Maryland

E. Gzenadine Gibb, State College of Iowa

Geraldine Green, Vetal School, Detroit, Michigan

William T: Guy, Jr:, University of Texas

4* Leon Haxlend, Kenwod School, Minneapolis, Mingesota

Clarence Ethel Hardgrove, Northern Illinois University

Royce S. Hargrove, Corpus Christi Public Schools, Texas

Max Hosien, State Col, lege of. Iowa

Henry G. Jacob, University of. Massachusetts

- Lenore John, University High School:, University of Chicago

George E. Knoblock, El Carmelo School, Palo Alto, Californfa.

Willtam G. Iister, State University of New York.

Lazia M. Maneely, Springèr School, Los Altos, California

John' "Markș, Sen Jose State Colizege.

Marthọ Meek, Glorietta Elementary School, Orinda, California

Mary MeDermott, Mt. Diablo' Unified School Distríft, Calif̣ornis

William K. McNabb, St. Mark's School, Dallas, Texas -

- Frances J. Mettler, Walter Hays Elementary School, . Palo Alto, California

Leon Rutiand, 'University of Colorado

Inene Sauble, Detrolt Public Schools

Hellen Schneider, LaGrange Public Schools, Illinois:

Witla J. Sessions, Hillsborough County. Piblic Schọls, Florida

- Rose Mary Shea, 'Edith C. Baker Śchool, Bróokline, Massachusetts'

"Wesley 'Thompson o Detroit Public Schapls

$\because$ Morgan Kard, California Institute of Technology

Ted Wassam, Ventura School, Pajo Alto, Cailfornia

J. Pred Weaver, Boston University 